\title{
TEMPO-Enabled Electrochemical Enantioselective Oxidative Coupling of Secondary Acyclic Amines with Ketones
}

Zhen-Hua Wang, ${ }^{\dagger}$ Pei-Sen Gao, ${ }^{\dagger}$ Xiu Wang,${ }^{\dagger}$ Jun-Qing Gao, ${ }^{\dagger}$ Xue-Tao Xu,${ }^{\dagger}$ Zeng He,${ }^{\dagger}$ Cong Ma, ${ }^{\dagger}$ and Tian-Sheng Mei ${ }^{*}, \dagger$

${ }^{\dagger}$ Key Laboratory of Organometallic Chemistry, Center for Excellence in Molecular Synthesis, Shanghai Institute of Organic Chemistry, University of Chinese Academy of Sciences, Chinese Academy of Science, 345 Lingling Road, Shanghai 200032, People's Republic of China

¥School of Biotechnology and Health Sciences, Wuyi University, Jiangmen, Guangdong 529020, People’s Republic of China 


\section{Table of Contents}

1. General Considerations

2. Preparation of Glycine Esters

3. Cyclic Voltammetry Studies

S15-S17

4. Optimization Details

S18-S25

5. Unsuccessful Substrates

S26-S27

6. Synthetic Procedures and Characterization Data

S28-S50

7. Large-Scale Synthesis and Mechanistic Studies

S51-S55

8. Crystallographic Data of Compound 4m

S56-S68

9. References

S69

10. Copies of NMR Spectra

S70-S146

11. Copies of HPLC Spectra

S147-S228 


\section{General Considerations}

All the electrochemical reactions were performed in an undivided cell equipped with two Pt electrodes $\left(1.0 \times 1.0 \mathrm{~cm}^{2}\right)$ unless otherwise noted. Solvents such as THF, MeCN, DMF, and DMSO were purchased from Sigma-Aldrich, or Innochem. Reagents were purchased from commercial sources and were used as received unless mentioned otherwise. Column chromatography was performed using 300-400 Mesh silica gel. Visualization of spots on TLC plate was accomplished with UV light (254 nm). All commercial reagents were purchased from Aladdin, Macklin, TCI, Sigma-Aldrich, Adamas-beta, 9-Ding chemistry and Energy Chemical of the highest purity grade. They were used without further purification unless specified. ${ }^{1} \mathrm{H}$ NMR and ${ }^{13} \mathrm{C}\left\{{ }^{1} \mathrm{H}\right\} \mathrm{NMR}$ spectra were recorded on Agilent AV 400, Varian Inova 400, or Bruker AV 500. ${ }^{19} \mathrm{~F}$ NMR spectra were recorded on Agilent AV 400, Varian Inova 400 (376 MHz) instrument and are reported relative to the $\mathrm{CFCl}_{3}$ as the internal standard. The peaks were internally referenced to TMS $(0.00 \mathrm{ppm})$ or residual undeuterated solvent signal. All ${ }^{1} \mathrm{H}$ NMR and ${ }^{13} \mathrm{C}\left\{{ }^{1} \mathrm{H}\right\}$ NMR chemical shifts were reported in ppm relative to 7.26 and 77.16, respectively. The NMR yields were determined using dibromomethane as an internal standard. Analytical and spectral data of all those known compounds were exactly matching with the reported values. HPLC was carried out on an Agilent 1260 infinity or Waters 2695 instrument using a chiralpak AS-H, AD-3, AD-H, IA, IB, IC, IG, cel 1, PA2, PC2, IC-3, IE3, Ij-3, or OZ-3 column. 


\section{Preparation of Glycine Esters}

The $N$-aryl glycine esters were synthesis followed with reported literatures ${ }^{1,2}$

Ethyl 2-((4-methoxyphenyl)amino)acetate (1a) ${ }^{1}$<smiles>CCOC(=O)CNc1ccc(OC)cc1</smiles>

${ }^{1} \mathbf{H}$ NMR $\left(400 \mathrm{MHz}, \mathrm{CDCl}_{3}\right) \delta 6.79(\mathrm{~d}, J=8.8 \mathrm{~Hz}, 2 \mathrm{H}), 6.59(\mathrm{~d}, J=8.8 \mathrm{~Hz}, 2 \mathrm{H}), 4.23(\mathrm{q}, J=7.2$ $\mathrm{Hz}, 2 \mathrm{H}), 4.03$ (br, 1H), $3.86(\mathrm{~s}, 2 \mathrm{H}), 3.74(\mathrm{~s}, 3 \mathrm{H}), 1.29(\mathrm{t}, J=7.2 \mathrm{~Hz}, 3 \mathrm{H}) .{ }^{13} \mathbf{C}\left\{{ }^{1} \mathbf{H}\right\}$ NMR $(101$ $\left.\mathrm{MHz}, \mathrm{CDCl}_{3}\right) \delta 171.5,152.7,141.4,115.0,114.5,61.3,55.8,46.9,14.3$.

Methyl 2-((4-methoxyphenyl)amino)acetate (1b $)^{3}$<smiles>COC(=O)CNc1ccc(OC)cc1</smiles>

${ }^{1} \mathrm{H}$ NMR $\left(400 \mathrm{MHz}, \mathrm{CDCl}_{3}\right) \delta 6.79(\mathrm{~d}, J=8.8 \mathrm{~Hz}, 2 \mathrm{H}), 6.59(\mathrm{~d}, J=8.8 \mathrm{~Hz}, 2 \mathrm{H}), 4.01(\mathrm{br}, 1 \mathrm{H}), 3.88$ (s, 2H), 3.77 (s, 3H), 3.75 (s, 3H). ${ }^{13} \mathbf{C}\left\{{ }^{1} \mathbf{H}\right\}$ NMR $\left(101 \mathrm{MHz}, \mathrm{CDCl}_{3}\right) \delta$ 172.0, 152.8, 141.3, 115.1, $114.5,55.9,52.3,46.8$.

Propyl 2-((4-isopropoxyphenyl)amino)acetate (1c) ${ }^{4}$<smiles>CCOC(=O)CNc1ccc(OC)cc1</smiles>

${ }^{1} \mathbf{H}$ NMR $\left(400 \mathrm{MHz}, \mathrm{CDCl}_{3}\right) \delta 6.79(\mathrm{~d}, J=8.8 \mathrm{~Hz}, 2 \mathrm{H}), 6.59(\mathrm{~d}, J=8.8 \mathrm{~Hz}, 2 \mathrm{H}), 4.13(\mathrm{t}, J=6.8$ Hz, 2H), 4.03 (br, 1H), 3.87 (s, 2H), 3.75 (s, 3H), $1.68(\mathrm{sex}, J=7.6 \mathrm{~Hz}, 2 \mathrm{H}), 0.94$ (t, $J=7.6 \mathrm{~Hz}$, 3H). ${ }^{13} \mathbf{C}\left\{{ }^{1} \mathbf{H}\right\} \mathbf{N M R}\left(101 \mathrm{MHz}, \mathrm{CDCl}_{3}\right) \delta 171.6,152.8,141.4,115.1,114.5,66.9,55.9,46.9,22.1$, 10.4. HR-MS (ESI) Calculated for $\mathrm{C}_{12} \mathrm{H}_{18} \mathrm{NO}_{3}\left([\mathrm{M}+\mathrm{H}]^{+}\right)$: 224.1281. Found: 224.1288. 
Butyl 2-((4-methoxyphenyl)amino)acetate (1d $)^{5}$<smiles>CCCCCCCC(=O)CNc1ccc(OC)cc1</smiles>

${ }^{1}$ H NMR $\left(400 \mathrm{MHz}, \mathrm{CDCl}_{3}\right) \delta 6.79(\mathrm{~d}, J=8.8 \mathrm{~Hz}, 2 \mathrm{H}), 6.59(\mathrm{~d}, J=8.8 \mathrm{~Hz}, 2 \mathrm{H}), 4.17(\mathrm{t}, J=6.8$ $\mathrm{Hz}, 2 \mathrm{H}$ ), 4.03 (br, 1H), 3.87 (s, 2H), 3.75 (s, 3H), 1.63 (quin, $J=6.8 \mathrm{~Hz}, 2 \mathrm{H}$ ), 1.37 (sex, $J=7.2 \mathrm{~Hz}$, 2H), $0.93(\mathrm{t}, J=7.2 \mathrm{~Hz}, 3 \mathrm{H}) . \quad{ }^{13} \mathbf{C}\left\{{ }^{1} \mathbf{H}\right\} \mathbf{N M R}\left(101 \mathrm{MHz}, \mathrm{CDCl}_{3}\right) \delta$ 171.6, 152.8, 141.4, 115.1, 114.5, 65.2, 55.9, 47.0, 30.8, 19.2, 13.8. HR-MS (ESI) Calculated for $\mathrm{C}_{13} \mathrm{H}_{20} \mathrm{NO}_{3}\left([\mathrm{M}+\mathrm{H}]^{+}\right)$: 238.1438. Found: 238.1444 .

Isopropyl 2-((4-methoxyphenyl)amino)acetate (1e $)^{5}$<smiles>CCOC(=O)CNc1ccc(OC)cc1</smiles>

${ }^{1} \mathbf{H}$ NMR $\left(400 \mathrm{MHz}, \mathrm{CDCl}_{3}\right) \delta 6.79(\mathrm{~d}, J=8.8 \mathrm{~Hz}, 2 \mathrm{H}), 6.58(\mathrm{~d}, J=8.8 \mathrm{~Hz}, 2 \mathrm{H}), 5.10(\mathrm{sep}, J=6.0$ $\mathrm{Hz}, 1 \mathrm{H}), 4.04(\mathrm{~s}, 1 \mathrm{H}), 3.83(\mathrm{~s}, 2 \mathrm{H}), 3.74(\mathrm{~s}, 3 \mathrm{H}), 1.26(\mathrm{~d}, J=6.4 \mathrm{~Hz}, 6 \mathrm{H}) .{ }^{13} \mathbf{C}\left\{{ }^{1} \mathbf{H}\right\}$ NMR $(101$ $\left.\mathrm{MHz}, \mathrm{CDCl}_{3}\right) \delta 171.0,152.7,141.4,115.0,114.5,69.0,55.9,47.2,21.9$.

Tert-butyl 2-((4-methoxyphenyl)amino)acetate (1f $)^{5}$<smiles>CCCCC(=O)CNc1ccc(OC)cc1</smiles>

${ }^{1} \mathbf{H}$ NMR $\left(400 \mathrm{MHz}, \mathrm{CDCl}_{3}\right) \delta 6.79(\mathrm{~d}, J=8.0 \mathrm{~Hz}, 2 \mathrm{H}), 6.57(\mathrm{~d}, J=8.4 \mathrm{~Hz}, 2 \mathrm{H}), 4.02(\mathrm{br}, 1 \mathrm{H}), 3.76$ (s, 2H), 3.74 (s, 3H), 1.48 (s, 9H). ${ }^{13} \mathbf{C}\left\{{ }^{1} \mathbf{H}\right\}$ NMR (101 MHz, $\left.\mathrm{CDCl}_{3}\right) \delta$ 170.6, 152.6, 141.6, 115.0, $114.4,81.9,55.9,47.6,28.2$.

Tert-pentyl 2-((4-methoxyphenyl)amino)acetate (1g)<smiles>CCC(C)(C)OC(=O)CNc1ccc(OC)cc1</smiles>

Yellow oil. ${ }^{1} \mathbf{H}$ NMR $\left(400 \mathrm{MHz}, \mathrm{CDCl}_{3}\right) \delta 6.78(\mathrm{~d}, J=8.8 \mathrm{~Hz}, 2 \mathrm{H}), 6.57(\mathrm{~d}, J=8.8 \mathrm{~Hz}, 2 \mathrm{H}), 3.77$ $(\mathrm{s}, 2 \mathrm{H}), 3.74(\mathrm{~s}, 3 \mathrm{H}), 1.80(\mathrm{q}, J=7.6 \mathrm{~Hz}, 2 \mathrm{H}), 1.44(\mathrm{~s}, 6 \mathrm{H}), 0.88(\mathrm{t}, J=7.6 \mathrm{~Hz}, 3 \mathrm{H}) . \quad{ }^{13} \mathbf{C}\left\{{ }^{1} \mathbf{H}\right\} \mathbf{N M R}$ $\left(101 \mathrm{MHz}, \mathrm{CDCl}_{3}\right) \delta 170.6,152.5,141.6,114.9,114.4,84.4,55.8,47.5,33.8,25.6,8.3$. IR (neat): 
$2975,1730,1513,1461,1359,1219,1149,1037,926,818,517 \mathrm{~cm}^{-1}$. HR-MS (ESI) Calculated for $\mathrm{C}_{14} \mathrm{H}_{22} \mathrm{NO}_{3}([\mathrm{M}+\mathrm{H}]+): 252.1594$ Found: 252.1594 .

Neopentyl 2-((4-methoxyphenyl)amino)acetate (1h)<smiles>COc1ccc(NCC(=O)OCC(C)(C)C)cc1</smiles>

White solid, m.p. $=54.6-56.1{ }^{\circ} \mathrm{C} .{ }^{1} \mathbf{H}$ NMR $\left(400 \mathrm{MHz}, \mathrm{CDCl}_{3}\right) \delta 6.79(\mathrm{~d}, J=8.8 \mathrm{~Hz}, 2 \mathrm{H}), 6.60(\mathrm{~d}$, $J=8.8 \mathrm{~Hz}, 2 \mathrm{H}), 4.04(\mathrm{br}, 1 \mathrm{H}), 3.90(\mathrm{~s}, 2 \mathrm{H}), 3.87(\mathrm{~s}, 2 \mathrm{H}), 3.75(\mathrm{~s}, 3 \mathrm{H}), 0.93(\mathrm{~s}, 9 \mathrm{H}) . \quad{ }^{13} \mathbf{C}\left\{{ }^{1} \mathbf{H}\right\} \mathbf{N M R}$ $\left(101 \mathrm{MHz}, \mathrm{CDCl}_{3}\right) \delta 171.7,152.8,141.4,115.1,114.5,74.6,55.9,46.9,31.5,26.5$. IR (neat): 3388, $2962,1727,1516,1443,1375,1349,1327,1232,1196,1177,1141,1037,986,941,819,804,756$, $600,518 \mathrm{~cm}^{-1}$. HR-MS (ESI) Calculated for $\mathrm{C}_{14} \mathrm{H}_{22} \mathrm{NO}_{3}\left([\mathrm{M}+\mathrm{H}]^{+}\right)$: 252.1594. Found: 252.1603 .

Cyclopropyl 2-((4-methoxyphenyl)amino)acetate (1i)<smiles>COc1ccc(NCC(=O)OC2CC2)cc1</smiles>

White solid, m.p. $=66.4-67.2{ }^{\circ} \mathrm{C} .{ }^{1} \mathbf{H}$ NMR $\left(400 \mathrm{MHz}, \mathrm{CDCl}_{3}\right) \delta 6.79(\mathrm{~d}, J=8.8 \mathrm{~Hz}, 2 \mathrm{H}), 6.57(\mathrm{~d}$, $J=8.8 \mathrm{~Hz}, 2 \mathrm{H}), 4.21(\mathrm{sep}, J=3.2 \mathrm{~Hz}, 1 \mathrm{H}), 4.00(\mathrm{br}, 1 \mathrm{H}), 3.83(\mathrm{~s}, 2 \mathrm{H}), 3.74(\mathrm{~s}, 3 \mathrm{H}), 0.77-0.68(\mathrm{~m}$, 4H). ${ }^{13} \mathbf{C}\left\{{ }^{1} \mathbf{H}\right\}$ NMR $\left(101 \mathrm{MHz}, \mathrm{CDCl}_{3}\right) \delta 172.4,152.8,141.3,115.1,114.6,55.9,49.8,46.9,5.2$. IR (neat): 3395, 2835, 1737, 1515, 1442, 1375, 1348, 1315, 1269, 1215, 1196, 1156, 1035, 956, 918, 809, 762, 599, $515 \mathrm{~cm}^{-1}$. HR-MS (ESI) Calculated for $\mathrm{C}_{12} \mathrm{H}_{16} \mathrm{NO}_{3}\left([\mathrm{M}+\mathrm{H}]^{+}\right): 222.1125$. Found: 222.1129 .

\section{Cyclobutyl 2-((4-methoxyphenyl)amino)acetate (1j)}<smiles>COc1ccc(NCC(=O)OC2CCC2)cc1</smiles>

White solid, m.p. $=40.5-41.3{ }^{\circ} \mathrm{C} .{ }^{1} \mathbf{H}$ NMR $\left(400 \mathrm{MHz}, \mathrm{CDCl}_{3}\right) \delta 6.79(\mathrm{~d}, J=8.8 \mathrm{~Hz}, 2 \mathrm{H}), 6.58(\mathrm{~d}$, $J=8.8 \mathrm{~Hz}, 2 \mathrm{H}), 5.07$ (quin, $J=7.6 \mathrm{~Hz}, 1 \mathrm{H}), 4.01(\mathrm{br}, 1 \mathrm{H}), 3.84(\mathrm{~s}, 2 \mathrm{H}), 3.75(\mathrm{~s}, 3 \mathrm{H}), 2.42-2.32(\mathrm{~m}$, 2H), 2.14-2.03 (m, 2H), $1.81(\mathrm{q}, J=9.6 \mathrm{~Hz}, 1 \mathrm{H}), 1.69-1.59(\mathrm{~m}, 1 \mathrm{H}) .{ }^{{ }^{13} \mathbf{C}}\left\{{ }^{1} \mathbf{H}\right\} \mathbf{N M R}(101 \mathrm{MHz}$, $\left.\mathrm{CDCl}_{3}\right) \delta 170.7,152.6,141.3,114.9,114.4,69.5,55.7,46.8,30.3,13.5$. IR (neat): 3388, 2992, 1729, 
$1522,1444,1377,1348,1302,1268,1234,1197,1145,1043,928,806,761,604,510 \mathrm{~cm}^{-1}$. HRMS (ESI) Calculated for $\mathrm{C}_{13} \mathrm{H}_{18} \mathrm{NO}_{3}\left([\mathrm{M}+\mathrm{H}]^{+}\right)$: 236.1281. Found: 236.1285 .

Cyclopentyl 2-((4-methoxyphenyl)amino)acetate (1k)<smiles>COc1ccc(NCC(=O)OC2CCCC2)cc1</smiles>

White solid, m.p. $=46.2-47.4{ }^{\circ} \mathrm{C} .{ }^{1} \mathbf{H}$ NMR $\left(400 \mathrm{MHz}, \mathrm{CDCl}_{3}\right) \delta 6.79(\mathrm{~d}, J=8.0 \mathrm{~Hz}, 2 \mathrm{H}), 6.58(\mathrm{~d}$, $J=8.0 \mathrm{~Hz}, 2 \mathrm{H}), 5.27-5.24(\mathrm{~m}, 1 \mathrm{H}), 4.03(\mathrm{~s}, 1 \mathrm{H}), 3.82(\mathrm{~s}, 2 \mathrm{H}), 3.74(\mathrm{~s}, 3 \mathrm{H}), 1.92-1.80(\mathrm{~m}, 2 \mathrm{H}), 1.74-$ $1.54(\mathrm{~m}, 6 \mathrm{H}) .{ }^{13} \mathbf{C}\left\{{ }^{1} \mathbf{H}\right\} \mathbf{N M R}\left(101 \mathrm{MHz}, \mathrm{CDCl}_{3}\right) \delta 171.3,152.7,141.5,115.0,114.5,78.3,55.9$, 47.2, 32.8, 23.8. IR (neat): 3365, 2972, 1728, 1515, 1439, 1418, 1375, 1353, 1308, 1251, 1232 , 1197, 1165, 1037, 965, 818, 776, 728,520 $\mathrm{cm}^{-1}$. HR-MS (ESI) Calculated for $\mathrm{C}_{14} \mathrm{H}_{19} \mathrm{NO}_{3} \mathrm{Na}$ $\left([\mathrm{M}+\mathrm{Na}]^{+}\right): 272.1257$. Found: 272.1260 .

Cyclohexyl 2-((4-methoxyphenyl)amino)acetate (11)<smiles>COc1ccc(NCC(=O)OC2CCCCC2)cc1</smiles>

White solid, m.p. $=68.6-70.8{ }^{\circ} \mathrm{C} .{ }^{1} \mathbf{H}$ NMR $\left(400 \mathrm{MHz}, \mathrm{CDCl}_{3}\right) \delta 6.79(\mathrm{~d}, J=8.4 \mathrm{~Hz}, 2 \mathrm{H}), 6.59(\mathrm{~d}$, $J=8.4 \mathrm{~Hz}, 2 \mathrm{H}), 4.88-4.82$ (m, 1H), 4.01 (br, 1H), 3.84 (s, 2H), 3.74 (s, 3H), 1.89-1.82 (m, 2H), 1.74-1.70 (m, 2H), 1.56-1.53 (m, 1H), $1.48-1.24(\mathrm{~m}, 5 \mathrm{H}) . \quad{ }^{13} \mathbf{C}\left\{{ }^{1} \mathbf{H}\right\}$ NMR $\left(101 \mathrm{MHz}, \mathrm{CDCl}_{3}\right) \delta$ 170.9, 152.7, 141.5, 115.0, 114.5, 73.9, 55.9, 47.3, 31.7, 25.4, 23.8. IR (neat): 3385, 2939, 1720, 1514, 1468, 1444, 1377, 1356, 1325, 1210, 1177, 1136, 1041, 1008, 965, 931, 893, 819, 802, 772, 608, $518 \mathrm{~cm}^{-1}$. HR-MS (ESI) Calculated for $\mathrm{C}_{15} \mathrm{H}_{21} \mathrm{NO}_{3} \mathrm{Na}$ ([M+Na] $\left.]^{+}\right)$: 286.1414. Found: 286.1422 . (3s,5s,7s)-Adamantan-1-yl 2-((4-methoxyphenyl)amino)acetate (1m)<smiles>COc1ccc(NCC(=O)OC23CC4CC(CC(C4)C2)C3)cc1</smiles>

Pale yellow solid, m.p. $=80.5-81.7^{\circ} \mathrm{C} .{ }^{1} \mathbf{H}$ NMR $\left(400 \mathrm{MHz}, \mathrm{CDCl}_{3}\right) \delta 6.78(\mathrm{~d}, J=8.4 \mathrm{~Hz}, 2 \mathrm{H}), 6.57$ (d, $J=8.4 \mathrm{~Hz}, 2 \mathrm{H}), 4.00(\mathrm{~s}, 1 \mathrm{H}), 3.76(\mathrm{~s}, 1 \mathrm{H}), 3.74$ (s, 3H), 2.17 (s, 3H), $2.12(\mathrm{~s}, 6 \mathrm{H}), 1.66(\mathrm{~s}, 6 \mathrm{H})$. ${ }^{13} \mathbf{C}\left\{{ }^{1} \mathbf{H}\right\}$ NMR (101 MHz, $\left.\mathrm{CDCl}_{3}\right) \delta 170.3,152.6,141.6,115.0,114.5,82.0,55.9,47.7,41.5,36.2,31.0$. 
IR (neat): 3380, 2911, 1725, 1511, 1440, 1352, 1301, 1264, 1206, 1138, 1103, 1035, 964, 889, 819, 767, $517 \mathrm{~cm}^{-1}$. HR-MS (ESI) Calculated for $\mathrm{C}_{19} \mathrm{H}_{25} \mathrm{NO}_{3} \mathrm{Na}\left([\mathrm{M}+\mathrm{Na}]^{+}\right)$: 338.1727. Found: 338.1732 .

2-Methoxyethyl (4-methoxyphenyl)glycinate (1n)<smiles>COCCOC(=O)CNc1ccc(OC)cc1</smiles>

Yellow oil. ${ }^{1} \mathbf{H}$ NMR $\left(400 \mathrm{MHz}, \mathrm{CDCl}_{3}\right) \delta 6.77(\mathrm{~d}, J=8.8 \mathrm{~Hz}, 2 \mathrm{H}), 6.57(\mathrm{~d}, J=9.2 \mathrm{~Hz}, 2 \mathrm{H}), 4.35$ $4.27(\mathrm{~m}, 2 \mathrm{H}), 4.00(\mathrm{br}, 1 \mathrm{H}), 3.90(\mathrm{~s}, 2 \mathrm{H}), 3.72(\mathrm{~d}, J=1.4 \mathrm{~Hz}, 3 \mathrm{H}), 3.59$ (t, $J=4.8 \mathrm{~Hz}, 2 \mathrm{H}), 3.37$ (s, 3H). ${ }^{13} \mathbf{C}\left\{{ }^{1} \mathbf{H}\right\}$ NMR $\left(101 \mathrm{MHz}, \mathrm{CDCl}_{3}\right) \delta 171.5,152.6,141.2,114.9,114.4,70.3,64.1,59.0,55.7$, 46.7. IR (neat): 3371, 2832, 1738, 1511, 1443, 1234, 1179, 1125, 1031, 820, $517 \mathrm{~cm}^{-1}$. HR-MS (ESI) Calculated for $\mathrm{C}_{12} \mathrm{H}_{18} \mathrm{NO}_{4}\left([\mathrm{M}+\mathrm{H}]^{+}\right)$: 240.1230. Found: 240.1229 .

\section{4-(4-Methoxyphenyl)butyl (4-methoxyphenyl)glycinate (1o)}<smiles>COc1ccc(CCCCOC(=O)CNc2ccc(OC)cc2)cc1</smiles>

White solid, m.p. $=53.6-55.1{ }^{\circ} \mathrm{C} .{ }^{1} \mathbf{H}$ NMR $\left(500 \mathrm{MHz}, \mathrm{CDCl}_{3}\right) \delta 7.10(\mathrm{~d}, J=8.5 \mathrm{~Hz}, 2 \mathrm{H}), 6.86(\mathrm{~d}$, $J=8.5 \mathrm{~Hz}, 2 \mathrm{H}), 6.80(\mathrm{~d}, J=9 \mathrm{~Hz}, 2 \mathrm{H}), 6.60(\mathrm{~d}, J=9 \mathrm{~Hz}, 2 \mathrm{H}), 4.20(\mathrm{t}, J=6.5 \mathrm{~Hz}, 2 \mathrm{H}), 3.87(\mathrm{~s}, 2 \mathrm{H})$, $3.80(\mathrm{~s}, 3 \mathrm{H}), 3.75(\mathrm{~s}, 3 \mathrm{H}), 2.59(\mathrm{t}, J=7.0 \mathrm{~Hz}, 2 \mathrm{H}), 1.71-1.63(\mathrm{~m}, 4 \mathrm{H}) .{ }^{13} \mathbf{C}\left\{{ }^{1} \mathbf{H}\right\}$ NMR $(126 \mathrm{MHz}$, $\left.\mathrm{CDCl}_{3}\right) \delta 171.5,157.9,152.7,141.3,134.0,129.3,114.9,114.4,113.8,65.1,55.7,55.3,46.8,34.5$, 28.2, 27.9. IR (neat): 3396, 2913, 1723, 1612, 1510, 1443, 1395, 1354, 1325, 1302, 1237, 1179, 1137, 1029, 932, 820, 753, 698, 571, $522 \mathrm{~cm}^{-1}$. HR-MS (ESI) Calculated for $\mathrm{C}_{20} \mathrm{H}_{26} \mathrm{NO}_{4}\left([\mathrm{M}+\mathrm{H}]^{+}\right)$: 344.1856. Found: 344.1852 .

\section{Benzyl 2-((4-methoxyphenyl)amino)acetate $(1 p)^{5}$}<smiles>COc1ccc(NCC(=O)OCc2ccccc2)cc1</smiles>

${ }^{1} \mathbf{H}$ NMR $\left(400 \mathrm{MHz}, \mathrm{CDCl}_{3}\right) \delta 7.43-7.29(\mathrm{~m}, 5 \mathrm{H}), 6.79(\mathrm{~d}, J=8.8 \mathrm{~Hz}, 2 \mathrm{H}), 6.61(\mathrm{~d}, J=8.8 \mathrm{~Hz}, 2 \mathrm{H})$, $5.20(\mathrm{~s}, 2 \mathrm{H}), 3.93(\mathrm{~s}, 2 \mathrm{H}), 3.75(\mathrm{~s}, 3 \mathrm{H}) . \quad{ }^{13} \mathbf{C}\left\{{ }^{1} \mathbf{H}\right\} \mathbf{N M R}\left(101 \mathrm{MHz}, \mathrm{CDCl}_{3}\right) \delta$ 171.4, 152.9, 141.2, $135.5,128.7,128.6,128.5,115.0,114.6,67.1,55.9,47.0$. 
4-Methoxybenzyl (4-methoxyphenyl)glycinate (1q)<smiles>COc1ccc(COC(=O)CNc2ccc(OC)cc2)cc1</smiles>

Yellow oil. ${ }^{1} \mathbf{H}$ NMR (400 MHz, CDCl $) \delta 7.29$ (d, $\left.J=8.4 \mathrm{~Hz}, 2 \mathrm{H}\right), 6.90$ (d, $\left.J=8.4 \mathrm{~Hz}, 2 \mathrm{H}\right), 6.79$ (d, $J=8.4 \mathrm{~Hz}, 2 \mathrm{H}), 6.58$ (d, J = 8.8 Hz, 2H), 5.14 (s, 2H), 4.07 (br, 1H), 3.89 (s, 2H), 3.82 (s, 3H), $3.75(\mathrm{~s}, 3 \mathrm{H}) .{ }^{13} \mathbf{C}\left\{{ }^{1} \mathbf{H}\right\} \mathbf{N M R}\left(126 \mathrm{MHz}, \mathrm{CDCl}_{3}\right) \delta 171.5,159.9,152.7,141.3,130.4,127.6,115.0$, 114.5, 114.1, 66. 9, 55.8, 55.4, 47.0. IR (neat): 2954,, 2835, 1732, 1612, 1511, 1461, 1380, 1242, 1170, 1030, 817, $517 \mathrm{~cm}^{-1}$. HR-MS (ESI) Calculated for $\mathrm{C}_{17} \mathrm{H}_{19} \mathrm{NO}_{4} \mathrm{Na}\left([\mathrm{M}+\mathrm{Na}]^{+}\right)$: 324.1206 . Found: 324.1206 .

\section{4-(Trifluoromethyl)benzyl (4-methoxyphenyl)glycinate (1r)}<smiles>COc1ccc(NCC(=O)OCc2ccc(C(F)(F)F)cc2)cc1</smiles>

White solid, m.p. $=70.8-72.3{ }^{\circ} \mathrm{C} .{ }^{1} \mathbf{H}$ NMR $\left(400 \mathrm{MHz}, \mathrm{CDCl}_{3}\right) \delta 7.62(\mathrm{~d}, J=7.6 \mathrm{~Hz}, 2 \mathrm{H}), 7.43(\mathrm{~d}$, $J=7.6 \mathrm{~Hz}, 2 \mathrm{H}), 6.79(\mathrm{~d}, J=8.8 \mathrm{~Hz}, 2 \mathrm{H}), 6.58(\mathrm{~d}, J=8.8 \mathrm{~Hz}, 2 \mathrm{H}, 2 \mathrm{H}), 5.25(\mathrm{~s}, 2 \mathrm{H}), 4.04(\mathrm{br}, 1 \mathrm{H})$, 3.96 (s, 2H), 3.75 (s, 3H). ${ }^{13} \mathbf{C}\left\{{ }^{1} \mathbf{H}\right\}$ NMR $\left(126 \mathrm{MHz}, \mathrm{CDCl}_{3}\right) \delta 171.3,152.8,141.1,139.5,130.5$ (q, $J=32.8 \mathrm{~Hz}), 128.3,125.6(\mathrm{q}, J=3.8 \mathrm{~Hz}), 124.0(\mathrm{q}, J=272.2 \mathrm{~Hz}), 115.0,114.5,65.9,55.7,46.9$. ${ }^{19} \mathrm{~F}$ NMR (376 MHz, $\mathrm{CDCl}_{3}$ ) $\delta$-62.66. IR (neat): 3378, 2835, 1733, 1621, 1516, 1467, 1437, 1381, 1327, 1285, 1269, 1236, 1211, 1192, 1148, 1109, 1065, 1035, 811, 752, 589, $511 \mathrm{~cm}^{-1}$. HR-MS (ESI) Calculated for $\mathrm{C}_{17} \mathrm{H}_{17} \mathrm{NO}_{3} \mathrm{~F}_{3}\left([\mathrm{M}+\mathrm{H}]^{+}\right)$: 340.1155 . Found: 340.1156 .

\section{4-Bromobenzyl (4-methoxyphenyl)glycinate (1s)}<smiles>COc1ccc(NCC(=O)OCc2ccc(Br)cc2)cc1</smiles>

Pink solid, m.p. $=104.2-105.9{ }^{\circ} \mathrm{C} .{ }^{1} \mathbf{H}$ NMR $\left(400 \mathrm{MHz}, \mathrm{CDCl}_{3}\right) \delta 7.48(\mathrm{~d}, J=8.4 \mathrm{~Hz}, 2 \mathrm{H}), 7.20(\mathrm{~d}$, $J=8.4 \mathrm{~Hz}, 2 \mathrm{H}), 6.79(\mathrm{~d}, J=9.2 \mathrm{~Hz}, 2 \mathrm{H}), 6.57(\mathrm{~d}, J=9.2 \mathrm{~Hz}, 2 \mathrm{H}), 5.14(\mathrm{~s}, 2 \mathrm{H}), 3.92(\mathrm{~s}, 2 \mathrm{H}), 3.75$ (s, 3H). ${ }^{13} \mathbf{C}\left\{{ }^{1} \mathbf{H}\right\}$ NMR $\left(126 \mathrm{MHz}, \mathrm{CDCl}_{3}\right) \delta 171.3,152.8,141.2,134.5,131.8,130.1,122.6,115.0$, 114.5, 66.2, 55.8, 46.9. IR (neat): 3380, 2971, 1729, 1511, 1440, 1383, 1349, 1313, 1236, 1203 , 
1138, 1067, 1034, 1012, 965, 941, 816, 773, 626, $514 \mathrm{~cm}^{-1}$. HR-MS (ESI) Calculated for $\mathrm{C}_{16} \mathrm{H}_{17} \mathrm{NO}_{3} \mathrm{Br}\left([\mathrm{M}+\mathrm{H}]^{+}\right): 350.0386$. Found: 350.0390 .

4-(4,4,5,5-Tetramethyl-1,3,2-dioxaborolan-2-yl)benzyl (4-methoxyphenyl)glycinate (1t)<smiles>COc1ccc(NCC(=O)OCc2ccc(Cc3ccccc3)cc2)cc1</smiles>

Yellow oil. ${ }^{1} \mathrm{H}$ NMR $\left(500 \mathrm{MHz}, \mathrm{CDCl}_{3}\right) \delta 7.82(\mathrm{~d}, J=8.0 \mathrm{~Hz}, 2 \mathrm{H}), 7.33(\mathrm{~d}, J=7.5 \mathrm{~Hz}, 2 \mathrm{H}), 6.79$ $(\mathrm{d}, J=9.0 \mathrm{~Hz}, 2 \mathrm{H}), 6.58(\mathrm{~d}, J=7.5 \mathrm{~Hz}, 2 \mathrm{H}), 5.21(\mathrm{~s}, 2 \mathrm{H}), 3.92(\mathrm{~s}, 2 \mathrm{H}), 3.74(\mathrm{~s}, 3 \mathrm{H}), 1.35$ (s, 12H). ${ }^{13} \mathbf{C}\left\{{ }^{1} \mathbf{H}\right\}$ NMR $\left(126 \mathrm{MHz}, \mathrm{CDCl}_{3}\right) \delta 171.4,152.8,141.2,138.4,135.1,127.5,115.0,114.5,84.0$, $66.9,55.8,46.9,24.9$. (The signal for the carbon that is attached to the boron atom was not observed). IR (neat): 2977, 1738, 1614, 1513, 1443, 1357, 1322, 1235, 1170, 1141, 1087, 1036, 961, 911, 857, 819, 730, 656, $518 \mathrm{~cm}^{-1}$. HR-MS (ESI) Calculated for $\mathrm{C}_{22} \mathrm{H}_{29} \mathrm{BNO}_{5}\left([\mathrm{M}+\mathrm{H}]^{+}\right)$: 398.2133. Found: 389.2137.

\section{4-Cyanobenzyl (4-methoxyphenyl)glycinate (1u)}<smiles>COc1ccc(NCC(=O)OCc2ccc(C#N)cc2)cc1</smiles>

White solid, m.p. $=140.8-142.4-72.3{ }^{\circ} \mathrm{C} .{ }^{1} \mathbf{H}$ NMR $\left(400 \mathrm{MHz}, \mathrm{CDCl}_{3}\right) \delta 7.63(\mathrm{~d}, J=7.6 \mathrm{~Hz}, 2 \mathrm{H})$, $7.40(\mathrm{~d}, J=8.0 \mathrm{~Hz}, 2 \mathrm{H}), 6.78(\mathrm{~d}, J=8.8 \mathrm{~Hz}, 2 \mathrm{H}), 6.58(\mathrm{~d}, J=8.8 \mathrm{~Hz}, 2 \mathrm{H}), 5.23(\mathrm{~s}, 2 \mathrm{H}), 3.96(\mathrm{~s}$, 2H), 3.74 (s, 3H). ${ }^{13} \mathbf{C}\left\{{ }^{1} \mathbf{H}\right\}$ NMR (101 MHz, $\left.\mathrm{CDCl}_{3}\right) \delta 171.3,152.9,141.0,140.7,132.5,128.5$, 118.6, 115.0, 114.6, 112.3, 65.7, 55.8, 46.9. IR (neat): 3384, 2838, 2229, 1734, 1513, 1442, 1384, 1350, 1319, 1253, 1233, 1202, 1141, 1034, 972, 872, 830, 572, $536 \mathrm{~cm}^{-1}$. HR-MS (ESI) Calculated for $\mathrm{C}_{17} \mathrm{H}_{16} \mathrm{~N}_{2} \mathrm{O}_{3}\left([\mathrm{M}+\mathrm{H}]^{+}\right)$: 297.1234. Found: 297.1239.

Hex-5-en-1-yl (4-methoxyphenyl)glycinate (1v)<smiles>C=CCCCCOC(=O)CNc1ccc(OC)cc1</smiles>

Yellow oil. ${ }^{1} \mathbf{H}$ NMR $\left(400 \mathrm{MHz}, \mathrm{CDCl}_{3}\right) \delta 6.79(\mathrm{~d}, J=9.2 \mathrm{~Hz}, 2 \mathrm{H}), 6.58(\mathrm{~d}, J=8.8 \mathrm{~Hz}, 2 \mathrm{H}), 5.84-$ $5.74(\mathrm{~m}, 1 \mathrm{H}), 5.05-4.96(\mathrm{~m}, 2 \mathrm{H}), 4.17(\mathrm{t}, J=6.4 \mathrm{~Hz}, 2 \mathrm{H}), 3.86(\mathrm{~s}, 2 \mathrm{H}), 3.74(\mathrm{~s}, 3 \mathrm{H}), 2.13-2.01(\mathrm{~m}$, 2H), 1.73-1.60 (m, 2H), 1.48-1.40 (m, 2H). ${ }^{13} \mathbf{C}\left\{{ }^{1} \mathbf{H}\right\}$ NMR (101 MHz, $\left.\mathrm{CDCl}_{3}\right) \delta 171.5,152.7,141.4$, 
138.2, 138.2, 114.9, 114.4, 65.1, 55.7, 46.8, 33.2, 28.0, 25.1. IR (neat): 2935, 1736, 1512, 1442, $1353,1234,1179,1137,1036,995,911,818,519 \mathrm{~cm}^{-1}$. HR-MS (ESI) Calculated for $\mathrm{C}_{15} \mathrm{H}_{21} \mathrm{NO}_{3}$ $\left([\mathrm{M}+\mathrm{H}]^{+}\right):$264.1594. Found: 264.1588.

\section{2-Methylbut-3-en-2-yl (4-methoxyphenyl)glycinate (1w)}<smiles>C=CC(C)(C)OC(=O)CNc1ccc(OC)cc1</smiles>

Yellow oil. ${ }^{1} \mathrm{H}$ NMR $\left(500 \mathrm{MHz}, \mathrm{CDCl}_{3}\right) \delta 6.78(\mathrm{~d}, J=9.0 \mathrm{~Hz}, 2 \mathrm{H}), 6.57(\mathrm{~d}, J=9.0 \mathrm{~Hz}, 2 \mathrm{H}), 6.08$ $(\mathrm{dd}, J=17.5,10.5 \mathrm{~Hz}, 1 \mathrm{H}), 5.23-5.08(\mathrm{~m}, 2 \mathrm{H}), 3.79(\mathrm{~s}, 2 \mathrm{H}), 3.74(\mathrm{~s}, 3 \mathrm{H}), 1.55(\mathrm{~s}, 6 \mathrm{H}) .{ }^{13} \mathbf{C}\left\{{ }^{1} \mathbf{H}\right\}$ NMR $\left(126 \mathrm{MHz}, \mathrm{CDCl}_{3}\right) \delta 170.3,152.6,142.0,141.5,115.0,114.4,113.3,82.2,55.8,47.5,26.5$. IR (neat): 2982, 1734, 1512, 1443, 1360, 1234, 1121, 1036, 993, 923, 818, $518 \mathrm{~cm}^{-1}$. HR-MS (DART) Calculated for $\mathrm{C}_{14} \mathrm{H}_{20} \mathrm{NO}_{3}\left([\mathrm{M}+\mathrm{H}]^{+}\right)$: 250.1429. Found: 250.1433.

\section{Ethyl 2-((4-ethoxyphenyl)amino)acetate (1x)}<smiles>CCOC(=O)CNc1ccc(OCC)cc1</smiles>

Yellow solid, m.p. $=45.1-45.8^{\circ} \mathrm{C} .{ }^{1} \mathbf{H}$ NMR $\left(400 \mathrm{MHz}, \mathrm{CDCl}_{3}\right) \delta 6.79(\mathrm{~d}, J=8.8 \mathrm{~Hz}, 2 \mathrm{H}), 6.57$ $(\mathrm{d}, J=8.8 \mathrm{~Hz}, 2 \mathrm{H}), 4.23(\mathrm{q}, J=7.2 \mathrm{~Hz}, 2 \mathrm{H}), 4.03(\mathrm{br}, 1 \mathrm{H}), 3.95(\mathrm{q}, J=7.2 \mathrm{~Hz}, 2 \mathrm{H}), 3.85(\mathrm{~s}, 2 \mathrm{H})$, $1.37(\mathrm{t}, J=7.2 \mathrm{~Hz}, 3 \mathrm{H}), 1.29(\mathrm{t}, J=7.2 \mathrm{~Hz}, 3 \mathrm{H}) .{ }^{13} \mathbf{C}\left\{{ }^{1} \mathbf{H}\right\} \mathbf{N M R}\left(101 \mathrm{MHz}, \mathrm{CDCl}_{3}\right) \delta 171.5,152.0$, 141.4, 115.9, 114.5, 64.1, 61.3, 47.0, 15.1, 14.3. IR (neat): 3389, 2977, 1725, 1513, 1477, 1442, $1391,1373,1353,1298,1261,1238,1202,1142,1049,1017,921,818,800,769,524 \mathrm{~cm}^{-1}$. HRMS (ESI) Calculated for $\mathrm{C}_{12} \mathrm{H}_{18} \mathrm{NO}_{3}\left([\mathrm{M}+\mathrm{H}]^{+}\right)$: 224.1281. Found: 224.1290 .

Ethyl 2-((4-isopropoxyphenyl)amino)acetate (1y)<smiles>CCOC(=O)CNc1ccc(OCC)cc1</smiles>

White solid, m.p. $=35.3-36.1{ }^{\circ} \mathrm{C} .{ }^{1} \mathbf{H}$ NMR $\left(400 \mathrm{MHz}, \mathrm{CDCl}_{3}\right) \delta 6.78(\mathrm{~d}, J=8.8 \mathrm{~Hz}, 2 \mathrm{H}), 6.56(\mathrm{~d}, J=$ $8.8 \mathrm{~Hz}, 2 \mathrm{H}), 4.37$ (p, $J=6.4 \mathrm{~Hz}, 1 \mathrm{H}), 4.23(\mathrm{q}, J=7.2 \mathrm{~Hz}, 2 \mathrm{H}), 4.04(\mathrm{br}, 1 \mathrm{H}), 3.86(\mathrm{~s}, 2 \mathrm{H}), 1.34-1.24(\mathrm{~m}$, 9H). ${ }^{13} \mathbf{C}\left\{{ }^{1} \mathbf{H}\right\}$ NMR $\left(101 \mathrm{MHz}, \mathrm{CDCl}_{3}\right) \delta 171.5,150.8,141.6,118.1,114.4,71.2,61.3,46.9,22.3$, 14.3. IR (neat): $3381,2969,1724,1617,1514,1451,1371,1314,1256,1203,1138,1023,952,882$, 
816, 770, 600, $524 \mathrm{~cm}^{-1}$. HR-MS (ESI) Calculated for $\mathrm{C}_{13} \mathrm{H}_{20} \mathrm{NO}_{3}\left([\mathrm{M}+\mathrm{H}]^{+}\right)$: 238.1438. Found: 238.1444 .

Ethyl 2-((4-butoxyphenyl)amino)acetate (1z) ${ }^{6}$<smiles>CCOC(=O)CNc1ccc(OCC)cc1</smiles>

${ }^{1} \mathbf{H}$ NMR $\left(400 \mathrm{MHz}, \mathrm{CDCl}_{3}\right) \delta 6.79(\mathrm{~d}, J=8.8 \mathrm{~Hz}, 2 \mathrm{H}), 6.57(\mathrm{~d}, J=8.4 \mathrm{~Hz}, 2 \mathrm{H}), 4.22(\mathrm{q}, J=7.2$ $\mathrm{Hz}, 2 \mathrm{H}), 4.07$ (br, 1H), 3.93-3.86 (m, 2H), 3.85 (s, 2H), 1.73 (quin, $J=6.8 \mathrm{~Hz}, 2 \mathrm{H}), 1.48$ (sex, $J=$ $7.2 \mathrm{~Hz}, 2 \mathrm{H}), 1.28(\mathrm{t}, J=7.2 \mathrm{~Hz}, 3 \mathrm{H}), 0.97(\mathrm{t}, J=7.2 \mathrm{~Hz}, 3 \mathrm{H}) .{ }^{13} \mathbf{C}\left\{{ }^{1} \mathbf{H}\right\} \mathbf{N M R}\left(101 \mathrm{MHz}, \mathrm{CDCl}_{3}\right) \delta$ 171.4, 152.1, 141.2, 115.7, 114.3, 68.3, 61.1, 46.8, 31.5, 19.3, 14.2, 13.9. HR-MS (ESI) Calculated for $\mathrm{C}_{14} \mathrm{H}_{22} \mathrm{NO}_{3}\left([\mathrm{M}+\mathrm{H}]^{+}\right)$: 252.1594. Found: 252.1601 .

Ethyl 2-((4-(benzyloxy)phenyl)amino)acetate (1aa) ${ }^{7}$<smiles>CCOC(=O)CNc1ccc(OCc2ccccc2)cc1</smiles>

${ }^{1} \mathbf{H}$ NMR $\left(400 \mathrm{MHz}, \mathrm{CDCl}_{3}\right) \delta 7.47(\mathrm{~d}, J=7.2 \mathrm{~Hz}, 2 \mathrm{H}), 7.41(\mathrm{t}, J=7.2 \mathrm{~Hz}, 2 \mathrm{H}), 7.35$ (t, $J=7.2 \mathrm{~Hz}$, 1H), $6.91(\mathrm{~d}, J=8.4 \mathrm{~Hz}, 2 \mathrm{H}), 6.61(\mathrm{~d}, J=8.4 \mathrm{~Hz}, 2 \mathrm{H}), 5.02(\mathrm{~s}, 2 \mathrm{H}), 4.27$ (q, $J=7.2 \mathrm{~Hz}, 2 \mathrm{H}), 4.13$ (br, 1H), $3.88(\mathrm{~s}, 2 \mathrm{H}), 1.32(\mathrm{t}, J=7.2 \mathrm{~Hz}, 3 \mathrm{H}) .{ }^{13} \mathbf{C}\left\{{ }^{1} \mathbf{H}\right\} \mathbf{N M R}\left(101 \mathrm{MHz}, \mathrm{CDCl}_{3}\right) \delta$ 171.35, 151.77, $141.57,137.55,128.48,127.76,127.47,116.12,114.26,70.72,61.18,46.71,14.21$. HR-MS (ESI) Calculated for $\mathrm{C}_{17} \mathrm{H}_{20} \mathrm{NO}_{3}\left([\mathrm{M}+\mathrm{H}]^{+}\right)$: 286.1438. Found: 286.1443 .

\section{Ethyl 2-((3-chloro-4-methoxyphenyl)amino)acetate (1ab)}<smiles>CCOC(=O)CNc1ccc(OC)c(Cl)c1</smiles>

White solid, m.p. $=109.8-110.4{ }^{\circ} \mathrm{C} .{ }^{1} \mathbf{H}$ NMR $\left(400 \mathrm{MHz}, \mathrm{CDCl}_{3}\right) \delta 6.81(\mathrm{~d}, J=8.8 \mathrm{~Hz}, 1 \mathrm{H}), 6.67$ (d, $J=2.8 \mathrm{~Hz}, 1 \mathrm{H}), 6.49$ (dd, $J=8.8,3.2 \mathrm{~Hz}, 1 \mathrm{H}), 4.24$ (q, $J=7.2 \mathrm{~Hz}, 2 \mathrm{H}), 4.10$ (br, 1H), 3.84 (s, 2H), 3.82 (s, 3H), 1.29 (t, $J=7.2 \mathrm{~Hz}, 3 \mathrm{H}) .{ }^{13} \mathbf{C}\left\{{ }^{1} \mathbf{H}\right\} \mathbf{N M R}\left(101 \mathrm{MHz}, \mathrm{CDCl}_{3}\right) \delta$ 171.1, 148.0, 142.0, 123.6, 115.4, 114.3, 112.4, 61.5, 57.1, 46.6, 14.3. IR (neat): 3370, 2990, 1725, 1613, 1503, 1444 , 
1211, 1143, 1058, 1015, 862, 788, 717, $585 \mathrm{~cm}^{-1}$. HR-MS (ESI) Calculated for $\mathrm{C}_{11} \mathrm{H}_{15} \mathrm{NO}_{3} \mathrm{Cl}$ $\left([\mathrm{M}+\mathrm{H}]^{+}\right): 244.0735$. Found: 244.0742 .

\section{Ethyl 2-(p-tolylamino)acetate (1ac) ${ }^{1}$}<smiles>CCOC(=O)CNc1ccc(C)cc1</smiles>

${ }^{1} \mathbf{H}$ NMR $\left(400 \mathrm{MHz}, \mathrm{CDCl}_{3}\right) \delta 7.02(\mathrm{~d}, J=8.0 \mathrm{~Hz}, 2 \mathrm{H}), 6.55(\mathrm{~d}, J=8.0 \mathrm{~Hz}, 2 \mathrm{H}), 4.25(\mathrm{q}, J=7.2$ $\mathrm{Hz}, 2 \mathrm{H}), 4.17$ (br, 1H), $3.89(\mathrm{~s}, 2 \mathrm{H}), 2.26(\mathrm{~s}, 3 \mathrm{H}), 1.31(\mathrm{t}, J=7.2 \mathrm{~Hz}, 3 \mathrm{H}) .{ }^{13} \mathbf{C}\left\{{ }^{1} \mathbf{H}\right\}$ NMR $(101$ $\left.\mathrm{MHz}, \mathrm{CDCl}_{3}\right) \delta 171.4,144.9,129.9,127.5,113.3,61.3,46.3,20.5,14.3$.

\section{Ethyl 2-((4-ethylphenyl)amino)acetate (1ad $)^{8}$}<smiles>CCOC(=O)CNc1ccc(CC)cc1</smiles>

${ }^{1} \mathbf{H}$ NMR $\left(400 \mathrm{MHz}, \mathrm{CDCl}_{3}\right) \delta 7.05(\mathrm{~d}, J=6.8 \mathrm{~Hz}, 2 \mathrm{H}), 6.58(\mathrm{~d}, J=6.8 \mathrm{~Hz}, 2 \mathrm{H}), 4.25(\mathrm{q}, J=7.2$ $\mathrm{Hz}, 2 \mathrm{H}), 3.90$ (s, 2H), 2.59-2.53 (m, 2H), 1.30 (dt, $J=6.4,1.6 \mathrm{~Hz}, 3 \mathrm{H}), 1.20(\mathrm{dt}, J=7.6,2.8 \mathrm{~Hz}$, 3H). ${ }^{13} \mathbf{C}\left\{{ }^{1} \mathbf{H}\right\} \mathbf{N M R}\left(101 \mathrm{MHz}, \mathrm{CDCl}_{3}\right) \delta$ 171.4, 145.1, 134.2, 128.7, 113.3, 61.4, 46.4, 28.1, 16.0, 14.3. HR-MS (ESI) Calculated for $\mathrm{C}_{12} \mathrm{H}_{18} \mathrm{NO}_{2}\left([\mathrm{M}+\mathrm{H}]^{+}\right)$: 208.1332. Found: 208.1337.

\section{Ethyl 2-((4-isopropylphenyl)amino)acetate (1ae $)^{9}$}<smiles>CCOC(=O)CNc1ccc(C(C)C)cc1</smiles>

${ }^{1} \mathbf{H}$ NMR $\left(400 \mathrm{MHz}, \mathrm{CDCl}_{3}\right) \delta 7.13(\mathrm{~d}, J=8.0 \mathrm{~Hz}, 2 \mathrm{H}), 6.62(\mathrm{~d}, J=8.8 \mathrm{~Hz}, 2 \mathrm{H}), 4.29(\mathrm{q}, J=7.2$ $\mathrm{Hz}, 3 \mathrm{H}), 3.93$ (s, 2H), 2.87 (sep, $J=6.8 \mathrm{~Hz}, 1 \mathrm{H}), 1.35$ (t, $J=7.2 \mathrm{~Hz}, 3 \mathrm{H}), 1.28$ (d, $J=7.2 \mathrm{~Hz}, 6 \mathrm{H}$ ). ${ }^{13} \mathbf{C}\left\{{ }^{1} \mathbf{H}\right\}$ NMR $\left(101 \mathrm{MHz}, \mathrm{CDCl}_{3}\right) \delta 171.3,145.1,138.5,127.1,113.1,61.1,46.1,33.2,24.2,14.2$. HR-MS (ESI) Calculated for $\mathrm{C}_{13} \mathrm{H}_{20} \mathrm{NO}_{2}\left([\mathrm{M}+\mathrm{H}]^{+}\right)$: 222.1489. Found: 222.1494 . 
Ethyl 2-((4-(tert-butyl)phenyl)amino)acetate (1ad) ${ }^{9}$<smiles>CCOC(=O)CNc1ccc(C(C)(C)C)cc1</smiles>

${ }^{1} \mathbf{H}$ NMR $\left(400 \mathrm{MHz}, \mathrm{CDCl}_{3}\right) \delta 7.21(\mathrm{~d}, J=8.8 \mathrm{~Hz}, 2 \mathrm{H}), 6.55(\mathrm{~d}, J=8.8 \mathrm{~Hz}, 2 \mathrm{H}), 4.26-4.20(\mathrm{~m}, 2 \mathrm{H})$, $4.19(\mathrm{br}, 1 \mathrm{H}), 3.86(\mathrm{~s}, 2 \mathrm{H}), 1.29-1.25(\mathrm{~m}, 12 \mathrm{H}) . \quad{ }^{13} \mathbf{C}\left\{{ }^{1} \mathbf{H}\right\} \mathbf{N M R}\left(101 \mathrm{MHz}, \mathrm{CDCl}_{3}\right) \delta 171.3,144.8$, $140.9,126.1,112.8,61.2,46.2,33.9,31.6,14.2$. HR-MS (ESI) Calculated for $\mathrm{C}_{14} \mathrm{H}_{22} \mathrm{NO}_{2}\left([\mathrm{M}+\mathrm{H}]^{+}\right)$: 236.1645. Found: 236.1652.

Ethyl 2-((3,4-dimethylphenyl)amino)acetate (1ag) ${ }^{7}$<smiles>CCOC(=O)CNc1ccc(C)c(C)c1</smiles>

${ }^{1} \mathrm{H}$ NMR $\left(400 \mathrm{MHz}, \mathrm{CDCl}_{3}\right) \delta 6.95(\mathrm{~d}, J=8.0 \mathrm{~Hz}, 1 \mathrm{H}), 6.46(\mathrm{~s}, 1 \mathrm{H}), 6.39(\mathrm{~d}, J=8.0 \mathrm{~Hz}, 1 \mathrm{H}), 4.24(\mathrm{q}$, $J=7.2 \mathrm{~Hz}, 2 \mathrm{H}), 4.10(\mathrm{br}, 1 \mathrm{H}), 3.88(\mathrm{~s}, 2 \mathrm{H}), 2.20(\mathrm{~s}, 3 \mathrm{H}), 2.15(\mathrm{~s}, 3 \mathrm{H}), 1.30(\mathrm{t}, J=7.2 \mathrm{~Hz}, 3 \mathrm{H}) . \quad{ }^{13} \mathbf{C}\left\{{ }^{1} \mathbf{H}\right\}$ NMR $\left(101 \mathrm{MHz}, \mathrm{CDCl}_{3}\right) \delta 171.5,145.4,137.5,130.4,126.3,115.1,110.6,61.3,46.4,20.1,18.8$, 14.3. HR-MS (ESI) Calculated for $\mathrm{C}_{12} \mathrm{H}_{18} \mathrm{NO}_{2}\left([\mathrm{M}+\mathrm{H}]^{+}\right)$: 208.1332. Found: 208.1338.

Ethyl 2-((3,5-dimethylphenyl)amino)acetate (1ah) ${ }^{1}$<smiles>CCOC(=O)CNc1cc(C)cc(C)c1</smiles>

${ }^{1}$ H NMR $\left(400 \mathrm{MHz}, \mathrm{CDCl}_{3}\right) \delta 6.43(\mathrm{~s}, 1 \mathrm{H}), 6.26(\mathrm{~s}, 2 \mathrm{H}), 4.25(\mathrm{q}, J=7.2 \mathrm{~Hz}, 2 \mathrm{H}), 4.19(\mathrm{~s}, 1 \mathrm{H})$, $3.89(\mathrm{~s}, 2 \mathrm{H}), 2.25(\mathrm{~s}, 6 \mathrm{H}), 1.31(\mathrm{t}, J=7.2 \mathrm{~Hz}, 3 \mathrm{H}) .{ }^{13} \mathbf{C}\left\{{ }^{1} \mathbf{H}\right\} \mathbf{N M R}\left(101 \mathrm{MHz}, \mathrm{CDCl}_{3}\right) \delta 171.4$, 147.3, 139.1, 120.3, 111.1, 61.4, 46.1, 21.6, 14.3. HR-MS (ESI) Calculated for $\mathrm{C}_{12} \mathrm{H}_{18} \mathrm{NO}_{2}$ $\left([\mathrm{M}+\mathrm{H}]^{+}\right):$208.1332. Found: 208.1336. 
Ethyl (4-(methylthio)phenyl)glycinate (1ai)<smiles>CCOC(=O)CNc1ccc(S(C)(=O)=O)cc1</smiles>

Light pink solid, m.p. $=47.4-48.4{ }^{\circ} \mathrm{C} .{ }^{1} \mathbf{H}$ NMR $\left(400 \mathrm{MHz}, \mathrm{CDCl}_{3}\right) \delta 7.22(\mathrm{~d}, J=8.4 \mathrm{~Hz}, 2 \mathrm{H}), 6.55$ (d, $J=8.4 \mathrm{~Hz}, 2 \mathrm{H}), 4.32(\mathrm{br}, 1 \mathrm{H}), 4.24$ (q, $J=7.2 \mathrm{~Hz}, 2 \mathrm{H}), 3.88$ (s, 2H), 2.54-2.26 (m, 3H), 1.29 (t, $J=7.2 \mathrm{~Hz}, 3 \mathrm{H}) .{ }^{13} \mathbf{C}\left\{{ }^{1} \mathbf{H}\right\} \mathbf{N M R}\left(101 \mathrm{MHz}, \mathrm{CDCl}_{3}\right) \delta 171.1,145.9,131.3,125.5,113.7,61.5,45.9$, 19.0, 14.3. IR (neat): 3377, 2918, 1721, 1603, 1500, 1446, 1366, 1345, 1320, 1293, 1251, 1212, 1186, 1147, 1120, 806, 597, $502 \mathrm{~cm}^{-1}$. HR-MS (ESI) Calculated for $\mathrm{C}_{11} \mathrm{H}_{16} \mathrm{NO}_{2} \mathrm{~S}\left([\mathrm{M}+\mathrm{H}]^{+}\right)$: 226.0896. Found: 226.0897. 


\section{Cyclic Voltammetry Studies}

All the voltammetric experiments were recorded with a CHI660E potentiostat at room temperature in $\mathrm{MeCN}^{n}{ }^{n} \mathrm{Bu}_{4} \mathrm{NPF}_{6}(0.1 \mathrm{M})$ was used as the supporting electrolyte, a Pt disk electrode and a platinum wire were used as working and counter electrodes, respectively. The working electrode potentials were measured versus $\mathrm{Ag} / \mathrm{AgNO}_{3}$ reference electrode (internal solution, $0.1 \mathrm{M} \mathrm{AgNO}_{3}$ in $\left.\mathrm{CH}_{3} \mathrm{CN}\right)$. The redox potential of ferrocene/ferrocenium $\left(\mathrm{Fc} / \mathrm{Fc}^{+}\right)$was measured (same experimental conditions) and used to provide an internal reference. The potential values were then adjusted relative to $\mathrm{Fc} / \mathrm{Fc}^{+}$, and electrochemical studies in organic solvents were recorded accordingly. The scan rate was $0.1 \mathrm{~V} \mathrm{~s}^{-1}$.

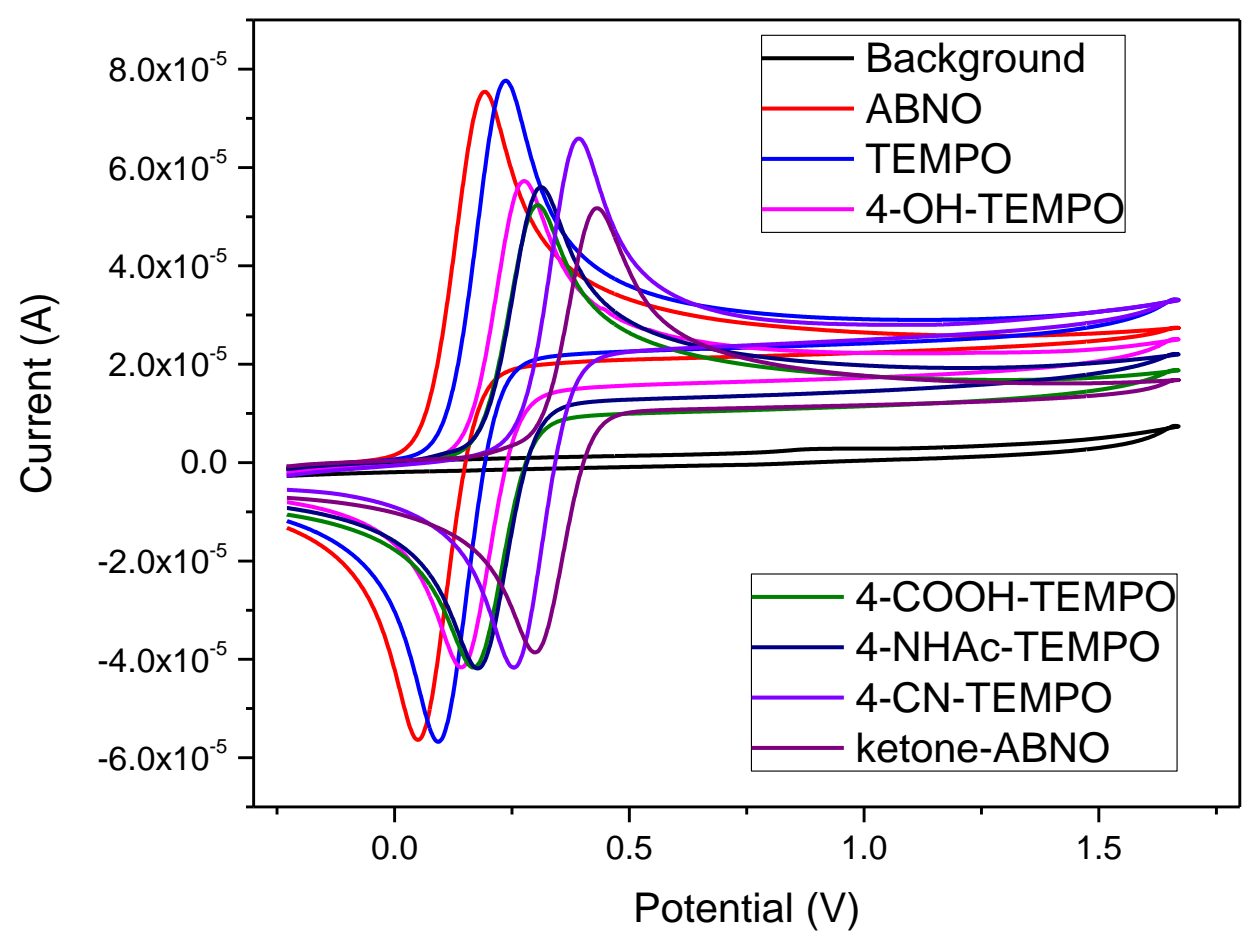

Figure S1. CVs of mediators $(5 \mathrm{mM})$ in acetonitrile with ${ }^{n} \mathrm{Bu}_{4} \mathrm{NPF}_{6}(0.1 \mathrm{M})$ as supporting electrolyte, $\mathrm{Pt}$ as working electrode and a platinum wire as counter electrode, scan rate $=100 \mathrm{mV} / \mathrm{s}$. 
<smiles>CCOC(=O)CNc1ccc(OC)cc1</smiles>

$1 \mathrm{a}$

$E_{o x}=0.295 \mathrm{~V}$<smiles>CC1(C)CC(C(=O)O)CC(C)(C)N1O</smiles>

4-COOH-TEMPO

$\mathrm{E}_{\mathrm{ox}}=0.305 \mathrm{~V}$<smiles>[O]N1C2CCCC1CCC2</smiles>

ABNO

$E_{o x}=0.192 \mathrm{~V}$<smiles>CC(C)(C)N1CC(C)(C)N(O)C(C)(C)C1</smiles>

O.<smiles>CC1(C)CCCC(C)(C)N1O</smiles>

TEMPO

$\mathrm{E}_{\mathrm{ox}}=0.235 \mathrm{~V}$<smiles>CC1(C)CC(C#N)CC(C)(C)N1O</smiles>

4-CN-TEMPO

$\mathrm{E}_{\mathrm{ox}}=0.392 \mathrm{~V}$<smiles>CC1(C)CC(O)CC(C)(C)N1[O]</smiles>

4-OH-TEMPO $E_{o x}=0.276 \mathrm{~V}$<smiles>O=C1CC2CCCC(C1)N2O</smiles>

keto-ABNO $E_{o x}=0.428 \mathrm{~V}$

Scheme S1. Oxidation potential of 1a and mediators.

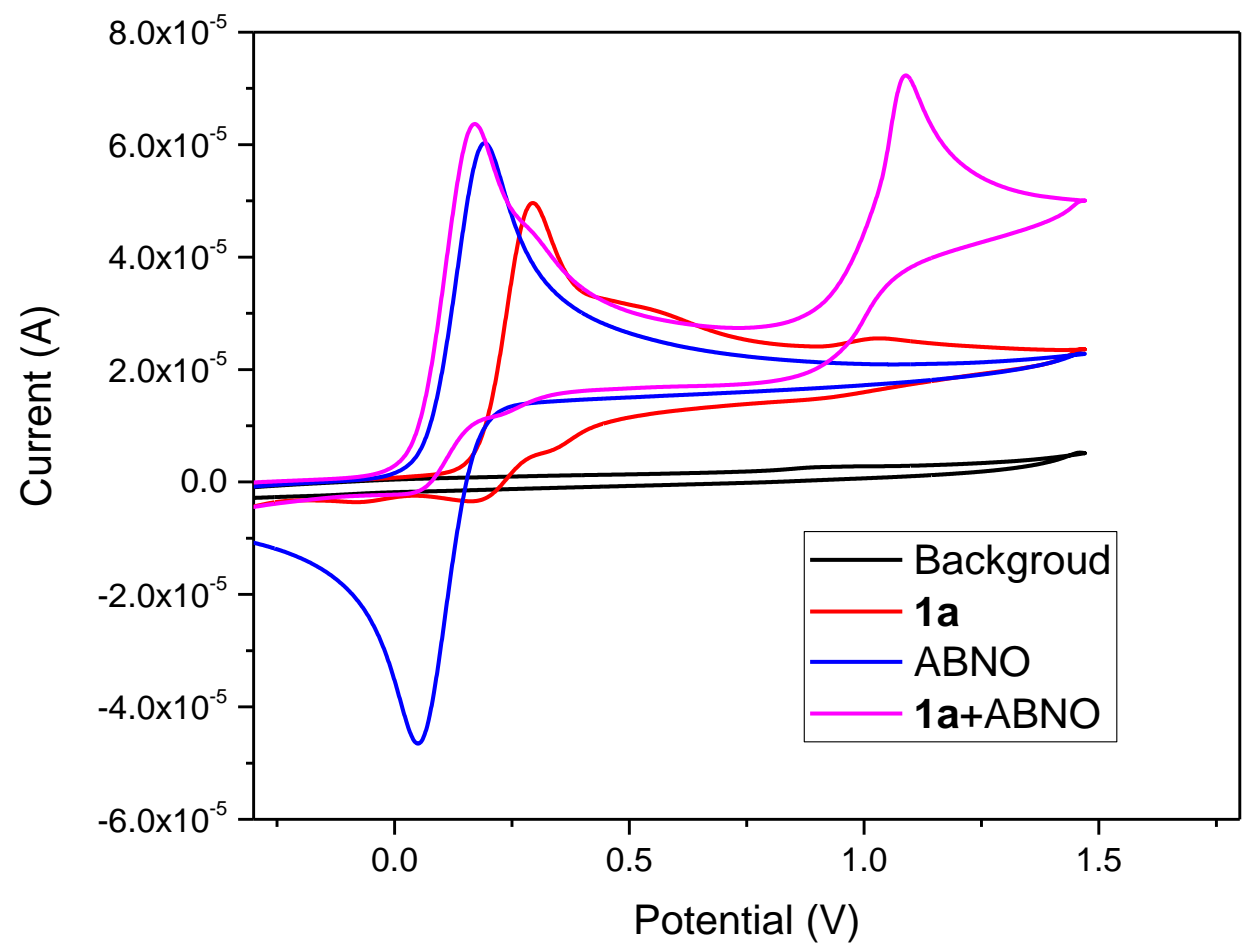

Figure S2. CV analysis on the interaction of $\mathbf{1 a}$ with $\mathrm{ABNO}$ in acetonitrile with ${ }^{n} \mathrm{Bu}_{4} \mathrm{NPF}_{6}(0.1 \mathrm{M})$ as supporting electrolyte, $\mathrm{Pt}$ as working electrode and a platinum wire as counter electrode, scan rate $=100 \mathrm{mV} / \mathrm{s}$. 


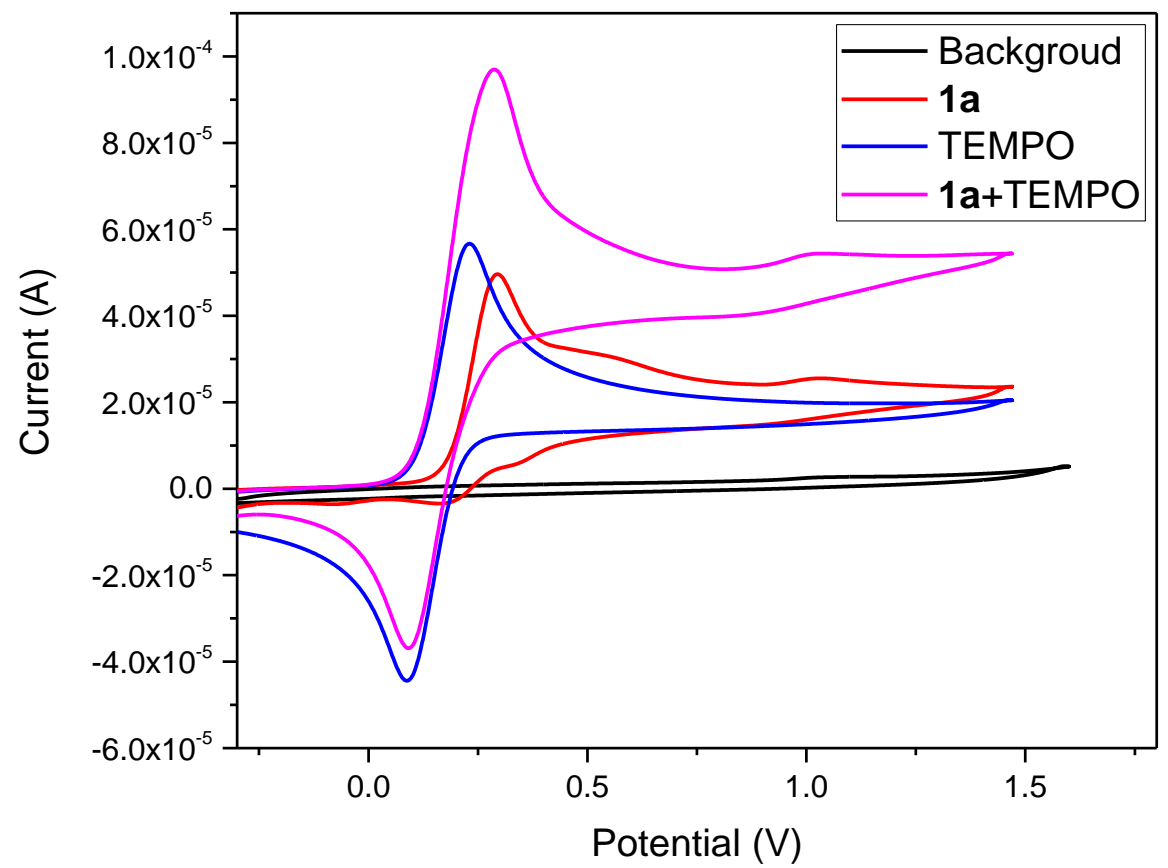

Figure S3. CV analysis on the interaction of $\mathbf{1 a}$ with TEMPO in acetonitrile with ${ }^{n} \mathrm{Bu}_{4} \mathrm{NPF}_{6}(0.1 \mathrm{M})$ as supporting electrolyte, $\mathrm{Pt}$ as working electrode and a platinum wire as counter electrode, scan rate $=100 \mathrm{mV} / \mathrm{s}$.

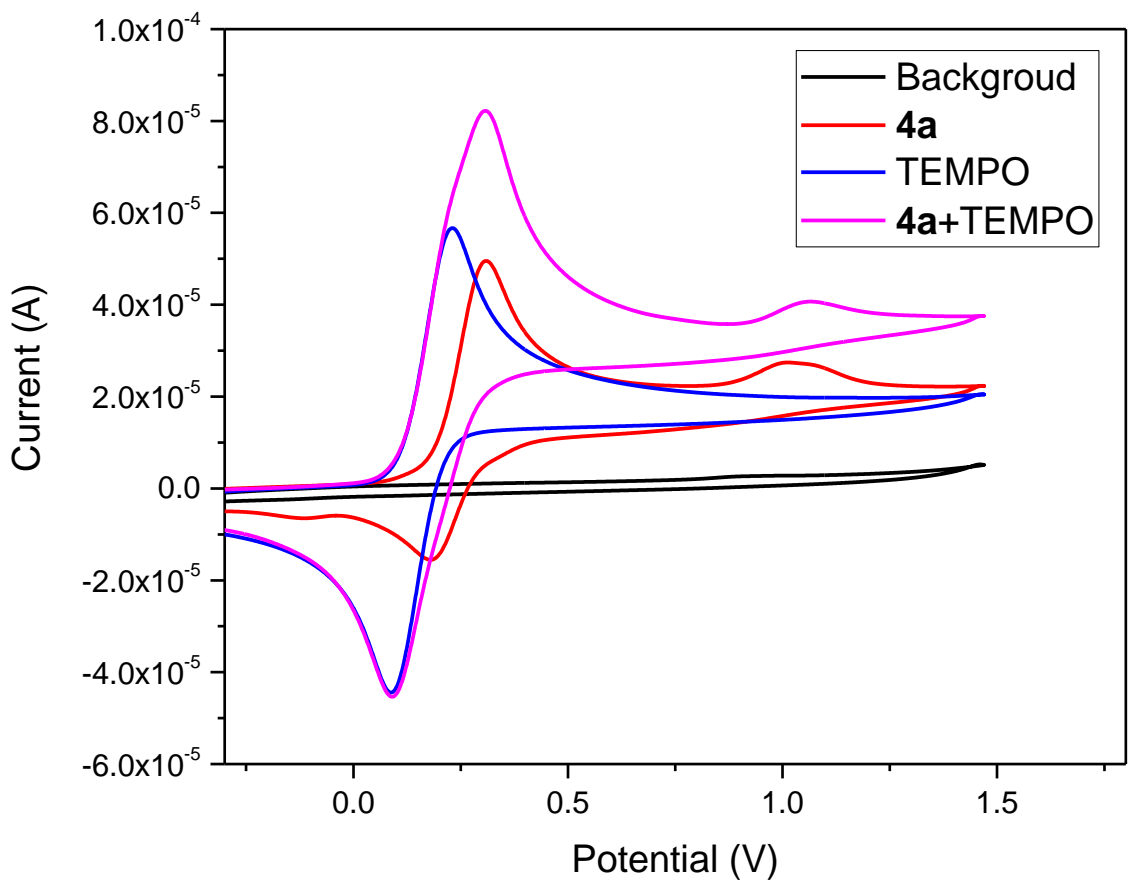

Figure S4. CV analysis on the interaction of $4 \mathbf{a}$ with TEMPO in acetonitrile with ${ }^{n} \mathrm{Bu}_{4} \mathrm{NPF}_{6}(0.1 \mathrm{M})$ as supporting electrolyte, $\mathrm{Pt}$ as working electrode and a platinum wire as counter electrode, scan rate $=100 \mathrm{mV} / \mathrm{s}$. 


\section{Optimization Details}

Table S1. Screening of Organocatalyst ${ }^{a, b}$

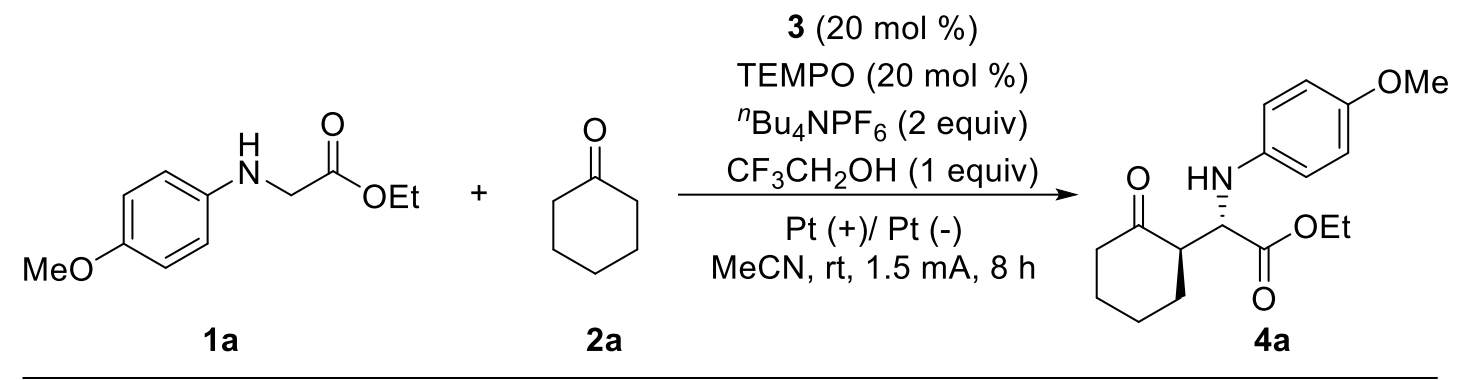

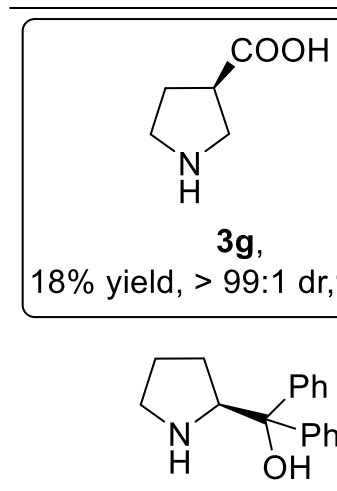

3d, trace<smiles>NCCNC1CCCCC1N</smiles>

3i, n.d.<smiles>[AlH]C1CCNC1</smiles>

3a, n.d.<smiles>O=C(O)C1CCCN1</smiles>

3e, n.d.<smiles>NC(c1ccccc1)C(N=P)c1ccccc1</smiles>

3j, n.d.<smiles>CN1C(=O)C(Cc2ccccc2)NC1(C)C</smiles>

3b, n.d.<smiles>O=C(O)[C@H]1CC(O)CN1</smiles>

3f, trace<smiles>COC(c1ccccc1)(c1ccccc1)C1CCCN1</smiles>

3c, trace<smiles>O=C(O)C1CCCNC1</smiles>

3h, n.d.

${ }^{a}$ Reaction conditions: 1a $(0.2 \mathrm{mmol}), \mathbf{2 a}\left(5\right.$ equiv), 3 (20 mol \%), TEMPO (20 mol \%), ${ }^{n} \mathrm{Bu}_{4} \mathrm{NPF}_{6}$ ( 2 equiv), $\mathrm{CF}_{3} \mathrm{CH}_{2} \mathrm{OH}$ (1 equiv), $\mathrm{MeCN}(4 \mathrm{~mL}$ ) in an undivided cell with two platinum electrodes (each $1.0^{*} 1.0 \mathrm{~cm}^{2}$ ) at $\mathrm{rt}$ in $1.5 \mathrm{~mA}$ for $8 \mathrm{~h} .{ }^{b}$ Yields were determined by ${ }^{1} \mathrm{H} \mathrm{NMR}$ using $\mathrm{CH}_{2} \mathrm{Br}_{2}$ as an internal standard. Enantioselectivities were determined by chiral HPLC analysis. 
Table S2. Screening of Solvent ${ }^{a, b}$

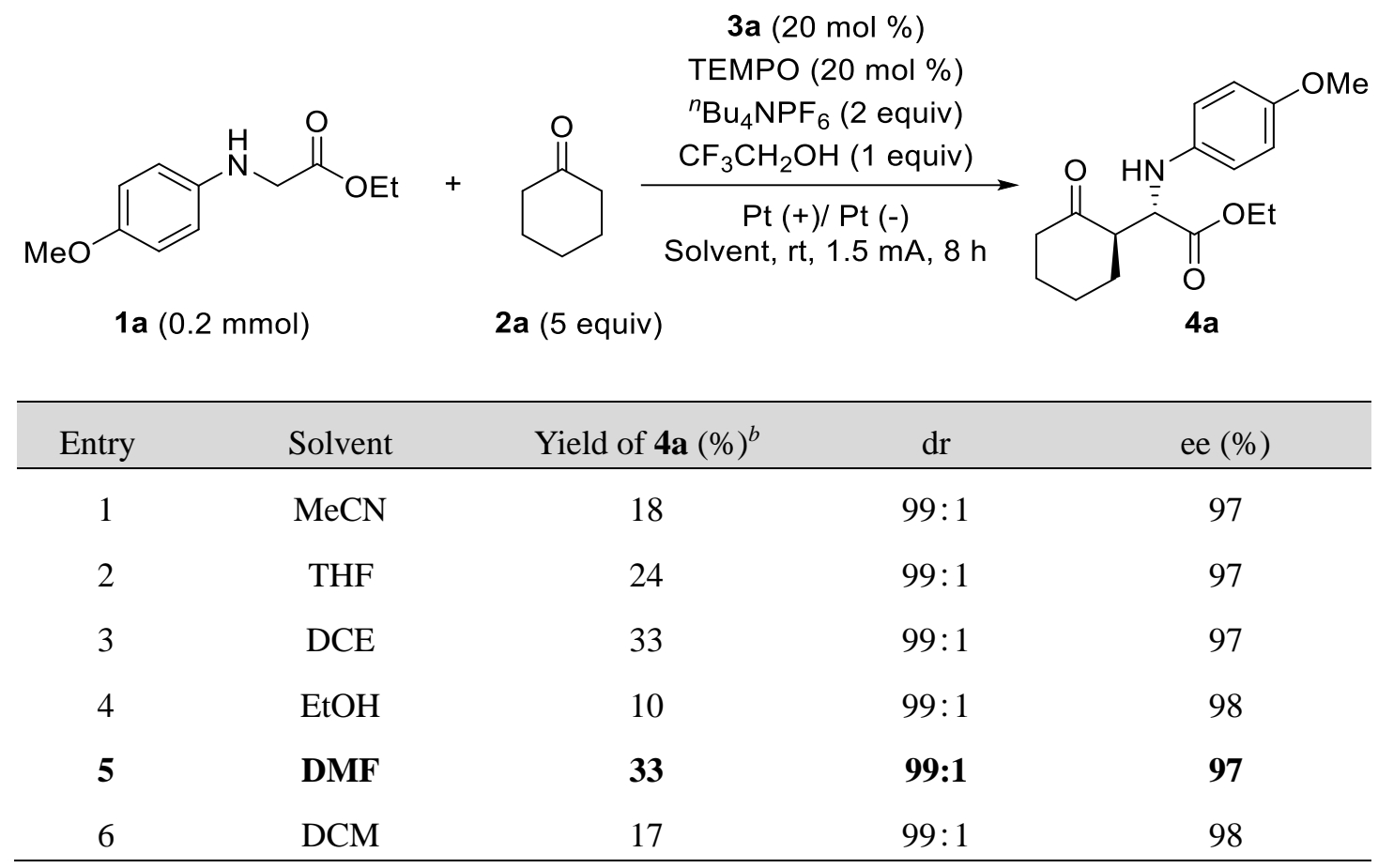

${ }^{a}$ Reaction conditions: 1a $(0.2 \mathrm{mmol}), \mathbf{2 a}\left(5\right.$ equiv), $\mathbf{3 a}(20 \mathrm{~mol} \%)$, TEMPO $(20 \mathrm{~mol} \%),{ }^{n} \mathrm{Bu}_{4} \mathrm{NPF}_{6}$ (2 equiv), $\mathrm{CF}_{3} \mathrm{CH}_{2} \mathrm{OH}$ (1 equiv), solvent ( $4 \mathrm{~mL}$ ) in an undivided cell with two platinum electrodes (each $1.0^{*} 1.0 \mathrm{~cm}^{2}$ ) at $\mathrm{rt}$ in $1.5 \mathrm{~mA}$ for $8 \mathrm{~h} .{ }^{b}$ Yields were determined by ${ }^{1} \mathrm{H} \mathrm{NMR}$ using $\mathrm{CH}_{2} \mathrm{Br}_{2}$ as an internal standard. Enantioselectivities were determined by chiral HPLC analysis. 
Table S3. Screening of Electrolyte ${ }^{a, b}$

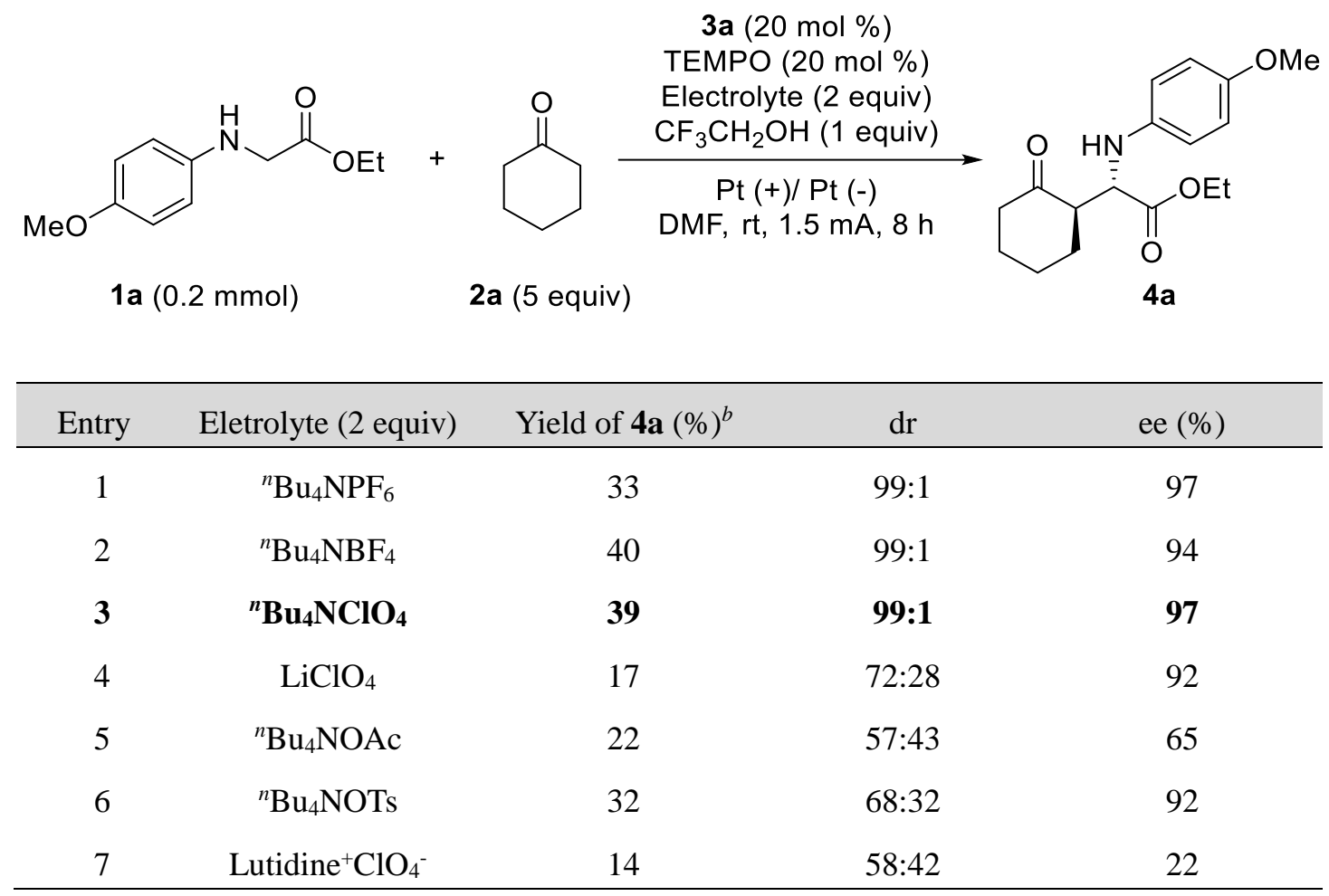

${ }^{a}$ Reaction conditions: 1a $(0.2 \mathrm{mmol}), \mathbf{2 a}(5$ equiv), $\mathbf{3 a}(20 \mathrm{~mol} \%)$, TEMPO (20 mol \%), electrolyte (2 equiv), $\mathrm{CF}_{3} \mathrm{CH}_{2} \mathrm{OH}$ (1 equiv), DMF ( $4 \mathrm{~mL}$ ) in an undivided cell with two platinum electrodes (each $1.0 * 1.0 \mathrm{~cm}^{2}$ ) at $\mathrm{rt}$ in $1.5 \mathrm{~mA}$ for $8 \mathrm{~h} .{ }^{b}$ Yields were determined by ${ }^{1} \mathrm{H} \mathrm{NMR}$ using $\mathrm{CH}_{2} \mathrm{Br}_{2}$ as an internal standard. Enantioselectivities were determined by chiral HPLC analysis. 
Table S4. Screening the Amount of $\mathrm{CF}_{3} \mathrm{CH}_{2} \mathrm{OH}^{a, b}$

\begin{tabular}{|c|c|c|c|c|}
\hline $\mathrm{eO}^{-}$ & 2a $(5$ & 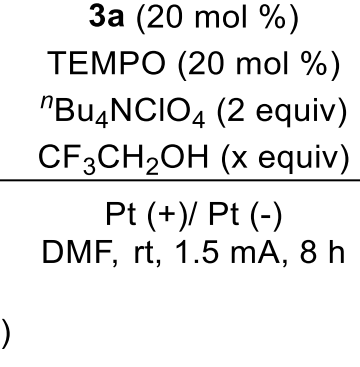 & & a \\
\hline Entry & $\mathrm{CF}_{3} \mathrm{CH}_{2} \mathrm{OH}$ (x equiv) & Yield of $\mathbf{4 a}(\%)^{b}$ & $\mathrm{dr}$ & ee $(\%)$ \\
\hline 1 & $\mathbf{0}$ & 43 & 99:1 & 97 \\
\hline 2 & 1 & 39 & $99: 1$ & 97 \\
\hline 3 & 2 & 41 & $99: 1$ & 98 \\
\hline 4 & 3 & 41 & $99: 1$ & 99 \\
\hline 5 & 4 & 45 & $99: 1$ & 96 \\
\hline 6 & 5 & 19 & $99: 1$ & 98 \\
\hline 7 & 10 & 24 & $99: 1$ & 97 \\
\hline 8 & 20 & 24 & $99: 1$ & 97 \\
\hline 9 & 66 & 14 & $99: 1$ & 97 \\
\hline
\end{tabular}

${ }^{a}$ Reaction conditions: 1a $(0.2 \mathrm{mmol}), \mathbf{2 a}\left(5\right.$ equiv), 3a (20 mol \%), TEMPO (20 mol \%), ${ }^{n} \mathrm{Bu}_{4} \mathrm{NClO}_{4}$ (2 equiv), $\mathrm{CF}_{3} \mathrm{CH}_{2} \mathrm{OH}$ (x equiv), DMF (4 mL) in an undivided cell with two platinum electrodes (each $1.0 * 1.0 \mathrm{~cm}^{2}$ ) at $\mathrm{rt}$ in $1.5 \mathrm{~mA}$ for $8 \mathrm{~h} .{ }^{b}$ Yields were determined by ${ }^{1} \mathrm{H} \mathrm{NMR}$ using $\mathrm{CH}_{2} \mathrm{Br}_{2}$ as an internal standard. Enantioselectivities were determined by chiral HPLC analysis. 
Table S5. Screening the Amount of 3a and TEMPO ${ }^{a, b}$

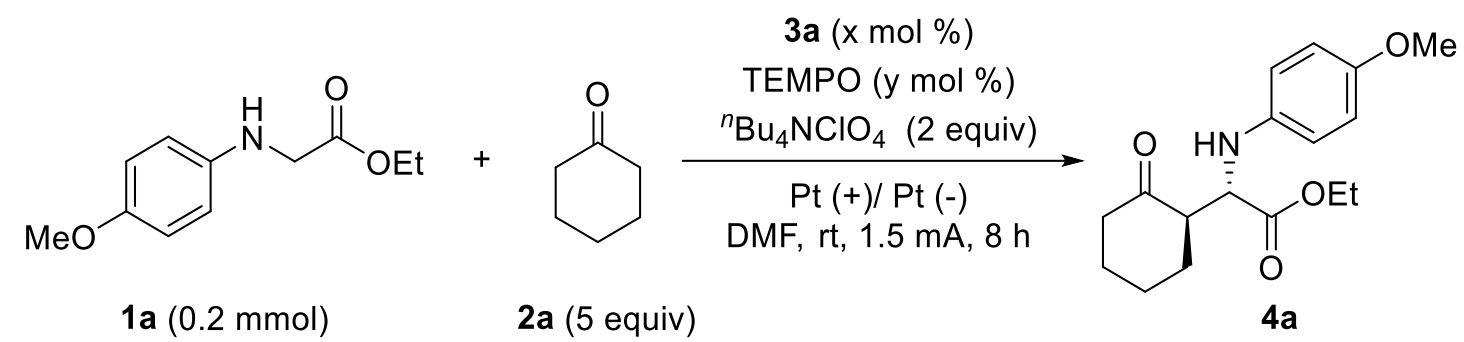

\begin{tabular}{cccccc}
\hline Entry & $\mathbf{3 a}(\mathrm{x} \mathrm{mol} \mathrm{\% )}$ & TEMPO $(\mathrm{y} \mathrm{mol} \mathrm{\% )}$ & Yield of $\mathbf{4 a}(\%)^{b}$ & $\mathrm{dr}$ & ee $(\%)$ \\
\hline $\mathbf{1}$ & $\mathbf{2 0}$ & $\mathbf{1 0}$ & $\mathbf{4 8}$ & $\mathbf{9 9 : 1}$ & $\mathbf{9 7}$ \\
2 & 20 & 20 & 43 & $99: 1$ & 97 \\
3 & 20 & 30 & 28 & $99: 1$ & 97 \\
4 & 10 & 20 & 22 & $99: 1$ & 93 \\
5 & 30 & 20 & 30 & $99: 1$ & 95 \\
\hline
\end{tabular}

${ }^{a}$ Reaction conditions: 1a $(0.2 \mathrm{mmol}), \mathbf{2 a}\left(5\right.$ equiv), 3a (x mol \%), TEMPO (y mol \%), ${ }^{n} \mathrm{Bu}_{4} \mathrm{NClO}_{4}$ (2 equiv), DMF ( $4 \mathrm{~mL}$ ) in an undivided cell with two platinum electrodes (each $1.0 * 1.0 \mathrm{~cm}^{2}$ ) at $\mathrm{rt}$ in $1.5 \mathrm{~mA}$ for $8 \mathrm{~h} .{ }^{b}$ Yields were determined by ${ }^{1} \mathrm{H}$ NMR using $\mathrm{CH}_{2} \mathrm{Br}_{2}$ as an internal standard. Enantioselectivities were determined by chiral HPLC analysis.

Table S6. Screening the Amount of ${ }^{n} \mathrm{Bu}_{4} \mathrm{NClO}_{4}{ }^{a, b}$

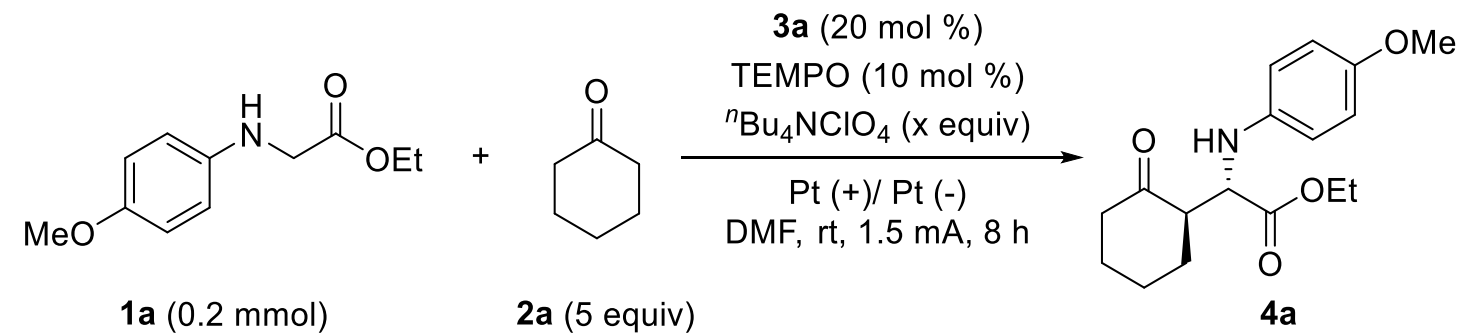

\begin{tabular}{ccccc}
\hline Entry & ${ }^{n} \mathrm{Bu}_{4} \mathrm{NClO}_{4}$ (x equiv) & Yield of $\mathbf{4 a}(\%)^{b}$ & $\mathrm{dr}$ & ee (\%) \\
\hline $\mathbf{1}$ & $\mathbf{0}$ & $\mathbf{6 0}$ & $\mathbf{9 9 : 1}$ & $\mathbf{9 7}$ \\
2 & 1 & 38 & $99: 1$ & 97 \\
3 & 2 & 48 & $99: 1$ & 97 \\
4 & 3 & 39 & $99: 1$ & 97 \\
\hline
\end{tabular}

${ }^{a}$ Reaction conditions: 1a $(0.1 \mathrm{mmol}), \mathbf{2 a}\left(5\right.$ equiv), 3a (20 mol \%), TEMPO $(10 \mathrm{~mol} \%),{ }^{n} \mathrm{Bu}_{4} \mathrm{NClO}_{4}$ (x equiv), DMF (4 mL) in an undivided cell with two platinum electrodes (each $1.0 * 1.0 \mathrm{~cm}^{2}$ ) at $\mathrm{rt}$ in $1.5 \mathrm{~mA}$ for 8 h. ${ }^{b}$ Yields were determined by ${ }^{1} \mathrm{H}$ NMR using $\mathrm{CH}_{2} \mathrm{Br}_{2}$ as an internal standard. Enantioselectivities were determined by chiral HPLC analysis. 
Table S7. Screening the Amount of $2^{a, b}$

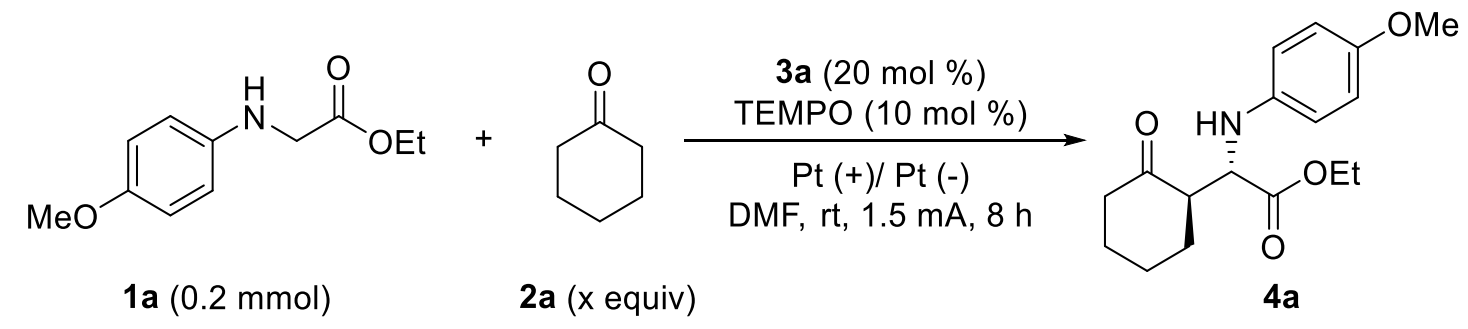

\begin{tabular}{ccccc}
\hline Entry & $\mathbf{2}$ (x equiv) & Yield of $\mathbf{4 a}(\%)^{b}$ & $\mathrm{dr}$ & ee (\%) \\
\hline 1 & 4 & 44 & $78: 22$ & 97 \\
2 & 5 & 60 & $97: 3$ & 97 \\
3 & 8 & 60 & $99: 1$ & 97 \\
$\mathbf{4}$ & $\mathbf{1 0}$ & $\mathbf{6 6}$ & $\mathbf{9 9 : 1}$ & $\mathbf{9 7}$ \\
5 & 15 & 55 & $99: 1$ & 97 \\
\hline
\end{tabular}

${ }^{a}$ Reaction conditions: 1a $(0.1 \mathrm{mmol}), \mathbf{2 a}$ (x equiv), 3a (20 mol \%), TEMPO (10 mol \%), DMF (4 $\mathrm{mL}$ ) in an undivided cell with two platinum electrodes (each $1.0 * 1.0 \mathrm{~cm}^{2}$ ) at $\mathrm{rt}$ in $1.5 \mathrm{~mA}$ for $8 \mathrm{~h}$. ${ }^{b}$ Yields were determined by ${ }^{1} \mathrm{H}$ NMR using $\mathrm{CH}_{2} \mathrm{Br}_{2}$ as an internal standard. Enantioselectivities were determined by chiral HPLC analysis. 
Table S8. Screening of Solvent $t^{a, b}$

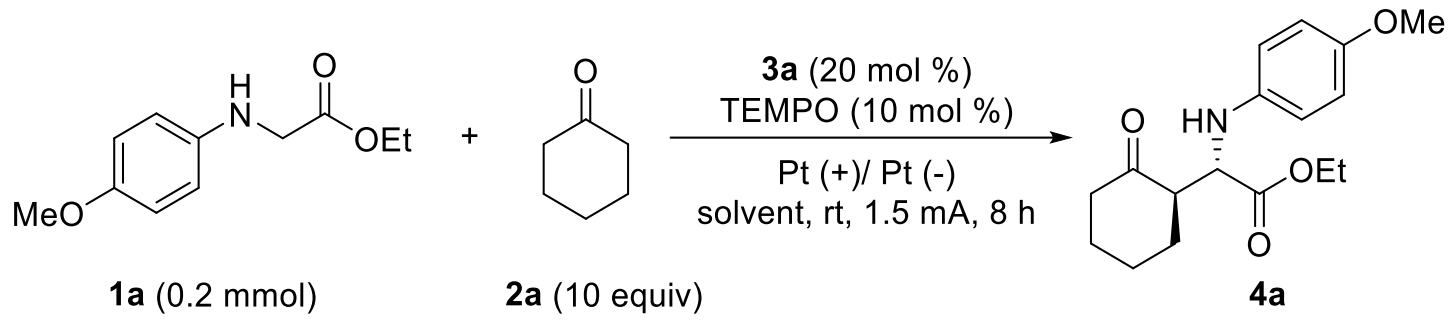

\begin{tabular}{ccccc}
\hline Entry & solvent & Yield of $\mathbf{4 a}(\%)^{b}$ & $\mathrm{dr}$ & ee (\%) \\
\hline 1 & DMF & 66 & $99: 1$ & 97 \\
2 & DMA & 25 & $94: 6$ & 98 \\
3 & DMI & 23 & $78: 22$ & 97 \\
4 & NMP & 24 & $61: 39$ & 91 \\
$5^{c}$ & DMF & 75 & $99: 1$ & 98 \\
$6^{d}$ & DMSO & $75(70)^{e}$ & $99: 1$ & 97 \\
\hline
\end{tabular}

${ }^{a}$ Reaction conditions: 1a $(0.2 \mathrm{mmol})$, 2a (10 equiv), 3a (20 mol \%), TEMPO (10 mol \%), solvent (4 $\mathrm{mL}$ ) in an undivided cell with two platinum electrodes (each $1.0 * 1.0 \mathrm{~cm}^{2}$ ) at $\mathrm{rt}$ in $1.5 \mathrm{~mA}$ for $8 \mathrm{~h}$. ${ }^{b}$ Yields were determined by ${ }^{1} \mathrm{H}$ NMR using $\mathrm{CH}_{2} \mathrm{Br}_{2}$ as an internal standard. Enantioselectivities were determined by chiral HPLC analysis. ${ }^{c} 3 \mathrm{a}(40 \mathrm{~mol} \%)$. ${ }^{d}$ Performed in $0.1 \mathrm{mmol}$ scale, $5 \mathrm{~h}$. ${ }^{e}$ Isolated yield was obtained by average of two parallel reactions. 
Table S9. Screening of Chemical Oxidant ${ }^{a, b}$<smiles>CCOC(=O)CNc1ccc(OC)cc1</smiles>

\begin{tabular}{ccccc}
\hline Entry & Chemical Oxidant (2 equiv) & Yield of $\mathbf{4 a}(\%)^{b}$ & $\mathrm{dr}$ & ee (\%) \\
\hline 1 & $\mathrm{DDQ}$ & 32 & $61: 39$ & 6 \\
2 & $\mathrm{PhI}(\mathrm{OAc})_{2}$ & 0 & - & - \\
3 & $\mathrm{Ag}_{2} \mathrm{CO}_{3}$ & 0 & - & - \\
4 & $\mathrm{Cu}(\mathrm{OAc})_{2} \bullet \mathrm{H}_{2} \mathrm{O}$ & trace & - & - \\
$6^{d}$ & $\mathrm{TEMPO}^{+} \mathrm{BF}_{4}^{-}$ & 26 & $63: 37$ & 13 \\
\hline
\end{tabular}

${ }^{a}$ Reaction conditions: 1a $(0.1 \mathrm{mmol}), \mathbf{2 a}(10$ equiv), 3a (20 mol \%), TEMPO (10 mol \%), DMSO $(1 \mathrm{~mL})$ in an $10 \mathrm{~mL}$ tube at $\mathrm{rt}$ for $60 \mathrm{~h} .{ }^{b}$ Yields were determined by ${ }^{1} \mathrm{H} \mathrm{NMR}$ using $\mathrm{CH}_{2} \mathrm{Br}_{2}$ as an internal standard. Enantioselectivities were determined by chiral HPLC analysis. 


\section{Unsuccessful Substrates}<smiles>CCOC(=O)C(Nc1ccccc1OC)C1CCCCC1=O</smiles>

$0 \%$<smiles>CCOC(=O)C(Nc1ccc(Oc2ccccc2)cc1)C1CCCCC1=O</smiles>

$30 \%$<smiles>CCOC(=O)C(Nc1cccc(C(=O)OC)c1)C1CCCCC1=O</smiles>

$18 \%$<smiles>CCOC(=O)C(Nc1ccc2ccccc2c1)C1CCCCC1=O</smiles>

$0 \%$<smiles>CCOC(=O)C(Nc1cccc(OC)c1)C1CCCCC1=O</smiles>

$21 \%$

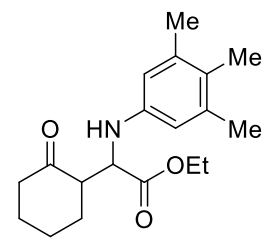

$0 \%$

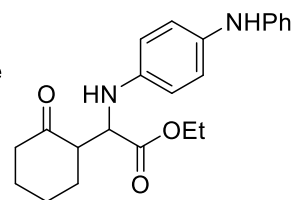

$45 \%,>99: 1 \mathrm{dr}, 65 \%$ ee

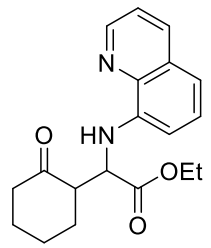

$17 \%$<smiles>CCOC(=O)C(Nc1ccc(OC)c(OC)c1)C1CCCCC1=O</smiles>

$0 \%$<smiles>CCOC(=O)C(Nc1ccc(Cl)cc1)C1CCCCC1=O</smiles>

trace

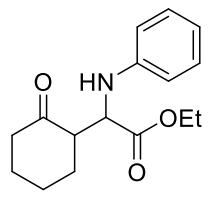

$23 \%$<smiles>CCOC(=O)C(NC12CC3CC(CC(C3)C1)C2)C1CCCCC1=O</smiles>

$0 \%$

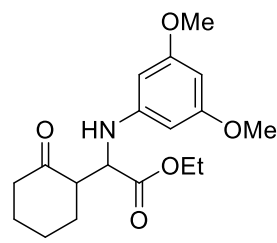

$0 \%$

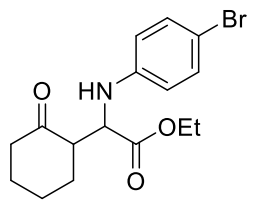

$28 \%$

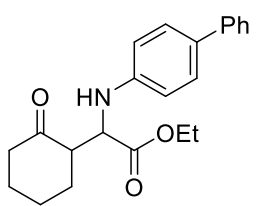

$22 \%$<smiles>CCOC(=O)C(Nc1ccc2c(c1)OCO2)C1CCCCC1=O</smiles>

$0 \%$

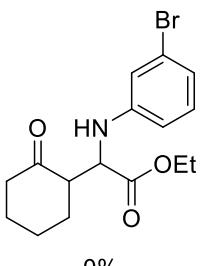

$0 \%$<smiles>CCOC(=O)C(Nc1cccc2ccccc12)C1CCCCC1=O</smiles>

$0 \%$

Scheme S2. Unsuccessful glycine esters.<smiles>CC(C)=O</smiles><smiles>CCC(=O)CC</smiles>

$0 \%$

$0 \%$<smiles>O=C1CCCC1</smiles>

$0 \%$<smiles>CC(=O)c1ccccc1</smiles>

$0 \%$<smiles>CC(=O)/C=C/c1ccccc1</smiles>

$0 \%$

Scheme S3. Unsuccessful ketones. 
<smiles>CCOC(=O)CNc1ccc(OC)cc1</smiles>

$1 a$<smiles>O=C1CCCC1</smiles>

cat. $(20 \mathrm{~mol} \%)$ TEMPO (10 mol \%)

$\mathrm{Pt}(+) / \mathrm{Pt}(-)$

DMSO, rt, $1.5 \mathrm{~mA}, 5 \mathrm{~h}$<smiles>CCOC(=O)[C@H](Nc1ccc(OC)cc1)[C@H]1CCCC1=O</smiles>

$6 a$<smiles>CC(C)(C)CC(N)C(=O)O</smiles><smiles>CCCC(N)C(=O)O</smiles><smiles>CCCCC(N)C(=O)O</smiles>

n.d<smiles>NC(C(=O)O)C(=O)O</smiles><smiles>NC(=[GeH2])C(=O)O</smiles><smiles>NC1CCCCC1NP</smiles><smiles>NC(c1ccccc1)[C@@H](N=P)c1ccccc1</smiles>

n.d

n.d.

n.d.

Scheme S4. Primary amine-catalyzed asymmetric Shono-type oxidative coupling with cyclopentanone.<smiles>CCOC(=O)CNc1cccc2ccccc12</smiles>

$\mathrm{E}_{\mathrm{ox}}=0.408 \mathrm{~V}$<smiles>CCOC(=O)CNC12CC3CC(CC(C3)C1)C2</smiles>

$E_{o x}=0.904 V$
$\mathrm{R}=4-\mathrm{OPh}, \mathrm{E}_{\mathrm{ox}}=0.421 \mathrm{~V}$

$\mathrm{R}=3,5-\mathrm{OMe}, \mathrm{E}_{\mathrm{ox}}=0.503 \mathrm{~V}$

$\mathrm{R}=4-\mathrm{Ph}, \mathrm{E}_{\mathrm{ox}}=0.506 \mathrm{~V}$

$\mathrm{R}=4-\mathrm{Br}, \mathrm{E}_{\mathrm{ox}}=0.642 \mathrm{~V}$

$\mathrm{R}=\mathrm{CO}_{2} \mathrm{Me}, \mathrm{E}_{\mathrm{ox}}=0.791 \mathrm{~V}$

Scheme S5. Selected examples for oxidation potential of unsuccessful glycine esters. 


\section{Synthetic Procedures and Characterization Data}

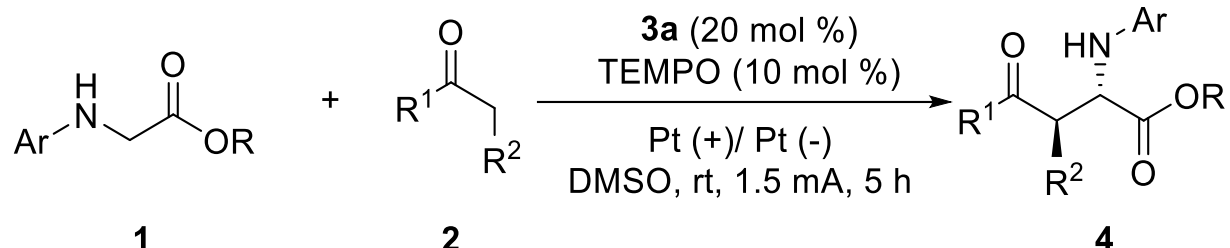

To a $15 \mathrm{~mL}$ hydrogenation tube charged with a proper stir bar was added $\mathbf{1}(0.1 \mathrm{mmol})$, TEMPO (0.01 mmol, $10 \mathrm{~mol} \%, 1.6 \mathrm{mg}), 3 a(0.02 \mathrm{mmol}, 20 \mathrm{~mol} \%, 2.4 \mathrm{mg}), 2$ (1 mmol, 10 equiv), and DMSO $(4 \mathrm{~mL})$. The tube was sealed with a septum equipped with two Pt electrodes $\left(1.0 \times 1.0 \mathrm{~cm}^{2}\right)$ and the reaction mixture was electrolyzed under a constant current of $1.5 \mathrm{~mA}$ for $5 \mathrm{~h}$. After the reaction was completed, the mixture was diluted with $20 \mathrm{~mL}$ of water and extracted with EtOAc $(3 \times 4 \mathrm{~mL})$. The organic layer was washed with saturated $\mathrm{NaCl}$ (aq.) $(10 \mathrm{~mL})$, dried over $\mathrm{Na}_{2} \mathrm{SO}_{4}$, filtered, and the solvent removed under reduced pressure. The crude product was purified by flash column chromatography (eluent: Petroleum ether: EtOAc= 5:1), affording the desired product 4 .

Ethyl 2-((4-methoxyphenyl)amino)-2-(2-oxocyclohexyl)acetate (4a) ${ }^{2}$<smiles>CCOC(=O)C(Nc1ccc(OC)cc1)C1CCCCC1=O</smiles>

Purification: column chromatography $\left(n-\mathrm{Hexane} / \mathrm{EtOAc}=5: 1, R_{\mathrm{f}}=0.39\right)$. Pale yellow oil. Isolated yield was $70 \%(21.5 \mathrm{mg}) . \quad{ }^{1} \mathrm{H}$ NMR $\left(400 \mathrm{MHz}, \mathrm{CDCl}_{3}\right) \delta 6.76(\mathrm{~d}, J=8.8 \mathrm{~Hz}, 2 \mathrm{H}), 6.63(\mathrm{~d}, J=$ $8.8 \mathrm{~Hz}, 2 \mathrm{H}), 4.29(\mathrm{br}, 1 \mathrm{H}), 4.20-4.08(\mathrm{~m}, 2 \mathrm{H}), 3.99(\mathrm{~d}, J=4.0 \mathrm{~Hz}, 1 \mathrm{H}), 3.73(\mathrm{~s}, 3 \mathrm{H}), 3.13-3.07(\mathrm{~m}$, 1H), 2.48-2.25 (m, 2H), $2.07(\mathrm{~m}, 2 \mathrm{H}), 1.99-1.85(\mathrm{~m}, 2 \mathrm{H}), 1.77-1.62(\mathrm{~m}, 2 \mathrm{H}), 1.21(\mathrm{t}, J=7.2 \mathrm{~Hz}$, 3H). ${ }^{13} \mathbf{C}\left\{{ }^{1} \mathbf{H}\right\}$ NMR $\left(101 \mathrm{MHz}, \mathrm{CDCl}_{3}\right) \delta 211.1,173.2,152.9,142.3,115.8,114.9,61.3,59.2$, $55.8,53.7,42.0,30.7,27.0,24.7,14.3 \cdot[\alpha]_{\mathrm{D}}^{27}=28.4\left(\mathrm{c} 0.5, \mathrm{CHCl}_{3}\right)$. The enantiomeric excess $(97 \%$ ee) was determined by HPLC with a Daicel Chiralpak AS-H column (Hexane/i-PrOH = 90:10, flow rate: $0.7 \mathrm{~mL} / \mathrm{min}, \lambda \max 214 \mathrm{~nm}): \mathrm{t}_{\mathrm{R}}$ (anti major enantiomer) $=15.19 \mathrm{~min} ; \mathrm{t}_{\mathrm{R}}($ anti minor enantiomer) $=21.33 \mathrm{~min}$. 


\section{Methyl 2-((4-methoxyphenyl)amino)-2-(2-oxocyclohexyl)acetate (4b) ${ }^{2}$}<smiles>COC(=O)C(Nc1ccc(OC)cc1)C1CCCCC1=O</smiles>

Purification: column chromatography $\left(n-\mathrm{Hexane} / \mathrm{EtOAc}=5: 1, R_{\mathrm{f}}=0.47\right)$. Pale yellow oil. Isolated yield was $60 \%(17.5 \mathrm{mg}) . \quad{ }^{1} \mathrm{H}$ NMR $\left(400 \mathrm{MHz}, \mathrm{CDCl}_{3}\right) \delta 6.76(\mathrm{~d}, J=8.8 \mathrm{~Hz}, 2 \mathrm{H}), 6.63(\mathrm{~d}, J=$ $8.8 \mathrm{~Hz}, 2 \mathrm{H}), 4.26(\mathrm{br}, 1 \mathrm{H}), 3.99(\mathrm{~d}, J=4.0 \mathrm{~Hz}, 1 \mathrm{H}), 3.73$ (s, 3H), 3.68 (s, 3H), 3.15-3.10 (m, 1H), 2.45-2.40 (m, 1H), 2.38-2.27 (m, 1H), 2.19-2.03 (m, 2H), 1.97-1.86 (m, 2H), 1.79-1.63 (m, 2H). ${ }^{13} \mathbf{C}\left\{{ }^{1} \mathbf{H}\right\}$ NMR $\left(101 \mathrm{MHz}, \mathrm{CDCl}_{3}\right) \delta 211.3,173.8,152.9,142.2,115.6,115.0,59.1,55.9,53.8,52.5$, 42.0, 30.8, 27.0, 24.8. $[\alpha]_{\mathrm{D}}{ }^{24}=32.5\left(\mathrm{c} 0.1, \mathrm{CHCl}_{3}\right)$. The enantiomeric excess $(89 \%$ ee) was determined by HPLC with a Daicel Chiralpak AS-H column (Hexane/i-PrOH $=90: 10$, flow rate: $0.7 \mathrm{~mL} / \mathrm{min}, \lambda \max 214 \mathrm{~nm}): t_{R}\left(\right.$ anti major enantiomer) $=20.12 \mathrm{~min} ; t_{R}$ (anti minor enantiomer $)=29.70 \mathrm{~min}$.

Propyl 2-((4-methoxyphenyl)amino)-2-(2-oxocyclohexyl)acetate (4c)<smiles>CCCOC(=O)C(Nc1ccc(OC)cc1)C1CCCCC1=O</smiles>

Purification: column chromatography $\left(n-\mathrm{Hexane} / \mathrm{EtOAc}=5: 1, R_{\mathrm{f}}=0.29\right)$. Pale yellow oil. Isolated yield was $58 \%(18.5 \mathrm{mg}) .{ }^{1} \mathbf{H}$ NMR $\left(400 \mathrm{MHz}, \mathrm{CDCl}_{3}\right) \delta 6.76(\mathrm{~d}, J=8.8 \mathrm{~Hz}, 2 \mathrm{H}), 6.63(\mathrm{~d}, J=8.8$ $\mathrm{Hz}, 2 \mathrm{H}), 4.24(\mathrm{br}, 1 \mathrm{H}), 4.04(\mathrm{td}, J=6.4,2.0 \mathrm{~Hz}, 2 \mathrm{H}), 3.99$ (d, $J=4.0 \mathrm{~Hz}, 1 \mathrm{H}), 3.73(\mathrm{~s}, 3 \mathrm{H}), 3.14-$ $3.08(\mathrm{~m}, 1 \mathrm{H}), 2.48-2.39(\mathrm{~m}, 1 \mathrm{H}), 2.36-2.28(\mathrm{~m}, 1 \mathrm{H}), 2.14-2.02(\mathrm{~m}, 2 \mathrm{H}), 1.99-1.85(\mathrm{~m}, 2 \mathrm{H}), 1.78-$ $1.56(\mathrm{~m}, 4 \mathrm{H}), 0.86(\mathrm{t}, J=7.6 \mathrm{~Hz}, 3 \mathrm{H}) .{ }^{13} \mathbf{C}\left\{{ }^{1} \mathbf{H}\right\} \mathbf{N M R}\left(101 \mathrm{MHz}, \mathrm{CDCl}_{3}\right) \delta 211.1,173.3,152.9$, 142.3, 115.7, 114.9, 66.9, 59.2, 55.9, 53.7, 42.0, 30.7, 27.0, 24.7, 22.0, 10.5. IR (neat): 2936, 1705, 1511, 1464, 1232, 1148, 1036, 821, $750 \mathrm{~cm}^{-1}$. HR-MS (ESI) Calculated for $\mathrm{C}_{18} \mathrm{H}_{26} \mathrm{NO}_{4}\left([\mathrm{M}+\mathrm{H}]^{+}\right)$: 320.1856. Found: $320.1854 . \quad[\alpha]_{\mathrm{D}}{ }^{27}=24.4\left(\mathrm{c} 0.5, \mathrm{CHCl}_{3}\right)$. The enantiomeric excess $(92 \%$ ee $)$ was determined by HPLC with a Daicel Chiralpak AS-H column (Hexane/i-PrOH $=90: 10$, flow rate: $0.7 \mathrm{~mL} / \min , \lambda \max 214 \mathrm{~nm}): \mathrm{t}_{\mathrm{R}}($ anti major enantiomer $)=16.69 \mathrm{~min} ; \mathrm{t}_{\mathrm{R}}($ anti minor enantiomer $)$ $=21.47 \mathrm{~min}$. 
Butyl 2-((4-methoxyphenyl)amino)-2-(2-oxocyclohexyl)acetate (4d)<smiles>CCCCOC(=O)C(Nc1ccc(OC)cc1)C1CCCCC1=O</smiles>

Purification: column chromatography $\left(n-\mathrm{Hexane} / \mathrm{EtOAc}=5: 1, R_{\mathrm{f}}=0.28\right)$. Pale yellow oil. Isolated yield was $72 \%$ (24 mg). ${ }^{1} \mathbf{H}$ NMR $\left(400 \mathrm{MHz}, \mathrm{CDCl}_{3}\right) \delta 6.76(\mathrm{~d}, J=8.8 \mathrm{~Hz}, 2 \mathrm{H}), 6.62(\mathrm{~d}, J=8.8$ Hz, 2H), $4.24(\mathrm{br}, 1 \mathrm{H}), 4.08$ (t, $J=6.4 \mathrm{~Hz}, 2 \mathrm{H}), 3.99$ (d, $J=4.0 \mathrm{~Hz}, 1 \mathrm{H}), 3.73(\mathrm{~s}, 3 \mathrm{H}), 3.13-3.08(\mathrm{~m}$, 1H), 2.47-2.39 (m, 1H), 2.36-2.28 (m, 1H), 2.14-2.02 (m, 2H), 1.98-1.87 (m, 2H), 1.79-1.64 (m, 2H), $1.58-1.52(\mathrm{~m}, 2 \mathrm{H}), 1.33-1.25(\mathrm{~m}, 2 \mathrm{H}), 0.88(\mathrm{t}, J=7.2 \mathrm{~Hz}, 3 \mathrm{H}) .{ }^{13} \mathbf{C}\left\{{ }^{1} \mathbf{H}\right\} \mathbf{N M R}(101 \mathrm{MHz}$, $\left.\mathrm{CDCl}_{3}\right) \delta 211.1,173.3,152.9,142.4,115.7,114.9,65.2,59.3,55.9,53.8,42.0,30.7,30.6,27.1$, 24.7, 19.2, 13.8. IR (neat): 3381, 2929, 1706, 1511, 1452, 1233, 1036, 820, $746 \mathrm{~cm}^{-1} . \quad$ HR-MS (ESI) Calculated for $\mathrm{C}_{19} \mathrm{H}_{27} \mathrm{NO}_{4} \mathrm{Na}\left([\mathrm{M}+\mathrm{Na}]^{+}\right)$: 356.1832. Found: 356.1831. $[\alpha]_{\mathrm{D}}{ }^{21}=18.6(\mathrm{c} 0.5$, $\mathrm{CHCl}_{3}$ ). The enantiomeric excess (99\% ee) was determined by HPLC with a Daicel Chiralpak AD$\mathrm{H}$ column (Hexane/i-PrOH $=90: 10$, flow rate: $0.7 \mathrm{~mL} / \mathrm{min}, \lambda \max 214 \mathrm{~nm}$ ): $\mathrm{t}_{\mathrm{R}}$ (anti minor enantiomer $)=24.59 \mathrm{~min} ; \mathrm{t}_{\mathrm{R}}($ anti major enantiomer $)=36.6 \mathrm{~min}$.

\section{Isopropyl 2-((4-methoxyphenyl)amino)-2-(2-oxocyclohexyl)acetate (4e) $)^{2}$}<smiles>COc1ccc(NC(C(=O)OC(C)C)C2CCCCC2=O)cc1</smiles>

Purification: column chromatography $\left(n-\mathrm{Hexane} / \mathrm{EtOAc}=5: 1, R_{\mathrm{f}}=0.50\right)$. Pale yellow oil. Isolated yield was $63 \%(20.2 \mathrm{mg}) . \quad{ }^{1} \mathbf{H}$ NMR $\left(400 \mathrm{MHz}, \mathrm{CDCl}_{3}\right) \delta 6.75(\mathrm{~d}, J=8.8 \mathrm{~Hz}, 2 \mathrm{H}), 6.63(\mathrm{~d}, J=$ $8.8 \mathrm{~Hz}, 2 \mathrm{H}), 4.99(\mathrm{~m}, 1 \mathrm{H}), 4.22(\mathrm{br}, 1 \mathrm{H}), 3.96(\mathrm{~d}, J=4.0 \mathrm{~Hz}, 1 \mathrm{H}), 3.73(\mathrm{~s}, 3 \mathrm{H}), 3.12-3.02(\mathrm{~m}, 1 \mathrm{H})$, 2.47-2.38 (m, 1H), 2.36-2.25 (m, 1H), 2.16-1.99 (m, 2H), 1.99-1.84 (m, 2H), 1.80-1.63 (m, 2H), $1.22(\mathrm{~d}, J=6.3 \mathrm{~Hz}, 3 \mathrm{H}), 1.14(\mathrm{~d}, J=6.2 \mathrm{~Hz}, 3 \mathrm{H}) .{ }^{13} \mathbf{C}\left\{{ }^{1} \mathbf{H}\right\} \mathbf{N M R}\left(101 \mathrm{MHz}, \mathrm{CDCl}_{3}\right) \delta 211.0$, $172.6,152.9,142.4,115.8,114.8,68.9,59.3,55.8,53.7,41.9,30.6,27.0,24.6,21.8,21.8 . \quad[\alpha]_{\mathrm{D}}{ }^{24}$ $=28.0\left(\mathrm{c} 0.52, \mathrm{CHCl}_{3}\right) . \quad$ The enantiomeric excess $(89 \%$ ee) was determined by HPLC with a Daicel Chiralpak AS-H column (Hexane/i-PrOH = 90:10, flow rate: $0.7 \mathrm{~mL} / \mathrm{min}, \lambda \max 214 \mathrm{~nm}): \mathrm{t}_{\mathrm{R}}($ anti major enantiomer $)=11.66 \mathrm{~min} ; \mathrm{t}_{\mathrm{R}}($ anti minor enantiomer $)=18.04 \mathrm{~min}$. 
<smiles>COc1ccc(NC(C(=O)OC(C)(C)C)C2CCCCC2=O)cc1</smiles>

Purification: column chromatography $\left(n-\mathrm{Hexane} / \mathrm{EtOAc}=5: 1, R_{\mathrm{f}}=0.67\right)$. Pale yellow oil. Isolated yield was $73 \%(24.3 \mathrm{mg}) . \quad{ }^{1} \mathbf{H}$ NMR $\left(400 \mathrm{MHz}, \mathrm{CDCl}_{3}\right) \delta 6.75(\mathrm{~d}, J=8.8 \mathrm{~Hz}, 2 \mathrm{H}), 6.61(\mathrm{~d}, J=$ $8.8 \mathrm{~Hz}, 2 \mathrm{H}), 4.18(\mathrm{br}, 1 \mathrm{H}), 3.92(\mathrm{~d}, J=4.0 \mathrm{~Hz}, 1 \mathrm{H}), 3.73(\mathrm{~s}, 3 \mathrm{H}), 3.08-2.98(\mathrm{~m}, 1 \mathrm{H}), 2.48-2.39(\mathrm{~m}$, 1H), 2.37-2.24 (m, 1H), 2.17-1.99 (m, 2H), 1.99-1.81 (m, 2H), 1.77-1.64 (m, 3H), 1.39 (s, 9H). ${ }^{13} \mathbf{C}\left\{{ }^{1} \mathbf{H}\right\}$ NMR $\left(101 \mathrm{MHz}, \mathrm{CDCl}_{3}\right) \delta 211.0,172.2,152.7,142.5,115.6,114.8,81.7,59.6,55.8,53.6$, $41.9,30.5,28.1,27.0,24.6 .[\alpha]_{D}{ }^{22}=21.8\left(\mathrm{c} 0.25, \mathrm{CHCl}_{3}\right)$. The enantiomeric excess $(89 \%$ ee $)$ was determined by HPLC with a Daicel Chiralpak AS-H column (Hexane/i-PrOH = 90:10, flow rate: $0.7 \mathrm{~mL} / \mathrm{min}, \lambda \max 214 \mathrm{~nm}$ ): $\mathrm{t}_{\mathrm{R}}$ (anti major enantiomer) $=7.86 \mathrm{~min} ; \mathrm{t}_{\mathrm{R}}$ (anti minor enantiomer) $=11.34 \mathrm{~min}$.

Tert-pentyl 2-((4-methoxyphenyl)amino)-2-(2-oxocyclohexyl)acetate (4g)<smiles>COc1ccc(NC(C(=O)OC(C)(C)C)C2CCCCC2=O)cc1</smiles>

Purification: column chromatography $\left(n\right.$-Hexane/EtOAc $\left.=5: 1, R_{\mathrm{f}}=0.52\right)$. Pale yellow oil. Isolated yield was $60 \%(20.8 \mathrm{mg}) . \quad{ }^{1} \mathbf{H}$ NMR $\left(400 \mathrm{MHz}, \mathrm{CDCl}_{3}\right) \delta 6.75(\mathrm{~d}, J=8.8 \mathrm{~Hz}, 2 \mathrm{H}), 6.60$ $(\mathrm{d}, J=8.8 \mathrm{~Hz}, 2 \mathrm{H}), 4.21(\mathrm{br}, 1 \mathrm{H}), 3.92(\mathrm{~d}, J=4.4 \mathrm{~Hz}, 1 \mathrm{H}), 3.73(\mathrm{~s}, 3 \mathrm{H}), 3.07-3.02(\mathrm{~m}, 1 \mathrm{H}), 2.45-$ $2.40(\mathrm{~m}, 1 \mathrm{H}), 2.35-2.27(\mathrm{~m}, 1 \mathrm{H}), 2.13-2.02(\mathrm{~m}, 2 \mathrm{H}), 1.96-1.86(\mathrm{~m}, 2 \mathrm{H}), 1.77-1.60(\mathrm{~m}, 4 \mathrm{H}), 1.36(\mathrm{~d}$, $J=8.8 \mathrm{~Hz}, 6 \mathrm{H}), 0.78(\mathrm{t}, J=7.6 \mathrm{~Hz}, 3 \mathrm{H}) .{ }^{13} \mathbf{C}\left\{{ }^{1} \mathbf{H}\right\} \mathbf{N M R}\left(101 \mathrm{MHz}, \mathrm{CDCl}_{3}\right) \delta 211.1,172.2,152.7$, $142.5,115.5,114.9,84.3,59.6,55.9,53.7,41.9,33.9,30.6,27.0,25.5,25.2,24.6,8.2$. IR (neat): 3372, 2926, 1731, 1709, 1508, 1234, 1213, 1143, 1107, 1074, 1036, 829, 755, $630 \mathrm{~cm}^{-1}$. HR-MS (ESI) Calculated for $\mathrm{C}_{20} \mathrm{H}_{30} \mathrm{NO}_{4}\left([\mathrm{M}+\mathrm{H}]^{+}\right)$: 348.2169. Found: $348.2163 .[\alpha]_{\mathrm{D}}^{30}=20.5$ (c 1.0, $\mathrm{CHCl}_{3}$ ). The enantiomeric excess (91\% ee) was determined by HPLC with a Daicel Chiralpak IB column $\left(\right.$ Hexane/i-PrOH $=98: 2$, flow rate: $0.7 \mathrm{~mL} / \mathrm{min}, \lambda \max 214 \mathrm{~nm}$ ): $\mathrm{t}_{\mathrm{R}}$ (anti minor enantiomer $)=11.93 \mathrm{~min} ; \mathrm{t}_{\mathrm{R}}($ anti major enantiomer $)=12.84 \mathrm{~min}$. 
Neopentyl 2-((4-methoxyphenyl)amino)-2-(2-oxocyclohexyl)acetate (4h)<smiles>COc1ccc(NC(C(=O)OCC(C)(C)C)C2CCCCC2=O)cc1</smiles>

Purification: column chromatography $\left(n-\mathrm{Hexane} / \mathrm{EtOAc}=5: 1, R_{\mathrm{f}}=0.44\right)$. Pale yellow oil. Isolated yield was $58 \%(20.2 \mathrm{mg}) . \quad{ }^{1} \mathbf{H}$ NMR $\left(400 \mathrm{MHz}, \mathrm{CDCl}_{3}\right) \delta 6.76(\mathrm{~d}, J=8.8 \mathrm{~Hz}, 2 \mathrm{H}), 6.62(\mathrm{~d}, J=8.8$ Hz, 2H), 4.29 (br, 1H), 4.03 (d, J = 3.6 Hz, 1H), 3.75 (s, 2H), 3.73 (s, 3H), 3.18-3.11 (m, 1H), 2.46 $-2.40(\mathrm{~m}, 1 \mathrm{H}), 2.37-2.28(\mathrm{~m}, 1 \mathrm{H}), 2.17-2.02(\mathrm{~m}, 2 \mathrm{H}), 2.00-1.90(\mathrm{~m}, 2 \mathrm{H}), 1.76-1.61(\mathrm{~m}, 2 \mathrm{H}), 0.85(\mathrm{~s}$, 9H). ${ }^{13} \mathbf{C}\left\{{ }^{1} \mathbf{H}\right\}$ NMR $\left(101 \mathrm{MHz}, \mathrm{CDCl}_{3}\right) \delta 211.2,173.4,152.8,142.4,115.4,115.0,74.8,59.0$, 55.9, 53.7, 42.0, 31.4, 30.7, 27.1, 26.5, 24.8. IR (neat): 2958, 1707, 1513, 1465, 1369, 1234, 1037, $796 \mathrm{~cm}^{-1}$. HR-MS (ESI) Calculated for $\mathrm{C}_{20} \mathrm{H}_{29} \mathrm{NO}_{4} \mathrm{Na}\left([\mathrm{M}+\mathrm{Na}]^{+}\right)$:370.1989. Found: 370.1989. $[\alpha]_{D}^{29}=17.6\left(\mathrm{c} 0.25, \mathrm{CHCl}_{3}\right)$. The enantiomeric excess (95\% ee) was determined by HPLC with a Daicel Chiralpak IB column (Hexane/i-PrOH = 98:2, flow rate: $0.7 \mathrm{~mL} / \mathrm{min}, \lambda \max 214$ $\mathrm{nm}): \mathrm{t}_{\mathrm{R}}($ anti minor enantiomer $)=13.41 \mathrm{~min} ; \mathrm{t}_{\mathrm{R}}($ anti major enantiomer $)=15.76 \mathrm{~min}$.

\section{Cyclopropyl 2-((4-methoxyphenyl)amino)-2-(2-oxocyclohexyl)acetate (4i)}<smiles>COc1ccc(NC(C(=O)OC2CC2)C2CCCCC2=O)cc1</smiles>

Purification: column chromatography $\left(n\right.$-Hexane/EtOAc $\left.=5: 1, R_{\mathrm{f}}=0.26\right)$. Pale yellow oil. Isolated yield was $58 \%(18.4 \mathrm{mg}) .{ }^{1} \mathbf{H}$ NMR $\left(400 \mathrm{MHz}, \mathrm{CDCl}_{3}\right) \delta 6.75(\mathrm{~d}, J=8.8 \mathrm{~Hz}, 2 \mathrm{H}), 6.60(\mathrm{~d}, J=8.8$ $\mathrm{Hz}, 2 \mathrm{H}), 4.17$ (br, 1H), 4.12-4.07 (m, 1H), $3.94(\mathrm{~d}, J=4.4 \mathrm{~Hz}, 1 \mathrm{H}), 3.73(\mathrm{~s}, 3 \mathrm{H}), 3.10-3.05(\mathrm{~m}, 1 \mathrm{H})$, 2.45-2.40 (m, 1H), 2.35-2.27 (m, 1H), 2.13-2.01 (m, 2H), 1.96-1.86 (m, 2H), 1.78-1.60 (m, 2H), 0.72-0.58 (m, 4H). ${ }^{13} \mathbf{C}\left\{{ }^{1} \mathbf{H}\right\}$ NMR $\left(101 \mathrm{MHz}, \mathrm{CDCl}_{3}\right) \delta 211.0,174.2,153.0,142.2,115.8,114.9$, 59.2, 55.8, 53.7, 49.7, 41.9, 30.7, 27.0, 24.7, 5.2, 5.1. IR (neat): 2935, 1704, 1511, 1450, 1232, 1180 , 1154, 1036, 820, 750, $665 \mathrm{~cm}^{-1}$. HR-MS (ESI) Calculated for $\mathrm{C}_{18} \mathrm{H}_{24} \mathrm{NO}_{4}\left([\mathrm{M}+\mathrm{H}]^{+}\right)$: 318.1700 . Found: $318.1702 . \quad[\alpha]_{\mathrm{D}}^{28}=18.9\left(\mathrm{c} 0.5, \mathrm{CHCl}_{3}\right)$. The enantiomeric excess $(89 \%$ ee $)$ was determined by HPLC with a Daicel Chiralpak IB column (Hexane/i-PrOH = 95:5, flow rate: $0.7 \mathrm{~mL} / \mathrm{min}, \lambda \max 214 \mathrm{~nm}): t_{R}($ anti minor enantiomer $)=17.18 \mathrm{~min} ; \mathrm{t}_{\mathrm{R}}($ anti major enantiomer $)$ 
$=18.05 \mathrm{~min}$.

Cyclobutyl 2-((4-methoxyphenyl)amino)-2-(2-oxocyclohexyl)acetate (4j)<smiles>COc1ccc(NC(C(=O)OC2CCC2)C2CCCCC2=O)cc1</smiles>

Purification: column chromatography $\left(n-\mathrm{Hexane} / \mathrm{EtOAc}=5: 1, R_{\mathrm{f}}=0.29\right)$. Pale yellow oil. Isolated yield was $50 \%(16.7 \mathrm{mg}) .{ }^{1} \mathbf{H}$ NMR $\left(400 \mathrm{MHz}, \mathrm{CDCl}_{3}\right) \delta 6.76(\mathrm{~d}, J=8.8 \mathrm{~Hz}, 2 \mathrm{H}), 6.63(\mathrm{~d}, J=8.8 \mathrm{~Hz}$, 2H), 4.95 (quin, $J=7.6 \mathrm{~Hz}, 1 \mathrm{H}), 4.22(\mathrm{br}, 1 \mathrm{H}), 3.95(\mathrm{~d}, J=4.0 \mathrm{~Hz}, 1 \mathrm{H}), 3.73(\mathrm{~s}, 3 \mathrm{H}), 3.12-3.06(\mathrm{~m}, 1 \mathrm{H})$, 2.46-2.40 (m, 1H), 2.36-2.25 (m, 3H), 2.12-1.87 (m, 6H), 1.78-1.57 (m, 4H). ${ }^{13} \mathbf{C}\left\{{ }^{1} \mathbf{H}\right\}$ NMR (101 MHz, $\left.\mathrm{CDCl}_{3}\right) \delta 211.0,172.6,152.9,142.3,115.8,114.9,69.8,59.2,55.8,53.7,41.9,30.6,30.3,30.2,27.0$, 24.7, 13.7. IR (neat): 2939, 1705, 1511, 1448, 1233, 1148, 1037, 821, $750 \mathrm{~cm}^{-1}$. HR-MS (ESI) Calculated for $\mathrm{C}_{19} \mathrm{H}_{26} \mathrm{NO}_{4}\left([\mathrm{M}+\mathrm{H}]^{+}\right)$: 332.1856. Found: 332.1853. $[\alpha]_{\mathrm{D}}^{25}=19.4$ (c 0.5, $\left.\mathrm{CHCl}_{3}\right)$. The enantiomeric excess ( $89 \%$ ee) was determined by HPLC with a Daicel Chiralpak PC2 column $($ Hexane/i-PrOH $=90: 10$, flow rate: $0.7 \mathrm{~mL} / \min , \lambda \max 214 \mathrm{~nm}): t_{R}($ anti minor enantiomer $)=16.90$ $\min ; \mathrm{t}_{\mathrm{R}}$ (anti major enantiomer) $=20.44 \mathrm{~min}$.

\section{Cyclopentyl 2-((4-methoxyphenyl)amino)-2-(2-oxocyclohexyl)acetate (4k)}<smiles>COc1ccc(NC(C(=O)OC2CCCC2)C2CCCCC2=O)cc1</smiles>

Purification: column chromatography $\left(n-\mathrm{Hexane} / \mathrm{EtOAc}=5: 1, R_{\mathrm{f}}=0.35\right)$. Pale yellow oil. Isolated yield was $56 \%(19.3 \mathrm{mg}) .{ }^{1} \mathbf{H}$ NMR $\left(400 \mathrm{MHz}, \mathrm{CDCl}_{3}\right) \delta 6.75(\mathrm{~d}, J=8.8 \mathrm{~Hz}, 2 \mathrm{H}), 6.61(\mathrm{~d}, J=8.8$ $\mathrm{Hz}, 2 \mathrm{H}), 5.20-5.09(\mathrm{~m}, 1 \mathrm{H}), 4.20(\mathrm{br}, 1 \mathrm{H}), 3.94(\mathrm{~d}, J=4.1 \mathrm{~Hz}, 1 \mathrm{H}), 3.73(\mathrm{~s}, 3 \mathrm{H}), 3.11-3.06(\mathrm{~m}, 1 \mathrm{H})$, 2.47-2.38 (m, 1H), 2.35-2.27 (m, 1H), 214-2.01 (m, 2H), 1.98-1.88 (m, 2H), 1.80-1.64 (m, 6H), 1.59 $-1.46(\mathrm{~m}, 4 \mathrm{H}) .{ }^{13} \mathbf{C}\left\{{ }^{1} \mathbf{H}\right\} \mathbf{N M R}\left(101 \mathrm{MHz}, \mathrm{CDCl}_{3}\right) \delta 211.1,172.9,152.9,142.5,115.8,114.9,78.3$, 59.4, 55.9, 53.7, 41.9, 32.6, 32.6, 30.6, 27.0, 24.7, 23.7, 23.7. IR (neat): 2936, 1705, 1511, 1450, 1233, 1150, 1036, 976, 820, $751 \mathrm{~cm}^{-1}$. HR-MS (ESI) Calculated for $\mathrm{C}_{20} \mathrm{H}_{28} \mathrm{NO}_{4}\left([\mathrm{M}+\mathrm{H}]^{+}\right)$: 346.2013. Found: $346.2013 . \quad[\alpha]_{\mathrm{D}}^{29}=21.9\left(\mathrm{c} 0.46, \mathrm{CHCl}_{3}\right) . \quad$ The enantiomeric excess $(90 \%$ ee $)$ 
was determined by HPLC with a Daicel Chiralpak AS-H column (Hexane/i-PrOH = 90:10, flow rate: $0.7 \mathrm{~mL} / \mathrm{min}, \lambda \max 214 \mathrm{~nm}): \mathrm{t}_{\mathrm{R}}($ anti major enantiomer $)=16.30 \mathrm{~min} ; \mathrm{t}_{\mathrm{R}}($ anti minor enantiomer) $=24.94 \mathrm{~min}$.

Cyclohexyl 2-((4-methoxyphenyl)amino)-2-(2-oxocyclohexyl)acetate (4l)<smiles>COc1ccc(NC(C(=O)OC2CCCCC2)C2CCCCC2=O)cc1</smiles>

Purification: column chromatography $\left(n\right.$-Hexane/EtOAc $\left.=5: 1, R_{\mathrm{f}}=0.38\right)$. Pale yellow oil. Isolated yield was 58\% (21 mg). ${ }^{1} \mathbf{H}$ NMR (400 MHz, $\left.\mathrm{CDCl}_{3}\right) \delta 6.75(\mathrm{~d}, J=8.8 \mathrm{~Hz}, 2 \mathrm{H}), 6.63(\mathrm{~d}, J=8.8 \mathrm{~Hz}$, 2H), 4.80-4.75 (m, 1H), 4.25 (br, 1H), 3.98 (d, J = 4.4 Hz, 1H), 3.73 (s, 3H), 3.12-3.07 (m, 1H), 2.45-2.41 (m, 1H), 2.36-2.28 (m, 1H), 2.15-2.01 (m, 2H), 1.98-1.86 (m, 2H), 1.78-1.65 (m, 5H), 1.59-1.53 (m, 1H), 1.50-1.40 (m, 2H), 1.38-1.24 (m, 4H). ${ }^{13} \mathbf{C}\left\{{ }^{1} \mathbf{H}\right\}$ NMR (101 MHz, CDCl $) \delta$ 211.1, 172.6, 152.9, 142.4, 115.8, 114.9, 73.5, 59.3, 55.9, 53.7, 41.9, 31.5, 31.4, 30.6, 27.0, 25.5, 24.7, 23.5, 23.5. IR (neat): 2932, 2858, 1706, 1511, 1450, 1233, 1149, 1036, 972, 820, $751 \mathrm{~cm}^{-1}$. HRMS (ESI) Calculated for $\mathrm{C}_{21} \mathrm{H}_{30} \mathrm{NO}_{4}\left([\mathrm{M}+\mathrm{H}]^{+}\right): 360.2169$. Found: $360.2178 . \quad[\alpha]_{\mathrm{D}}^{24}=12.0(\mathrm{c}$ 0.275, $\left.\mathrm{CHCl}_{3}\right)$. The enantiomeric excess $(88 \%$ ee) was determined by HPLC with a Daicel Chiralpak PC2 column (Hexane/i-PrOH $=90: 10$, flow rate: $0.7 \mathrm{~mL} / \mathrm{min}, \lambda \max 214 \mathrm{~nm}$ ): $\mathrm{t}_{\mathrm{R}}$ (anti minor enantiomer $)=14.35 \mathrm{~min} ; \mathrm{t}_{\mathrm{R}}($ anti major enantiomer $)=18.49 \mathrm{~min}$.

(1s,3s)-Adamantan-1-yl 2-((4-methoxyphenyl)amino)-2-(2-oxocyclohexyl)acetate (4m)<smiles>COc1ccc(NC(C(=O)OC23CC4CC(CC(C4)C2)C3)C2CCCCC2=O)cc1</smiles>

Purification: column chromatography $\left(n\right.$-Hexane/EtOAc $\left.=5: 1, R_{\mathrm{f}}=0.52\right)$. Pale yellow soild, m.p. $=122.3-123.5^{\circ} \mathrm{C}$. Isolated yield was $60 \%(24.8 \mathrm{mg}) . \quad{ }^{1} \mathbf{H} \mathbf{N M R}\left(400 \mathrm{MHz}, \mathrm{CDCl}_{3}\right) \delta 6.75(\mathrm{~d}, J=$ $8.8 \mathrm{~Hz}, 2 \mathrm{H}), 6.61$ (d, $J=8.8 \mathrm{~Hz}, 2 \mathrm{H}), 4.18$ (br, 1H), 3.93 (d, $J=4.4 \mathrm{~Hz}, 1 \mathrm{H}), 3.73$ (s, 3H), 3.04$2.98(\mathrm{~m}, 1 \mathrm{H}), 2.46-2.41(\mathrm{~m}, 1 \mathrm{H}), 2.35-2.24(\mathrm{~m}, 1 \mathrm{H}), 2.13-2.00(\mathrm{~m}, 11 \mathrm{H}), 1.97-1.85(\mathrm{~m}, 2 \mathrm{H}), 1.75-$ $1.61(\mathrm{~m}, 8 \mathrm{H}) .{ }^{13} \mathbf{C}\left\{{ }^{1} \mathbf{H}\right\} \mathbf{N M R}\left(101 \mathrm{MHz}, \mathrm{CDCl}_{3}\right) \delta 211.0,171.9,152.8,142.5,115.7,114.9,81.8$, 
59.7, 55.9, 53.6, 41.9, 41.3, 36.2, 30.9, 30.4, 27.0, 24.6. IR (neat): 2908, 1733, 1704, 1507, 1234, $1202,1146,1051,968,820,788,750,607,521 \mathrm{~cm}^{-1}$. HR-MS (ESI) Calculated for $\mathrm{C}_{25} \mathrm{H}_{34} \mathrm{NO}_{4}$ $\left([\mathrm{M}+\mathrm{H}]^{+}\right): 412.2482$. Found: $412.2490 .[\alpha]_{\mathrm{D}}^{23}=7.3\left(\mathrm{c} 0.1, \mathrm{CHCl}_{3}\right)$. The enantiomeric excess $(91 \%$ ee) was determined by HPLC with a Daicel Chiralpak Ij-3 column $\left(\mathrm{CO}_{2} / \mathrm{MeOH}=95: 5\right.$, flow rate: $1.3 \mathrm{~mL} / \mathrm{min}, \lambda \max 254 \mathrm{~nm}): t_{R}$ (anti major enantiomer) $=25.131 \mathrm{~min} ; \mathrm{t}_{\mathrm{R}}$ (anti minor enantiomer) $=26.298 \mathrm{~min}$.

\section{2-Methoxyethyl 2-((4-methoxyphenyl)amino)-2-(2-oxocyclohexyl)acetate (4n)}<smiles>COCCOC(=O)C(Nc1ccc(OC)cc1)C1CCCCC1=O</smiles>

Purification: column chromatography $\left(n\right.$-Hexane/EtOAc $\left.=3: 1, R_{\mathrm{f}}=0.28\right)$. Pale yellow oil. Isolated yield was $62 \%$ (20.8 mg). ${ }^{1} \mathbf{H}$ NMR $\left(400 \mathrm{MHz}, \mathrm{CDCl}_{3}\right) \delta 6.75(\mathrm{~d}, J=8.4 \mathrm{~Hz}, 2 \mathrm{H}), 6.64(\mathrm{~d}, J=8.8$ $\mathrm{Hz}, 2 \mathrm{H}), 4.28-4.18(\mathrm{~m}, 2 \mathrm{H}), 4.02(\mathrm{~d}, J=4.0 \mathrm{~Hz}, 1 \mathrm{H}), 3.73(\mathrm{~s}, 3 \mathrm{H}), 3.52(\mathrm{t}, J=4.8 \mathrm{~Hz}, 2 \mathrm{H}), 3.31(\mathrm{~s}$, $3 \mathrm{H}), 3.16-3.10(\mathrm{~m}, 1 \mathrm{H}), 2.45-2.41(\mathrm{~m}, 1 \mathrm{H}), 2.36-2.28(\mathrm{~m}, 1 \mathrm{H}), 2.14-2.02(\mathrm{~m}, 2 \mathrm{H}), 1.99-1.88(\mathrm{~m}$, 2H), 1.78-1.62 (m, 2H). ${ }^{13} \mathbf{C}\left\{{ }^{1} \mathbf{H}\right\}$ NMR (101 MHz, $\left.\mathrm{CDCl}_{3}\right) \delta 211.2,173.1,152.9,142.2,115.8$, 114.9, 70.4, 64.2, 59.1, 59.0, 55.8, 53.7, 41.9, 30.7, 27.0, 24.7. IR (neat): 2933, 1704, 1512, 1450, 1233, 1195, 1127, 1033, 822, 732, $499 \mathrm{~cm}^{-1}$. HR-MS (ESI) Calculated for $\mathrm{C}_{18} \mathrm{H}_{25} \mathrm{NO}_{5} \mathrm{Na}$ $\left([\mathrm{M}+\mathrm{Na}]^{+}\right): 358.1625$. Found: $358.1628 .[\alpha]_{\mathrm{D}}{ }^{24}=31.62\left(\mathrm{c} 0.25, \mathrm{CHCl}_{3}\right)$. The enantiomeric excess (91\% ee) was determined by HPLC with a Daicel Chiralpak Ij-3 column $\left(\mathrm{CO}_{2} / \mathrm{MeOH}=98: 2\right.$, flow rate: $1.3 \mathrm{~mL} / \mathrm{min}, \lambda \max 254 \mathrm{~nm}): t_{R}($ anti major enantiomer $)=22.171 \mathrm{~min} ; \mathrm{t}_{\mathrm{R}}($ anti minor enantiomer $)$ $=25.074 \mathrm{~min}$.

4-(4-Methoxyphenyl)butyl 2-((4-methoxyphenyl)amino)-2-(2-oxocyclohexyl)acetate (4o)<smiles>COc1ccc(CCCCOC(=O)C(Nc2ccc(OC)cc2)C2CCCCC2=O)cc1</smiles>

Purification: column chromatography $\left(n\right.$-Hexane/EtOAc $\left.=3: 1, R_{\mathrm{f}}=0.34\right)$. Pale yellow oil. Isolated yield was $62 \%(27.3 \mathrm{mg}) . \quad{ }^{1} \mathbf{H}$ NMR $\left(400 \mathrm{MHz}, \mathrm{CDCl}_{3}\right) \delta 7.05(\mathrm{~d}, J=8.4 \mathrm{~Hz}, 2 \mathrm{H}), 6.82(\mathrm{~d}, J=$ 
$8.8 \mathrm{~Hz}, 2 \mathrm{H}), 6.75(\mathrm{~d}, J=8.8 \mathrm{~Hz}, 2 \mathrm{H}), 6.62(\mathrm{~d}, J=9.2 \mathrm{~Hz}, 2 \mathrm{H}), 4.30-4.20(\mathrm{~m}, 1 \mathrm{H}), 4.09(\mathrm{t}, J=6.4$ $\mathrm{Hz}, 2 \mathrm{H}), 3.98(\mathrm{~d}, J=4.0 \mathrm{~Hz}, 1 \mathrm{H}), 3.79(\mathrm{~s}, 3 \mathrm{H}), 3.72(\mathrm{~s}, 3 \mathrm{H}), 3.14-3.08(\mathrm{~m}, 1 \mathrm{H}), 2.52$ (t, $J=7.2 \mathrm{~Hz}$, $2 \mathrm{H}), 2.45-2.38(\mathrm{~m}, 1 \mathrm{H}), 2.35-2.26(\mathrm{~m}, 1 \mathrm{H}), 2.17-2.00(\mathrm{~m}, 2 \mathrm{H}), 1.98-1.87(\mathrm{~m}, 2 \mathrm{H}), 1.74-1.53(\mathrm{~m}$, 6H). ${ }^{13} \mathbf{C}\left\{{ }^{1} \mathbf{H}\right\}$ NMR $\left(101 \mathrm{MHz}, \mathrm{CDCl}_{3}\right) \delta 211.2,173.4,157.8,152.9,142.3,134.2,129.3,115.7$, $114.9,113.8,65.2,59.2,55.8,55.45,53.7,42.0,34.5,30.7,28.1,28.0,27.0,24.7 . \quad$ IR (neat): 2935 , $1705,1612,1511,1643,1238,1177,1034,821,732,512 \mathrm{~cm}^{-1}$. HR-MS (ESI) Calculated for $\mathrm{C}_{26} \mathrm{H}_{34} \mathrm{NO}_{5}\left([\mathrm{M}+\mathrm{H}]^{+}\right): 440.2432$. Found: 440.2435. $[\alpha]_{\mathrm{D}}^{22}=10.1$ (c $\left.0.25, \mathrm{CHCl}_{3}\right)$. The enantiomeric excess (87\% ee) was determined by HPLC with a Daicel Chiralpak Ij-3 column $\left(\mathrm{CO}_{2} / \mathrm{MeOH}=95: 5\right.$, flow rate: $\left.1.0 \mathrm{~mL} / \mathrm{min}, \lambda \max 214 \mathrm{~nm}\right): \mathrm{t}_{\mathrm{R}}($ anti major enantiomer $)=18.357$ $\min ; \mathrm{t}_{\mathrm{R}}($ anti minor enantiomer $)=24.136 \mathrm{~min}$.

\section{Benzyl 2-((4-methoxyphenyl)amino)-2-(2-oxocyclohexyl)acetate $(4 p)^{1}$}<smiles>COc1ccc(NC(C(=O)OCc2ccccc2)C2CCCCC2=O)cc1</smiles>

Purification: column chromatography $\left(n\right.$-Hexane/EtOAc $\left.=5: 1, R_{\mathrm{f}}=0.36\right)$. Pale yellow oil. Isolated yield was $60 \%$ (22.0 mg). ${ }^{1} \mathbf{H} \mathbf{N M R}\left(400 \mathrm{MHz}, \mathrm{CDCl}_{3}\right) \delta$ 7.36-7.31 (m, 3H), 7.25-7.23 (m, 2H), $6.77(\mathrm{~d}, J=8.8 \mathrm{~Hz}, 2 \mathrm{H}), 6.64(\mathrm{~d}, J=8.8 \mathrm{~Hz}, 2 \mathrm{H}), 5.17-5.10(\mathrm{~m}, 2 \mathrm{H}), 4.32(\mathrm{br}, 1 \mathrm{H}), 4.07$ (d, $J=4.0$ $\mathrm{Hz}, 1 \mathrm{H}), 3.76$ (s, 3H), 3.16-3.11 (m, 1H), 2.48-2.41 (m, 1H), 2.35-2.27 (m, 1H), 2.13-2.02 (m, 2H), 1.96-1.85 (m, 2H), 1.77-1.62 (m, 2H). ${ }^{13} \mathbf{C}\left\{{ }^{1} \mathbf{H}\right\} \mathbf{N M R}\left(101 \mathrm{MHz}, \mathrm{CDCl}_{3}\right) \delta$ 211.0, 173.0, 152.9, $142.1,135.7,128.5,128.2,128.1,115.8,114.9,67.0,59.1,55.8,53.6,41.9,30.6,26.9,24.6 .[\alpha]_{\mathrm{D}}{ }^{22}$ $=17.9\left(\mathrm{c} 0.2, \mathrm{CHCl}_{3}\right)$. The enantiomeric excess (99\% ee) was determined by HPLC with a Daicel Chiralpak IA column (Hexane/i-PrOH $=90: 10$, flow rate: $0.7 \mathrm{~mL} / \mathrm{min}, \lambda \max 214 \mathrm{~nm}$ ): $t_{R}($ anti major enantiomer $)=43.78 \mathrm{~min} ; t_{R}($ anti minor enantiomer $)=64.66 \mathrm{~min}$. 
<smiles>COc1ccc(COC(=O)C(Nc2ccc(OC)cc2)C2CCCCC2=O)cc1</smiles>

Purification: column chromatography $\left(n\right.$-Hexane/EtOAc $\left.=5: 1, R_{\mathrm{f}}=0.19\right)$. Pale yellow oil. Isolated yield was $58 \%(23.1 \mathrm{mg}) . \quad{ }^{1} \mathbf{H}$ NMR $\left(400 \mathrm{MHz}, \mathrm{CDCl}_{3}\right) \delta 7.17(\mathrm{~d}, J=8.4 \mathrm{~Hz}, 2 \mathrm{H}), 6.84(\mathrm{~d}, J=$ $8.4 \mathrm{~Hz}, 2 \mathrm{H}, 2 \mathrm{H}), 6.75(\mathrm{~d}, J=9.2 \mathrm{~Hz}, 2 \mathrm{H}), 6.61(\mathrm{~d}, J=8.4 \mathrm{~Hz}, 2 \mathrm{H}), 5.05(\mathrm{~s}, 2 \mathrm{H}), 4.27(\mathrm{br}, 1 \mathrm{H}), 4.03$ $(\mathrm{d}, J=4.0 \mathrm{~Hz}, 1 \mathrm{H}), 3.80(\mathrm{~s}, 3 \mathrm{H}), 3.74(\mathrm{~s}, 3 \mathrm{H}), 3.11-3.06(\mathrm{~m}, 1 \mathrm{H}), 2.43-2.39(\mathrm{~m}, 1 \mathrm{H}), 2.33 .2 .25(\mathrm{~m}$, 1H), 2.09-2.0 (m, 2H), 1.93-1.82 (m, 2H), 1.73-1.59 (m, 2H). ${ }^{13} \mathbf{C}\left\{{ }^{1} \mathbf{H}\right\}$ NMR (101 MHz, $\left.\mathrm{CDCl}_{3}\right)$ $\delta 211.0,173.1,159.7,153.0,142.2,130.0,127.9,115.8,114.9,114.0,66.9,59.2,55.9,55.4,53.6$, 41.9, 30.6, 27.0, 24.6. IR (neat): 2936, 1704, 1612, 1511, 1450, 1233, 1173, 1146, 1032, 911, 819, 729, $513 \mathrm{~cm}^{-1}$. HR-MS (ESI) Calculated for $\mathrm{C}_{23} \mathrm{H}_{27} \mathrm{NO}_{5} \mathrm{Na}\left([\mathrm{M}+\mathrm{Na}]^{+}\right)$: 420.1781. Found: 420.1791. $[\alpha]_{\mathrm{D}}^{23}=9.6\left(\mathrm{c} 0.25, \mathrm{CHCl}_{3}\right)$. The enantiomeric excess (91\% ee) was determined by HPLC with a Daicel Chiralpak Ij-3 column $\left(\mathrm{CO}_{2} / \mathrm{EtOH}=60: 40\right.$, flow rate: $\left.1.0 \mathrm{~mL} / \mathrm{min}, \lambda \max 254 \mathrm{~nm}\right): \mathrm{t}_{\mathrm{R}}$ $($ anti major enantiomer $)=8.522 \mathrm{~min} ; \mathrm{t}_{\mathrm{R}}($ anti minor enantiomer $)=11.553 \mathrm{~min}$.

\section{4-(Trifluoromethyl)benzyl 2-((4-methoxyphenyl)amino)-2-(2-oxocyclohexyl)acetate (4r)}<smiles>COc1ccc(NC(C(=O)OCc2ccc(C(F)(F)F)cc2)C2CCCCC2=O)cc1</smiles>

Purification: column chromatography $\left(n\right.$-Hexane/EtOAc $\left.=3: 1, R_{\mathrm{f}}=0.38\right)$. Pale yellow oil. Isolated yield was $61 \%(26.6 \mathrm{mg}) . \quad{ }^{1} \mathbf{H}$ NMR $\left(400 \mathrm{MHz}, \mathrm{CDCl}_{3}\right) \delta 7.55(\mathrm{~d}, J=8.0 \mathrm{~Hz}, 2 \mathrm{H}), 7.30(\mathrm{~d}, J=$ $8.0 \mathrm{~Hz}, 2 \mathrm{H}), 6.76(\mathrm{~d}, J=8.8 \mathrm{~Hz}, 2 \mathrm{H}), 6.62(\mathrm{~d}, J=8.8 \mathrm{~Hz}, 2 \mathrm{H}), 5.16(\mathrm{~s}, 2 \mathrm{H}), 4.29$ (br, $1 \mathrm{H}), 4.03(\mathrm{~d}$, $J=3.6 \mathrm{~Hz}, 1 \mathrm{H}), 3.74(\mathrm{~s}, 3 \mathrm{H}), 3.21-3.13(\mathrm{~m}, 1 \mathrm{H}), 2.46-2.40$ (m, 1H), 2.35-2.27 (m, 1H), 2.14-2.02 (m, 2H), $1.98-1.89(\mathrm{~m}, 2 \mathrm{H}), 1.79-1.58(\mathrm{~m}, 2 \mathrm{H}) .{ }^{13} \mathbf{C}\left\{{ }^{1} \mathbf{H}\right\}$ NMR $\left(126 \mathrm{MHz}, \mathrm{CDCl}_{3}\right) \delta$ 211.3, 173.1, 153.1, 142.1, $130.4(\mathrm{q}, J=32.8 \mathrm{~Hz}), 128.0,125.5(\mathrm{q}, J=3.8 \mathrm{~Hz}), 124.1(\mathrm{q}, J=272.2 \mathrm{~Hz}), 115.8$, 115.0, 66.0, 59.2, 55.9, 53.8, 42.0, 30.8, 27.0, 24.7. ${ }^{19} \mathbf{F}$ NMR $\left(376 \mathrm{MHz}, \mathrm{CDCl}_{3}\right) \delta-62.63$. IR (neat): 2934, 1739, 1512, 1450, 1324, 1162, 1112, 1065, 1017, 819, $592 \mathrm{~cm}^{-1}$. HR-MS (ESI) Calculated for $\mathrm{C}_{23} \mathrm{H}_{25} \mathrm{NO}_{4} \mathrm{~F}_{3}\left([\mathrm{M}+\mathrm{H}]^{+}\right)$: 436.1730. Found: 436.1739. $[\alpha]_{\mathrm{D}}^{23}=4.2\left(\mathrm{c} 0.5, \mathrm{CHCl}_{3}\right)$. The 
enantiomeric excess $(87 \%$ ee) was determined by HPLC with a Daicel Chiralpak Ij-3 column $\left(\mathrm{CO}_{2} / \mathrm{MeOH}=90: 10\right.$, flow rate: $\left.1.0 \mathrm{~mL} / \mathrm{min}, \lambda \max 214 \mathrm{~nm}\right): \mathrm{t}_{\mathrm{R}}($ anti major enantiomer $)=14.128$ $\min ; t_{R}($ anti minor enantiomer $)=18.437 \mathrm{~min}$.

\section{4-Bromobenzyl 2-((4-methoxyphenyl)amino)-2-(2-oxocyclohexyl)acetate (4s)}<smiles>COc1ccc(NC(C(=O)OCc2ccc(Br)cc2)C2CCCCC2=O)cc1</smiles>

Purification: column chromatography $\left(n-\mathrm{Hexane} / \mathrm{EtOAc}=5: 1, R_{\mathrm{f}}=0.28\right)$. Pale yellow oil. Isolated yield was $60 \%(26.7 \mathrm{mg}) .{ }^{1} \mathbf{H}$ NMR $\left(400 \mathrm{MHz}, \mathrm{CDCl}_{3}\right) \delta 7.42(\mathrm{~d}, J=8.0 \mathrm{~Hz}, 2 \mathrm{H}), 7.07(\mathrm{~d}, J=8.0$ $\mathrm{Hz}, 2 \mathrm{H}), 6.75$ (d, $J=8.4 \mathrm{~Hz}, 2 \mathrm{H}), 6.61(\mathrm{~d}, J=8.4 \mathrm{~Hz}, 2 \mathrm{H}), 5.05$ (s, 2H), 4.29 (br, 1H), 4.01 (d, $J=$ $4.0 \mathrm{~Hz}, 1 \mathrm{H}), 3.74(\mathrm{~s}, 3 \mathrm{H}), 3.17-3.08(\mathrm{~m}, 1 \mathrm{H}), 2.43-2.40(\mathrm{~m}, 1 \mathrm{H}), 2.34-2.26(\mathrm{~m}, 1 \mathrm{H}), 2.11-2.01(\mathrm{~m}$, 2H), 1.96-1.87 (m, 2H), 1.74-1.61 (m, 2H). ${ }^{13} \mathbf{C}\left\{{ }^{1} \mathbf{H}\right\}$ NMR (126 MHz, $\left.\mathrm{CDCl}_{3}\right) \delta 211.2,173.1,153.0$, 142.1, 134.8, 131.7, 129.8, 122.3, 115.8, 114.9, 66.2, 59.2, 55.9, 53.7, 42.0, 30.7, 27.0, 24.7. IR (neat): 2932, 1704, 1512, 1450, 1370, 1233, 1147, 1070, 1036, 1010, 820, 747, $511 \mathrm{~cm}^{-1}$. HR-MS (ESI) Calculated for $\mathrm{C}_{22} \mathrm{H}_{25} \mathrm{NO}_{4} \mathrm{Br}\left([\mathrm{M}+\mathrm{H}]^{+}\right)$: 446.0962. Found: 446.0952. $[\alpha]_{\mathrm{D}}^{23}=12.04$ (c 0.25, $\left.\mathrm{CHCl}_{3}\right)$. The enantiomeric excess (92\% ee) was determined by HPLC with a Daicel Chiralpak cel 1 column $\left(\mathrm{CO}_{2} / \mathrm{EtOH}=90: 10\right.$, flow rate: $\left.1.0 \mathrm{~mL} / \mathrm{min}, \lambda \max 214 \mathrm{~nm}\right): \mathrm{t}_{\mathrm{R}}($ anti minor enantiomer $)=$ $5.818 \mathrm{~min} ; \mathrm{t}_{\mathrm{R}}($ anti major enantiomer $)=6.123 \mathrm{~min}$.

\section{4-(4,4,5,5-Tetramethyl-1,3,2-dioxaborolan-2-yl)benzyl 2-((4-methoxyphenyl)amino)-2-(2- oxocyclohexyl)acetate (4t)}<smiles>COc1ccc(NC(C(=O)OCc2ccc(Cc3ccccc3)cc2)C2CCCCC2=O)cc1</smiles>

Purification: column chromatography $\left(n\right.$-Hexane/EtOAc $\left.=4: 1, R_{\mathrm{f}}=0.0 .47\right)$. Pale yellow oil. Isolated yield was $57 \%(28.1 \mathrm{mg}) .{ }^{1} \mathbf{H}$ NMR $\left(500 \mathrm{MHz}, \mathrm{CDCl}_{3}\right) \delta 7.75(\mathrm{~d}, J=7.0 \mathrm{~Hz}, 2 \mathrm{H}), 7.22(\mathrm{~d}$, $J=7.5 \mathrm{~Hz}, 2 \mathrm{H}), 6.75(\mathrm{~d}, J=8.5 \mathrm{~Hz}, 2 \mathrm{H}), 6.62(\mathrm{~d}, J=8.5 \mathrm{~Hz}, 2 \mathrm{H}), 5.18-5.07(\mathrm{~m}, 2 \mathrm{H}), 4.03(\mathrm{~d}, J=$ $4.0 \mathrm{~Hz}, 1 \mathrm{H}), 3.74(\mathrm{~s}, 3 \mathrm{H}), 3.13-3.10(\mathrm{~m}, 1 \mathrm{H}), 2.45-2.39(\mathrm{~m}, 1 \mathrm{H}), 2.33-2.26(\mathrm{~m}, 1 \mathrm{H}), 2.11-2.01(\mathrm{~m}$, 2H), 1.94-1.87 (m, 2H), 1.72-1.64 (m, 2H), $1.34(\mathrm{~s}, 12 \mathrm{H}) .{ }^{13} \mathbf{C}\left\{{ }^{1} \mathbf{H}\right\}$ NMR (101 MHz, CDCl 3 ) $\delta 211.1$, 
173.1, 153.0, 142.2, 138.7, 135.1, 127.3, 115.8, 115.0, 84.0, 66.9, 59.3, 55.9, 53.7, 42.0, 30.7, 27.0, 25.0, 24.7. IR (neat): 2923, 1706, 1512, 1465, 1398, 1359, 1322, 1235, 1142, 1087, 1022, 962, 858, 819, 728, 657, $514 \mathrm{~cm}^{-1}$. HR-MS (ESI) Calculated for $\mathrm{C}_{28} \mathrm{H}_{37} \mathrm{BNO}_{6}\left([\mathrm{M}+\mathrm{H}]^{+}\right)$: 494.2708. Found: 494.2705. $[\alpha]_{\mathrm{D}}^{24}=13.87\left(\mathrm{c} 0.25, \mathrm{CHCl}_{3}\right)$. The enantiomeric excess $(56 \%$ ee) was determined by HPLC with a Daicel Chiralpak cel 1 column $\left(\mathrm{CO}_{2} / \mathrm{EtOH}=90: 10\right.$, flow rate: $1.0 \mathrm{~mL} / \mathrm{min}, \lambda \max 214$ $\mathrm{nm}): \mathrm{t}_{\mathrm{R}}($ anti minor enantiomer $)=4.895 \mathrm{~min} ; \mathrm{t}_{\mathrm{R}}($ anti major enantiomer $)=5.197 \mathrm{~min}$.

\section{4-Cyanobenzyl 2-((4-methoxyphenyl)amino)-2-(2-oxocyclohexyl)acetate (4u)}<smiles>COc1ccc(NC(C(=O)OCc2ccc(C#N)cc2)C2CCCCC2=O)cc1</smiles>

Purification: column chromatography $\left(n\right.$-Hexane/EtOAc $\left.=3: 1, R_{\mathrm{f}}=0.47\right)$. Pale yellow oil. Isolated yield was $64 \%(25.2 \mathrm{mg}) .{ }^{1} \mathbf{H}$ NMR $\left(400 \mathrm{MHz}, \mathrm{CDCl}_{3}\right) \delta 7.57(\mathrm{~d}, J=8.0 \mathrm{~Hz}, 2 \mathrm{H}), 7.27(\mathrm{~d}, J=6.4$ $\mathrm{Hz}, 2 \mathrm{H}), 6.76(\mathrm{~d}, J=8.8 \mathrm{~Hz}, 2 \mathrm{H}), 6.61(\mathrm{~d}, J=8.8 \mathrm{~Hz}, 2 \mathrm{H}), 5.19-5.12(\mathrm{~m}, 2 \mathrm{H}), 4.30(\mathrm{br}, 1 \mathrm{H}), 4.02$ (d, $J=3.6 \mathrm{~Hz}, 1 \mathrm{H}), 3.74$ (s, 3H), 3.21-3.15 (m, 1H), 2.44-2.41 (m, 1H), 2.35-2.27 (m, 1H), 2.15$2.04(\mathrm{~m}, 2 \mathrm{H}), 1.97-1.92(\mathrm{~m}, 2 \mathrm{H}), 1.76-1.62(\mathrm{~m}, 2 \mathrm{H}) .{ }^{13} \mathbf{C}\left\{{ }^{1} \mathbf{H}\right\} \mathbf{N M R}\left(101 \mathrm{MHz}, \mathrm{CDCl}_{3}\right) \delta 211.3$, 173.0, 152.9, 142.0, 140.9, 132.2, 128.0, 118.6, 115.5, 114.8, 111.8, 65.6, 59.0, 55.7, 53.6, 41.9, 30.6, 26.9, 24.6. IR (neat): 2935, 2229, 1703, 1151, 1450, 1375, 1233, 1178, 1035, 951, 819, 732, $546 \mathrm{~cm}^{-1}$. HR-MS (ESI) Calculated for $\mathrm{C}_{23} \mathrm{H}_{24} \mathrm{~N}_{2} \mathrm{O}_{4} \mathrm{Na}\left([\mathrm{M}+\mathrm{Na}]^{+}\right)$: 415.1628. Found: 415.1620 . $[\alpha]_{\mathrm{D}}^{23}=-3.76\left(\mathrm{c} 0.25, \mathrm{CHCl}_{3}\right)$. The enantiomeric excess $(85 \%$ ee) was determined by HPLC with a Daicel Chiralpak cel 1 column $\left(\mathrm{CO}_{2} / \mathrm{EtOH}=90: 10\right.$, flow rate: $\left.1.0 \mathrm{~mL} / \mathrm{min}, \lambda \max 214 \mathrm{~nm}\right): \mathrm{t}_{\mathrm{R}}$ (anti minor enantiomer $)=6.010 \mathrm{~min} ; \mathrm{t}_{\mathrm{R}}($ anti major enantiomer $)=6.275 \mathrm{~min}$.

\section{Hex-5-en-1-yl 2-((4-methoxyphenyl)amino)-2-(2-oxocyclohexyl)acetate (v)}<smiles>C=CCCCCOC(=O)C(Nc1ccc(OC)cc1)C1CCCCC1=O</smiles>

Purification: column chromatography $\left(n-\mathrm{Hexane} / \mathrm{EtOAc}=5: 1, R_{\mathrm{f}}=0.39\right)$. Pale yellow oil. Isolated yield was 58\% (21 mg). $\quad{ }^{1} \mathbf{H} \mathbf{~ N M R}\left(400 \mathrm{MHz}, \mathrm{CDCl}_{3}\right) \delta 6.75(\mathrm{~d}, J=9.2 \mathrm{~Hz}, 2 \mathrm{H}), 6.62(\mathrm{~d}, J=8.8$ 
$\mathrm{Hz}, 2 \mathrm{H}), 5.80-5.69(\mathrm{~m}, 1 \mathrm{H}), 5.03-4.91(\mathrm{~m}, 2 \mathrm{H}), 4.24(\mathrm{br}, 1 \mathrm{H}), 4.07(\mathrm{t}, J=6.4 \mathrm{~Hz}, 2 \mathrm{H}), 3.98(\mathrm{~d}, J=$ $4.0 \mathrm{~Hz}, 1 \mathrm{H}), 3.73(\mathrm{~s}, 3 \mathrm{H}), 3.14-3.08(\mathrm{~m}, 1 \mathrm{H}), 2.45-2.40(\mathrm{~m}, 1 \mathrm{H}), 2.36-2.28(\mathrm{~m}, 1 \mathrm{H}), 2.12-1.88(\mathrm{~m}$, $6 \mathrm{H}$ ), 1.76-1.56 (m, 4H), 1.34 (quin, $J=7.6 \mathrm{~Hz}, 2 \mathrm{H}) .{ }^{13} \mathbf{C}\left\{{ }^{1} \mathbf{H}\right\} \mathbf{N M R}\left(101 \mathrm{MHz}, \mathrm{CDCl}_{3}\right) \delta 211.2$, $173.4,152.9,142.3,138.4,115.7,114.9,65.2,59.2,55.8,53.7,42.0,33.3,30.7,28.0,27.0,25.2$, 24.7. IR (neat): 2935, 1706, 1511, 1451, 1233, 1036, 911, 820, 745, 637, $509 \mathrm{~cm}^{-1}$. HR-MS (ESI) Calculated for $\mathrm{C}_{21} \mathrm{H}_{29} \mathrm{NO}_{4} \mathrm{Na}\left([\mathrm{M}+\mathrm{Na}]^{+}\right)$: 382.1989. Found: $382.1996 .[\alpha]_{\mathrm{D}}^{22}=1.7$ (c $\left.0.25, \mathrm{CHCl}_{3}\right)$. The enantiomeric excess (90\% ee) was determined by HPLC with a Daicel Chiralpak Ij-3 column $\left(\mathrm{CO}_{2} / \mathrm{MeOH}=95: 5\right.$, flow rate: $\left.1.0 \mathrm{~mL} / \mathrm{min}, \lambda \max 214 \mathrm{~nm}\right): \mathrm{t}_{\mathrm{R}}($ anti major enantiomer $)=12.786$ $\min ; t_{R}($ anti minor enantiomer $)=14.733 \mathrm{~min}$.

\section{2-Methylbut-3-en-2-yl 2-((4-methoxyphenyl)amino)-2-(2-oxocyclohexyl)acetate (4w)}<smiles>C=CC(C)(C)OC(=O)C(Nc1ccc(OC)cc1)C1CCCCC1=O</smiles>

Purification: column chromatography $\left(n\right.$-Hexane/EtOAc $\left.=5: 1, R_{\mathrm{f}}=0.48\right)$. Pale yellow oil. Isolated yield was $44 \%(15.2 \mathrm{mg}) . \quad{ }^{1} \mathbf{H}$ NMR $\left(400 \mathrm{MHz}, \mathrm{CDCl}_{3}\right) \delta 6.75(\mathrm{~d}, J=8.8 \mathrm{~Hz}, 2 \mathrm{H}), 6.61(\mathrm{~d}, J=$ 8.4 Hz, 2H), 6.03-5.95 (m, 1H), 5.15-4.99 (m, 2H), $4.21(\mathrm{br}, 1 \mathrm{H}), 3.94(\mathrm{~d}, J=4.0 \mathrm{~Hz}, 1 \mathrm{H}), 3.73(\mathrm{~d}$, $J=1.2 \mathrm{~Hz}, 3 \mathrm{H}), 3.08-3.03(\mathrm{~m}, 1 \mathrm{H}), 2.47-2.39$ (m, 1H), 2.35-2.27 (m, 1H), 2.17-1.99 (m, 2H), 1.98$1.84(\mathrm{~m}, 2 \mathrm{H}), 1.80-1.59(\mathrm{~m}, 2 \mathrm{H}), 1.45(\mathrm{~d}, J=3.6 \mathrm{~Hz}, 6 \mathrm{H}) .{ }^{13} \mathbf{C}\left\{{ }^{1} \mathbf{H}\right\} \mathbf{N M R}\left(101 \mathrm{MHz}, \mathrm{CDCl}_{3}\right) \delta$ 211.1, 171.9, 152.8, 142.4, 142.3, 115.6, 114.9, 113.0, 82.1, 59.6, 55.9, 53.7, 41.9, 30.5, 27.0, 26.5, 26.4, 24.6. IR (neat): 2936, 1706, 1511, 1451, 1234, 1122, 1037, 918, 821, 730, $513 \mathrm{~cm}^{-1}$. HR-MS (ESI) Calculated for $\mathrm{C}_{20} \mathrm{H}_{27} \mathrm{NO}_{4} \mathrm{Na}\left([\mathrm{M}+\mathrm{Na}]^{+}\right)$: 368.1823 . Found: 368.1837. $[\alpha]_{\mathrm{D}}^{23}=-5.8(\mathrm{c} 0.25$, $\left.\mathrm{CHCl}_{3}\right)$. The enantiomeric excess (92\% ee) was determined by HPLC with a Daicel Chiralpak Ij-3 column $\left(\mathrm{CO}_{2} / \mathrm{MeOH}=98: 2\right.$, flow rate: $\left.1.0 \mathrm{~mL} / \mathrm{min}, \lambda \max 214 \mathrm{~nm}\right): \mathrm{t}_{\mathrm{R}}($ anti major enantiomer $)=$ $16.656 \mathrm{~min} ; \mathrm{t}_{\mathrm{R}}($ anti minor enantiomer $)=19.141 \mathrm{~min}$. 
Ethyl 2-((4-ethoxyphenyl)amino)-2-(2-oxocyclohexyl)acetate (4x)<smiles>CCOC(=O)C(Nc1ccc(OCC)cc1)C1CCCCC1=O</smiles>

Purification: column chromatography $\left(n-\mathrm{Hexane} / \mathrm{EtOAc}=5: 1, R_{\mathrm{f}}=0.25\right)$. Pale yellow oil. Isolated yield was $68 \%(21.8 \mathrm{mg}) . \quad{ }^{1} \mathbf{H}$ NMR $\left(400 \mathrm{MHz}, \mathrm{CDCl}_{3}\right) \delta 6.75(\mathrm{~d}, J=8.8 \mathrm{~Hz}, 2 \mathrm{H}), 6.62(\mathrm{~d}, J=$ $8.8 \mathrm{~Hz}, 2 \mathrm{H}), 4.23(\mathrm{br}, 1 \mathrm{H}), 4.18-4.10(\mathrm{~m}, 2 \mathrm{H}), 3.98(\mathrm{~d}, J=4.4 \mathrm{~Hz}, 1 \mathrm{H}), 3.94(\mathrm{q}, J=6.8 \mathrm{~Hz}, 2 \mathrm{H})$, 3.12-3.07 (m, 1H), 2.46-2.40 (m, 1H), 2.36-2.28 (m, 1H), 2.14-2.01 (m, 2H), 1.97-1.86 (m, 2H), 1.78-1.60 (m, 2H), $1.36(\mathrm{t}, J=7.2 \mathrm{~Hz}, 3 \mathrm{H}), 1.21(\mathrm{t}, J=7.2 \mathrm{~Hz}, 3 \mathrm{H}) .{ }^{13} \mathbf{C}\left\{{ }^{1} \mathbf{H}\right\} \mathbf{N M R}(101 \mathrm{MHz}$, $\left.\mathrm{CDCl}_{3}\right) \delta 211.1,173.2,152.2,142.2,115.8,115.7,64.1,61.3,59.2,53.7,42.0,30.7,27.0,24.7$, 15.1, 14.3. IR (neat): 2930, 1706, 1511, 1230, 1148, 1028, 818, $752 \mathrm{~cm}^{-1} . \quad$ HR-MS (ESI) Calculated for $\mathrm{C}_{18} \mathrm{H}_{26} \mathrm{NO}_{4}\left([\mathrm{M}+\mathrm{H}]^{+}\right): 320.1856$. Found: $320.1851 .[\alpha]_{\mathrm{D}}^{26}=22.3\left(\mathrm{c} 0.48, \mathrm{CHCl}_{3}\right)$. The enantiomeric excess $(89 \%$ ee) was determined by HPLC with a Daicel Chiralpak PA2 column $($ Hexane/i-PrOH $=70: 30$, flow rate: $0.7 \mathrm{~mL} / \mathrm{min}, \lambda \max 214 \mathrm{~nm}): t_{R}($ anti minor enantiomer $)=18.40$ $\min ; t_{R}($ anti major enantiomer $)=74.14 \min$.

\section{Ethyl 2-((4-isopropoxyphenyl)amino)-2-(2-oxocyclohexyl)acetate (4y)}<smiles>CCCOc1ccc(NC(C(=O)OCC)C2CCCCC2=O)cc1</smiles>

Purification: column chromatography $\left(n-\mathrm{Hexane} / \mathrm{EtOAc}=5: 1, R_{\mathrm{f}}=0.31\right)$. Pale yellow oil. Isolated yield was $59 \%(19.7 \mathrm{mg}) . \quad{ }^{1} \mathbf{H} \mathbf{N M R}\left(400 \mathrm{MHz}, \mathrm{CDCl}_{3}\right) \delta 6.75(\mathrm{~d}, J=8.8 \mathrm{~Hz}, 2 \mathrm{H}), 6.60(\mathrm{~d}, J=$ $8.8 \mathrm{~Hz}, 2 \mathrm{H}), 4.35(\mathrm{sep}, J=6.0 \mathrm{~Hz}, 1 \mathrm{H}), 4.24(\mathrm{br}, 1 \mathrm{H}), 4.14(\mathrm{qd}, J=7.2,2.4 \mathrm{~Hz}, 2 \mathrm{H}), 3.99$ (d, $J=$ $4.0 \mathrm{~Hz}, 1 \mathrm{H}), 3.12-3.07(\mathrm{~m}, 1 \mathrm{H}), 2.47-2.38(\mathrm{~m}, 1 \mathrm{H}), 2.36-2.28(\mathrm{~m}, 1 \mathrm{H}), 2.14-2.02(\mathrm{~m}, 2 \mathrm{H}), 1.96-1.86$ $(\mathrm{m}, 2 \mathrm{H}), 1.79-1.61(\mathrm{~m}, 2 \mathrm{H}), 1.27(\mathrm{~d}, J=6.0 \mathrm{~Hz}, 6 \mathrm{H}), 1.21(\mathrm{t}, J=7.2 \mathrm{~Hz}, 3 \mathrm{H}) .{ }^{13} \mathbf{C}\left\{{ }^{1} \mathbf{H}\right\}$ NMR $(101$ $\left.\mathrm{MHz}, \mathrm{CDCl}_{3}\right) \delta 211.1,173.2,151.0,142.4,117.8,115.6,71.1,61.3,59.1,53.7,42.0,30.7,27.0$, 24.7, 22.3, 22.3, 14.3. IR (neat): 2934, 1706, 1508, 1369, 1229, 1117, 1027, 954, 822, $752 \mathrm{~cm}^{-1}$. HR-MS (ESI) Calculated for $\mathrm{C}_{19} \mathrm{H}_{28} \mathrm{NO}_{4}\left([\mathrm{M}+\mathrm{H}]^{+}\right): 334.2013$. Found: $334.2011 .[\alpha]_{\mathrm{D}}^{29}=27.0(\mathrm{c}$ 0.68, $\left.\mathrm{CHCl}_{3}\right)$. The enantiomeric excess $(99 \%$ ee) was determined by HPLC with a Daicel 
Chiralpak PC2 column (Hexane/i-PrOH $=90: 10$, flow rate: $0.7 \mathrm{~mL} / \mathrm{min}, \lambda \max 214 \mathrm{~nm}): \mathrm{t}_{\mathrm{R}}$ (anti minor enantiomer $)=11.62 \mathrm{~min} ; \mathrm{t}_{\mathrm{R}}($ anti major enantiomer $)=12.47 \mathrm{~min}$.

\section{Ethyl 2-((4-butoxyphenyl)amino)-2-(2-oxocyclohexyl)acetate (4z)}<smiles>CCCCOc1ccc(NC(C(=O)OCC)C2CCCCC2=O)cc1</smiles>

Purification: column chromatography $\left(n-\mathrm{Hexane} / \mathrm{EtOAc}=5: 1, R_{\mathrm{f}}=0.28\right)$. Pale yellow oil. Isolated yield was $65 \%(22.6 \mathrm{mg}) . \quad{ }^{1} \mathbf{H} \mathbf{N M R}\left(400 \mathrm{MHz}, \mathrm{CDCl}_{3}\right) \delta 6.75(\mathrm{~d}, J=8.8 \mathrm{~Hz}, 2 \mathrm{H}), 6.62(\mathrm{~d}, J=$ $8.8 \mathrm{~Hz}, 2 \mathrm{H}), 4.20(\mathrm{br}, 1 \mathrm{H}), 4.18-4.10(\mathrm{~m}, 2 \mathrm{H}), 3.98(\mathrm{~d}, J=4.0 \mathrm{~Hz}, 1 \mathrm{H}), 3.87$ (t, $J=6.4 \mathrm{~Hz}, 2 \mathrm{H})$, 3.12-3.07 (m, 1H), 2.47-2.38 (m, 1H), 2.36-2.28 (m, 1H), 2.16-2.01 (m, 2H), 1.97-1.87 (m, 2H), 1.75-1.64 (m, 4H), $1.46(\mathrm{sex}, J=7.6 \mathrm{~Hz}, 2 \mathrm{H}), 1.21(\mathrm{t}, J=7.2 \mathrm{~Hz}, 3 \mathrm{H}), 0.95$ (t, $J=7.2 \mathrm{~Hz}, 3 \mathrm{H})$. ${ }^{13} \mathbf{C}\left\{{ }^{1} \mathbf{H}\right\}$ NMR (101 MHz, $\left.\mathrm{CDCl}_{3}\right) \delta 211.1,173.2,152.5,142.2,115.8,115.7,68.5,61.3,59.3,53.8$, 42.0, 31.6, 30.7, 27.0, 24.7, 19.4, 14.3, 14.0. IR (neat): 2930, 2866, 1706, 1510, 1233, 1148, 1071, 1027, 820, $500 \mathrm{~cm}^{-1}$. HR-MS (ESI) Calculated for $\mathrm{C}_{20} \mathrm{H}_{30} \mathrm{NO}_{4}\left([\mathrm{M}+\mathrm{H}]^{+}\right)$: 348.2169. Found: 348.2171. $[\alpha]_{\mathrm{D}}{ }^{22}=19.0\left(\mathrm{c} 0.25, \mathrm{CHCl}_{3}\right)$. The enantiomeric excess (92\% ee) was determined by HPLC with a Daicel Chiralpak Ij-3 column $\left(\mathrm{CO}_{2} / \mathrm{MeOH}=98: 2\right.$, flow rate: $1.3 \mathrm{~mL} / \mathrm{min}, \lambda \max 254$ $\mathrm{nm}): \mathrm{t}_{\mathrm{R}}($ anti major enantiomer $)=19.706 \mathrm{~min} ; \mathrm{t}_{\mathrm{R}}($ anti minor enantiomer $)=21.787 \mathrm{~min}$.

\section{Ethyl 2-((4-(benzyloxy)phenyl)amino)-2-(2-oxocyclohexyl)acetate (4aa)}<smiles>CCOC(=O)C(Nc1ccc(OCC)cc1)C1CCCCC1=O</smiles>

Purification: column chromatography $\left(n\right.$-Hexane/EtOAc $\left.=5: 1, R_{\mathrm{f}}=0.36\right)$. Pale yellow oil. Isolated yield was $68 \%(26.1 \mathrm{mg}) . \quad{ }^{1} \mathbf{H}$ NMR $\left(400 \mathrm{MHz}, \mathrm{CDCl}_{3}\right) \delta 7.45-7.28(\mathrm{~m}, 5 \mathrm{H}), 6.83(\mathrm{~d}, J=8.8 \mathrm{~Hz}$, 2H), $6.63(\mathrm{~d}, J=8.8 \mathrm{~Hz}, 2 \mathrm{H}), 4.98(\mathrm{~s}, 2 \mathrm{H}), 4.27(\mathrm{br}, 1 \mathrm{H}), 4.19-4.11(\mathrm{~m}, 2 \mathrm{H}), 3.99(\mathrm{~d}, J=4.0 \mathrm{~Hz}$, 1H), 3.14-3.09 (m, 1H), 2.46-2.41 (m, 1H), 2.37-2.28 (m, 1H), 2.15-2.02 (m, 2H), 1.97-1.86 (m, 2H), 1.79-1.62 (m, 2H), $1.22(\mathrm{t}, J=7.2 \mathrm{~Hz}, 3 \mathrm{H}) .{ }^{13} \mathbf{C}\left\{{ }^{1} \mathbf{H}\right\} \mathbf{N M R}\left(101 \mathrm{MHz}, \mathrm{CDCl}_{3}\right) \delta 211.1,173.2$, 152.1, 142.5, 137.7, 128.6, 127.9, 127.6, 116.1, 115.6, 70.8, 61.4, 59.1, 53.7, 42.0, 30. 7, 27.0, 24.70, 
14.3. IR (neat): 2922, 2854, 1706, 1510, 1453, 1376, 1229, 1024, 819, 756, $697 \mathrm{~cm}^{-1}$. HR-MS (ESI) Calculated for $\mathrm{C}_{23} \mathrm{H}_{27} \mathrm{NO}_{4} \mathrm{Na}\left([\mathrm{M}+\mathrm{Na}]^{+}\right)$: 404.1832. Found: 404.1843. [ $\left.\alpha\right]_{\mathrm{D}}^{23}=7.3$ (c 0.7, $\left.\mathrm{CHCl}_{3}\right)$. The enantiomeric excess $(92 \%$ ee) was determined by HPLC with a Daicel Chiralpak IG column (Hexane/i-PrOH $=90: 10$, flow rate: $0.7 \mathrm{~mL} / \mathrm{min}, \lambda \max 214 \mathrm{~nm}$ ): $\mathrm{t}_{\mathrm{R}}$ (anti major enantiomer $)=114.67 \mathrm{~min} ; t_{R}($ anti minor enantiomer $)=127.22 \mathrm{~min}$.

Ethyl 2-((3-chloro-4-methoxyphenyl)amino)-2-(2-oxocyclohexyl)acetate (4ab)<smiles>CCOC(=O)C(Nc1ccc(OC)c(Cl)c1)C1CCCCC1=O</smiles>

Purification: column chromatography $\left(n\right.$-Hexane/EtOAc $\left.=5: 1, R_{\mathrm{f}}=0.2\right)$. Pale yellow oil. Isolated yield was $41 \%(14 \mathrm{mg}) .{ }^{1} \mathbf{H}$ NMR $\left(400 \mathrm{MHz}, \mathrm{CDCl}_{3}\right) \delta 6.77(\mathrm{~d}, J=8.8 \mathrm{~Hz}, 1 \mathrm{H}), 6.71(\mathrm{~s}, 1 \mathrm{H}), 6.54(\mathrm{~d}$, $J=8.4 \mathrm{~Hz}, 1 \mathrm{H}), 4.31(\mathrm{br}, 1 \mathrm{H}), 4.19-4.09$ (m, 2H), 3.90 (s, 1H), 3.80 (s, 3H), 3.16-3.10 (m, 1H), 2.43$2.28(\mathrm{~m}, 2 \mathrm{H}), 2.12-2.04(\mathrm{~m}, 2 \mathrm{H}), 1.96-1.83(\mathrm{~m}, 2 \mathrm{H}), 1.78-1.60(\mathrm{~m}, 2 \mathrm{H}), 1.21(\mathrm{t}, J=7.2 \mathrm{~Hz}, 3 \mathrm{H}) . \quad{ }^{13} \mathbf{C}\left\{{ }^{1} \mathbf{H}\right\}$ $\operatorname{NMR}\left(101 \mathrm{MHz}, \mathrm{CDCl}_{3}\right) \delta 211.2,172.8,148.1,143.0,123.3,116.4,114.0,113.6,61.5,58.8,57.0,53.6$, 42.0, 30.8, 27.0, 24.7, 14.2. IR (neat): 3380, 2936, 1704, 1503, 1229, 1202, 1131, 1060, 1020, 801, $751 \mathrm{~cm}^{-1}$. HR-MS (ESI) Calculated for $\mathrm{C}_{17} \mathrm{H}_{22} \mathrm{NO}_{4} \mathrm{Na}\left([\mathrm{M}+\mathrm{Na}]^{+}\right)$: 362.1130 . Found: 362.1134 . $[\alpha]_{\mathrm{D}}^{21}=23.7\left(\mathrm{c} 0.5, \mathrm{CHCl}_{3}\right) . \quad$ The enantiomeric excess $(85 \%$ ee) was determined by HPLC with a Daicel Chiralpak AD-H column (Hexane/i-PrOH = 90:10, flow rate: $0.7 \mathrm{~mL} / \mathrm{min}, \lambda \max 214 \mathrm{~nm}$ ): $t_{R}($ anti minor enantiomer $)=24.36 \mathrm{~min} ; t_{R}($ anti major enantiomer $)=43.31 \mathrm{~min}$.

\section{Ethyl 2-(2-oxocyclohexyl)-2-(p-tolylamino)acetate (4ac) ${ }^{1}$}<smiles>CCOC(=O)C(Nc1ccc(C)cc1)C1CCCCC1=O</smiles>

Purification: column chromatography $\left(n\right.$-Hexane/EtOAc $\left.=5: 1, R_{\mathrm{f}}=0.43\right)$. Pale yellow oil. Isolated yield was $60 \%(17.4 \mathrm{mg}) . \quad{ }^{1} \mathbf{H}$ NMR $\left(400 \mathrm{MHz}, \mathrm{CDCl}_{3}\right) \delta 6.98(\mathrm{~d}, J=8.0 \mathrm{~Hz}, 2 \mathrm{H}), 6.58(\mathrm{~d}, J=8.0$ $\mathrm{Hz}, 2 \mathrm{H}), 4.36$ (br, 1H), 4.15 (t, $J=7.2 \mathrm{~Hz}, 2 \mathrm{H}), 4.08(\mathrm{~s}, 1 \mathrm{H}), 3.16-3.10(\mathrm{~m}, 1 \mathrm{H}), 2.48-2.39$ (m, 1H), 2.36$2.28(\mathrm{~m}, 1 \mathrm{H}), 2.23(\mathrm{~s}, 3 \mathrm{H}), 2.17-2.00(\mathrm{~m}, 2 \mathrm{H}), 1.98-1.82(\mathrm{~m}, 2 \mathrm{H}), 1.78-1.61(\mathrm{~m}, 2 \mathrm{H}), 1.22(\mathrm{t}, J=7.2 \mathrm{~Hz}$, 
3H). ${ }^{13} \mathbf{C}\left\{{ }^{1} \mathbf{H}\right\}$ NMR $\left(101 \mathrm{MHz}, \mathrm{CDCl}_{3}\right) \delta 211.11,173.01,145.80,129.83,127.67,114.10,61.33,58.09$, $53.71,41.91,30.54,26.93,24.65,20.48,14.23 . \quad[\alpha]_{\mathrm{D}}^{27}=36.4\left(\mathrm{c} 0.5, \mathrm{CHCl}_{3}\right) . \quad$ The enantiomeric excess (93\% ee) was determined by HPLC with a Daicel Chiralpak IC column $(\mathrm{Hexane} / \mathrm{i}-\mathrm{PrOH}=$ 95:5, flow rate: $0.7 \mathrm{~mL} / \mathrm{min}, \lambda \max 254 \mathrm{~nm}$ ): $\mathrm{t}_{\mathrm{R}}$ (anti minor enantiomer) $=24.28 \mathrm{~min} ; \mathrm{t}_{\mathrm{R}}$ (anti major enantiomer $)=29.57 \mathrm{~min}$.

\section{Ethyl 2-((4-ethylphenyl)amino)-2-(2-oxocyclohexyl)acetate (4ad)}<smiles>CCOC(=O)C(Nc1ccc(CC)cc1)C1CCCCC1=O</smiles>

Purification: column chromatography $\left(n\right.$-Hexane/EtOAc $\left.=5: 1, R_{\mathrm{f}}=0.38\right)$. Pale yellow oil. Isolated yield was $51 \%(15.5 \mathrm{mg}) . \quad{ }^{1} \mathbf{H}$ NMR $\left(400 \mathrm{MHz}, \mathrm{CDCl}_{3}\right) \delta 7.00(\mathrm{~d}, J=8.4 \mathrm{~Hz}, 2 \mathrm{H}), 6.59(\mathrm{~d}, J=$ $8.4 \mathrm{~Hz}, 2 \mathrm{H}), 4.42(\mathrm{br}, 1 \mathrm{H}), 4.19-4.11(\mathrm{~m}, 2 \mathrm{H}), 4.08(\mathrm{~d}, J=3.6 \mathrm{~Hz}, 1 \mathrm{H}), 3.16-3.10(\mathrm{~m}, 1 \mathrm{H}), 2.53(\mathrm{q}$, $J=7.6 \mathrm{~Hz}, 2 \mathrm{H}), 2.48-2.38(\mathrm{~m}, 1 \mathrm{H}), 2.36-2.28(\mathrm{~m}, 1 \mathrm{H}), 2.17-2.00(\mathrm{~m}, 2 \mathrm{H}), 1.99-1.82(\mathrm{~m}, 2 \mathrm{H}), 1.77-$ $1.60(\mathrm{~m}, 2 \mathrm{H}), 1.25-1.16(\mathrm{~m}, 6 \mathrm{H}) . \quad{ }^{13} \mathbf{C}\left\{{ }^{1} \mathbf{H}\right\} \mathbf{N M R}\left(101 \mathrm{MHz}, \mathrm{CDCl}_{3}\right) \delta 211.2,173.1,146.0,134.3$, 128.7, 114.1, 61.4, 58.1, 53.8, 42.0, 30.6, 28.0, 27.0, 24.7, 16.0, 14.3. IR (neat): 2933, 2864,1705, $1615,1517,1449,1367,1255,1200,1150,1026,963,822,742 \mathrm{~cm}^{-1} . \quad$ HR-MS (ESI) Calculated for $\mathrm{C}_{18} \mathrm{H}_{25} \mathrm{NO}_{3} \mathrm{Na}\left([\mathrm{M}+\mathrm{Na}]^{+}\right)$: 326.1727. Found: 326.1729. $[\alpha]_{\mathrm{D}}{ }^{25}=11.5\left(\mathrm{c} 0.5, \mathrm{CHCl}_{3}\right)$. The enantiomeric excess $(86 \%$ ee) was determined by HPLC with a Daicel Chiralpak IE3 column $(\mathrm{Hexane} / \mathrm{i}-\mathrm{PrOH}=80: 20$, flow rate: $0.7 \mathrm{~mL} / \mathrm{min}, \lambda \max 214 \mathrm{~nm}): \mathrm{t}_{\mathrm{R}}($ anti minor enantiomer $)=15.21$ $\min ; t_{R}($ anti major enantiomer $)=23.90 \mathrm{~min}$.

\section{Ethyl 2-((4-isopropylphenyl)amino)-2-(2-oxocyclohexyl)acetate (4ae)}<smiles>CCOC(=O)C(Nc1ccc(C(C)C)cc1)C1CCCCC1=O</smiles>

Purification: column chromatography $\left(n\right.$-Hexane/EtOAc $\left.=5: 1, R_{\mathrm{f}}=0.34\right)$. Pale yellow oil. Isolated yield was 54\% (17.1 mg). ${ }^{1} \mathbf{H}$ NMR $\left(400 \mathrm{MHz}, \mathrm{CDCl}_{3}\right) \delta 7.03(\mathrm{~d}, J=8.4 \mathrm{~Hz}, 2 \mathrm{H}), 6.60(\mathrm{~d}, J=8.4$ $\mathrm{Hz}, 2 \mathrm{H}), 4.40$ (br, 1H), 4.22-4.06 (m, 3H), 3.16-3.10 (m, 1H), 2.80 (sep, $J=6.8 \mathrm{~Hz}, 1 \mathrm{H}), 2.48-2.39$ 
(m, 1H), 2.36-2.28 (m, 1H), 2.15-2.02 (m, 2H), 1.98-1.82 (m, 2H), 1.78-1.63 (m, 2H), 1.25-1.15 (m, 9H). ${ }^{13} \mathbf{C}\left\{{ }^{1} \mathbf{H}\right\}$ NMR $\left(101 \mathrm{MHz}, \mathrm{CDCl}_{3}\right) \delta 211.1,173.0,146.1,139.0,127.2,114.0,61.4,58.0,53.8$, 42.0, 33.3, 30.6, 26.9, 24.7, 24.3, 24.3, 14.3. IR (neat): 2933, 1707, 1614, 1518, 1449, 1252, 1185 , 1026, 821, 752, $665 \mathrm{~cm}^{-1}$. HR-MS (ESI) Calculated for $\mathrm{C}_{19} \mathrm{H}_{27} \mathrm{NO}_{3} \mathrm{Na}\left([\mathrm{M}+\mathrm{Na}]^{+}\right)$: 340.1883 . Found: $340.1889 .[\alpha]_{\mathrm{D}}{ }^{25}=21.1\left(\mathrm{c} 0.25, \mathrm{CHCl}_{3}\right)$. The enantiomeric excess $(83 \%$ ee $)$ was determined by HPLC with a Daicel Chiralpak PA2 column (Hexane/i-PrOH = 90:10, flow rate: 0.7 $\mathrm{mL} / \min , \lambda \max 214 \mathrm{~nm}): t_{R}($ anti minor enantiomer $)=19.94 \mathrm{~min} ; \mathrm{t}_{\mathrm{R}}($ anti major enantiomer $)=92.19$ $\min$.

Ethyl 2-((4-(tert-butyl)phenyl)amino)-2-(2-oxocyclohexyl)acetate (4af)<smiles>CCOC(=O)C(Nc1ccc(C(C)(C)C)cc1)C1CCCCC1=O</smiles>

Purification: column chromatography $\left(n\right.$-Hexane/EtOAc $\left.=5: 1, R_{\mathrm{f}}=0.34\right)$. Pale yellow oil. Isolated yield was $53 \%$ (17.6 mg). ${ }^{1} \mathbf{H}$ NMR $\left(400 \mathrm{MHz}, \mathrm{CDCl}_{3}\right) \delta 7.19(\mathrm{~d}, J=8.8 \mathrm{~Hz}, 2 \mathrm{H}), 6.60(\mathrm{~d}, J=8.8$ $\mathrm{Hz}, 2 \mathrm{H}), 4.41$ (br, 1H), 4.20-4.07 (m, 3H), 3.18-3.09 (m, 1H), 2.47-2.39 (m, 1H), 2.36-2.29 (m, 1H), 2.15-2.01 (m, 2H), 1.98-1.81 (m, 2H), 1.78-1.60 (m, 2H), $1.26(\mathrm{~s}, 9 \mathrm{H}), 1.22(\mathrm{t}, J=7.2 \mathrm{~Hz}, 3 \mathrm{H})$. ${ }^{13} \mathbf{C}\left\{{ }^{1} \mathbf{H}\right\}$ NMR (101 MHz, $\left.\mathrm{CDCl}_{3}\right) \delta 211.1,173.0,145.7,141.2,126.2,113.6,61.4,57.9,53.8,42.0$, 34.0, 31.7, 30.5, 26.9, 24.7, 14.3. IR (neat): 2922, 2854, 1710, 1614, 1519, 1457, 1376, 1257, 1193, 1028, 821, $757 \mathrm{~cm}^{-1}$. HR-MS (ESI) Calculated for $\mathrm{C}_{20} \mathrm{H}_{30} \mathrm{NO}_{3}\left([\mathrm{M}+\mathrm{H}]^{+}\right): 332.2220$. Found: 332.2226. $[\alpha]_{\mathrm{D}}{ }^{25}=19.3\left(\mathrm{c} 0.25, \mathrm{CHCl}_{3}\right)$. The enantiomeric excess ( $87 \%$ ee) was determined by HPLC with a Daicel Chiralpak PA2 column (Hexane/i-PrOH $=90: 10$, flow rate: $0.7 \mathrm{~mL} / \mathrm{min}, \lambda \max$ $214 \mathrm{~nm}): \mathrm{t}_{\mathrm{R}}($ anti minor enantiomer $)=18.78 \mathrm{~min} ; \mathrm{t}_{\mathrm{R}}($ anti major enantiomer $)=59.54 \mathrm{~min}$. 
Ethyl 2-((3,4-dimethylphenyl)amino)-2-(2-oxocyclohexyl)acetate (4ag)<smiles>CCOC(=O)C(Nc1ccc(C)c(C)c1)C1CCCCC1=O</smiles>

Purification: column chromatography $\left(n\right.$-Hexane/EtOAc $\left.=5: 1, R_{\mathrm{f}}=0.4\right)$. Pale yellow oil. Isolated yield was $40 \%(12.2 \mathrm{mg}) .{ }^{1} \mathbf{H}$ NMR $\left(400 \mathrm{MHz}, \mathrm{CDCl}_{3}\right) \delta 6.92(\mathrm{~d}, J=8.0 \mathrm{~Hz}, 1 \mathrm{H}), 6.48(\mathrm{~s}, 1 \mathrm{H}), 6.41$ (dd, $J=8.0,2.4 \mathrm{~Hz}, 1 \mathrm{H}), 4.33(\mathrm{br}, 1 \mathrm{H}), 4.21-4.09(\mathrm{~m}, 2 \mathrm{H}), 4.07(\mathrm{~d}, J=4.0 \mathrm{~Hz}, 1 \mathrm{H}), 3.16-3.10(\mathrm{~m}, 1 \mathrm{H})$, 2.46-2.40 (m, 1H), 2.36-2.28 (m, 1H), $2.18(\mathrm{~s}, 3 \mathrm{H}), 2.13(\mathrm{~s}, 3 \mathrm{H}), 2.12-1.99(\mathrm{~m}, 2 \mathrm{H}), 1.97-1.82(\mathrm{~m}, 2 \mathrm{H})$, 1.79-1.62 (m, 2H), $1.22(\mathrm{t}, J=7.2 \mathrm{~Hz}, 3 \mathrm{H}) .{ }^{13} \mathbf{C}\left\{{ }^{1} \mathbf{H}\right\} \mathbf{N M R}\left(101 \mathrm{MHz}, \mathrm{CDCl}_{3}\right) \delta 211.1,173.1,146.2$, $137.5,130.4,126.5,115.9,111.2,61.4,58.0,53.8,42.0,30.6,27.0,24.7,20.2,18.9,14.3$. IR (neat): 2924, 2859, 1707, 1617, 1511, 1449, 1314, 1256, 1201, 1025, 803, $703 \mathrm{~cm}^{-1}$. HR-MS (ESI) Calculated for $\mathrm{C}_{18} \mathrm{H}_{26} \mathrm{NO}_{3}\left([\mathrm{M}+\mathrm{H}]^{+}\right)$: 304.1907. Found: 304.1913. $[\alpha]_{\mathrm{D}}^{30}=44.2\left(\mathrm{c} 0.1, \mathrm{CHCl}_{3}\right)$. The enantiomeric excess (99\% ee) was determined by HPLC with a Daicel Chiralpak AS-H column $($ Hexane/i-PrOH $=80: 20$, flow rate: $0.7 \mathrm{~mL} / \mathrm{min}, \lambda \max 214 \mathrm{~nm}): \mathrm{t}_{\mathrm{R}}($ anti major enantiomer $)=7.15$ $\min ; t_{R}($ anti minor enantiomer $)=7.98$ min .

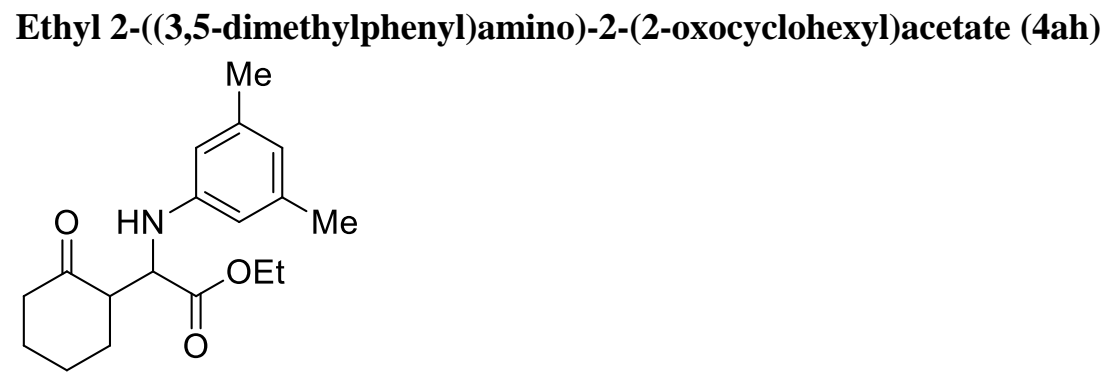

Purification: column chromatography $\left(n\right.$-Hexane/EtOAc $\left.=5: 1, R_{\mathrm{f}}=0.4\right)$. Pale yellow oil. Isolated yield was 50\% (15.2 mg). ${ }^{1} \mathrm{H}$ NMR (400 MHz, $\left.\mathrm{CDCl}_{3}\right) \delta 6.39(\mathrm{~s}, 1 \mathrm{H}), 6.28(\mathrm{~s}, 2 \mathrm{H}), 4.40(\mathrm{br}, 1 \mathrm{H})$, 4.21-4.06 (m, 3H), 3.18-3.08 (m, 1H), 2.44-2.41 (m, 1H), 2.37-2.28 (m, 1H), $2.22(\mathrm{~s}, 6 \mathrm{H}), 2.14-2.01$ $(\mathrm{m}, 2 \mathrm{H}), 1.96-1.81(\mathrm{~m}, 2 \mathrm{H}), 1.76-1.62(\mathrm{~m}, 2 \mathrm{H}), 1.23(\mathrm{t}, J=7.2 \mathrm{~Hz}, 3 \mathrm{H}) .{ }^{13} \mathbf{C}\left\{{ }^{1} \mathbf{H}\right\}$ NMR $(101 \mathrm{MHz}$, $\left.\mathrm{CDCl}_{3}\right) \delta 211.1,173.0,148.1,139.0,120.5,111.8,61.4,57.5,53.8,42.0,30.6,27.0,24.7,21.6$, 14.3. IR (neat): 2924, 2859, 1707, 1617, 1511, 1449, 1368, 1256, 1201, 1025, 803, $703 \mathrm{~cm}^{-1}$. HRMS (ESI) Calculated for $\mathrm{C}_{18} \mathrm{H}_{26} \mathrm{NO}_{3}\left([\mathrm{M}+\mathrm{H}]^{+}\right)$: 304.1907. Found: 304.1913. $[\alpha]_{\mathrm{D}}^{29}=50.8(\mathrm{c} 0.1$, $\left.\mathrm{CHCl}_{3}\right)$. The enantiomeric excess (99\% ee) was determined by HPLC with a Daicel Chiralpak IE3 
column (Hexane/i-PrOH $=80: 20$, flow rate: $0.5 \mathrm{~mL} / \mathrm{min}, \lambda \max 240 \mathrm{~nm}$ ): $\mathrm{t}_{\mathrm{R}}$ (anti major enantiomer) $=26.33 \mathrm{~min} ; \mathrm{t}_{\mathrm{R}}($ anti minor enantiomer $)=39.26 \mathrm{~min}$.

Ethyl 2-((4-(methylthio)phenyl)amino)-2-(2-oxocyclohexyl)acetate (4ai)<smiles>CCOC(=O)C(Nc1ccc(C)cc1)C1CCCCC1=O</smiles>

Purification: column chromatography $\left(n\right.$-Hexane/EtOAc $\left.=3: 1, R_{\mathrm{f}}=0.44\right)$. Pale yellow oil. Isolated yield was $42 \%(13.5 \mathrm{mg}) .{ }^{1} \mathbf{H}$ NMR $\left(400 \mathrm{MHz}, \mathrm{CDCl}_{3}\right) \delta 7.19(\mathrm{~d}, J=8.4 \mathrm{~Hz}, 2 \mathrm{H}), 6.59(\mathrm{~d}, J=8.4$ Hz, 2H), 4.52 (br, 1H), 4.19-4.11 (m, 2H), 4.07 (s, 1H), 3.18-3.13 (m, 1H), 2.49-2.26 (m, 5H), 2.15$2.02(\mathrm{~m}, 2 \mathrm{H}), 1.95-1.62(\mathrm{~m}, 4 \mathrm{H}), 1.21(\mathrm{t}, J=7.2 \mathrm{~Hz}, 3 \mathrm{H}) .{ }^{13} \mathbf{C}\left\{{ }^{1} \mathbf{H}\right\} \mathbf{N M R}\left(101 \mathrm{MHz}, \mathrm{CDCl}_{3}\right) \delta$ $211.2,172.7,146.9,131.3,125.5,114.5,61.5,57.6,53.7,42.0,30.7,27.0,24.7,19.0,14.3 . \quad$ IR (neat): 3368, 2920, 1704, 1631, 1501, 1312, 1287, 1235, 1184, 1026, 965, 816,735, 704, $498 \mathrm{~cm}^{-1}$. HR-MS (ESI) Calculated for $\mathrm{C}_{17} \mathrm{H}_{23} \mathrm{NO}_{3} \mathrm{NaS}\left([\mathrm{M}+\mathrm{Na}]^{+}\right)$: 334.1291. Found: 334.1288 . $[\alpha]_{\mathrm{D}}^{25}=14.4$ (c $0.5, \mathrm{CHCl}_{3}$ ). The enantiomeric excess $(92 \%$ ee) was determined by HPLC with a Daicel Chiralpak IE3 column (Hexane/i-PrOH = 80:20, flow rate: $0.7 \mathrm{~mL} / \mathrm{min}, \lambda \max 214 \mathrm{~nm}$ ): $\mathrm{t}_{\mathrm{R}}$ (anti minor enantiomer $)=24.13 \mathrm{~min} ; \mathrm{t}_{\mathrm{R}}($ anti major enantiomer $)=40.89 \mathrm{~min}$.

Ethyl 2-((4-methoxyphenyl)amino)-2-(5-methyl-2-oxocyclohexyl)acetate (4aj) ${ }^{10}$<smiles>CCOC(=O)C(Nc1ccc(OC)cc1)C1CC(C)CCC1=O</smiles>

Purification: column chromatography $\left(n\right.$-Hexane/EtOAc $\left.=5: 1, R_{\mathrm{f}}=0.4\right)$. Pale yellow oil. Isolated yield was 33\% (10.6 mg). ${ }^{1} \mathbf{H}$ NMR $\left(400 \mathrm{MHz}, \mathrm{CDCl}_{3}\right) \delta 6.75(\mathrm{~d}, J=8.8 \mathrm{~Hz}, 2 \mathrm{H}), 6.62(\mathrm{~d}, J=8.8$ Hz, 2H), 4.29-4.01 (m, 4H), 3.73 (d, J = 2.4 Hz, 3H), 3.19-2.99 (m, 1H), 2.45-2.33 (m, 2H), 2.24$2.19(\mathrm{~m}, 1 \mathrm{H}), 2.08-1.93(\mathrm{~m}, 2 \mathrm{H}), 1.74-1.60(\mathrm{~m}, 2 \mathrm{H}), 1.21(\mathrm{t}, J=7.2 \mathrm{~Hz}, 3 \mathrm{H}), 1.08(\mathrm{~d}, J=6.8 \mathrm{~Hz}$, 3H). ${ }^{13} \mathbf{C}\left\{{ }^{1} \mathbf{H}\right\}$ NMR $\left(101 \mathrm{MHz}, \mathrm{CDCl}_{3}\right) \delta 211.6,173.0,153.1,141.5,115.7,114.9,61.4,59.2,55.8$, 50.6, 37.9, 36.5, 33.5, 27.0, 19.6, 14.3. $[\alpha]_{\mathrm{D}}^{25}=1.85\left(\mathrm{c} 0.25, \mathrm{CHCl}_{3}\right)$. The enantiomeric excess (85\% ee) was determined by HPLC with a Daicel Chiralpak AS-H column (Hexane/i-PrOH = 98:2, 
flow rate: $0.7 \mathrm{~mL} / \mathrm{min}, \lambda \max 230 \mathrm{~nm}$ ): $\mathrm{t}_{\mathrm{R}}$ (anti major enantiomer) $=18.383 \mathrm{~min}$; $\mathrm{t}_{\mathrm{R}}$ (anti minor enantiomer) $=36.761 \mathrm{~min}$.

Ethyl 2-((4-methoxyphenyl)amino)-2-(4-oxotetrahydro-2H-pyran-3-yl)acetate (4ak)<smiles>CCOC(=O)C(Nc1ccc(OC)cc1)C1COCCC1=O</smiles>

Purification: column chromatography $\left(n\right.$-Hexane/EtOAc $\left.=3: 1, R_{\mathrm{f}}=0.4\right)$. Pale yellow oil. Isolated yield was $50 \%(15.4 \mathrm{mg}) . \mathrm{dr}($ anti/syn $)=1: 1$. Mixture of two diastereomers: ${ }^{1} \mathbf{H} \mathbf{N M R}\left(\mathrm{CDCl}_{3}, 400 \mathrm{MHz}\right)$ 6.79-6.76 (m, 2 H), 6.73-6.70 (m, 1 H), 6.63-6.61 (m, 1 H), 4.26-3.78 (m, 8 H), 3.74 (d, J = 2 Hz, 3 H), 3.27-3.22 (m, 0.5 H), 2.94-2.89 (m, 0.50 H), 2.65-2.45 (m, $2 \mathrm{H}), 1.23-1.20(\mathrm{~m}, 3 \mathrm{H}) .[\alpha]_{\mathrm{D}}{ }^{25}=-0.35(\mathrm{c}$ $\left.0.25, \mathrm{CHCl}_{3}\right)$. The enantiomeric excess $(13 \%$ ee) was determined by HPLC with a Daicel Chiralpak IC-3 column (Hexane/i-PrOH $=90: 10$, flow rate: $1.0 \mathrm{~mL} / \mathrm{min}, \lambda \max 254 \mathrm{~nm}): \mathrm{t}_{\mathrm{R}}=22.736 \mathrm{~min} ; \mathrm{t}_{\mathrm{R}}$ $=24.713 \mathrm{~min}$.

Ethyl 2-((4-methoxyphenyl)amino)-2-(4-oxotetrahydro-2H-thiopyran-3-yl)acetate (4al) ${ }^{2}$<smiles>CCOC(=O)C(Nc1ccc(OC)cc1)C1CSCCC1=O</smiles>

Purification: column chromatography $\left(n\right.$-Hexane/EtOAc $\left.=5: 1, R_{\mathrm{f}}=0.42\right)$. Pale yellow oil. Isolated yield was $45 \%(14.6 \mathrm{mg}) .{ }^{1} \mathbf{H}$ NMR $\left(400 \mathrm{MHz}, \mathrm{CDCl}_{3}\right) \delta 6.76(\mathrm{~d}, J=8.0 \mathrm{~Hz}, 2 \mathrm{H}), 6.64(\mathrm{~d}, J=8.4$ Hz, 2H), 4.24 (d, $J=4.8 \mathrm{~Hz}, 1 \mathrm{H}), 4.14(\mathrm{q}, J=7.2 \mathrm{~Hz}, 3 \mathrm{H}), 3.73$ (s, 3H), 3.39-3.34 (m, 1H), 3.17$3.11(\mathrm{~m}, 1 \mathrm{H}), 3.02-2.88(\mathrm{~m}, 3 \mathrm{H}), 2.79-2.67(\mathrm{~m}, 2 \mathrm{H}), 1.21(\mathrm{t}, J=7.2 \mathrm{~Hz}, 3 \mathrm{H}) .{ }^{13} \mathbf{C}\left\{{ }^{1} \mathbf{H}\right\}$ NMR $(126$ $\left.\mathrm{MHz}, \mathrm{CDCl}_{3}\right) \delta 208.2,172.3,153.2,141.5,116.0,114.9,61.6,59.1,55.8,55.4,43.9,32.9,30.0$, 14.2. $[\alpha]_{\mathrm{D}}^{26}=35.19\left(\mathrm{c} 0.25, \mathrm{CHCl}_{3}\right)$. The enantiomeric excess (74\% ee) was determined by HPLC with a Daicel Chiralpak OZ-3 column (Hexane/i-PrOH = 98:2, flow rate: $0.7 \mathrm{~mL} / \mathrm{min}, \lambda \max 230$ $\mathrm{nm}): \mathrm{t}_{\mathrm{R}}($ anti minor enantiomer $)=22.736 \mathrm{~min} ; \mathrm{t}_{\mathrm{R}}($ anti major enantiomer $)=24.713 \mathrm{~min}$. 
Ethyl 2-((4-methoxyphenyl)amino)-2-(2-oxocycloheptyl)acetate (4am) $)^{2}$<smiles>CCOC(=O)C(Nc1ccc(OC)cc1)C1CCCCCC1=O</smiles>

Purification: column chromatography $\left(n\right.$-Hexane/EtOAc $\left.=5: 1, R_{\mathrm{f}}=0.46\right)$. Pale yellow oil. Isolated yield was $42 \%(13.4 \mathrm{mg}) .{ }^{1} \mathbf{H}$ NMR $\left(400 \mathrm{MHz}, \mathrm{CDCl}_{3}\right) \delta 6.77(\mathrm{~d}, J=8.8 \mathrm{~Hz}, 2 \mathrm{H}), 6.67(\mathrm{~d}, J=8.8$ $\mathrm{Hz}, 2 \mathrm{H}), 4.31(\mathrm{~d}, J=4.8 \mathrm{~Hz}, 2 \mathrm{H}), 4.16(\mathrm{q}, J=7.2 \mathrm{~Hz}, 2 \mathrm{H}), 3.73(\mathrm{~s}, 3 \mathrm{H}), 3.05-3.00(\mathrm{~m}, 1 \mathrm{H}), 2.60-$ $2.46(\mathrm{~m}, 2 \mathrm{H}), 2.04-1.85(\mathrm{~m}, 4 \mathrm{H}), 1.59-1.48(\mathrm{~m}, 2 \mathrm{H}), 1.41-1.32(\mathrm{~m}, 2 \mathrm{H}), 1.23(\mathrm{t}, J=7.2 \mathrm{~Hz}, 3 \mathrm{H})$. ${ }^{13} \mathbf{C}\left\{{ }^{1} \mathbf{H}\right\}$ NMR $\left(101 \mathrm{MHz}, \mathrm{CDCl}_{3}\right) \delta 214.4,172.7,152.8,141.1,115.3,115.0,61.5,60.8,55.9,54.5$, $44.0,30.1,29.2,27.3,24.4,14.3 .[\alpha]_{\mathrm{D}}^{25}=55.85\left(\mathrm{c} 0.25, \mathrm{CHCl}_{3}\right)$. The enantiomeric excess $(89 \%$ ee $)$ was determined by HPLC with a Daicel Chiralpak AS-H column (Hexane/i-PrOH = 98:2, flow rate: $0.7 \mathrm{~mL} / \mathrm{min}, \lambda \max 230 \mathrm{~nm}): \mathrm{t}_{\mathrm{R}}($ anti major enantiomer $)=19.552 \mathrm{~min} ; \mathrm{t}_{\mathrm{R}}($ anti minor enantiomer $)=$ $33.642 \mathrm{~min}$.

Propyl 2-((4-methoxyphenyl)amino)-2-(2-oxocycloheptyl)acetate (4an)<smiles>CCCOC(=O)C(Nc1ccc(OC)cc1)C1CCCCCC1=O</smiles>

Purification: column chromatography $\left(n\right.$-Hexane/EtOAc $\left.=8: 1, R_{\mathrm{f}}=0.56\right)$. Pale yellow oil. Isolated yield was $44 \%(14.7 \mathrm{mg}) .{ }^{1} \mathbf{H}$ NMR $\left(400 \mathrm{MHz}, \mathrm{CDCl}_{3}\right) \delta 6.77(\mathrm{~d}, J=8.8 \mathrm{~Hz}, 2 \mathrm{H}), 6.66(\mathrm{~d}, J=8.8$ $\mathrm{Hz}, 2 \mathrm{H}), 4.30(\mathrm{~d}, J=4.8 \mathrm{~Hz}, 1 \mathrm{H}), 4.07-4.03(\mathrm{~m}, 2 \mathrm{H}), 3.73(\mathrm{~s}, 3 \mathrm{H}), 3.07-3.03(\mathrm{~m}, 1 \mathrm{H}), 2.66-2.37(\mathrm{~m}$, 2H), 2.0-1.87 (m, 4H), 1.65-1.54 (m, 4H), 1.37 (q, $J=9.6 \mathrm{~Hz}, 2 \mathrm{H}), 0.88(\mathrm{t}, J=7.6 \mathrm{~Hz}, 3 \mathrm{H}) .{ }^{13} \mathbf{C}\left\{{ }^{1} \mathbf{H}\right\}$ NMR $\left(126 \mathrm{MHz}, \mathrm{CDCl}_{3}\right) \delta 214.3,172.9,152.9,141.2,115.4,115.0,67.1,60.9,55.9,54.5,44.0$, 30.0, 29.2, 27.5, 24.3, 22.1, 10.5. IR (neat): 2927, 1694, 1454, 1235, 1035, 936, 821, 754, $516 \mathrm{~cm}^{-}$ 1. HR-MS (ESI) Calculated for $\mathrm{C}_{19} \mathrm{H}_{27} \mathrm{NO}_{4} \mathrm{Na}\left([\mathrm{M}+\mathrm{Na}]^{+}\right)$: 356.1832. Found: $356.1838 .[\alpha]_{\mathrm{D}}{ }^{23}=$ 27.8 (c $\left.0.25, \mathrm{CHCl}_{3}\right)$. The enantiomeric excess $(90 \%$ ee) was determined by HPLC with a Daicel Chiralpak AD-3 column (Hexane/i-PrOH = 90:10, flow rate: $0.7 \mathrm{~mL} / \mathrm{min}, \lambda \max 254 \mathrm{~nm}): \mathrm{t}_{\mathrm{R}}($ anti minor enantiomer $)=20.269 \mathrm{~min} ; \mathrm{t}_{\mathrm{R}}($ anti major enantiomer $)=25.092 \mathrm{~min}$.

Butyl 2-((4-methoxyphenyl)amino)-2-(2-oxocycloheptyl)acetate (4ao) 
<smiles>CCOC(=O)C(Nc1ccc(OC)cc1)C1CCCCCC1=O</smiles>

Purification: column chromatography $\left(n\right.$-Hexane/EtOAc $\left.=8: 1, R_{\mathrm{f}}=0.6\right)$. Pale yellow oil. Isolated yield was $40 \%$ (14.0 mg). ${ }^{1} \mathbf{H}$ NMR $\left(400 \mathrm{MHz}, \mathrm{CDCl}_{3}\right) \delta 6.77(\mathrm{~d}, J=8.8 \mathrm{~Hz}, 2 \mathrm{H}), 6.66(\mathrm{~d}, J=8.8$ $\mathrm{Hz}, 2 \mathrm{H}), 4.30$ (d, J=4.8 Hz, 1H), 4.07-4.03 (m, 2H), 3.73 (s, 3H), 3.07-3.03 (m, 1H), 2.66-2.37 (m, 2H), 2.0-1.87 (m, 4H), 1.65-1.54 (m, 6H), 1.37 (q, $J=9.6 \mathrm{~Hz}, 2 \mathrm{H}), 0.88(\mathrm{t}, J=7.6 \mathrm{~Hz}, 3 \mathrm{H}) .{ }^{13} \mathbf{C}\left\{{ }^{1} \mathbf{H}\right\}$ NMR $\left(126 \mathrm{MHz}, \mathrm{CDCl}_{3}\right) \delta 214.3,172.9,152.9,141.2,115.4,115.0,65.3,60.9,55.9,54.5,44.0$, 30.7, 30.0, 29.2, 27.5, 24.3, 19.2, 13.8. IR (neat): 2926, 1695, 1512, 1454, 1236, 1036, 938, 820, $516 \mathrm{~cm}^{-1}$. HR-MS (ESI) Calculated for $\mathrm{C}_{20} \mathrm{H}_{29} \mathrm{NO}_{4} \mathrm{Na}\left([\mathrm{M}+\mathrm{Na}]^{+}\right)$: 370.1989 . Found: 370.1991 . $[\alpha]_{\mathrm{D}}{ }^{24}=33.3\left(\mathrm{c} 0.25, \mathrm{CHCl}_{3}\right)$. The enantiomeric excess (90\% ee) was determined by HPLC with a Daicel Chiralpak AD-3 column (Hexane/i-PrOH = 95:5, flow rate: $0.7 \mathrm{~mL} / \mathrm{min}, \lambda \max 254 \mathrm{~nm}$ ): $\mathrm{t}_{\mathrm{R}}$ $($ anti minor enantiomer $)=29.847 \mathrm{~min} ; \mathrm{t}_{\mathrm{R}}($ anti major enantiomer $)=38.374 \mathrm{~min}$. 


\section{Large-Scale Synthesis and Mechanistic Studies}

(a) Large-scale synthesis of $4 \mathrm{~m}$ and $4 \mathrm{a}$<smiles>COc1ccc(NCC(=O)O[Na])cc1</smiles>

$1 \mathrm{~m}(2 \mathrm{mmol})$<smiles>O=C1CCCCC1</smiles>

2a
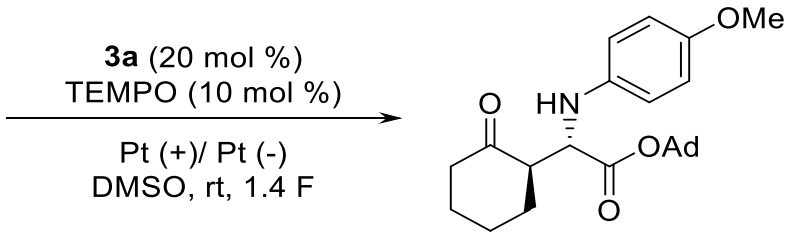

$4 \mathrm{~m}, 60 \%(496 \mathrm{mg})>99: 1 \mathrm{dr}, 98 \%$ ee

To a $100 \mathrm{~mL}$ hydrogenation tube charged with a proper stir bar was added $\mathbf{1 m}$ ( $2 \mathrm{mmol})$, TEMPO (0.2 mmol, $10 \mathrm{~mol} \%, 31.2 \mathrm{mg})$, 3a (0.4 mmol, $20 \mathrm{~mol} \%, 48.5 \mathrm{mg}), 2 \mathbf{a}(20 \mathrm{mmol}, 10$ equiv, 2066 $\mu \mathrm{L})$, and DMSO $(80 \mathrm{~mL})$. The tube was sealed with a septum equipped with two Pt electrodes (4.0 $\times 4.0 \mathrm{~cm}^{2}$ ) and the reaction mixture was electrolyzed under a constant current of $24 \mathrm{~mA}$ for $6.25 \mathrm{~h}$. After the reaction was completed, the mixture was diluted with $200 \mathrm{~mL}$ of water and extracted with EtOAc $(3 \times 40 \mathrm{~mL})$. The organic layer was washed with saturated $\mathrm{NaCl}$ (aq.) $(120 \mathrm{~mL})$, dried over $\mathrm{Na}_{2} \mathrm{SO}_{4}$, filtered, and the solvent removed under reduced pressure. The crude product was purified by flash column chromatography (eluent: Petroleum ether: EtOAc= 5:1), affording the desired product $\mathbf{4 m}$ in 60\% (496 mg) isolated yield (> 99:1 dr, 98\% ee).

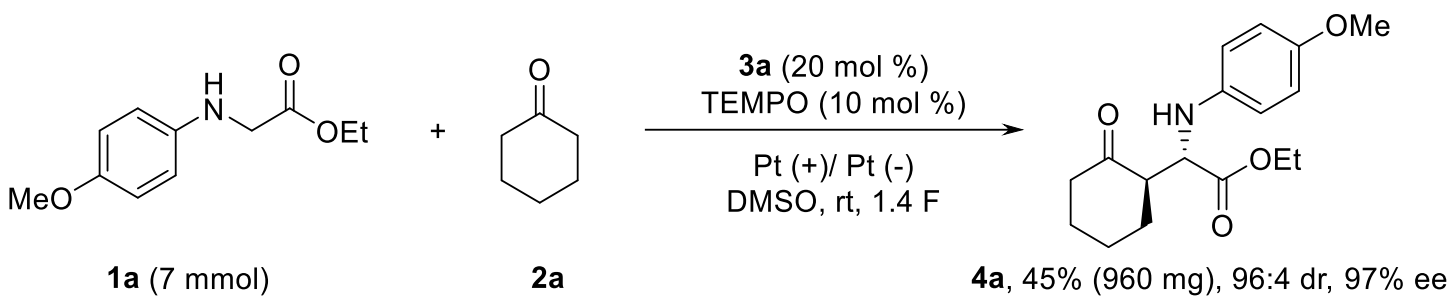

To a $100 \mathrm{~mL}$ hydrogenation tube charged with a proper stir bar was added $\mathbf{1 a}(7 \mathrm{mmol})$, TEMPO (0.7 mmol, $10 \mathrm{~mol} \%, 109.4 \mathrm{mg}), 3 a(1.4 \mathrm{mmol}, 20 \mathrm{~mol} \%, 178.6 \mathrm{mg}), \mathbf{2 a}(7 \mathrm{mmol}, 10$ equiv, 7231 $\mu \mathrm{L})$, and DMSO (80 mL). The tube was sealed with a septum equipped with two Pt electrodes (4.0 $\times 4.0 \mathrm{~cm}^{2}$ ) and the reaction mixture was electrolyzed under a constant current of $24 \mathrm{~mA}$ for $21.5 \mathrm{~h}$. After the reaction was completed, the mixture was diluted with $200 \mathrm{~mL}$ of water and extracted with EtOAc $(3 \times 40 \mathrm{~mL})$. The organic layer was washed with saturated $\mathrm{NaCl}$ (aq.) $(120 \mathrm{~mL})$, dried over $\mathrm{Na}_{2} \mathrm{SO}_{4}$, filtered, and the solvent removed under reduced pressure. The crude product was purified by flash column chromatography (eluent: Petroleum ether: EtOAc= 5:1), affording the desired product $4 \mathbf{a}$ in $45 \%(960 \mathrm{mg})$ isolated yield (96:4 dr, 97\% ee). 


\section{(b) Control experiment.}<smiles>CCOC(=O)CNc1ccc(OC)cc1</smiles>

$1 \mathrm{a}$<smiles>O=C1CCCCC1</smiles>

$2 a$<smiles>CCOC(=O)C(Nc1ccc(OC)cc1)C1CCCCC1=O</smiles>

$4 a$

$35 \%, 63: 37 \mathrm{dr}, \mathrm{rac}$

The procedure for the CDC reaction of $N$-PMP glycine ester with cyclohexane was followed the reported literature. ${ }^{[2]}$ To a solution of cyclohexane (15.0 equiv) and 3a (5.4 mg, $0.045 \mathrm{mmol}, 30$ $\mathrm{mol} \%$, ) in $\mathrm{CHCl}_{3}(1.0 \mathrm{~mL})$ was added $N$-PMP glycine ester $\mathbf{1 a}(0.15 \mathrm{mmol})$ and $\mathrm{Cu}(\mathrm{OAc})_{2} \cdot \mathrm{H}_{2} \mathrm{O}(3.0$ $\mathrm{mg}, 10 \mathrm{~mol} \%)$, and the reaction mixture was stirred at $0^{\circ} \mathrm{C}$ for 5-10 min. Then DDQ (34.1 $\mathrm{mg}, 0.15$ mmol, 1.0 equiv) was added at $0{ }^{\circ} \mathrm{C}$ in portions. The reaction mixture was stirred at $0-5^{\circ} \mathrm{C}$ for $6 \mathrm{~h}$, and at $\mathrm{rt}$ for $18 \mathrm{~h}$ under air. After the reaction was finished, the mixture was filtered through a short pad of silica gel (diethyl ether as eluent). The filtrate was concentrated, and the residue was analysised by proton NMR. The dr and ee values were determined by chiral HPLC analysis of the pure product.

\section{(c) Chemical oxidant instead of electric current}<smiles>CCOC(=O)CNc1ccc(NC(C(=O)OCC)C2CCCCC2C(=O)OCC)cc1</smiles>

To a $15 \mathrm{~mL}$ hydrogenation tube charged with a proper stir bar was added $\mathbf{1 a}(0.1 \mathrm{mmol})$, TEMPO (0.01 mmol, $10 \mathrm{~mol} \%, 1.6 \mathrm{mg}), 3 \mathrm{a}$ (0.02 mmol, $20 \mathrm{~mol} \%, 2.4 \mathrm{mg})$, oxidant (2 equiv), $\mathbf{2 a}$ ( $1 \mathrm{mmol}$, 10 equiv, $108 \mu \mathrm{L}$ ), and DMSO (4 mL). The tube was sealed with a septum at rt for $60 \mathrm{~h}$. After the reaction was completed, the mixture was diluted with $20 \mathrm{~mL}$ of water and extracted with EtOAc $(3 \times 4 \mathrm{~mL})$. The organic layer was washed with saturated $\mathrm{NaCl}$ (aq.) $(12 \mathrm{~mL})$, dried over $\mathrm{Na}_{2} \mathrm{SO}_{4}$, filtered, and the solvent removed under reduced pressure. The filtrate was concentrated, and the residue was analysised by proton NMR. The dr and ee values were determined by chiral HPLC analysis of the pure product. 
(d) The effect of proton additive.

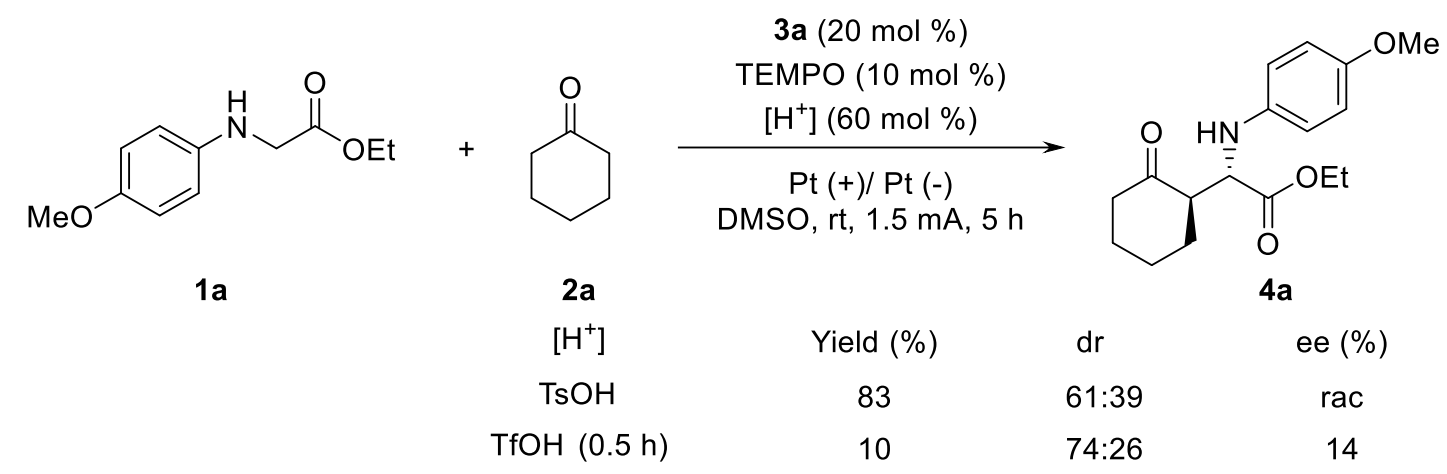

To a $15 \mathrm{~mL}$ hydrogenation tube charged with a proper stir bar was added $\mathbf{1}(0.1 \mathrm{mmol})$, TEMPO (0.01 mmol, $10 \mathrm{~mol} \%, 1.6 \mathrm{mg}), 3 a(0.02 \mathrm{mmol}, 20 \mathrm{~mol} \%, 2.4 \mathrm{mg}),\left[\mathrm{H}^{+}\right](60 \mathrm{~mol} \%), 2 \mathrm{a}(1 \mathrm{mmol}$, 10 equiv, $108 \mu \mathrm{L}$ ), and DMSO (4 mL). The tube was sealed with a septum equipped with two $\mathrm{Pt}$ electrodes $\left(1.0 \times 1.0 \mathrm{~cm}^{2}\right)$ and the reaction mixture was electrolyzed under a constant current of 1.5 $\mathrm{mA}$ for $5 \mathrm{~h}$. After the reaction was completed, the mixture was diluted with $20 \mathrm{~mL}$ of water and extracted with EtOAc $(3 \times 4 \mathrm{~mL})$. The organic layer was washed with saturated $\mathrm{NaCl}$ (aq.) $(10 \mathrm{~mL})$, dried over $\mathrm{Na}_{2} \mathrm{SO}_{4}$, filtered, and the solvent removed under reduced pressure. The filtrate was concentrated, and the residue was analysised by proton NMR. The dr and ee values were determined by chiral HPLC analysis of the pure product.

(e) The stability of $4 a$ in the presence of proton.<smiles>CCOC(=O)C(Nc1ccc(OC)cc1)C1CCCCC1=O</smiles>

$4 a$

$96: 4 \mathrm{dr}, 97 \%$ ee

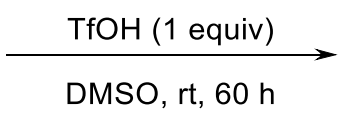<smiles>CCOC(=O)C(Nc1ccc(OC)cc1)C1CCCCC1=O</smiles>

$4 a$ $61 \%, 87: 13 \mathrm{dr}, 92 \%$ ee<smiles>CCOC(=O)C=C1CCCCC1=O</smiles>

5a, $39 \%$

To a $15 \mathrm{~mL}$ hydrogenation tube charged with a proper stir bar was added $\mathbf{4 a}(0.1 \mathrm{mmol})$, TfOH (1 equiv), and DMSO (2 mL). The tube was sealed with a septum at $\mathrm{rt}$ for $60 \mathrm{~h}$. After the reaction was completed, the mixture was diluted with $10 \mathrm{~mL}$ of water and extracted with EtOAc $(3 \times 3 \mathrm{~mL})$. The organic layer was washed with saturated $\mathrm{NaCl}$ (aq.) $\left(9 \mathrm{~mL}\right.$ ), dried over $\mathrm{Na}_{2} \mathrm{SO}_{4}$, filtered, and the solvent removed under reduced pressure. The filtrate was concentrated, and the residue was analysised by proton NMR. The dr and ee values were determined by chiral HPLC analysis of the pure product. 
(f) Stability of imine III under electrochemical conditions.

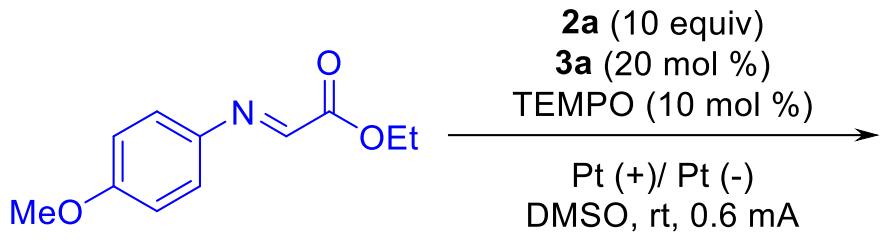

III $(0.1 \mathrm{mmol})$<smiles>CCOC(=O)[C@H](Nc1ccc(OC)cc1)[C@H]1CCCCC1=O</smiles>

$4 a$

$\begin{array}{cc}\text { reaction time }(\mathrm{h}) & \text { yield }(\%) \\ 2 & 62 \\ 5 & 50\end{array}$

To a $15 \mathrm{~mL}$ hydrogenation tube charged with a proper stir bar was added III $(0.1 \mathrm{mmol}, 20.7 \mathrm{mg})$, TEMPO (0.01 mmol, $10 \mathrm{~mol} \%, 1.6 \mathrm{mg}), 3 \mathbf{a}(0.02 \mathrm{mmol}, 20 \mathrm{~mol} \%, 2.4 \mathrm{mg}), \mathbf{2 a}(1 \mathrm{mmol}, 10$ equiv, $108 \mu \mathrm{L}$ ), and DMSO (4 mL). The tube was sealed with a septum equipped with two Pt electrodes $\left(1.0 \times 1.0 \mathrm{~cm}^{2}\right)$ and the reaction mixture was electrolyzed under a constant current of $0.6 \mathrm{~mA}$. After 2 or $5 \mathrm{~h}$, the mixture was diluted with $20 \mathrm{~mL}$ of water and extracted with EtOAc $(3 \times 4 \mathrm{~mL})$. The organic layer was washed with saturated $\mathrm{NaCl}$ (aq.) $\left(10 \mathrm{~mL}\right.$ ), dried over $\mathrm{Na}_{2} \mathrm{SO}_{4}$, filtered, and the solvent removed under reduced pressure. The filtrate was concentrated, and the residue was analysised by proton NMR.

(g) Stability of product 4 a under electrochemical conditions.<smiles>CCOC(=O)[C@H](Nc1ccc(OC)cc1)[C@H]1CCCCC1=O</smiles>

$4 \mathrm{a}(0.1 \mathrm{mmol})$

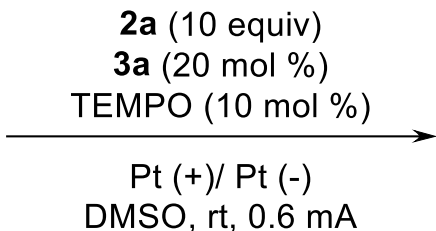

DMSO, rt, 0.6 mA<smiles>CCOC(=O)[C@H](Nc1ccc(OC)cc1)[C@H]1CCCCC1=O</smiles>

4a

reaction time $(\mathrm{h}) \quad$ yield $(\%)$

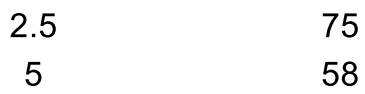

To a $15 \mathrm{~mL}$ hydrogenation tube charged with a proper stir bar was added $\mathbf{4 a}(0.1 \mathrm{mmol}, 30.5 \mathrm{mg})$, TEMPO (0.01 mmol, $10 \mathrm{~mol} \%, 1.6 \mathrm{mg}), 3 \mathbf{a}(0.02 \mathrm{mmol}, 20 \mathrm{~mol} \%, 2.4 \mathrm{mg}), \mathbf{2 a}(1 \mathrm{mmol}, 10$ equiv, $108 \mu \mathrm{L}$ ), and DMSO (4 mL). The tube was sealed with a septum equipped with two Pt electrodes $\left(1.0 \times 1.0 \mathrm{~cm}^{2}\right)$ and the reaction mixture was electrolyzed under a constant current of $0.6 \mathrm{~mA}$. After 2.5 or $5 \mathrm{~h}$, the mixture was diluted with $20 \mathrm{~mL}$ of water and extracted with EtOAc $(3 \times 4 \mathrm{~mL})$. The organic layer was washed with saturated $\mathrm{NaCl}$ (aq.) $(10 \mathrm{~mL})$, dried over $\mathrm{Na}_{2} \mathrm{SO}_{4}$, filtered, and the solvent removed under reduced pressure. The filtrate was concentrated, and the residue was 
analysised by proton NMR.

(h) Reaction of III, 2a, and 3a under non-electrochemical reaction conditions.

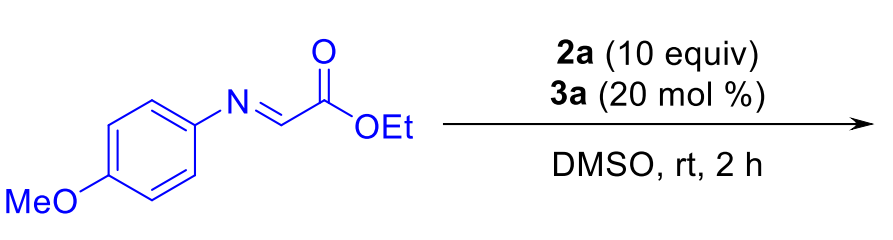

III $(0.1 \mathrm{mmol})$<smiles>CCOC(=O)[C@H](Nc1ccc(OC)cc1)[C@H]1CCCCC1=O</smiles>

$92 \%, 99: 1 \mathrm{dr}, 97 \%$ ee

To a $15 \mathrm{~mL}$ hydrogenation tube charged with a proper stir bar was added III $(0.1 \mathrm{mmol}, 20.7 \mathrm{mg})$, TEMPO (0.01 mmol, $10 \mathrm{~mol} \%, 1.6 \mathrm{mg}), 3 \mathbf{3}(0.02 \mathrm{mmol}, 20 \mathrm{~mol} \%, 2.4 \mathrm{mg}), \mathbf{2 a}(1 \mathrm{mmol}, 10$ equiv, $108 \mu \mathrm{L}$ ), and DMSO (4 mL). After the reaction was completed, the mixture was diluted with $20 \mathrm{~mL}$ of water and extracted with EtOAc $(3 \times 4 \mathrm{~mL})$. The organic layer was washed with saturated $\mathrm{NaCl}$ (aq.) $(10 \mathrm{~mL})$, dried over $\mathrm{Na}_{2} \mathrm{SO}_{4}$, filtered, and the solvent removed under reduced pressure. The filtrate was concentrated, and the residue was analysised by proton NMR. The dr and ee values were determined by chiral HPLC analysis of the pure product. 


\section{Crystallographic Data of Compound 4m}

Table S10. Crystal data and structure refinement for $\mathbf{4 m}$.

Identification code

Empirical formula

Formula weight

Temperature

Wavelength

Crystal system

Space group

Unit cell dimensions

Volume

$\mathrm{Z}$

Density (calculated)

Absorption coefficient

$\mathrm{F}(000)$

Crystal size

Theta range for data collection

Index ranges

Reflections collected

Independent reflections

Completeness to theta $=25.242^{\circ}$

Absorption correction

Max. and min. transmission

Refinement method

Data / restraints / parameters

Goodness-of-fit on $\mathrm{F}^{2}$

Final R indices [I>2sigma(I)]

$\mathrm{R}$ indices (all data)

Absolute structure parameter

Extinction coefficient

Largest diff. peak and hole mo_d8v21326_0m

$\mathrm{C} 25 \mathrm{H} 33 \mathrm{~N} \mathrm{O} 4$

411.52

213(2) K

$0.71073 \AA$

Orthorhombic

P 212121

$a=6.8297(2) \AA \quad a=90^{\circ}$.

$\mathrm{b}=15.2381(4) \AA \quad \mathrm{b}=90^{\circ}$.

$\mathrm{c}=20.7344(7) \AA \quad \mathrm{g}=90^{\circ}$.

2157.86(11) $\AA^{3}$

4

$1.267 \mathrm{Mg} / \mathrm{m}^{3}$

$0.085 \mathrm{~mm}^{-1}$

888

$0.200 \times 0.150 \times 0.120 \mathrm{~mm}^{3}$

2.376 to $25.996^{\circ}$.

$-8<=\mathrm{h}<=8,-18<=\mathrm{k}<=18,-25<=\mathrm{l}<=25$

16394

$4220[\mathrm{R}(\mathrm{int})=0.0463]$

$99.3 \%$

Semi-empirical from equivalents

0.7456 and 0.5522

Full-matrix least-squares on $\mathrm{F}^{2}$

4220 / 0 / 277

1.044

$\mathrm{R} 1=0.0347, \mathrm{wR} 2=0.0793$

$\mathrm{R} 1=0.0418, \mathrm{wR} 2=0.0847$

$0.5(5)$

$0.022(4)$

0.153 and -0.125 e. $\AA^{-3}$ 
Table S11. Atomic coordinates ( x 104) and equivalent isotropic displacement parameters $\left(\AA^{2} \mathrm{x}\right.$ $10^{3}$ ) for $\mathbf{4 m}$. U(eq) is defined as one third of the trace of the orthogonalized $\mathrm{U}^{\mathrm{ij}}$ tensor.

\begin{tabular}{|c|c|c|c|c|}
\hline- & $\mathrm{x}$ & $\mathrm{y}$ & $\mathrm{z}$ & $\mathrm{U}(\mathrm{eq})$ \\
\hline $\mathrm{O}(1)$ & $6182(2)$ & $3544(1)$ & $4562(1)$ & $51(1)$ \\
\hline $\mathrm{O}(2)$ & $6060(2)$ & $5386(1)$ & $5215(1)$ & $42(1)$ \\
\hline $\mathrm{O}(3)$ & $4857(2)$ & $5658(1)$ & $4217(1)$ & $33(1)$ \\
\hline $\mathrm{O}(4)$ & $-769(2)$ & $5646(1)$ & $7608(1)$ & $47(1)$ \\
\hline $\mathrm{N}(1)$ & $3125(3)$ & $4220(1)$ & $5483(1)$ & $36(1)$ \\
\hline $\mathrm{C}(1)$ & $4718(3)$ & $3398(1)$ & $4254(1)$ & $34(1)$ \\
\hline$C(2)$ & $4579(3)$ & $2634(2)$ & 3798(1) & $43(1)$ \\
\hline$C(3)$ & 2662(4) & $2128(2)$ & $3866(1)$ & $43(1)$ \\
\hline $\mathrm{C}(4)$ & $933(4)$ & 2749(2) & $3824(1)$ & $48(1)$ \\
\hline C(5) & $1055(3)$ & $3447(1)$ & $4347(1)$ & $43(1)$ \\
\hline$C(6)$ & 2941(3) & 3988(1) & 4303(1) & $32(1)$ \\
\hline$C(7)$ & $3113(3)$ & $4651(1)$ & $4856(1)$ & $32(1)$ \\
\hline$C(8)$ & $4880(3)$ & $5264(1)$ & 4793(1) & $32(1)$ \\
\hline $\mathrm{C}(9)$ & 6511(3) & $6205(1)$ & 3996(1) & $28(1)$ \\
\hline $\mathrm{C}(10)$ & 6893(3) & $6986(1)$ & 4441(1) & $34(1)$ \\
\hline $\mathrm{C}(11)$ & $8534(3)$ & 7543(1) & $4145(1)$ & $39(1)$ \\
\hline$C(12)$ & 10387(3) & 6983(2) & $4080(1)$ & $42(1)$ \\
\hline$C(13)$ & $9966(3)$ & $6208(1)$ & $3632(1)$ & $36(1)$ \\
\hline $\mathrm{C}(14)$ & $9327(4)$ & $6534(2)$ & $2969(1)$ & $44(1)$ \\
\hline$C(15)$ & 7481(4) & 7094(2) & 3042(1) & $42(1)$ \\
\hline$C(16)$ & $5856(3)$ & $6531(1)$ & $3340(1)$ & $36(1)$ \\
\hline$C(17)$ & $8340(3)$ & $5645(1)$ & 3929(1) & $33(1)$ \\
\hline $\mathrm{C}(18)$ & 7917(4) & $7873(2)$ & $3485(1)$ & $47(1)$ \\
\hline$C(19)$ & 2099(3) & $4600(1)$ & $6000(1)$ & $32(1)$ \\
\hline $\mathrm{C}(20)$ & $217(3)$ & 4930(1) & $5935(1)$ & $36(1)$ \\
\hline $\mathrm{C}(21)$ & $-794(3)$ & $5278(1)$ & $6458(1)$ & $36(1)$ \\
\hline $\mathrm{C}(22)$ & $63(3)$ & $5290(1)$ & $7060(1)$ & $36(1)$ \\
\hline$C(23)$ & 1919(3) & $4940(1)$ & 7139(1) & $39(1)$ \\
\hline $\mathrm{C}(24)$ & 2926(3) & $4603(1)$ & $6616(1)$ & $37(1)$ \\
\hline $\mathrm{C}(25)$ & $-2543(4)$ & $6112(2)$ & $7526(1)$ & $54(1)$ \\
\hline
\end{tabular}


Table S12. Bond lengths $[\AA]$ and angles $\left[{ }^{\circ}\right]$ for $\mathbf{4 m}$.

\begin{tabular}{|c|c|}
\hline $\mathrm{O}(1)-\mathrm{C}(1)$ & $1.207(3)$ \\
\hline $\mathrm{O}(2)-\mathrm{C}(8)$ & $1.203(2)$ \\
\hline $\mathrm{O}(3)-\mathrm{C}(8)$ & $1.337(2)$ \\
\hline $\mathrm{O}(3)-\mathrm{C}(9)$ & $1.477(2)$ \\
\hline $\mathrm{O}(4)-\mathrm{C}(22)$ & $1.382(3)$ \\
\hline $\mathrm{O}(4)-\mathrm{C}(25)$ & $1.415(3)$ \\
\hline $\mathrm{N}(1)-\mathrm{C}(19)$ & $1.405(3)$ \\
\hline $\mathrm{N}(1)-\mathrm{C}(7)$ & $1.456(3)$ \\
\hline $\mathrm{N}(1)-\mathrm{H}(1)$ & $0.85(3)$ \\
\hline $\mathrm{C}(1)-\mathrm{C}(2)$ & $1.503(3)$ \\
\hline$C(1)-C(6)$ & $1.514(3)$ \\
\hline $\mathrm{C}(2)-\mathrm{C}(3)$ & $1.525(3)$ \\
\hline $\mathrm{C}(2)-\mathrm{H}(2 \mathrm{~A})$ & 0.9800 \\
\hline $\mathrm{C}(2)-\mathrm{H}(2 \mathrm{~B})$ & 0.9800 \\
\hline$C(3)-C(4)$ & $1.516(3)$ \\
\hline $\mathrm{C}(3)-\mathrm{H}(3 \mathrm{~A})$ & 0.9800 \\
\hline $\mathrm{C}(3)-\mathrm{H}(3 \mathrm{~B})$ & 0.9800 \\
\hline $\mathrm{C}(4)-\mathrm{C}(5)$ & $1.520(3)$ \\
\hline $\mathrm{C}(4)-\mathrm{H}(4 \mathrm{~A})$ & 0.9800 \\
\hline $\mathrm{C}(4)-\mathrm{H}(4 \mathrm{~B})$ & 0.9800 \\
\hline$C(5)-C(6)$ & $1.531(3)$ \\
\hline $\mathrm{C}(5)-\mathrm{H}(5 \mathrm{~A})$ & 0.9800 \\
\hline $\mathrm{C}(5)-\mathrm{H}(5 \mathrm{~B})$ & 0.9800 \\
\hline$C(6)-C(7)$ & $1.533(3)$ \\
\hline $\mathrm{C}(6)-\mathrm{H}(6)$ & 0.9900 \\
\hline $\mathrm{C}(7)-\mathrm{C}(8)$ & $1.532(3)$ \\
\hline $\mathrm{C}(7)-\mathrm{H}(7)$ & 0.9900 \\
\hline $\mathrm{C}(9)-\mathrm{C}(16)$ & $1.517(3)$ \\
\hline $\mathrm{C}(9)-\mathrm{C}(17)$ & $1.519(3)$ \\
\hline $\mathrm{C}(9)-\mathrm{C}(10)$ & $1.528(3)$ \\
\hline$C(10)-C(11)$ & $1.533(3)$ \\
\hline $\mathrm{C}(10)-\mathrm{H}(10 \mathrm{~A})$ & 0.9800 \\
\hline $\mathrm{C}(10)-\mathrm{H}(10 \mathrm{~B})$ & 0.9800 \\
\hline $\mathrm{C}(11)-\mathrm{C}(18)$ & $1.518(3)$ \\
\hline $\mathrm{C}(11)-\mathrm{C}(12)$ & $1.533(3)$ \\
\hline $\mathrm{C}(11)-\mathrm{H}(11)$ & 0.9900 \\
\hline
\end{tabular}




\begin{tabular}{|c|c|}
\hline $\mathrm{C}(12)-\mathrm{C}(13)$ & $1.529(3)$ \\
\hline $\mathrm{C}(12)-\mathrm{H}(12 \mathrm{~A})$ & 0.9800 \\
\hline $\mathrm{C}(12)-\mathrm{H}(12 \mathrm{~B})$ & 0.9800 \\
\hline $\mathrm{C}(13)-\mathrm{C}(14)$ & $1.524(3)$ \\
\hline $\mathrm{C}(13)-\mathrm{C}(17)$ & $1.533(3)$ \\
\hline $\mathrm{C}(13)-\mathrm{H}(13)$ & 0.9900 \\
\hline $\mathrm{C}(14)-\mathrm{C}(15)$ & $1.530(3)$ \\
\hline $\mathrm{C}(14)-\mathrm{H}(14 \mathrm{~A})$ & 0.9800 \\
\hline $\mathrm{C}(14)-\mathrm{H}(14 \mathrm{~B})$ & 0.9800 \\
\hline $\mathrm{C}(15)-\mathrm{C}(18)$ & $1.530(4)$ \\
\hline$C(15)-C(16)$ & $1.534(3)$ \\
\hline $\mathrm{C}(15)-\mathrm{H}(15)$ & 0.9900 \\
\hline $\mathrm{C}(16)-\mathrm{H}(16 \mathrm{~A})$ & 0.9800 \\
\hline $\mathrm{C}(16)-\mathrm{H}(16 \mathrm{~B})$ & 0.9800 \\
\hline $\mathrm{C}(17)-\mathrm{H}(17 \mathrm{~A})$ & 0.9800 \\
\hline $\mathrm{C}(17)-\mathrm{H}(17 \mathrm{~B})$ & 0.9800 \\
\hline $\mathrm{C}(18)-\mathrm{H}(18 \mathrm{~A})$ & 0.9800 \\
\hline $\mathrm{C}(18)-\mathrm{H}(18 \mathrm{~B})$ & 0.9800 \\
\hline$C(19)-C(20)$ & $1.387(3)$ \\
\hline$C(19)-C(24)$ & $1.396(3)$ \\
\hline$C(20)-C(21)$ & $1.392(3)$ \\
\hline $\mathrm{C}(20)-\mathrm{H}(20)$ & 0.9400 \\
\hline$C(21)-C(22)$ & $1.377(3)$ \\
\hline $\mathrm{C}(21)-\mathrm{H}(21)$ & 0.9400 \\
\hline$C(22)-C(23)$ & $1.386(3)$ \\
\hline$C(23)-C(24)$ & $1.384(3)$ \\
\hline $\mathrm{C}(23)-\mathrm{H}(23)$ & 0.9400 \\
\hline $\mathrm{C}(24)-\mathrm{H}(24)$ & 0.9400 \\
\hline $\mathrm{C}(25)-\mathrm{H}(25 \mathrm{~A})$ & 0.9700 \\
\hline $\mathrm{C}(25)-\mathrm{H}(25 \mathrm{~B})$ & 0.9700 \\
\hline $\mathrm{C}(25)-\mathrm{H}(25 \mathrm{C})$ & 0.9700 \\
\hline $\mathrm{C}(8)-\mathrm{O}(3)-\mathrm{C}(9)$ & $121.41(16)$ \\
\hline $\mathrm{C}(22)-\mathrm{O}(4)-\mathrm{C}(25)$ & $116.73(19)$ \\
\hline $\mathrm{C}(19)-\mathrm{N}(1)-\mathrm{C}(7)$ & $119.51(17)$ \\
\hline $\mathrm{C}(19)-\mathrm{N}(1)-\mathrm{H}(1)$ & $110.7(17)$ \\
\hline $\mathrm{C}(7)-\mathrm{N}(1)-\mathrm{H}(1)$ & $110.1(18)$ \\
\hline $\mathrm{O}(1)-\mathrm{C}(1)-\mathrm{C}(2)$ & $121.9(2)$ \\
\hline $\mathrm{O}(1)-\mathrm{C}(1)-\mathrm{C}(6)$ & $121.2(2)$ \\
\hline
\end{tabular}




\begin{tabular}{|c|c|}
\hline$C(2)-C(1)-C(6)$ & $116.85(19)$ \\
\hline $\mathrm{C}(1)-\mathrm{C}(2)-\mathrm{C}(3)$ & $112.79(19)$ \\
\hline $\mathrm{C}(1)-\mathrm{C}(2)-\mathrm{H}(2 \mathrm{~A})$ & 109.0 \\
\hline $\mathrm{C}(3)-\mathrm{C}(2)-\mathrm{H}(2 \mathrm{~A})$ & 109.0 \\
\hline $\mathrm{C}(1)-\mathrm{C}(2)-\mathrm{H}(2 \mathrm{~B})$ & 109.0 \\
\hline $\mathrm{C}(3)-\mathrm{C}(2)-\mathrm{H}(2 \mathrm{~B})$ & 109.0 \\
\hline $\mathrm{H}(2 \mathrm{~A})-\mathrm{C}(2)-\mathrm{H}(2 \mathrm{~B})$ & 107.8 \\
\hline $\mathrm{C}(4)-\mathrm{C}(3)-\mathrm{C}(2)$ & $110.35(18)$ \\
\hline $\mathrm{C}(4)-\mathrm{C}(3)-\mathrm{H}(3 \mathrm{~A})$ & 109.6 \\
\hline $\mathrm{C}(2)-\mathrm{C}(3)-\mathrm{H}(3 \mathrm{~A})$ & 109.6 \\
\hline $\mathrm{C}(4)-\mathrm{C}(3)-\mathrm{H}(3 \mathrm{~B})$ & 109.6 \\
\hline $\mathrm{C}(2)-\mathrm{C}(3)-\mathrm{H}(3 \mathrm{~B})$ & 109.6 \\
\hline $\mathrm{H}(3 \mathrm{~A})-\mathrm{C}(3)-\mathrm{H}(3 \mathrm{~B})$ & 108.1 \\
\hline $\mathrm{C}(3)-\mathrm{C}(4)-\mathrm{C}(5)$ & $110.76(19)$ \\
\hline $\mathrm{C}(3)-\mathrm{C}(4)-\mathrm{H}(4 \mathrm{~A})$ & 109.5 \\
\hline $\mathrm{C}(5)-\mathrm{C}(4)-\mathrm{H}(4 \mathrm{~A})$ & 109.5 \\
\hline $\mathrm{C}(3)-\mathrm{C}(4)-\mathrm{H}(4 \mathrm{~B})$ & 109.5 \\
\hline $\mathrm{C}(5)-\mathrm{C}(4)-\mathrm{H}(4 \mathrm{~B})$ & 109.5 \\
\hline $\mathrm{H}(4 \mathrm{~A})-\mathrm{C}(4)-\mathrm{H}(4 \mathrm{~B})$ & 108.1 \\
\hline $\mathrm{C}(4)-\mathrm{C}(5)-\mathrm{C}(6)$ & $112.4(2)$ \\
\hline $\mathrm{C}(4)-\mathrm{C}(5)-\mathrm{H}(5 \mathrm{~A})$ & 109.1 \\
\hline $\mathrm{C}(6)-\mathrm{C}(5)-\mathrm{H}(5 \mathrm{~A})$ & 109.1 \\
\hline $\mathrm{C}(4)-\mathrm{C}(5)-\mathrm{H}(5 \mathrm{~B})$ & 109.1 \\
\hline $\mathrm{C}(6)-\mathrm{C}(5)-\mathrm{H}(5 \mathrm{~B})$ & 109.1 \\
\hline $\mathrm{H}(5 \mathrm{~A})-\mathrm{C}(5)-\mathrm{H}(5 \mathrm{~B})$ & 107.9 \\
\hline $\mathrm{C}(1)-\mathrm{C}(6)-\mathrm{C}(5)$ & $111.04(15)$ \\
\hline$C(1)-C(6)-C(7)$ & $112.34(17)$ \\
\hline$C(5)-C(6)-C(7)$ & $112.02(18)$ \\
\hline $\mathrm{C}(1)-\mathrm{C}(6)-\mathrm{H}(6)$ & 107.0 \\
\hline $\mathrm{C}(5)-\mathrm{C}(6)-\mathrm{H}(6)$ & 107.0 \\
\hline $\mathrm{C}(7)-\mathrm{C}(6)-\mathrm{H}(6)$ & 107.0 \\
\hline $\mathrm{N}(1)-\mathrm{C}(7)-\mathrm{C}(8)$ & $110.27(17)$ \\
\hline $\mathrm{N}(1)-\mathrm{C}(7)-\mathrm{C}(6)$ & $111.81(16)$ \\
\hline $\mathrm{C}(8)-\mathrm{C}(7)-\mathrm{C}(6)$ & $113.50(17)$ \\
\hline $\mathrm{N}(1)-\mathrm{C}(7)-\mathrm{H}(7)$ & 107.0 \\
\hline $\mathrm{C}(8)-\mathrm{C}(7)-\mathrm{H}(7)$ & 107.0 \\
\hline $\mathrm{C}(6)-\mathrm{C}(7)-\mathrm{H}(7)$ & 107.0 \\
\hline $\mathrm{O}(2)-\mathrm{C}(8)-\mathrm{O}(3)$ & $126.0(2)$ \\
\hline
\end{tabular}




\begin{tabular}{|c|c|}
\hline $\mathrm{O}(2)-\mathrm{C}(8)-\mathrm{C}(7)$ & $124.05(19)$ \\
\hline $\mathrm{O}(3)-\mathrm{C}(8)-\mathrm{C}(7)$ & 109.91(17) \\
\hline $\mathrm{O}(3)-\mathrm{C}(9)-\mathrm{C}(16)$ & $103.71(15)$ \\
\hline $\mathrm{O}(3)-\mathrm{C}(9)-\mathrm{C}(17)$ & 109.91(14) \\
\hline$C(16)-C(9)-C(17)$ & $110.10(17)$ \\
\hline $\mathrm{O}(3)-\mathrm{C}(9)-\mathrm{C}(10)$ & $112.56(16)$ \\
\hline $\mathrm{C}(16)-\mathrm{C}(9)-\mathrm{C}(10)$ & $109.67(17)$ \\
\hline $\mathrm{C}(17)-\mathrm{C}(9)-\mathrm{C}(10)$ & $110.67(16)$ \\
\hline $\mathrm{C}(9)-\mathrm{C}(10)-\mathrm{C}(11)$ & $108.36(17)$ \\
\hline $\mathrm{C}(9)-\mathrm{C}(10)-\mathrm{H}(10 \mathrm{~A})$ & 110.0 \\
\hline $\mathrm{C}(11)-\mathrm{C}(10)-\mathrm{H}(10 \mathrm{~A})$ & 110.0 \\
\hline $\mathrm{C}(9)-\mathrm{C}(10)-\mathrm{H}(10 \mathrm{~B})$ & 110.0 \\
\hline $\mathrm{C}(11)-\mathrm{C}(10)-\mathrm{H}(10 \mathrm{~B})$ & 110.0 \\
\hline $\mathrm{H}(10 \mathrm{~A})-\mathrm{C}(10)-\mathrm{H}(10 \mathrm{~B})$ & 108.4 \\
\hline $\mathrm{C}(18)-\mathrm{C}(11)-\mathrm{C}(12)$ & $109.45(19)$ \\
\hline $\mathrm{C}(18)-\mathrm{C}(11)-\mathrm{C}(10)$ & 109.91(19) \\
\hline $\mathrm{C}(12)-\mathrm{C}(11)-\mathrm{C}(10)$ & $109.31(17)$ \\
\hline $\mathrm{C}(18)-\mathrm{C}(11)-\mathrm{H}(11)$ & 109.4 \\
\hline $\mathrm{C}(12)-\mathrm{C}(11)-\mathrm{H}(11)$ & 109.4 \\
\hline $\mathrm{C}(10)-\mathrm{C}(11)-\mathrm{H}(11)$ & 109.4 \\
\hline $\mathrm{C}(13)-\mathrm{C}(12)-\mathrm{C}(11)$ & $109.20(18)$ \\
\hline $\mathrm{C}(13)-\mathrm{C}(12)-\mathrm{H}(12 \mathrm{~A})$ & 109.8 \\
\hline $\mathrm{C}(11)-\mathrm{C}(12)-\mathrm{H}(12 \mathrm{~A})$ & 109.8 \\
\hline $\mathrm{C}(13)-\mathrm{C}(12)-\mathrm{H}(12 \mathrm{~B})$ & 109.8 \\
\hline $\mathrm{C}(11)-\mathrm{C}(12)-\mathrm{H}(12 \mathrm{~B})$ & 109.8 \\
\hline $\mathrm{H}(12 \mathrm{~A})-\mathrm{C}(12)-\mathrm{H}(12 \mathrm{~B})$ & 108.3 \\
\hline $\mathrm{C}(14)-\mathrm{C}(13)-\mathrm{C}(12)$ & $110.47(19)$ \\
\hline $\mathrm{C}(14)-\mathrm{C}(13)-\mathrm{C}(17)$ & 109.71(18) \\
\hline$C(12)-C(13)-C(17)$ & $108.93(18)$ \\
\hline $\mathrm{C}(14)-\mathrm{C}(13)-\mathrm{H}(13)$ & 109.2 \\
\hline $\mathrm{C}(12)-\mathrm{C}(13)-\mathrm{H}(13)$ & 109.2 \\
\hline $\mathrm{C}(17)-\mathrm{C}(13)-\mathrm{H}(13)$ & 109.2 \\
\hline$C(13)-C(14)-C(15)$ & $109.22(19)$ \\
\hline $\mathrm{C}(13)-\mathrm{C}(14)-\mathrm{H}(14 \mathrm{~A})$ & 109.8 \\
\hline $\mathrm{C}(15)-\mathrm{C}(14)-\mathrm{H}(14 \mathrm{~A})$ & 109.8 \\
\hline $\mathrm{C}(13)-\mathrm{C}(14)-\mathrm{H}(14 \mathrm{~B})$ & 109.8 \\
\hline $\mathrm{C}(15)-\mathrm{C}(14)-\mathrm{H}(14 \mathrm{~B})$ & 109.8 \\
\hline $\mathrm{H}(14 \mathrm{~A})-\mathrm{C}(14)-\mathrm{H}(14 \mathrm{~B})$ & 108.3 \\
\hline
\end{tabular}




\begin{tabular}{|c|c|}
\hline $\mathrm{C}(14)-\mathrm{C}(15)-\mathrm{C}(18)$ & $109.3(2)$ \\
\hline $\mathrm{C}(14)-\mathrm{C}(15)-\mathrm{C}(16)$ & 108.91(17) \\
\hline$C(18)-C(15)-C(16)$ & $109.41(19)$ \\
\hline $\mathrm{C}(14)-\mathrm{C}(15)-\mathrm{H}(15)$ & 109.7 \\
\hline $\mathrm{C}(18)-\mathrm{C}(15)-\mathrm{H}(15)$ & 109.7 \\
\hline $\mathrm{C}(16)-\mathrm{C}(15)-\mathrm{H}(15)$ & 109.7 \\
\hline$C(9)-C(16)-C(15)$ & $109.36(17)$ \\
\hline $\mathrm{C}(9)-\mathrm{C}(16)-\mathrm{H}(16 \mathrm{~A})$ & 109.8 \\
\hline $\mathrm{C}(15)-\mathrm{C}(16)-\mathrm{H}(16 \mathrm{~A})$ & 109.8 \\
\hline $\mathrm{C}(9)-\mathrm{C}(16)-\mathrm{H}(16 \mathrm{~B})$ & 109.8 \\
\hline $\mathrm{C}(15)-\mathrm{C}(16)-\mathrm{H}(16 \mathrm{~B})$ & 109.8 \\
\hline $\mathrm{H}(16 \mathrm{~A})-\mathrm{C}(16)-\mathrm{H}(16 \mathrm{~B})$ & 108.3 \\
\hline $\mathrm{C}(9)-\mathrm{C}(17)-\mathrm{C}(13)$ & $108.55(15)$ \\
\hline $\mathrm{C}(9)-\mathrm{C}(17)-\mathrm{H}(17 \mathrm{~A})$ & 110.0 \\
\hline $\mathrm{C}(13)-\mathrm{C}(17)-\mathrm{H}(17 \mathrm{~A})$ & 110.0 \\
\hline $\mathrm{C}(9)-\mathrm{C}(17)-\mathrm{H}(17 \mathrm{~B})$ & 110.0 \\
\hline $\mathrm{C}(13)-\mathrm{C}(17)-\mathrm{H}(17 \mathrm{~B})$ & 110.0 \\
\hline $\mathrm{H}(17 \mathrm{~A})-\mathrm{C}(17)-\mathrm{H}(17 \mathrm{~B})$ & 108.4 \\
\hline $\mathrm{C}(11)-\mathrm{C}(18)-\mathrm{C}(15)$ & $109.86(18)$ \\
\hline $\mathrm{C}(11)-\mathrm{C}(18)-\mathrm{H}(18 \mathrm{~A})$ & 109.7 \\
\hline $\mathrm{C}(15)-\mathrm{C}(18)-\mathrm{H}(18 \mathrm{~A})$ & 109.7 \\
\hline $\mathrm{C}(11)-\mathrm{C}(18)-\mathrm{H}(18 \mathrm{~B})$ & 109.7 \\
\hline $\mathrm{C}(15)-\mathrm{C}(18)-\mathrm{H}(18 \mathrm{~B})$ & 109.7 \\
\hline $\mathrm{H}(18 \mathrm{~A})-\mathrm{C}(18)-\mathrm{H}(18 \mathrm{~B})$ & 108.2 \\
\hline $\mathrm{C}(20)-\mathrm{C}(19)-\mathrm{C}(24)$ & $117.6(2)$ \\
\hline $\mathrm{C}(20)-\mathrm{C}(19)-\mathrm{N}(1)$ & $122.5(2)$ \\
\hline $\mathrm{C}(24)-\mathrm{C}(19)-\mathrm{N}(1)$ & $119.79(19)$ \\
\hline $\mathrm{C}(19)-\mathrm{C}(20)-\mathrm{C}(21)$ & $121.4(2)$ \\
\hline $\mathrm{C}(19)-\mathrm{C}(20)-\mathrm{H}(20)$ & 119.3 \\
\hline $\mathrm{C}(21)-\mathrm{C}(20)-\mathrm{H}(20)$ & 119.3 \\
\hline $\mathrm{C}(22)-\mathrm{C}(21)-\mathrm{C}(20)$ & $120.0(2)$ \\
\hline $\mathrm{C}(22)-\mathrm{C}(21)-\mathrm{H}(21)$ & 120.0 \\
\hline $\mathrm{C}(20)-\mathrm{C}(21)-\mathrm{H}(21)$ & 120.0 \\
\hline $\mathrm{C}(21)-\mathrm{C}(22)-\mathrm{O}(4)$ & $125.2(2)$ \\
\hline $\mathrm{C}(21)-\mathrm{C}(22)-\mathrm{C}(23)$ & $119.4(2)$ \\
\hline $\mathrm{O}(4)-\mathrm{C}(22)-\mathrm{C}(23)$ & $115.4(2)$ \\
\hline$C(24)-C(23)-C(22)$ & $120.3(2)$ \\
\hline $\mathrm{C}(24)-\mathrm{C}(23)-\mathrm{H}(23)$ & 119.8 \\
\hline
\end{tabular}




$\begin{array}{ll}\mathrm{C}(22)-\mathrm{C}(23)-\mathrm{H}(23) & 119.8 \\ \mathrm{C}(23)-\mathrm{C}(24)-\mathrm{C}(19) & 121.1(2) \\ \mathrm{C}(23)-\mathrm{C}(24)-\mathrm{H}(24) & 119.4 \\ \mathrm{C}(19)-\mathrm{C}(24)-\mathrm{H}(24) & 119.4 \\ \mathrm{O}(4)-\mathrm{C}(25)-\mathrm{H}(25 \mathrm{~A}) & 109.5 \\ \mathrm{O}(4)-\mathrm{C}(25)-\mathrm{H}(25 \mathrm{~B}) & 109.5 \\ \mathrm{H}(25 \mathrm{~A})-\mathrm{C}(25)-\mathrm{H}(25 \mathrm{~B}) & 109.5 \\ \mathrm{O}(4)-\mathrm{C}(25)-\mathrm{H}(25 \mathrm{C}) & 109.5 \\ \mathrm{H}(25 \mathrm{~A})-\mathrm{C}(25)-\mathrm{H}(25 \mathrm{C}) & 109.5 \\ \mathrm{H}(25 \mathrm{~B})-\mathrm{C}(25)-\mathrm{H}(25 \mathrm{C}) & 109.5\end{array}$

Symmetry transformations used to generate equivalent atoms: 
Table S13. Anisotropic displacement parameters $\left(\AA^{2} \times 10^{3}\right)$ for $\mathbf{4 m}$. The anisotropic displacement factor exponent takes the form: $\quad-2 p^{2}\left[h^{2} a^{* 2} U^{11}+\ldots+2 h k a^{*} b^{*} U^{12}\right]$

\begin{tabular}{|c|c|c|c|c|c|c|}
\hline & $\mathrm{U}^{11}$ & $\mathrm{U}^{22}$ & $\mathrm{U}^{33}$ & $\mathrm{U}^{23}$ & $\mathrm{U}^{13}$ & $\mathrm{U}^{12}$ \\
\hline $\mathrm{O}(1)$ & $30(1)$ & $58(1)$ & $64(1)$ & $-11(1)$ & $-8(1)$ & $6(1)$ \\
\hline $\mathrm{O}(2)$ & $43(1)$ & $48(1)$ & $36(1)$ & $7(1)$ & $-10(1)$ & $-6(1)$ \\
\hline $\mathrm{O}(3)$ & $31(1)$ & $36(1)$ & $32(1)$ & $7(1)$ & $-4(1)$ & $-6(1)$ \\
\hline $\mathrm{O}(4)$ & $49(1)$ & $54(1)$ & $39(1)$ & $0(1)$ & $4(1)$ & $15(1)$ \\
\hline $\mathrm{N}(1)$ & $36(1)$ & $38(1)$ & $34(1)$ & $10(1)$ & $3(1)$ & $8(1)$ \\
\hline $\mathrm{C}(1)$ & $28(1)$ & $40(1)$ & $35(1)$ & $7(1)$ & $2(1)$ & $-2(1)$ \\
\hline $\mathrm{C}(2)$ & $40(1)$ & $46(1)$ & $44(1)$ & $-5(1)$ & $2(1)$ & $5(1)$ \\
\hline$C(3)$ & $47(1)$ & $35(1)$ & $48(2)$ & $-1(1)$ & $-4(1)$ & $1(1)$ \\
\hline $\mathrm{C}(4)$ & $38(1)$ & $38(1)$ & $67(2)$ & $5(1)$ & $-13(1)$ & $-6(1)$ \\
\hline$C(5)$ & $27(1)$ & $37(1)$ & $65(2)$ & $3(1)$ & $-1(1)$ & $0(1)$ \\
\hline$C(6)$ & $27(1)$ & $33(1)$ & $35(1)$ & $7(1)$ & $-4(1)$ & $-1(1)$ \\
\hline$C(7)$ & $28(1)$ & $32(1)$ & $34(1)$ & $7(1)$ & $0(1)$ & $3(1)$ \\
\hline $\mathrm{C}(8)$ & $32(1)$ & $31(1)$ & $32(1)$ & $3(1)$ & $-2(1)$ & $3(1)$ \\
\hline $\mathrm{C}(9)$ & $27(1)$ & $29(1)$ & $27(1)$ & $3(1)$ & $1(1)$ & $-2(1)$ \\
\hline$C(10)$ & $35(1)$ & $31(1)$ & $37(1)$ & $-5(1)$ & $5(1)$ & $1(1)$ \\
\hline$C(11)$ & $40(1)$ & $28(1)$ & $49(2)$ & $-6(1)$ & $6(1)$ & $-4(1)$ \\
\hline$C(12)$ & $30(1)$ & $45(1)$ & $50(2)$ & $-4(1)$ & $0(1)$ & $-4(1)$ \\
\hline $\mathrm{C}(13)$ & $30(1)$ & $38(1)$ & $42(1)$ & $-5(1)$ & $3(1)$ & $6(1)$ \\
\hline$C(14)$ & $42(1)$ & $50(1)$ & $38(1)$ & $1(1)$ & $11(1)$ & $0(1)$ \\
\hline$C(15)$ & $42(1)$ & $49(1)$ & $35(1)$ & $13(1)$ & $3(1)$ & $2(1)$ \\
\hline$C(16)$ & $32(1)$ & $41(1)$ & $34(1)$ & $7(1)$ & $-2(1)$ & $1(1)$ \\
\hline$C(17)$ & $38(1)$ & $29(1)$ & $33(1)$ & $-3(1)$ & $-5(1)$ & $6(1)$ \\
\hline $\mathrm{C}(18)$ & $44(1)$ & $34(1)$ & $64(2)$ & $12(1)$ & $14(1)$ & $2(1)$ \\
\hline$C(19)$ & $31(1)$ & $28(1)$ & $36(1)$ & $8(1)$ & $3(1)$ & $-3(1)$ \\
\hline$C(20)$ & $32(1)$ & $41(1)$ & $35(1)$ & $4(1)$ & $-2(1)$ & $-3(1)$ \\
\hline $\mathrm{C}(21)$ & $27(1)$ & $40(1)$ & $41(1)$ & $6(1)$ & $1(1)$ & $1(1)$ \\
\hline$C(22)$ & $36(1)$ & $34(1)$ & $36(1)$ & $4(1)$ & $5(1)$ & $0(1)$ \\
\hline$C(23)$ & $40(1)$ & $45(1)$ & $33(1)$ & $8(1)$ & $-4(1)$ & $3(1)$ \\
\hline$C(24)$ & $32(1)$ & $40(1)$ & $39(1)$ & $10(1)$ & $-1(1)$ & $4(1)$ \\
\hline$C(25)$ & $48(2)$ & $56(2)$ & $57(2)$ & $-3(1)$ & $7(1)$ & $16(1)$ \\
\hline
\end{tabular}


Table S14. Hydrogen coordinates ( x 10 $)$ and isotropic displacement parameters $\left(\AA^{2} \times 10^{3}\right)$ for $4 \mathrm{~m}$.

\begin{tabular}{|c|c|c|c|c|}
\hline & $\mathrm{x}$ & $\mathrm{y}$ & $\mathrm{z}$ & $\mathrm{U}(\mathrm{eq})$ \\
\hline $\mathrm{H}(2 \mathrm{~A})$ & 5676 & 2234 & 3878 & 52 \\
\hline $\mathrm{H}(2 \mathrm{~B})$ & 4696 & 2849 & 3354 & 52 \\
\hline $\mathrm{H}(3 \mathrm{~A})$ & 2568 & 1688 & 3522 & 52 \\
\hline $\mathrm{H}(3 \mathrm{~B})$ & 2640 & 1821 & 4281 & 52 \\
\hline $\mathrm{H}(4 \mathrm{~A})$ & -287 & 2417 & 3874 & 57 \\
\hline $\mathrm{H}(4 \mathrm{~B})$ & 916 & 3031 & 3400 & 57 \\
\hline $\mathrm{H}(5 \mathrm{~A})$ & 990 & 3164 & 4770 & 51 \\
\hline $\mathrm{H}(5 \mathrm{~B})$ & -74 & 3841 & 4308 & 51 \\
\hline $\mathrm{H}(6)$ & 2872 & 4329 & 3897 & 38 \\
\hline $\mathrm{H}(7)$ & 1927 & 5024 & 4841 & 38 \\
\hline $\mathrm{H}(10 \mathrm{~A})$ & 7283 & 6779 & 4870 & 41 \\
\hline $\mathrm{H}(10 \mathrm{~B})$ & 5701 & 7339 & 4486 & 41 \\
\hline $\mathrm{H}(11)$ & 8808 & 8050 & 4430 & 47 \\
\hline $\mathrm{H}(12 \mathrm{~A})$ & 11454 & 7339 & 3902 & 50 \\
\hline $\mathrm{H}(12 \mathrm{~B})$ & 10791 & 6767 & 4504 & 50 \\
\hline $\mathrm{H}(13)$ & 11166 & 5849 & 3585 & 44 \\
\hline $\mathrm{H}(14 \mathrm{~A})$ & 9059 & 6033 & 2686 & 52 \\
\hline $\mathrm{H}(14 \mathrm{~B})$ & 10376 & 6884 & 2775 & 52 \\
\hline $\mathrm{H}(15)$ & 7060 & 7310 & 2613 & 51 \\
\hline $\mathrm{H}(16 \mathrm{~A})$ & 4658 & 6880 & 3385 & 43 \\
\hline $\mathrm{H}(16 \mathrm{~B})$ & 5571 & 6031 & 3058 & 43 \\
\hline $\mathrm{H}(17 \mathrm{~A})$ & 8069 & 5138 & 3652 & 40 \\
\hline $\mathrm{H}(17 \mathrm{~B})$ & 8751 & 5428 & 4353 & 40 \\
\hline $\mathrm{H}(18 \mathrm{~A})$ & 8966 & 8231 & 3299 & 57 \\
\hline $\mathrm{H}(18 \mathrm{~B})$ & 6747 & 8240 & 3526 & 57 \\
\hline $\mathrm{H}(20)$ & -391 & 4917 & 5528 & 43 \\
\hline $\mathrm{H}(21)$ & -2062 & 5505 & 6402 & 43 \\
\hline $\mathrm{H}(23)$ & 2497 & 4930 & 7550 & 47 \\
\hline $\mathrm{H}(24)$ & 4189 & 4372 & 6676 & 44 \\
\hline $\mathrm{H}(25 \mathrm{~A})$ & -2343 & 6589 & 7224 & 81 \\
\hline $\mathrm{H}(25 \mathrm{~B})$ & -2963 & 6347 & 7938 & 81 \\
\hline $\mathrm{H}(25 \mathrm{C})$ & -3540 & 5718 & 7360 & 81 \\
\hline $\mathrm{H}(1)$ & $4290(40)$ & 4097(17) & $5593(12)$ & $50(8)$ \\
\hline
\end{tabular}


Table S15. Torsion angles $\left[^{\circ}\right]$ for $\mathbf{4 m}$.

\begin{tabular}{|c|c|}
\hline $\mathrm{O}(1)-\mathrm{C}(1)-\mathrm{C}(2)-\mathrm{C}(3)$ & $134.7(2)$ \\
\hline $\mathrm{C}(6)-\mathrm{C}(1)-\mathrm{C}(2)-\mathrm{C}(3)$ & $-46.2(3)$ \\
\hline $\mathrm{C}(1)-\mathrm{C}(2)-\mathrm{C}(3)-\mathrm{C}(4)$ & $51.8(3)$ \\
\hline $\mathrm{C}(2)-\mathrm{C}(3)-\mathrm{C}(4)-\mathrm{C}(5)$ & $-58.5(3)$ \\
\hline$C(3)-C(4)-C(5)-C(6)$ & $58.6(3)$ \\
\hline $\mathrm{O}(1)-\mathrm{C}(1)-\mathrm{C}(6)-\mathrm{C}(5)$ & $-136.5(2)$ \\
\hline $\mathrm{C}(2)-\mathrm{C}(1)-\mathrm{C}(6)-\mathrm{C}(5)$ & $44.4(3)$ \\
\hline $\mathrm{O}(1)-\mathrm{C}(1)-\mathrm{C}(6)-\mathrm{C}(7)$ & $-10.1(3)$ \\
\hline $\mathrm{C}(2)-\mathrm{C}(1)-\mathrm{C}(6)-\mathrm{C}(7)$ & $170.77(18)$ \\
\hline $\mathrm{C}(4)-\mathrm{C}(5)-\mathrm{C}(6)-\mathrm{C}(1)$ & $-49.9(3)$ \\
\hline $\mathrm{C}(4)-\mathrm{C}(5)-\mathrm{C}(6)-\mathrm{C}(7)$ & $-176.39(19)$ \\
\hline $\mathrm{C}(19)-\mathrm{N}(1)-\mathrm{C}(7)-\mathrm{C}(8)$ & $92.5(2)$ \\
\hline $\mathrm{C}(19)-\mathrm{N}(1)-\mathrm{C}(7)-\mathrm{C}(6)$ & $-140.22(19)$ \\
\hline $\mathrm{C}(1)-\mathrm{C}(6)-\mathrm{C}(7)-\mathrm{N}(1)$ & $-65.7(2)$ \\
\hline $\mathrm{C}(5)-\mathrm{C}(6)-\mathrm{C}(7)-\mathrm{N}(1)$ & $60.1(2)$ \\
\hline $\mathrm{C}(1)-\mathrm{C}(6)-\mathrm{C}(7)-\mathrm{C}(8)$ & $59.8(2)$ \\
\hline$C(5)-C(6)-C(7)-C(8)$ & $-174.41(17)$ \\
\hline $\mathrm{C}(9)-\mathrm{O}(3)-\mathrm{C}(8)-\mathrm{O}(2)$ & $9.7(3)$ \\
\hline $\mathrm{C}(9)-\mathrm{O}(3)-\mathrm{C}(8)-\mathrm{C}(7)$ & $-172.72(15)$ \\
\hline $\mathrm{N}(1)-\mathrm{C}(7)-\mathrm{C}(8)-\mathrm{O}(2)$ & $-2.2(3)$ \\
\hline $\mathrm{C}(6)-\mathrm{C}(7)-\mathrm{C}(8)-\mathrm{O}(2)$ & $-128.5(2)$ \\
\hline $\mathrm{N}(1)-\mathrm{C}(7)-\mathrm{C}(8)-\mathrm{O}(3)$ & $-179.86(17)$ \\
\hline $\mathrm{C}(6)-\mathrm{C}(7)-\mathrm{C}(8)-\mathrm{O}(3)$ & $53.8(2)$ \\
\hline $\mathrm{C}(8)-\mathrm{O}(3)-\mathrm{C}(9)-\mathrm{C}(16)$ & $-178.73(17)$ \\
\hline $\mathrm{C}(8)-\mathrm{O}(3)-\mathrm{C}(9)-\mathrm{C}(17)$ & $63.6(2)$ \\
\hline $\mathrm{C}(8)-\mathrm{O}(3)-\mathrm{C}(9)-\mathrm{C}(10)$ & $-60.3(2)$ \\
\hline $\mathrm{O}(3)-\mathrm{C}(9)-\mathrm{C}(10)-\mathrm{C}(11)$ & $-176.12(16)$ \\
\hline $\mathrm{C}(16)-\mathrm{C}(9)-\mathrm{C}(10)-\mathrm{C}(11)$ & $-61.2(2)$ \\
\hline $\mathrm{C}(17)-\mathrm{C}(9)-\mathrm{C}(10)-\mathrm{C}(11)$ & $60.5(2)$ \\
\hline $\mathrm{C}(9)-\mathrm{C}(10)-\mathrm{C}(11)-\mathrm{C}(18)$ & $60.4(2)$ \\
\hline $\mathrm{C}(9)-\mathrm{C}(10)-\mathrm{C}(11)-\mathrm{C}(12)$ & $-59.8(2)$ \\
\hline $\mathrm{C}(18)-\mathrm{C}(11)-\mathrm{C}(12)-\mathrm{C}(13)$ & $-59.3(2)$ \\
\hline $\mathrm{C}(10)-\mathrm{C}(11)-\mathrm{C}(12)-\mathrm{C}(13)$ & $61.1(3)$ \\
\hline$C(11)-C(12)-C(13)-C(14)$ & $59.2(2)$ \\
\hline $\mathrm{C}(11)-\mathrm{C}(12)-\mathrm{C}(13)-\mathrm{C}(17)$ & $-61.3(2)$ \\
\hline $\mathrm{C}(12)-\mathrm{C}(13)-\mathrm{C}(14)-\mathrm{C}(15)$ & $-59.4(2)$ \\
\hline
\end{tabular}




$\begin{array}{lc}\mathrm{C}(17)-\mathrm{C}(13)-\mathrm{C}(14)-\mathrm{C}(15) & 60.7(2) \\ \mathrm{C}(13)-\mathrm{C}(14)-\mathrm{C}(15)-\mathrm{C}(18) & 59.4(2) \\ \mathrm{C}(13)-\mathrm{C}(14)-\mathrm{C}(15)-\mathrm{C}(16) & -60.1(2) \\ \mathrm{O}(3)-\mathrm{C}(9)-\mathrm{C}(16)-\mathrm{C}(15) & -178.36(16) \\ \mathrm{C}(17)-\mathrm{C}(9)-\mathrm{C}(16)-\mathrm{C}(15) & -60.8(2) \\ \mathrm{C}(10)-\mathrm{C}(9)-\mathrm{C}(16)-\mathrm{C}(15) & 61.2(2) \\ \mathrm{C}(14)-\mathrm{C}(15)-\mathrm{C}(16)-\mathrm{C}(9) & 60.1(2) \\ \mathrm{C}(18)-\mathrm{C}(15)-\mathrm{C}(16)-\mathrm{C}(9) & -59.4(2) \\ \mathrm{O}(3)-\mathrm{C}(9)-\mathrm{C}(17)-\mathrm{C}(13) & 174.04(16) \\ \mathrm{C}(16)-\mathrm{C}(9)-\mathrm{C}(17)-\mathrm{C}(13) & 60.4(2) \\ \mathrm{C}(10)-\mathrm{C}(9)-\mathrm{C}(17)-\mathrm{C}(13) & -61.0(2) \\ \mathrm{C}(14)-\mathrm{C}(13)-\mathrm{C}(17)-\mathrm{C}(9) & -60.3(2) \\ \mathrm{C}(12)-\mathrm{C}(13)-\mathrm{C}(17)-\mathrm{C}(9) & 60.7(2) \\ \mathrm{C}(12)-\mathrm{C}(11)-\mathrm{C}(18)-\mathrm{C}(15) & 60.4(2) \\ \mathrm{C}(10)-\mathrm{C}(11)-\mathrm{C}(18)-\mathrm{C}(15) & -59.7(2) \\ \mathrm{C}(14)-\mathrm{C}(15)-\mathrm{C}(18)-\mathrm{C}(11) & -60.5(2) \\ \mathrm{C}(16)-\mathrm{C}(15)-\mathrm{C}(18)-\mathrm{C}(11) & 58.7(2) \\ \mathrm{C}(7)-\mathrm{N}(1)-\mathrm{C}(19)-\mathrm{C}(20) & 45.0(3) \\ \mathrm{C}(7)-\mathrm{N}(1)-\mathrm{C}(19)-\mathrm{C}(24) & -139.3(2) \\ \mathrm{C}(24)-\mathrm{C}(19)-\mathrm{C}(20)-\mathrm{C}(21) & 2.1(3) \\ \mathrm{N}(1)-\mathrm{C}(19)-\mathrm{C}(20)-\mathrm{C}(21) & 178.0(2) \\ \mathrm{C}(19)-\mathrm{C}(20)-\mathrm{C}(21)-\mathrm{C}(22) & -0.9(3) \\ \mathrm{C}(20)-\mathrm{C}(21)-\mathrm{C}(22)-\mathrm{O}(4) & -177.7(2) \\ \mathrm{C}(20)-\mathrm{C}(21)-\mathrm{C}(22)-\mathrm{C}(23) & -1.1(3) \\ \mathrm{C}(25)-\mathrm{O}(4)-\mathrm{C}(22)-\mathrm{C}(21) & -7.0(3) \\ \mathrm{C}(25)-\mathrm{O}(4)-\mathrm{C}(22)-\mathrm{C}(23) & 171.83(19) \\ \mathrm{C}(21)-\mathrm{C}(22)-\mathrm{C}(23)-\mathrm{C}(24) & 1.8(3) \\ \mathrm{O}(4)-\mathrm{C}(22)-\mathrm{C}(23)-\mathrm{C}(24) & -177.0(2) \\ \mathrm{C}(22)-\mathrm{C}(23)-\mathrm{C}(24)-\mathrm{C}(19) & \\ \mathrm{C}(20)-\mathrm{C}(19)-\mathrm{C}(24)-\mathrm{C}(23) & \\ \mathrm{N}(1)-\mathrm{C}(19)-\mathrm{C}(24)-\mathrm{C}(23) & \\ & \\ & \end{array}$

Symmetry transformations used to generate equivalent atoms: 
Table S16. Hydrogen bonds for $\mathbf{4 m}\left[\AA\right.$ and $\left.{ }^{\circ}\right]$.

$\begin{array}{lllll}\text { D-H...A } & \text { d(D-H) } & \text { d(H...A }) & \text { d(D...A })\end{array}$




\section{References}

[1] Rohlmann, R.; Stopka, T.; Richter, H. and Mancheño, O. G. J. Org. Chem. 2013, 78, 60506064.

[2] Xie, J. and Huang, Z.-Z. Angew. Chem., Int. Ed. 2010, 49, 10181-10185.

[3] Inokuma, T., Jichu, T.; Nishida, K.; Shigenaga, A. and Otaka, A. Chem. Pharm. Bull. 2017, 65, 573-581.

[4] Jia, X.; Hou, W.; Shao, Y.; Yuan, Y.; Chen, Q.; Li, P.; Liu, X. and Ji, H. Chem. Eur. J. 2017, 23, 12980-12984.

[5] Jiang, W.; Wan, S.; Su, Y. and Huo, C. J. Org. Chem. 2019, 84, 8232-8241.

[6] Joshi, P. C.; Parmar, S. S.; Rastogi, V. K. J. Heterocycl. Chem. 1979, 16, 607-608.

[7] Stanton, J. L.; Gruenfeld, N.; Babiarz, J. E.; Ackerman, M. H.; Friedmann, R. C.; Yuan, A. M. and Macchia, W. J. Med. Chem. 1983, 26, 1267-1277.

[8] Tien, N. B.; Buu-Hoï, N. P. and Xuong, N. D. J. Org. Chem. 1958, 23, 186-188.

[9] Li, X.-M.; Tang, L.; Qian, Z.-M.; He, Y.-H.; Guan, Z. Tetrahedron Lett. 2020, 61, 152346.

[10] Yang, X.; Xie, Z.; Li, Y. and Zhang, Y. Chem. Sci. 2020, 11, 4741-4746. 


\section{Copies of NMR Spectra}

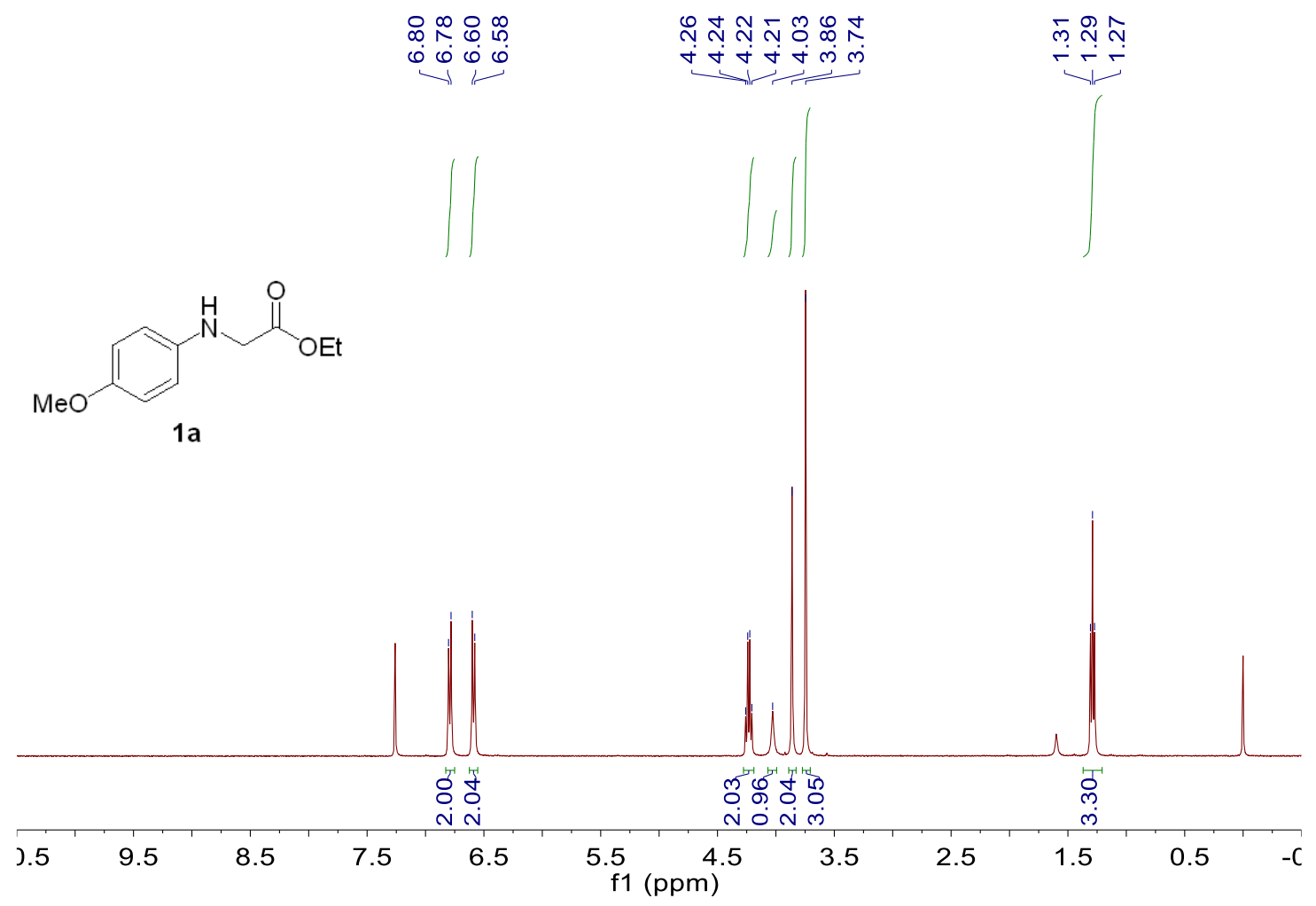

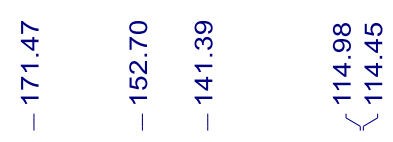

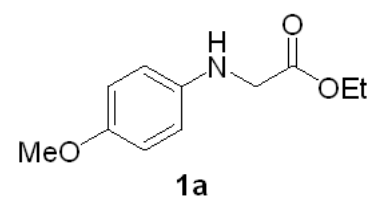

1a

${ }^{1} \mathrm{H}$ NMR $(400 \mathrm{MHz})$ and ${ }^{13} \mathrm{C}\left\{{ }^{1} \mathrm{H}\right\}$ NMR $(101 \mathrm{MHz})$ spectra of $\mathbf{1 a}\left(\mathrm{CDCl}_{3}, \mathrm{rt}\right)$. 

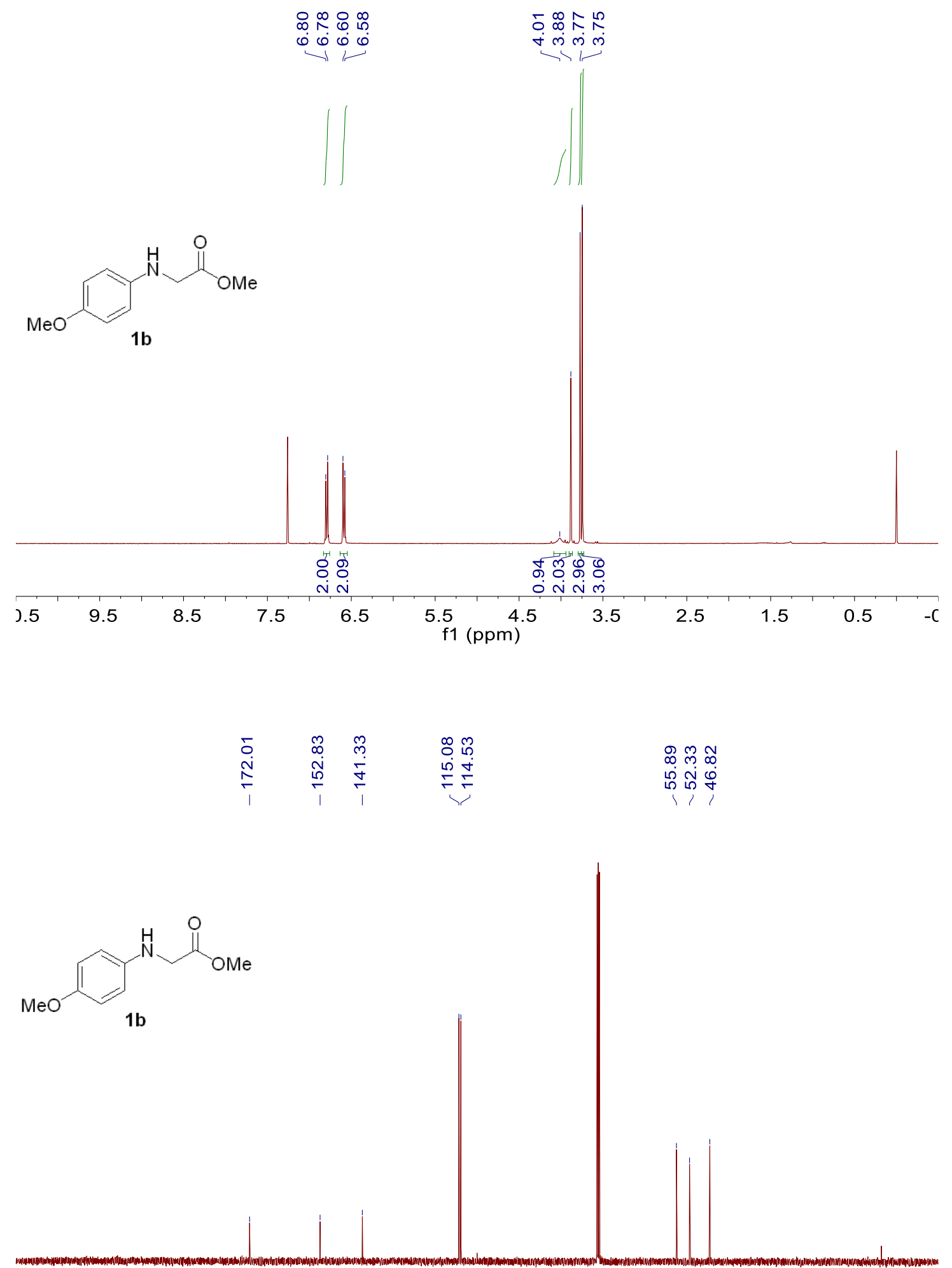

$230 \quad 210 \quad 190 \quad 170 \quad 150 \quad 130 \begin{gathered}110 \\ \mathrm{f} 1(\mathrm{ppm})\end{gathered}$

${ }^{1} \mathrm{H}$ NMR (400 MHz) and ${ }^{13} \mathrm{C}\left\{{ }^{1} \mathrm{H}\right\}$ NMR $(101 \mathrm{MHz})$ spectra of $\mathbf{1 b}\left(\mathrm{CDCl}_{3}, \mathrm{rt}\right)$. 


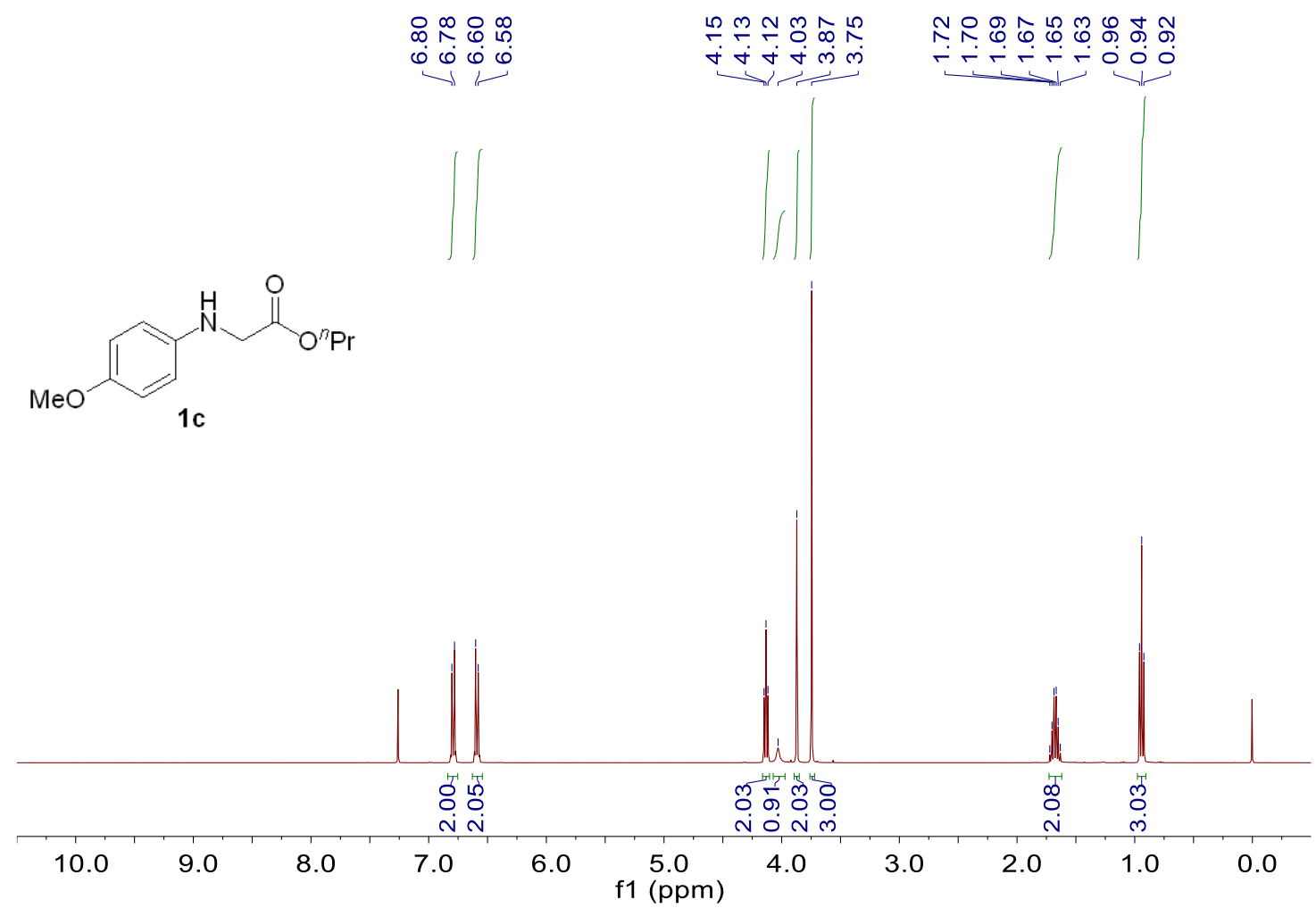

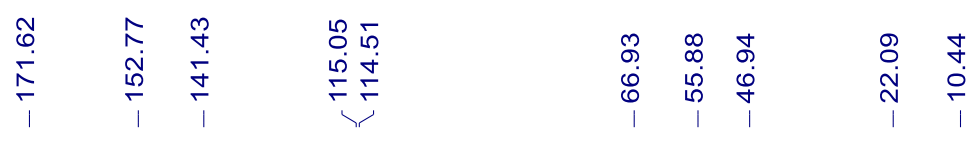<smiles>CCCOC(=O)CNc1ccc(OC)cc1</smiles>

$1 \mathrm{c}$

230

$210 \quad 190 \quad 170$

150

130

110
$\mathrm{f} 1(\mathrm{ppm})$

70

50

30

$10-10$

${ }^{1} \mathrm{H}$ NMR (400 MHz) and ${ }^{13} \mathrm{C}\left\{{ }^{1} \mathrm{H}\right\} \mathrm{NMR}(101 \mathrm{MHz})$ spectra of $\mathbf{1 c}\left(\mathrm{CDCl}_{3}, \mathrm{rt}\right)$. 


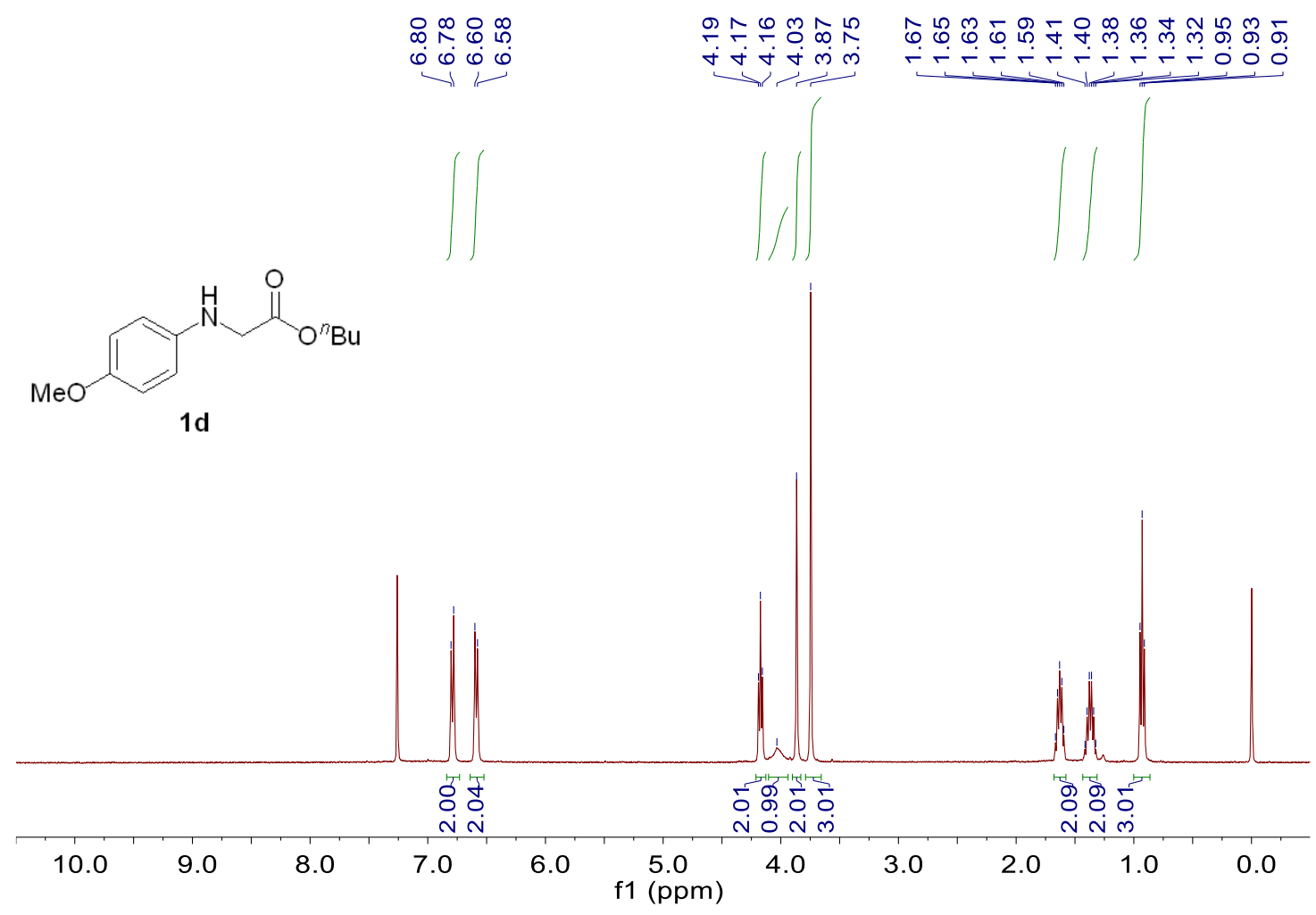

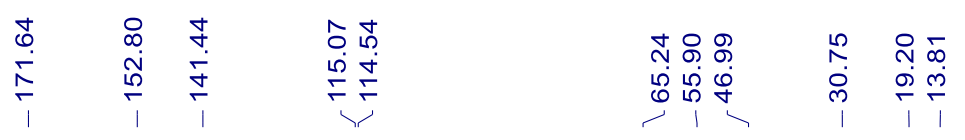<smiles>CCOC(=O)CNc1ccc(OC)cc1</smiles>

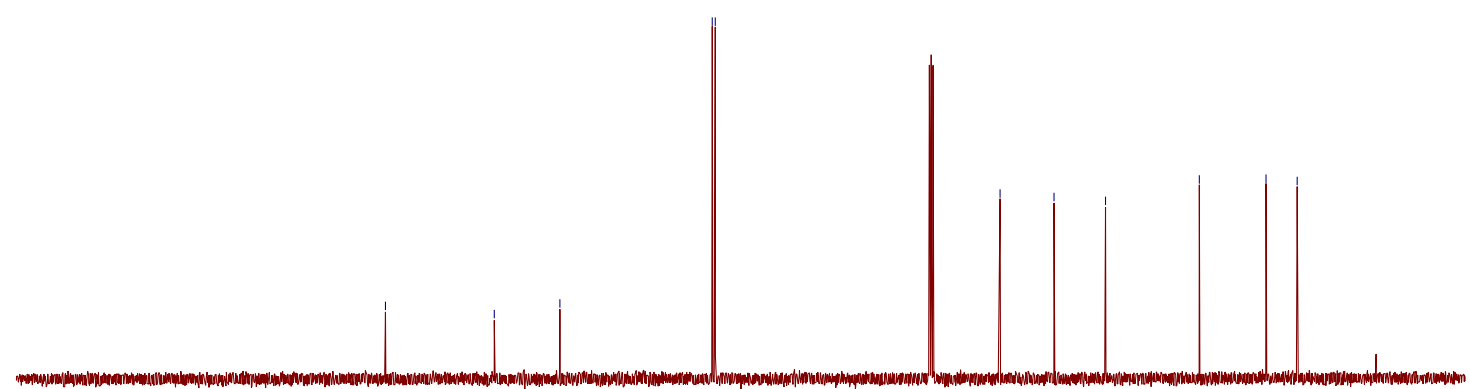

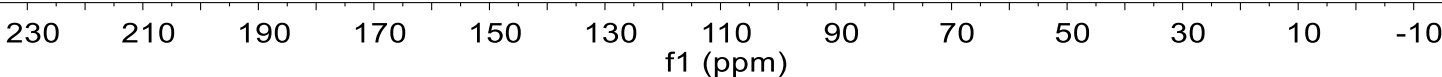

${ }^{1} \mathrm{H}$ NMR $(400 \mathrm{MHz})$ and ${ }^{13} \mathrm{C}\left\{{ }^{1} \mathrm{H}\right\}$ NMR $(101 \mathrm{MHz})$ spectra of $\mathbf{1 d}\left(\mathrm{CDCl}_{3}, \mathrm{rt}\right)$. 


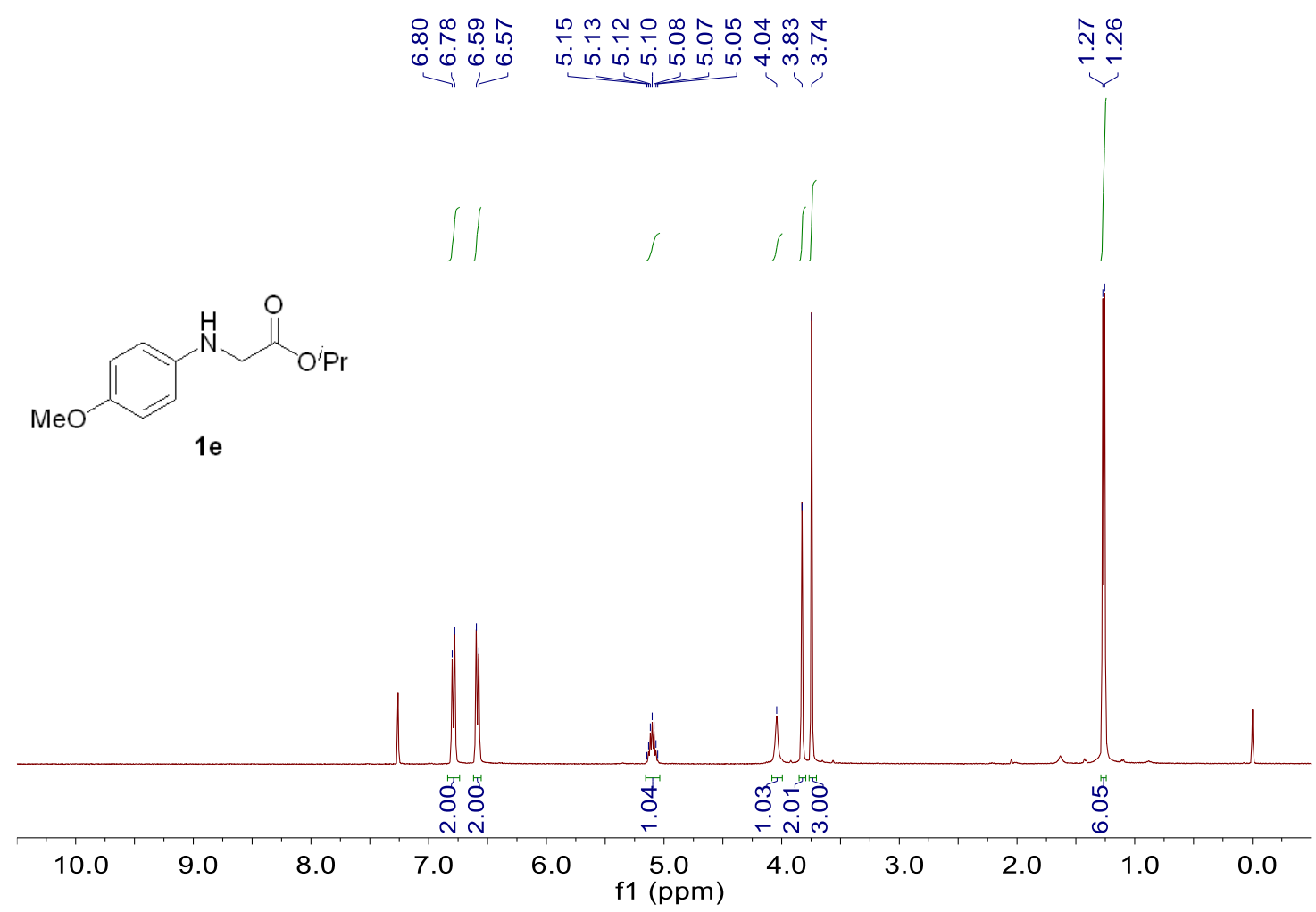

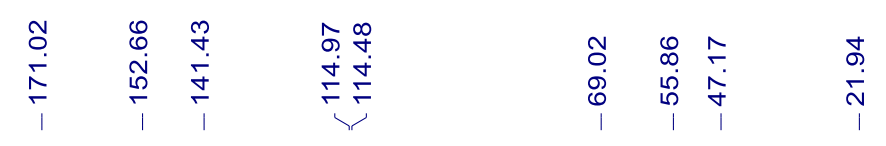<smiles>CCCOC(=O)CNc1ccc(OC)cc1</smiles>

$1 e$

\begin{tabular}{|c|c|c|c|c|c|c|c|c|c|c|c|c|}
\hline 240 & 220 & 200 & 180 & 160 & 140 & $\begin{array}{c}120 \\
f 1\end{array}$ & $\begin{array}{l}100 \\
m)\end{array}$ & 80 & 60 & 40 & 20 & 0 \\
\hline
\end{tabular}

${ }^{1} \mathrm{H}$ NMR $(400 \mathrm{MHz})$ and ${ }^{13} \mathrm{C}\left\{{ }^{1} \mathrm{H}\right\}$ NMR $(101 \mathrm{MHz})$ spectra of $\mathbf{1 e}\left(\mathrm{CDCl}_{3}, \mathrm{rt}\right)$. 


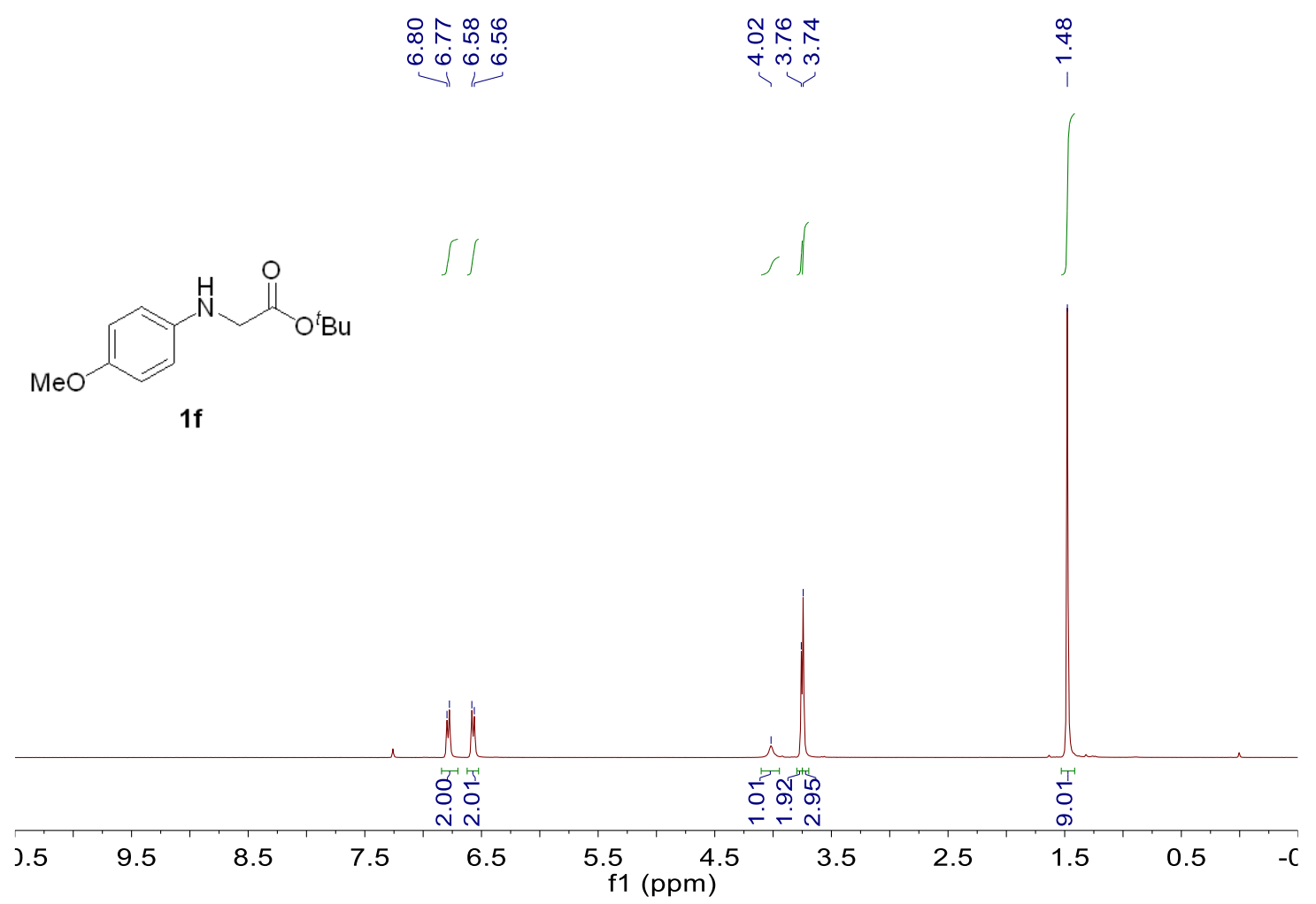

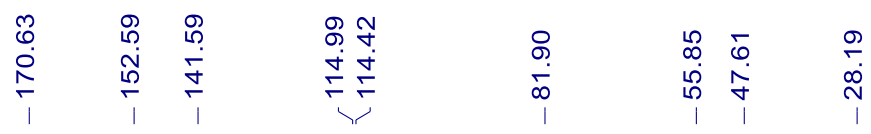

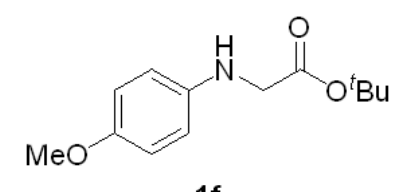

$1 f$

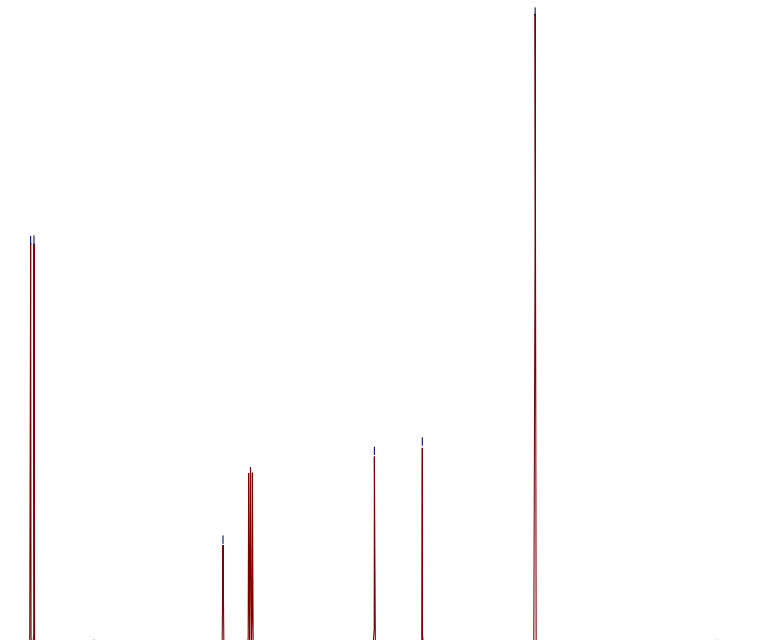

230

210

$190 \quad 170 \quad 150$

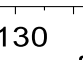

f1 110

${ }^{1} \mathrm{H}$ NMR (400 MHz) and ${ }^{13} \mathrm{C}\left\{{ }^{1} \mathrm{H}\right\}$ NMR (101 MHz) spectra of $\mathbf{1 f}\left(\mathrm{CDCl}_{3}, \mathrm{rt}\right)$. 


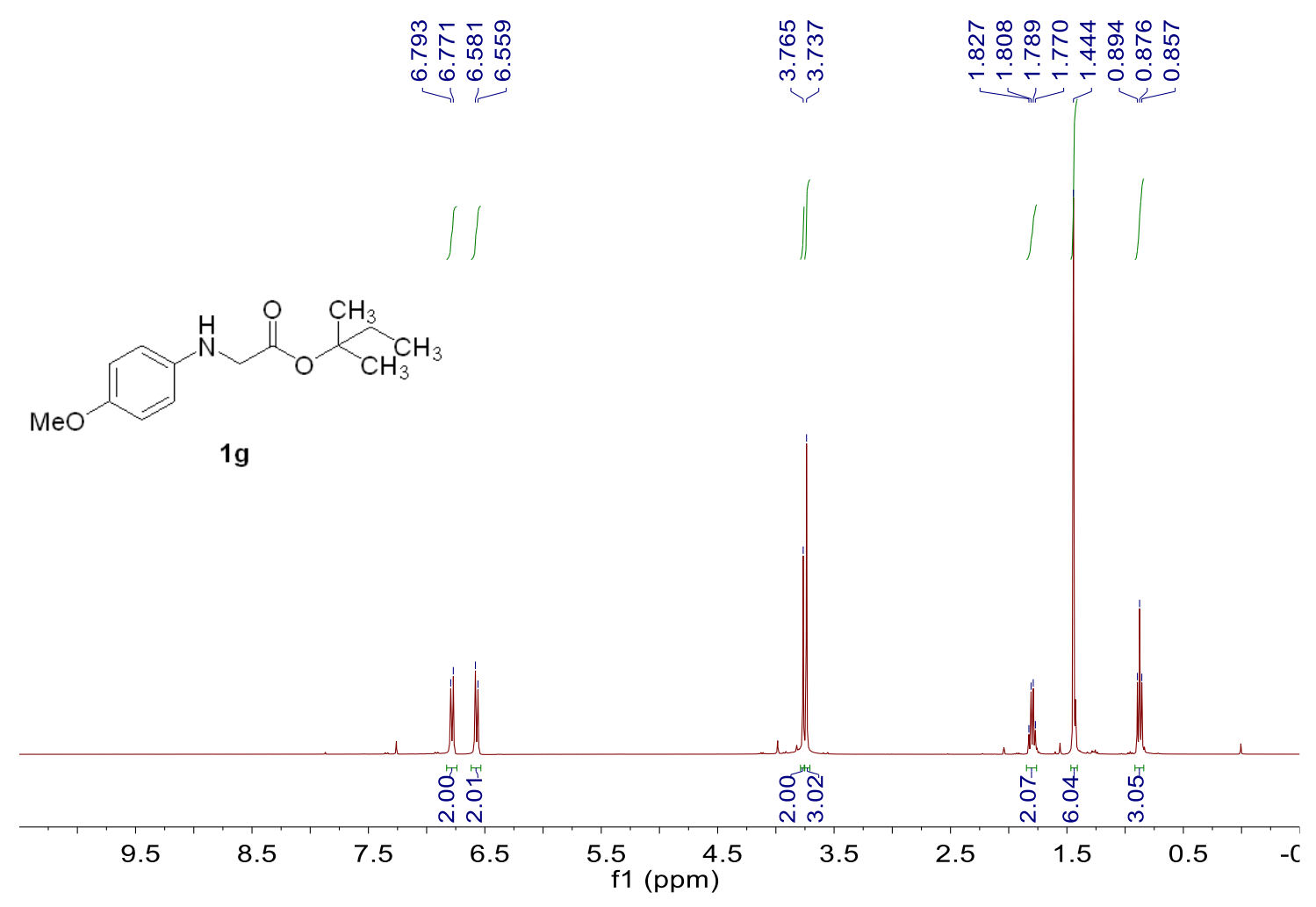

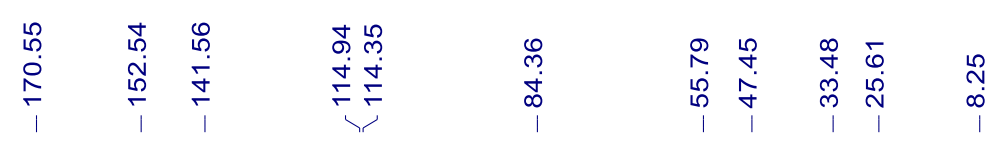
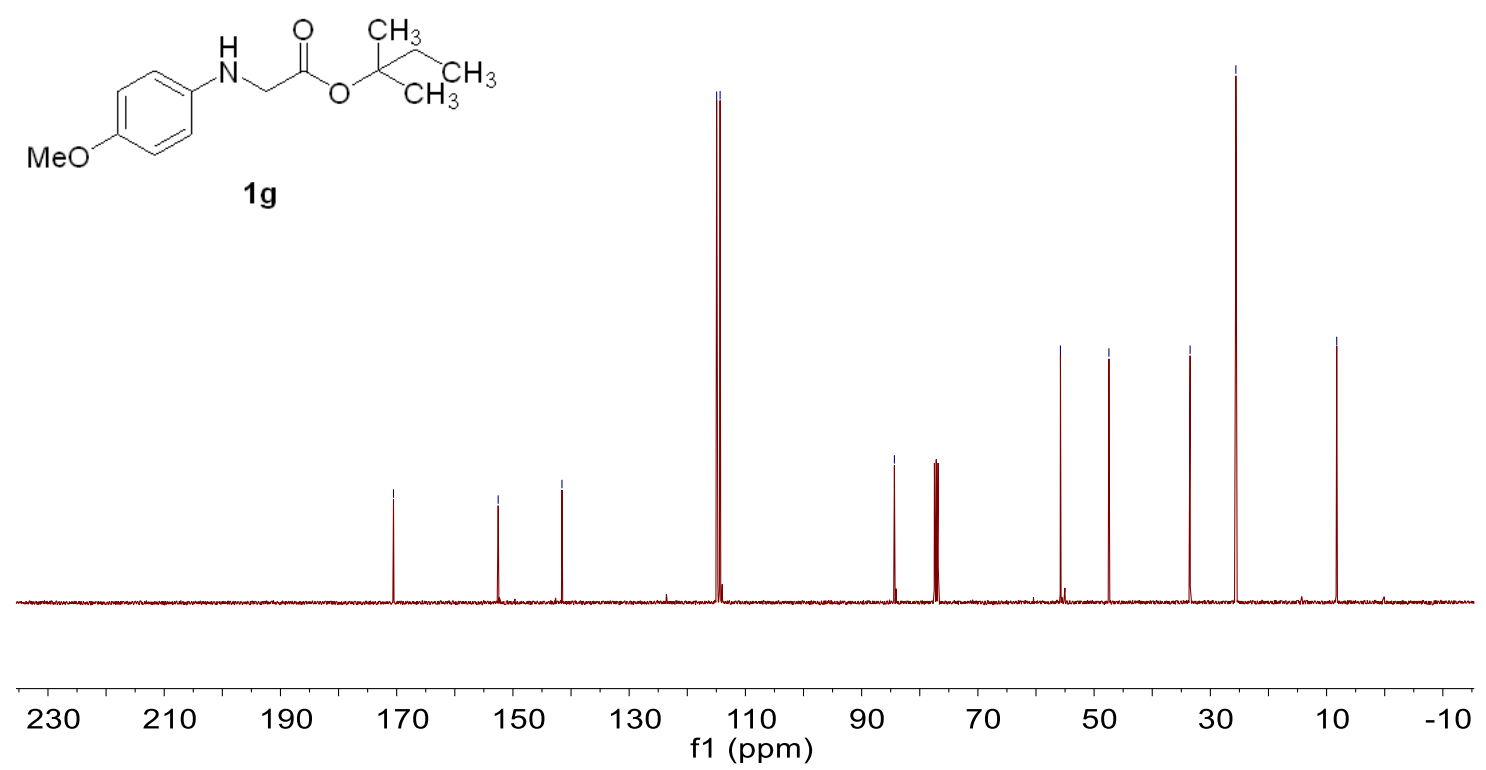

${ }^{1} \mathrm{H}$ NMR (400 MHz) and ${ }^{13} \mathrm{C}\left\{{ }^{1} \mathrm{H}\right\}$ NMR (101 MHz) spectra of $\mathbf{1 g}\left(\mathrm{CDCl}_{3}, \mathrm{rt}\right)$. 

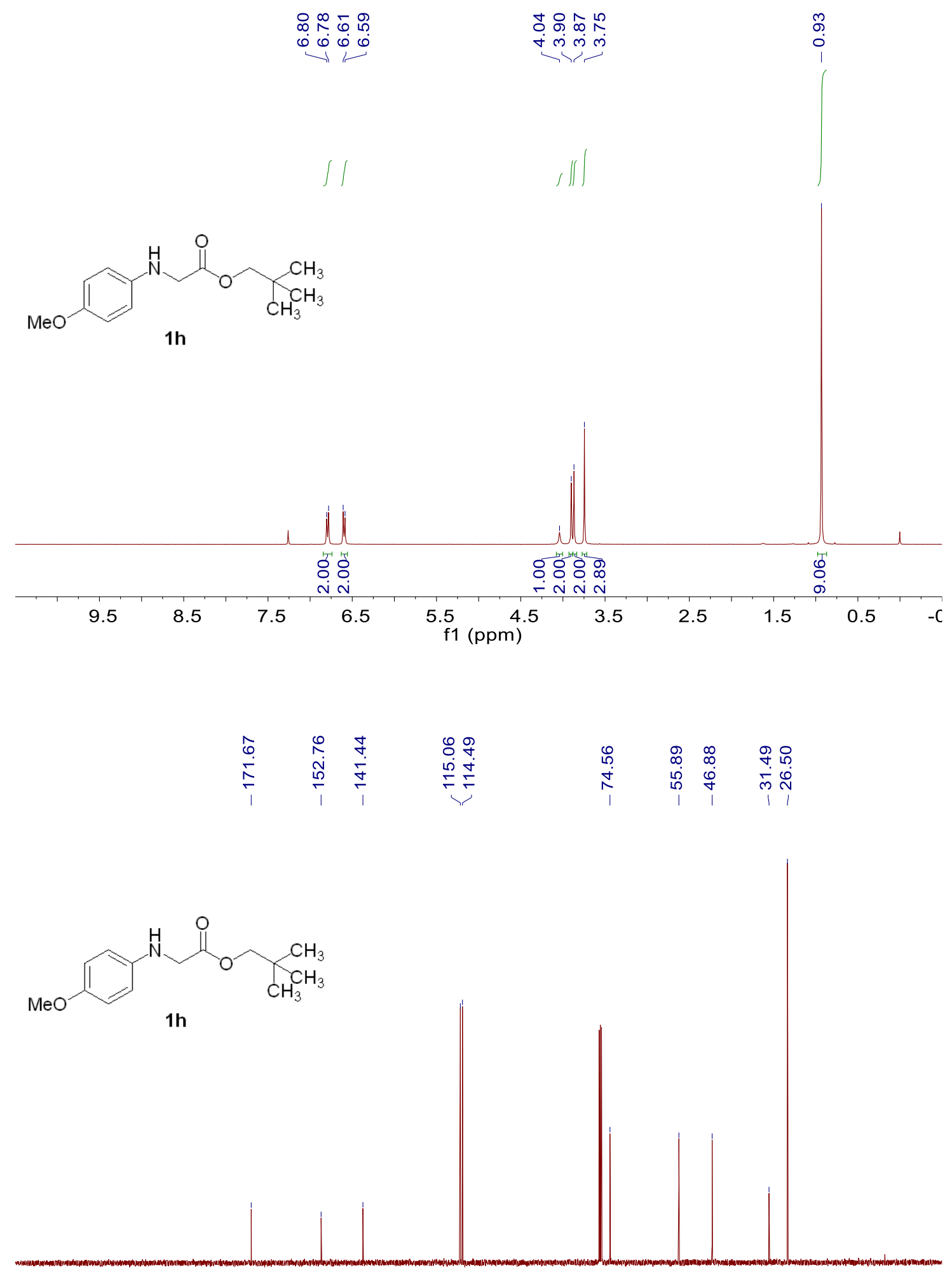

$230 \quad 210 \quad 190 \quad 170 \quad 150 \quad 130 \begin{gathered}110 \\ \mathrm{f} 1(\mathrm{ppm})\end{gathered}$

${ }^{1} \mathrm{H}$ NMR (400 MHz) and ${ }^{13} \mathrm{C}\left\{{ }^{1} \mathrm{H}\right\}$ NMR $(101 \mathrm{MHz})$ spectra of $\mathbf{1 h}\left(\mathrm{CDCl}_{3}, \mathrm{rt}\right)$. 


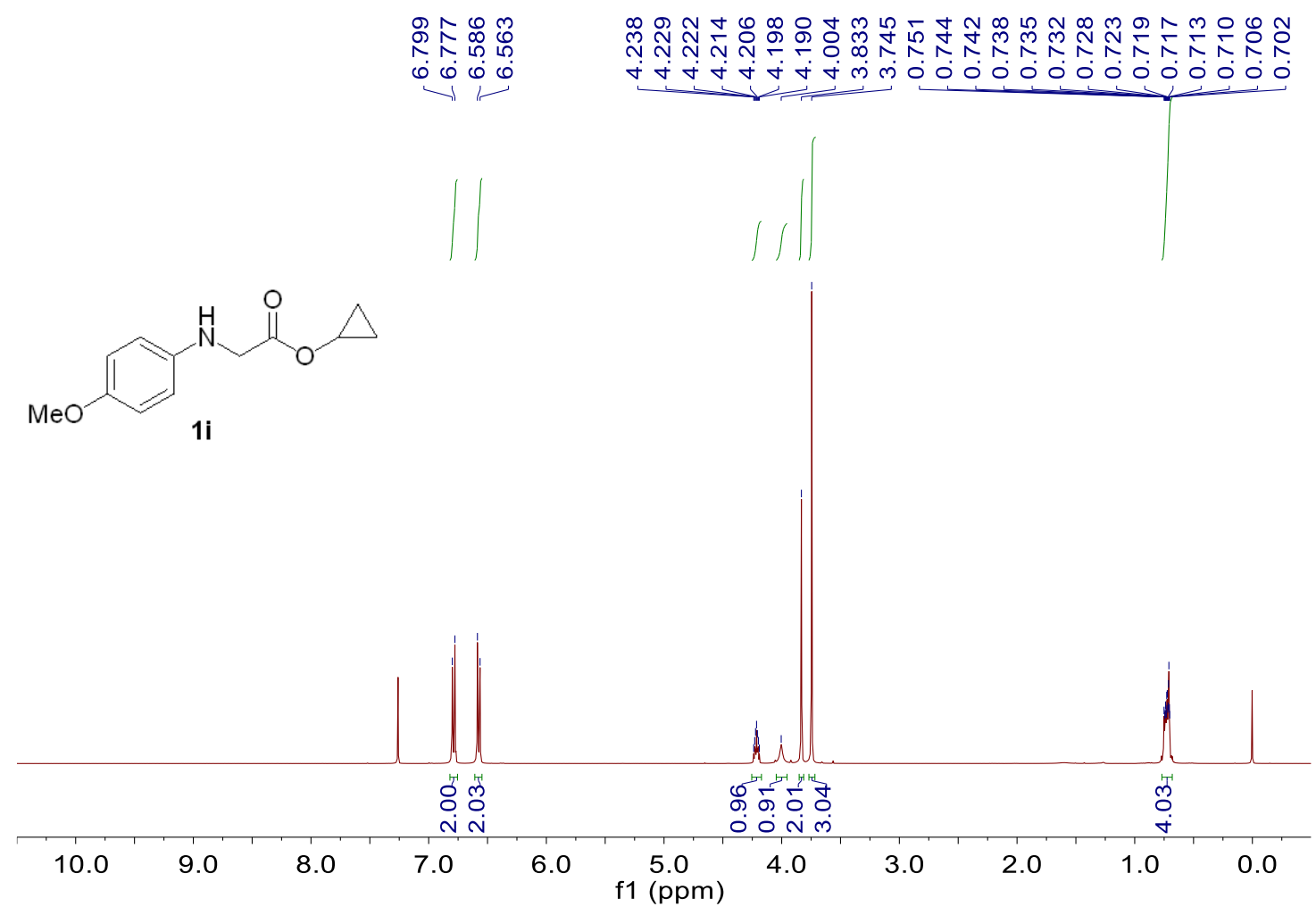

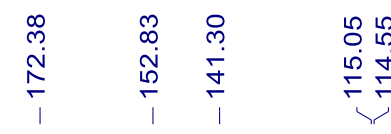

\section{的势}

它守守

$\underset{\substack{\bar{n} \\ \text { in }}}{1}$<smiles>COc1ccc(NCC(=O)OC2CC2)cc1</smiles>

$1 i$

230

210190

170150

130

f1 (ppm)

$1<1$

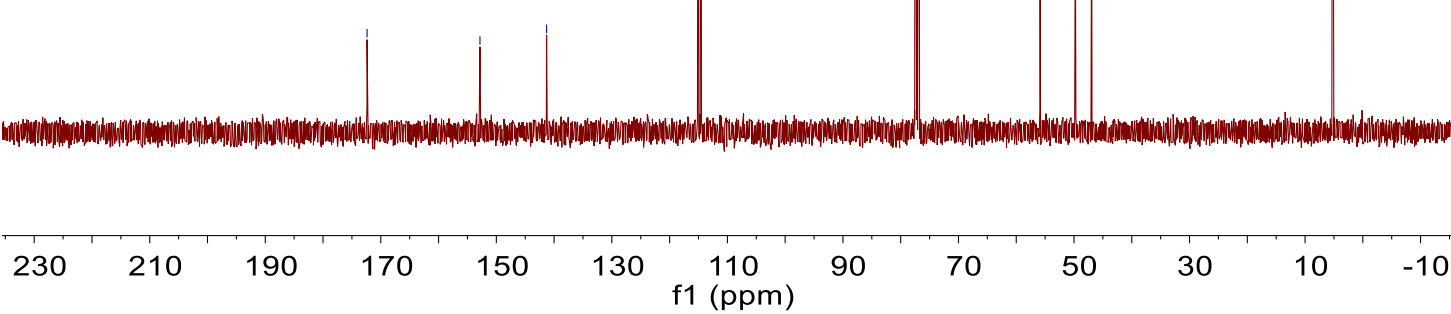

${ }^{1} \mathrm{H}$ NMR $(400 \mathrm{MHz})$ and ${ }^{13} \mathrm{C}\left\{{ }^{1} \mathrm{H}\right\} \mathrm{NMR}(101 \mathrm{MHz})$ spectra of $\mathbf{1 i}\left(\mathrm{CDCl}_{3}, \mathrm{rt}\right)$. 


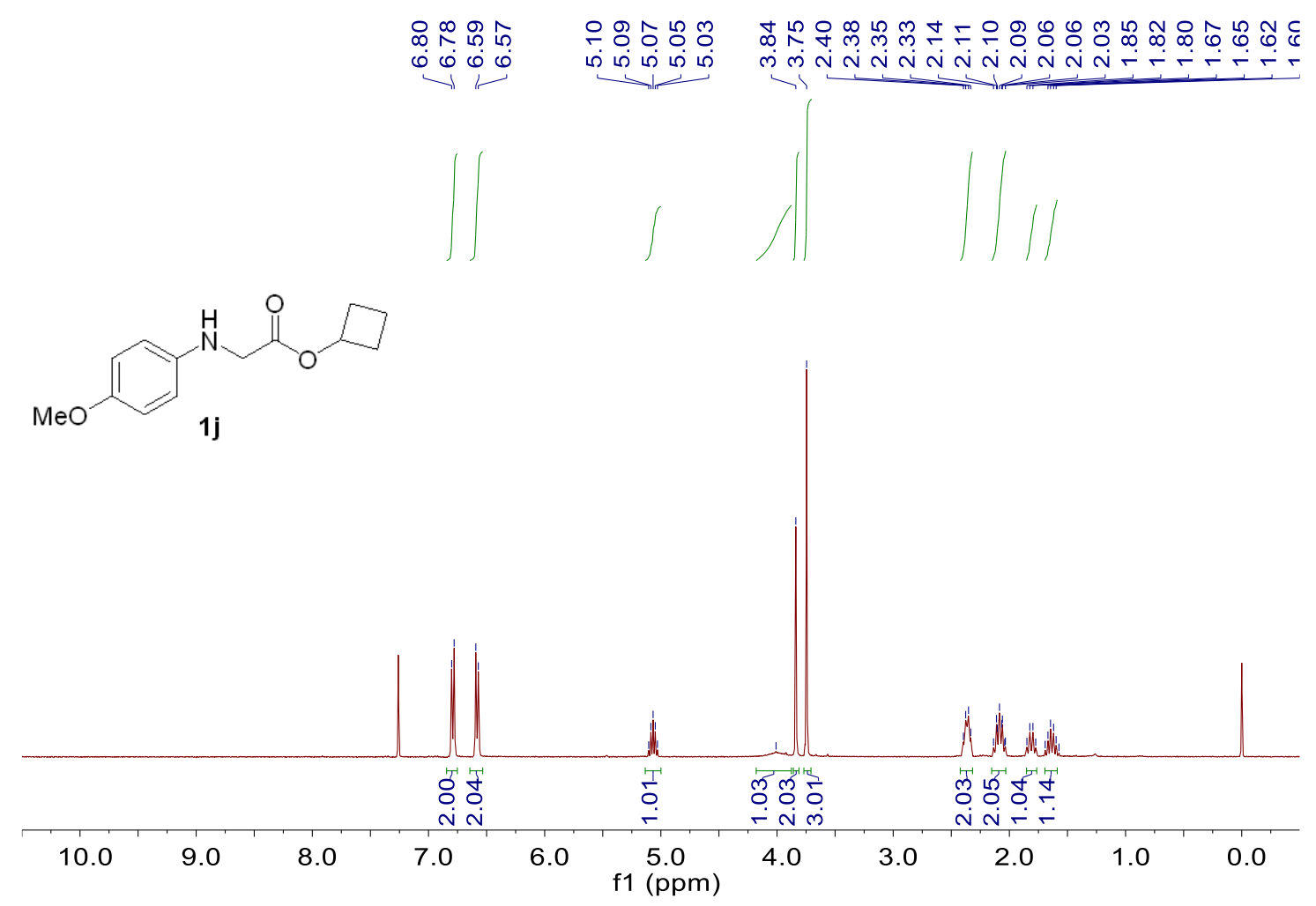

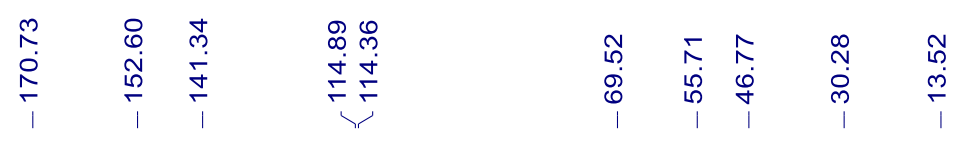
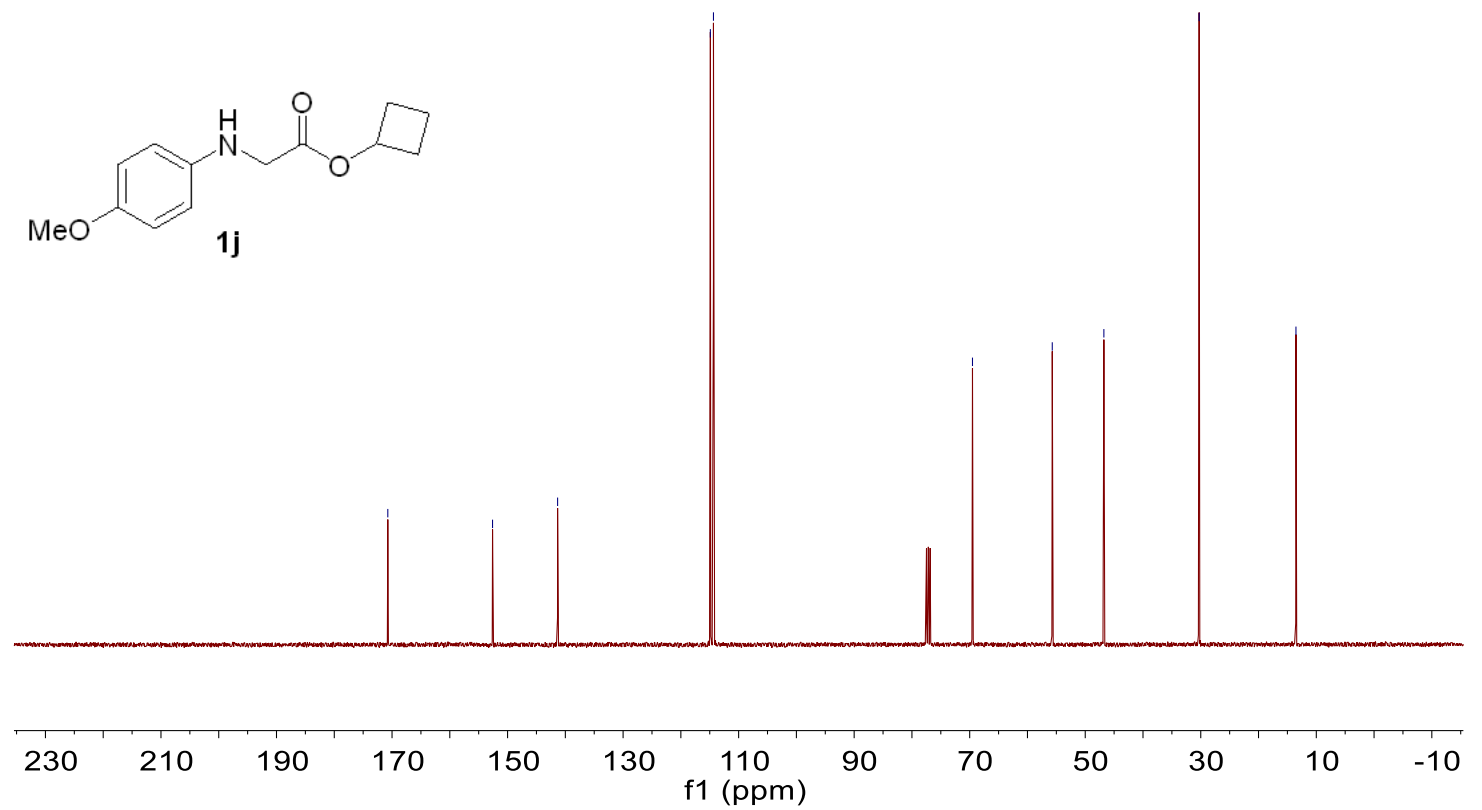

${ }^{1} \mathrm{H}$ NMR (400 MHz) and ${ }^{13} \mathrm{C}\left\{{ }^{1} \mathrm{H}\right\}$ NMR $(101 \mathrm{MHz})$ spectra of $\mathbf{1} \mathbf{j}\left(\mathrm{CDCl}_{3}, \mathrm{rt}\right)$. 


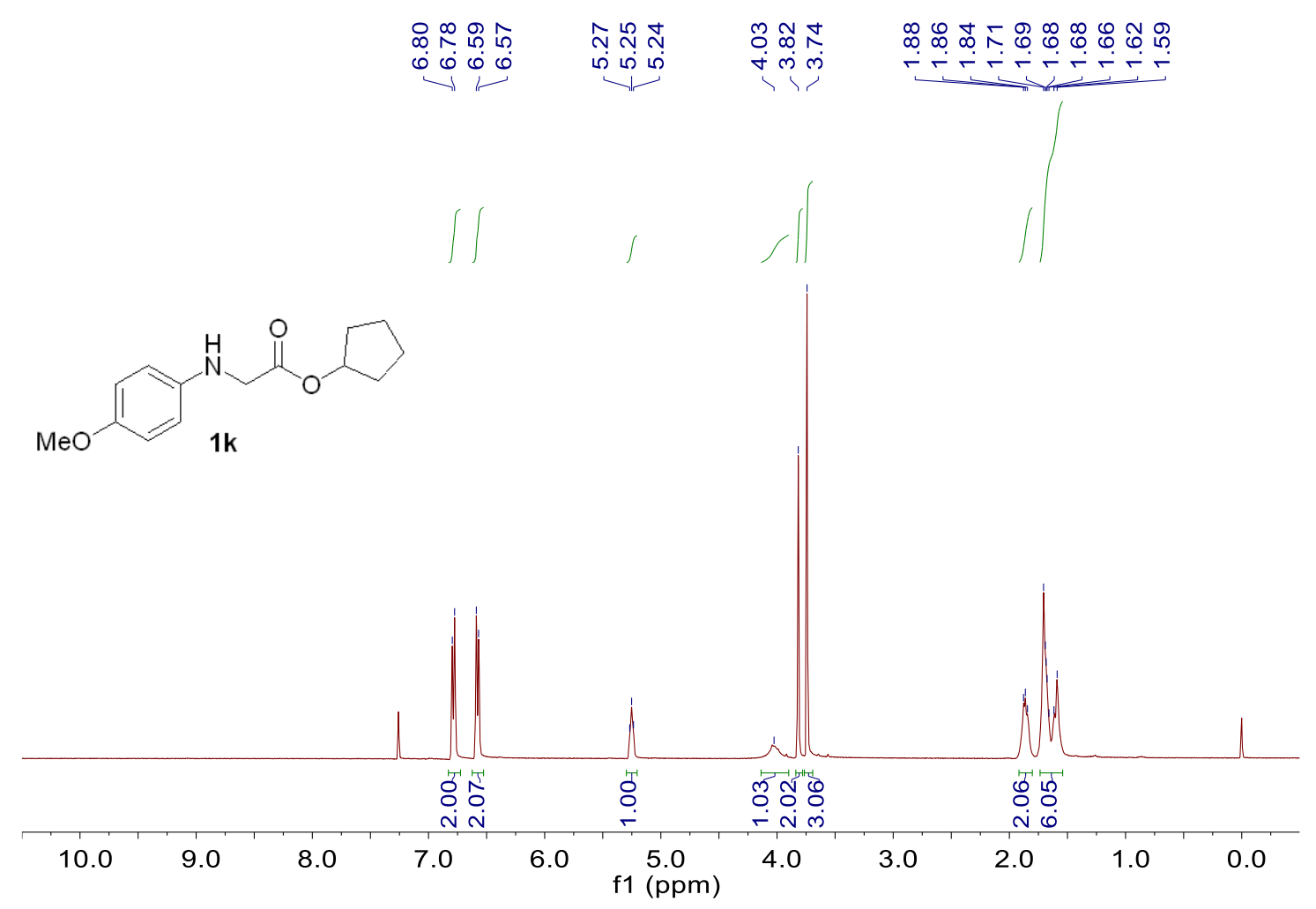

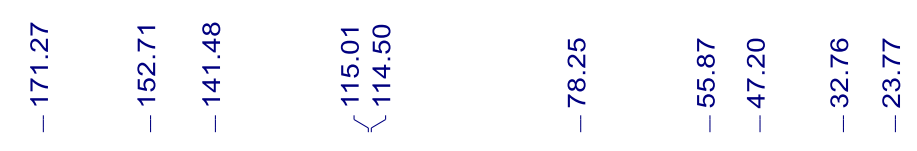
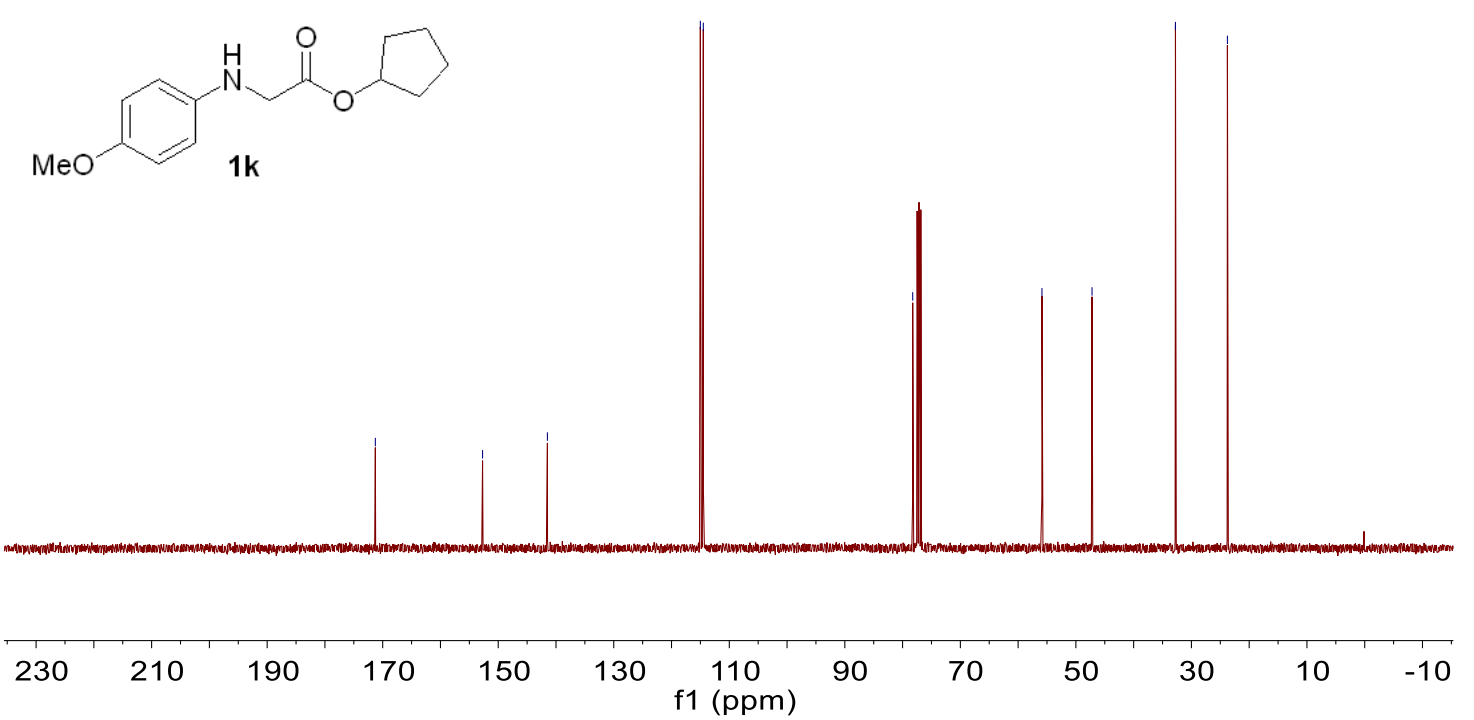

${ }^{1} \mathrm{H}$ NMR $(400 \mathrm{MHz})$ and ${ }^{13} \mathrm{C}\left\{{ }^{1} \mathrm{H}\right\}$ NMR $(101 \mathrm{MHz})$ spectra of $\mathbf{1 k}\left(\mathrm{CDCl}_{3}, \mathrm{rt}\right)$. 
<smiles>COc1ccc(NCC(=O)OC2CCCCC2)cc1</smiles><smiles>C1=CC=C1</smiles>
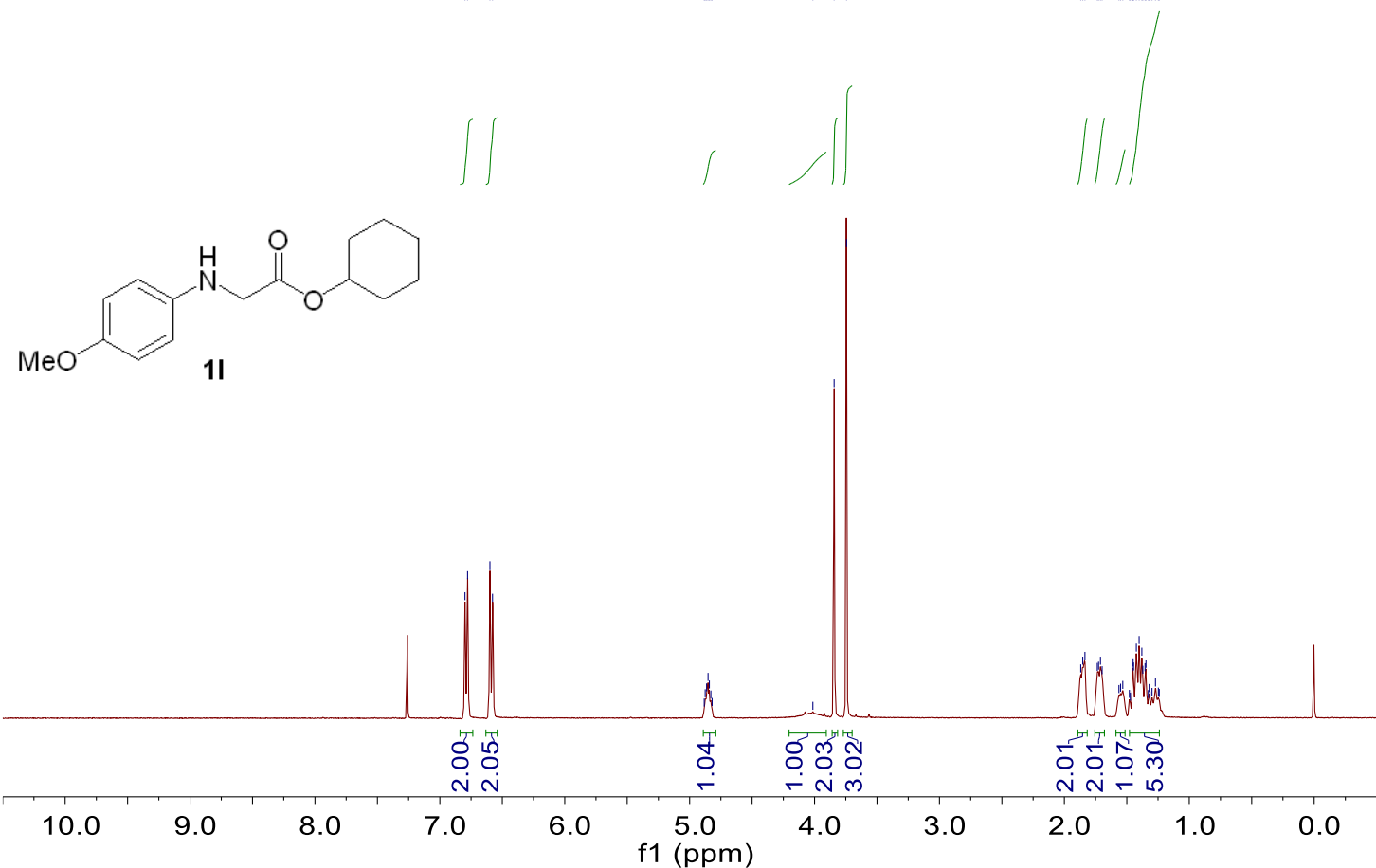

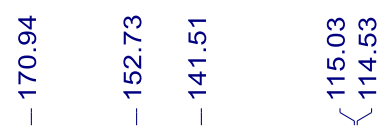
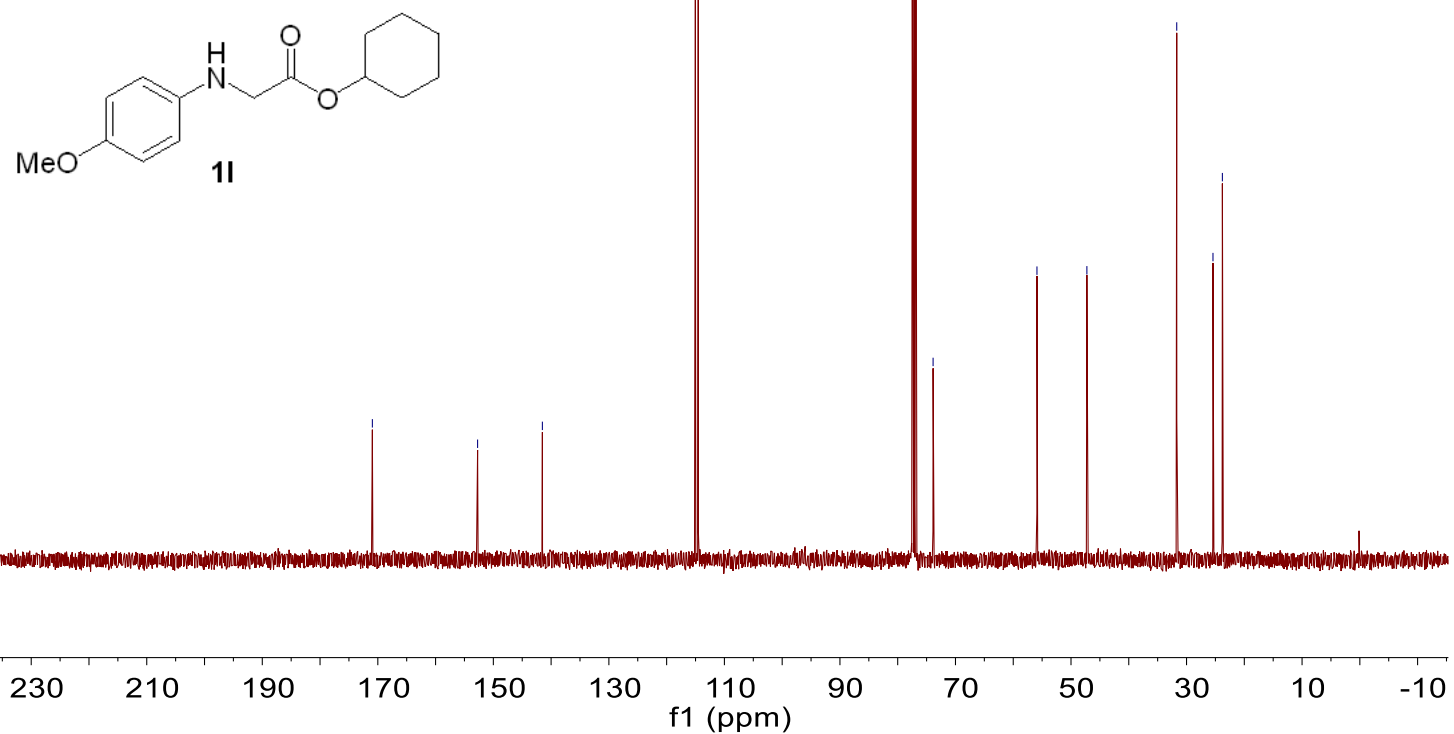

${ }^{1} \mathrm{H}$ NMR (400 MHz) and ${ }^{13} \mathrm{C}\left\{{ }^{1} \mathrm{H}\right\}$ NMR $(101 \mathrm{MHz})$ spectra of $\mathbf{1 l}\left(\mathrm{CDCl}_{3}, \mathrm{rt}\right)$. 


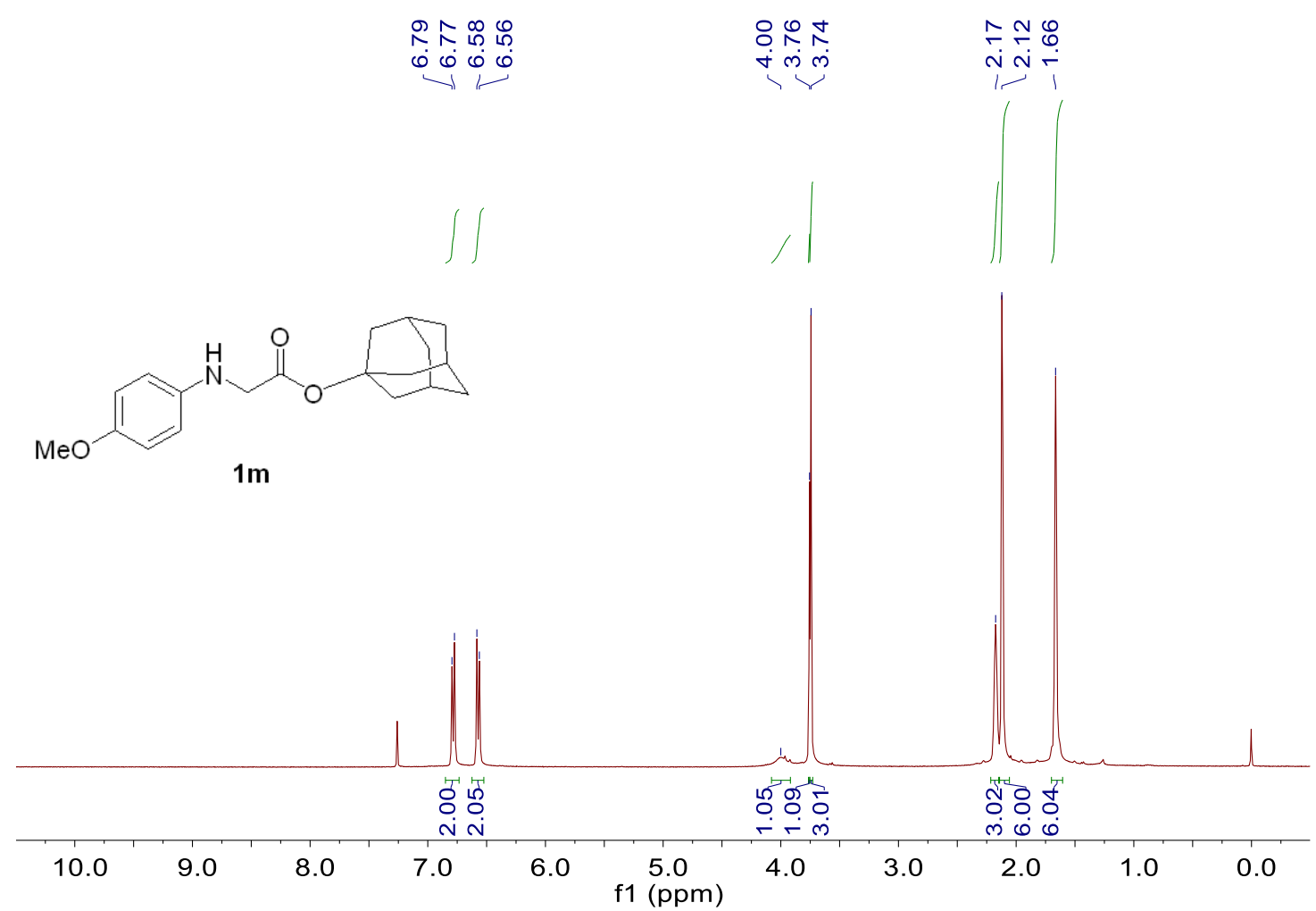

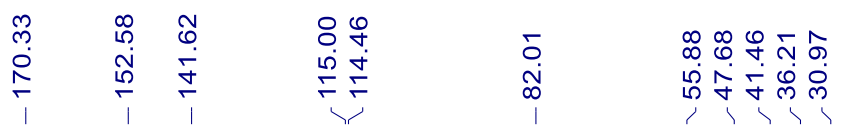
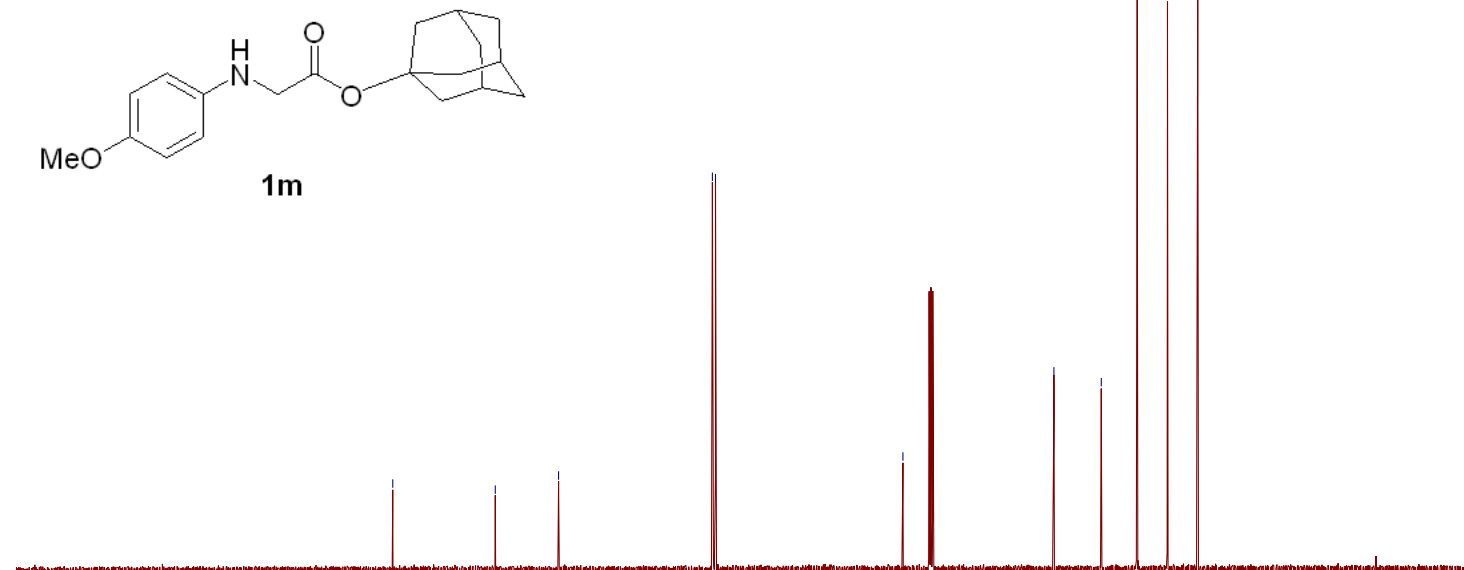

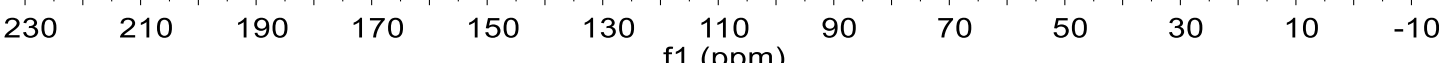

${ }^{1} \mathrm{H}$ NMR (400 MHz) and ${ }^{13} \mathrm{C}\left\{{ }^{1} \mathrm{H}\right\}$ NMR (101 MHz) spectra of $\mathbf{1 m}\left(\mathrm{CDCl}_{3}, \mathrm{rt}\right)$. 


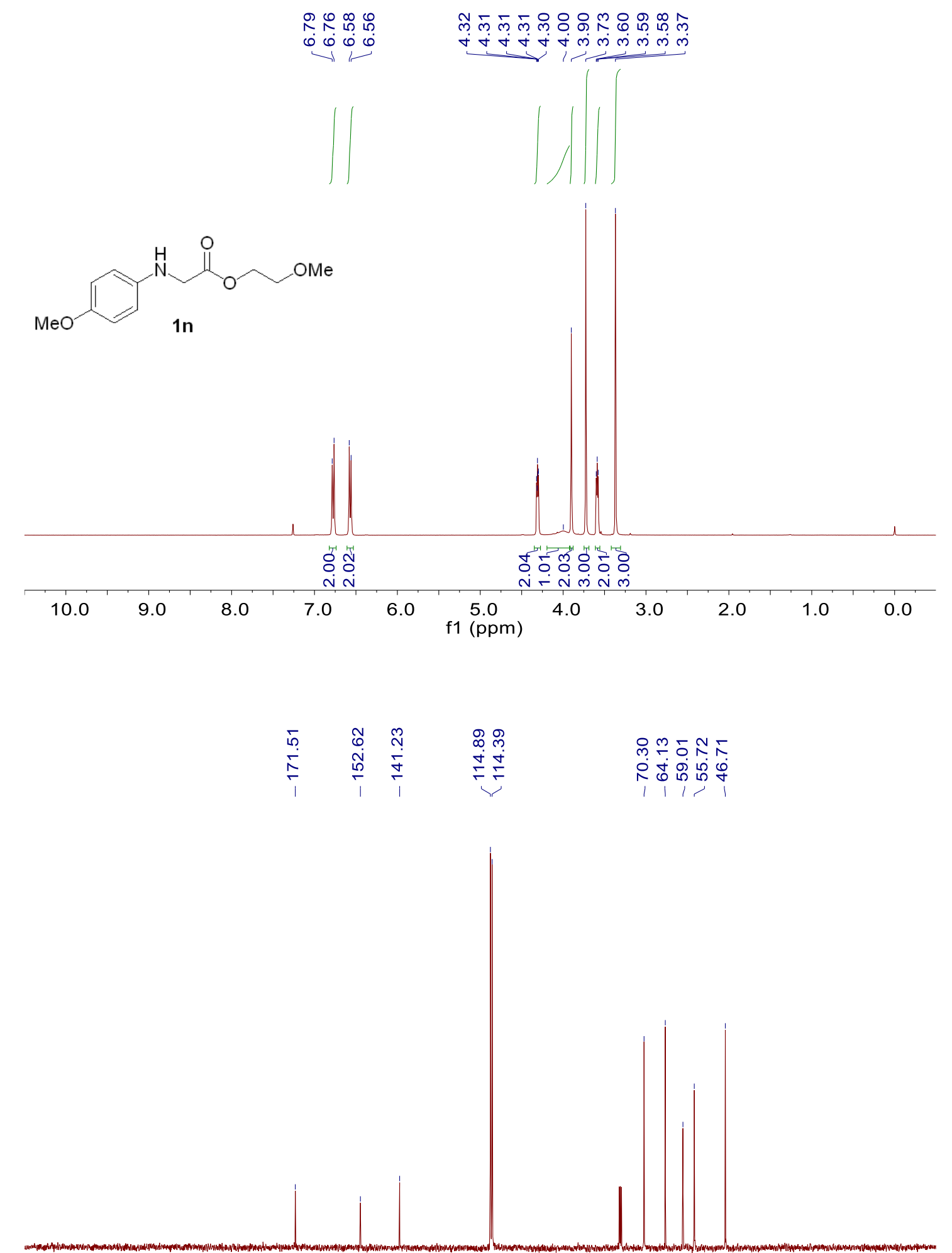

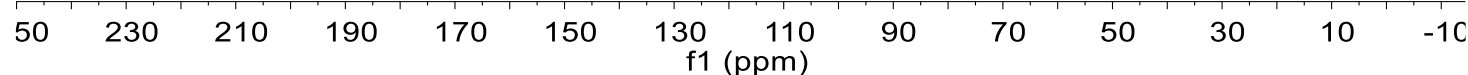

${ }^{1} \mathrm{H}$ NMR (400 MHz) and ${ }^{13} \mathrm{C}\left\{{ }^{1} \mathrm{H}\right\}$ NMR $(101 \mathrm{MHz})$ spectra of 1 n $\left(\mathrm{CDCl}_{3}, \mathrm{rt}\right)$. 


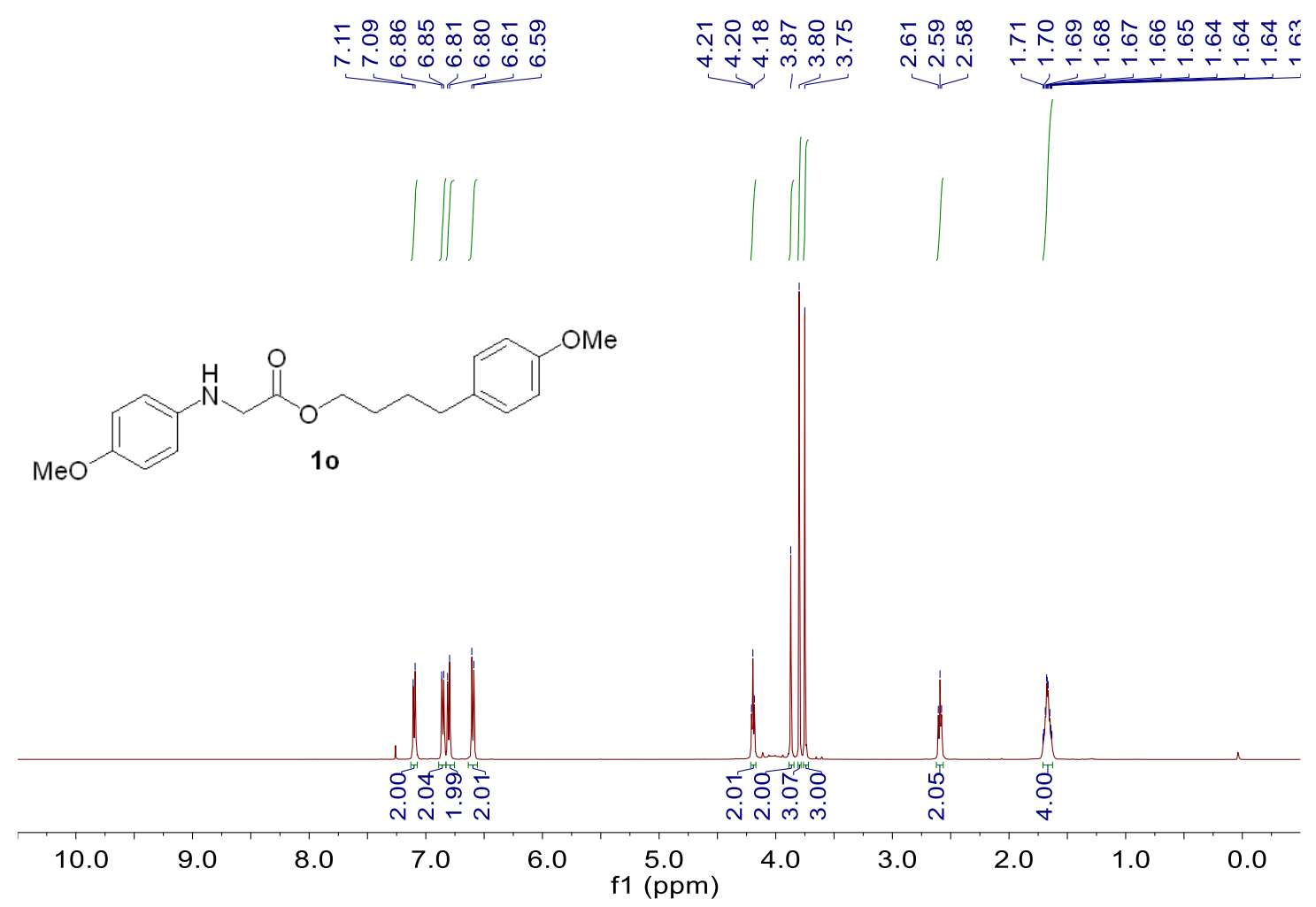

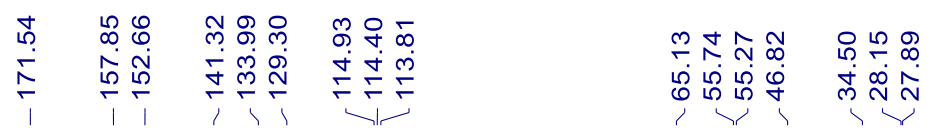

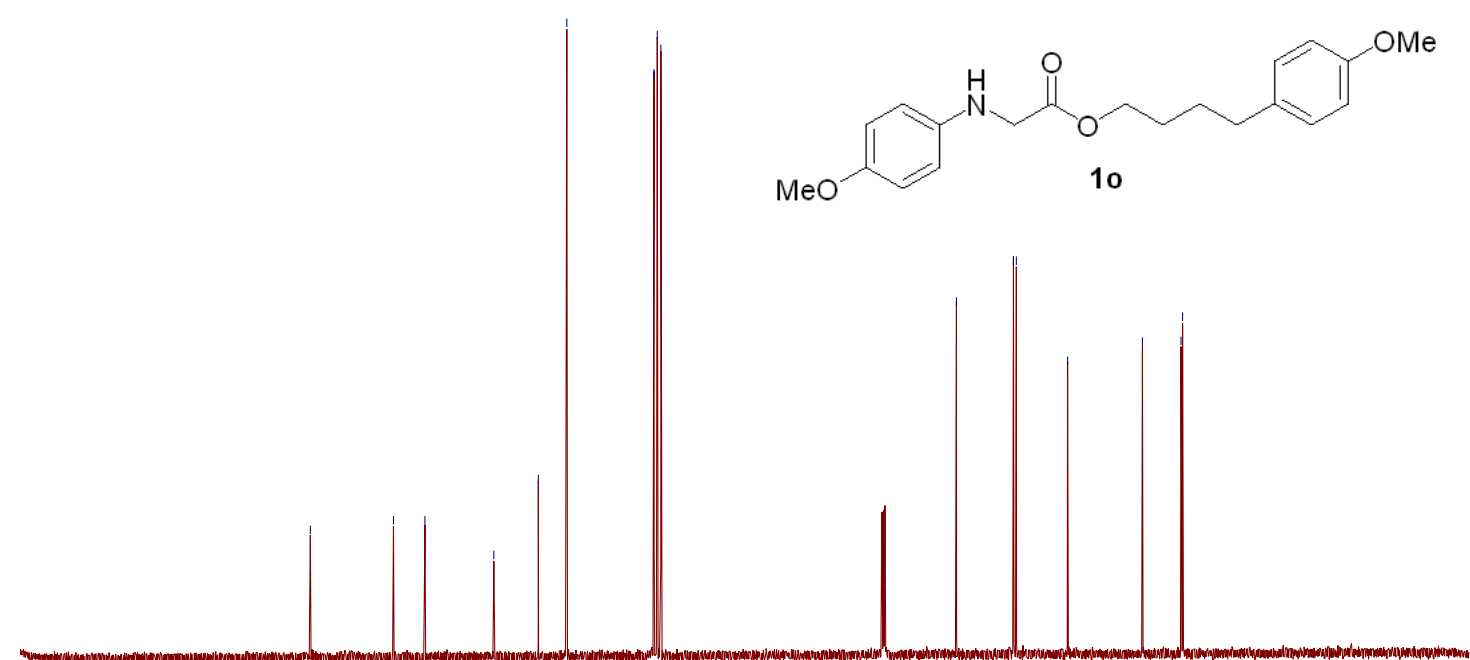

$21020019018017016015014013012011010090 \quad 80 \quad 70 \quad 60 \quad 50 \quad 40 \quad 30 \quad 20 \quad 10 \quad 0 \quad-10$ f1 (ppm)

${ }^{1} \mathrm{H}$ NMR $(500 \mathrm{MHz})$ and ${ }^{13} \mathrm{C}\left\{{ }^{1} \mathrm{H}\right\} \mathrm{NMR}(126 \mathrm{MHz})$ spectra of $\mathbf{1 o}\left(\mathrm{CDCl}_{3}, \mathrm{rt}\right)$. 


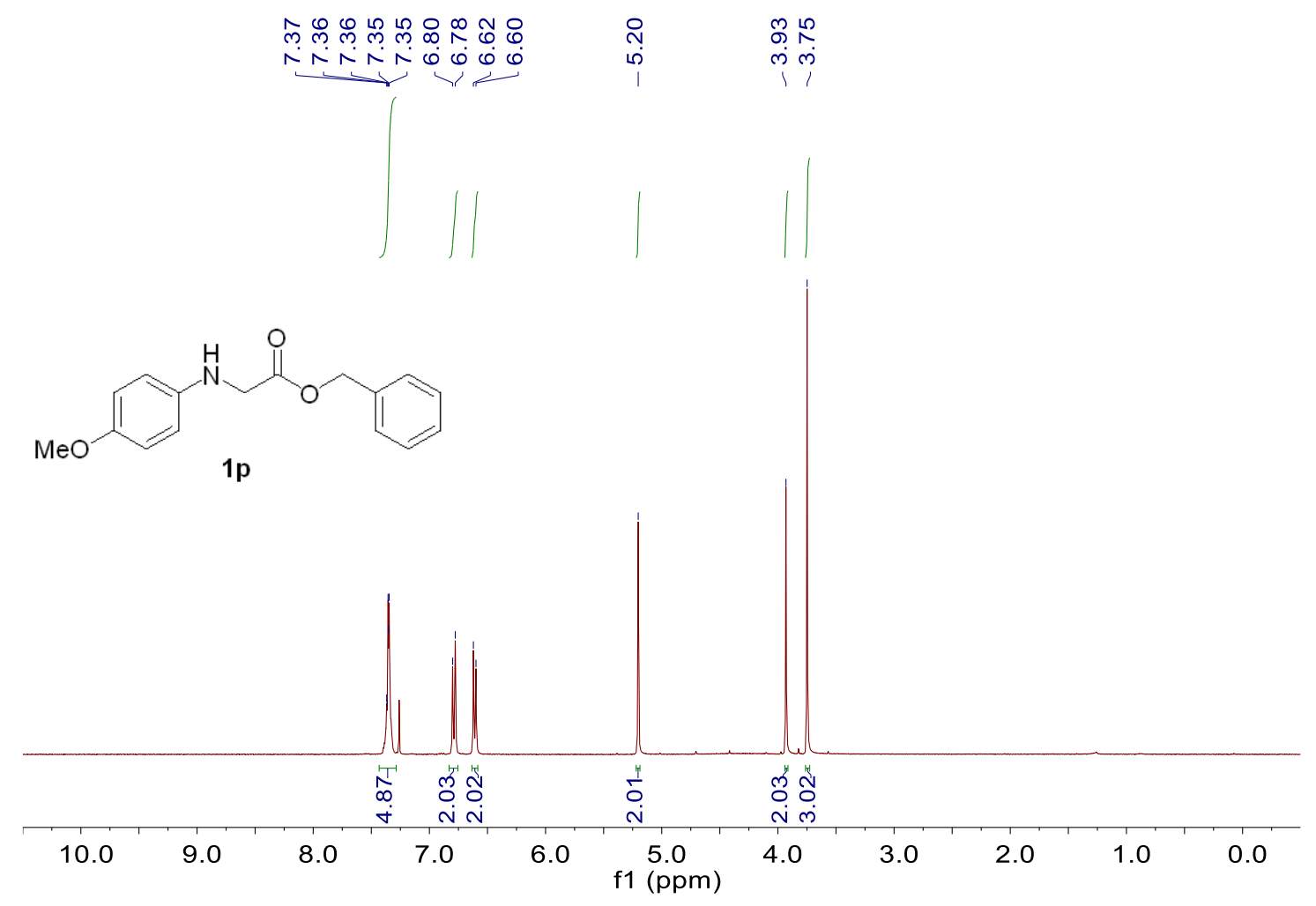

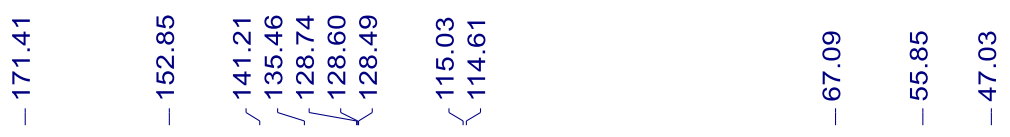<smiles>COc1ccc(NCC(=O)OCc2ccccc2)cc1</smiles>

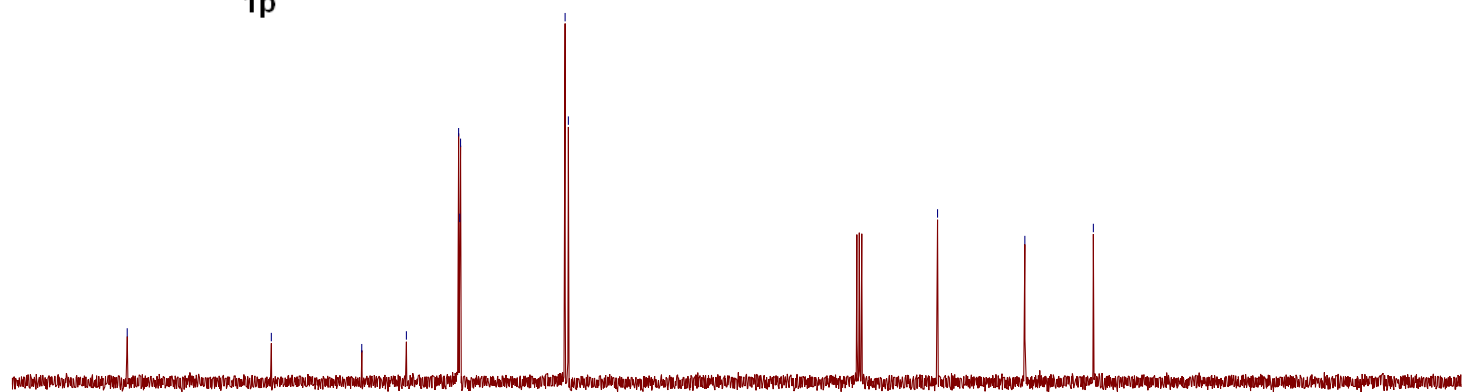

$\begin{array}{llllllllllllllllll}180 & 170 & 160 & 150 & 140 & 130 & 120 & 110 & \begin{array}{c}100 \\ \mathrm{f} 1\end{array}(\mathrm{ppm}) & 90 & 80 & 70 & 60 & 50 & 40 & 30 & 20 & 10\end{array}$

${ }^{1} \mathrm{H}$ NMR $(400 \mathrm{MHz})$ and ${ }^{13} \mathrm{C}\left\{{ }^{1} \mathrm{H}\right\}$ NMR $(101 \mathrm{MHz})$ spectra of $\mathbf{1 p}\left(\mathrm{CDCl}_{3}, \mathrm{rt}\right)$. 

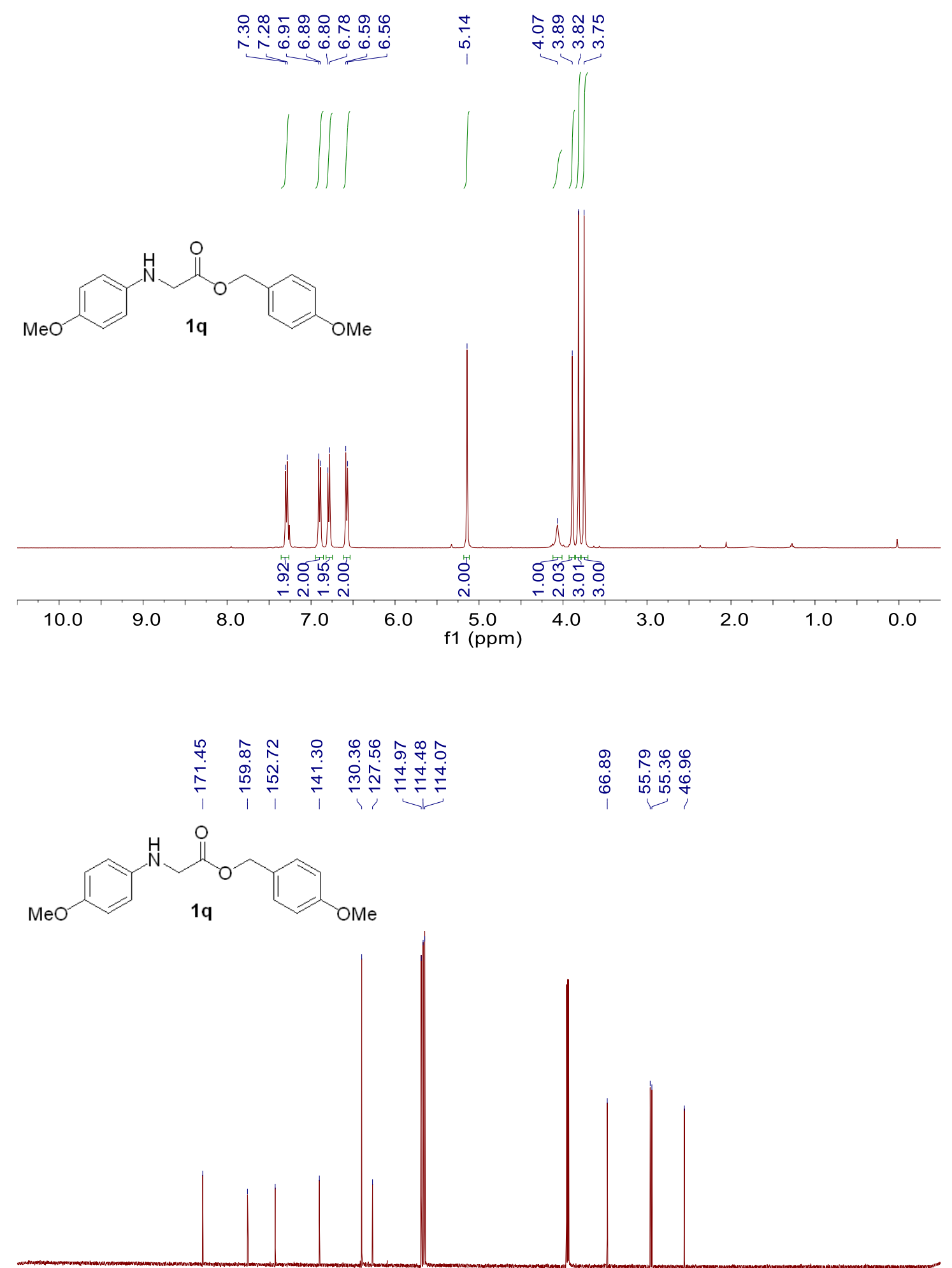

$21020019018017016015014013012011010090 \quad 80 \quad 70 \quad 60 \quad 50 \quad 40 \quad 30 \quad 20 \quad 10 \quad 0 \quad-10$ f1 (ppm)

${ }^{1} \mathrm{H}$ NMR $(400 \mathrm{MHz})$ and ${ }^{13} \mathrm{C}\left\{{ }^{1} \mathrm{H}\right\} \mathrm{NMR}(126 \mathrm{MHz})$ spectra of $\mathbf{1 q}\left(\mathrm{CDCl}_{3}, \mathrm{rt}\right)$. 


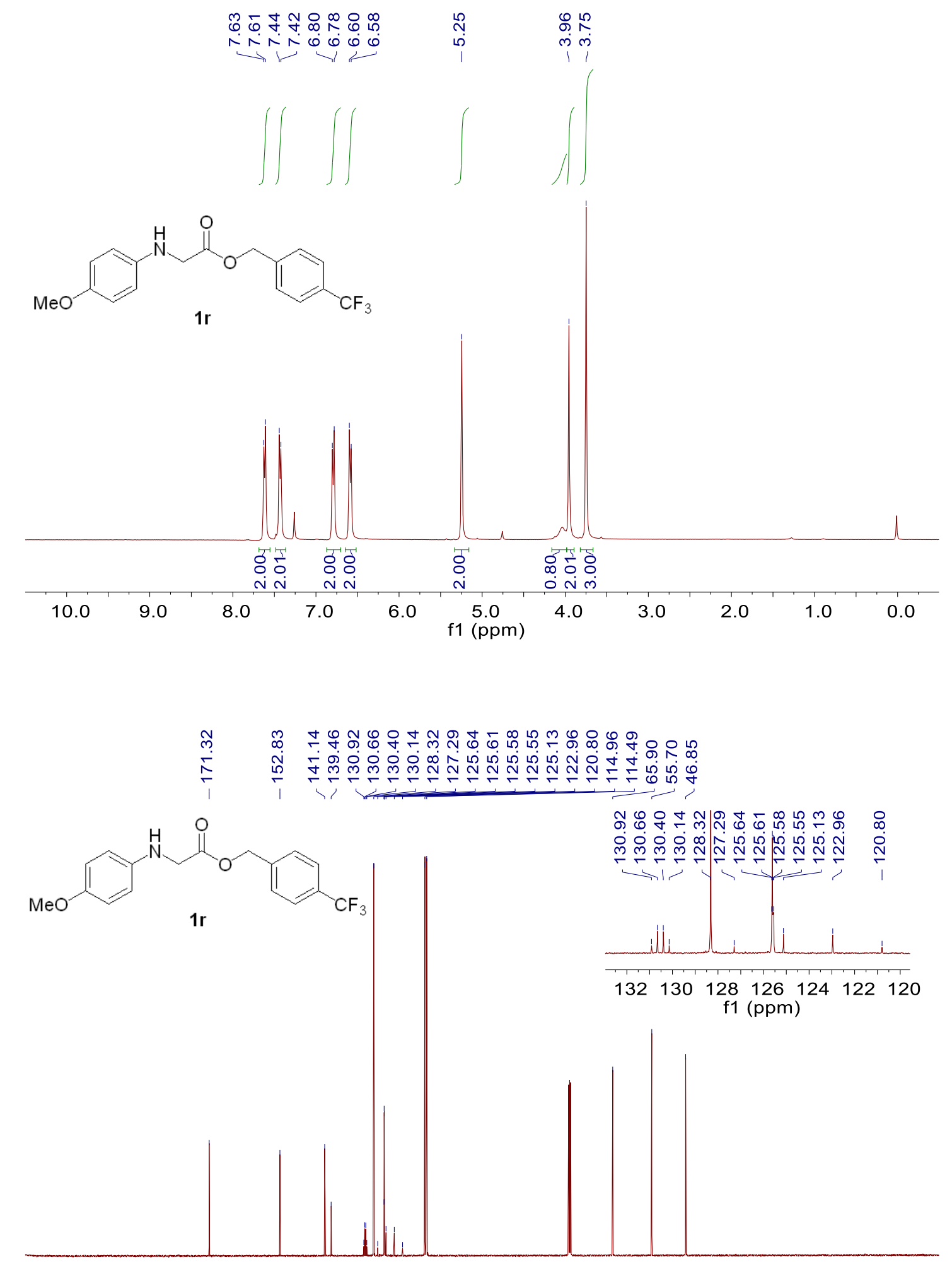

$21020019018017016015014013012011010090 \quad 80 \quad 70 \quad 60 \quad 50 \quad 40 \quad 30 \quad 20 \quad 10 \quad 0 \quad-10$ f1 (ppm) 
<smiles>COc1ccc(NCC(=O)OCc2ccc(C(F)(F)F)cc2)cc1</smiles>

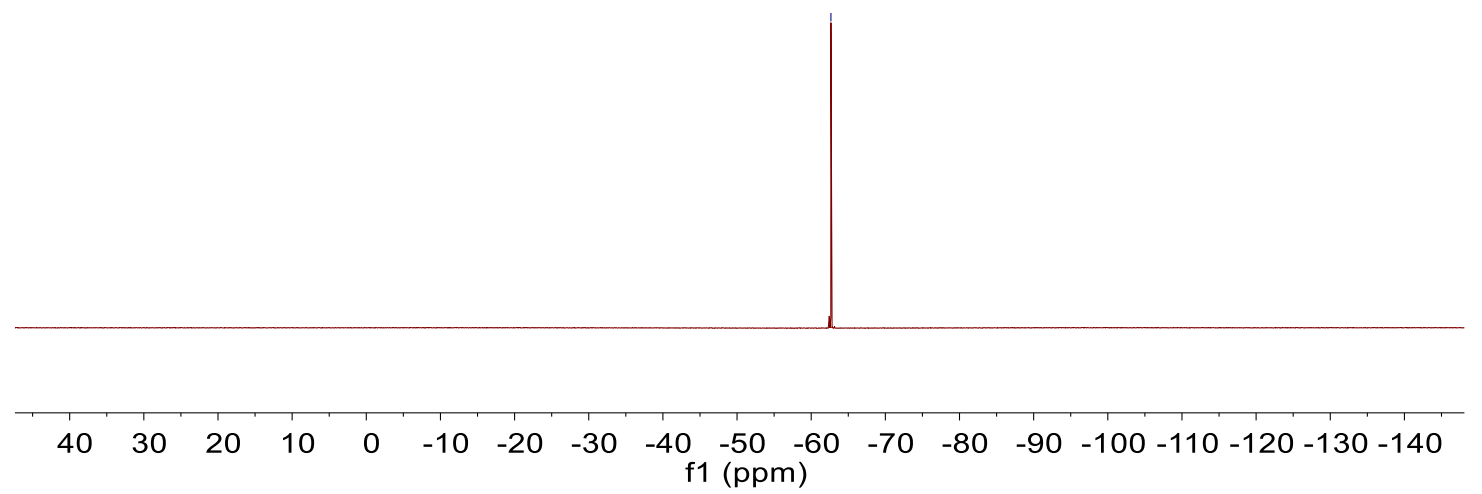

${ }^{1} \mathrm{H}$ NMR (400 MHz), ${ }^{13} \mathrm{C}\left\{{ }^{1} \mathrm{H}\right\}$ NMR(126 MHz), and ${ }^{19} \mathrm{~F}$ NMR (376 MHz)spectra of $\mathbf{1 r}\left(\mathrm{CDCl}_{3}, \mathrm{rt}\right)$. 


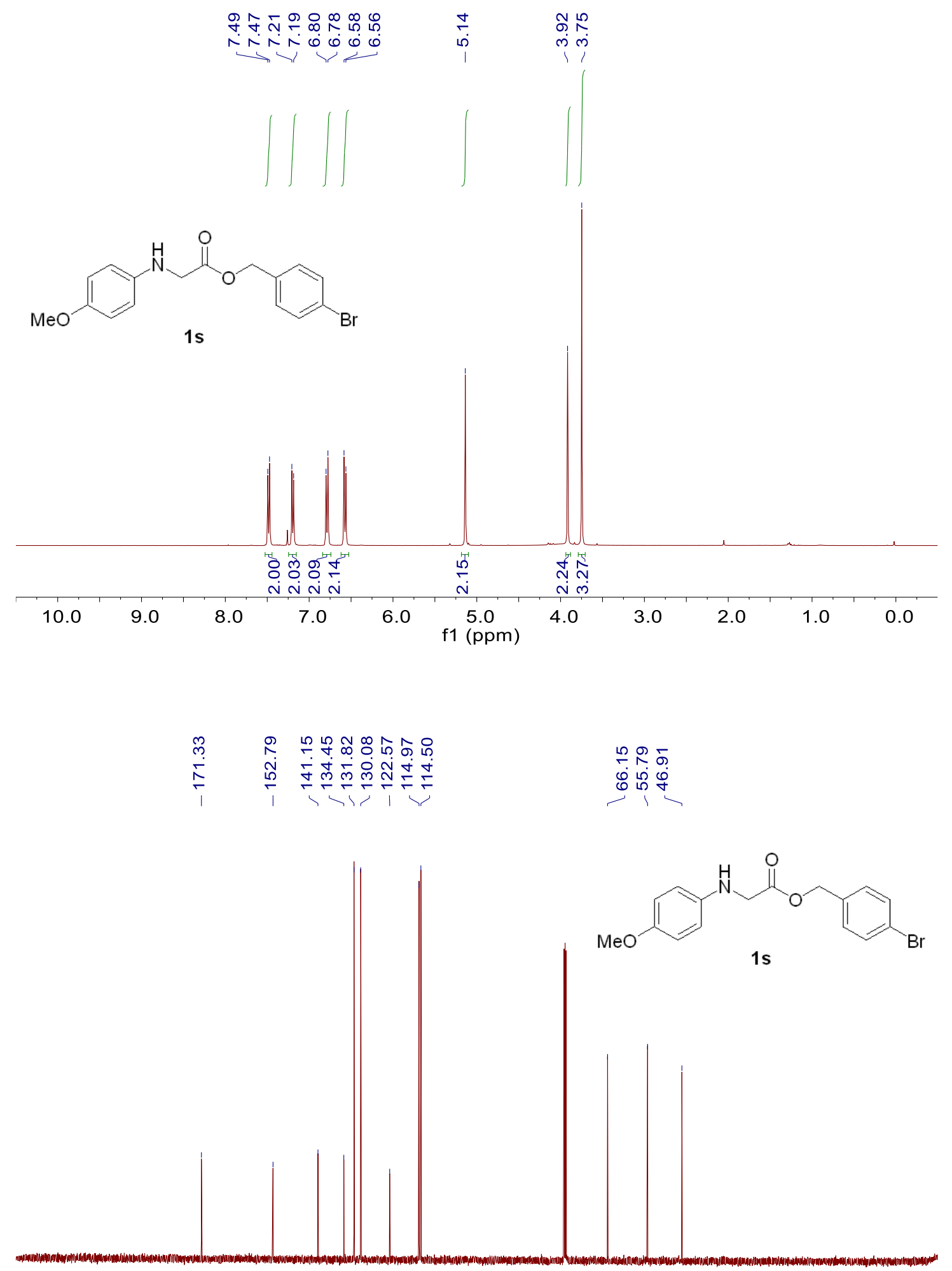

$21020019018017016015014013012011010090 \quad 80 \quad 70 \quad 60 \quad 50 \quad 40 \quad 30 \quad 20 \quad 10 \quad 0 \quad-10$ f1 (ppm)

${ }^{1} \mathrm{H}$ NMR (400 MHz) and ${ }^{13} \mathrm{C}\left\{{ }^{1} \mathrm{H}\right\}$ NMR $(126 \mathrm{MHz})$ spectra of $\mathbf{1 s}\left(\mathrm{CDCl}_{3}, \mathrm{rt}\right)$. 


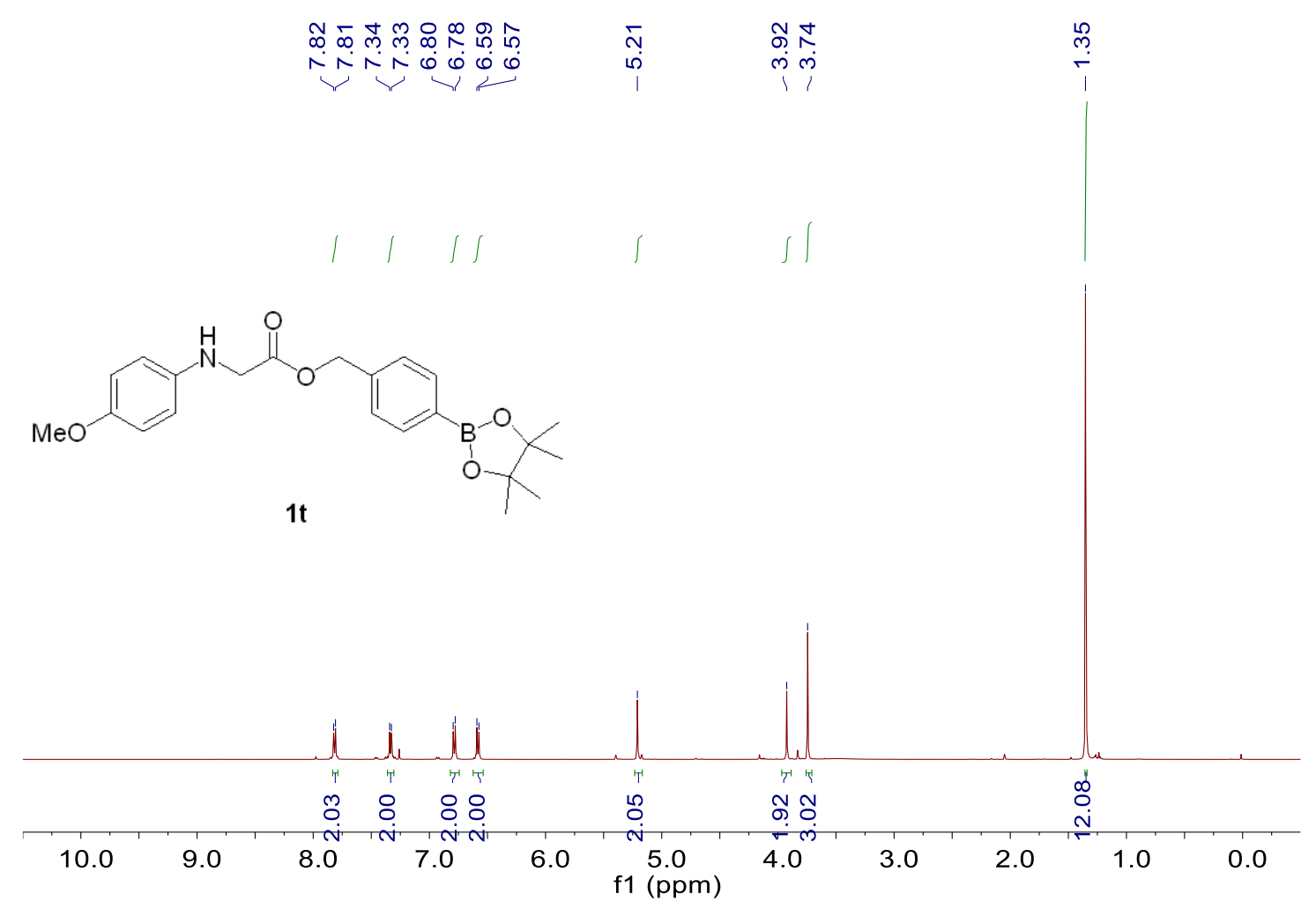

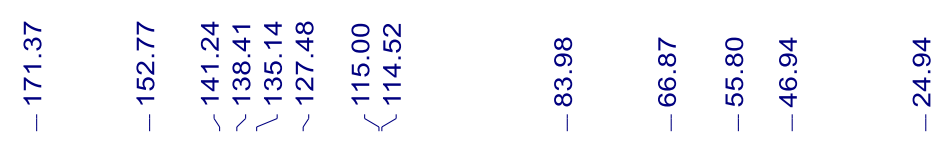<smiles>COc1ccc(NCC(=O)OCc2ccc(B3OC(C)(C)C(C)(C)O3)cc2)cc1</smiles>

1t f1 (ppm)

${ }^{1} \mathrm{H}$ NMR $(500 \mathrm{MHz})$ and ${ }^{13} \mathrm{C}\left\{{ }^{1} \mathrm{H}\right\}$ NMR $(126 \mathrm{MHz})$ spectra of $\mathbf{1 t}\left(\mathrm{CDCl}_{3}, \mathrm{rt}\right)$. 


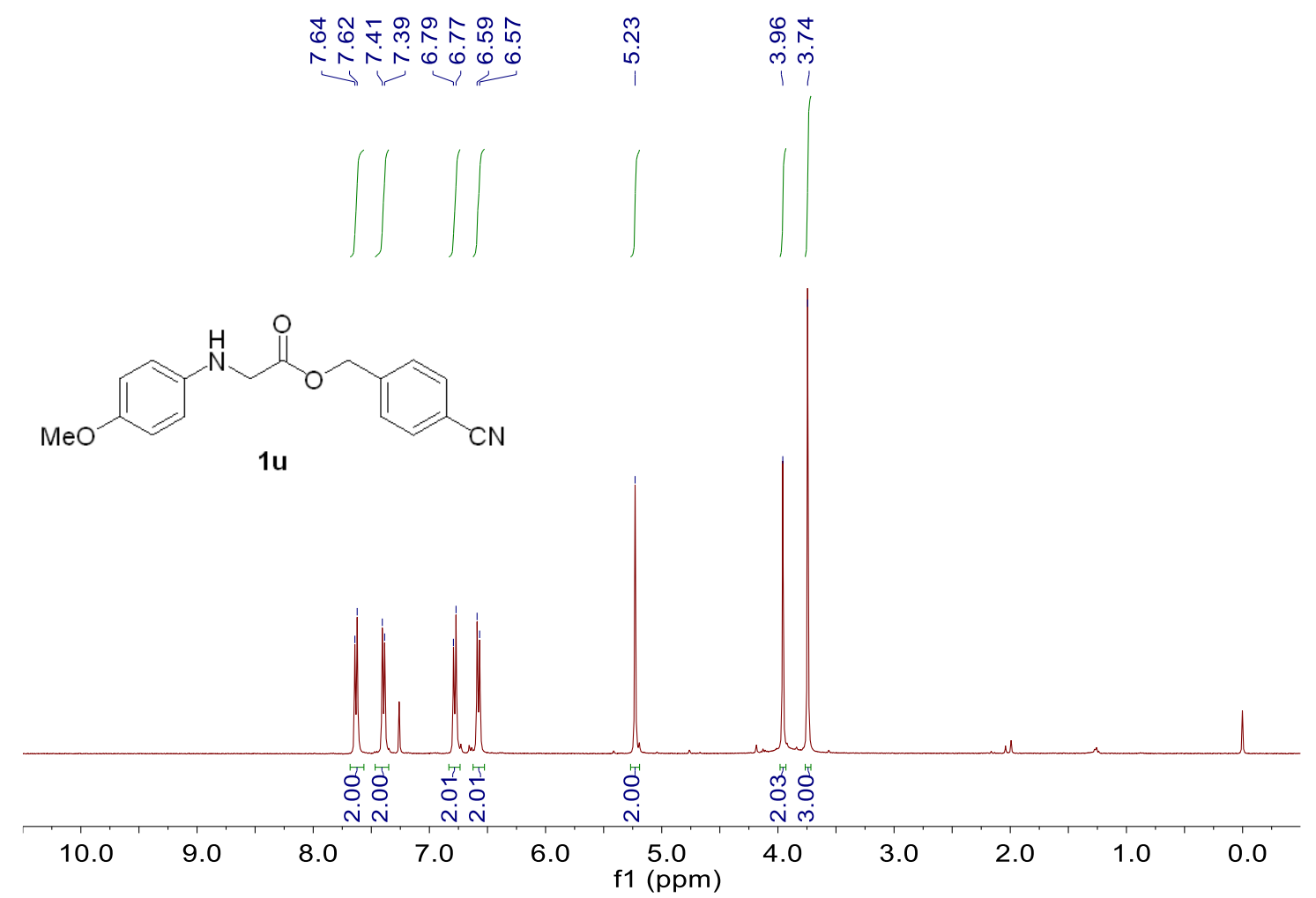

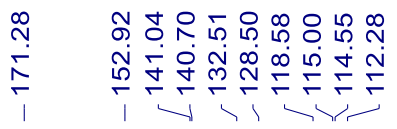

둥

它绾守<smiles>COc1ccc(NCC(=O)OCc2ccc(C#N)cc2)cc1</smiles>

1u

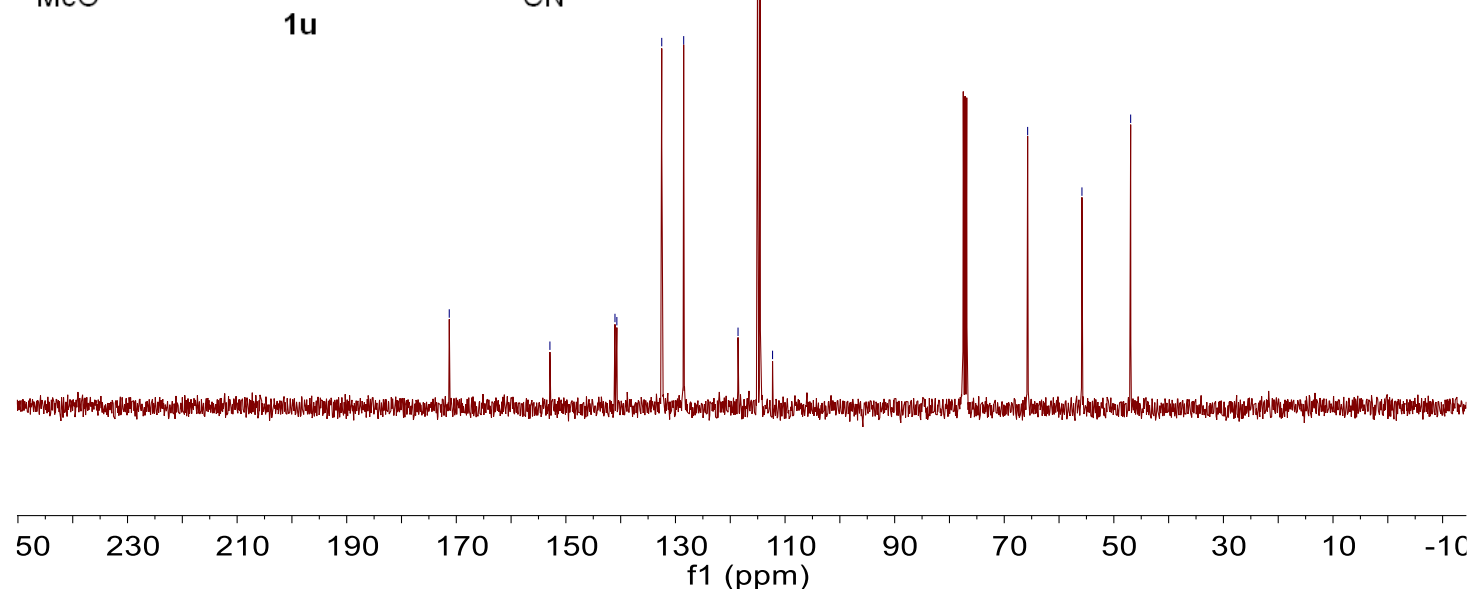

${ }^{1} \mathrm{H}$ NMR $(400 \mathrm{MHz})$ and ${ }^{13} \mathrm{C}\left\{{ }^{1} \mathrm{H}\right\}$ NMR $(101 \mathrm{MHz})$ spectra of $\mathbf{1 u}\left(\mathrm{CDCl}_{3}, \mathrm{rt}\right)$. 


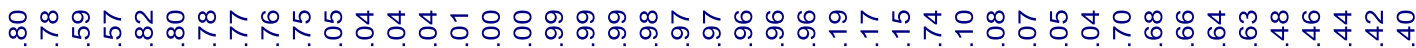
0 0
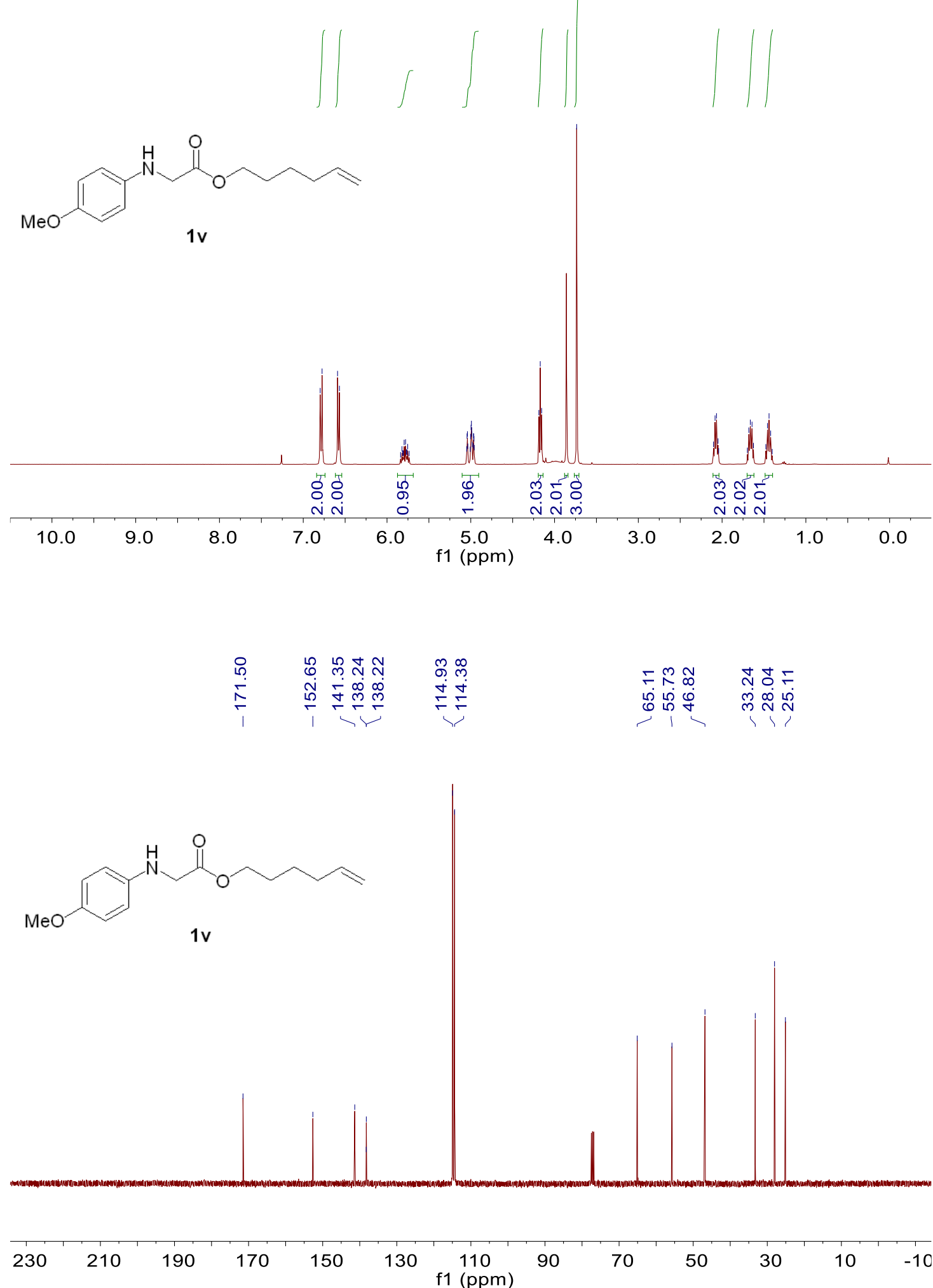

${ }^{1} \mathrm{H}$ NMR (400 MHz) and ${ }^{13} \mathrm{C}\left\{{ }^{1} \mathrm{H}\right\}$ NMR (101 MHz) spectra of $\mathbf{1 v}\left(\mathrm{CDCl}_{3}, \mathrm{rt}\right)$. 


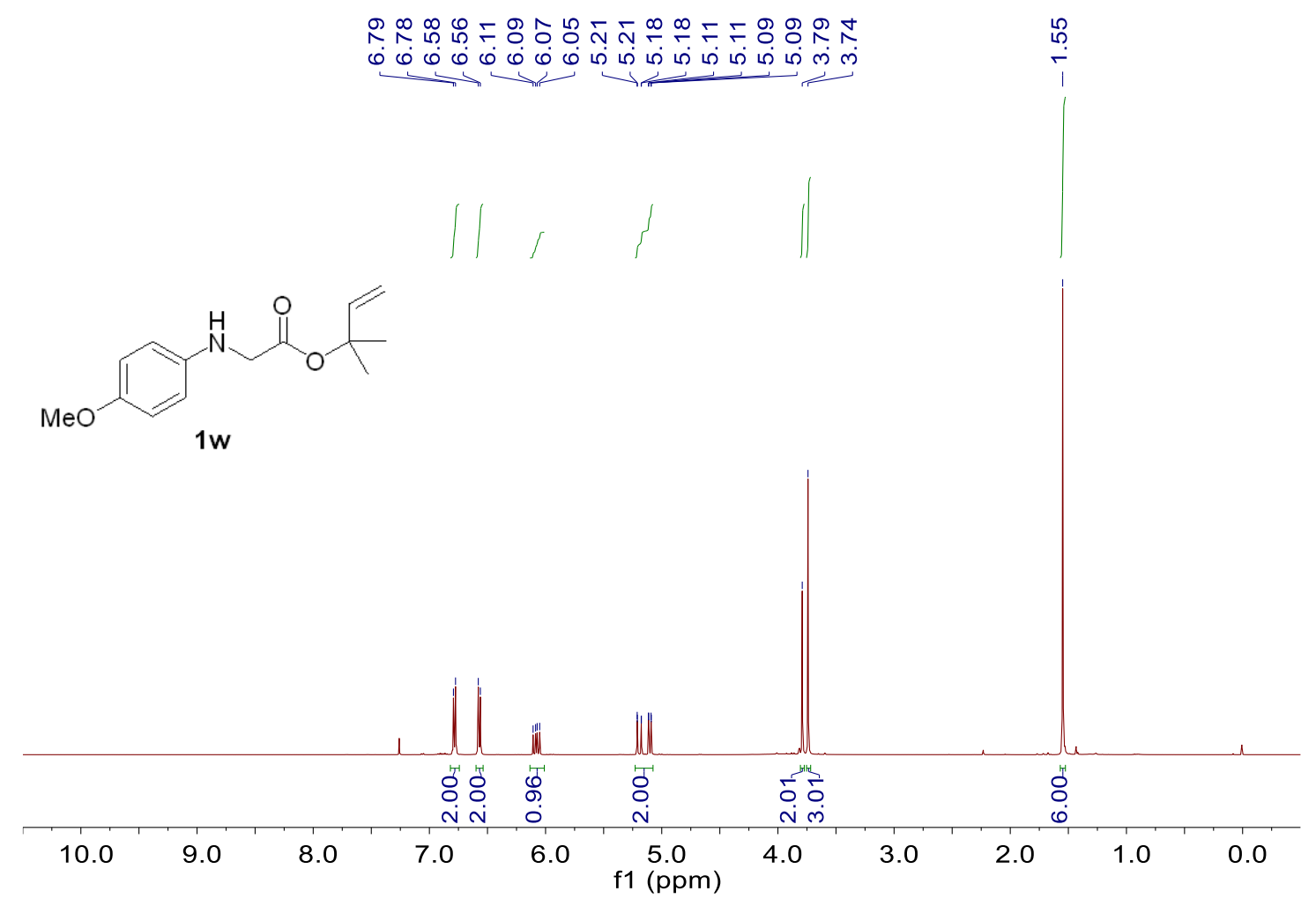

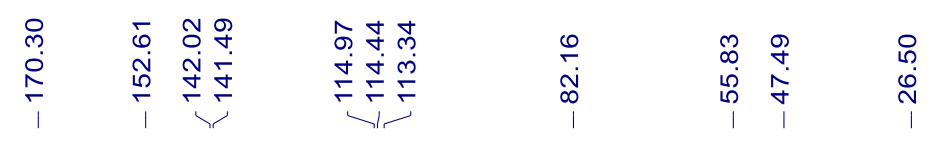

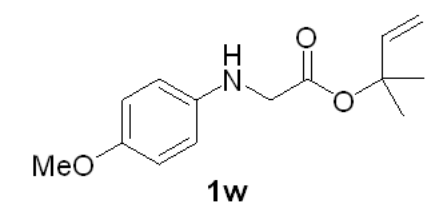

$21020019018017016015014013012011010090 \quad 80 \quad 70 \quad 60 \quad 50 \quad 40 \quad 30 \quad 20 \quad 10 \quad 0 \quad-10$ f1 (ppm)

${ }^{1} \mathrm{H}$ NMR $(500 \mathrm{MHz})$ and ${ }^{13} \mathrm{C}\left\{{ }^{1} \mathrm{H}\right\} \mathrm{NMR}(126 \mathrm{MHz})$ spectra of $\mathbf{1 w}\left(\mathrm{CDCl}_{3}, \mathrm{rt}\right)$. 


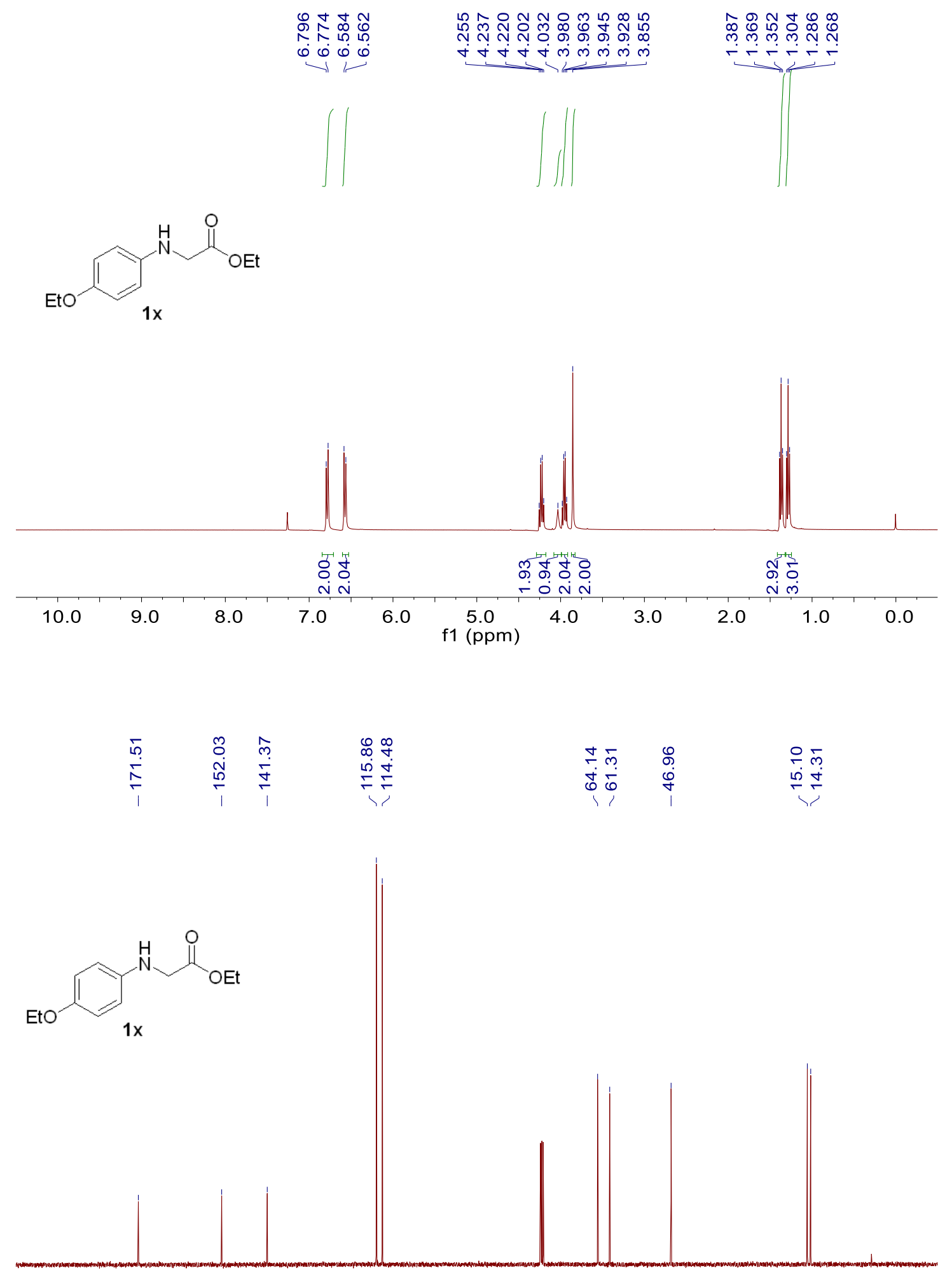
$00190180170160150140130120110 \begin{gathered}100 \quad \begin{array}{l}90 \\ \mathrm{f} 1(\mathrm{ppm})\end{array} \\ 00\end{gathered}$

${ }^{1} \mathrm{H}$ NMR $(400 \mathrm{MHz})$ and ${ }^{13} \mathrm{C}\left\{{ }^{1} \mathrm{H}\right\}$ NMR $(101 \mathrm{MHz})$ spectra of $\mathbf{1 x}\left(\mathrm{CDCl}_{3}, \mathrm{rt}\right)$. 


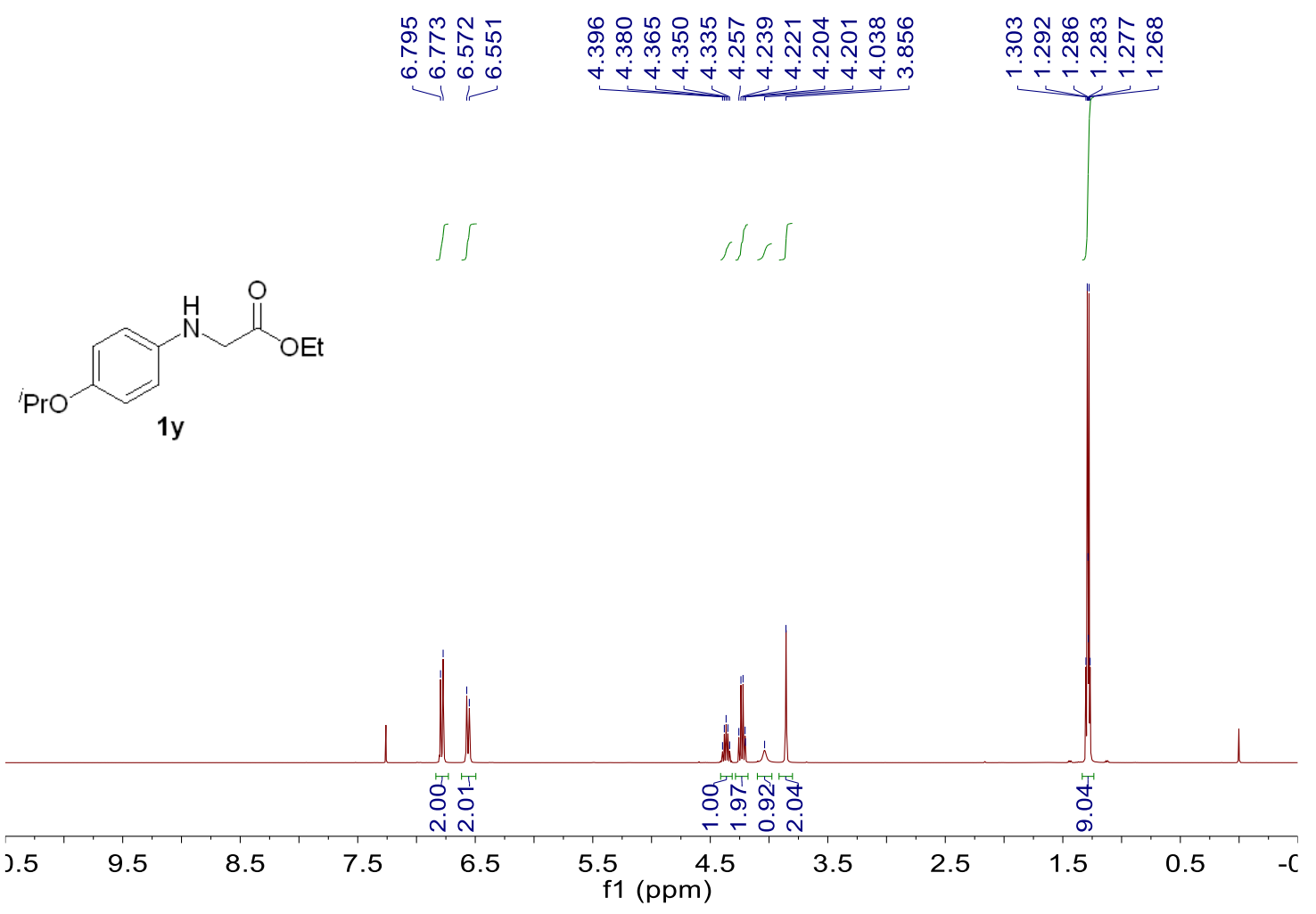

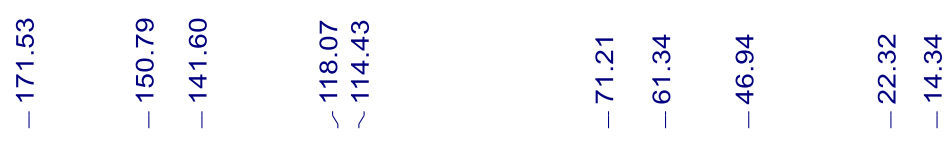<smiles>CCCOc1ccc(NCC(=O)OCC)cc1</smiles>

230

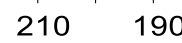

$170 \quad 150$

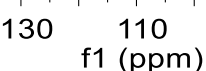

90

$70 \quad 50$

$30 \quad 10 \quad-10$

${ }^{1} \mathrm{H}$ NMR $(400 \mathrm{MHz})$ and ${ }^{13} \mathrm{C}\left\{{ }^{1} \mathrm{H}\right\}$ NMR $(101 \mathrm{MHz})$ spectra of $\mathbf{1 y}\left(\mathrm{CDCl}_{3}, \mathrm{rt}\right)$. 


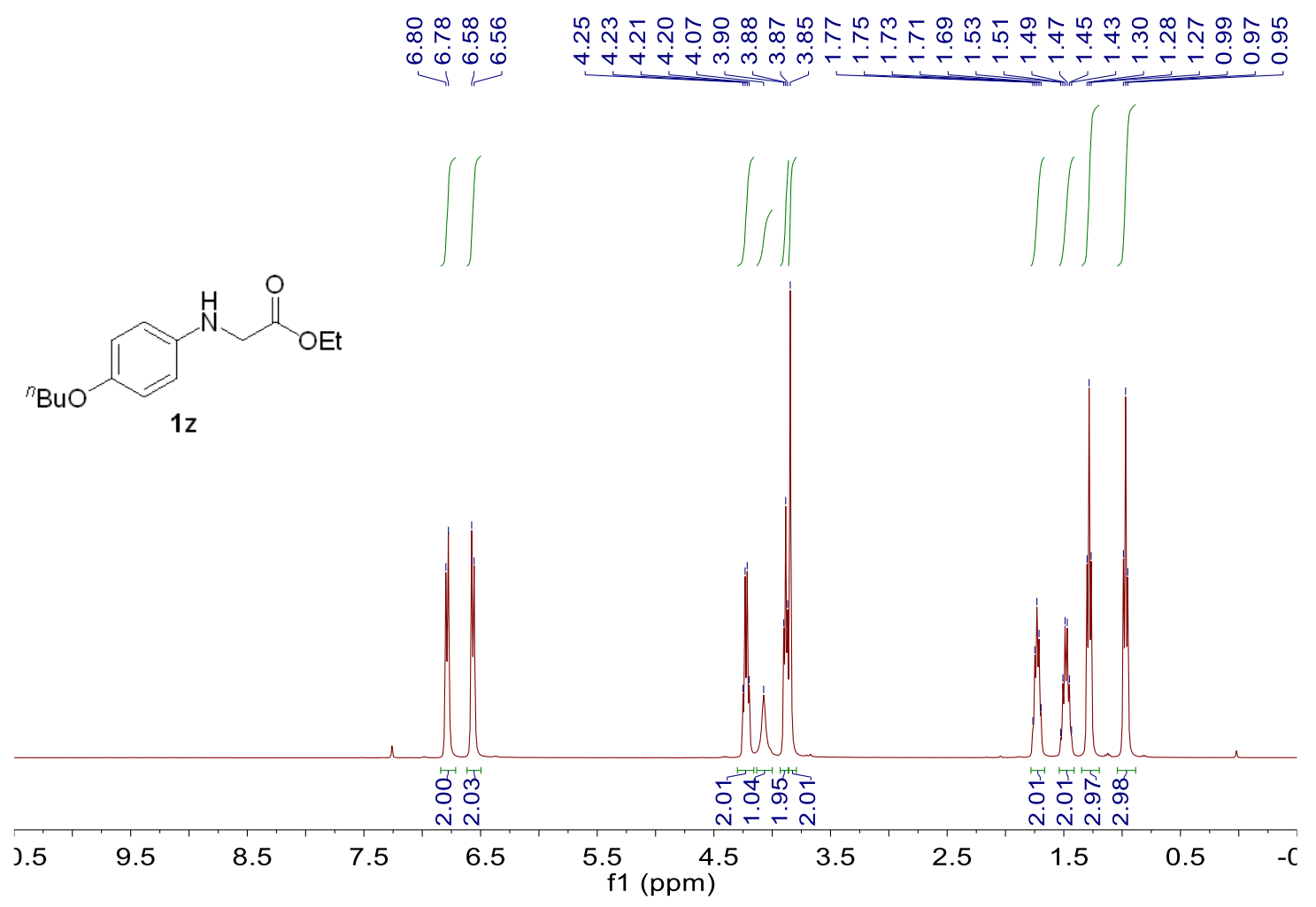

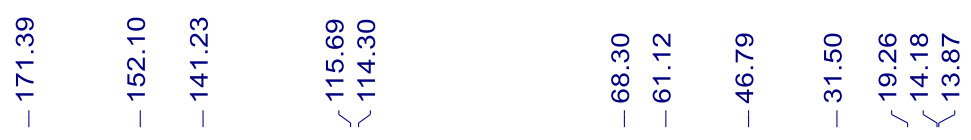<smiles>CCOC(=O)CNc1ccc(OC(C)(C)C)cc1</smiles>

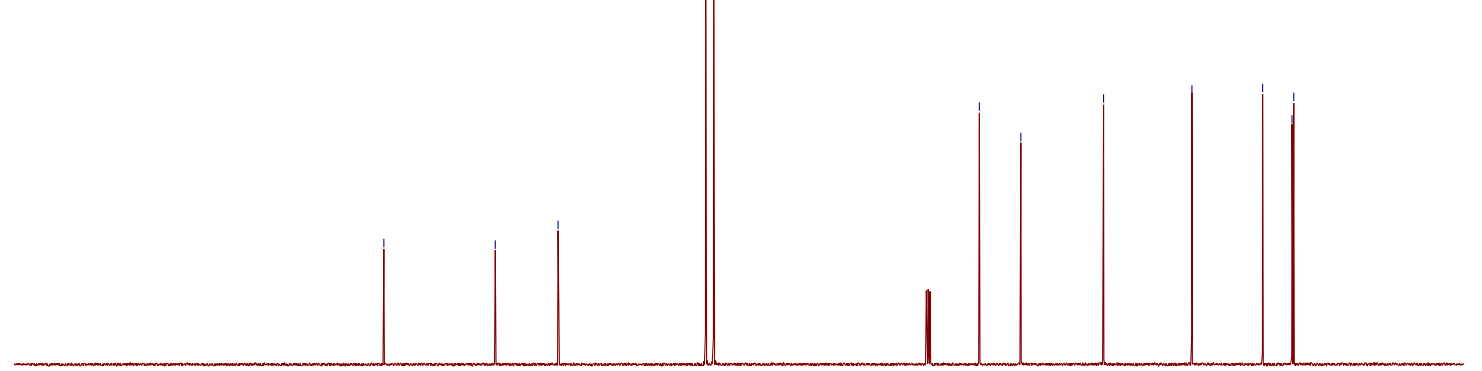

$230 \quad 210 \quad 190 \quad 170 \quad 150 \quad 130 \begin{gathered}110 \\ \mathrm{f} 1(\mathrm{ppm})\end{gathered}$

${ }^{1} \mathrm{H}$ NMR (400 MHz) and ${ }^{13} \mathrm{C}\left\{{ }^{1} \mathrm{H}\right\}$ NMR (101 MHz) spectra of $\mathbf{1 z}\left(\mathrm{CDCl}_{3}, \mathrm{rt}\right)$. 

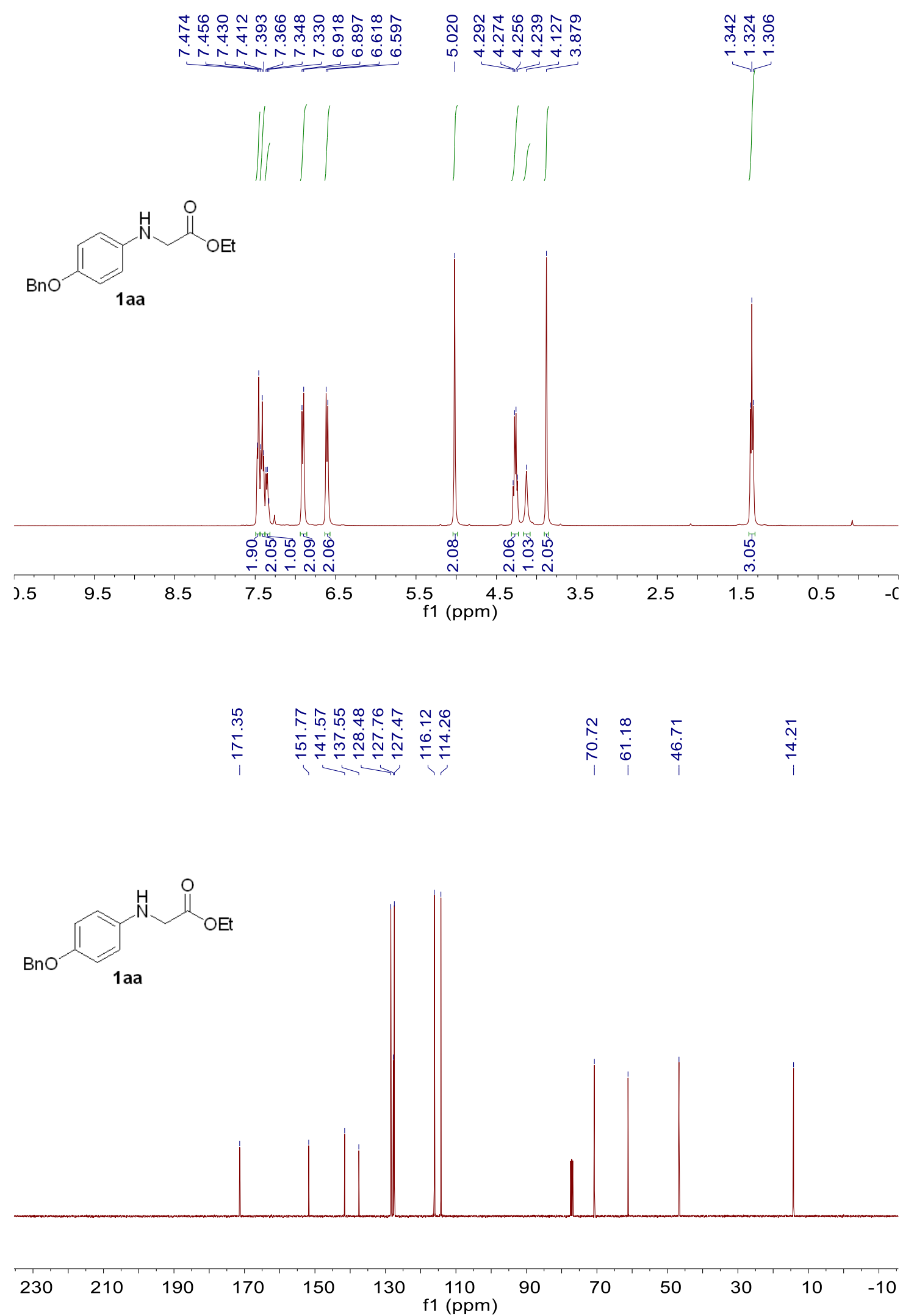

${ }^{1} \mathrm{H}$ NMR (400 MHz) and ${ }^{13} \mathrm{C}\left\{{ }^{1} \mathrm{H}\right\}$ NMR (101 MHz) spectra of $\mathbf{1 a a}\left(\mathrm{CDCl}_{3}, \mathrm{rt}\right)$. 


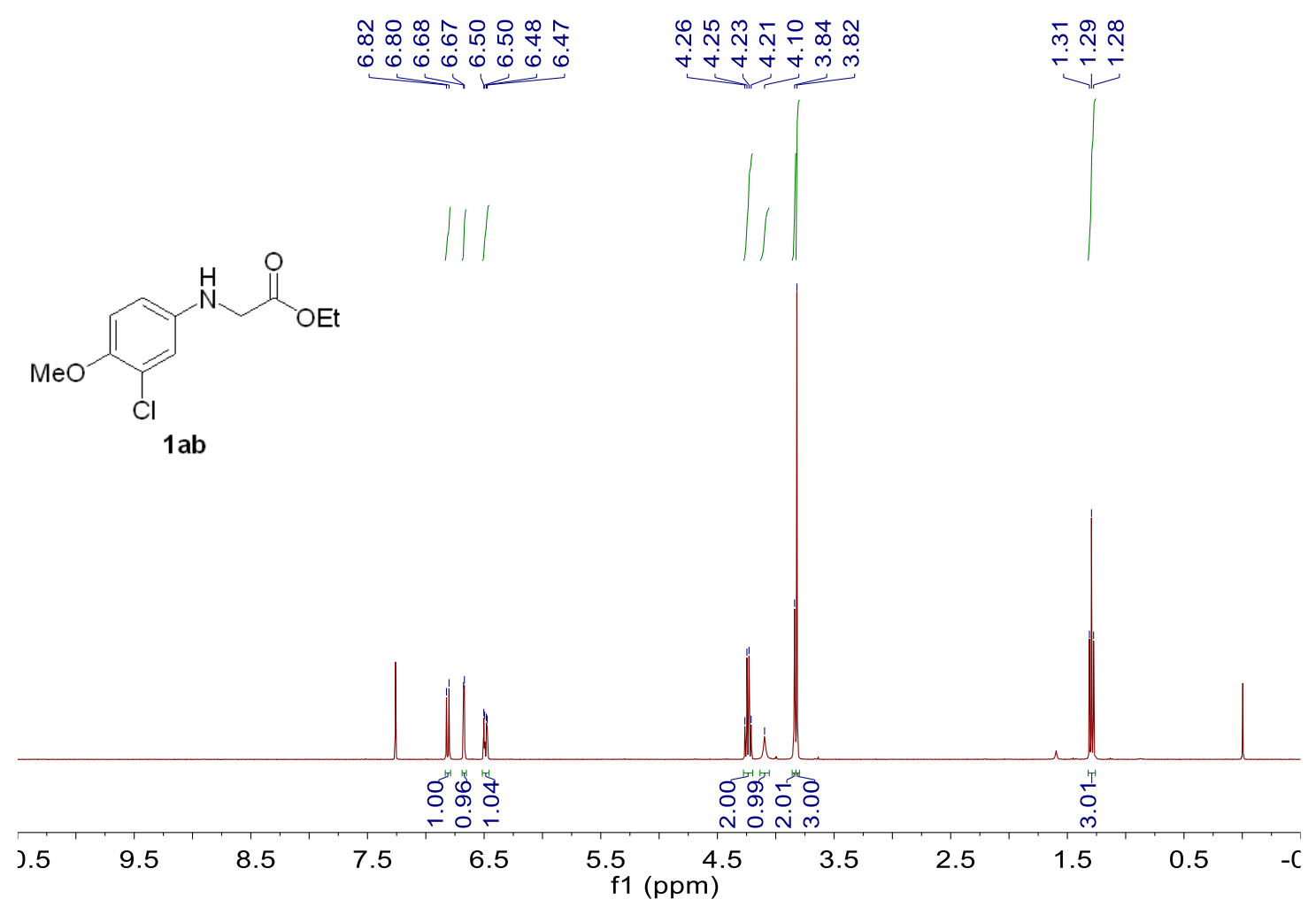

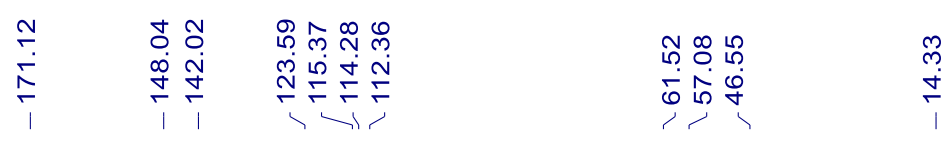<smiles>CCOC(=O)CNc1ccc(OC)c(Cl)c1</smiles>

1 ab

${ }^{1} \mathrm{H}$ NMR (400 MHz) and ${ }^{13} \mathrm{C}\left\{{ }^{1} \mathrm{H}\right\}$ NMR (101 MHz) spectra of $\mathbf{1 a b}\left(\mathrm{CDCl}_{3}, \mathrm{rt}\right)$. 

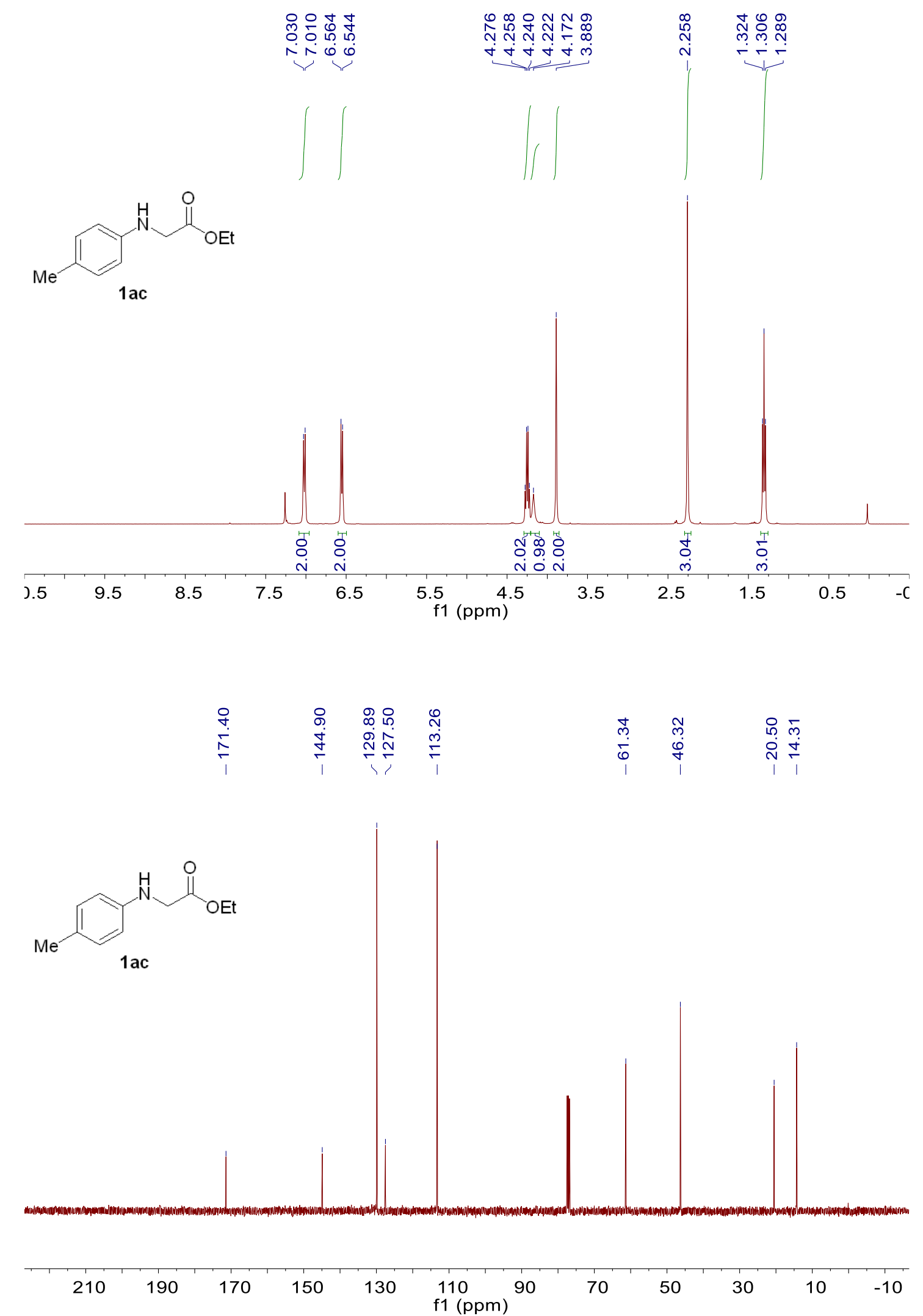

${ }^{1} \mathrm{H}$ NMR (400 MHz) and ${ }^{13} \mathrm{C}\left\{{ }^{1} \mathrm{H}\right\}$ NMR $(101 \mathrm{MHz})$ spectra of $\mathbf{1 a c}\left(\mathrm{CDCl}_{3}, \mathrm{rt}\right)$. 


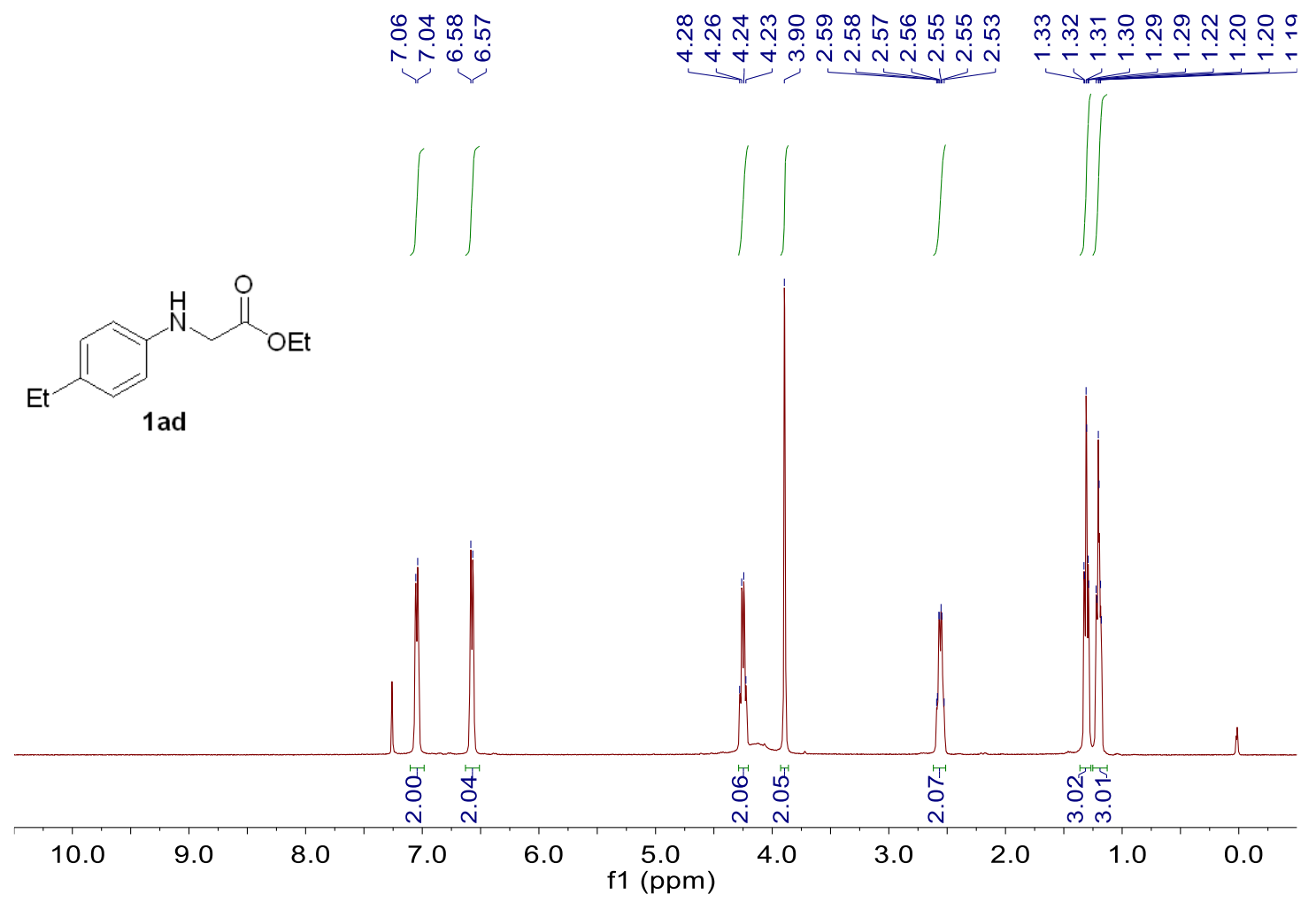

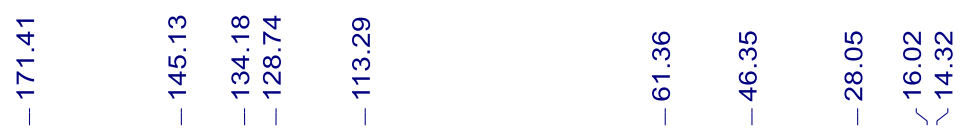<smiles>CCOC(=O)CNc1ccc(CC)cc1</smiles>

${ }^{1} \mathrm{H}$ NMR (400 MHz) and ${ }^{13} \mathrm{C}\left\{{ }^{1} \mathrm{H}\right\}$ NMR (101 MHz) spectra of $\mathbf{1 a d}\left(\mathrm{CDCl}_{3}, \mathrm{rt}\right)$. 


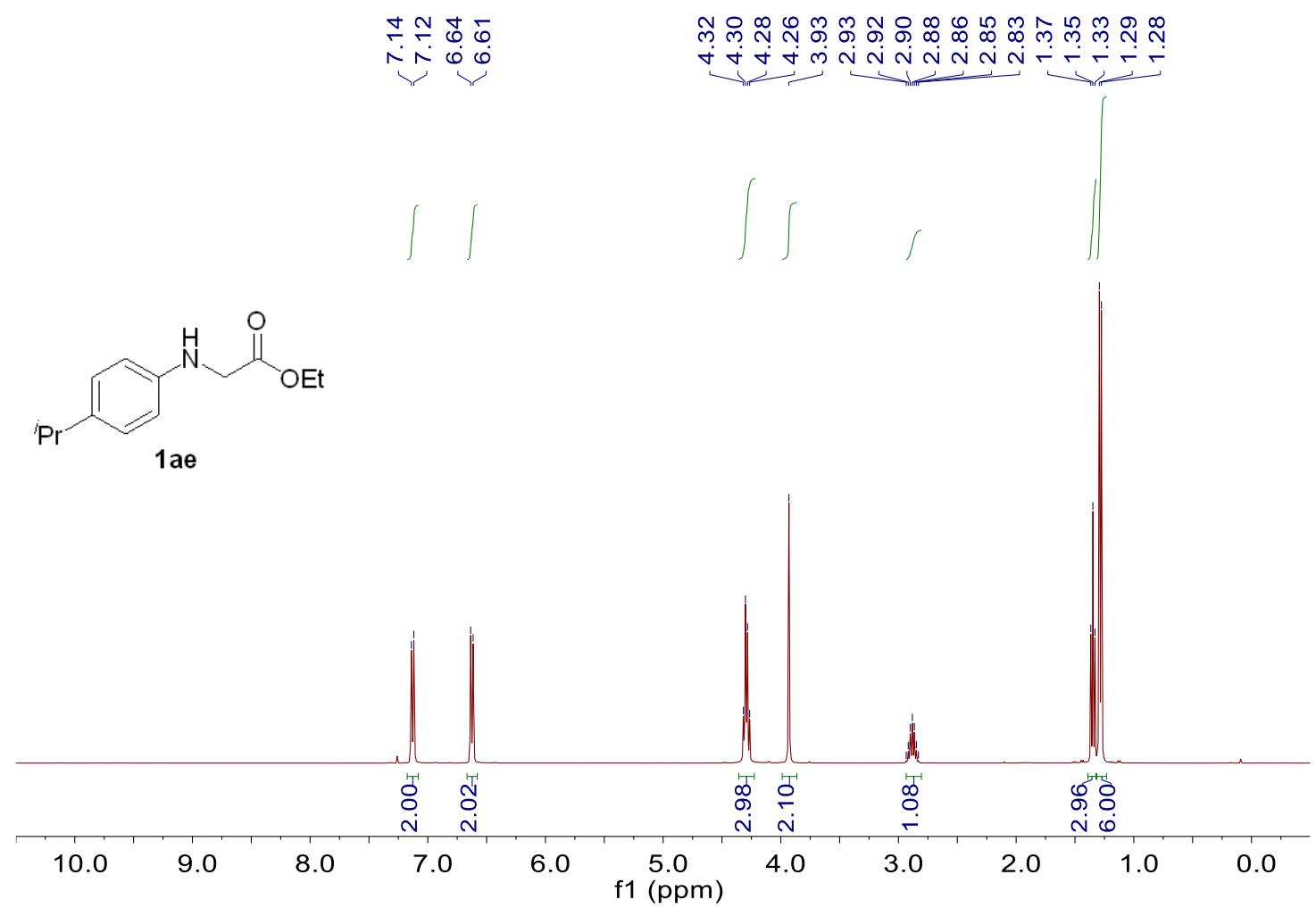

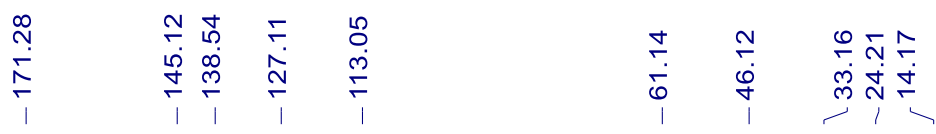

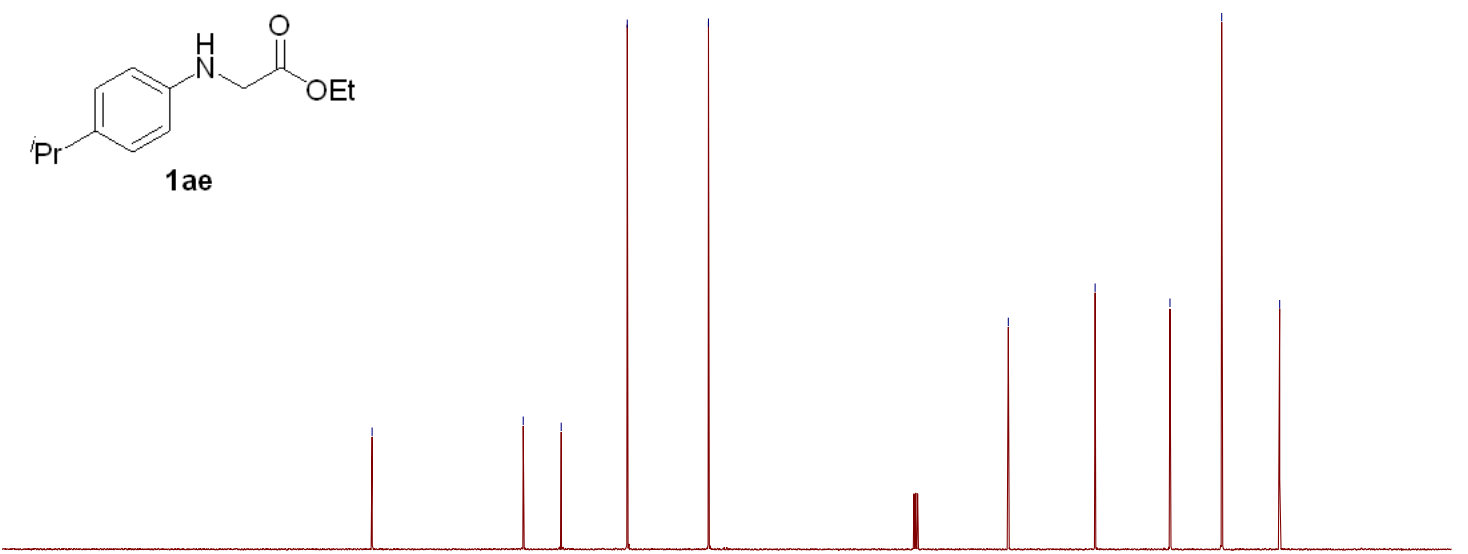

$230 \quad 210 \quad 190 \quad 170 \quad 150 \quad 130 \begin{gathered}110 \\ \mathrm{f} 1(\mathrm{ppm})\end{gathered}$

${ }^{1} \mathrm{H}$ NMR $(400 \mathrm{MHz})$ and ${ }^{13} \mathrm{C}\left\{{ }^{1} \mathrm{H}\right\}$ NMR $(101 \mathrm{MHz})$ spectra of $\mathbf{1 a e}\left(\mathrm{CDCl}_{3}, \mathrm{rt}\right)$. 


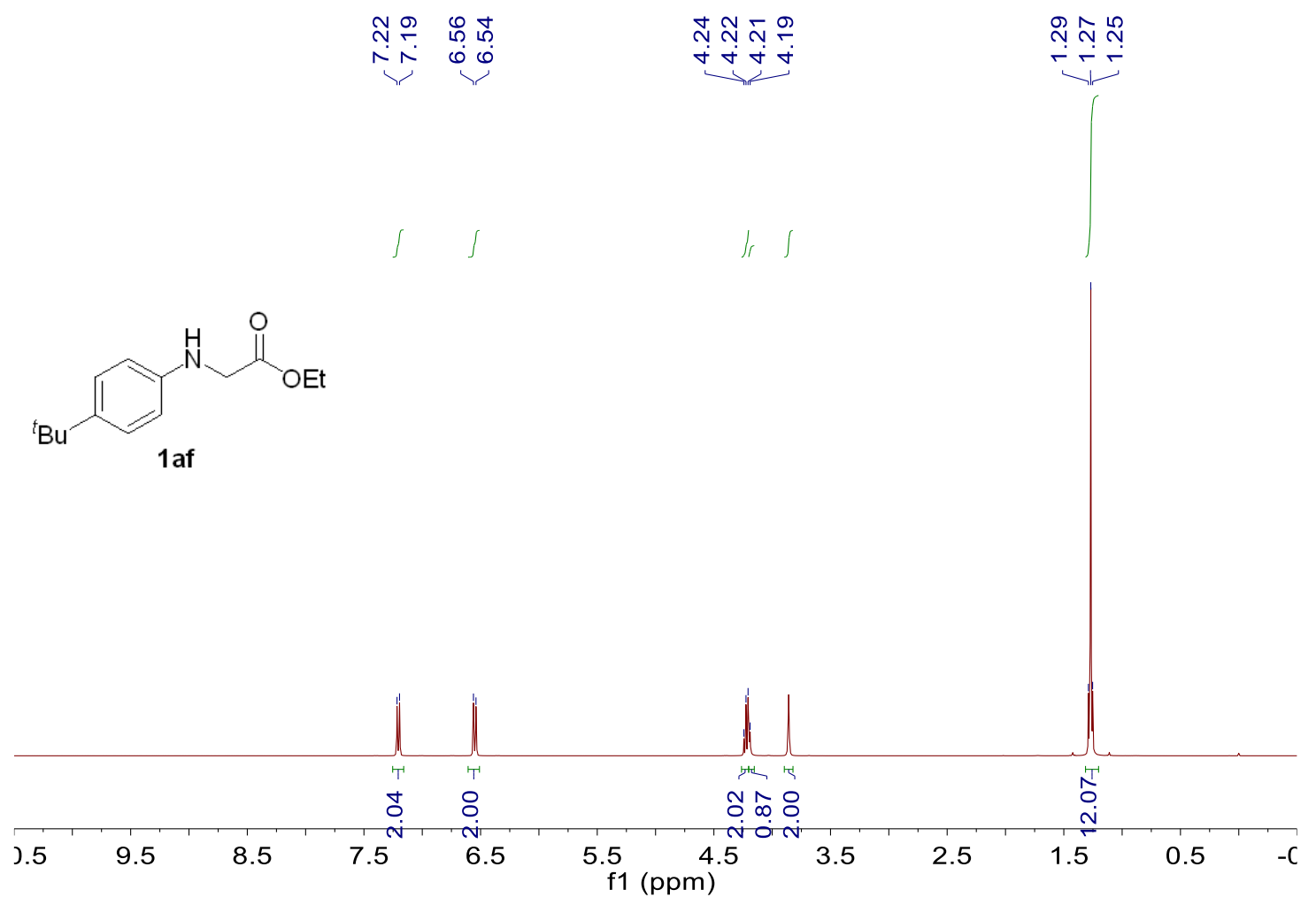

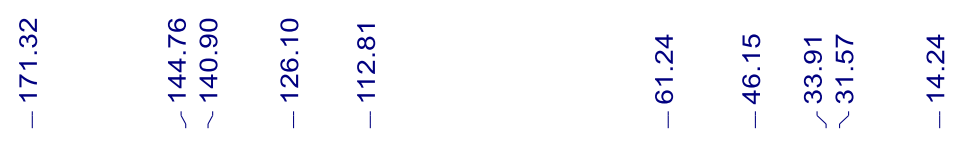
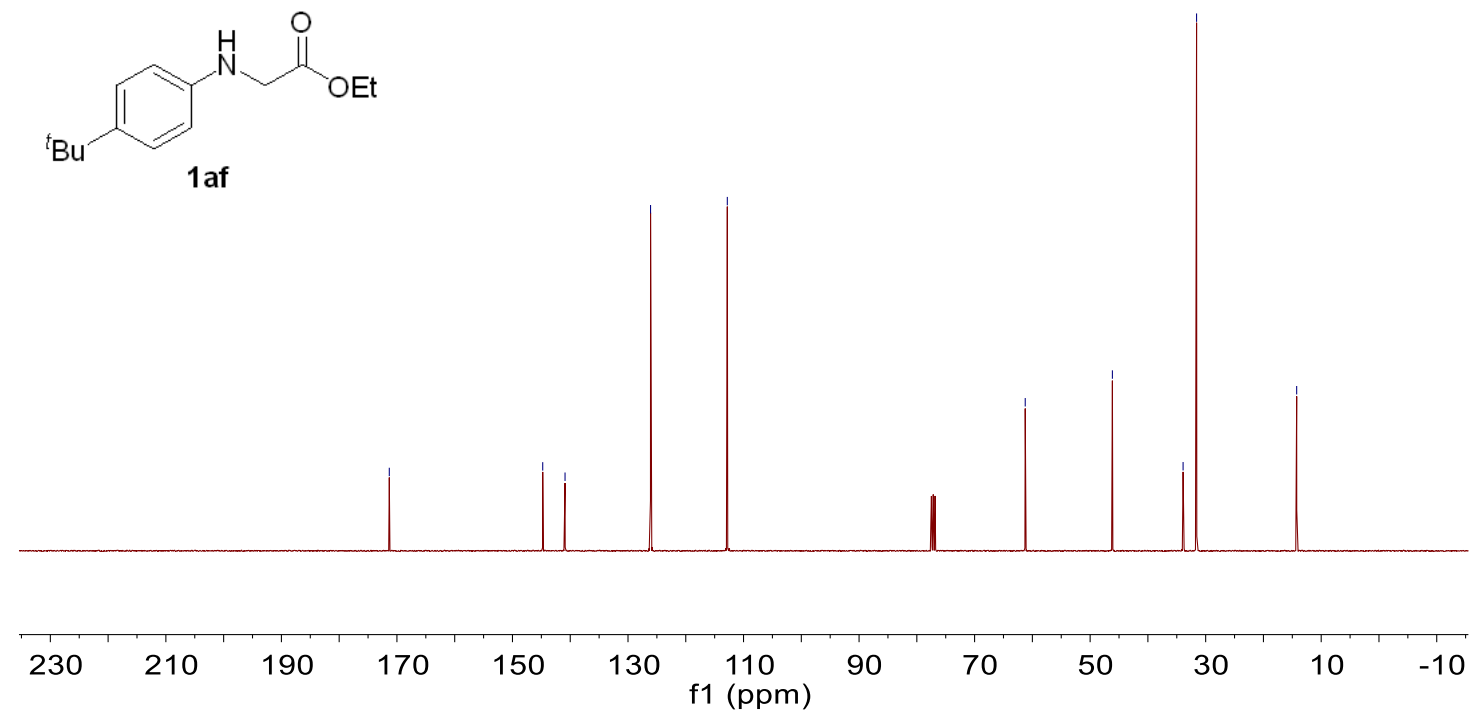

${ }^{1} \mathrm{H}$ NMR (400 MHz) and ${ }^{13} \mathrm{C}\left\{{ }^{1} \mathrm{H}\right\}$ NMR (101 MHz) spectra of $\mathbf{1 a f}\left(\mathrm{CDCl}_{3}, \mathrm{rt}\right)$. 

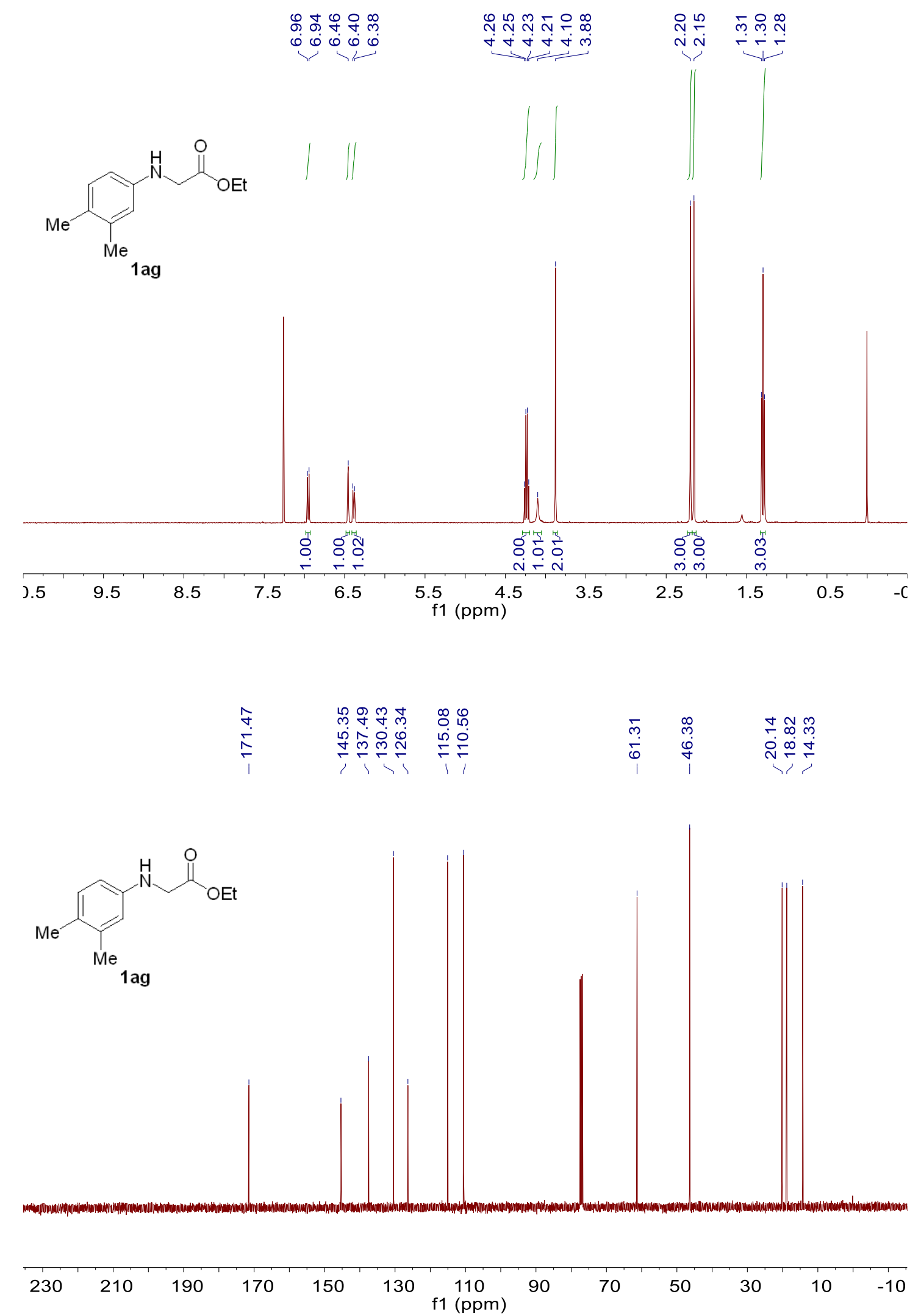

${ }^{1} \mathrm{H}$ NMR (400 MHz) and ${ }^{13} \mathrm{C}\left\{{ }^{1} \mathrm{H}\right\}$ NMR (101 MHz) spectra of $\mathbf{1 a g}\left(\mathrm{CDCl}_{3}, \mathrm{rt}\right)$. 


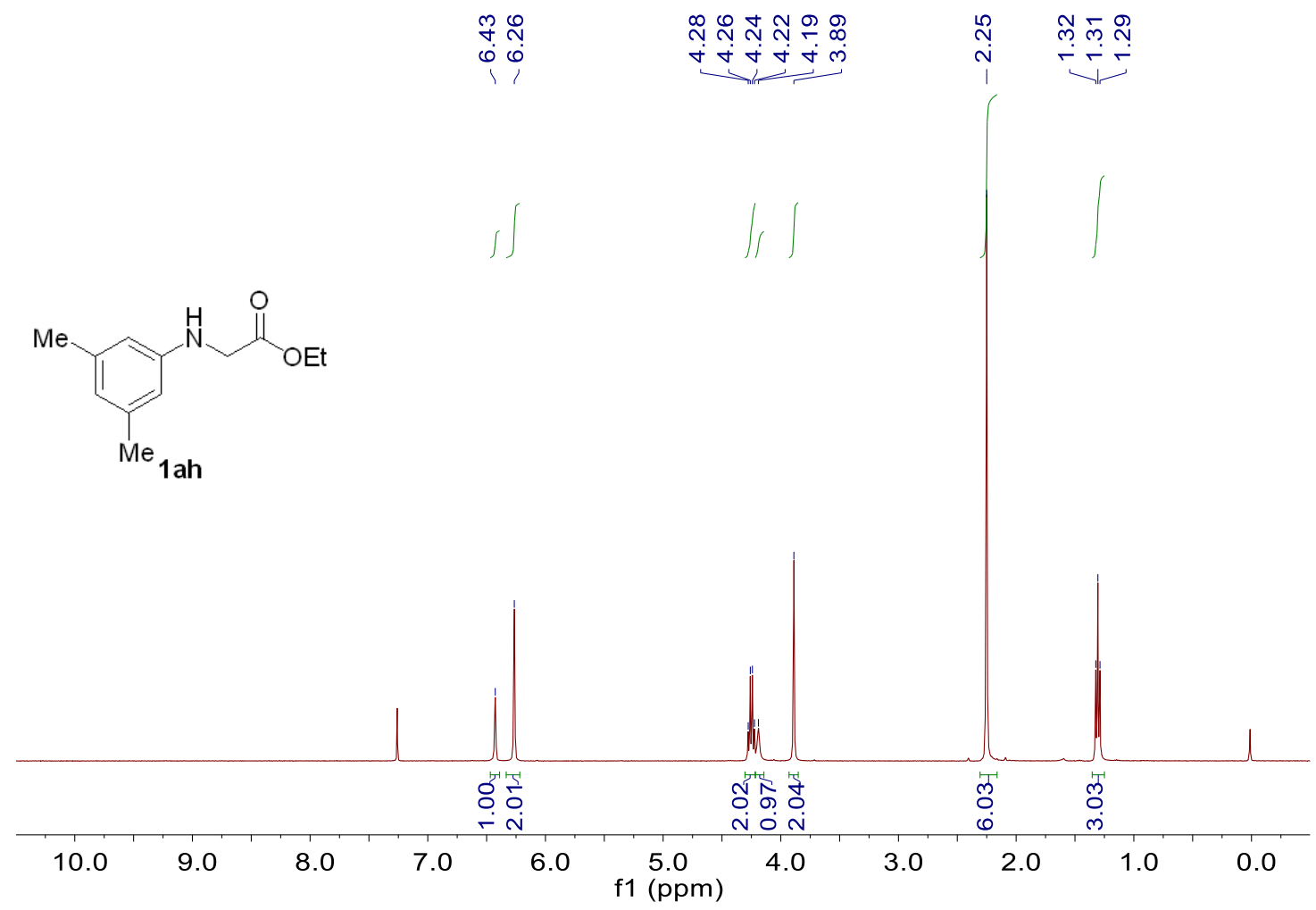

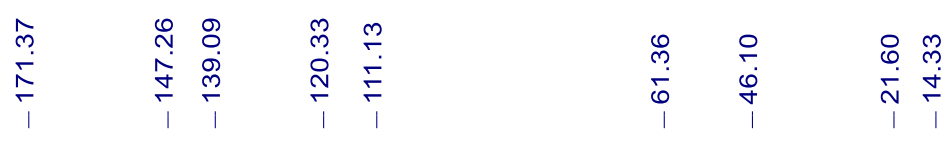

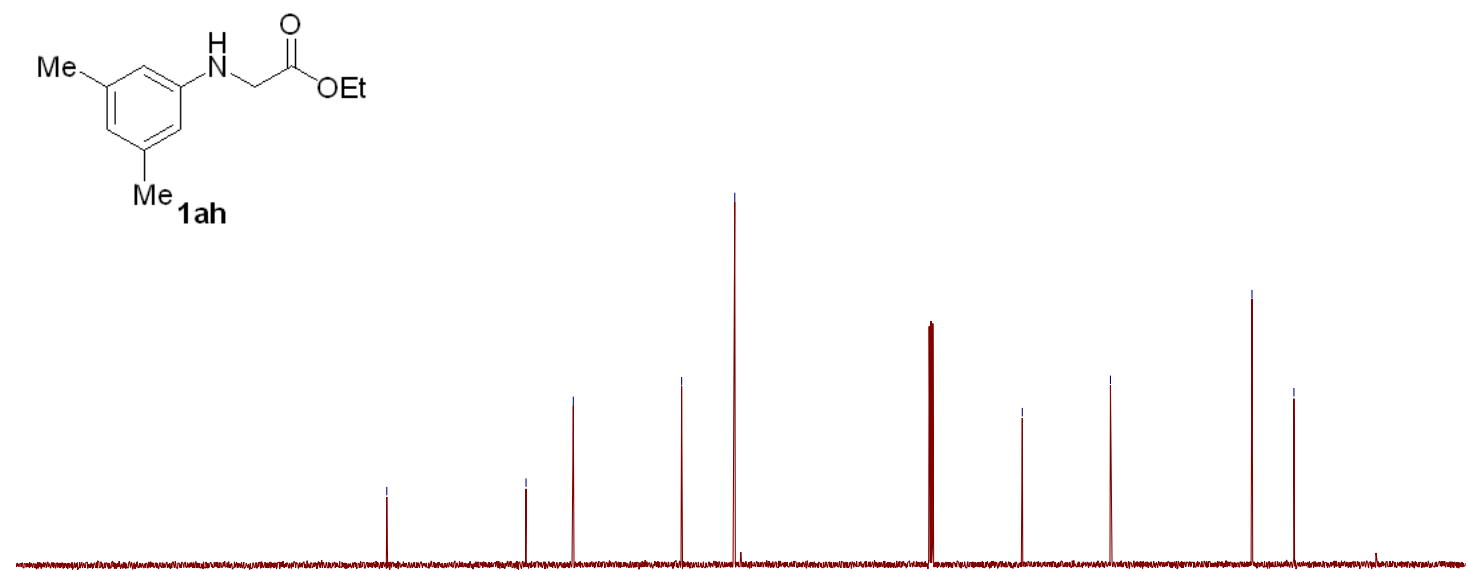

$230 \quad 210 \quad 190 \quad 170 \quad 150 \quad 130 \begin{gathered}110 \\ \mathrm{f} 1(\mathrm{ppm})\end{gathered}$

${ }^{1} \mathrm{H}$ NMR (400 MHz) and ${ }^{13} \mathrm{C}\left\{{ }^{1} \mathrm{H}\right\}$ NMR (101 MHz) spectra of $\mathbf{1 a h}\left(\mathrm{CDCl}_{3}, \mathrm{rt}\right)$. 


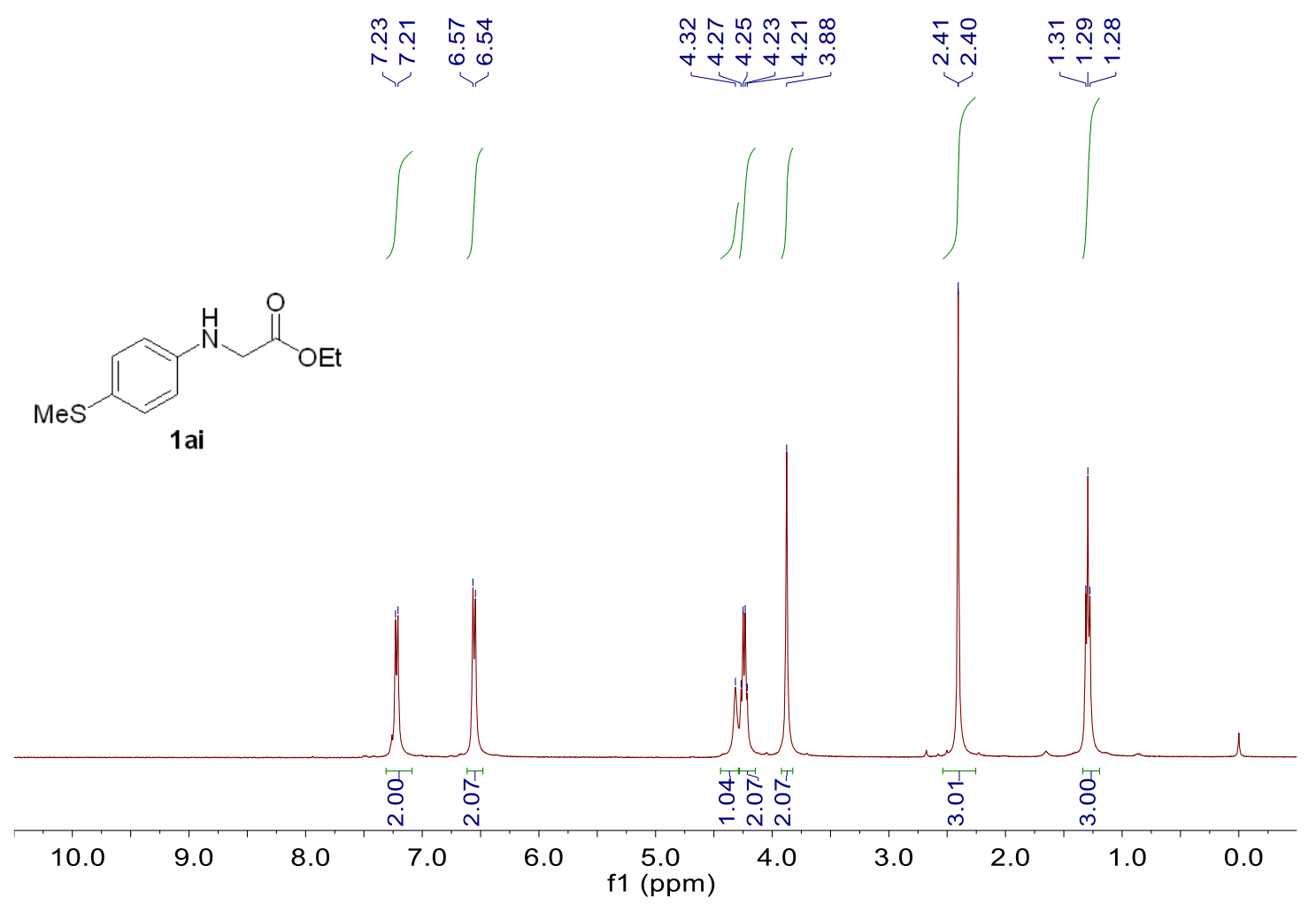

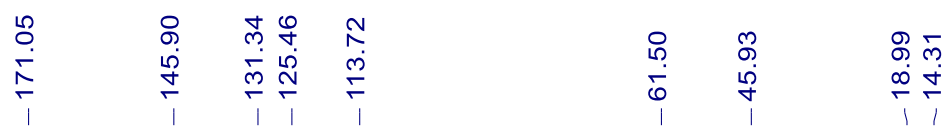

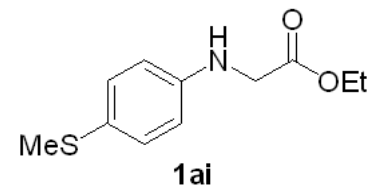

230

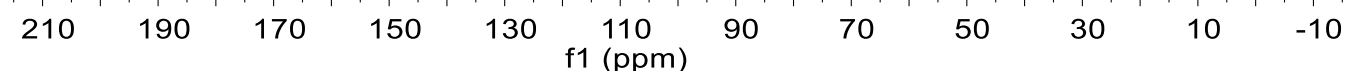

${ }^{1} \mathrm{H}$ NMR $(400 \mathrm{MHz})$ and ${ }^{13} \mathrm{C}\left\{{ }^{1} \mathrm{H}\right\}$ NMR $(101 \mathrm{MHz})$ spectra of 1ai $\left(\mathrm{CDCl}_{3}, \mathrm{rt}\right)$. 


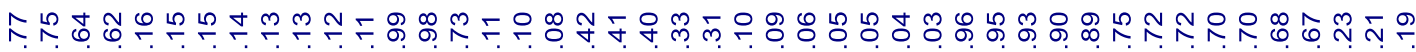

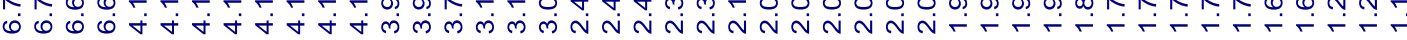<smiles>CCOC(=O)C(Nc1ccc(OC)cc1)C1CCCCC1=O</smiles>

$4 a$
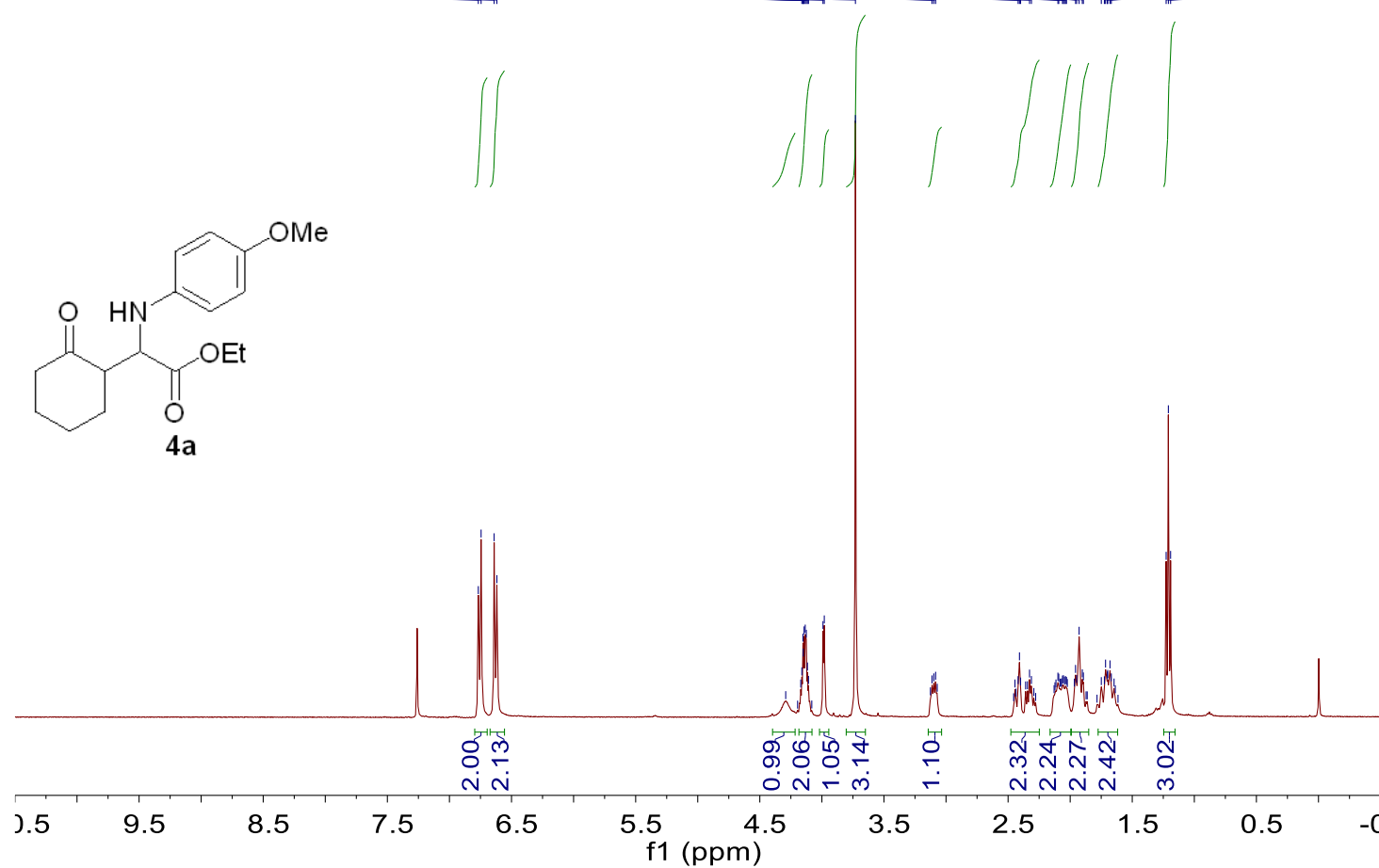

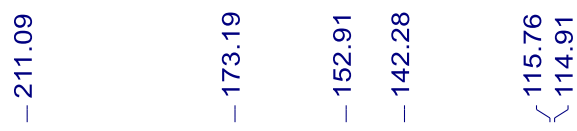

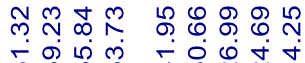

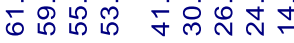<smiles>CCOC(=O)C(Nc1ccc(OC)cc1)C1CCCCC1=O</smiles>

$4 a$

它

it

ois,
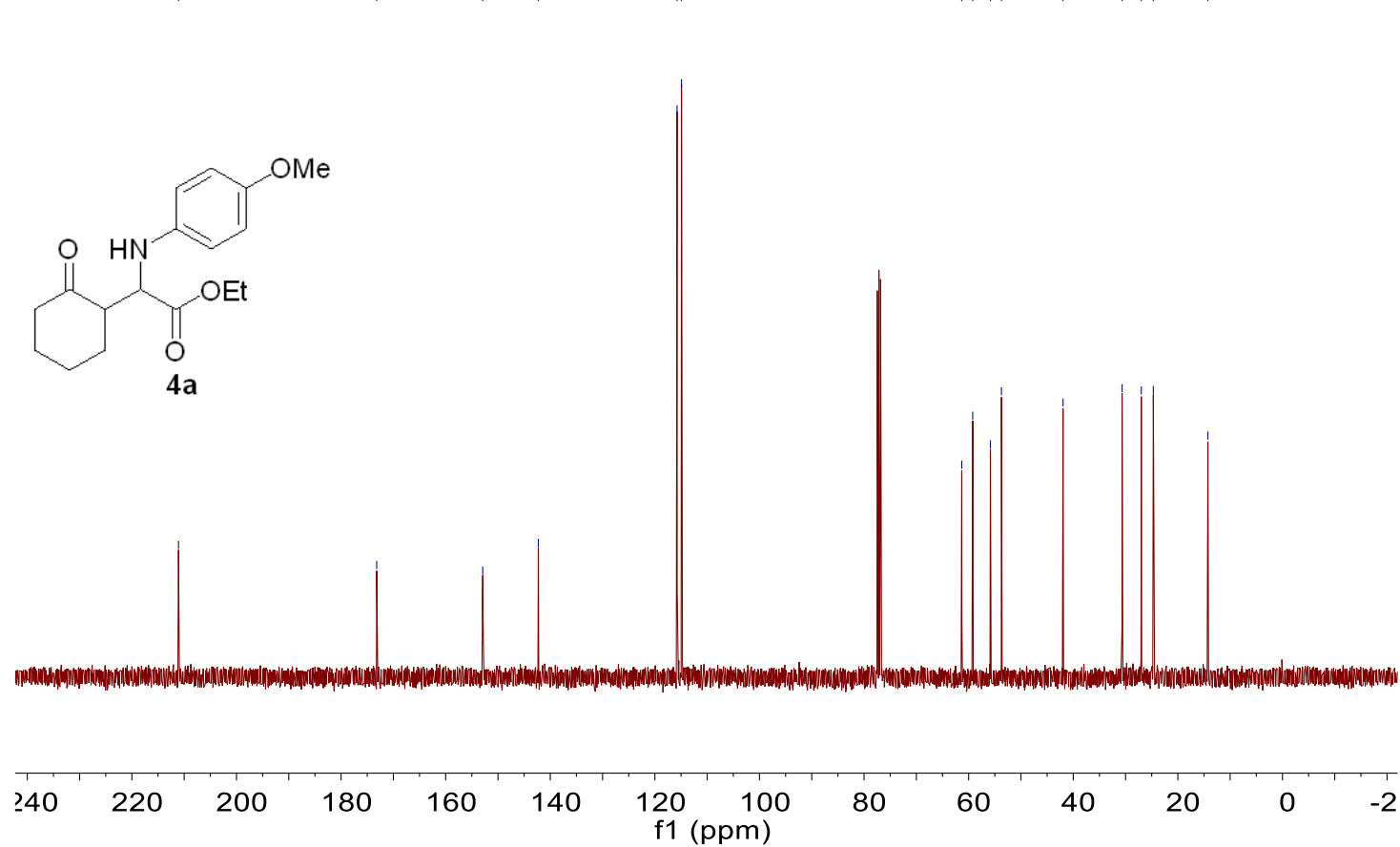

${ }^{1} \mathrm{H}$ NMR (400 MHz) and ${ }^{13} \mathrm{C}\left\{{ }^{1} \mathrm{H}\right\} \mathrm{NMR}(101 \mathrm{MHz})$ spectra of $\mathbf{4 a}\left(\mathrm{CDCl}_{3}, \mathrm{rt}\right)$. 
<smiles>COC(=O)C(Nc1ccc(OC)cc1)C1CCCCC1=O</smiles>

$4 b$
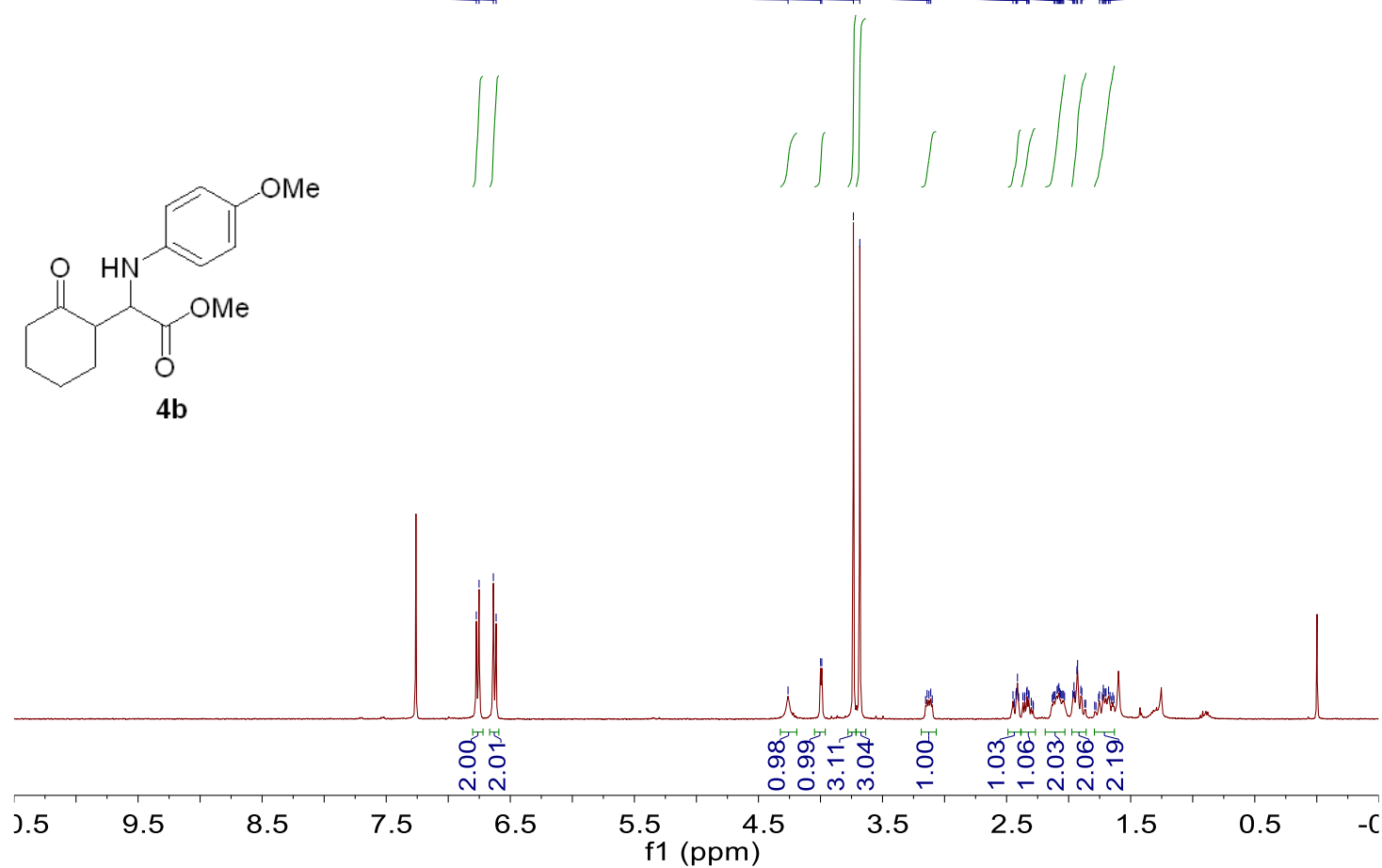

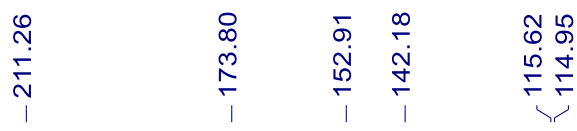

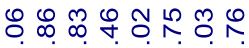

官占<smiles>COC(=O)C(Nc1ccc(OC)cc1)C1CCCCC1=O</smiles>

$4 b$

240

220

200

180160

140

f1 (ppm)

,

${ }^{1} \mathrm{H}$ NMR (400 MHz) and ${ }^{13} \mathrm{C}\left\{{ }^{1} \mathrm{H}\right\} \mathrm{NMR}(101 \mathrm{MHz})$ spectra of $\mathbf{4 b}\left(\mathrm{CDCl}_{3}, \mathrm{rt}\right)$. 


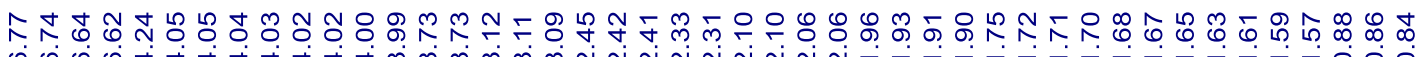

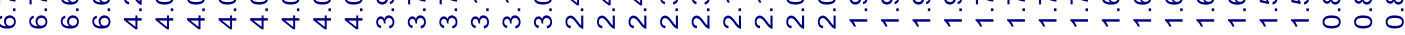

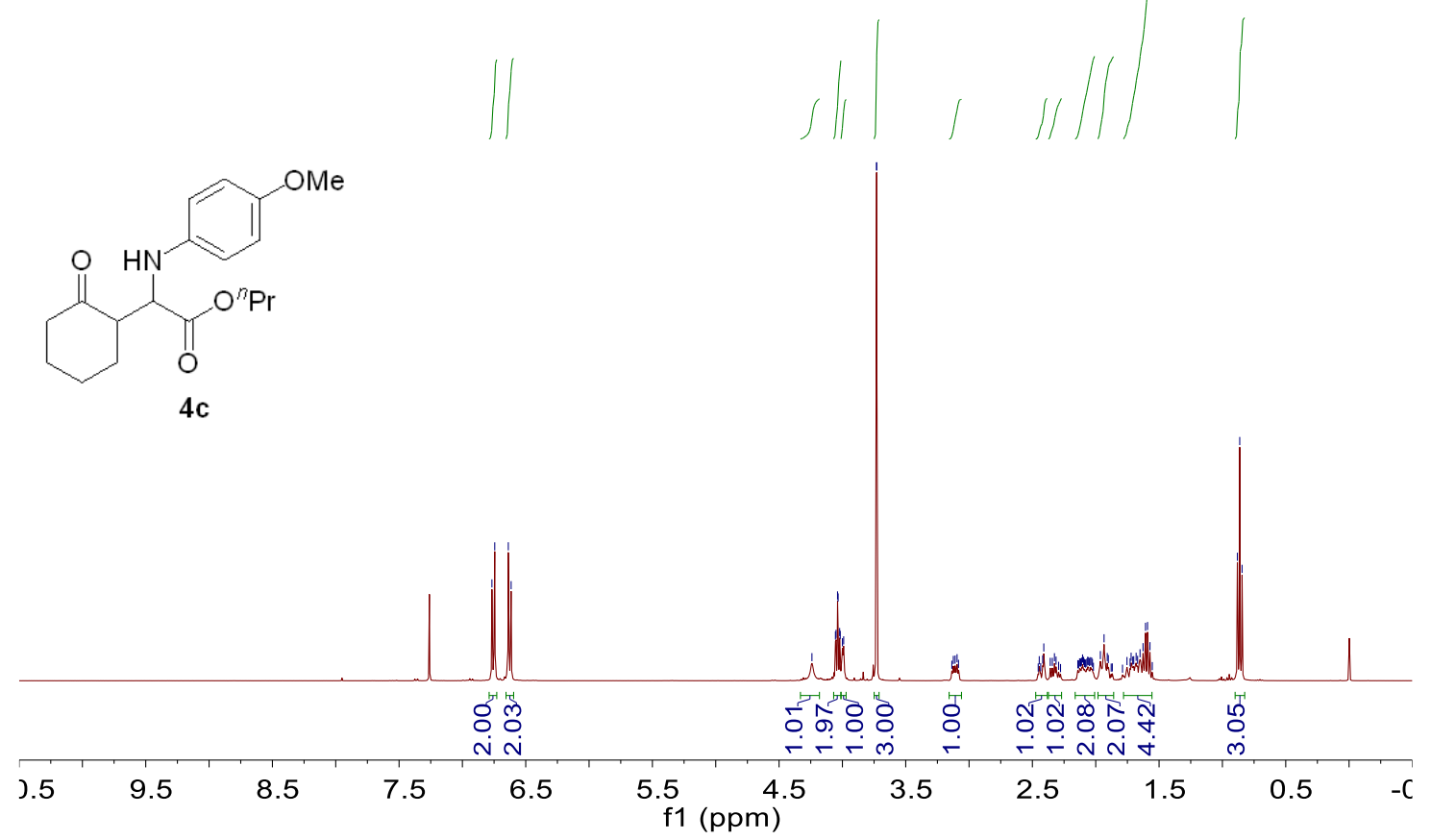

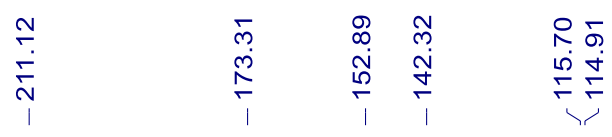

gกำ

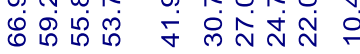<smiles>CCCOC(=O)C(Nc1ccc(OC)cc1)C1CCCCC1=O</smiles>

230

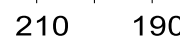

$90 \quad 170$

150

130

f1 $(\mathrm{ppm})$

${ }^{1} \mathrm{H}$ NMR $(400 \mathrm{MHz})$ and ${ }^{13} \mathrm{C}\left\{{ }^{1} \mathrm{H}\right\}$ NMR $(101 \mathrm{MHz})$ spectra of $4 \mathbf{c}\left(\mathrm{CDCl}_{3}, \mathrm{rt}\right)$. 
순

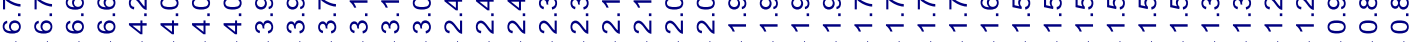
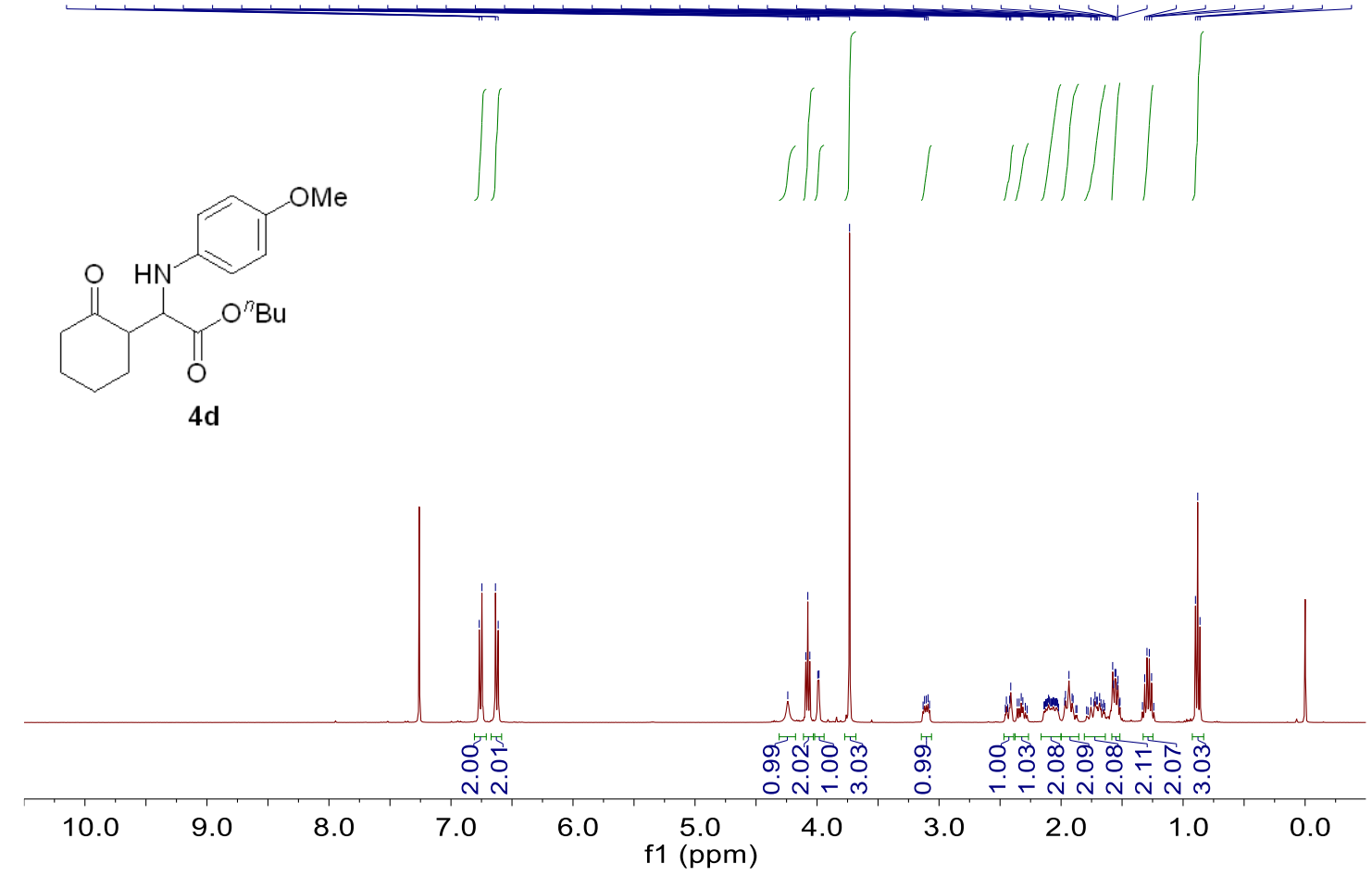

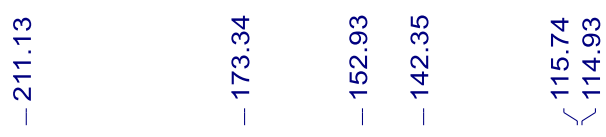

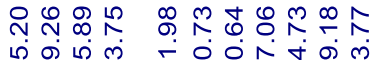

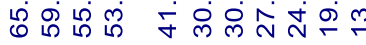

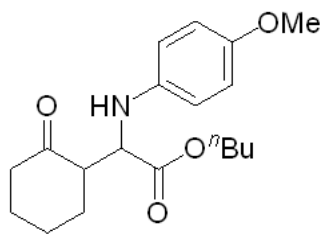

$4 d$

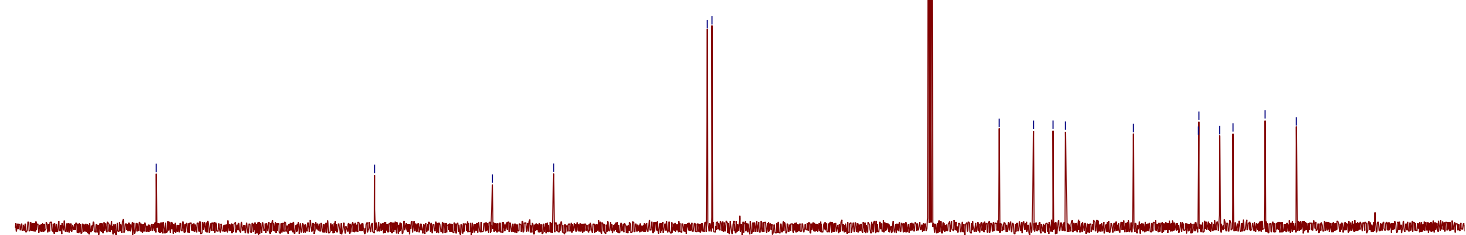

230

210190

$170 \quad 150$

$30 \begin{array}{cc}110 \\ \mathrm{f} 1(\mathrm{ppm})\end{array} 90$

70

50

$30 \quad 10 \quad-10$

${ }^{1} \mathrm{H}$ NMR $(400 \mathrm{MHz})$ and ${ }^{13} \mathrm{C}\left\{{ }^{1} \mathrm{H}\right\}$ NMR $(101 \mathrm{MHz})$ spectra of $4 \mathbf{d}\left(\mathrm{CDCl}_{3}, \mathrm{rt}\right)$. 


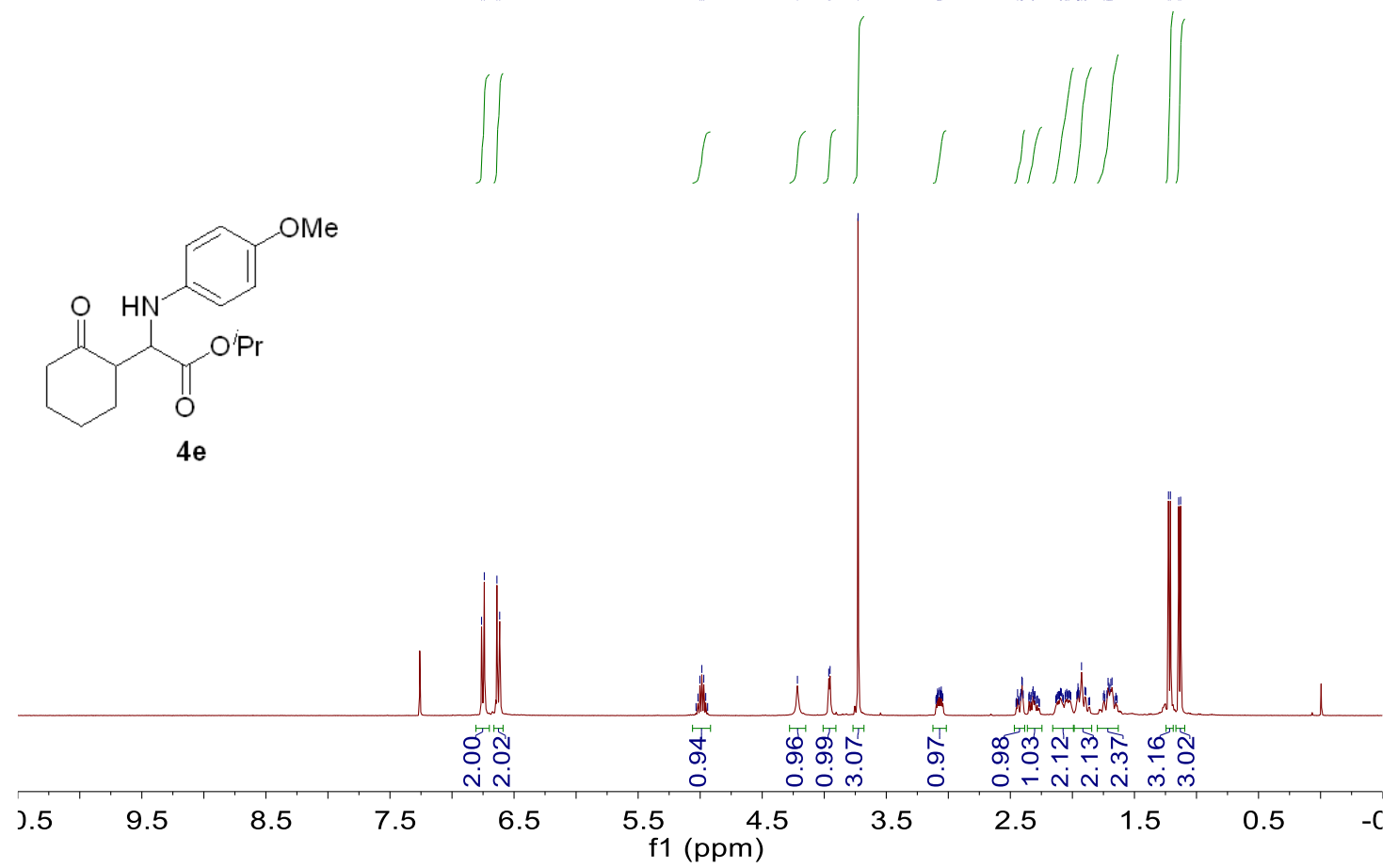

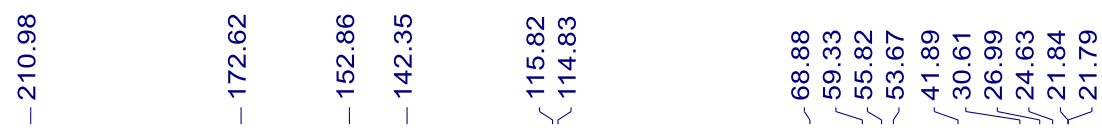

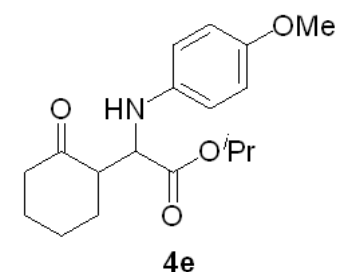

$4 e$

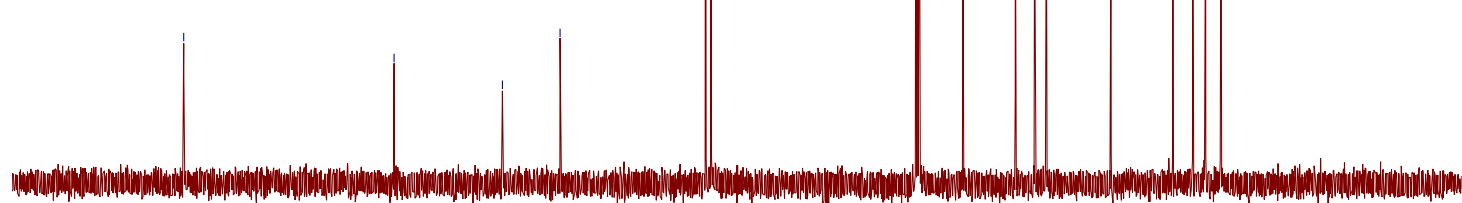

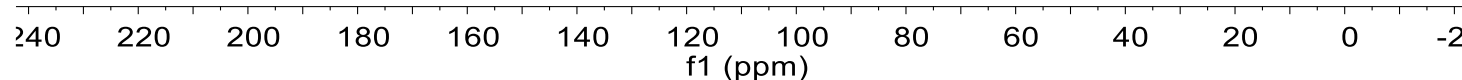

${ }^{1} \mathrm{H}$ NMR (400 MHz) and ${ }^{13} \mathrm{C}\left\{{ }^{1} \mathrm{H}\right\} \mathrm{NMR}(101 \mathrm{MHz})$ spectra of $4 \mathbf{e}\left(\mathrm{CDCl}_{3}, \mathrm{rt}\right)$. 

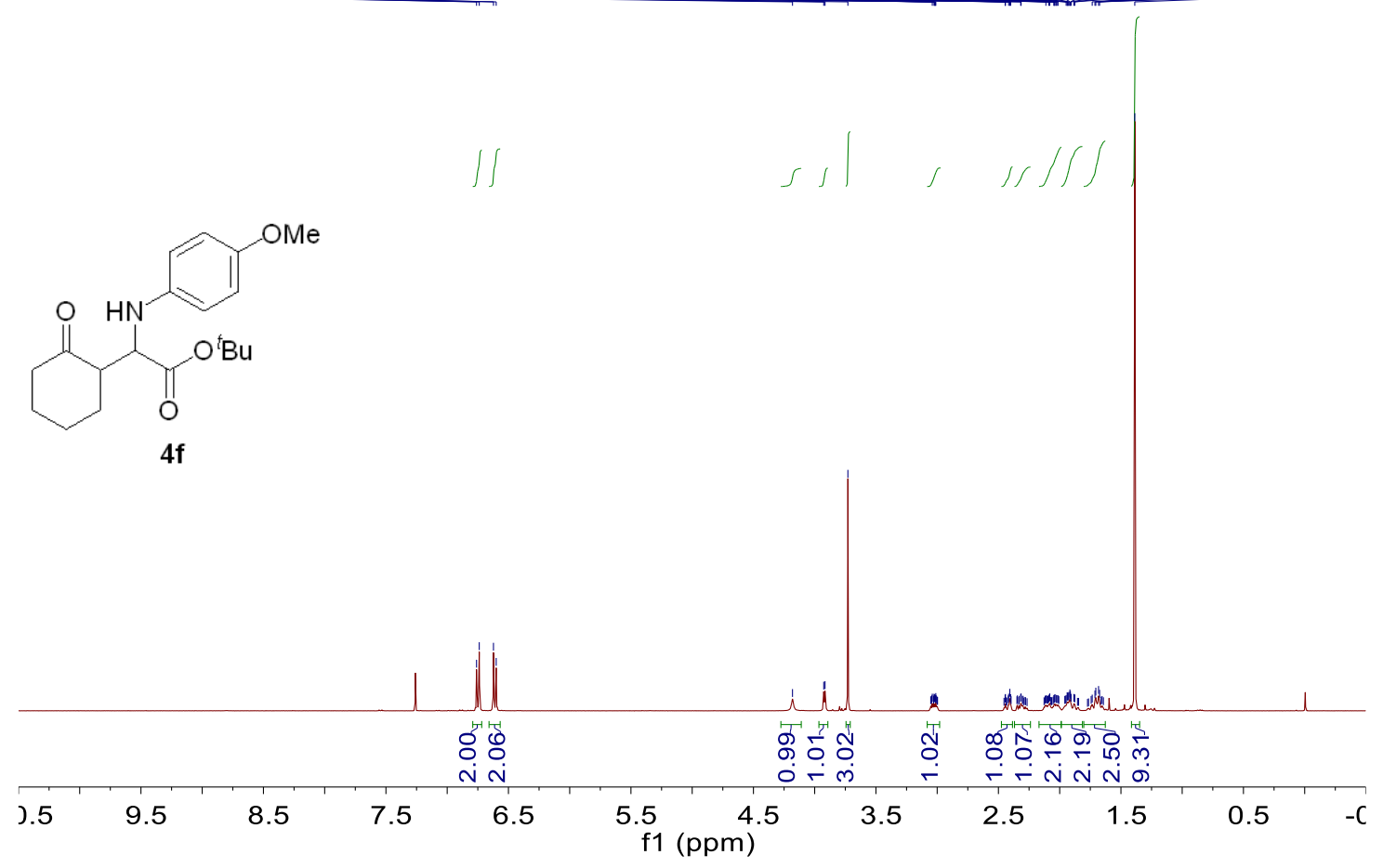

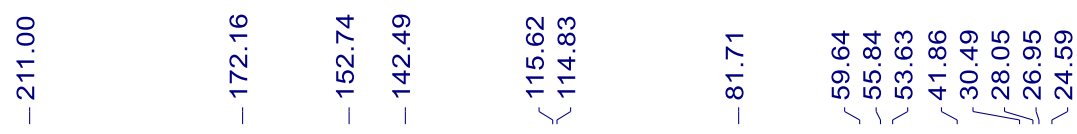
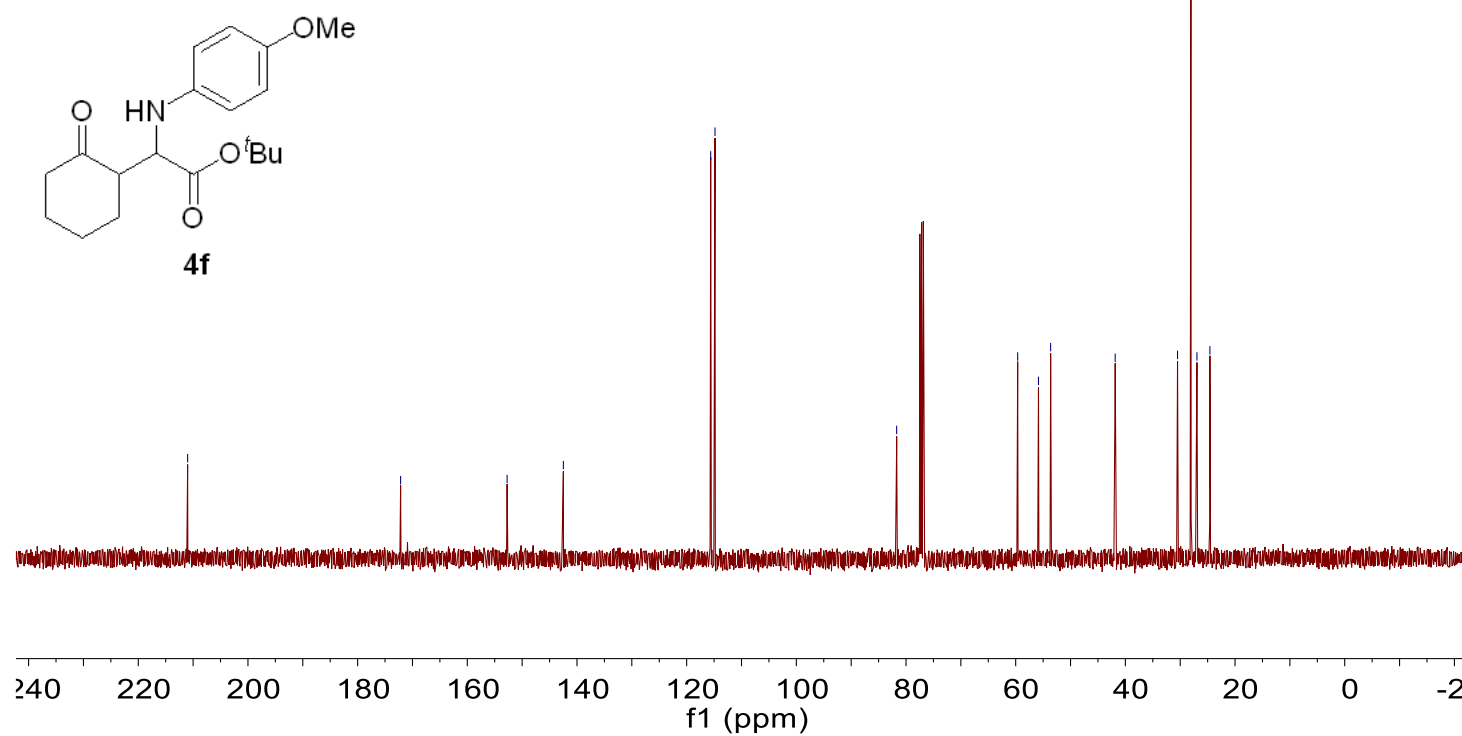

${ }^{1} \mathrm{H}$ NMR (400 MHz) and ${ }^{13} \mathrm{C}\left\{{ }^{1} \mathrm{H}\right\}$ NMR (101 MHz) spectra of $\mathbf{4 f}\left(\mathrm{CDCl}_{3}, \mathrm{rt}\right)$. 
<smiles>COc1ccc(NC(C(=O)OC(C)(C)C)C2CCCCC2=O)cc1</smiles><smiles>C1=CC=C1</smiles><smiles>CC1CCCC1</smiles>
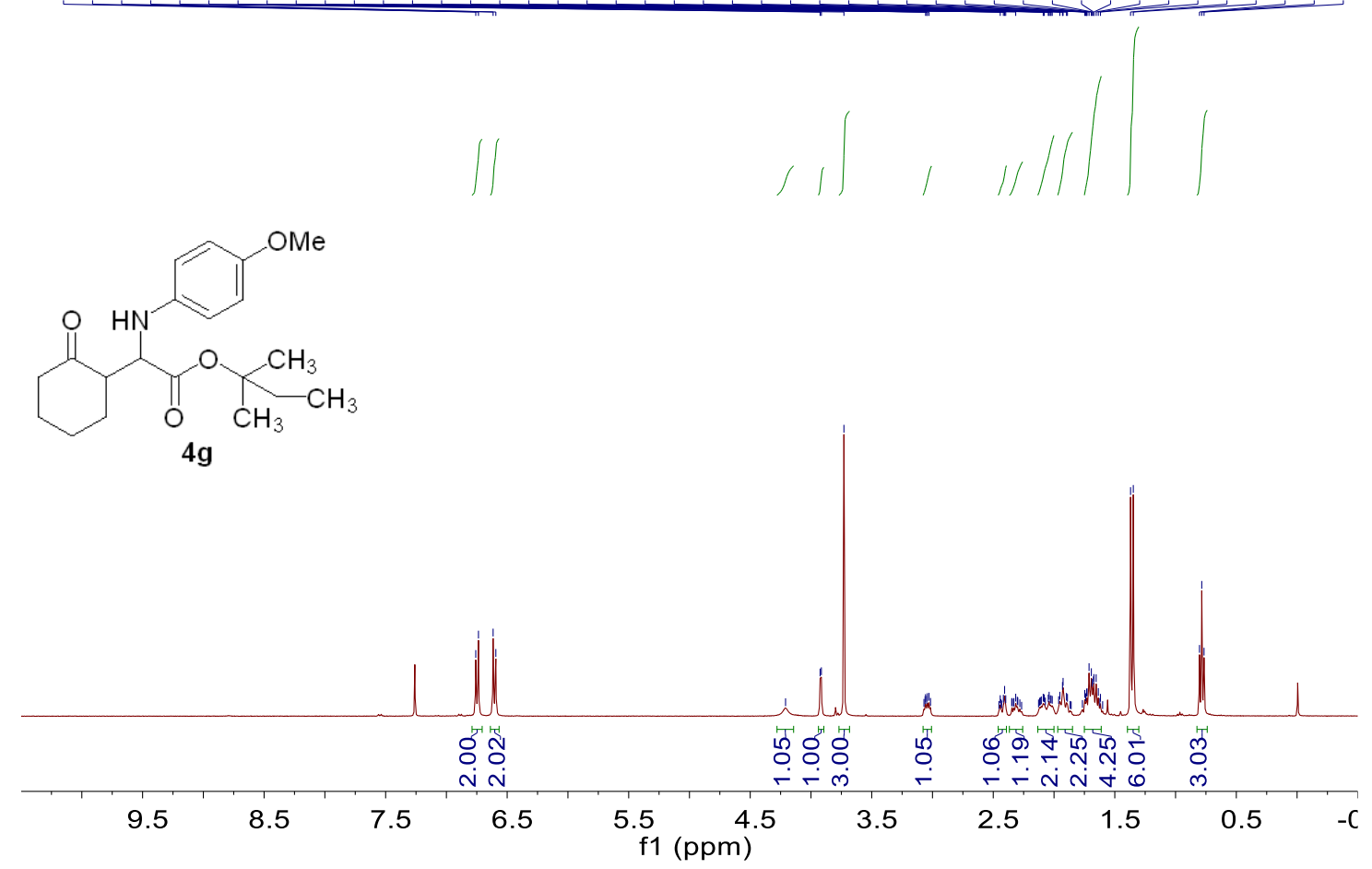

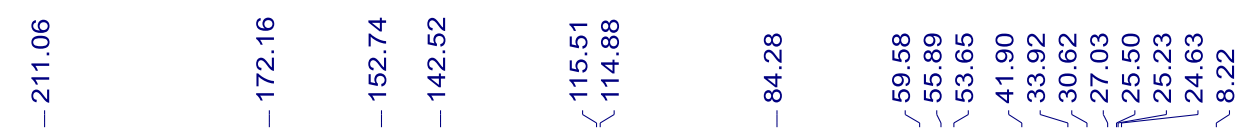<smiles>COc1ccc(NC(C(=O)OC(C)(C)C)C2CCCCC2=O)cc1</smiles>

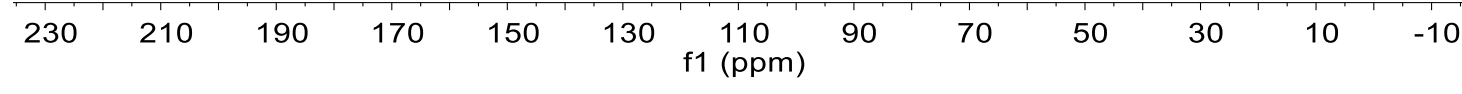

${ }^{1} \mathrm{H}$ NMR $(400 \mathrm{MHz})$ and ${ }^{13} \mathrm{C}\left\{{ }^{1} \mathrm{H}\right\} \mathrm{NMR}(101 \mathrm{MHz})$ spectra of $\mathbf{4 g}\left(\mathrm{CDCl}_{3}, \mathrm{rt}\right)$. 
<smiles>COc1ccc(NC(C(=O)OCC(C)(C)C)C2CCCCC2=O)cc1</smiles>

$4 \mathrm{~h}$
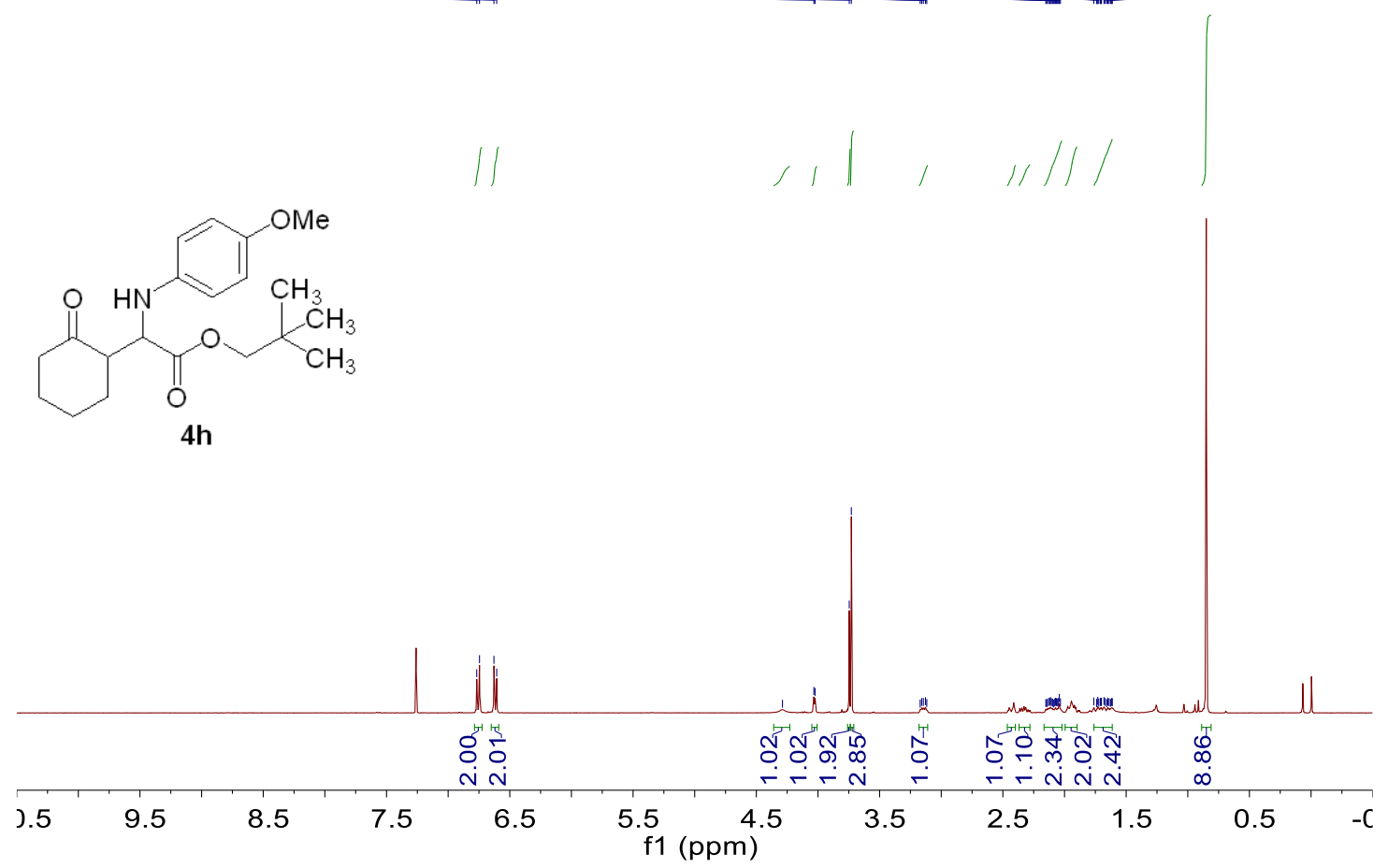

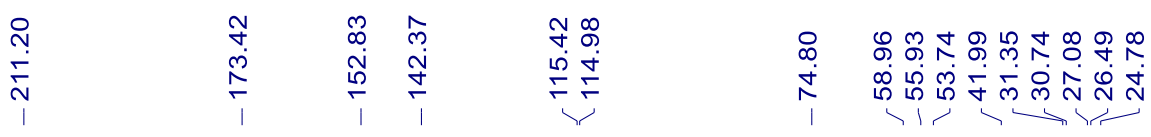<smiles>COc1ccc(NC(C(=O)OCC(C)(C)C)C2CCCCC2=O)cc1</smiles>

4h

${ }^{1} \mathrm{H}$ NMR (400 MHz) and ${ }^{13} \mathrm{C}\left\{{ }^{1} \mathrm{H}\right\} \mathrm{NMR}(101 \mathrm{MHz})$ spectra of $\mathbf{4 h}\left(\mathrm{CDCl}_{3}, \mathrm{rt}\right)$. 


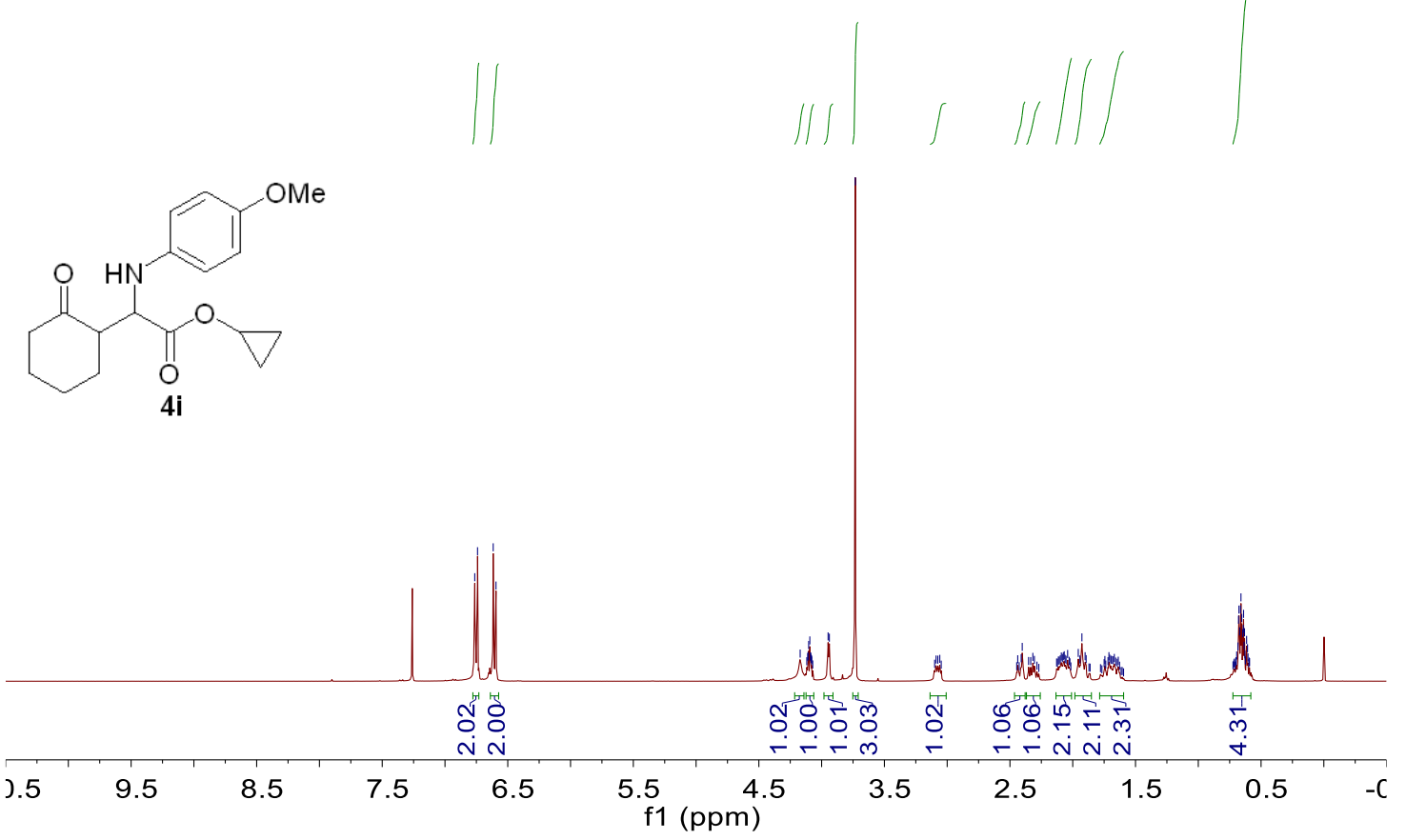

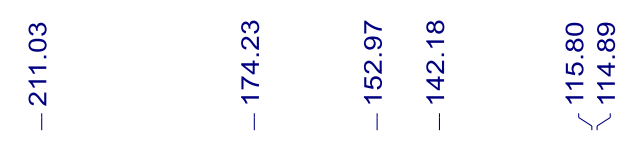

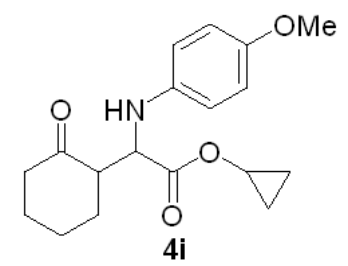

${ }^{1} \mathrm{H}$ NMR (400 MHz) and ${ }^{13} \mathrm{C}\left\{{ }^{1} \mathrm{H}\right\} \mathrm{NMR}(101 \mathrm{MHz})$ spectra of $\mathbf{4 i}\left(\mathrm{CDCl}_{3}, \mathrm{rt}\right)$. 


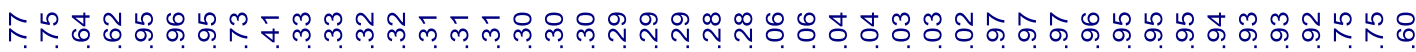

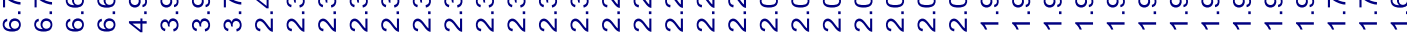
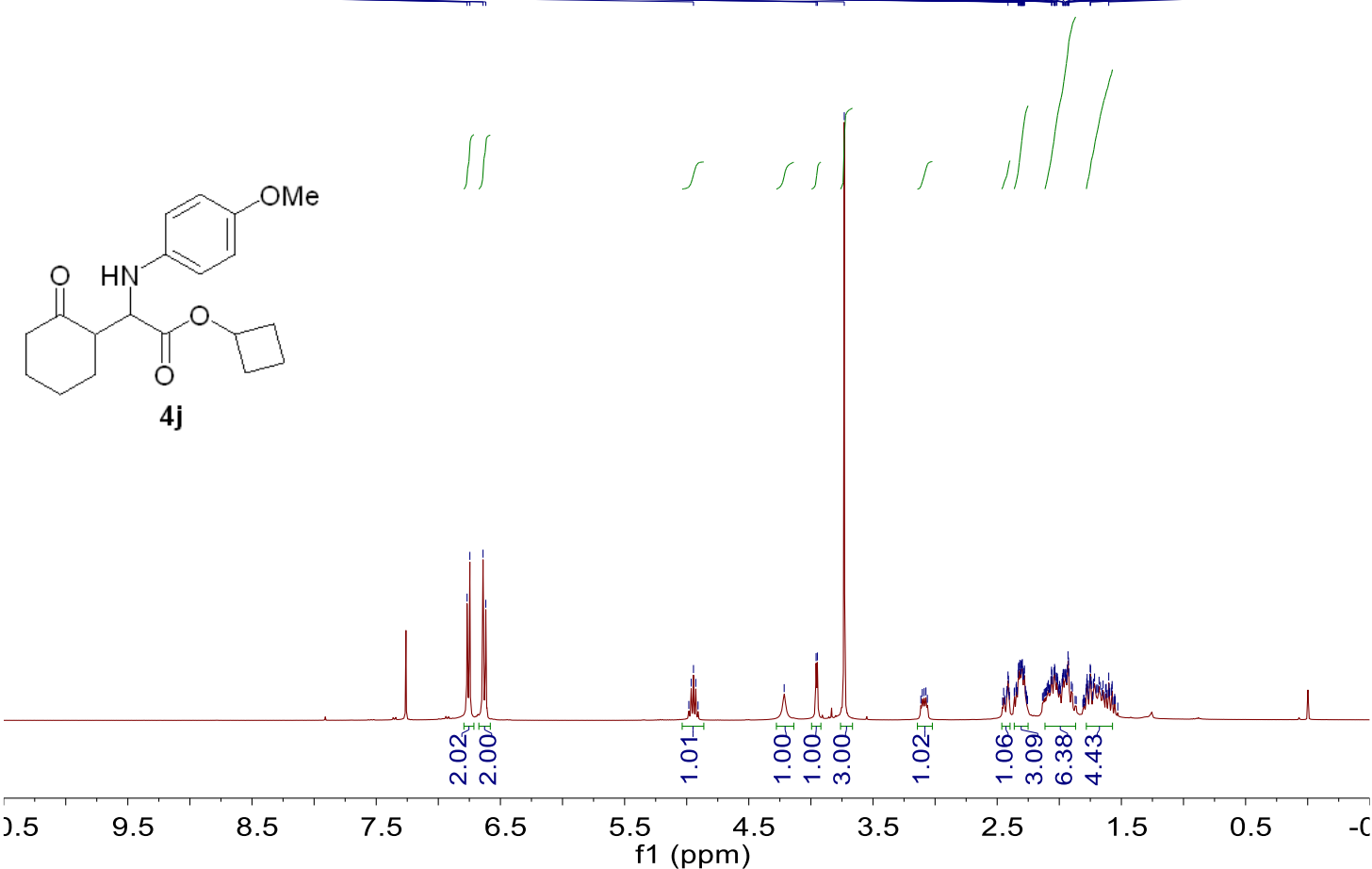

\begin{tabular}{|c|c|c|c|c|}
\hline ָั & 10 & ৪ & $\bar{m}$ & 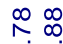 \\
\hline 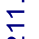 & $\stackrel{N}{N}$ & กี & $\stackrel{\sim}{\forall}$ & $\stackrel{\circ 0}{\leftarrow}$ \\
\hline$N$ & - & $r$ & $r$ & $r$ \\
\hline
\end{tabular}

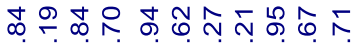
ஓंठ்

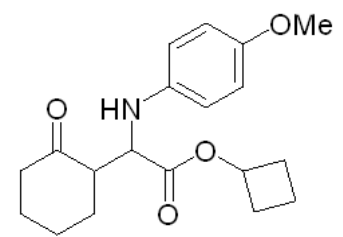

$4 \mathbf{j}$

230
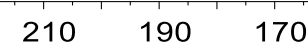

150

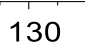

110
f1 $(p p m)$

90

70

50

$30 \quad 10 \quad-10$

${ }^{1} \mathrm{H}$ NMR $(400 \mathrm{MHz})$ and ${ }^{13} \mathrm{C}\left\{{ }^{1} \mathrm{H}\right\} \mathrm{NMR}(101 \mathrm{MHz})$ spectra of $\mathbf{4} \mathbf{j}\left(\mathrm{CDCl}_{3}, \mathrm{rt}\right)$. 


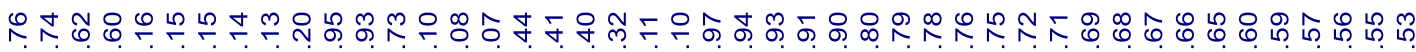

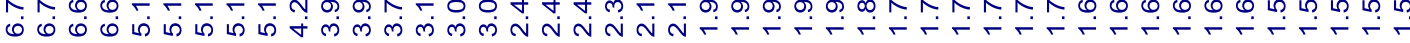

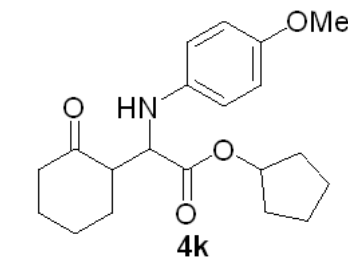<smiles>c1ccccc1</smiles>
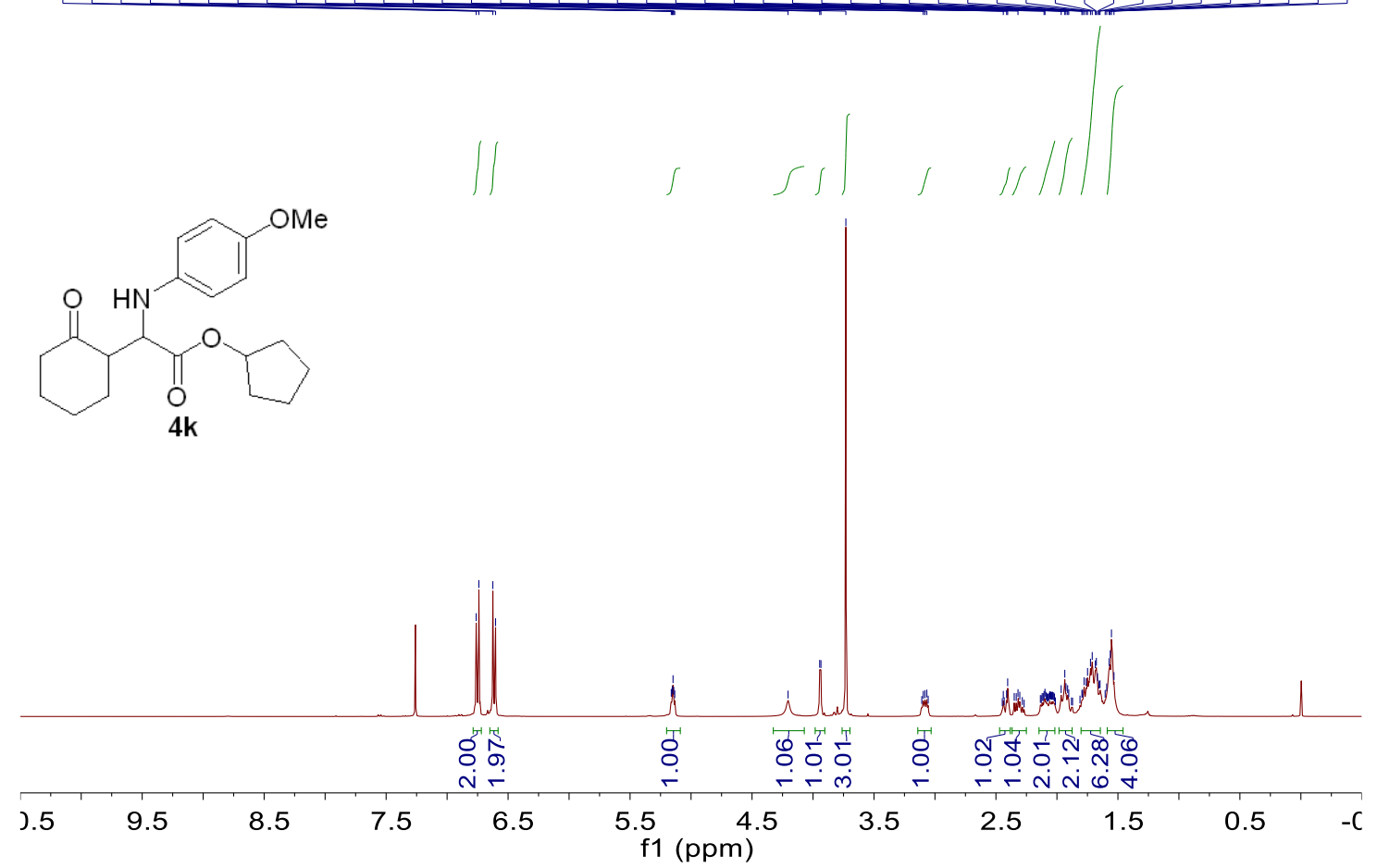

\begin{tabular}{|c|c|c|c|c|}
\hline$\stackrel{m}{\leftarrow}$ & ฮ้ & న & 守 & $\Omega_{\Lambda}$ \\
\hline$\check{E}$ & $\stackrel{N}{N}$ & N & $\stackrel{\sim}{\sim}$ & மீ \\
\hline $\bar{N}$ & 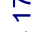 & $\stackrel{\text { L }}{2}$ & $\stackrel{\sim}{\sim}$ & $\mp F$ \\
\hline | & 1 & 1 & | & \\
\hline
\end{tabular}
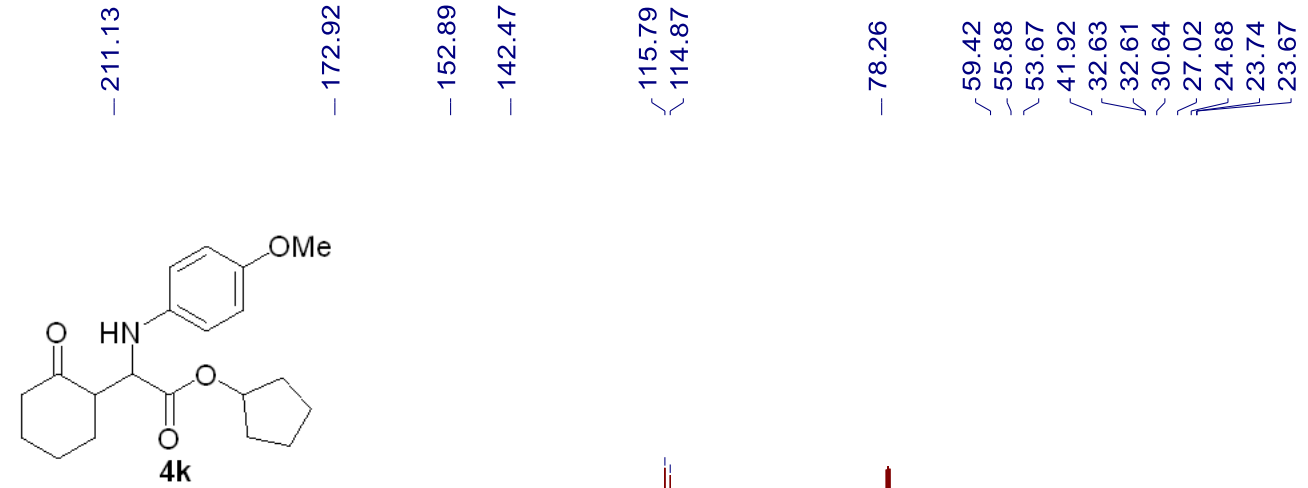

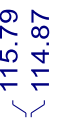

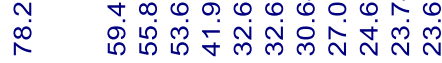
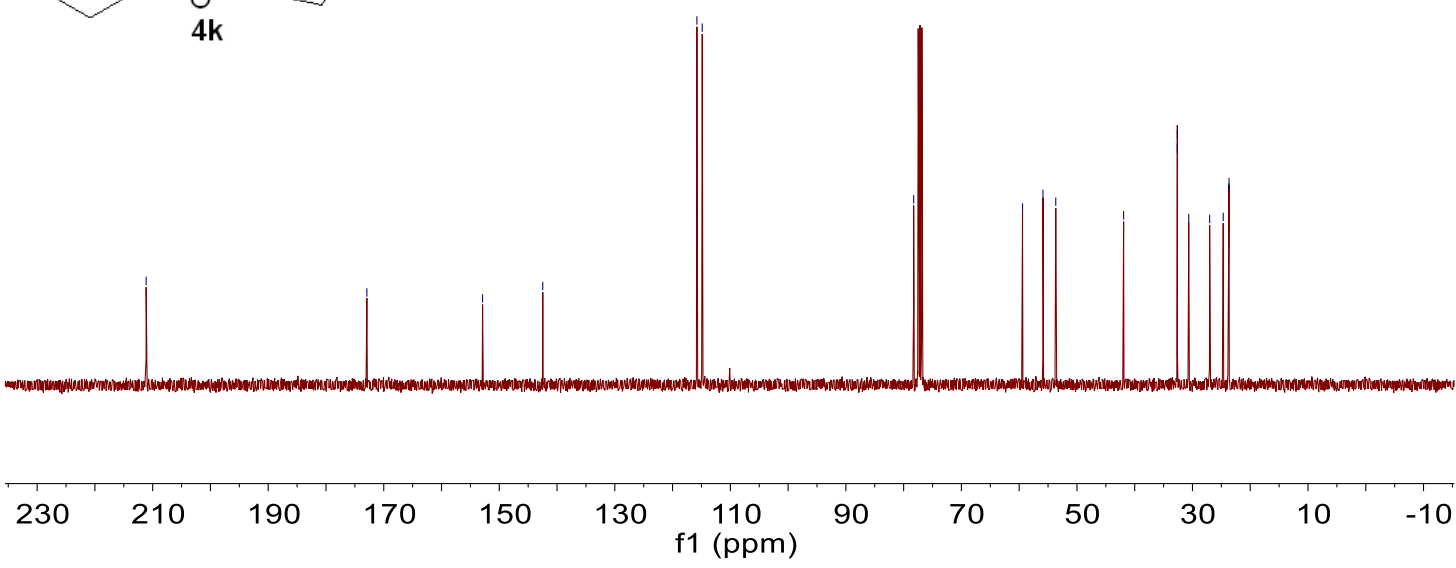

${ }^{1} \mathrm{H}$ NMR $(400 \mathrm{MHz})$ and ${ }^{13} \mathrm{C}\left\{{ }^{1} \mathrm{H}\right\} \mathrm{NMR}(101 \mathrm{MHz})$ spectra of $\mathbf{4 k}\left(\mathrm{CDCl}_{3}, \mathrm{rt}\right)$. 

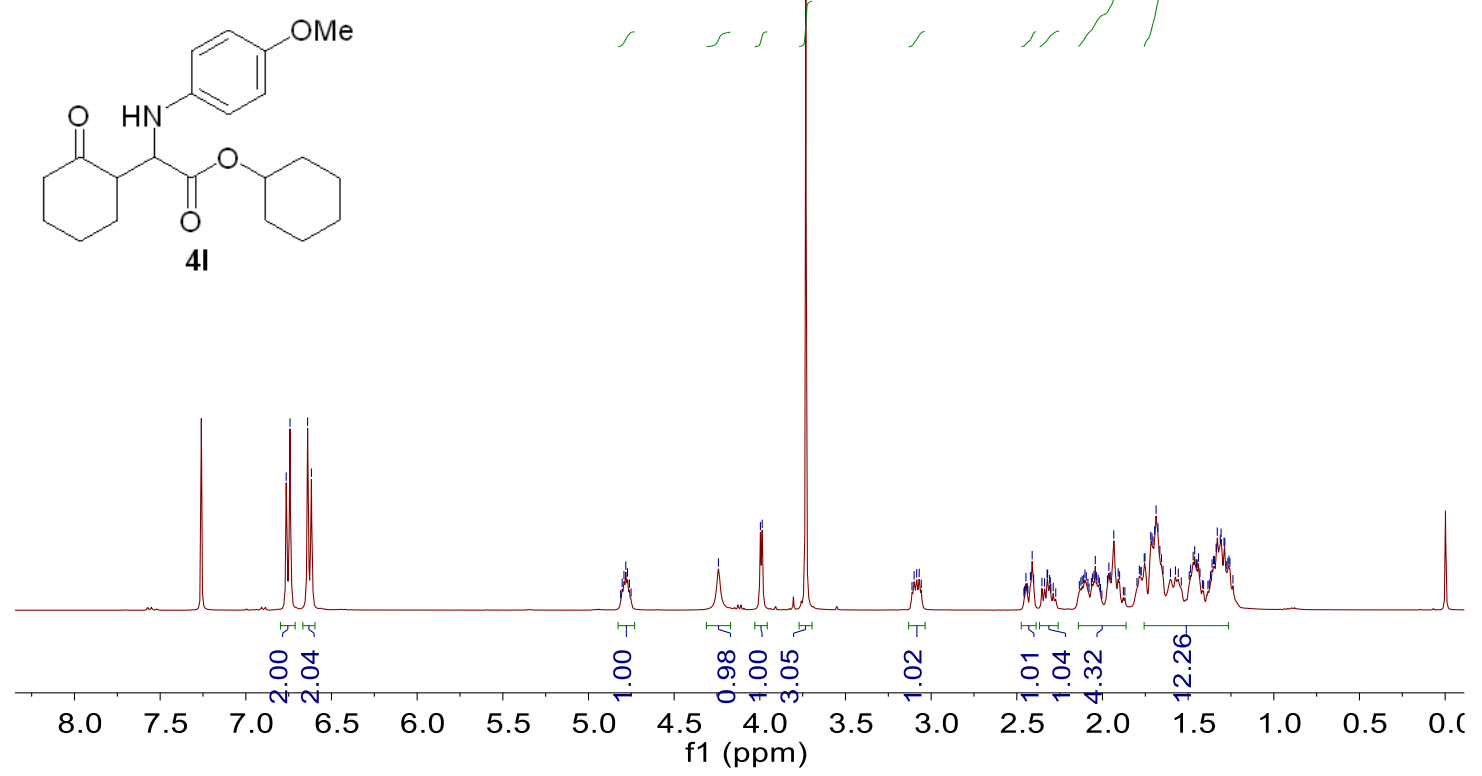

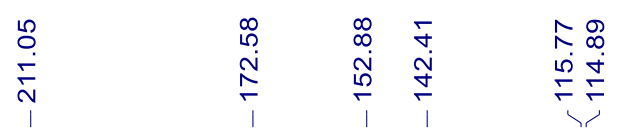

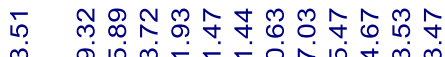

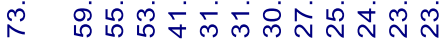
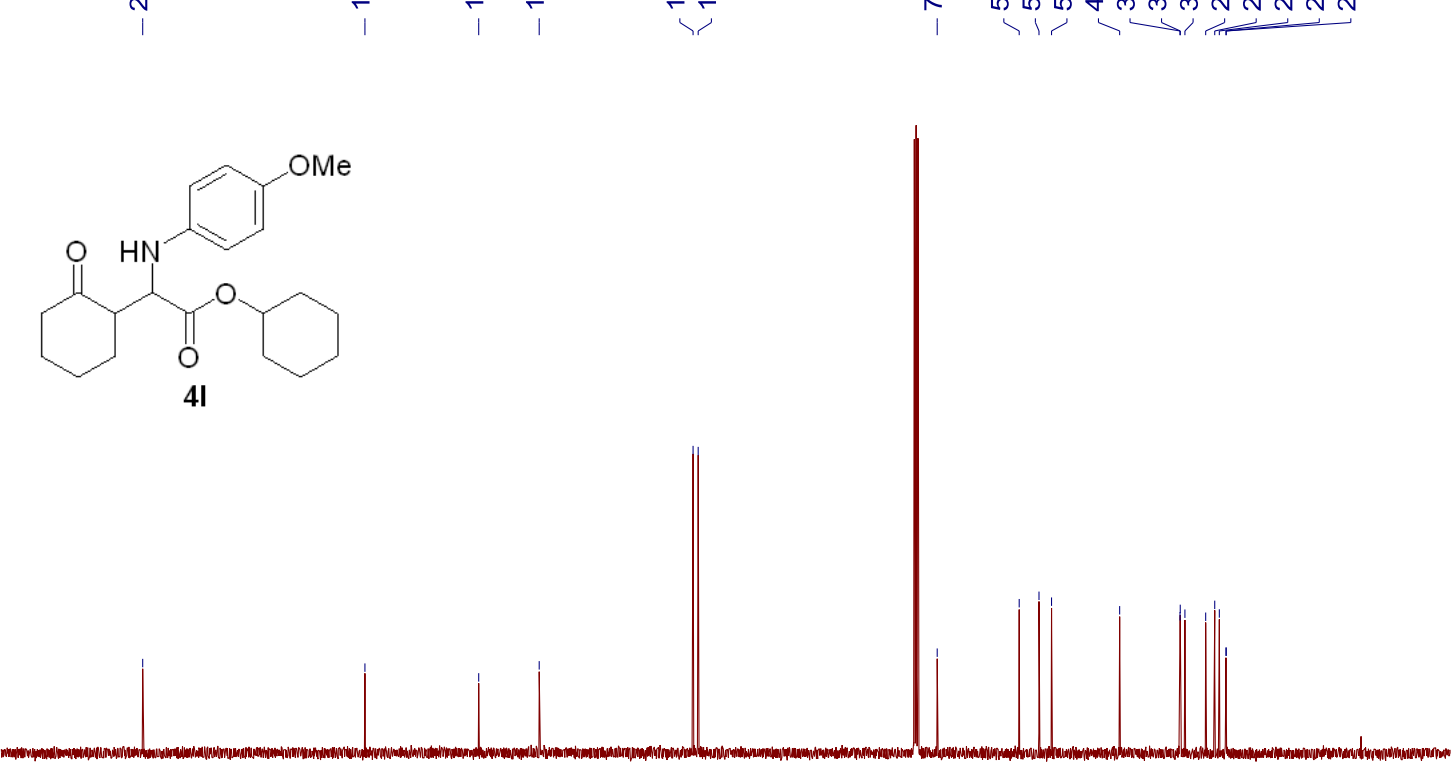

$230 \quad 210 \quad 190 \quad 170 \quad 150 \quad 130 \begin{gathered}110 \\ \mathrm{f} 1(\mathrm{ppm})\end{gathered}$

${ }^{1} \mathrm{H}$ NMR (400 MHz) and ${ }^{13} \mathrm{C}\left\{{ }^{1} \mathrm{H}\right\}$ NMR (101 MHz) spectra of $4 \mathbf{l}\left(\mathrm{CDCl}_{3}, \mathrm{rt}\right)$. 

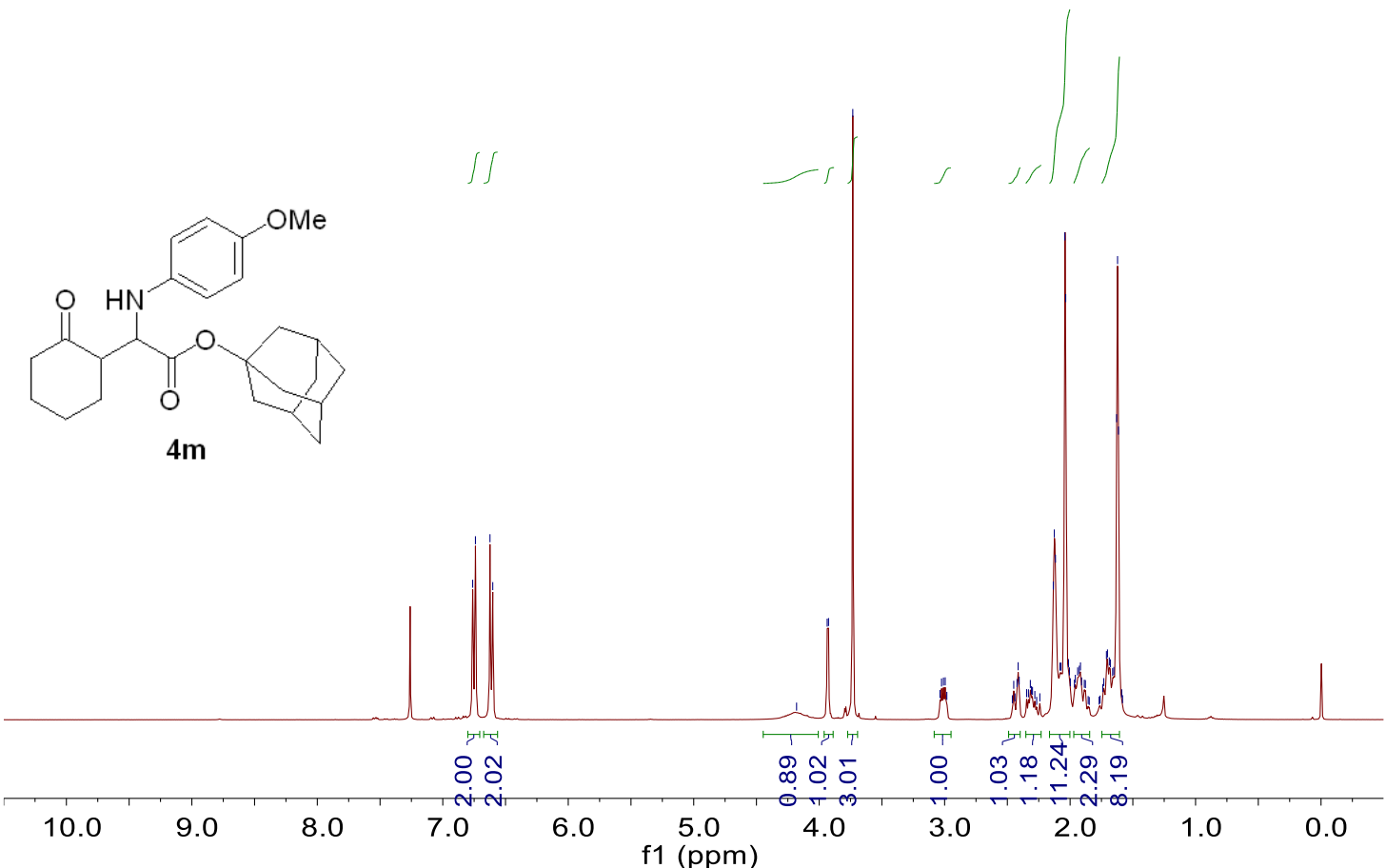

\begin{tabular}{|c|c|c|c|c|c|}
\hline 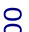 & 央 & $\hat{\Lambda}$ & 00 & & \\
\hline & $\stackrel{\sim}{\sim}$ & i & $\stackrel{\infty}{\infty}$ & $\stackrel{\infty}{N}$ & 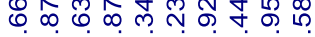 \\
\hline$V$ & 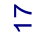 & $\stackrel{0}{\sim}$ & $\div$ & $\dot{\infty}$ & 官 மீ \\
\hline 1 & 1 & I & 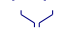 & I & $41 ;>$ \\
\hline
\end{tabular}
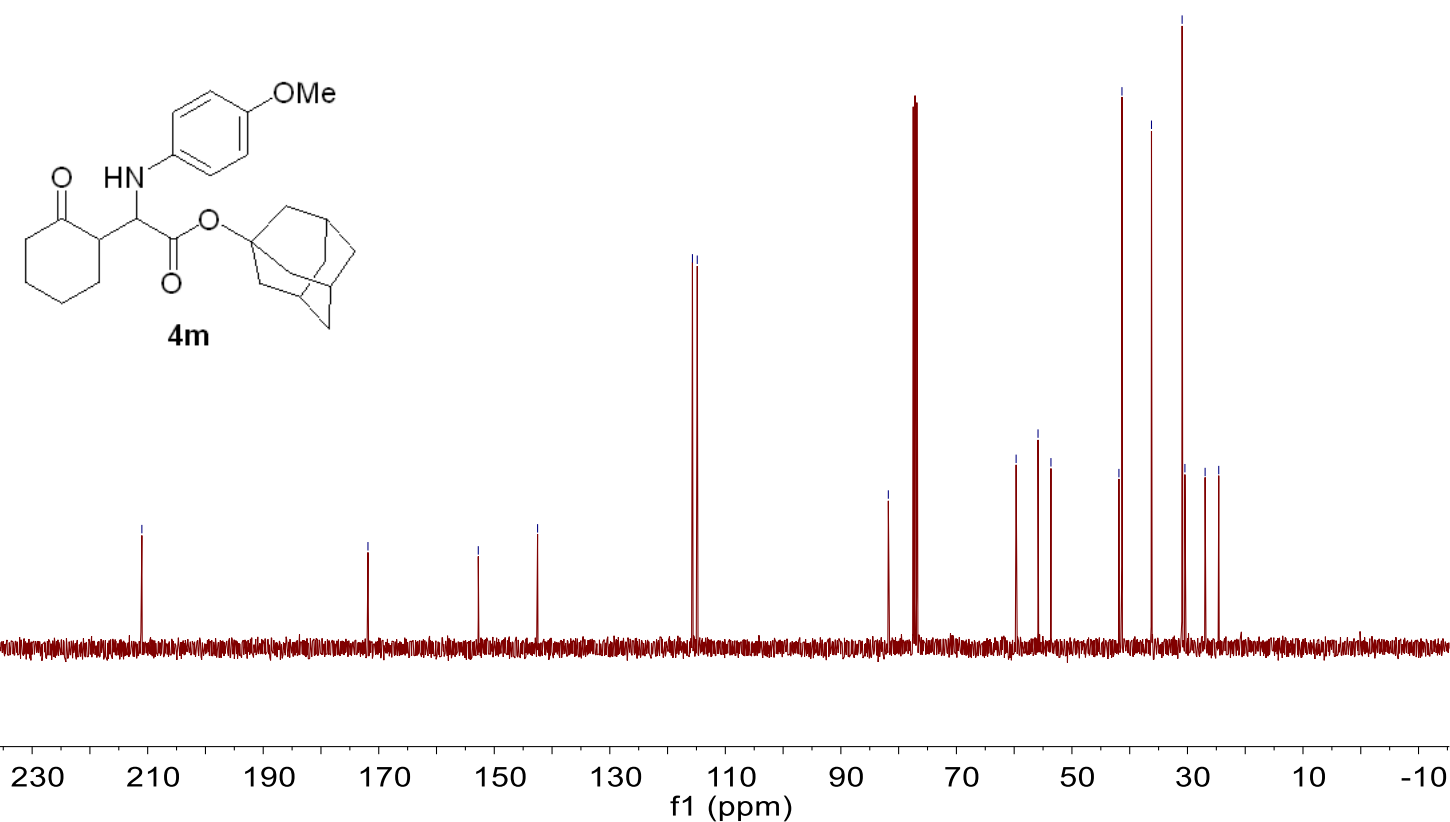

${ }^{1} \mathrm{H}$ NMR $(400 \mathrm{MHz})$ and ${ }^{13} \mathrm{C}\left\{{ }^{1} \mathrm{H}\right\}$ NMR $(101 \mathrm{MHz})$ spectra of $\mathbf{4 m}\left(\mathrm{CDCl}_{3}, \mathrm{rt}\right)$ 


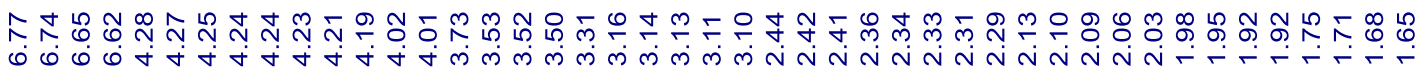

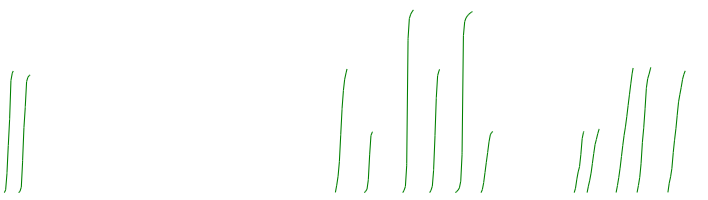<smiles>COCCOC(=O)C(Nc1ccc(OC)cc1)C1CCCCC1=O</smiles>

$4 n$

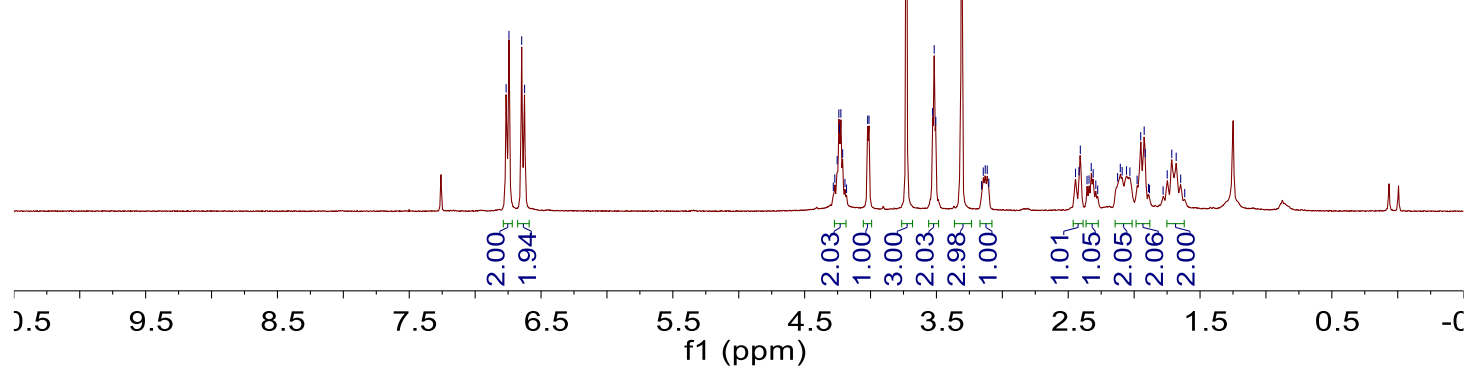

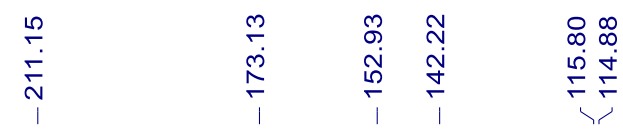

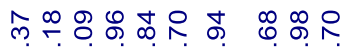

रें<smiles>COCCOC(=O)C(Nc1ccc(OC)cc1)C1CCCCC1=O</smiles>

$4 n$

${ }^{1} \mathrm{H}$ NMR (400 MHz) and ${ }^{13} \mathrm{C}\left\{{ }^{1} \mathrm{H}\right\}$ NMR $(101 \mathrm{MHz})$ spectra of $4 \mathbf{n}\left(\mathrm{CDCl}_{3}, \mathrm{rt}\right)$. 
<smiles>C#CC#CCCCCOC(=O)C(Nc1ccc(OC)cc1)C1CCCCC1=O</smiles>

40

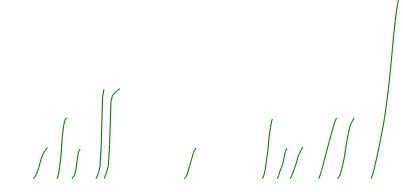

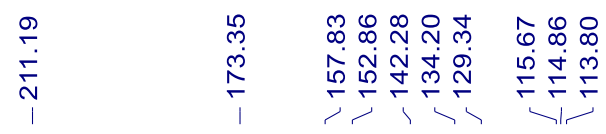

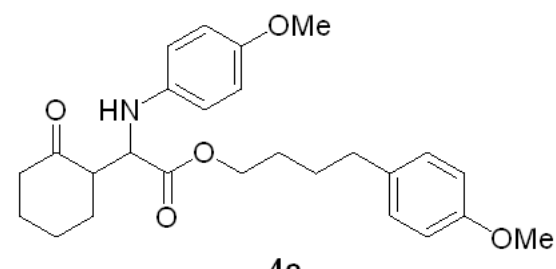

40
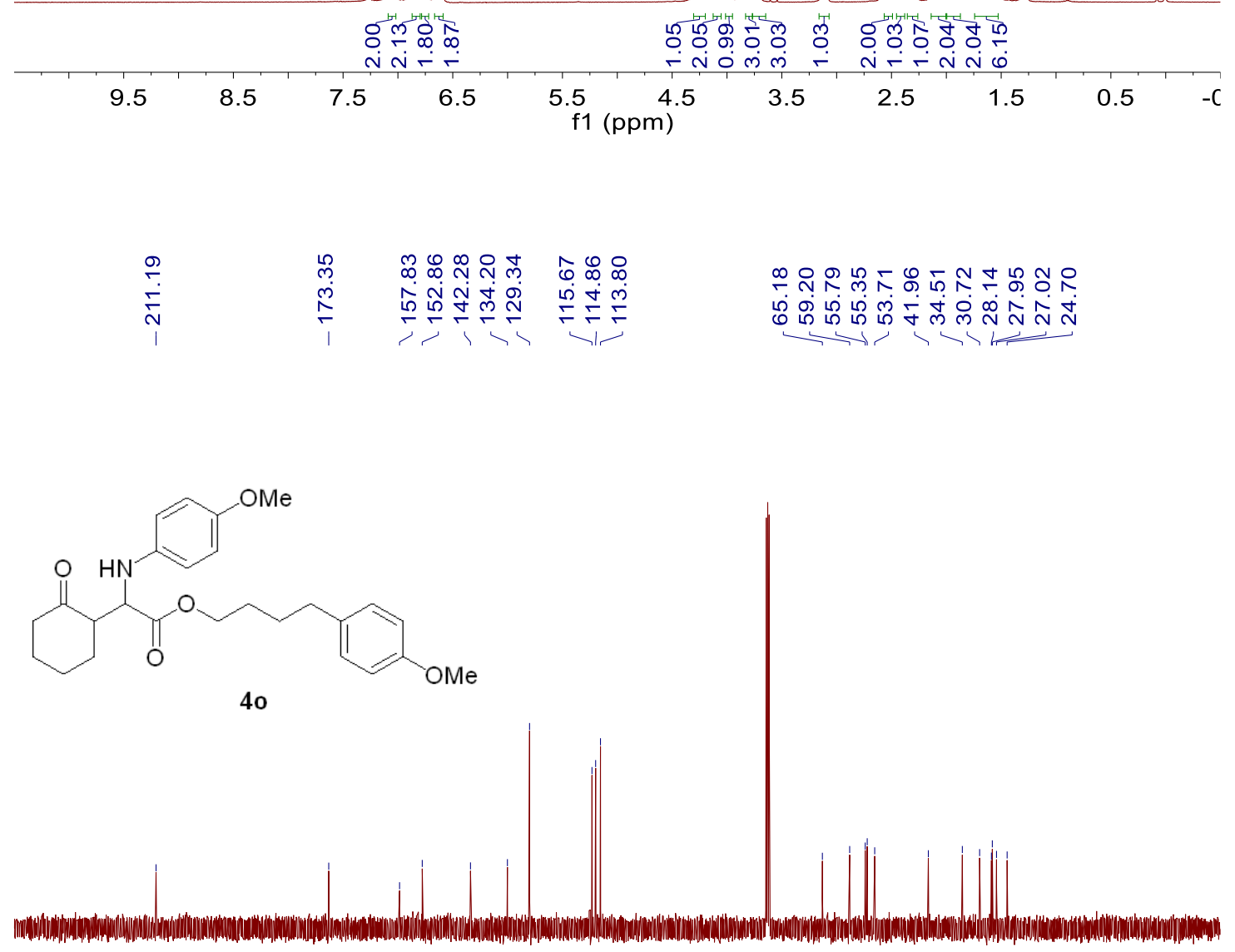

$240 \quad 220 \quad 200 \quad 180 \quad 160 \quad \begin{gathered}140 \\ \begin{array}{c}120 \\ \mathrm{f} 1(\mathrm{ppm})\end{array}\end{gathered}$

${ }^{1} \mathrm{H}$ NMR $(400 \mathrm{MHz})$ and ${ }^{13} \mathrm{C}\left\{{ }^{1} \mathrm{H}\right\} \mathrm{NMR}(101 \mathrm{MHz})$ spectra of $\mathbf{4 o}\left(\mathrm{CDCl}_{3}, \mathrm{rt}\right)$. 
芦

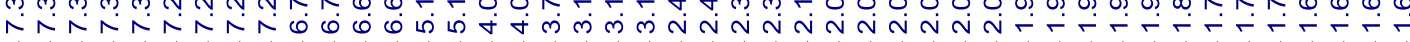

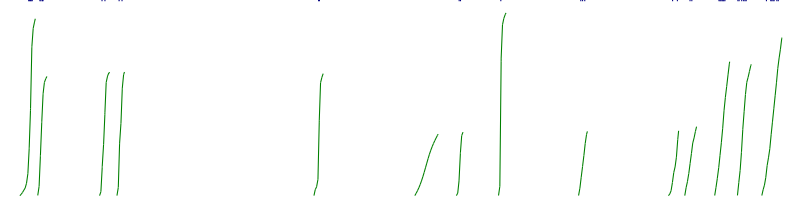<smiles>COc1ccc(NC(C(=O)OCc2ccccc2)C2CCCCC2=O)cc1</smiles>

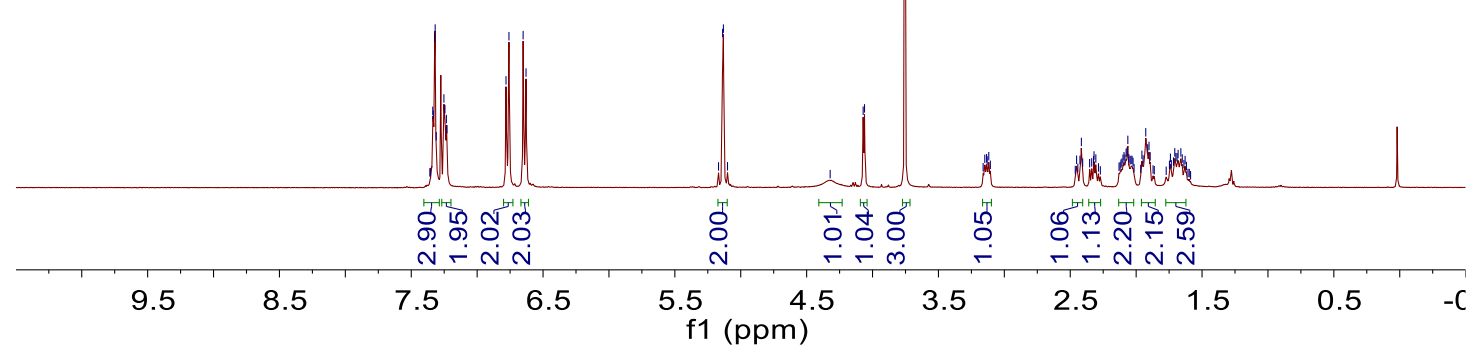

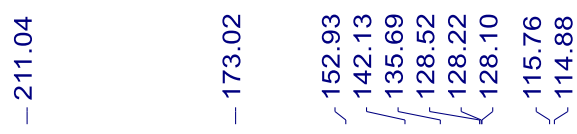

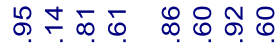

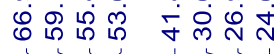

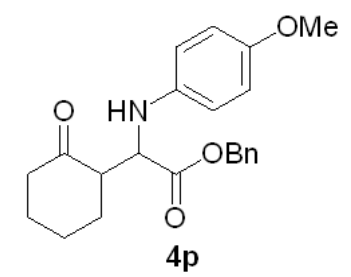

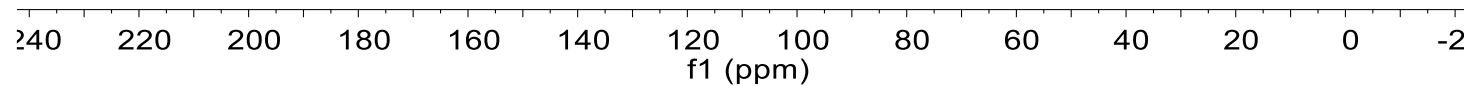

${ }^{1} \mathrm{H}$ NMR $(400 \mathrm{MHz})$ and ${ }^{13} \mathrm{C}\left\{{ }^{1} \mathrm{H}\right\}$ NMR $(101 \mathrm{MHz})$ spectra of $\mathbf{4 p}\left(\mathrm{CDCl}_{3}, \mathrm{rt}\right)$. 


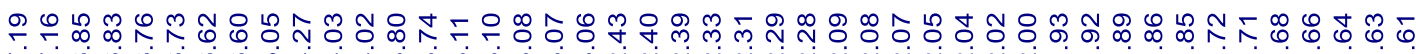

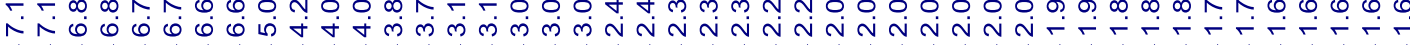

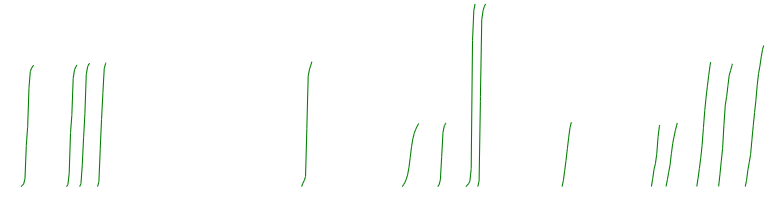<smiles>COc1ccc(COC(=O)C(Nc2ccc(OC)cc2)C2CCCCC2=O)cc1</smiles>

$4 q$

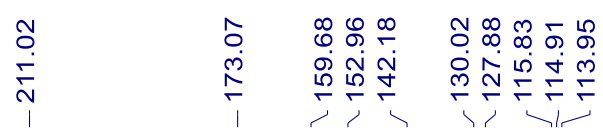<smiles>COc1ccc(COC(=O)C(Nc2ccc(OC)cc2)C2CCCCC2=O)cc1</smiles>

$4 q$

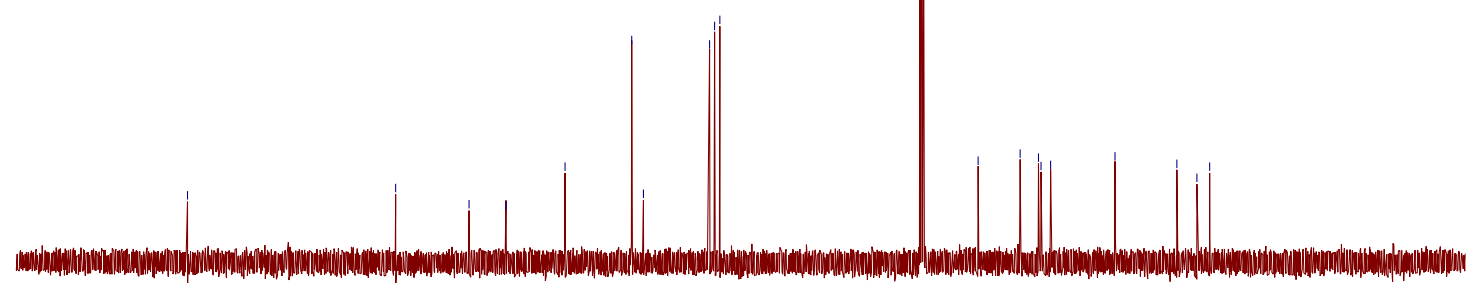

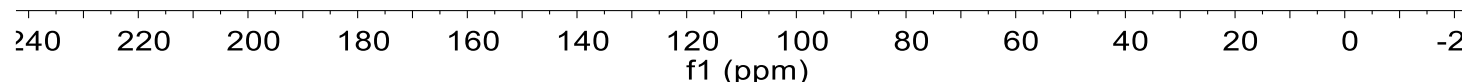

${ }^{1} \mathrm{H}$ NMR $(400 \mathrm{MHz})$ and ${ }^{13} \mathrm{C}\left\{{ }^{1} \mathrm{H}\right\}$ NMR $(101 \mathrm{MHz})$ spectra of $\mathbf{4 q}\left(\mathrm{CDCl}_{3}, \mathrm{rt}\right)$. 


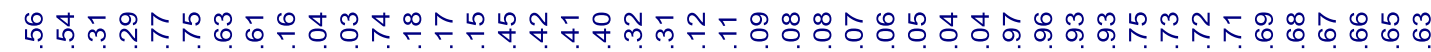

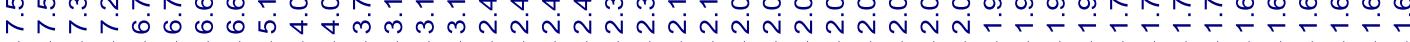

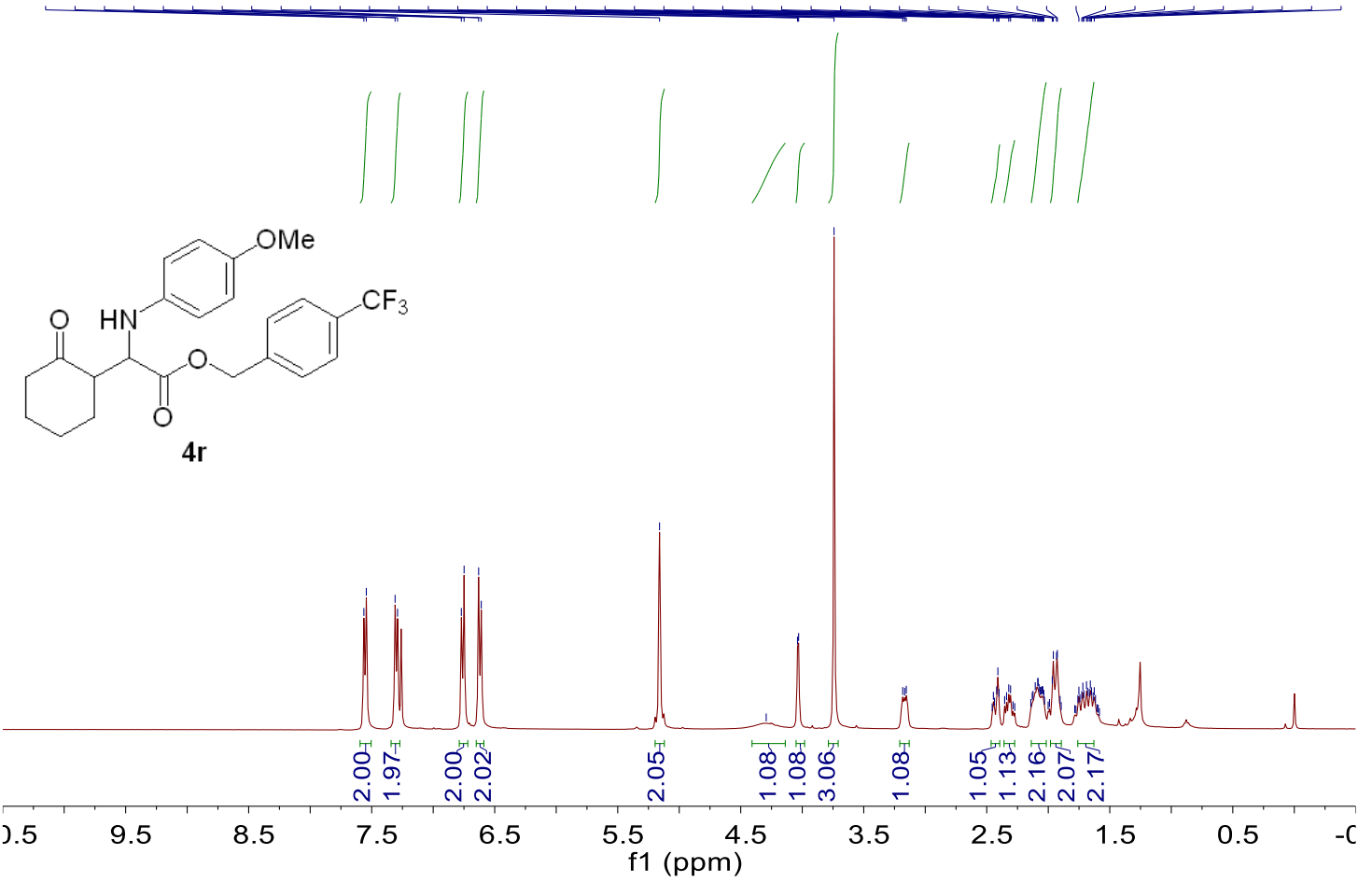

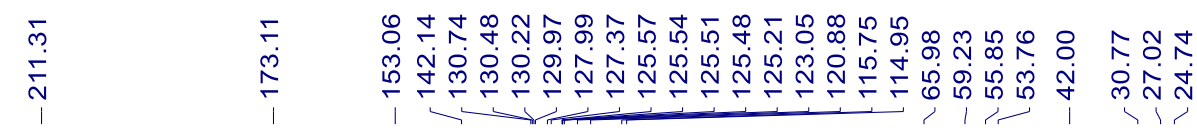

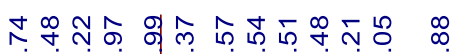

官官官怘

$21020019018017016015014013012011010090 \quad 80 \quad 70 \quad 60 \quad 50 \quad 40 \quad 30 \quad 20 \quad 10 \quad 0 \quad-10$ f1 (ppm) 


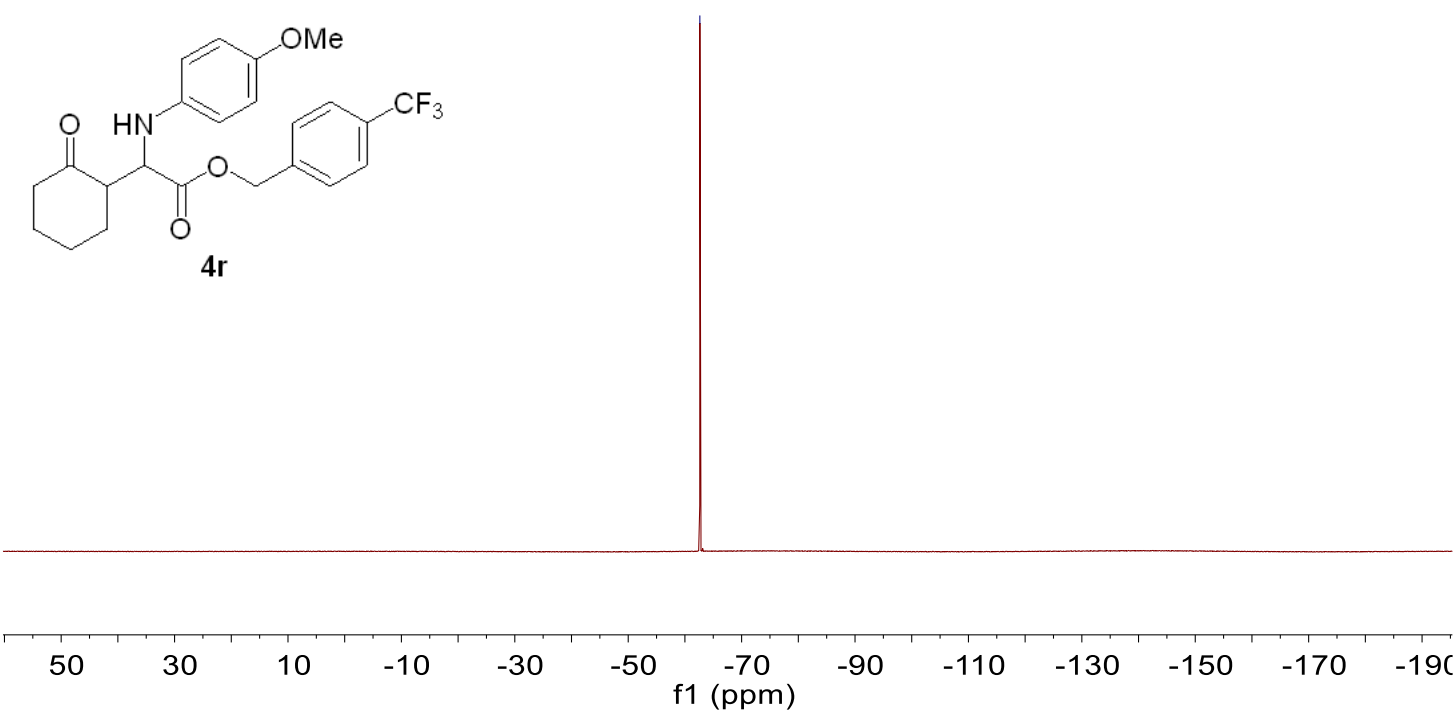

${ }^{1} \mathrm{H}$ NMR (400 MHz), ${ }^{13} \mathrm{C}\left\{{ }^{1} \mathrm{H}\right\}$ NMR (126 MHz), and ${ }^{19} \mathrm{~F} \mathrm{NMR}(376 \mathrm{MHz})$ spectra of $4 \mathbf{r}\left(\mathrm{CDCl}_{3}, \mathrm{rt}\right)$. 


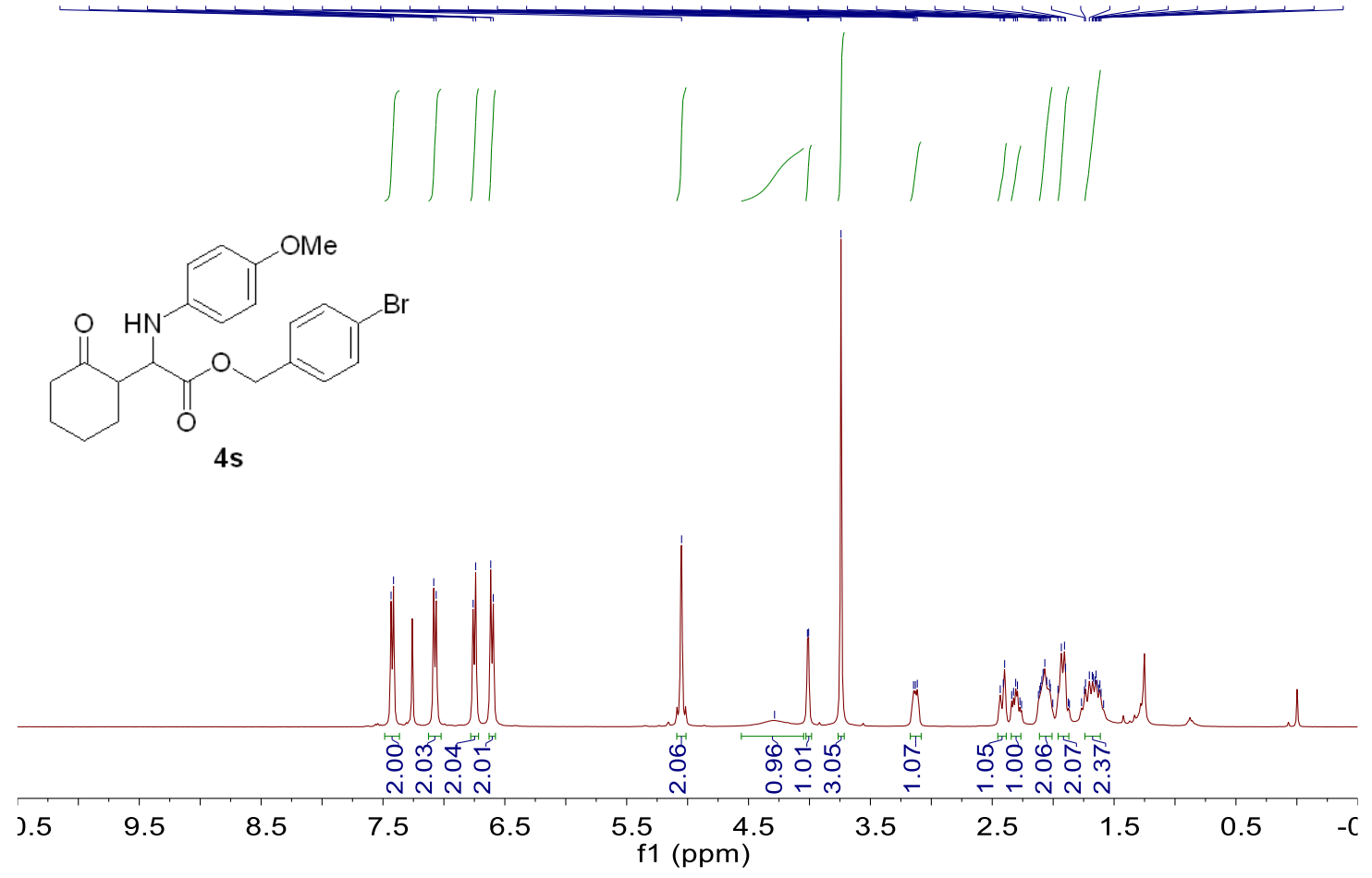

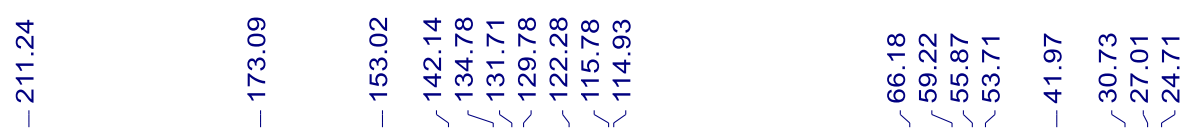<smiles>COc1ccc(NC(C(=O)OCc2ccc(Br)cc2)C2CCCCC2=O)cc1</smiles>

4s

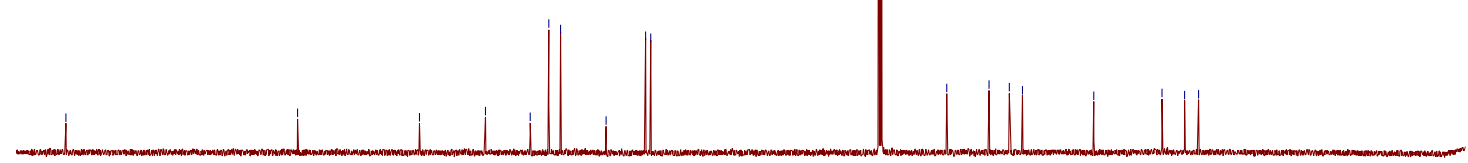

$21020019018017016015014013012011010090 \quad 80 \quad 70 \quad 60 \quad 50 \quad 40 \quad 30 \quad 20 \quad 10 \quad 0 \quad-10$ f1 (ppm)

${ }^{1} \mathrm{H}$ NMR $(400 \mathrm{MHz})$ and ${ }^{13} \mathrm{C}\left\{{ }^{1} \mathrm{H}\right\} \mathrm{NMR}(126 \mathrm{MHz})$ spectra of $4 \mathbf{s}\left(\mathrm{CDCl}_{3}, \mathrm{rt}\right)$. 


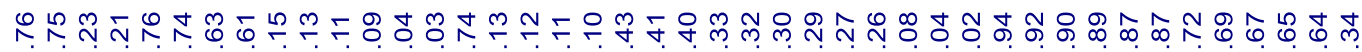

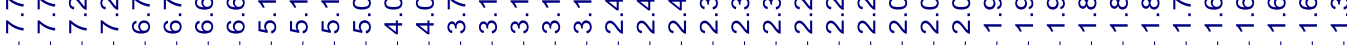
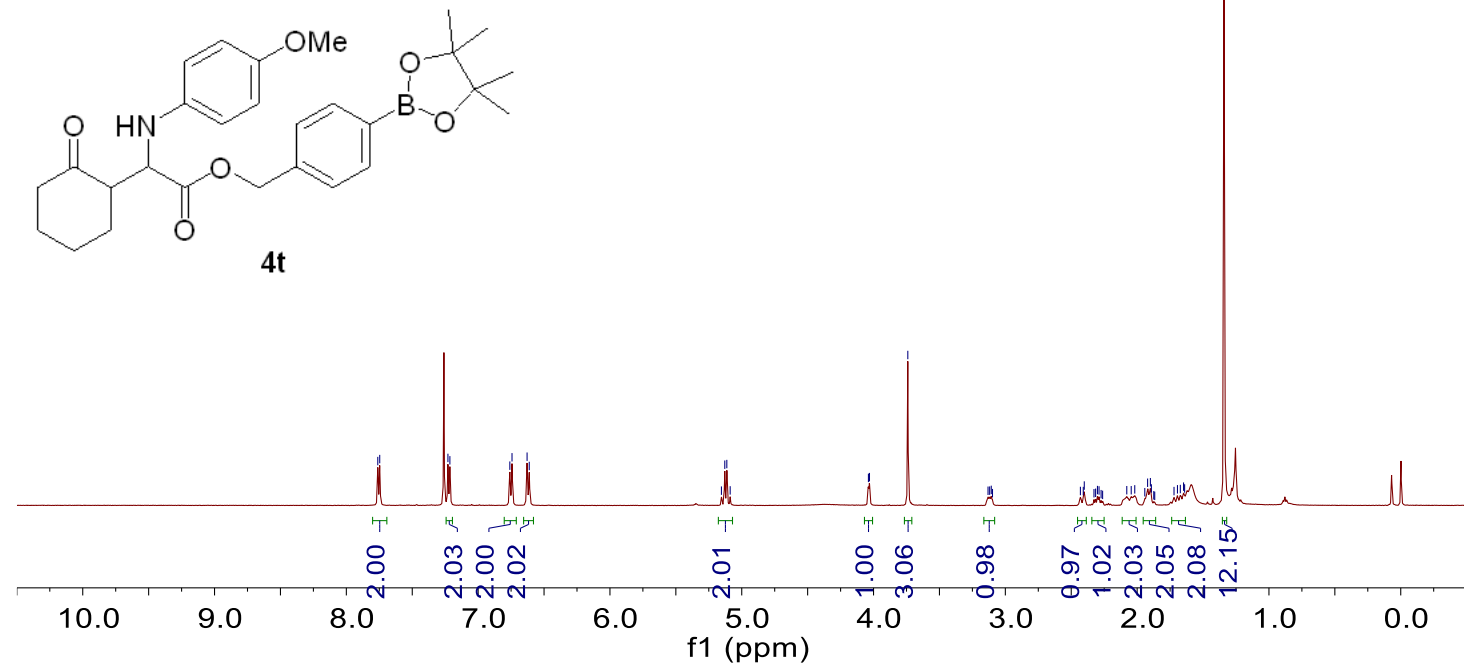

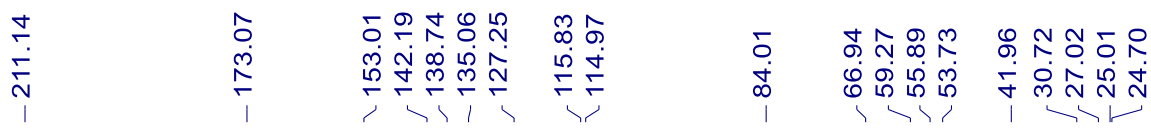

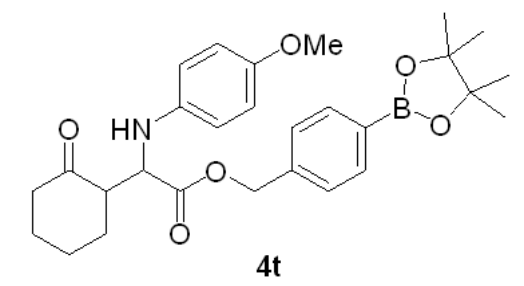

$4 \mathbf{t}$

230

$210 \quad 190 \quad 170 \quad 150$

$\begin{array}{ll}110 \\ \mathrm{f} 1 & (\mathrm{ppm})\end{array}$
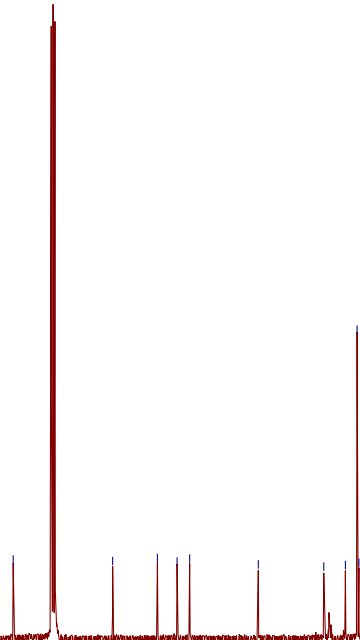

${ }^{1} \mathrm{H}$ NMR (500 MHz) and ${ }^{13} \mathrm{C}\left\{{ }^{1} \mathrm{H}\right\}$ NMR (101 MHz) spectra of $4 \mathbf{t}\left(\mathrm{CDCl}_{3}, \mathrm{rt}\right)$. 

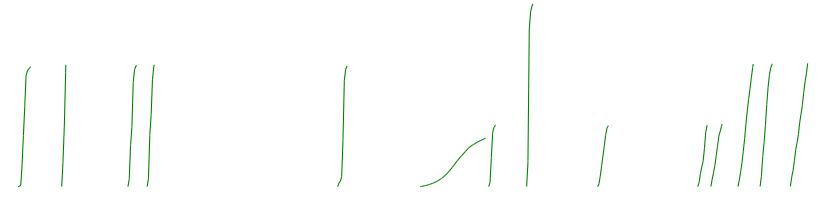<smiles>COc1ccc(NC(C(=O)OCc2ccc(C#N)cc2)C2CCCCC2=O)cc1</smiles>

$4 \mathrm{u}$
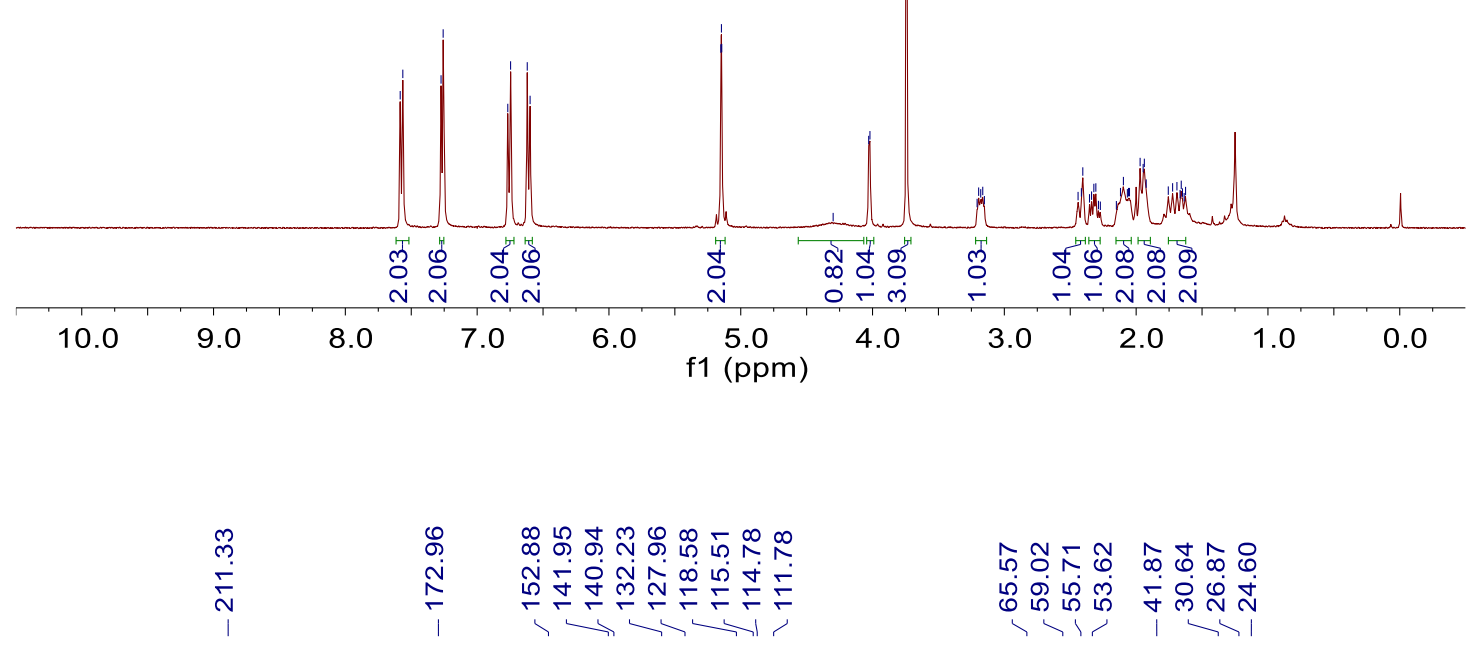

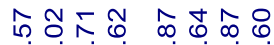

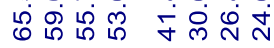<smiles>COc1ccc(NC(C(=O)OCc2ccc(C#N)cc2)C2CCCCC2=O)cc1</smiles>

$4 \mathrm{u}$

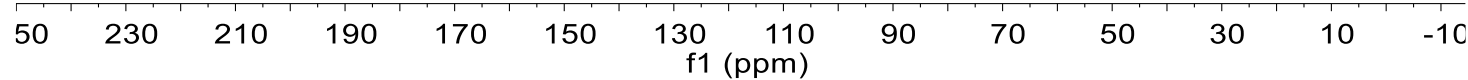

${ }^{1} \mathrm{H}$ NMR $(400 \mathrm{MHz})$ and ${ }^{13} \mathrm{C}\left\{{ }^{1} \mathrm{H}\right\}$ NMR $(101 \mathrm{MHz})$ spectra of $\mathbf{4 u}\left(\mathrm{CDCl}_{3}, \mathrm{rt}\right)$. 

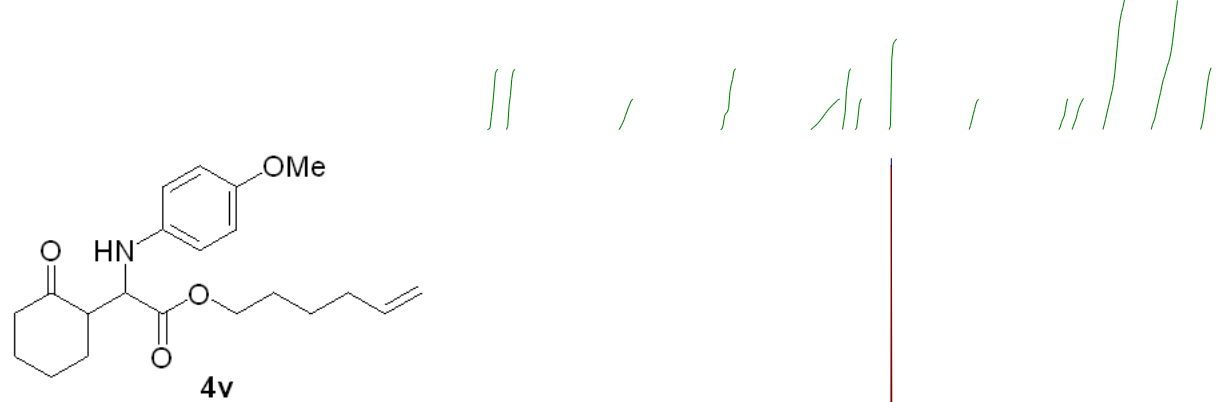

$4 v$

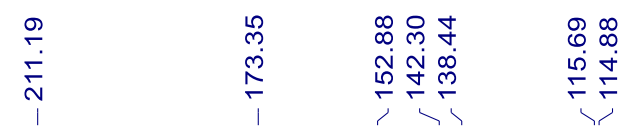

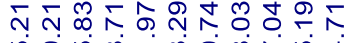

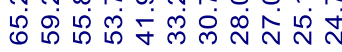<smiles>C=CCCCCOC(=O)C(Nc1ccc(OC)cc1)C1CCCCC1=O</smiles>

$4 v$

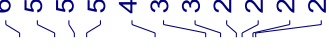

minth

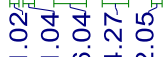
$\ulcorner-0$,

1 (ppm)
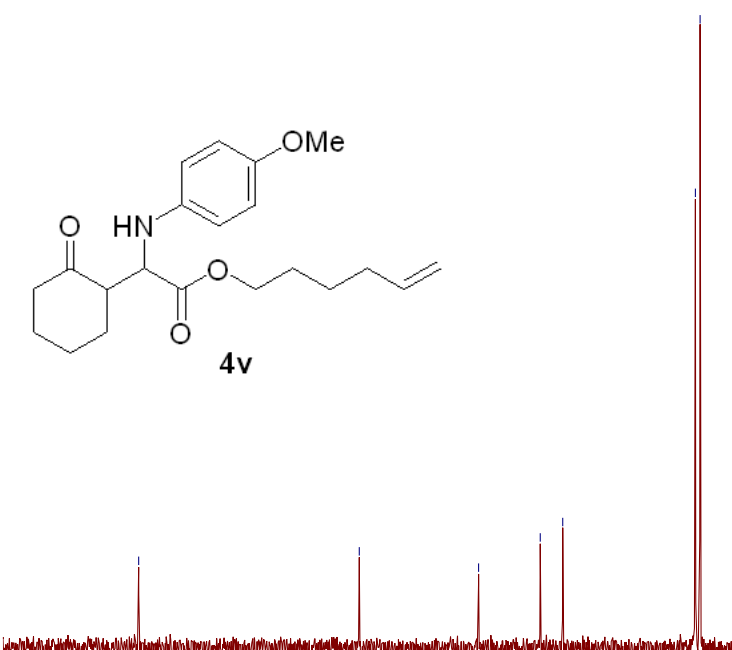

${ }^{1} \mathrm{H}$ NMR (400 MHz) and ${ }^{13} \mathrm{C}\left\{{ }^{1} \mathrm{H}\right\} \mathrm{NMR}(101 \mathrm{MHz})$ spectra of $\mathbf{4 v}\left(\mathrm{CDCl}_{3}, \mathrm{rt}\right)$. 

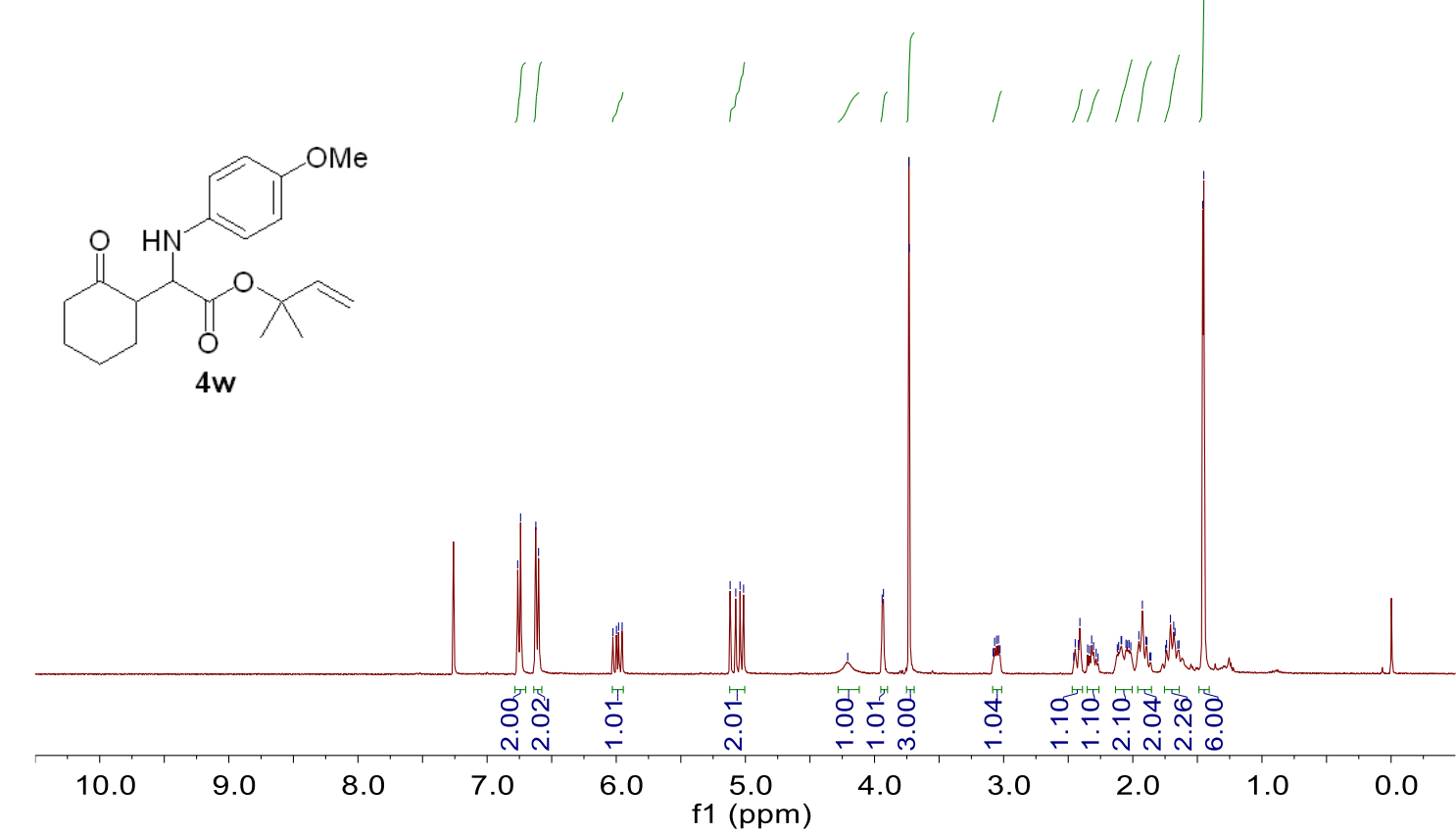

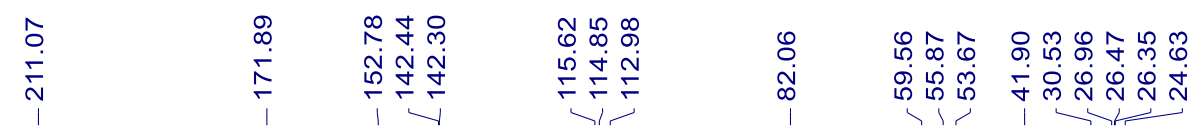

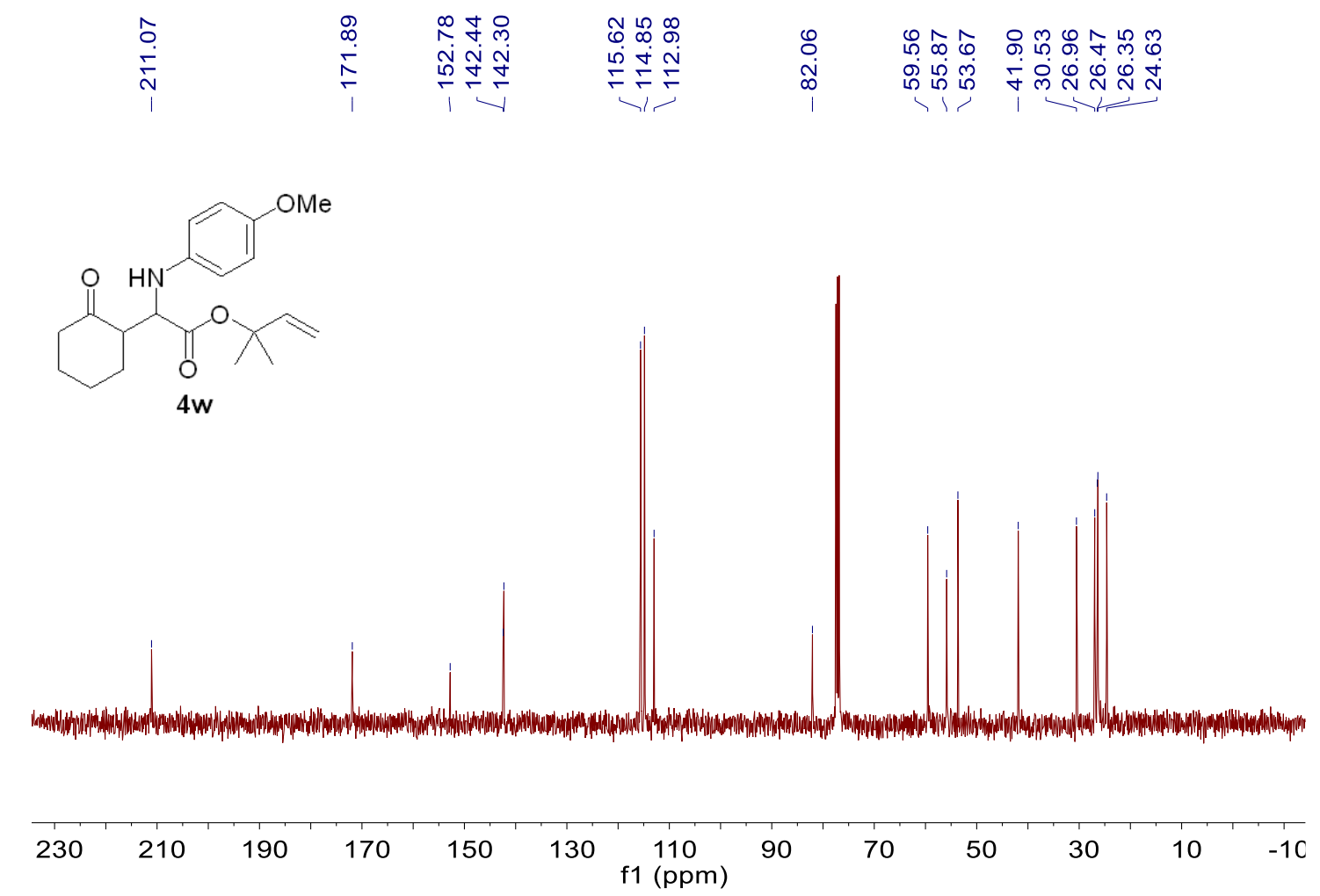

f1 (ppm)

${ }^{1} \mathrm{H}$ NMR $(400 \mathrm{MHz})$ and ${ }^{13} \mathrm{C}\left\{{ }^{1} \mathrm{H}\right\}$ NMR $(101 \mathrm{MHz})$ spectra of $\mathbf{4 w}\left(\mathrm{CDCl}_{3}, \mathrm{rt}\right)$. 

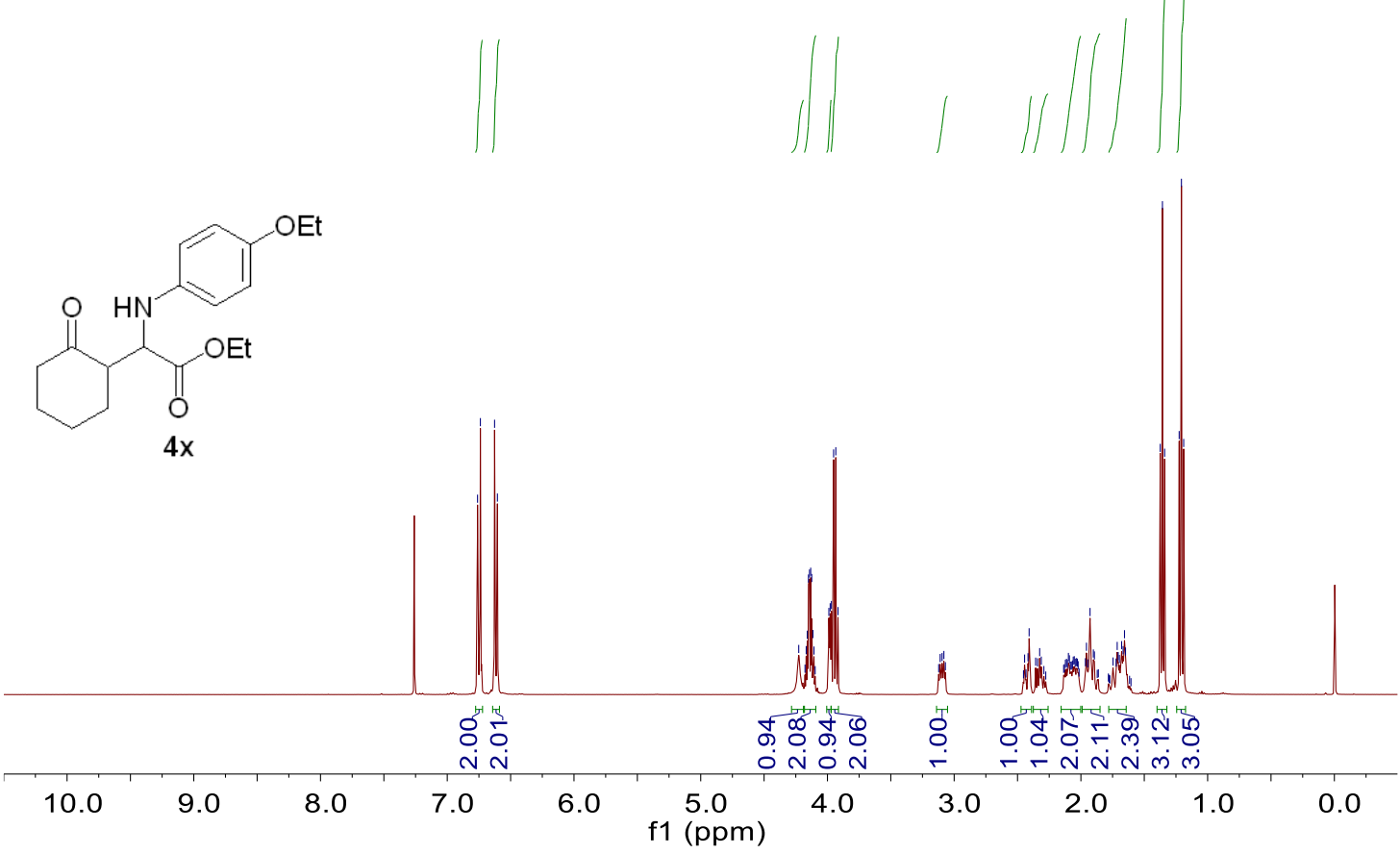

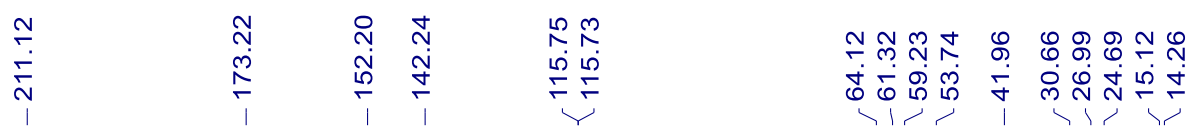

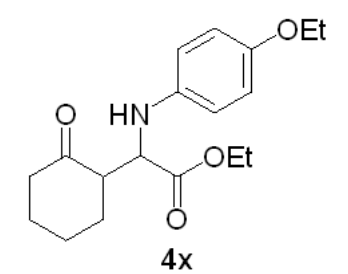

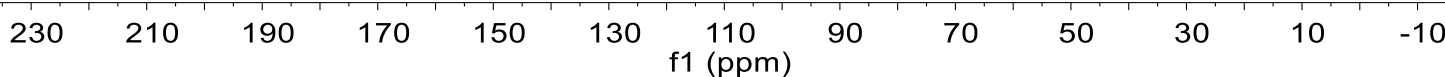

${ }^{1} \mathrm{H}$ NMR $(400 \mathrm{MHz})$ and ${ }^{13} \mathrm{C}\left\{{ }^{1} \mathrm{H}\right\}$ NMR $(101 \mathrm{MHz})$ spectra of $\mathbf{4 x}\left(\mathrm{CDCl}_{3}, \mathrm{rt}\right)$. 


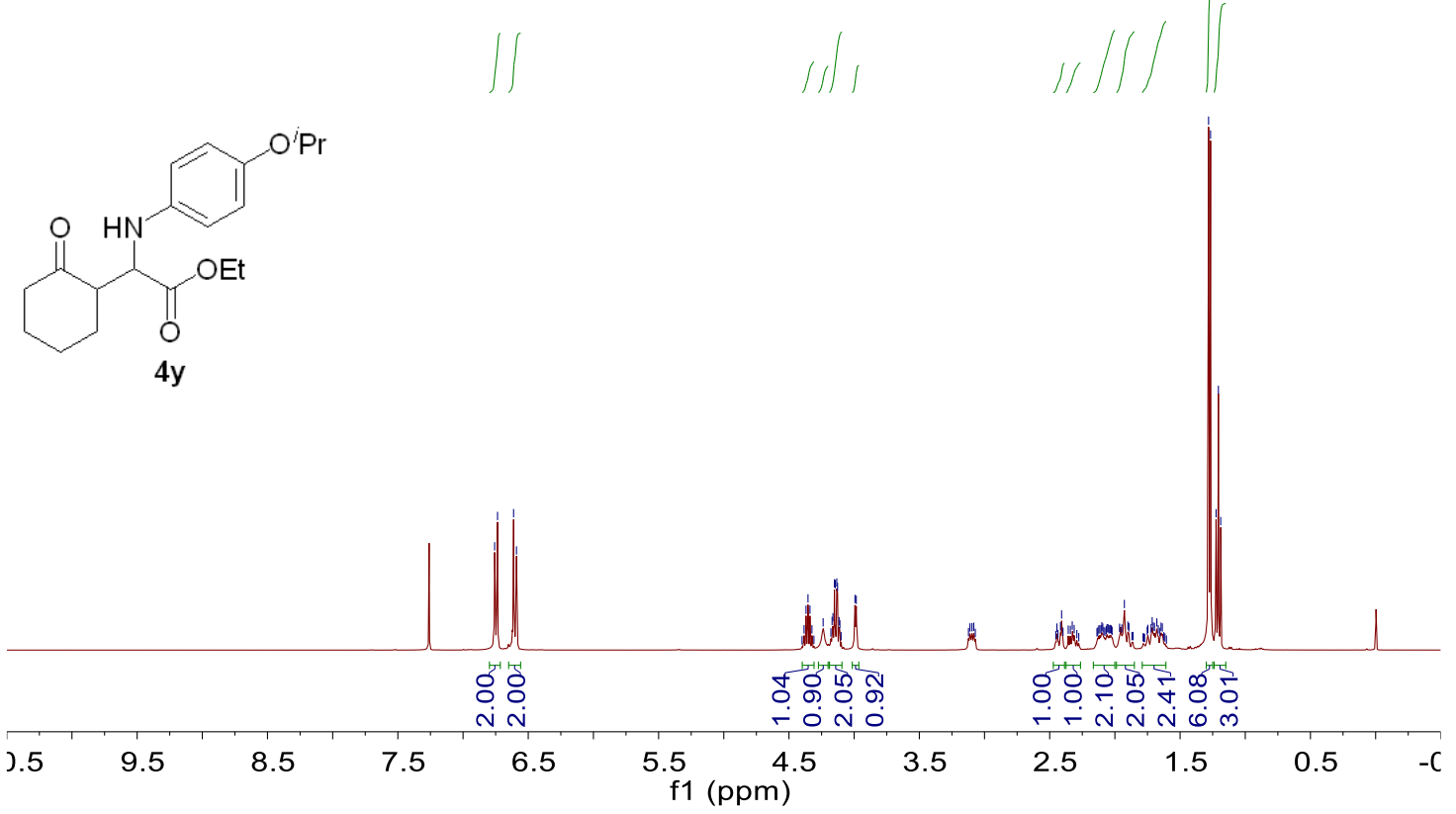

\begin{tabular}{|c|c|c|c|c|}
\hline $\bar{\Omega}$ & 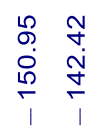 & 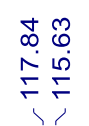 & 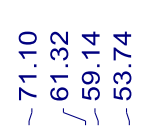 & 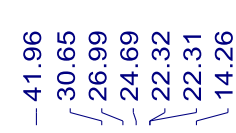 \\
\hline
\end{tabular}

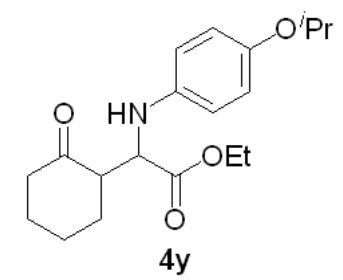

$230 \quad 210 \quad 190 \quad 170 \quad 150 \quad 130 \begin{gathered}110 \\ \mathrm{f} 1(\mathrm{ppm})\end{gathered}$

${ }^{1} \mathrm{H}$ NMR (400 MHz) and ${ }^{13} \mathrm{C}\left\{{ }^{1} \mathrm{H}\right\} \mathrm{NMR}(101 \mathrm{MHz})$ spectra of $\mathbf{4 y}\left(\mathrm{CDCl}_{3}, \mathrm{rt}\right)$. 

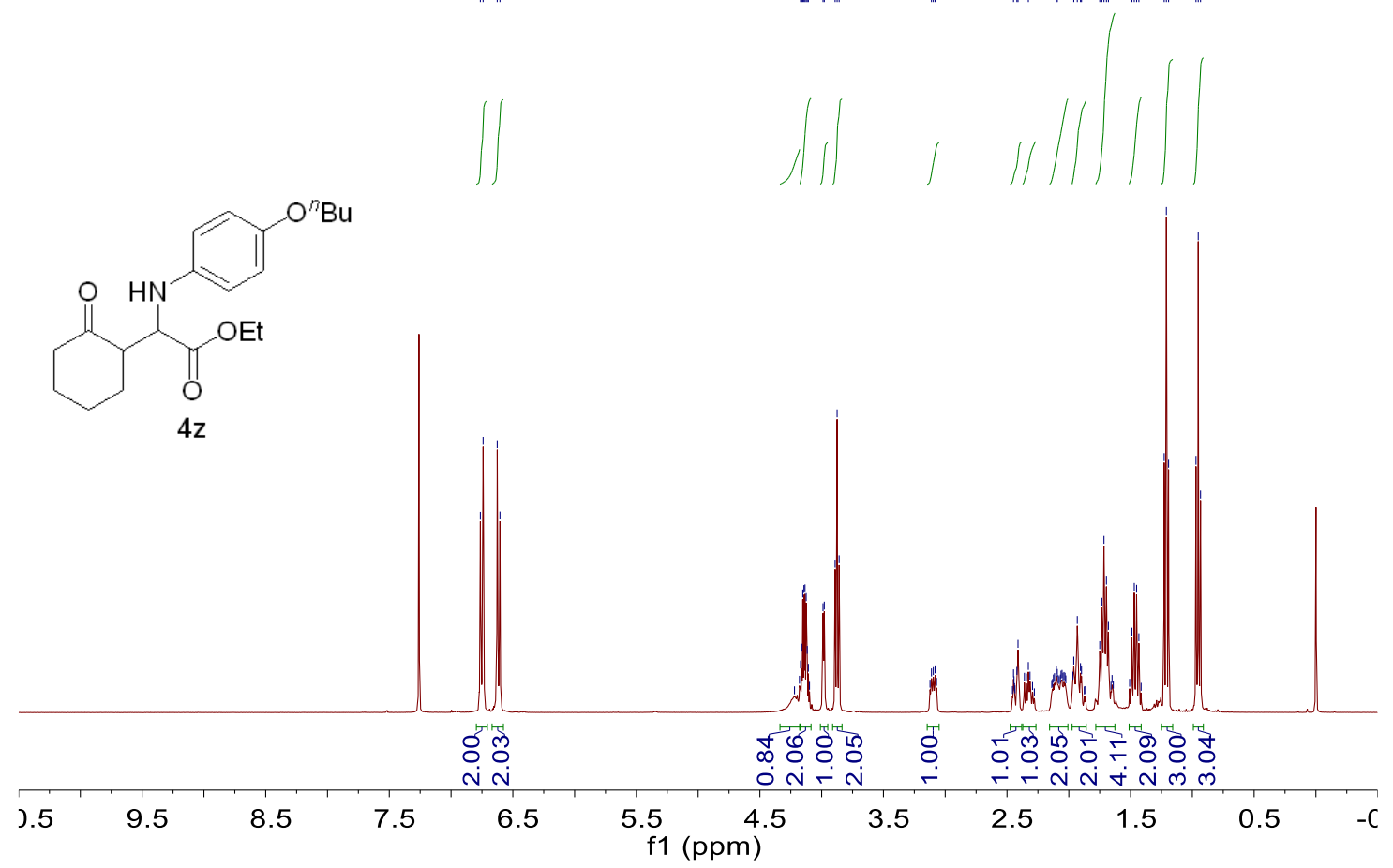

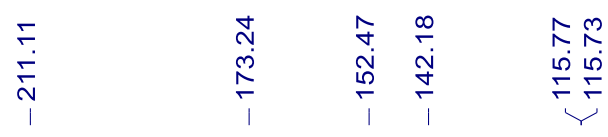

\section{ஸ m}

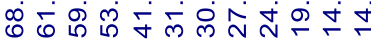
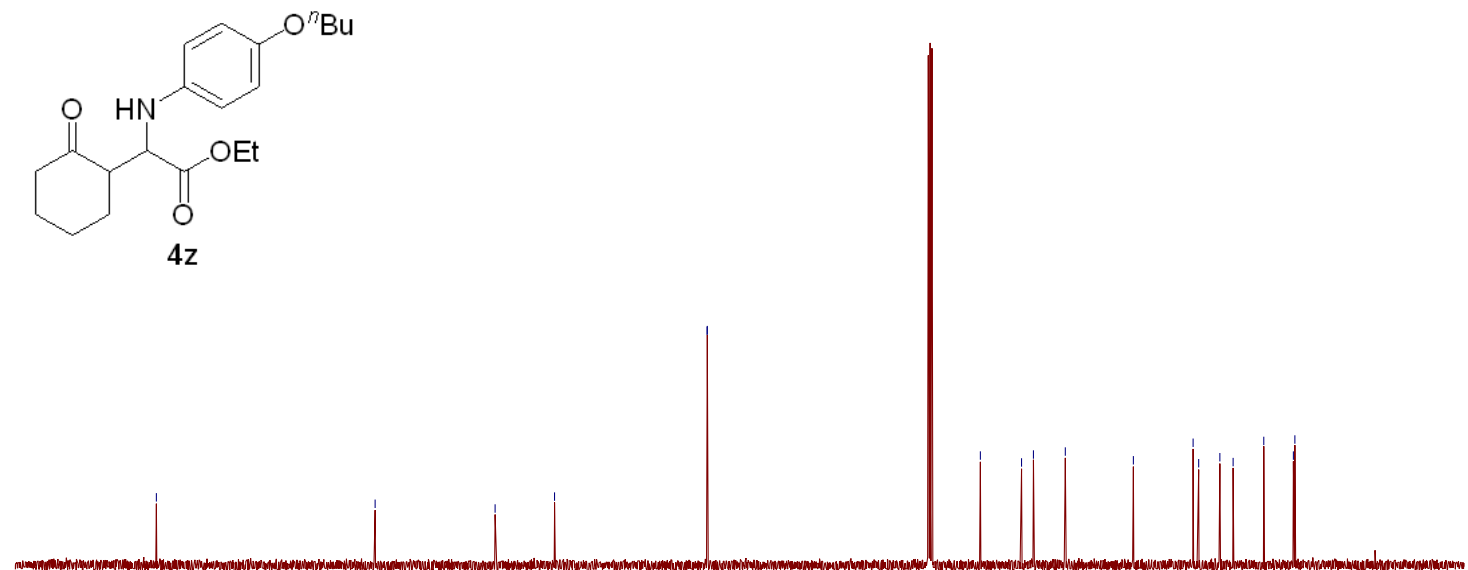

$230 \quad 210 \quad 190 \quad 170 \quad 150 \quad 130 \begin{gathered}110 \\ \mathrm{f} 1(\mathrm{ppm})\end{gathered}$

${ }^{1} \mathrm{H}$ NMR $(400 \mathrm{MHz})$ and ${ }^{13} \mathrm{C}\left\{{ }^{1} \mathrm{H}\right\}$ NMR $(101 \mathrm{MHz})$ spectra of $\mathbf{4 z}\left(\mathrm{CDCl}_{3}, \mathrm{rt}\right)$. 


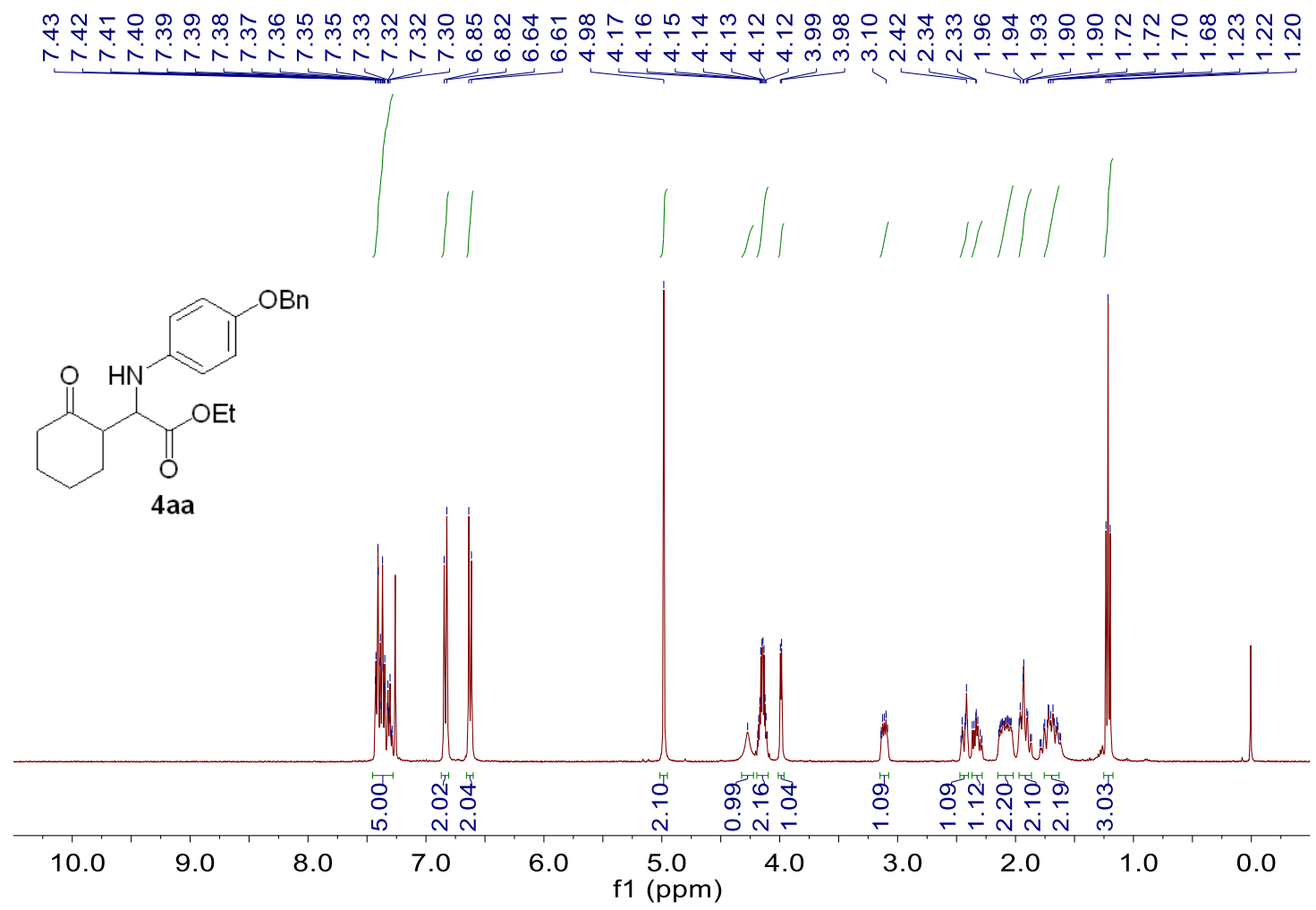

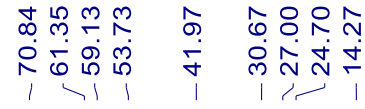<smiles>CCCCOc1ccc(NC(C(=O)OCC)C2CCCCC2=O)cc1</smiles>

${ }^{1} \mathrm{H}$ NMR $(400 \mathrm{MHz})$ and ${ }^{13} \mathrm{C}\left\{{ }^{1} \mathrm{H}\right\}$ NMR $(101 \mathrm{MHz})$ spectra of $4 \mathbf{a a}\left(\mathrm{CDCl}_{3}, \mathrm{rt}\right)$. 


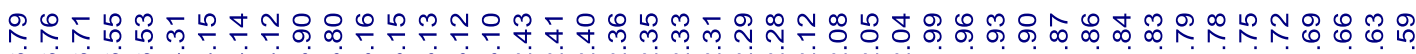

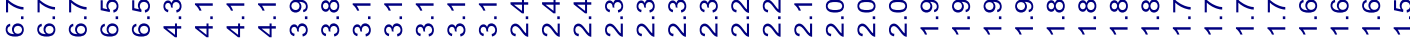<smiles>CCOC(=O)C(Nc1ccc(OC)c(Cl)c1)C1CCCCC1=O</smiles>
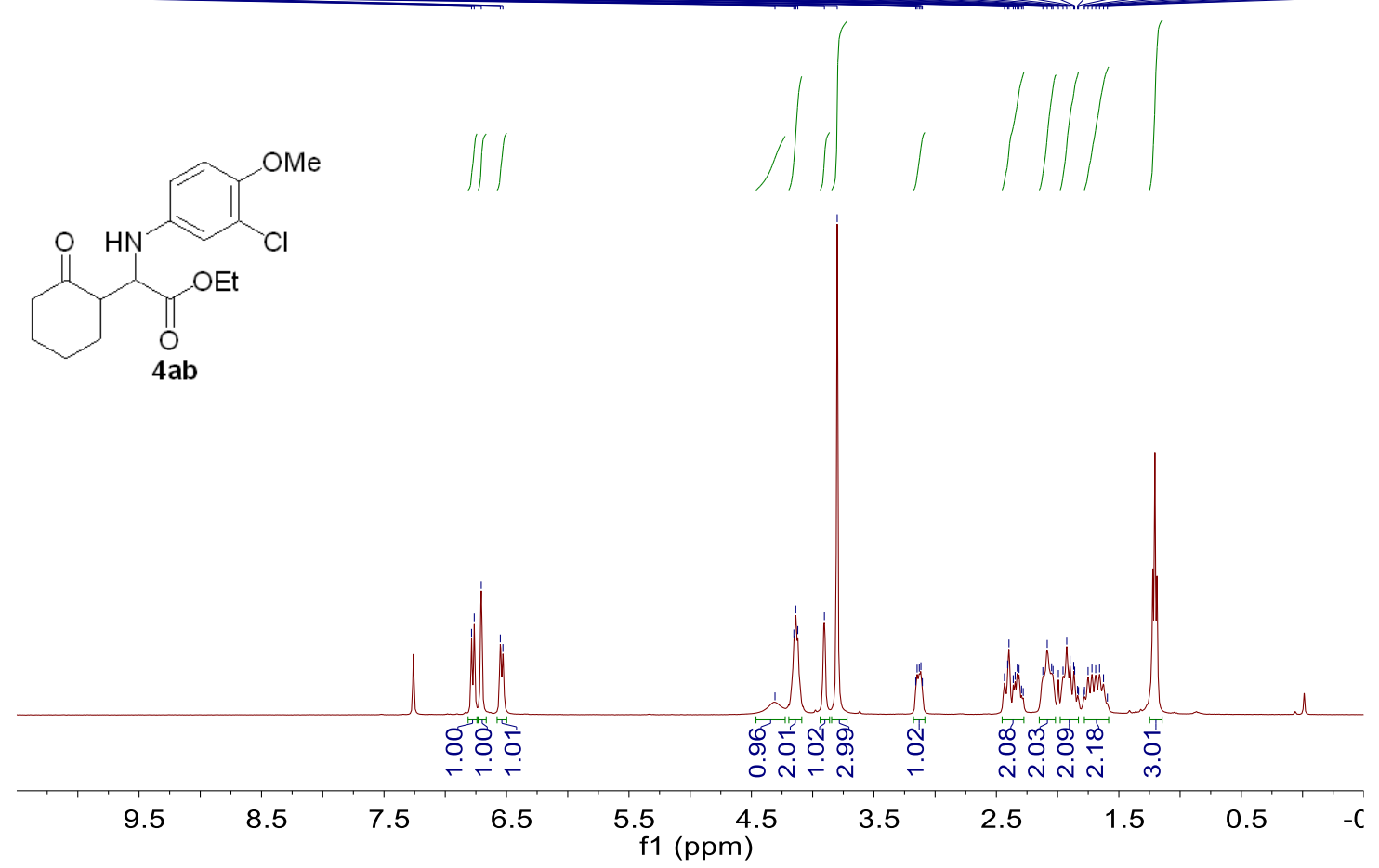

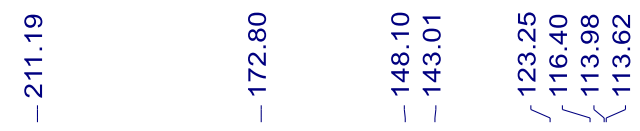<smiles>CCOC(=O)C(Nc1ccc(OC)c(Cl)c1)C1CCCCC1=O</smiles>

$4 a b$

${ }^{1} \mathrm{H}$ NMR (400 MHz) and ${ }^{13} \mathrm{C}\left\{{ }^{1} \mathrm{H}\right\}$ NMR (101 MHz) spectra of 4ab $\left(\mathrm{CDCl}_{3}, \mathrm{rt}\right)$. 


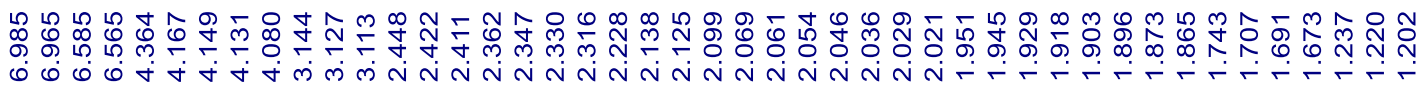

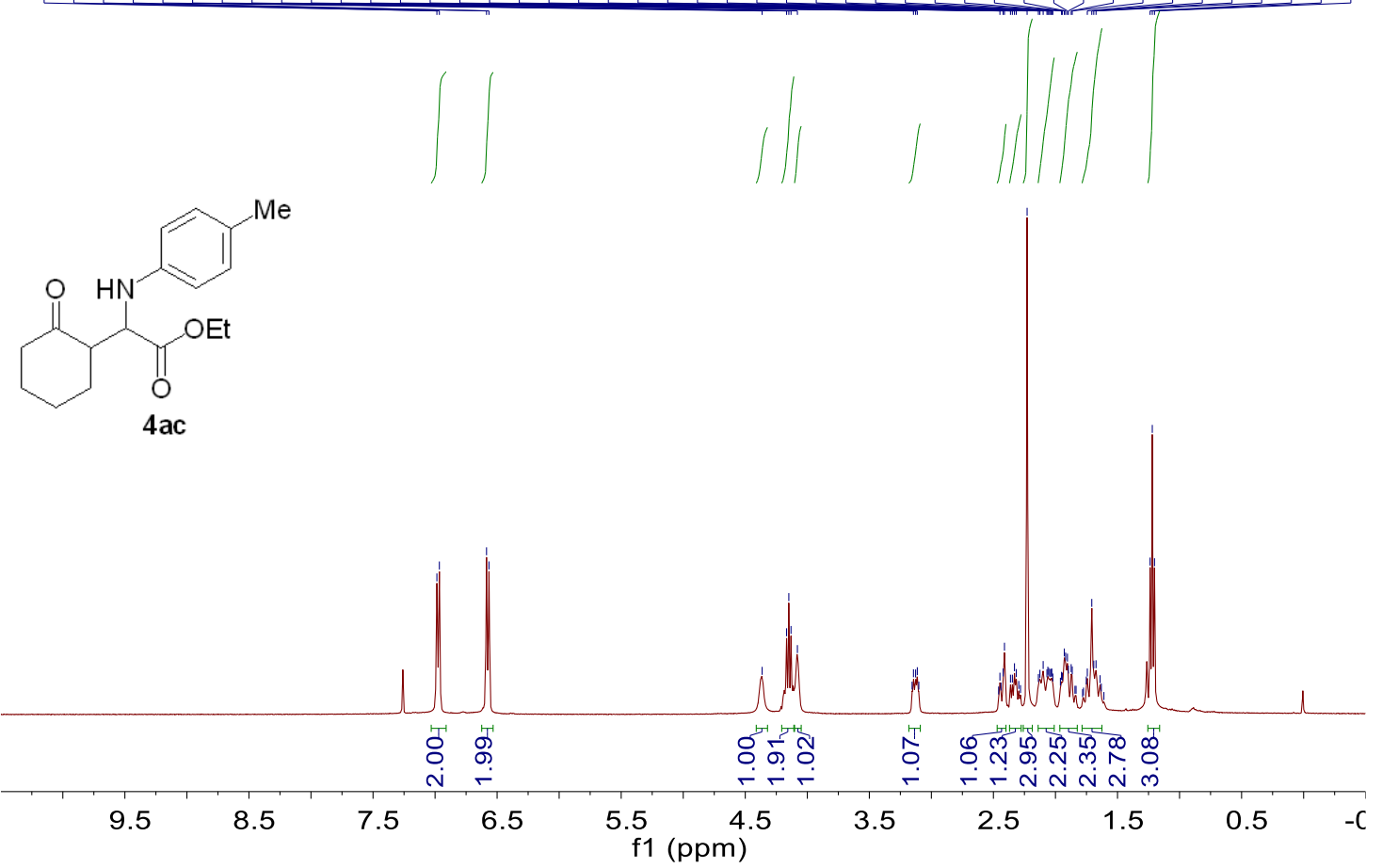

mु.

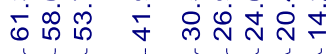<smiles>CCOC(=O)C(Nc1ccc(C)cc1)C1CCCCC1=O</smiles>
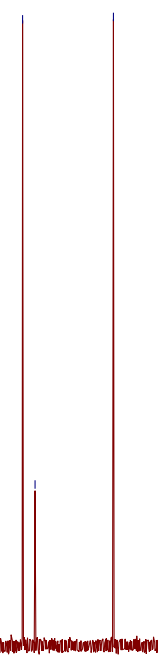

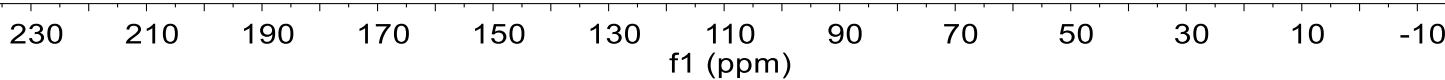

${ }^{1} \mathrm{H}$ NMR $(400 \mathrm{MHz})$ and ${ }^{13} \mathrm{C}\left\{{ }^{1} \mathrm{H}\right\}$ NMR $(101 \mathrm{MHz})$ spectra of $\mathbf{4 a c}\left(\mathrm{CDCl}_{3}, \mathrm{rt}\right)$. 
<smiles>CCOC(=O)C(Nc1ccc(CC)cc1)C1CCCCC1=O</smiles>
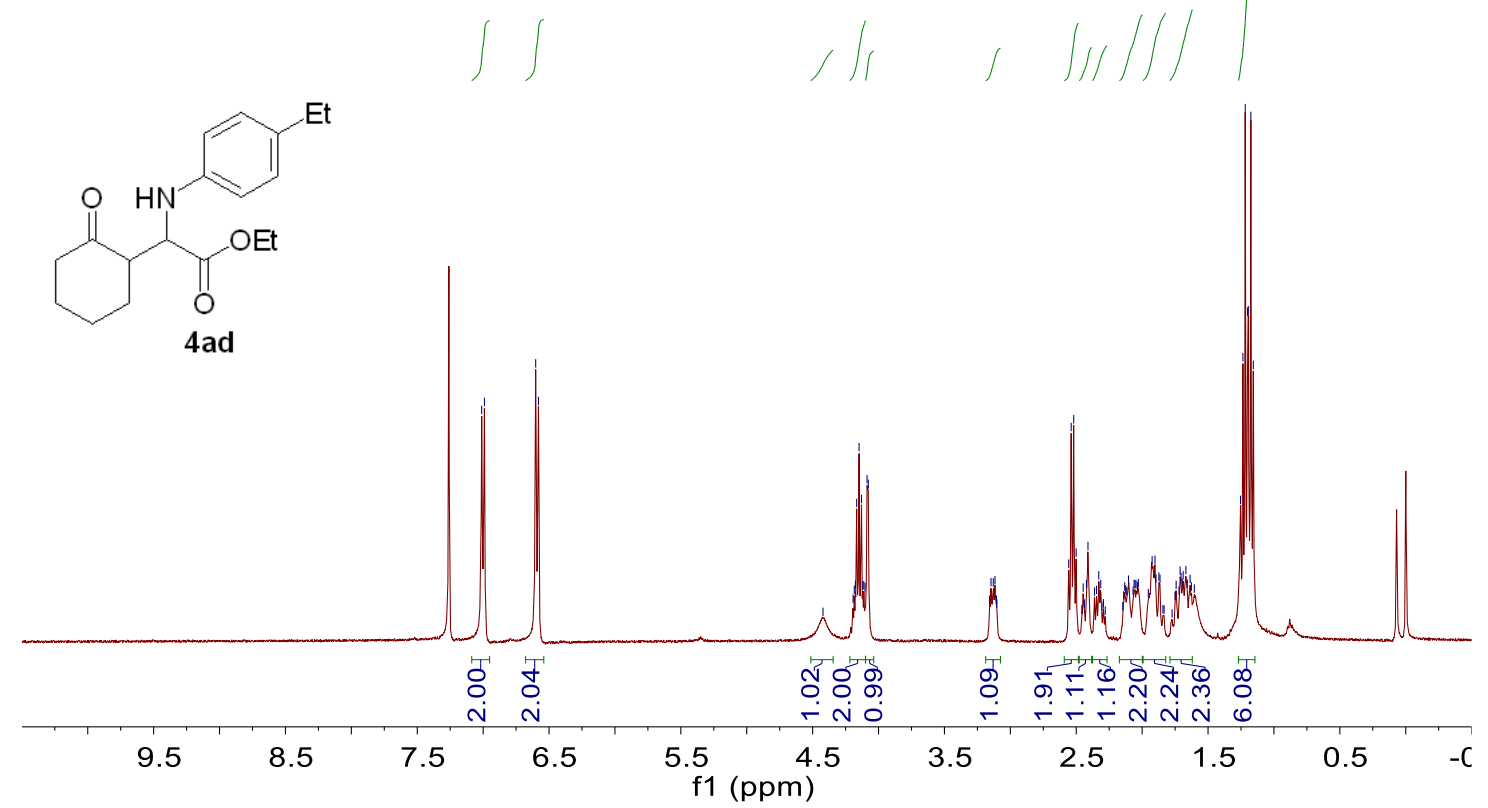

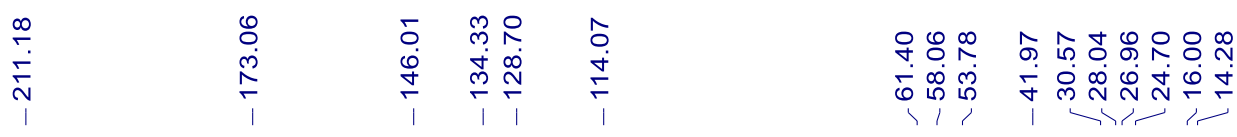<smiles>CCOC(=O)C(Nc1ccc(CC)cc1)C1CCCCC1=O</smiles>

$210 \quad 190 \quad 170$

150

$130 \quad 110$ f1 (ppm)

${ }^{1} \mathrm{H}$ NMR $(400 \mathrm{MHz})$ and ${ }^{13} \mathrm{C}\left\{{ }^{1} \mathrm{H}\right\}$ NMR $(101 \mathrm{MHz})$ spectra of $\mathbf{4 a d}\left(\mathrm{CDCl}_{3}, \mathrm{rt}\right)$. 

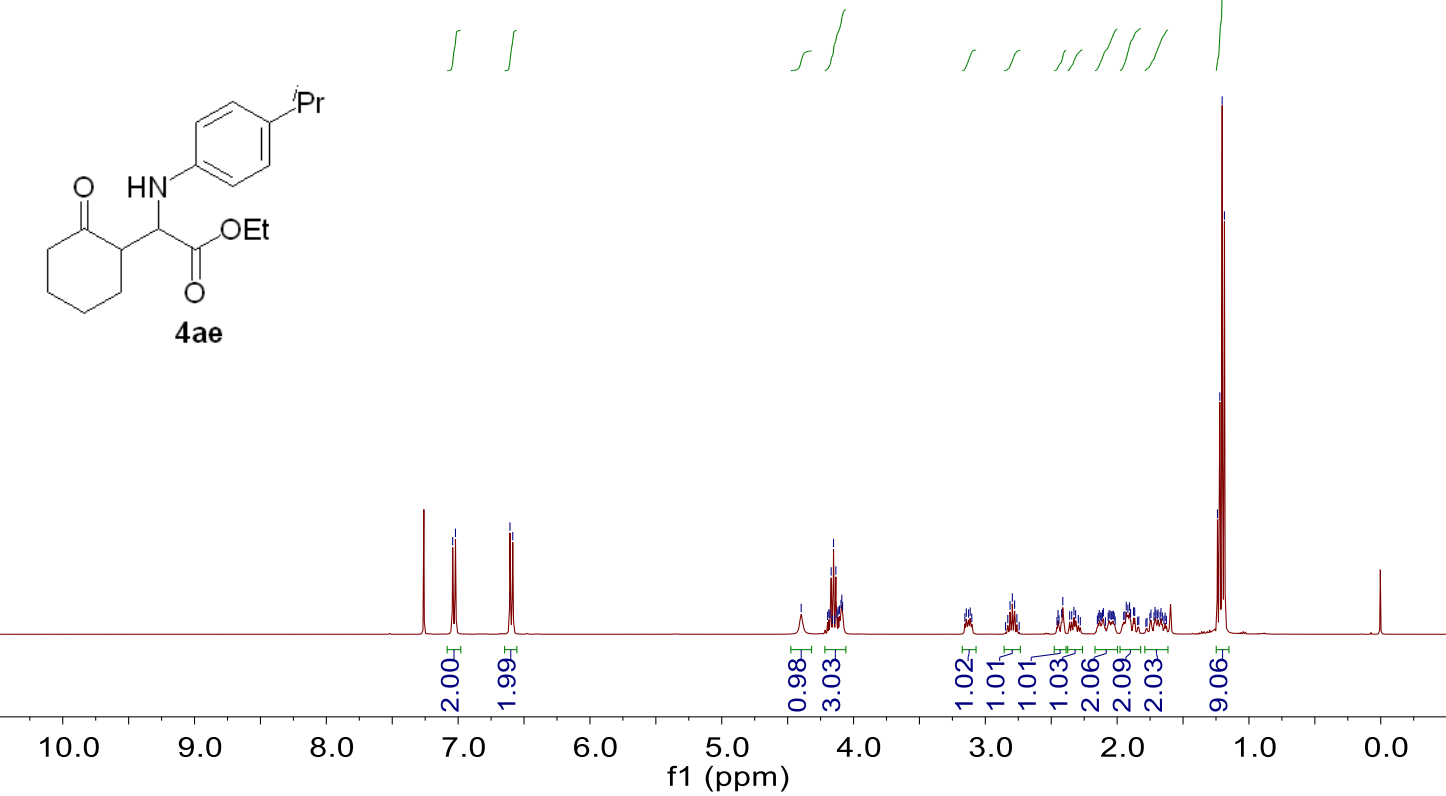

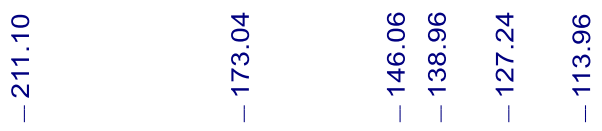

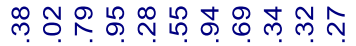

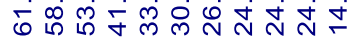<smiles>CCCc1ccc(NC(C(=O)OCC)C2CCCCC2=O)cc1</smiles>

${ }^{1} \mathrm{H}$ NMR $(400 \mathrm{MHz})$ and ${ }^{13} \mathrm{C}\left\{{ }^{1} \mathrm{H}\right\}$ NMR $(101 \mathrm{MHz})$ spectra of $4 \mathbf{a e}\left(\mathrm{CDCl}_{3}, \mathrm{rt}\right)$. 
<smiles>CCOC(=O)C(Nc1ccc(C(C)(C)C)cc1)C1CCCCC1=O</smiles><smiles>C1=CCC=C1</smiles>

4af

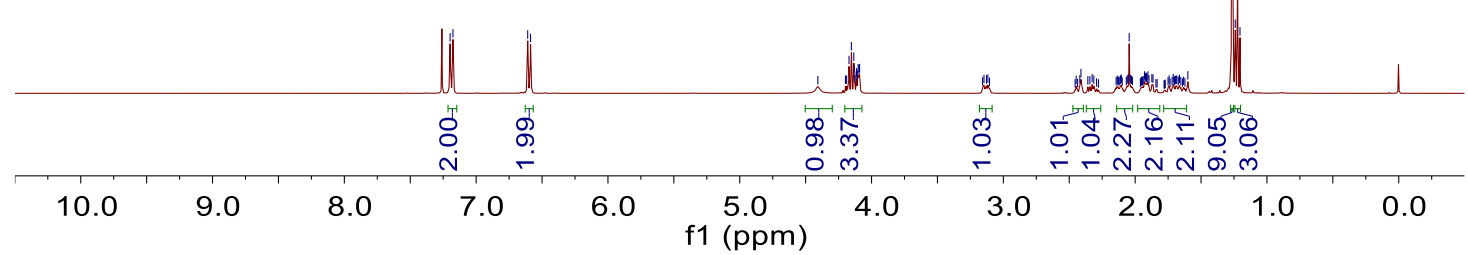

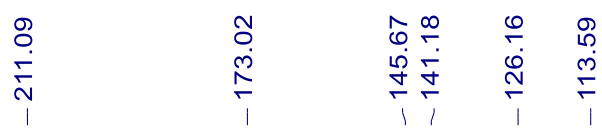

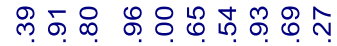

ঢ்

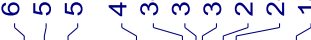<smiles>CCOC(=O)C(Nc1ccc(C(C)(C)C)cc1)C1CCCCC1=O</smiles>
4af

${ }^{1} \mathrm{H}$ NMR (400 MHz) and ${ }^{13} \mathrm{C}\left\{{ }^{1} \mathrm{H}\right\}$ NMR (101 MHz) spectra of 4af $\left(\mathrm{CDCl}_{3}, \mathrm{rt}\right)$. 


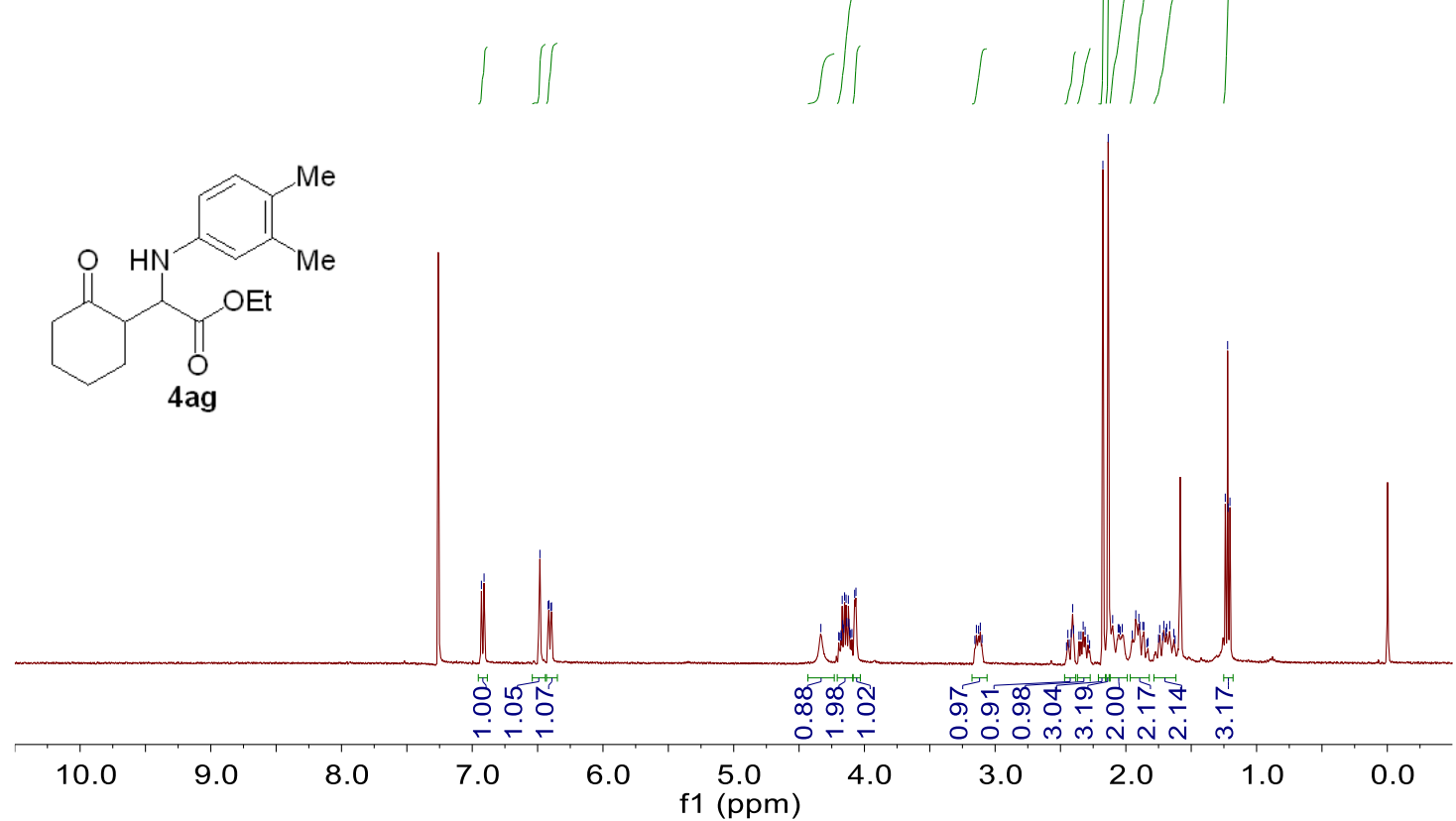

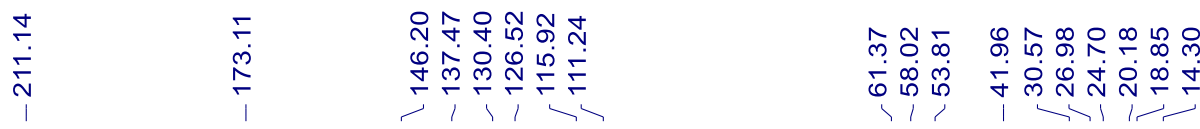<smiles>CCOC(=O)C(Nc1ccc(C)c(C)c1)C1CCCCC1=O</smiles>

${ }^{1} \mathrm{H}$ NMR $(400 \mathrm{MHz})$ and ${ }^{13} \mathrm{C}\left\{{ }^{1} \mathrm{H}\right\}$ NMR $(101 \mathrm{MHz})$ spectra of $4 \mathbf{a g}\left(\mathrm{CDCl}_{3}, \mathrm{rt}\right)$. 
<smiles>CCOC(=O)C(Nc1cc(C)cc(C)c1)C1CCCCC1=O</smiles>

4ah
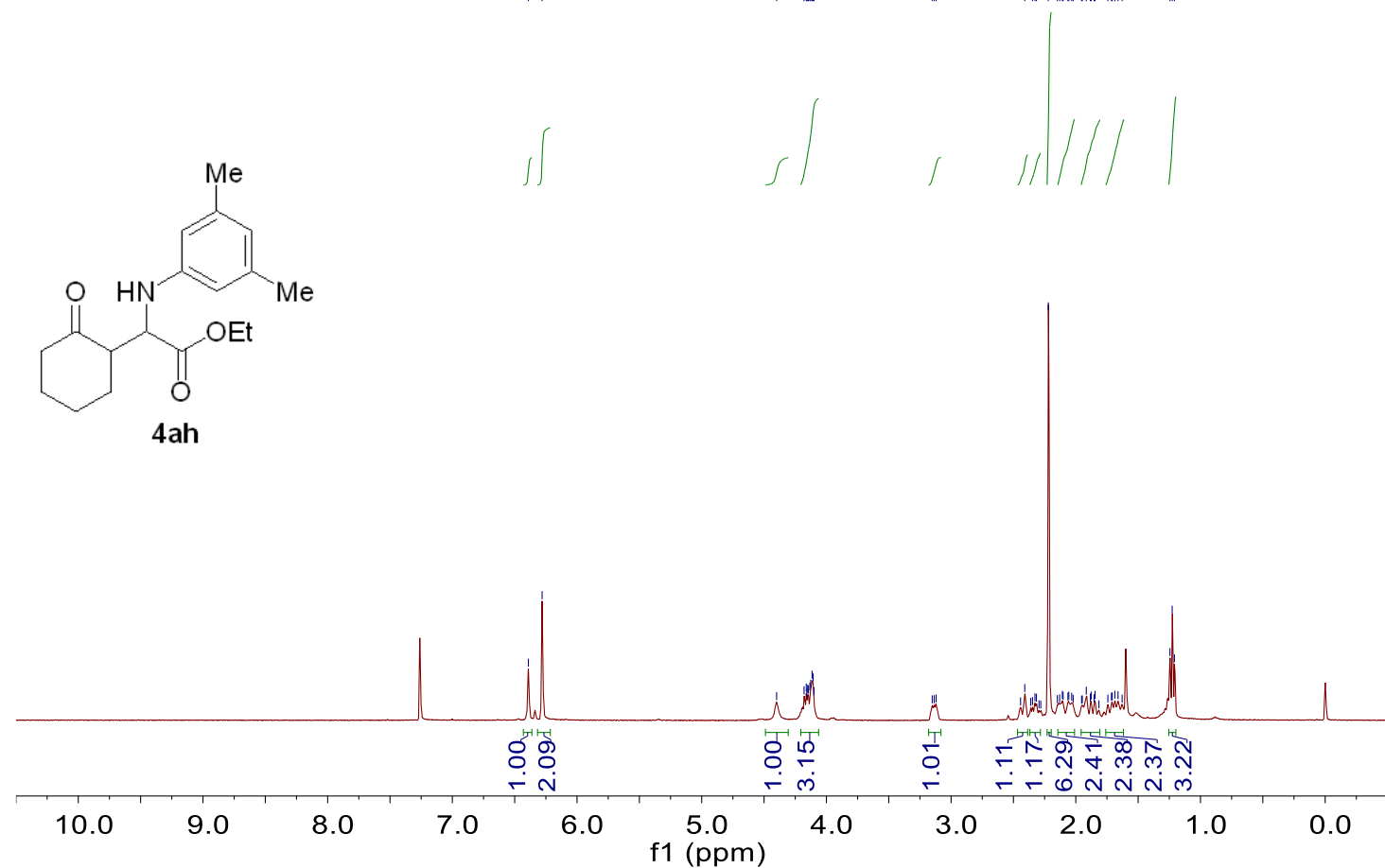

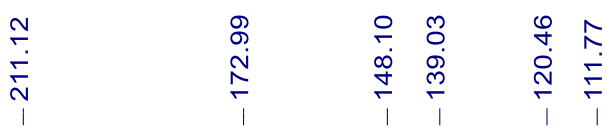

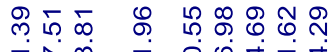

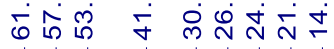<smiles>CCOC(=O)C(Nc1cc(C)cc(C)c1)C1CCCCC1=O</smiles>

4 ah

${ }^{1} \mathrm{H}$ NMR $(400 \mathrm{MHz})$ and ${ }^{13} \mathrm{C}\left\{{ }^{1} \mathrm{H}\right\}$ NMR $(101 \mathrm{MHz})$ spectra of 4ah $\left(\mathrm{CDCl}_{3}, \mathrm{rt}\right)$. 


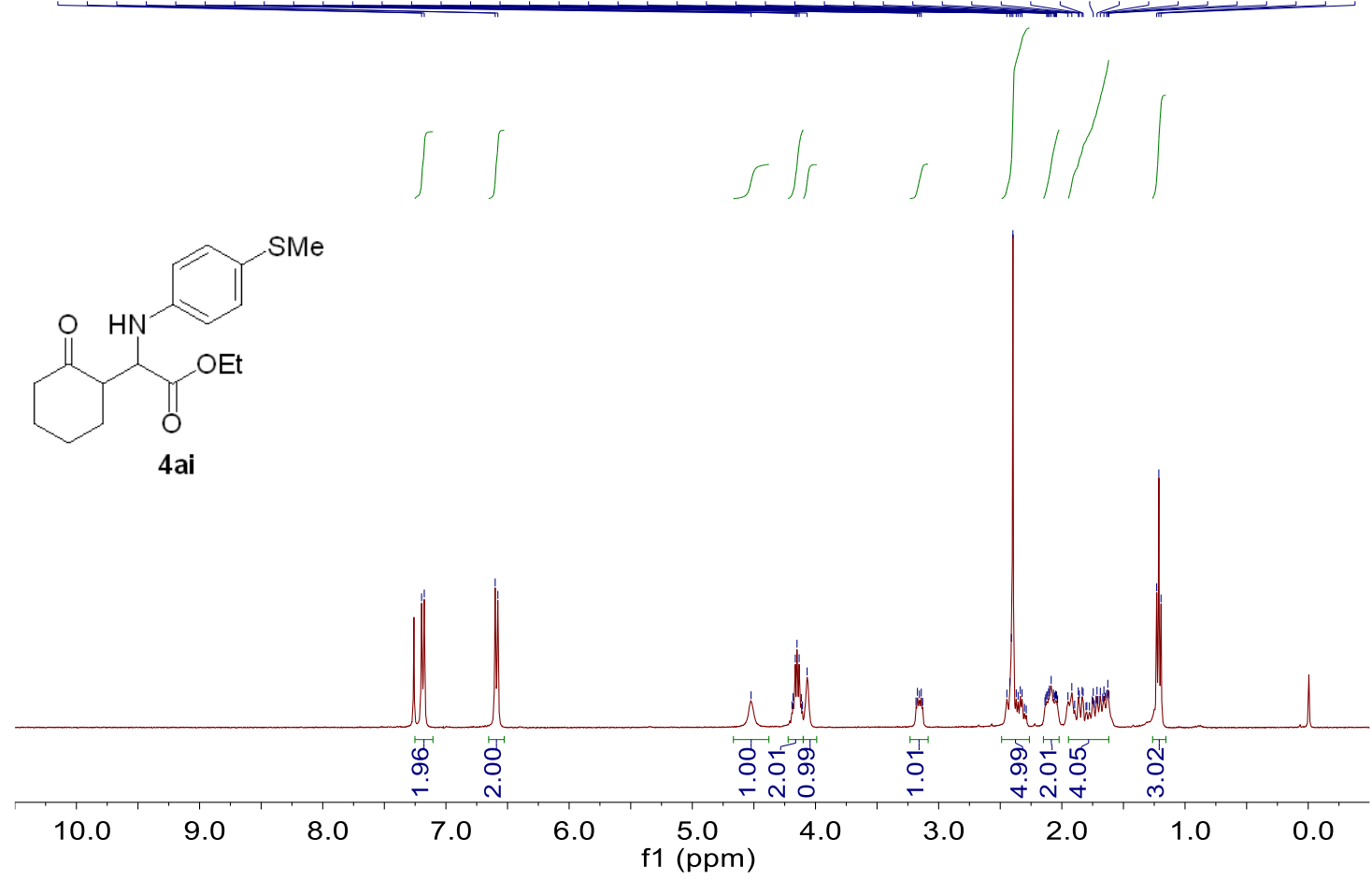

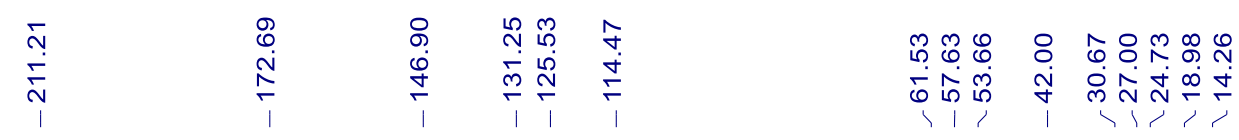

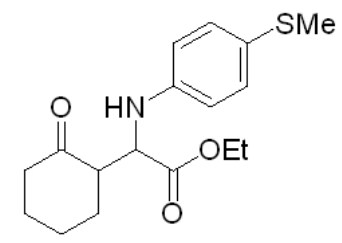

4ai

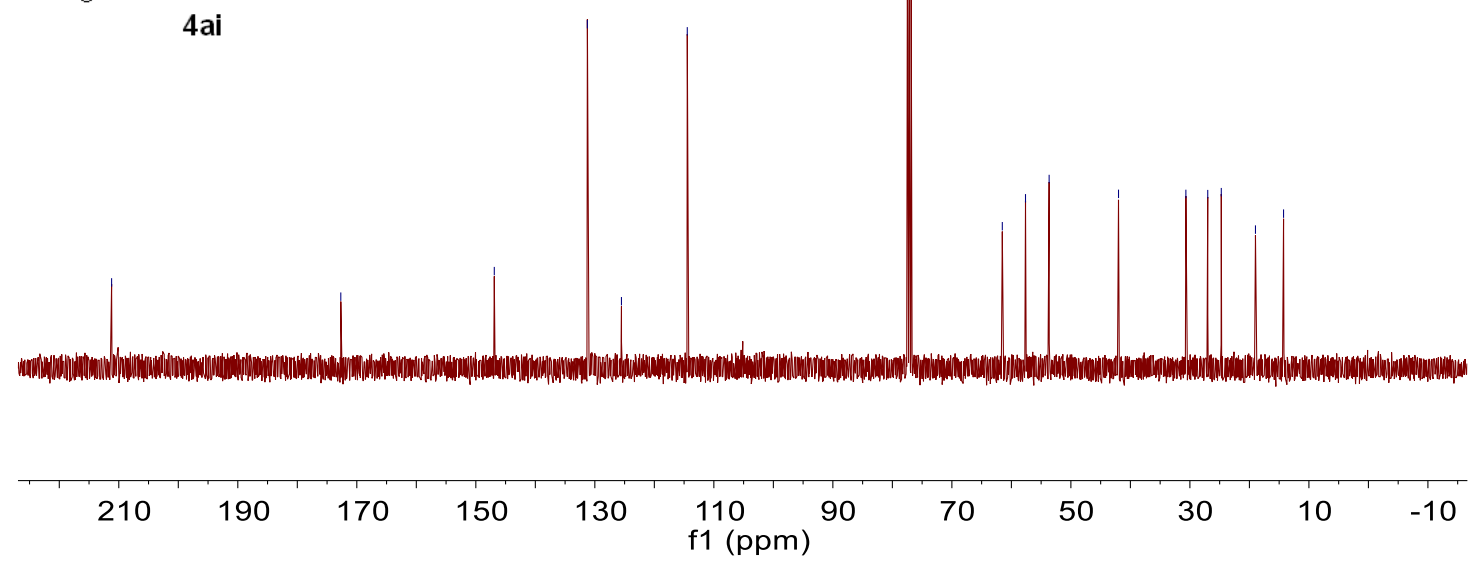

${ }^{1} \mathrm{H}$ NMR $(400 \mathrm{MHz})$ and ${ }^{13} \mathrm{C}\left\{{ }^{1} \mathrm{H}\right\}$ NMR $(101 \mathrm{MHz})$ spectra of 4ai $\left(\mathrm{CDCl}_{3}, \mathrm{rt}\right)$. 


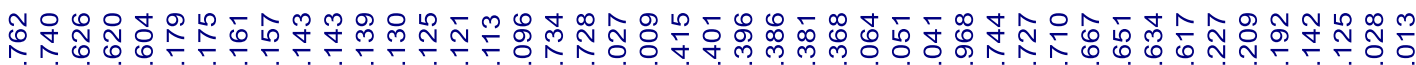

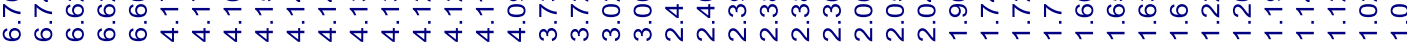

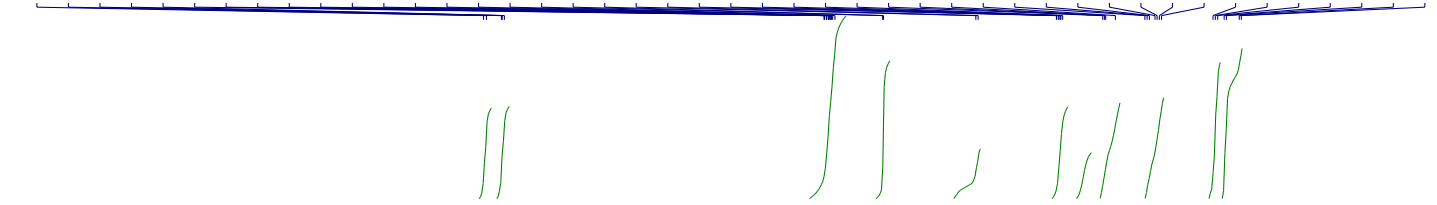<smiles>CCOC(=O)C(Nc1ccc(OC)cc1)C1CC(C)CCC1=O</smiles>

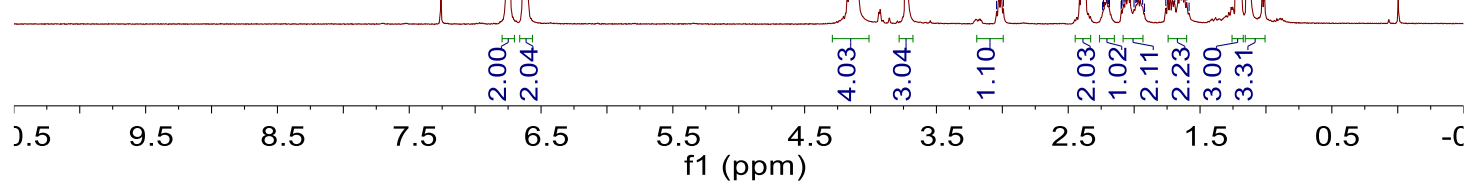

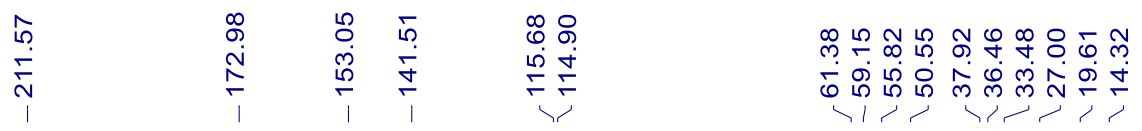

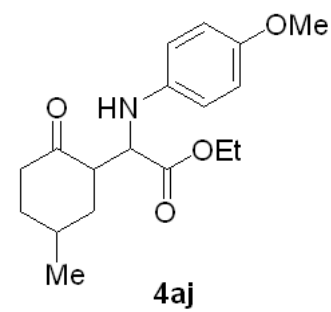

4aj

240

220

$200 \quad 180 \quad 160$

140

120
$\mathrm{f} 1(\mathrm{ppm})$

$80 \quad 60$

20

$-2$

${ }^{1} \mathrm{H}$ NMR (400 MHz) and ${ }^{13} \mathrm{C}\left\{{ }^{1} \mathrm{H}\right\}$ NMR (101 MHz) spectra of 4aj $\left(\mathrm{CDCl}_{3}, \mathrm{rt}\right)$. 


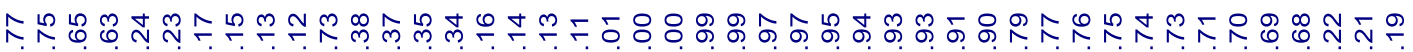

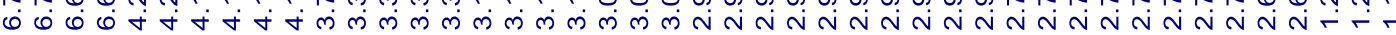<smiles>CCOC(=O)C(Nc1ccc(OC)cc1)C1CSCCC1=O</smiles>
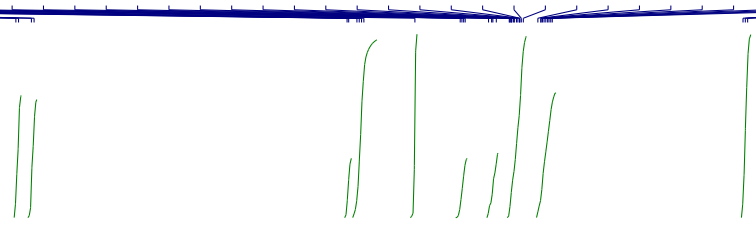

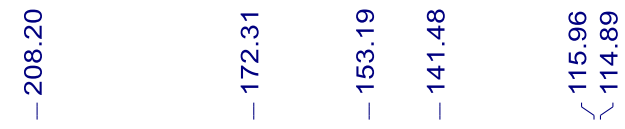

ก๊ำ

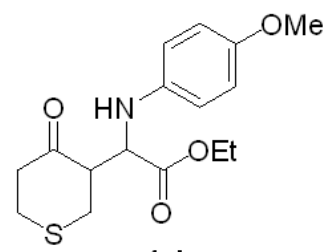

4al
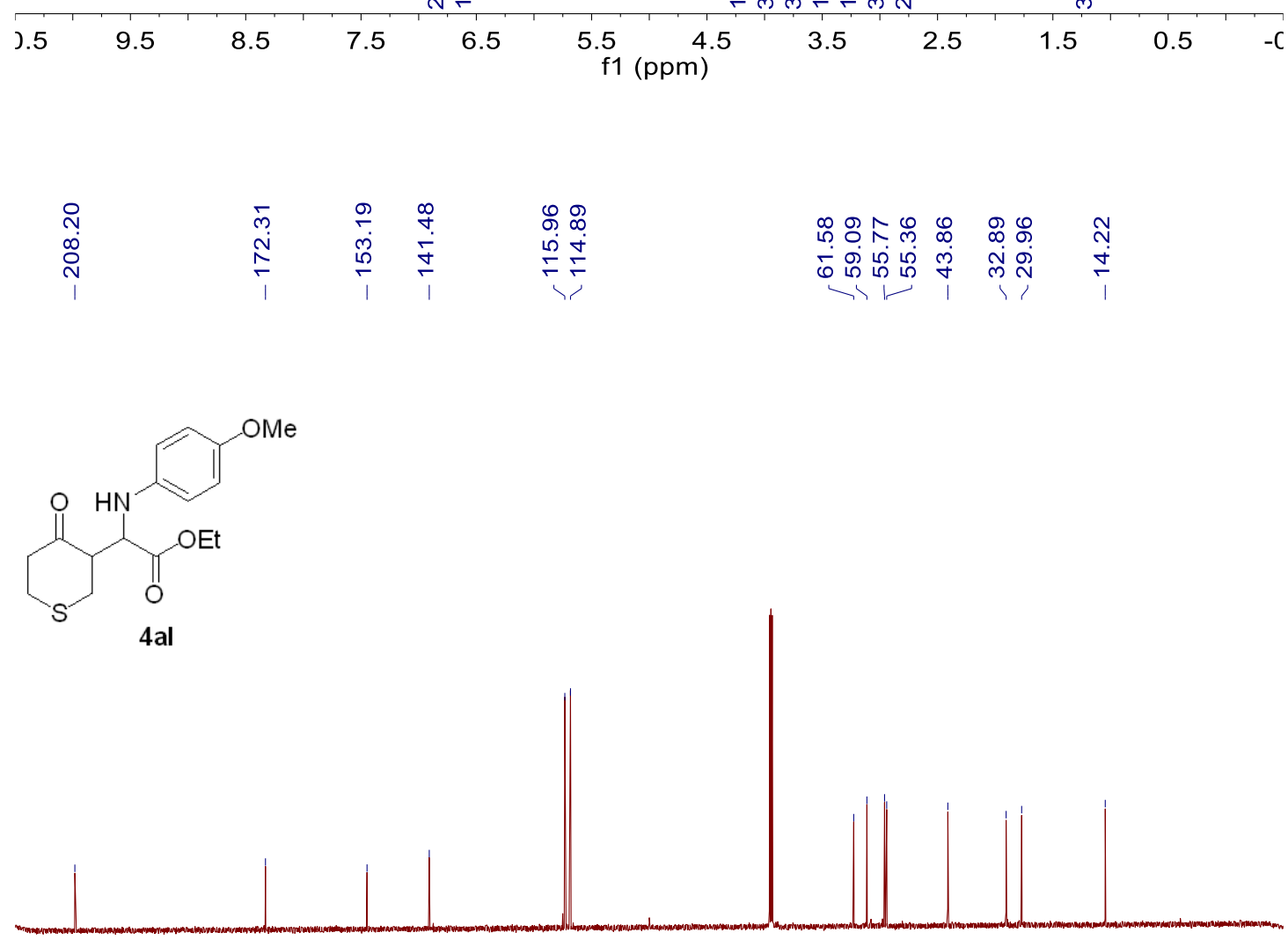

$21020019018017016015014013012011010090 \quad 80 \quad 70 \quad 60 \quad 50 \quad 40 \quad 30 \quad 20 \quad 10 \quad 0 \quad-10$ f1 (ppm)

${ }^{1} \mathrm{H}$ NMR $(400 \mathrm{MHz})$ and ${ }^{13} \mathrm{C}\left\{{ }^{1} \mathrm{H}\right\} \mathrm{NMR}(126 \mathrm{MHz})$ spectra of $\mathbf{4 a l}\left(\mathrm{CDCl}_{3}, \mathrm{rt}\right)$. 
<smiles>CCOC(=O)C(Nc1ccc(OC)cc1)C1CCCCCC1=O</smiles>

4am
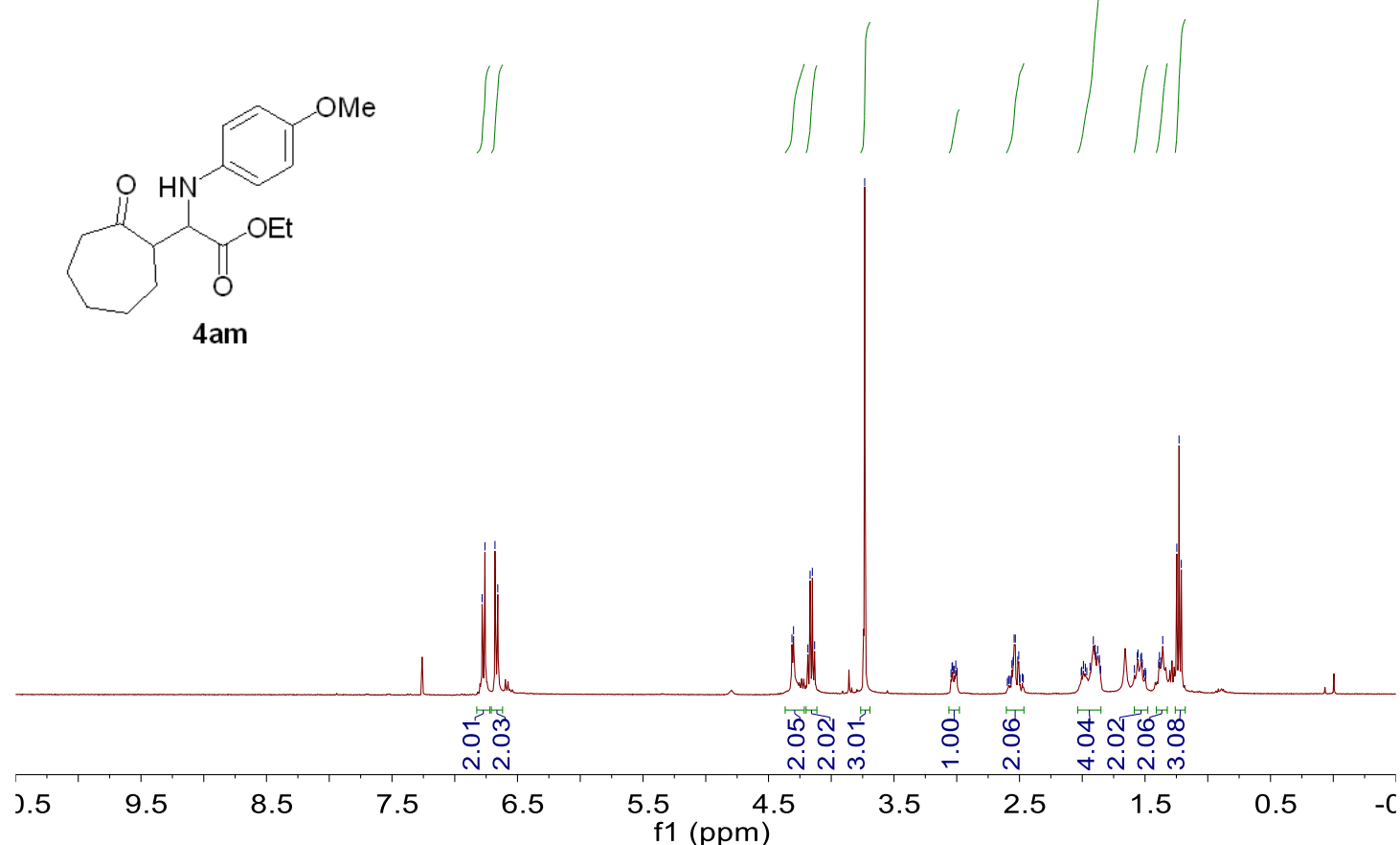

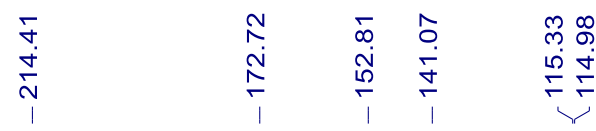

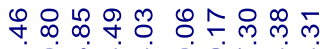

ธo

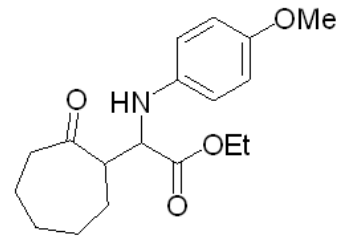

$4 \mathrm{am}$

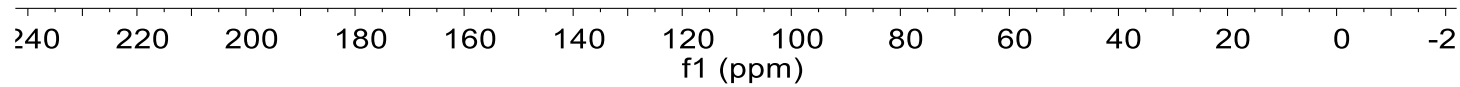

${ }^{1} \mathrm{H}$ NMR (400 MHz) and ${ }^{13} \mathrm{C}\left\{{ }^{1} \mathrm{H}\right\}$ NMR $(101 \mathrm{MHz})$ spectra of 4am $\left(\mathrm{CDCl}_{3}, \mathrm{rt}\right)$. 


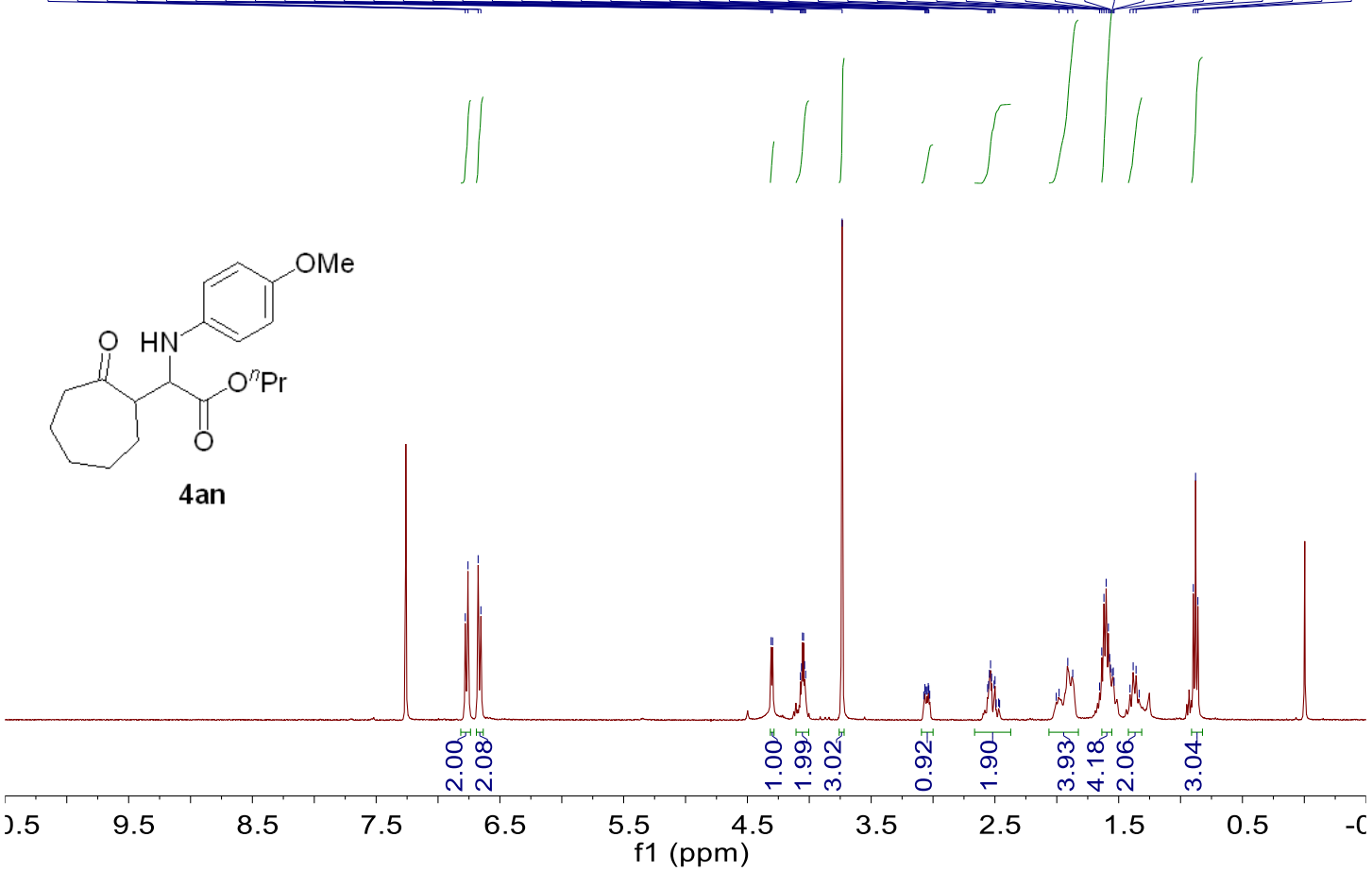

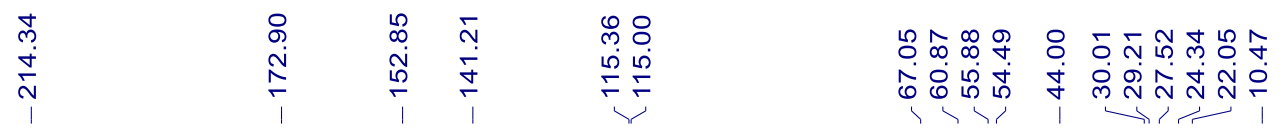

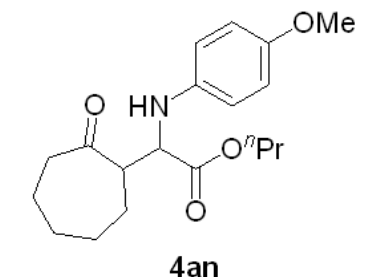

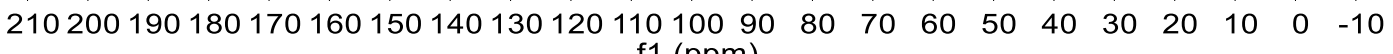
f1 (ppm)

${ }^{1} \mathrm{H}$ NMR $(400 \mathrm{MHz})$ and ${ }^{13} \mathrm{C}\left\{{ }^{1} \mathrm{H}\right\} \mathrm{NMR}(126 \mathrm{MHz})$ spectra of 4 an $\left(\mathrm{CDCl}_{3}, \mathrm{rt}\right)$. 

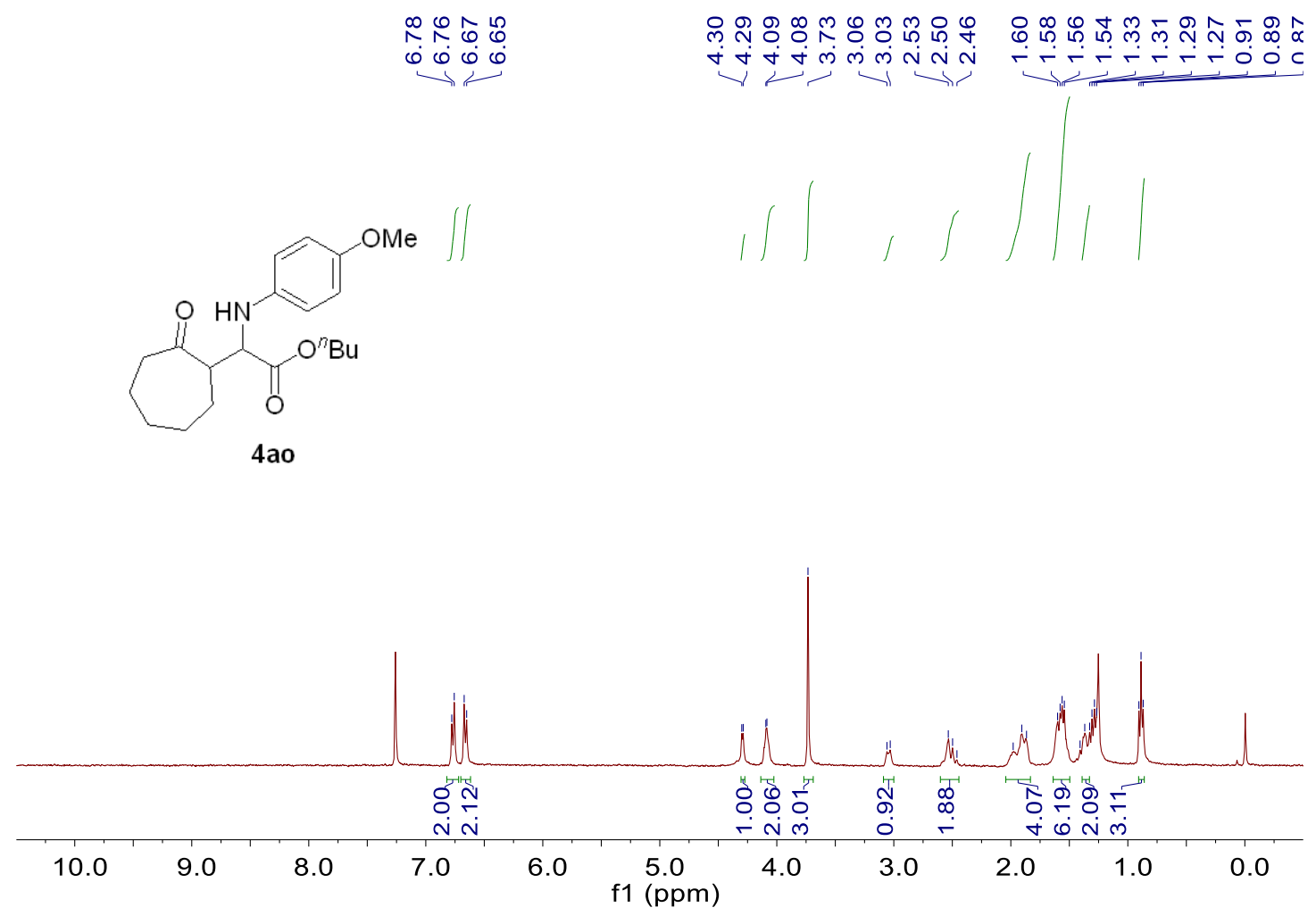

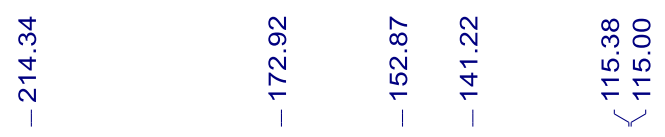

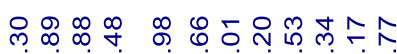

๒ి
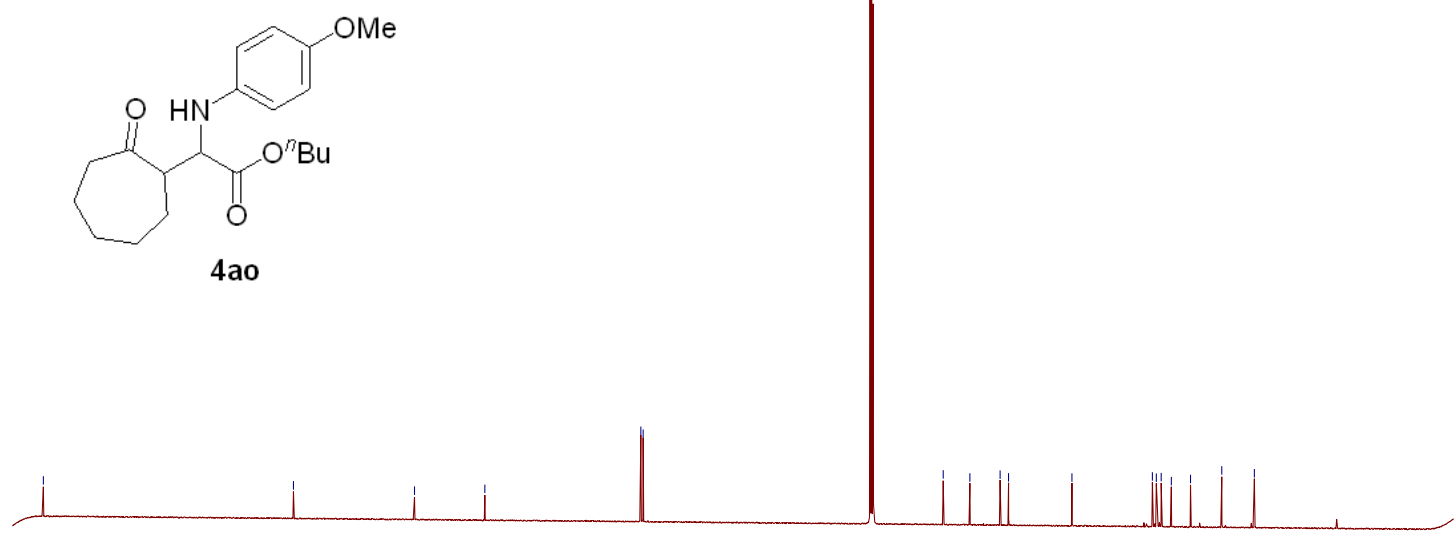

$21020019018017016015014013012011010090 \quad 80 \quad 70 \quad 60 \quad 50 \quad 40 \quad 30 \quad 20 \quad 10 \quad 0 \quad-10$ f1 (ppm)

${ }^{1} \mathrm{H}$ NMR $(400 \mathrm{MHz})$ and ${ }^{13} \mathrm{C}\left\{{ }^{1} \mathrm{H}\right\}$ NMR $(126 \mathrm{MHz})$ spectra of $4 \mathbf{a o}\left(\mathrm{CDCl}_{3}, \mathrm{rt}\right)$. 


\section{Copies of HPLC Spectra}

\section{WZH-1106-1 ASH 912140.7}

Sample Name: Vial Number:

Sample Type:

Control Program:

Quantif. Method:

Recording Time:

Run Time (min):
WZH-1106-1 ASH 912140.7

RE5

unknown

test-dad3

20170608

2020-11-10 21:46

45.00
Injection Volume:

Channel:

Wavelength:

Bandwidth:

Dilution Factor:

Sample Weight:

Sample Amount:
2.0 UV_VIS_2 214.0

4

1.0000

1.0000 1.0000

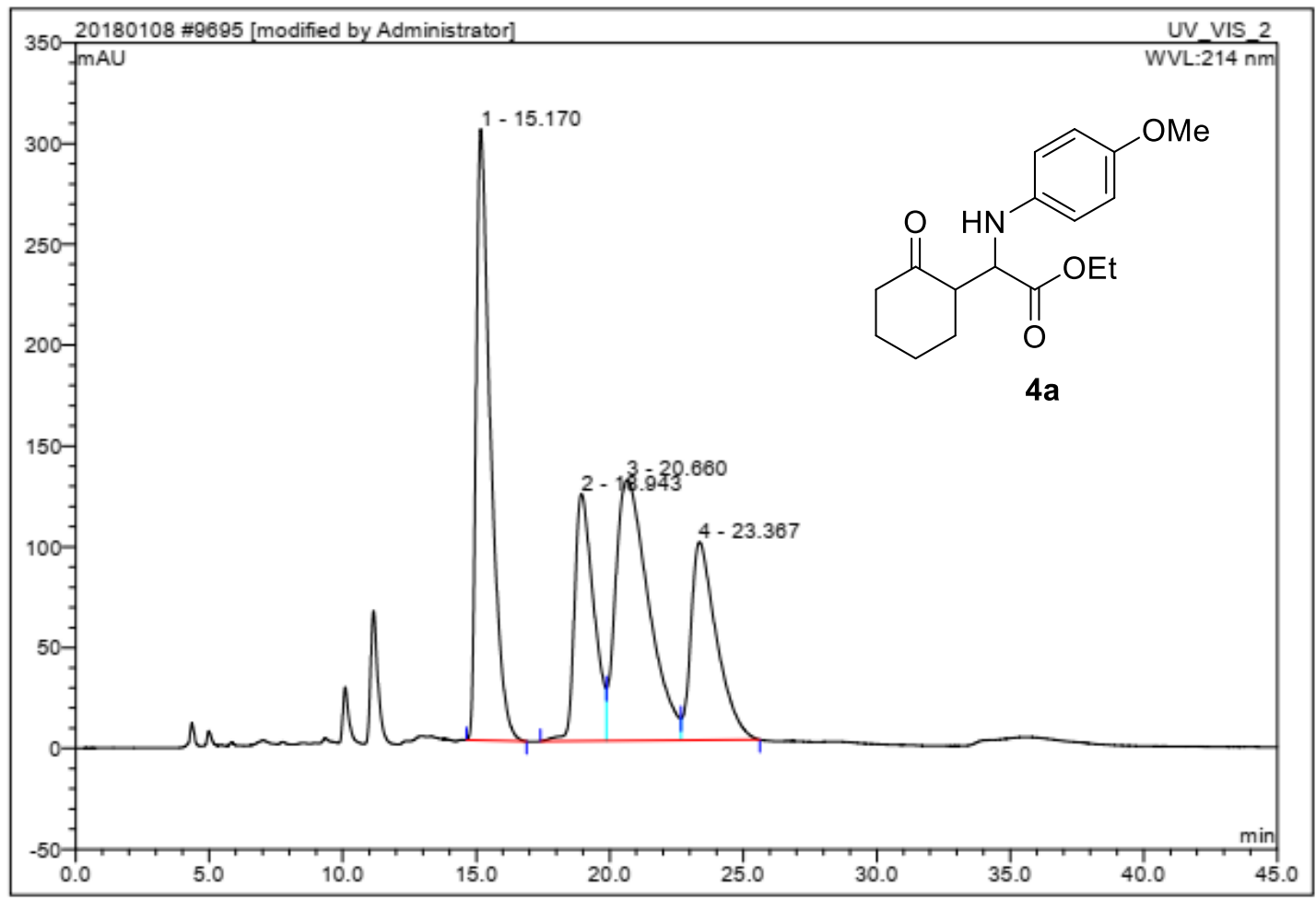

\begin{tabular}{|r|ccccccc|}
\hline No. & $\begin{array}{c}\text { Ret.Time } \\
\text { min }\end{array}$ & Peak Name & $\begin{array}{c}\text { Height } \\
\text { mAU }\end{array}$ & $\begin{array}{c}\text { Area } \\
\text { mAU*min }\end{array}$ & $\begin{array}{c}\text { Rel.Area } \\
\%\end{array}$ & Amount & Type \\
\hline 1 & 15.17 & n.a. & 302.874 & 189.485 & 32.22 & n.a. & BMB \\
2 & 18.94 & n.a. & 122.533 & 102.516 & 17.43 & n.a. & BM \\
3 & 20.66 & n.a. & 129.354 & 185.974 & 31.63 & n.a. & M \\
4 & 23.37 & n.a. & 98.297 & 110.053 & 18.72 & n.a. & MB \\
\hline Total: & & & 653.059 & 588.028 & 100.00 & 0.000 & \\
\hline
\end{tabular}




\section{WZH-1023-5 ASH 912140.7}

\begin{tabular}{llll}
\hline Sample Name: & WZH-1023-5 ASH 91 214 0.7 & Injection Volume: & $\mathbf{5 . 0}$ \\
Vial Number: & RC6 & Channel: & UV_VIS_2 \\
Sample Type: & unknown & Wavelength: & $\mathbf{2 1 4 . 0}$ \\
Control Program: & test-dad2 & Bandwidth: & 4 \\
Quantif. Method: & $\mathbf{2 0 1 7 0 6 0 8}$ & Dilution Factor: & $\mathbf{1 . 0 0 0 0}$ \\
Recording Time: & $\mathbf{2 0 2 0 - 1 0 - 2 8 ~ 1 5 : 1 0}$ & Sample Weight: & $\mathbf{1 . 0 0 0 0}$ \\
Run Time (min): & $\mathbf{6 0 . 0 0}$ & Sample Amount: & $\mathbf{1 . 0 0 0 0}$ \\
\hline
\end{tabular}

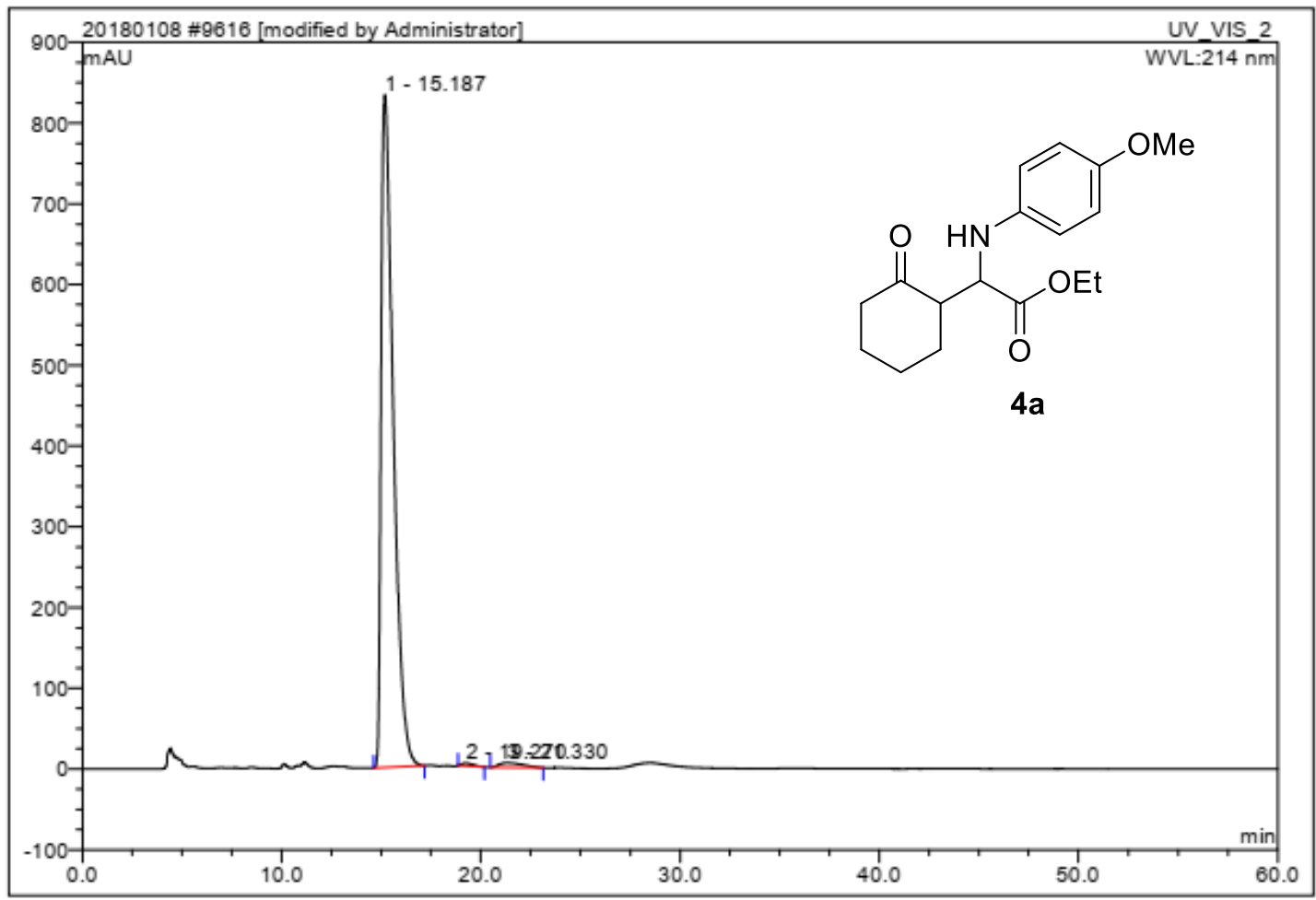

\begin{tabular}{|r|ccrrrrr|}
\hline No. & $\begin{array}{c}\text { Ret.Time } \\
\text { min }\end{array}$ & Peak Name & $\begin{array}{c}\text { Height } \\
\text { mAU }\end{array}$ & $\begin{array}{c}\text { Area } \\
\text { mAU*min }\end{array}$ & $\begin{array}{r}\text { Rel.Area } \\
\%\end{array}$ & Amount & Type \\
\hline 1 & 15.19 & n.a. & 833.159 & 588.766 & 98.28 & n.a. & BMB \\
2 & 19.27 & n.a. & 3.523 & 2.355 & 0.39 & n.a. & BMB $^{\star}$ \\
3 & 21.33 & n.a. & 5.723 & 7.961 & 1.33 & n.a. & BMB \\
\hline Total: & & & 842.405 & 599.082 & 100.00 & 0.000 & \\
\hline
\end{tabular}


9656 WZH-1029-1-1+1-2 ASH 912140.7

\begin{tabular}{llll}
\hline Sample Name: & WZH-1029-1-1+1-2 ASH 912140.7 & Injection Volume: & 3.0 \\
Vial Number: & RB5 & Channel: & UV_VIS_2 \\
Sample Type: & unknown & Wavelength: & $\mathbf{2 1 4 . 0}$ \\
Control Program: & test-dad3 & Bandwidth: & 4 \\
Quantif. Method: & $\mathbf{2 0 1 7 0 6 0 8}$ & Dilution Factor: & $\mathbf{1 . 0 0 0 0}$ \\
Recording Time: & $\mathbf{2 0 2 0 - 1 1 - 4 ~ 1 4 : 3 5}$ & Sample Weight: & $\mathbf{1 . 0 0 0 0}$ \\
Run Time (min): & $\mathbf{6 2 . 5 1}$ & Sample Amount: & $\mathbf{1 . 0 0 0 0}$ \\
\hline
\end{tabular}

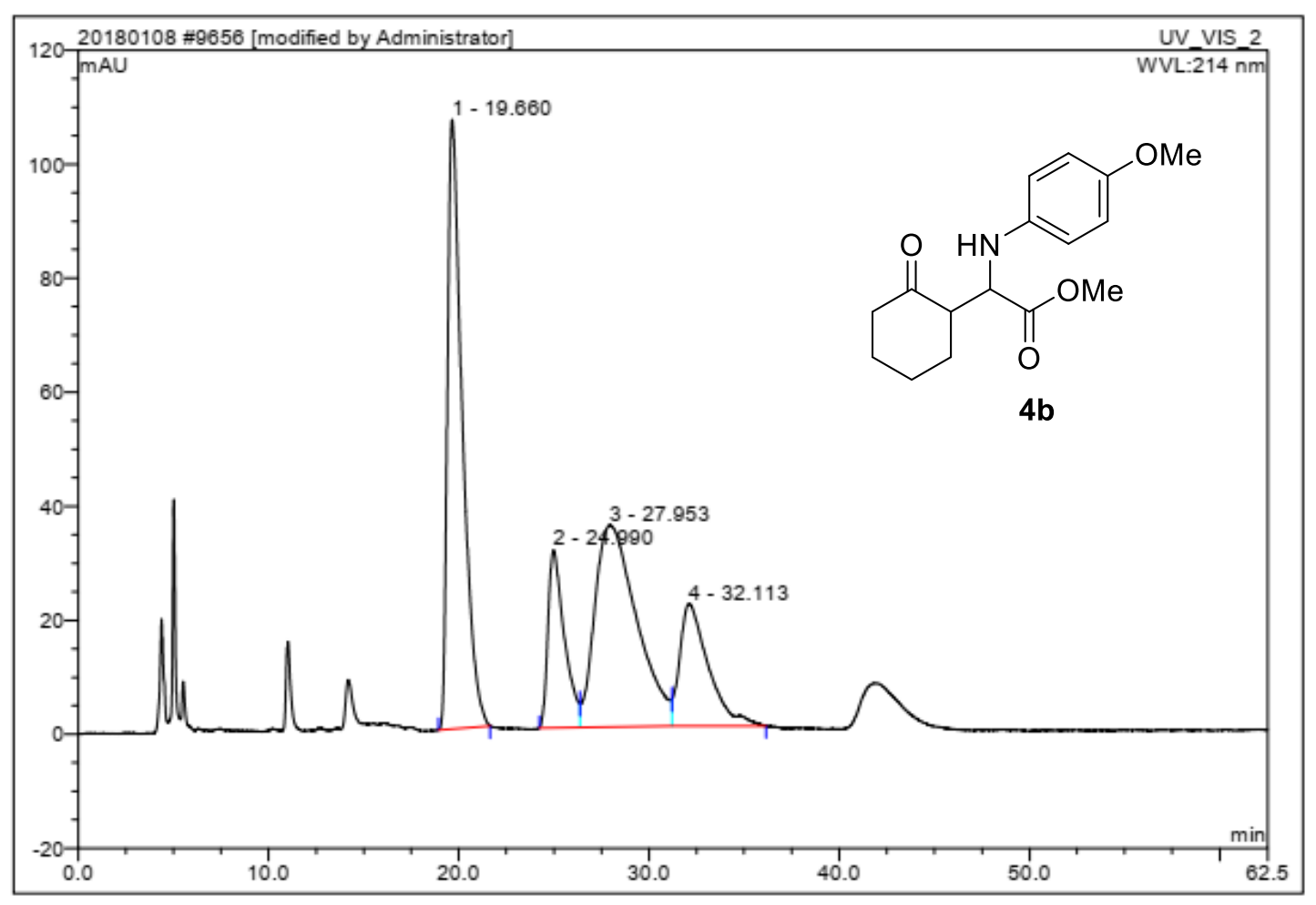

\begin{tabular}{|r|ccrrrrr|}
\hline No. & $\begin{array}{c}\text { Ret.Time } \\
\text { min }\end{array}$ & Peak Name & $\begin{array}{c}\text { Height } \\
\text { mAU }\end{array}$ & $\begin{array}{c}\text { Area } \\
\text { mAU*min }\end{array}$ & $\begin{array}{c}\text { Rel.Area } \\
\%\end{array}$ & Amount & Type \\
\hline 1 & 19.66 & n.a. & 106.955 & 96.690 & 37.78 & n.a. & BMB \\
2 & 24.99 & n.a. & 31.198 & 31.233 & 12.20 & n.a. & BM $^{*}$ \\
3 & 27.95 & n.a. & 35.430 & 90.520 & 35.37 & n.a. & M $^{*}$ \\
4 & 32.11 & n.a. & 21.468 & 37.515 & 14.66 & n.a. & MB $^{*}$ \\
\hline Total: & & & 195.051 & 255.958 & 100.00 & 0.000 & \\
\hline
\end{tabular}




\section{WZH-1109-1 ASH 912140.7}

\begin{tabular}{llll}
\hline Sample Name: & WZH-1109-1 ASH 91 214 0.7 & Injection Volume: & 2.0 \\
Vial Number: & RE7 & Channel: & UV_VIS_2 \\
Sample Type: & unknown & Wavelength: & $\mathbf{2 1 4 . 0}$ \\
Control Program: & test-dad2 & Bandwidth: & 4 \\
Quantif. Method: & $\mathbf{2 0 1 7 0 6 0 8}$ & Dilution Factor: & 1.0000 \\
Recording Time: & $\mathbf{2 0 2 0 - 1 1 - 1 2 ~ 1 0 : 1 9 ~}$ & Sample Weight: & 1.0000 \\
Run Time (min): & $\mathbf{6 0 . 0 0}$ & Sample Amount: & $\mathbf{1 . 0 0 0 0}$ \\
\hline
\end{tabular}

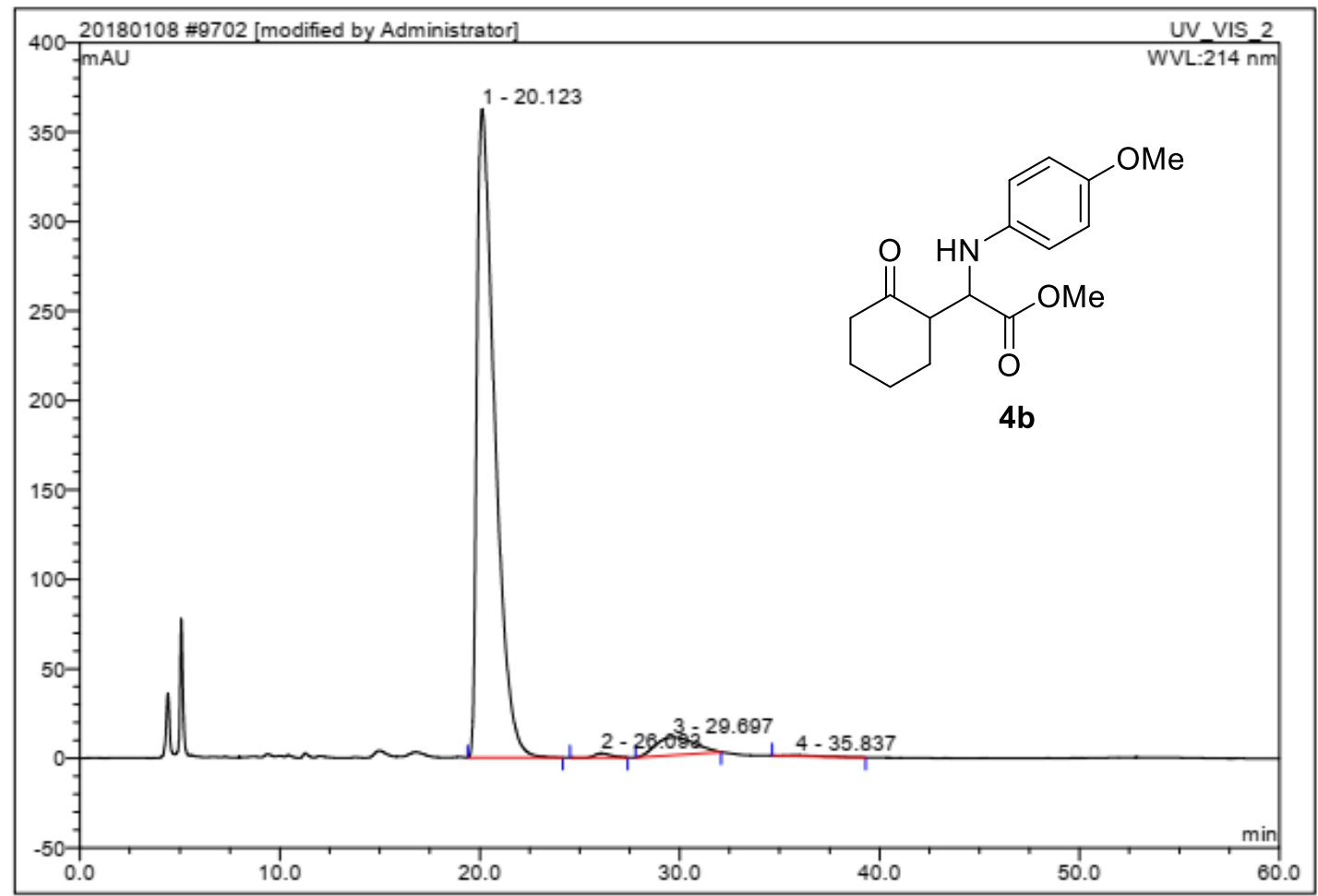

\begin{tabular}{|r|ccrrrrr|}
\hline No. & $\begin{array}{c}\text { Ret.Time } \\
\text { min }\end{array}$ & Peak Name & $\begin{array}{c}\text { Height } \\
\text { mAU }\end{array}$ & $\begin{array}{c}\text { Area } \\
\text { mAU*min }\end{array}$ & $\begin{array}{r}\text { Rel.Area } \\
\%\end{array}$ & Amount & Type \\
\hline 1 & 20.12 & n.a. & 362.679 & 376.731 & 93.73 & n.a. & BMB $^{\star}$ \\
2 & 26.09 & n.a. & 2.419 & 2.098 & 0.52 & n.a. & BMB $^{\star}$ \\
3 & 29.70 & n.a. & 9.893 & 22.231 & 5.53 & n.a. & BMB $^{\star}$ \\
4 & 35.84 & n.a. & 0.527 & 0.867 & 0.22 & n.a. & BMB $^{\star}$ \\
\hline Total: & & & 375.517 & 401.927 & 100.00 & 0.000 & \\
\hline
\end{tabular}




\section{WZH1201-2+- ASH 912140.7}

\begin{tabular}{llll}
\hline Sample Name: & WZH1201-2+- ASH 91 214 0.7 & Injection Volume: & 3.0 \\
Vial Number: & RE5 & Channel: & UV_VIS_2 \\
Sample Type: & unknown & Wavelength: & $\mathbf{2 1 4 . 0}$ \\
Control Program: & test-dad3 & Bandwidth: & 4 \\
Quantif. Method: & $\mathbf{2 0 1 7 0 6 0 8}$ & Dilution Factor: & $\mathbf{1 . 0 0 0 0}$ \\
Recording Time: & $\mathbf{2 0 2 0 - 1 2 - 1 5 ~ 1 7 : 2 1 ~}$ & Sample Weight: & $\mathbf{1 . 0 0 0 0}$ \\
Run Time (min): & $\mathbf{4 0 . 0 0}$ & Sample Amount: & $\mathbf{1 . 0 0 0 0}$ \\
\hline
\end{tabular}

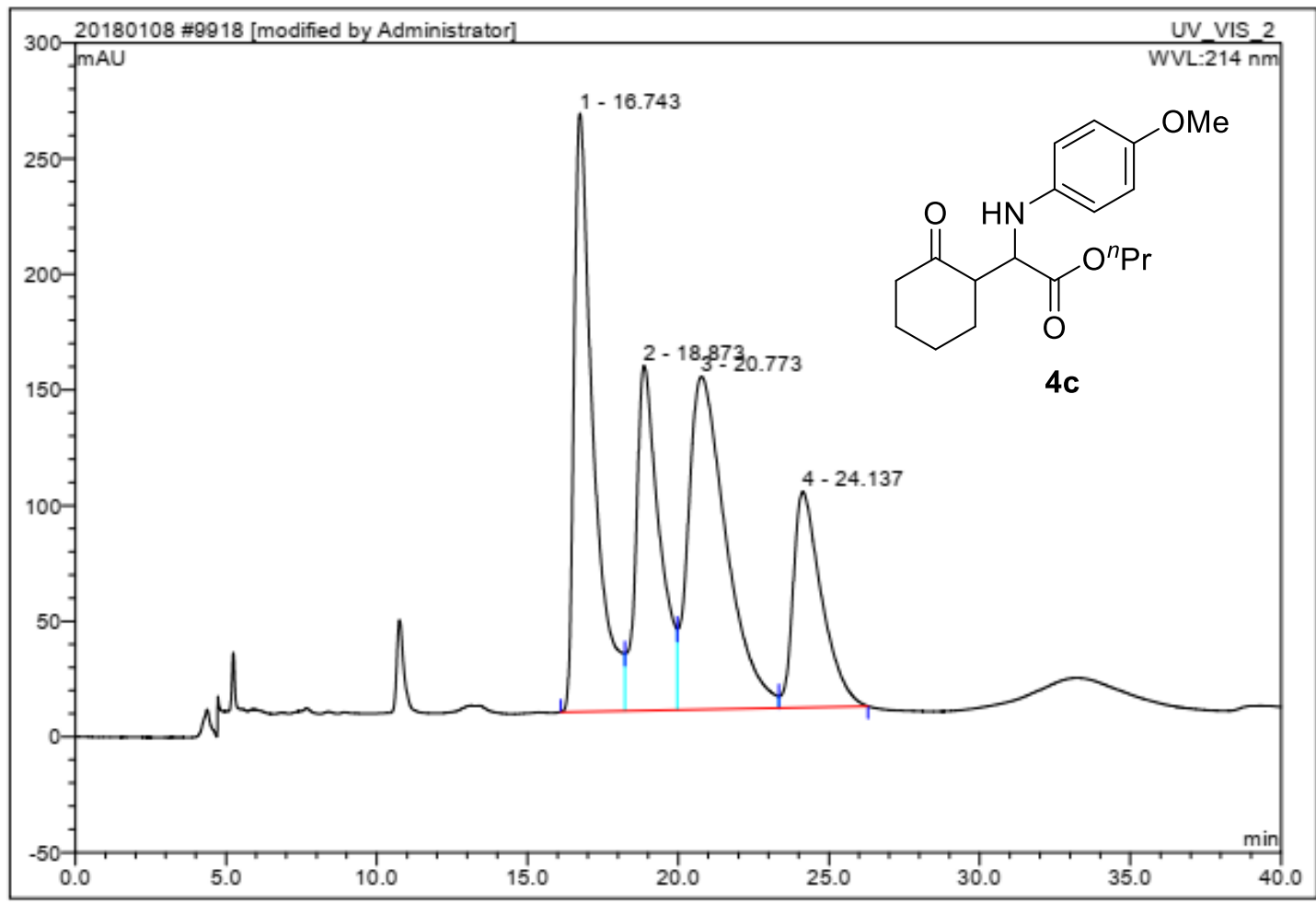

\begin{tabular}{|r|ccccccc|}
\hline No. & $\begin{array}{c}\text { Ret.Time } \\
\text { min }\end{array}$ & Peak Name & $\begin{array}{c}\text { Height } \\
\text { mAU }\end{array}$ & $\begin{array}{c}\text { Area } \\
\text { mAU*min }\end{array}$ & $\begin{array}{c}\text { Rel.Area } \\
\%\end{array}$ & Amount & Type \\
\hline 1 & 16.74 & n.a. & 258.835 & 193.312 & 30.09 & n.a. & BM \\
2 & 18.87 & n.a. & 149.199 & 135.671 & 21.12 & n.a. & M \\
3 & 20.77 & n.a. & 143.931 & 211.096 & 32.86 & n.a. & M \\
4 & 24.14 & n.a. & 93.436 & 102.411 & 15.94 & n.a. & MB \\
\hline Total: & & & 645.400 & 642.491 & 100.00 & 0.000 & \\
\hline
\end{tabular}




\section{\#\#\# WZH-1202-1 ASH 912140.7}

\begin{tabular}{llll}
\hline Sample Name: & WZH-1202-1 ASH 91 214 0.7 & Injection Volume: & $\mathbf{1 . 0}$ \\
Vial Number: & RD7 & Channel: & UV_VIS_2 \\
Sample Type: & unknown & Wavelength: & $\mathbf{2 4 0 . 0}$ \\
Control Program: & test-dad2 & Bandwidth: & 4 \\
Quantif. Method: & $\mathbf{2 0 1 7 0 6 0 8}$ & Dilution Factor: & $\mathbf{1 . 0 0 0 0}$ \\
Recording Time: & $\mathbf{2 0 2 0 - 1 2 - 3 0 ~ 8 : 5 7}$ & Sample Weight: & $\mathbf{1 . 0 0 0 0}$ \\
Run Time (min): & $\mathbf{4 1 . 1 8}$ & Sample Amount: & $\mathbf{1 . 0 0 0 0}$ \\
\hline
\end{tabular}

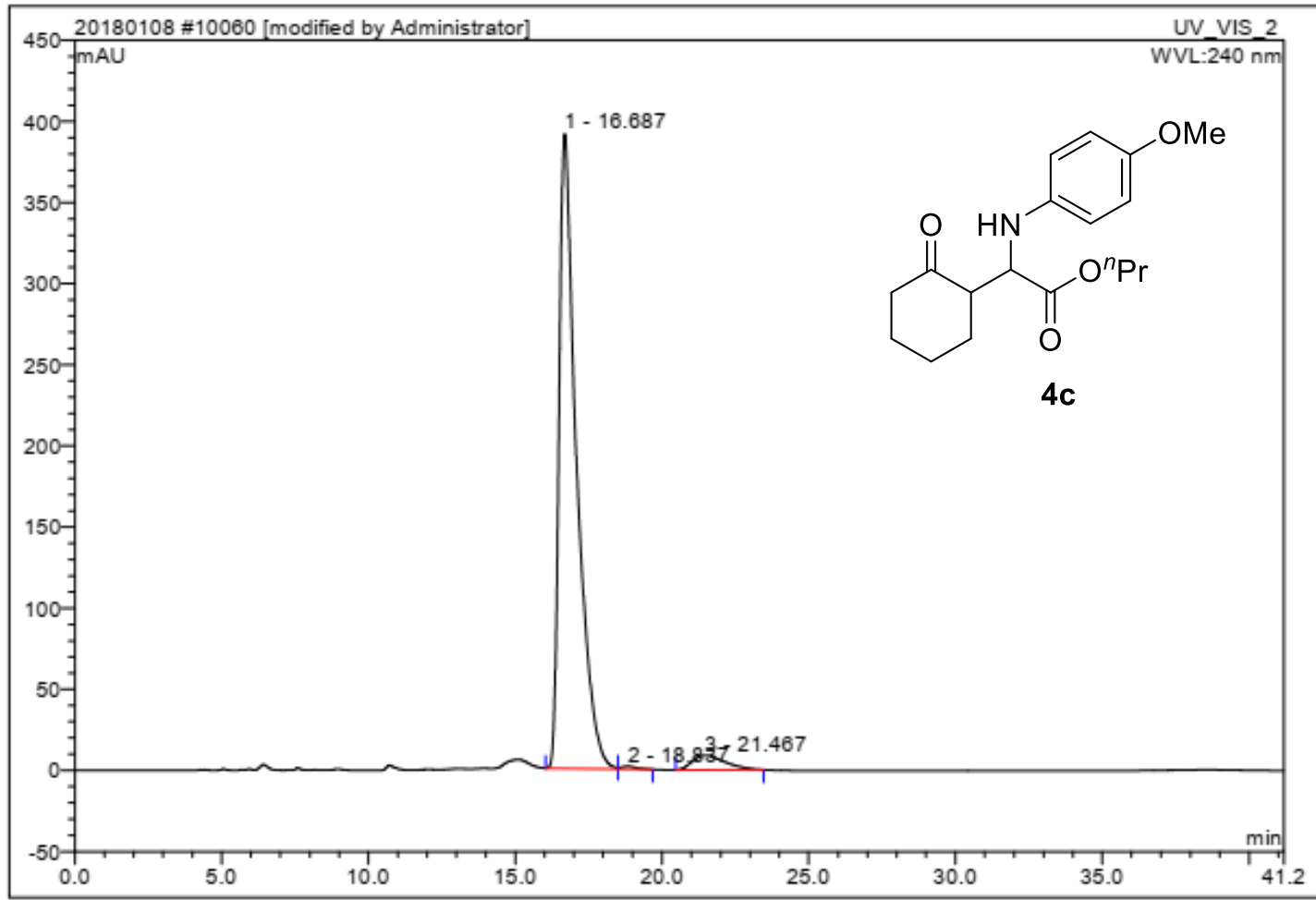

\begin{tabular}{|r|ccrrrrr|}
\hline No. & $\begin{array}{c}\text { Ret.Time } \\
\text { min }\end{array}$ & Peak Name & $\begin{array}{c}\text { Height } \\
\text { mAU }\end{array}$ & $\begin{array}{r}\text { Area } \\
\text { mAU*min }\end{array}$ & $\begin{array}{r}\text { Rel.Area } \\
\%\end{array}$ & Amount & Type \\
\hline 1 & 16.69 & n.a. & 391.276 & 269.298 & 95.52 & n.a. & BM $^{*}$ \\
2 & 18.84 & n.a. & 1.730 & 1.044 & 0.37 & n.a. & MB $^{*}$ \\
3 & 21.47 & n.a. & 8.912 & 11.578 & 4.11 & n.a. & BMB \\
\hline Total: & & & 401.918 & 281.920 & 100.00 & 0.000 & \\
\hline
\end{tabular}




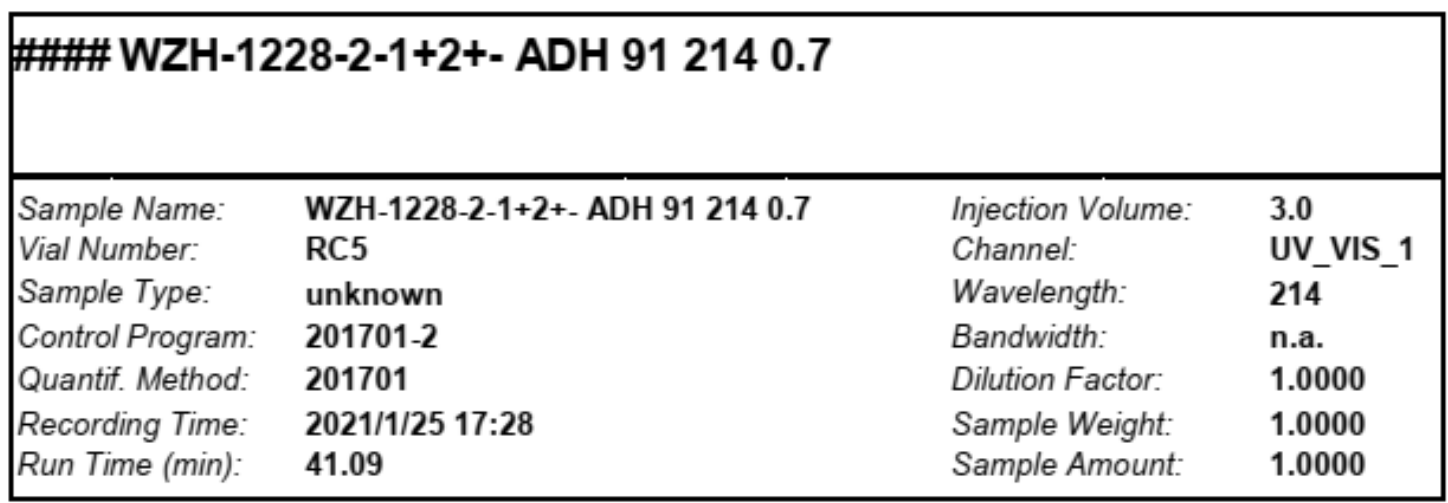

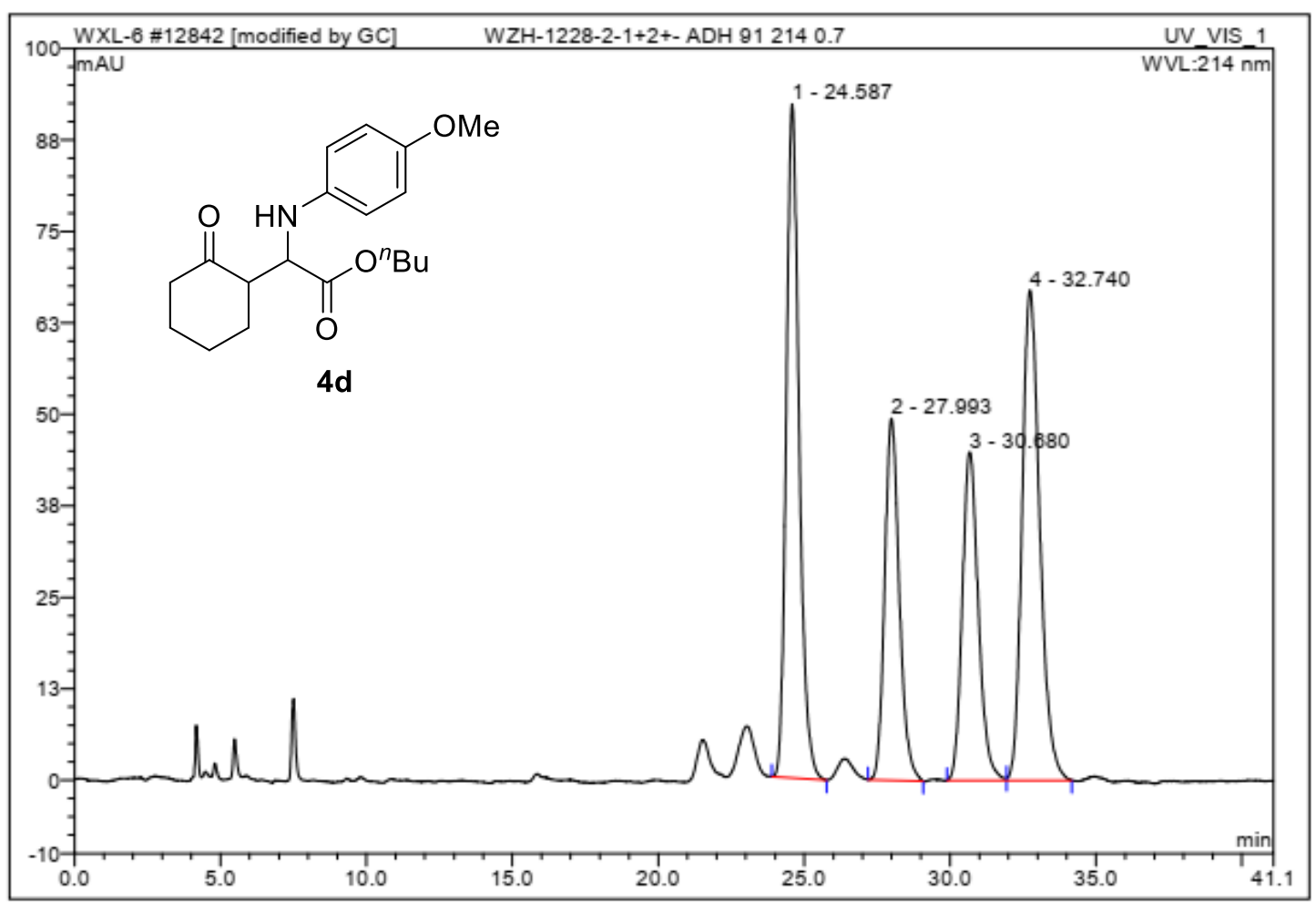

\begin{tabular}{|c|c|c|c|c|c|c|c|}
\hline No. & $\begin{array}{c}\text { Ret.Time } \\
\text { min }\end{array}$ & Peak Name & $\begin{array}{c}\text { Height } \\
\text { mAU }\end{array}$ & $\begin{array}{c}\text { Area } \\
\mathrm{mAU}^{*} \min \end{array}$ & $\begin{array}{c}\text { Rel.Area } \\
\%\end{array}$ & Amount & Type \\
\hline 1 & 24.59 & n.a. & 92.100 & 47.078 & 31.06 & n.a. & $\mathrm{BMB}^{*}$ \\
\hline 2 & 27.99 & n.a. & 49.452 & 28.750 & 18.97 & n.a. & BMB $^{*}$ \\
\hline 3 & 30.68 & n.a. & 44.902 & 28.684 & 18.92 & n.a. & BM \\
\hline 4 & 32.74 & n.a. & 66.997 & 47.069 & 31.05 & n.a. & $\mathrm{MB}$ \\
\hline Total: & & & 253.452 & 151.580 & 100.00 & 0.000 & \\
\hline
\end{tabular}




\begin{tabular}{|lllll|}
\hline \#\#\# WZH-0104-2 ADH 912140.7 & & \\
& & & \\
\hline Sample Name: & WZH-0104-2 ADH 912140.7 & Injection Volume: & 3.0 \\
Vial Number: & RA7 & Channel: & UV_VIS_1 \\
Sample Type: & unknown & Wavelength: & $\mathbf{2 1 4}$ \\
Control Program: & $\mathbf{2 0 1 7 0 1 - 6}$ & Bandwidth: & n.a. \\
Quantif. Method: & $\mathbf{2 0 1 7 0 1}$ & Dilution Factor: & $\mathbf{1 . 0 0 0 0}$ \\
Recording Time: & $\mathbf{2 0 2 1 / 2 / 2 4}$ 17:20 & Sample Weight: & $\mathbf{1 . 0 0 0 0}$ \\
Run Time (min): & $\mathbf{6 0 . 0 0}$ & Sample Amount: & $\mathbf{1 . 0 0 0 0}$ \\
\hline
\end{tabular}

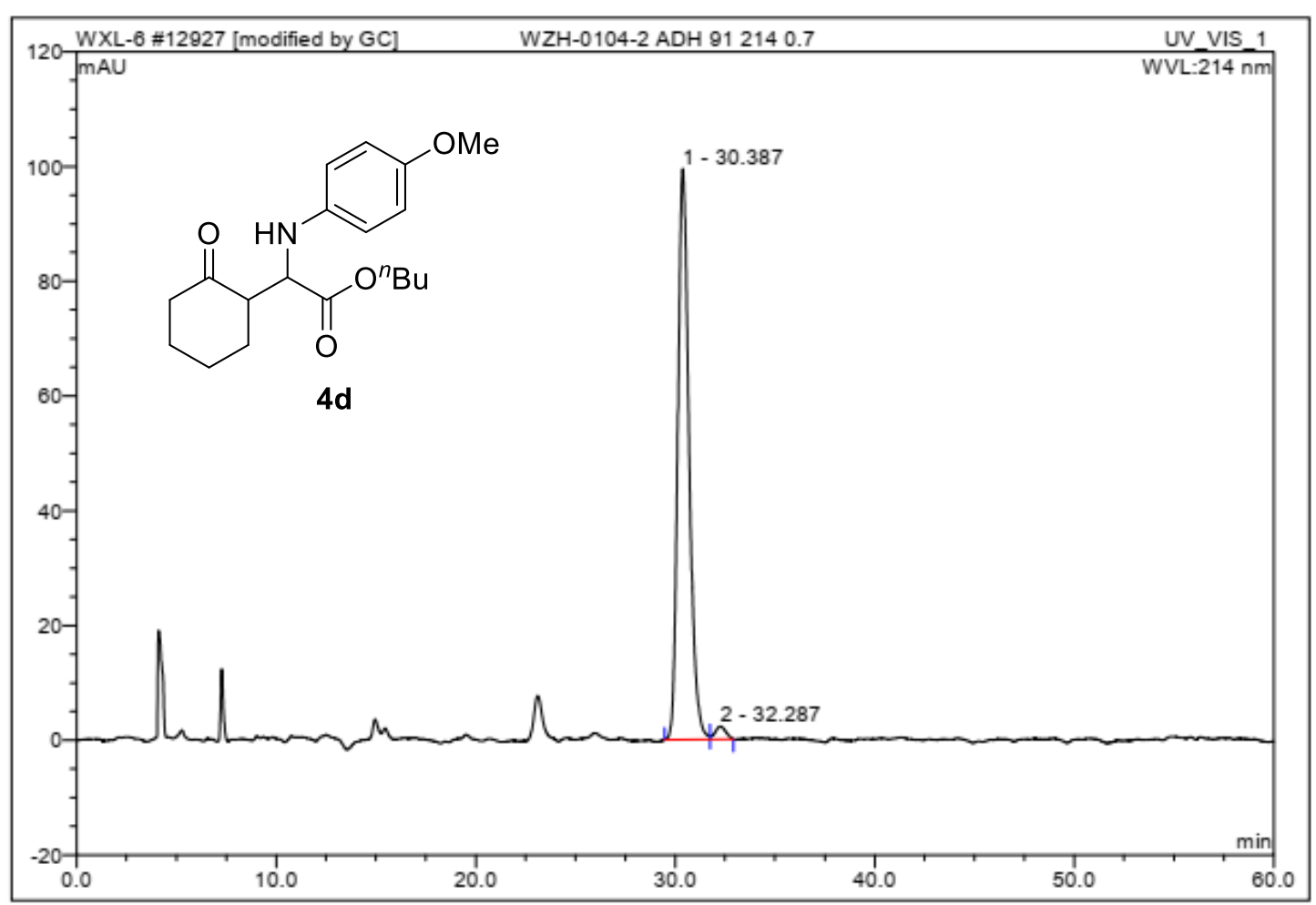

\begin{tabular}{|r|ccrrrrr|}
\hline No. & $\begin{array}{c}\text { Ret.Time } \\
\text { min }\end{array}$ & Peak Name & $\begin{array}{c}\text { Height } \\
\text { mAU }\end{array}$ & $\begin{array}{c}\text { Area } \\
\text { mAU*min }\end{array}$ & $\begin{array}{r}\text { Rel.Area } \\
\%\end{array}$ & Amount & Type \\
\hline 1 & 30.39 & n.a. & 99.552 & 65.127 & 97.86 & n.a. & BM \\
2 & 32.29 & n.a. & 2.268 & 1.421 & 2.14 & n.a. & MB \\
\hline Total: & & & 101.820 & 66.548 & 100.00 & 0.000 & \\
\hline
\end{tabular}


9726 WZH-1106-22 ASH 912140.7

\begin{tabular}{llll}
\hline Sample Name: & WZH-1106-22 ASH 91 214 0.7 & Injection Volume: & 2.0 \\
Vial Number: & RE6 & Channel: & UV_VIS_2 \\
Sample Type: & unknown & Wavelength: & $\mathbf{2 1 4 . 0}$ \\
Control Program: & test-dad2 & Bandwidth: & 4 \\
Quantif. Method: & $\mathbf{2 0 1 7 0 6 0 8}$ & Dilution Factor: & $\mathbf{1 . 0 0 0 0}$ \\
Recording Time: & $\mathbf{2 0 2 0 - 1 1 - 1 6 ~ 1 5 : 4 6}$ & Sample Weight: & $\mathbf{1 . 0 0 0 0}$ \\
Run Time (min): & $\mathbf{4 0 . 7 0}$ & Sample Amount: & $\mathbf{1 . 0 0 0 0}$
\end{tabular}

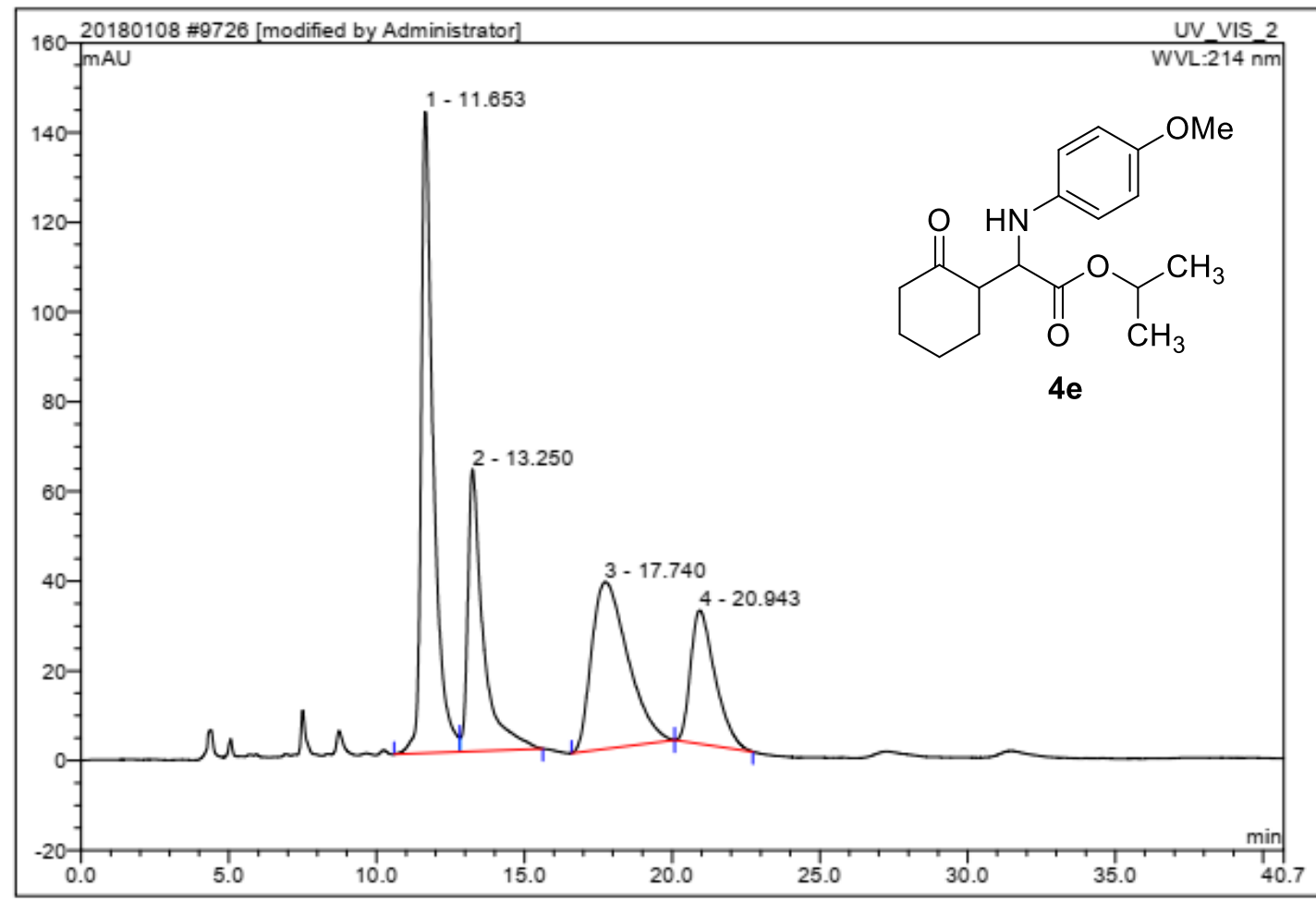

\begin{tabular}{|r|ccrrrrr|}
\hline No. & $\begin{array}{c}\text { Ret.Time } \\
\text { min }\end{array}$ & Peak Name & $\begin{array}{c}\text { Height } \\
\text { mAU }\end{array}$ & $\begin{array}{c}\text { Area } \\
\text { mAU*min }\end{array}$ & $\begin{array}{c}\text { Rel.Area } \\
\%\end{array}$ & Amount & Type \\
\hline 1 & 11.65 & n.a. & 142.928 & 68.431 & 36.36 & n.a. & BM \\
2 & 13.25 & n.a. & 62.958 & 38.075 & 20.23 & n.a. & $\begin{array}{c}\text { MB } \\
3\end{array}$ \\
17.74 & n.a. & 37.268 & 52.994 & 28.16 & n.a. & BMb* \\
4 & 20.94 & n.a. & 29.798 & 28.708 & 15.25 & n.a. & bMB \\
\hline Total: & & & 272.952 & 188.207 & 100.00 & 0.000 & \\
\hline
\end{tabular}




\section{WZH-1109-2 ASH 912140.7}

\begin{tabular}{llll|}
\hline Sample Name: & WZH-1109-2 ASH 912140.7 & Injection Volume: & $\mathbf{1 . 0}$ \\
Vial Number: & RE7 & Channel: & UV_VIS_2 \\
Sample Type: & unknown & Wavelength: & $\mathbf{2 1 4 . 0}$ \\
Control Program: & test-dad2 & Bandwidth: & 4 \\
Quantif. Method: & $\mathbf{2 0 1 7 0 6 0 8}$ & Dilution Factor: & $\mathbf{1 . 0 0 0 0}$ \\
Recording Time: & $\mathbf{2 0 2 0 - 1 1 - 1 1 ~ 1 5 : 1 8}$ & Sample Weight: & $\mathbf{1 . 0 0 0 0}$ \\
Run Time (min): & $\mathbf{2 8 . 4 9}$ & Sample Amount: & $\mathbf{1 . 0 0 0 0}$
\end{tabular}

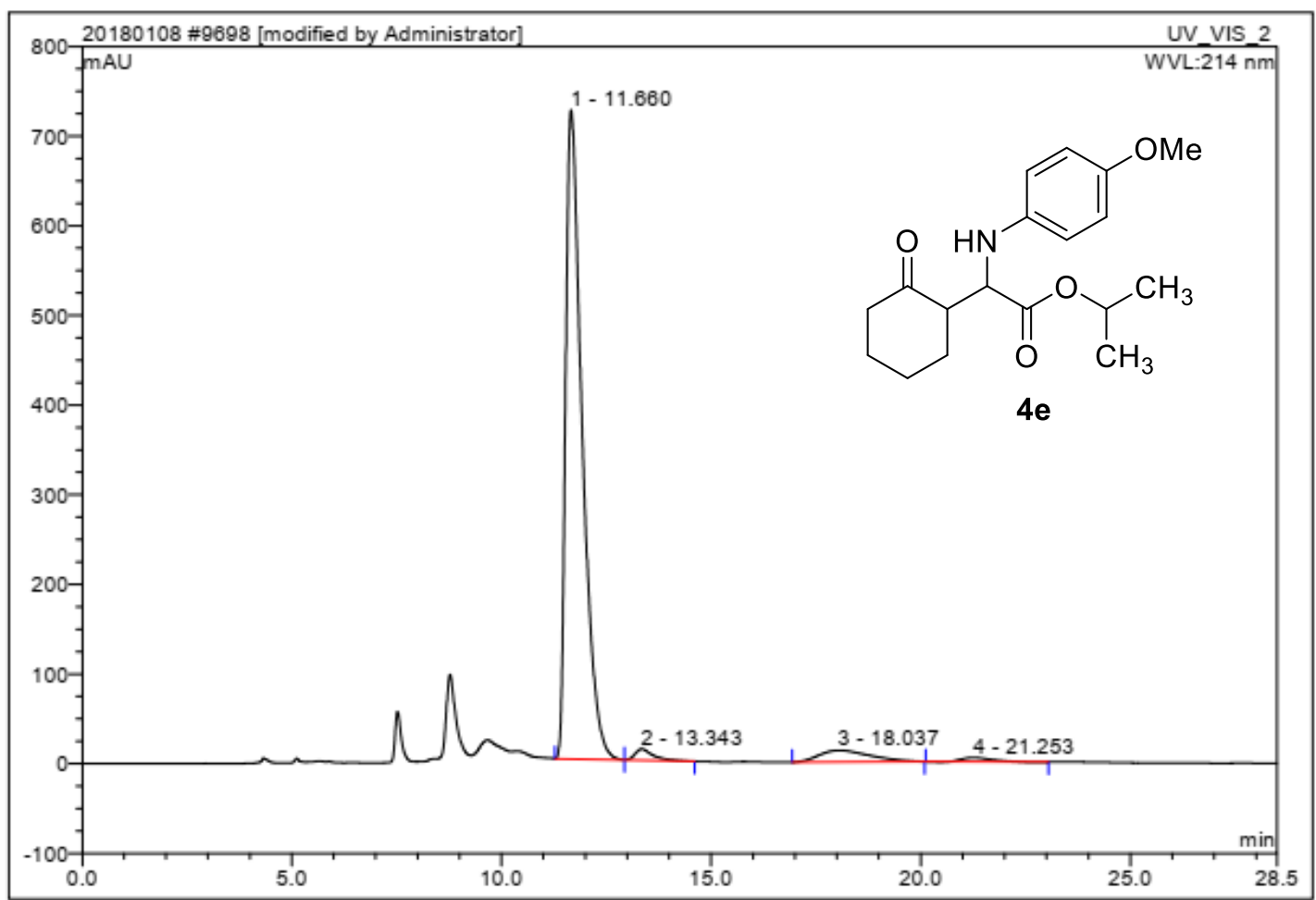

\begin{tabular}{|r|ccrrrrr|}
\hline No. & $\begin{array}{c}\text { Ret.Time } \\
\text { min }\end{array}$ & Peak Name & $\begin{array}{c}\text { Height } \\
\text { mAU }\end{array}$ & $\begin{array}{c}\text { Area } \\
\text { mAU*min }\end{array}$ & $\begin{array}{r}\text { Rel.Area } \\
\%\end{array}$ & Amount & Type \\
\hline 1 & 11.66 & n.a. & 724.223 & 330.015 & 92.06 & n.a. & BM $^{*}$ \\
2 & 13.34 & n.a. & 12.682 & 6.391 & 1.78 & n.a. & MB $^{*}$ \\
3 & 18.04 & n.a. & 12.921 & 18.348 & 5.12 & n.a. & BMB $^{*}$ \\
4 & 21.25 & n.a. & 4.641 & 3.719 & 1.04 & n.a. & BMB $^{*}$ \\
\hline Total: & & & 754.467 & 358.473 & 100.00 & 0.000 & \\
\hline
\end{tabular}


9700 WZH-1106-3+- ASH 912140.7

\begin{tabular}{llll}
\hline Sample Name: & WZH-1106-3+- ASH 912140.7 & Injection Volume: & 2.0 \\
Vial Number: & RC5 & Channel: & UV_VIS_2 \\
Sample Type: & unknown & Wavelength: & $\mathbf{2 1 4 . 0}$ \\
Control Program: & test-dad2 & Bandwidth: & 4 \\
Quantif. Method: & $\mathbf{2 0 1 7 0 6 0 8}$ & Dilution Factor: & 1.0000 \\
Recording Time: & $\mathbf{2 0 2 0 - 1 1 - 1 1 ~ 1 1 : 4 6}$ & Sample Weight: & 1.0000 \\
Run Time (min): & $\mathbf{2 8 . 6 9}$ & Sample Amount: & $\mathbf{1 . 0 0 0 0}$
\end{tabular}

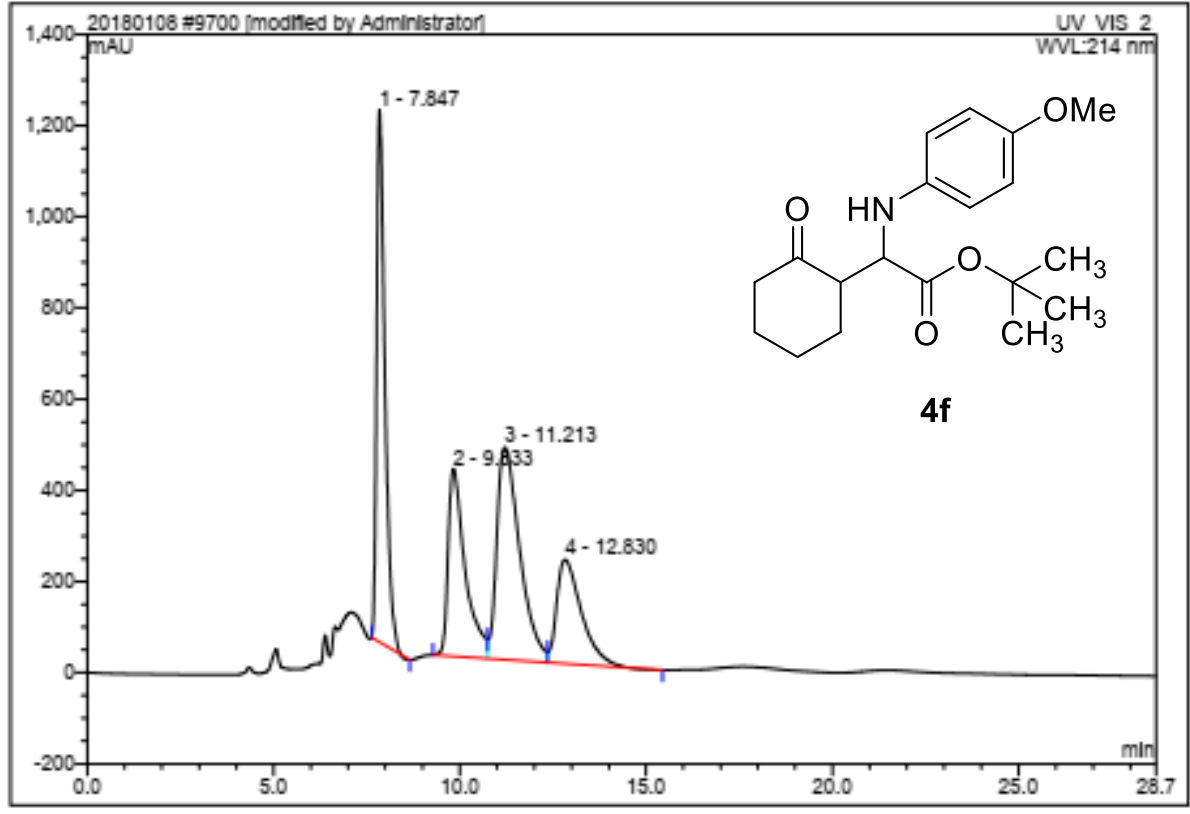

\begin{tabular}{|r|rrrrrrr|}
\hline No. & $\begin{array}{r}\text { Ret.Time } \\
\text { min }\end{array}$ & Peak Name & $\begin{array}{c}\text { Height } \\
\text { mAU }\end{array}$ & $\begin{array}{c}\text { Area } \\
\text { mAU*min }\end{array}$ & $\begin{array}{c}\text { Rel_Area } \\
\%\end{array}$ & Amount & Type \\
\hline 1 & 7.85 & n.a. & 1167.129 & 302.744 & 29.36 & n.a. & BMB $^{*}$ \\
2 & 9.83 & n.a. & 411.465 & 219.162 & 21.26 & n.a. & BM * $^{*}$ \\
3 & 11.21 & n.a. & 466.231 & 333.059 & 32.30 & n.a. & $M^{*}$ \\
4 & 12.83 & n.a. & 228.477 & 176.125 & 17.08 & n.a. & MB $^{*}$ \\
\hline Total: & & & 2273.301 & 1031.090 & 100.00 & 0.000 & \\
\hline
\end{tabular}




\section{WZH-1109-33 ASH 912140.7}

\begin{tabular}{llll}
\hline Sample Name: & WZH-1109-33 ASH 912140.7 & Injection Volume: & $\mathbf{2 . 0}$ \\
Vial Number: & RE5 & Channel: & UV_VIS_2 \\
Sample Type: & unknown & Wavelength: & $\mathbf{2 1 4 . 0}$ \\
Control Program: & test-dad2 & Bandwidth: & 4 \\
Quantif. Method: & $\mathbf{2 0 1 7 0 6 0 8}$ & Dilution Factor: & $\mathbf{1 . 0 0 0 0}$ \\
Recording Time: & $\mathbf{2 0 2 0 - 1 1 - 1 6 ~ 1 6 : 2 8}$ & Sample Weight: & 1.0000 \\
Run Time (min): & $\mathbf{3 0 . 6 3}$ & Sample Amount: & $\mathbf{1 . 0 0 0 0}$ \\
\hline
\end{tabular}

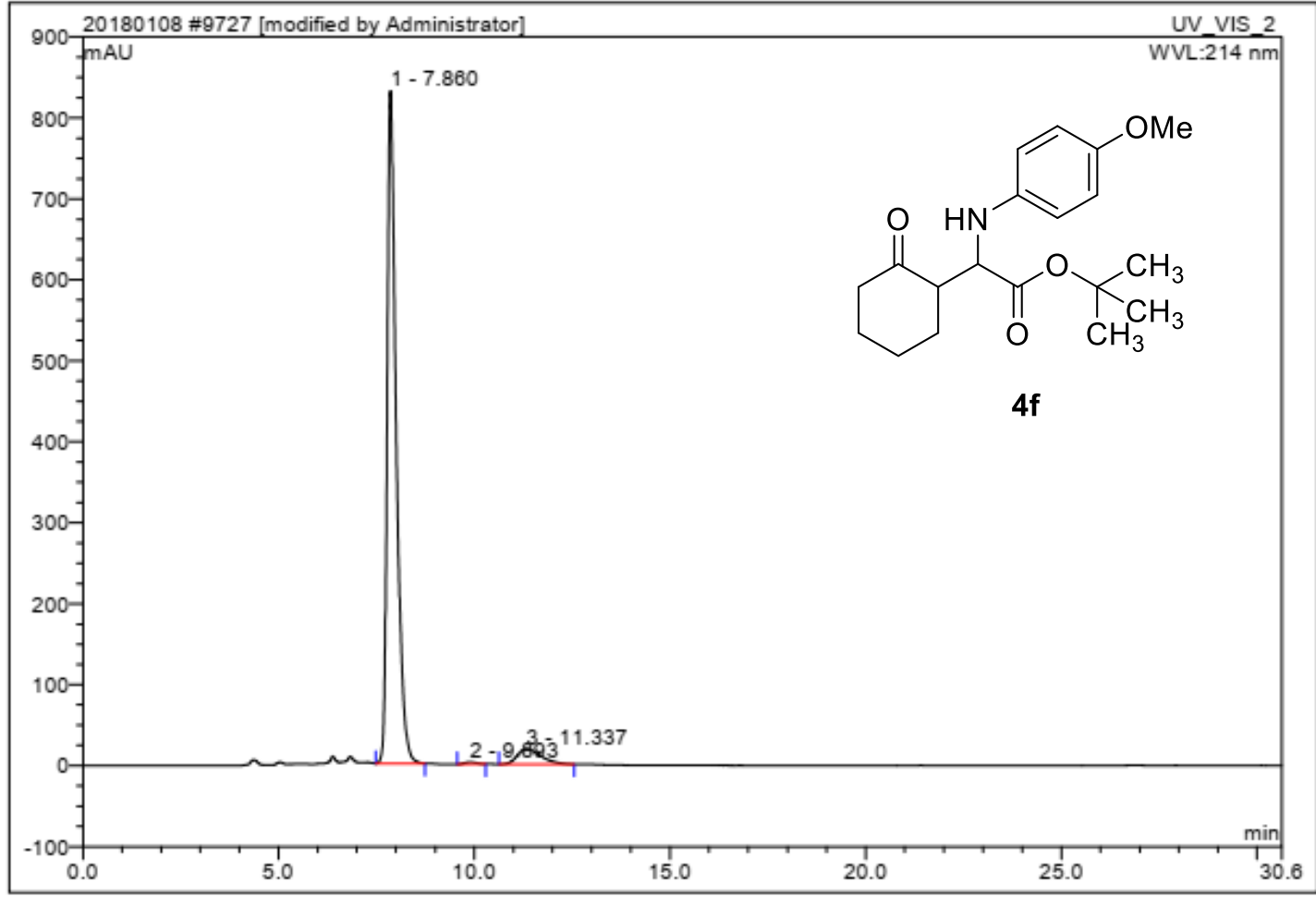

\begin{tabular}{|r|rrrrrrr|}
\hline No. & $\begin{array}{r}\text { Ret.Time } \\
\text { min }\end{array}$ & Peak Name & $\begin{array}{c}\text { Height } \\
\text { mAU }\end{array}$ & $\begin{array}{r}\text { Area } \\
\text { mAU*min }\end{array}$ & $\begin{array}{r}\text { Rel.Area } \\
\%\end{array}$ & Amount & Type \\
\hline 1 & 7.86 & n.a. & 831.175 & 221.982 & 93.95 & n.a. & BMB $^{\star}$ \\
2 & 9.89 & n.a. & 2.478 & 0.887 & 0.38 & n.a. & BMB \\
3 & 11.34 & n.a. & 19.121 & 13.403 & 5.67 & n.a. & BMB \\
\hline Total: & & & 852.775 & 236.272 & 100.00 & 0.000 & \\
\hline
\end{tabular}




\section{WZH1201-1+- IB 9822140.7}

\begin{tabular}{llll}
\hline Sample Name: & WZH1201-1+- IB 982 214 0.7 & Injection Volume: & 3.0 \\
Vial Number: & RE6 & Channel: & UV_VIS_2 \\
Sample Type: & unknown & Wavelength: & $\mathbf{2 1 4 . 0}$ \\
Control Program: & test-dad3 & Bandwidth: & 4 \\
Quantif. Method: & $\mathbf{2 0 1 7 0 6 0 8}$ & Dilution Factor: & $\mathbf{1 . 0 0 0 0}$ \\
Recording Time: & $\mathbf{2 0 2 0 - 1 2 - 1 5 ~ 1 6 : 4 5}$ & Sample Weight: & $\mathbf{1 . 0 0 0 0}$ \\
Run Time (min): & $\mathbf{2 1 . 7 2}$ & Sample Amount: & $\mathbf{1 . 0 0 0 0}$
\end{tabular}

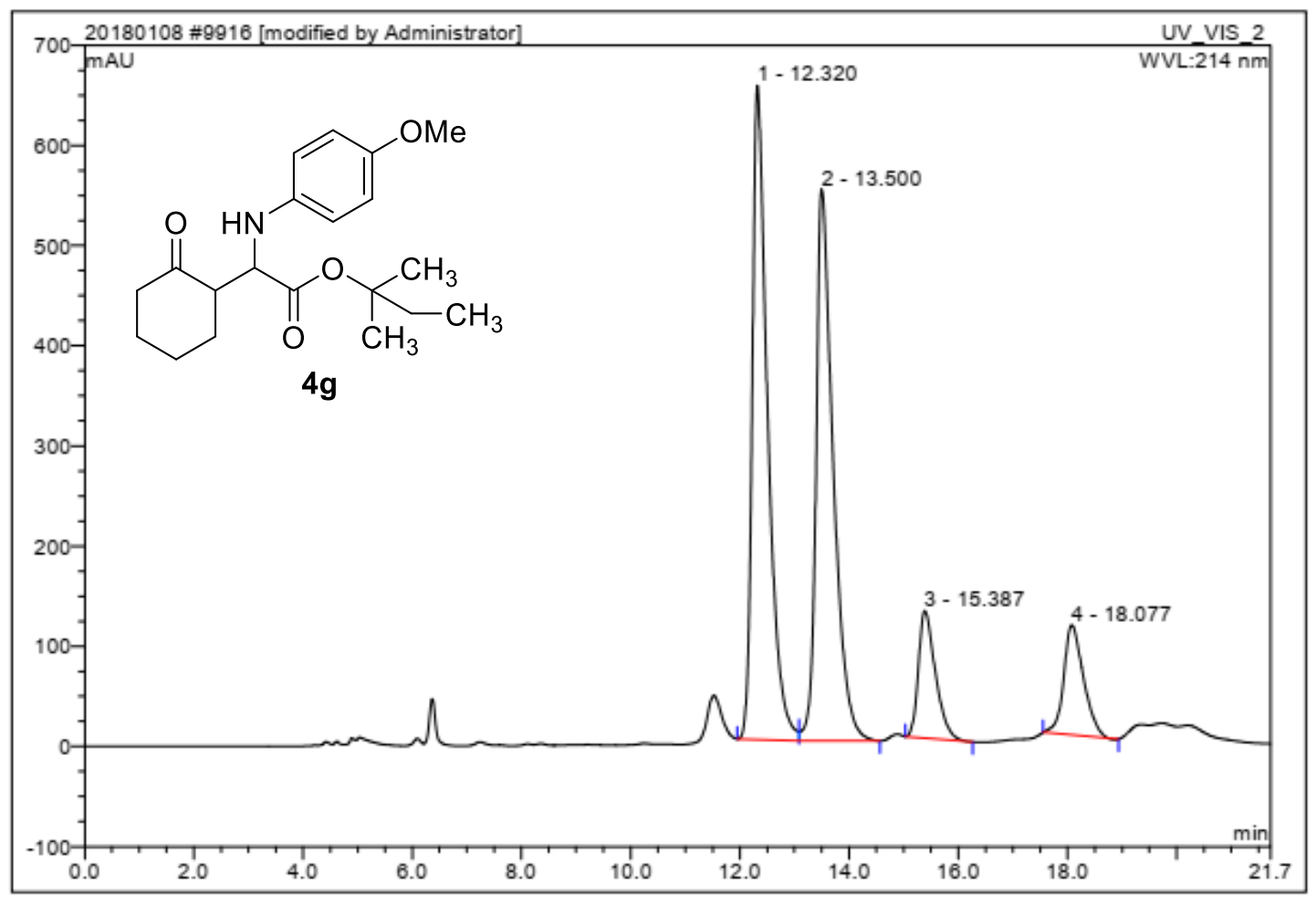

\begin{tabular}{|r|ccrrrrr|}
\hline No. & $\begin{array}{c}\text { Ret.Time } \\
\text { min }\end{array}$ & Peak Name & $\begin{array}{c}\text { Height } \\
\text { mAU }\end{array}$ & $\begin{array}{c}\text { Area } \\
\text { mAU*min }\end{array}$ & $\begin{array}{r}\text { Rel.Area } \\
\%\end{array}$ & Amount & Type \\
\hline 1 & 12.32 & n.a. & 653.141 & 212.788 & 42.15 & n.a. & BM $^{*}$ \\
2 & 13.50 & n.a. & 550.873 & 199.847 & 39.59 & n.a. & MB $^{*}$ \\
3 & 15.39 & n.a. & 127.299 & 46.191 & 9.15 & n.a. & BMB $^{*}$ \\
4 & 18.08 & n.a. & 109.897 & 45.963 & 9.11 & n.a. & BMB $^{*}$ \\
\hline Total: & & & 1441.209 & 504.789 & 100.00 & 0.000 & \\
\hline
\end{tabular}




\section{\#\#\# WZH-1118-2 IB 9822140.7}

\begin{tabular}{llll|}
\hline Sample Name: & WZH-1118-2 IB 982 214 0.7 & Injection Volume: & 2.0 \\
Vial Number: & RD7 & Channel: & UV_VIS_2 \\
Sample Type: & unknown & Wavelength: & $\mathbf{2 4 0 . 0}$ \\
Control Program: & test-dad2 & Bandwidth: & 4 \\
Quantif. Method: & $\mathbf{2 0 1 7 0 6 0 8}$ & Dilution Factor: & 1.0000 \\
Recording Time: & $\mathbf{2 0 2 0 - 1 2 - 2 9 ~ 1 0 : 5 8}$ & Sample Weight: & 1.0000 \\
Run Time (min): & $\mathbf{5 1 . 4 4}$ & Sample Amount: & $\mathbf{1 . 0 0 0 0}$ \\
\hline
\end{tabular}

2.50020180108 \#10051 [modified by Administrator] $\quad$ UV_VIS_2

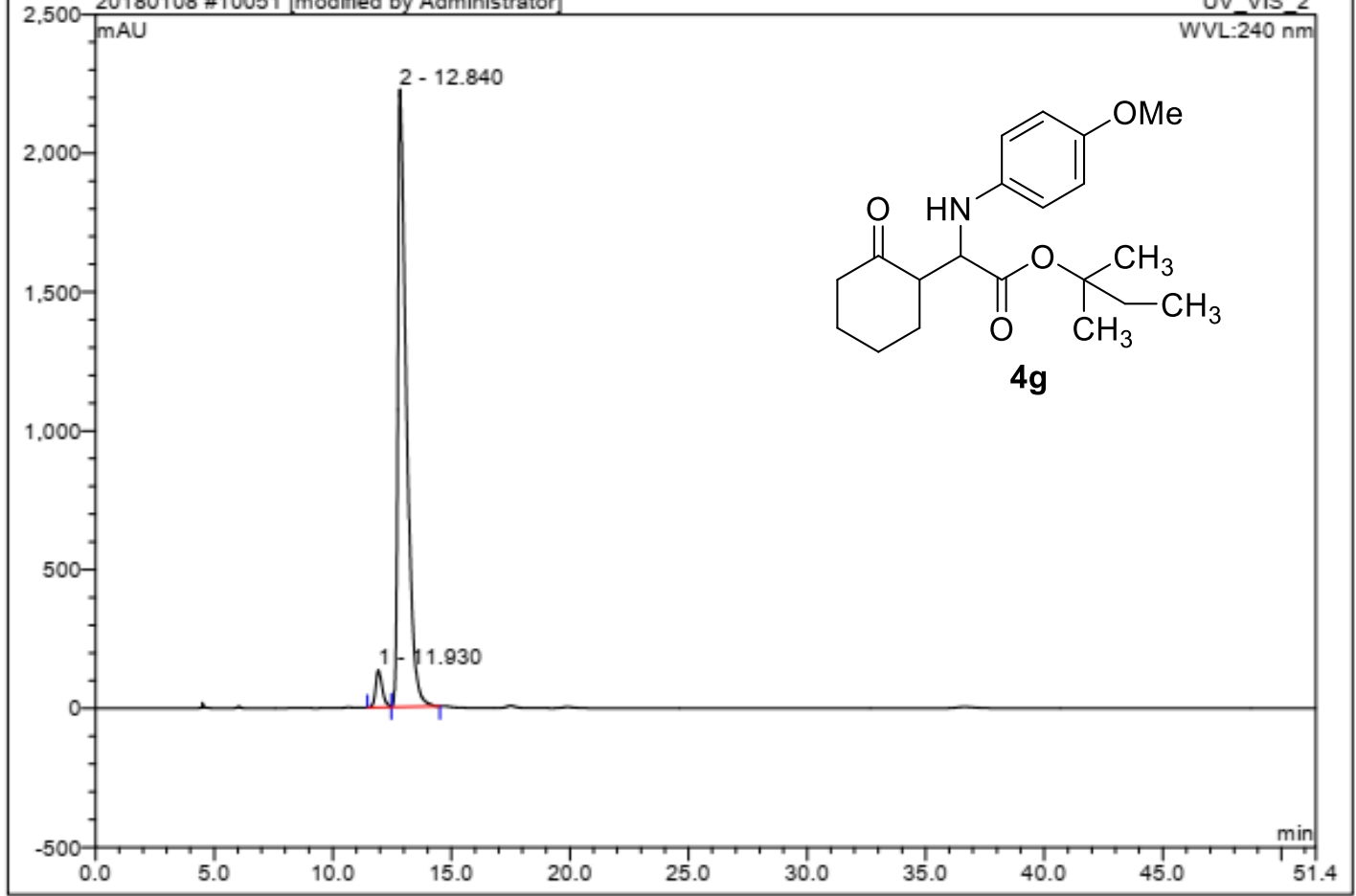

\begin{tabular}{|r|ccrrrrr|}
\hline No. & $\begin{array}{c}\text { Ret.Time } \\
\text { min }\end{array}$ & Peak Name & $\begin{array}{c}\text { Height } \\
\text { mAU }\end{array}$ & $\begin{array}{c}\text { Area } \\
\text { mAU*min }\end{array}$ & $\begin{array}{r}\text { Rel.Area } \\
\%\end{array}$ & Amount & Type \\
\hline 1 & 11.93 & n.a. & 135.377 & 44.177 & 4.54 & n.a. & BM \\
2 & 12.84 & n.a. & 2225.812 & 928.351 & 95.46 & n.a. & MB \\
\hline Total: & & & 2361.189 & 972.528 & 100.00 & 0.000 & \\
\hline
\end{tabular}


9881 WZH-1125-3-1+3-2 ASH 912140.7

\begin{tabular}{llll}
\hline Sample Name: & WZH-1125-3-1+3-2 ASH 912140.7 & Injection Volume: & $\mathbf{1 . 0}$ \\
Vial Number: & RA7 & Channel: & UV_VIS_2 \\
Sample Type: & unknown & Wavelength: & $\mathbf{2 1 4 . 0}$ \\
Control Program: & test-dad3 & Bandwidth: & 4 \\
Quantif. Method: & $\mathbf{2 0 1 7 0 6 0 8}$ & Dilution Factor: & $\mathbf{1 . 0 0 0 0}$ \\
Recording Time: & $\mathbf{2 0 2 0 - 1 2 - 9 ~ 1 7 : 0 1}$ & Sample Weight: & $\mathbf{1 . 0 0 0 0}$ \\
Run Time (min): & $\mathbf{3 0 . 7 9}$ & Sample Amount: & $\mathbf{1 . 0 0 0 0}$ \\
\hline
\end{tabular}

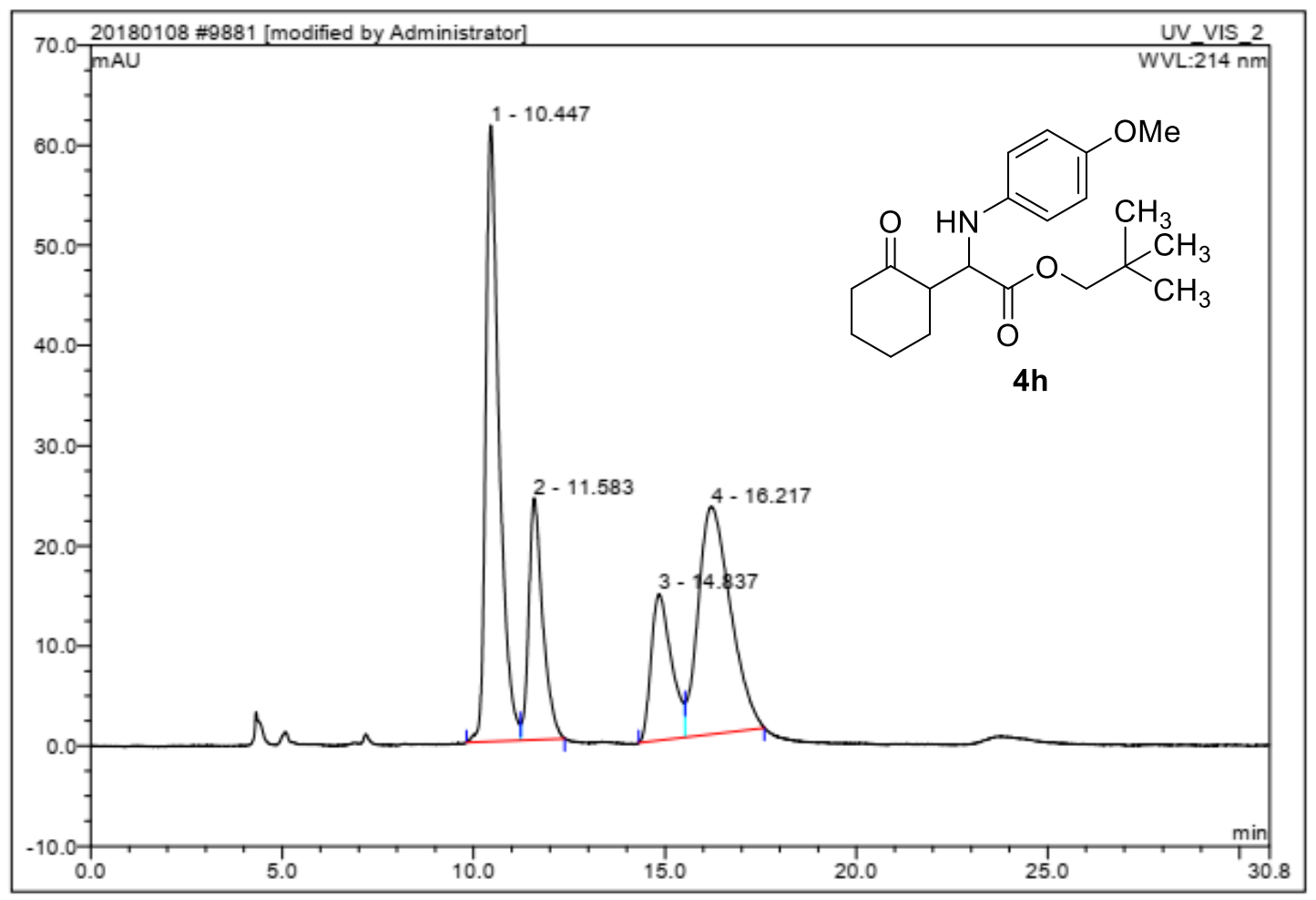

\begin{tabular}{|r|ccrrrrr|}
\hline No. & $\begin{array}{c}\text { Ret.Time } \\
\text { min }\end{array}$ & Peak Name & $\begin{array}{c}\text { Height } \\
\text { mAU }\end{array}$ & $\begin{array}{c}\text { Area } \\
\text { mAU*min }\end{array}$ & $\begin{array}{c}\text { Rel.Area } \\
\%\end{array}$ & Amount & Type \\
\hline 1 & 10.45 & n.a. & 61.575 & 25.080 & 37.62 & n.a. & BM \\
2 & 11.58 & n.a. & 24.165 & 10.207 & 15.31 & n.a. & MB \\
3 & 14.84 & n.a. & 14.637 & 9.032 & 13.55 & n.a. & BM \\
4 & 16.22 & n.a. & 22.725 & 22.354 & 33.53 & n.a. & MB \\
\hline Total: & & & 123.101 & 66.673 & 100.00 & 0.000 & \\
\hline
\end{tabular}




\begin{tabular}{|lllll|}
\hline \#\#\# WZH-1125-1 IB 9822140.7 & & \\
& & & \\
\hline Sample Name: & WZH-1125-1 IB 982 214 0.7 & Injection Volume: & 2.0 \\
Vial Number: & RD2 & Channel: & UV_VIS_2 \\
Sample Type: & unknown & Wavelength: & $\mathbf{2 1 4 . 0}$ \\
Control Program: & test-dad3 & Bandwidth: & 4 \\
Quantif. Method: & 20170608 & Dilution Factor: & 1.0000 \\
Recording Time: & $2020-12-23$ 18:42 & Sample Weight: & 1.0000 \\
Run Time (min): & 35.00 & Sample Amount: & $\mathbf{1 . 0 0 0 0}$ \\
\hline
\end{tabular}

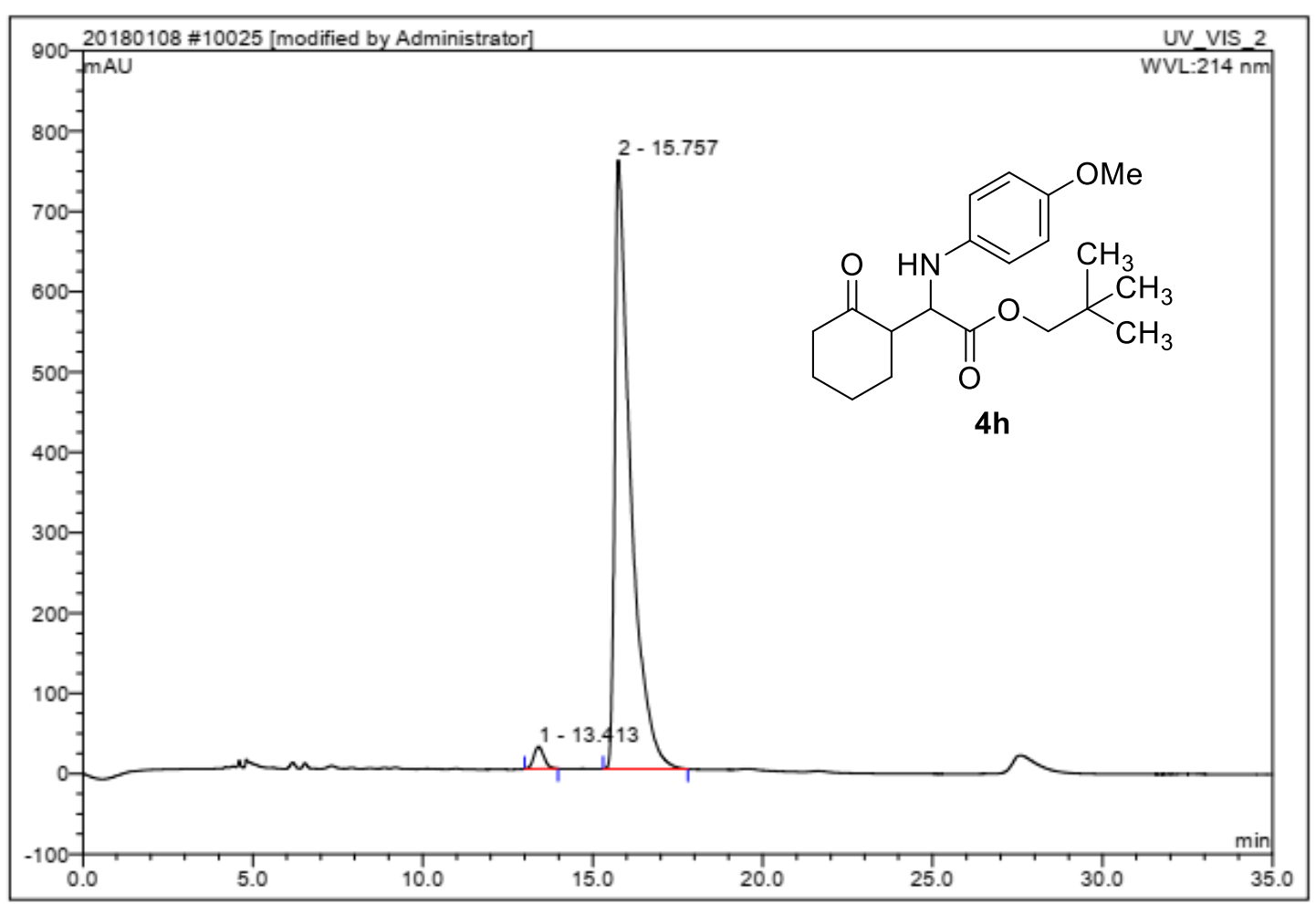

\begin{tabular}{|r|ccrrrrr|}
\hline No. & $\begin{array}{c}\text { Ret.Time } \\
\text { min }\end{array}$ & Peak Name & $\begin{array}{c}\text { Height } \\
\text { mAU }\end{array}$ & $\begin{array}{c}\text { Area } \\
\text { mAU*min }\end{array}$ & $\begin{array}{c}\text { Rel.Area } \\
\%\end{array}$ & Amount & Type \\
\hline 1 & 13.41 & n.a. & 27.671 & 9.609 & 2.28 & n.a. & BMB \\
2 & 15.76 & n.a. & 758.259 & 412.060 & 97.72 & n.a. & BMB \\
\hline Total: & & & 785.931 & 421.669 & 100.00 & 0.000 & \\
\hline
\end{tabular}


9917 WZH1201-3+- IB 9552140.7

\begin{tabular}{|llll|}
\hline Sample Name: & WZH1201-3+- IB 955 214 0.7 & Injection Volume: & 3.0 \\
Vial Number: & RD5 & Channel: & UV_VIS_2 \\
Sample Type: & unknown & Wavelength: & $\mathbf{2 1 4 . 0}$ \\
Control Program: & test-dad3 & Bandwidth: & 4 \\
Quantif. Method: & $\mathbf{2 0 1 7 0 6 0 8}$ & Dilution Factor: & 1.0000 \\
Recording Time: & $\mathbf{2 0 2 0 - 1 2 - 1 5 ~ 9 : 1 8}$ & Sample Weight: & $\mathbf{1 . 0 0 0 0}$ \\
Run Time (min): & $\mathbf{4 2 . 9 8}$ & Sample Amount: & $\mathbf{1 . 0 0 0 0}$ \\
\hline
\end{tabular}

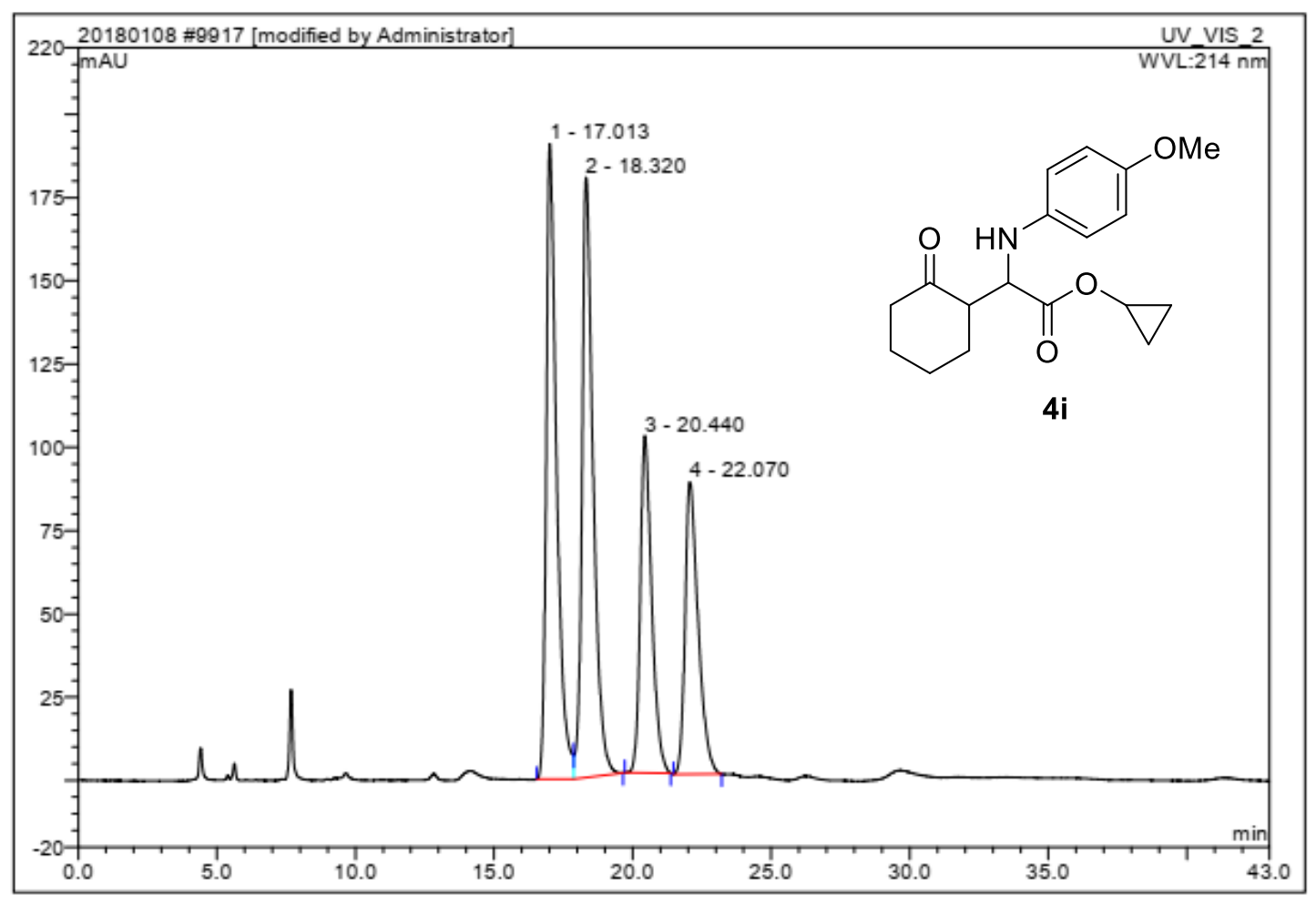

\begin{tabular}{|r|ccrrrrr|}
\hline No. & $\begin{array}{c}\text { Ret.Time } \\
\text { min }\end{array}$ & Peak Name & $\begin{array}{c}\text { Height } \\
\text { mAU }\end{array}$ & $\begin{array}{c}\text { Area } \\
\text { mAU*min }\end{array}$ & $\begin{array}{c}\text { Rel.Area } \\
\%\end{array}$ & Amount & Type \\
\hline 1 & 17.01 & n.a. & 190.781 & 82.980 & 31.48 & n.a. & BM $^{*}$ \\
2 & 18.32 & n.a. & 180.274 & 84.033 & 31.88 & n.a. & MB $^{*}$ \\
3 & 20.44 & n.a. & 101.301 & 48.478 & 18.39 & n.a. & BMB $^{*}$ \\
4 & 22.07 & n.a. & 87.787 & 48.081 & 18.24 & n.a. & BMB \\
\hline Total: & & & 560.142 & 263.572 & 100.00 & 0.000 & \\
\hline
\end{tabular}




\begin{tabular}{|lllll|}
\hline \#\#\# WZH-1202-2 IB 9552140.7 & & \\
& & & \\
\hline Sample Name: & WZH-1202-2 IB 9552140.7 & Injection Volume: & 2.0 \\
Vial Number: & RC2 & Channel: & UV_VIS_2 \\
Sample Type: & unknown & Wavelength: & $\mathbf{2 4 0 . 0}$ \\
Control Program: & test-dad2 & Bandwidth: & 4 \\
Quantif. Method: & $\mathbf{2 0 1 7 0 6 0 8}$ & Dilution Factor: & $\mathbf{1 . 0 0 0 0}$ \\
Recording Time: & $\mathbf{2 0 2 0 - 1 2 - 2 3 ~ 2 0 : 3 0}$ & Sample Weight: & $\mathbf{1 . 0 0 0 0}$ \\
Run Time (min): & $\mathbf{4 5 . 0 0}$ & Sample Amount: & $\mathbf{1 . 0 0 0 0}$ \\
\hline
\end{tabular}

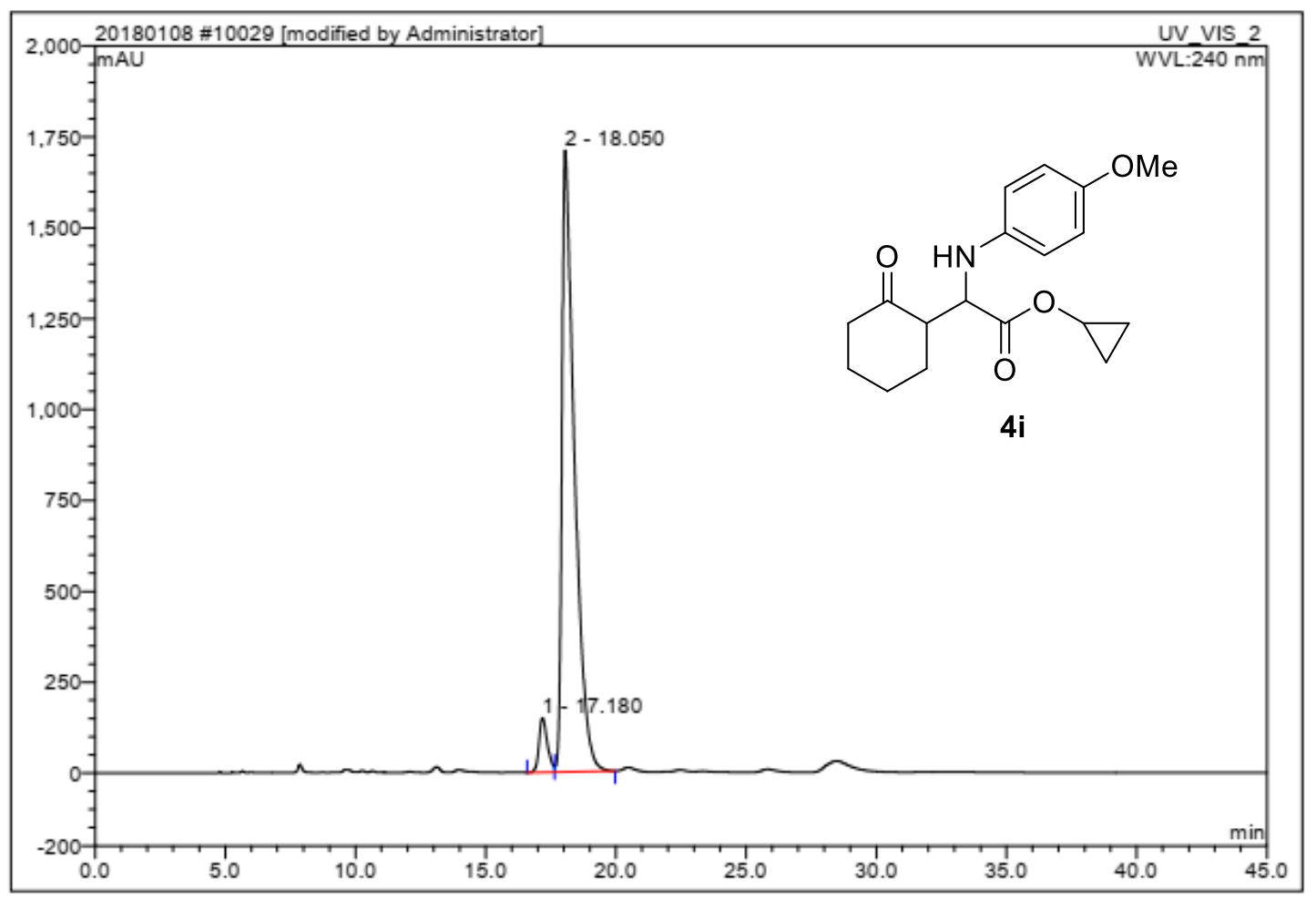

\begin{tabular}{|r|ccrrrrr|}
\hline No. & $\begin{array}{c}\text { Ret.Time } \\
\text { min }\end{array}$ & Peak Name & \multicolumn{1}{c}{$\begin{array}{c}\text { Height } \\
\text { mAU }\end{array}$} & $\begin{array}{c}\text { Area } \\
\text { mAU*min }\end{array}$ & $\begin{array}{r}\text { Rel.Area } \\
\%\end{array}$ & Amount & Type \\
\hline 1 & 17.18 & n.a. & 149.187 & 56.165 & 5.57 & n.a. & BM $^{*}$ \\
2 & 18.05 & n.a. & 1710.589 & 952.372 & 94.43 & n.a. & MB $^{*}$ \\
\hline Total: & & & 1859.775 & 1008.537 & 100.00 & 0.000 & \\
\hline
\end{tabular}


\#\#\# WZH-1201-4+- PC2 912140.7

\begin{tabular}{llll}
\hline Sample Name: & WZH-1201-4+- PC2 912140.7 & Injection Volume: & $\mathbf{2 . 0}$ \\
Vial Number: & RE4 & Channel: & UV_VIS_2 \\
Sample Type: & unknown & Wavelength: & $\mathbf{2 1 4 . 0}$ \\
Control Program: & test-dad4 & Bandwidth: & 4 \\
Quantif. Method: & $\mathbf{2 0 1 7 0 6 0 8}$ & Dilution Factor: & $\mathbf{1 . 0 0 0 0}$ \\
Recording Time: & $\mathbf{2 0 2 0 - 1 2 - 2 2 ~ 1 2 : 2 3}$ & Sample Weight: & 1.0000 \\
Run Time (min): & $\mathbf{7 0 . 8 4}$ & Sample Amount: & $\mathbf{1 . 0 0 0 0}$ \\
\hline
\end{tabular}

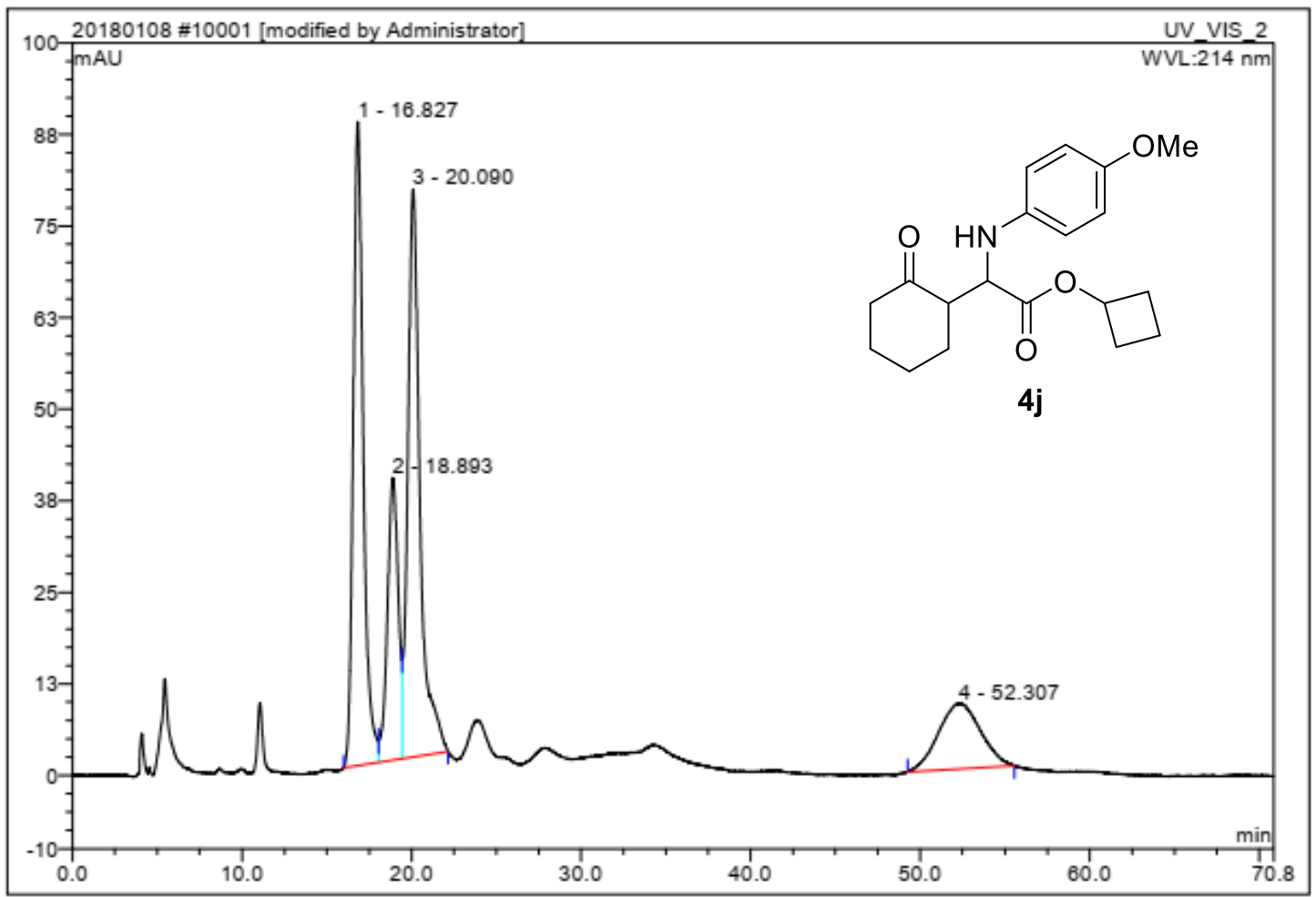

\begin{tabular}{|r|ccrrrrc|}
\hline No. & $\begin{array}{c}\text { Ret.Time } \\
\text { min }\end{array}$ & Peak Name & $\begin{array}{c}\text { Height } \\
\text { mAU }\end{array}$ & $\begin{array}{c}\text { Area } \\
\text { mAU*min }\end{array}$ & $\begin{array}{c}\text { Rel.Area } \\
\%\end{array}$ & Amount & Type \\
\hline 1 & 16.83 & n.a. & 87.869 & 60.198 & 33.58 & n.a. & BM \\
2 & 18.89 & n.a. & 38.522 & 28.651 & 15.98 & n.a. & M \\
3 & 20.09 & n.a. & 77.471 & 64.745 & 36.11 & n.a. & MB \\
4 & 52.31 & n.a. & 8.987 & 25.696 & 14.33 & n.a. & BMB $^{\star}$ \\
\hline Total: & & & 212.849 & 179.290 & 100.00 & 0.000 & \\
\hline
\end{tabular}


\#\#\# WZH-1202-3+- PC2 912140.7

Sample Name: $\quad$ WZH-1202-3+- PC2 912140.7

Vial Number:

Sample Type:

Control Program:

Quantif. Method:

Recording Time:

Run Time (min):

\section{RB6}

unknown

test-dad2

20170608

2020-12-29 16:48

70.00
Injection Volume:

Channel:

Wavelength:

Bandwidth:

Dilution Factor:

Sample Weight:

Sample Amount:
1.0 UV_VIS_2 240.0

4

1.0000

1.0000

1.0000

35020180108 \#10058 [modified by Administrator] $\quad$ UV_VIS_2

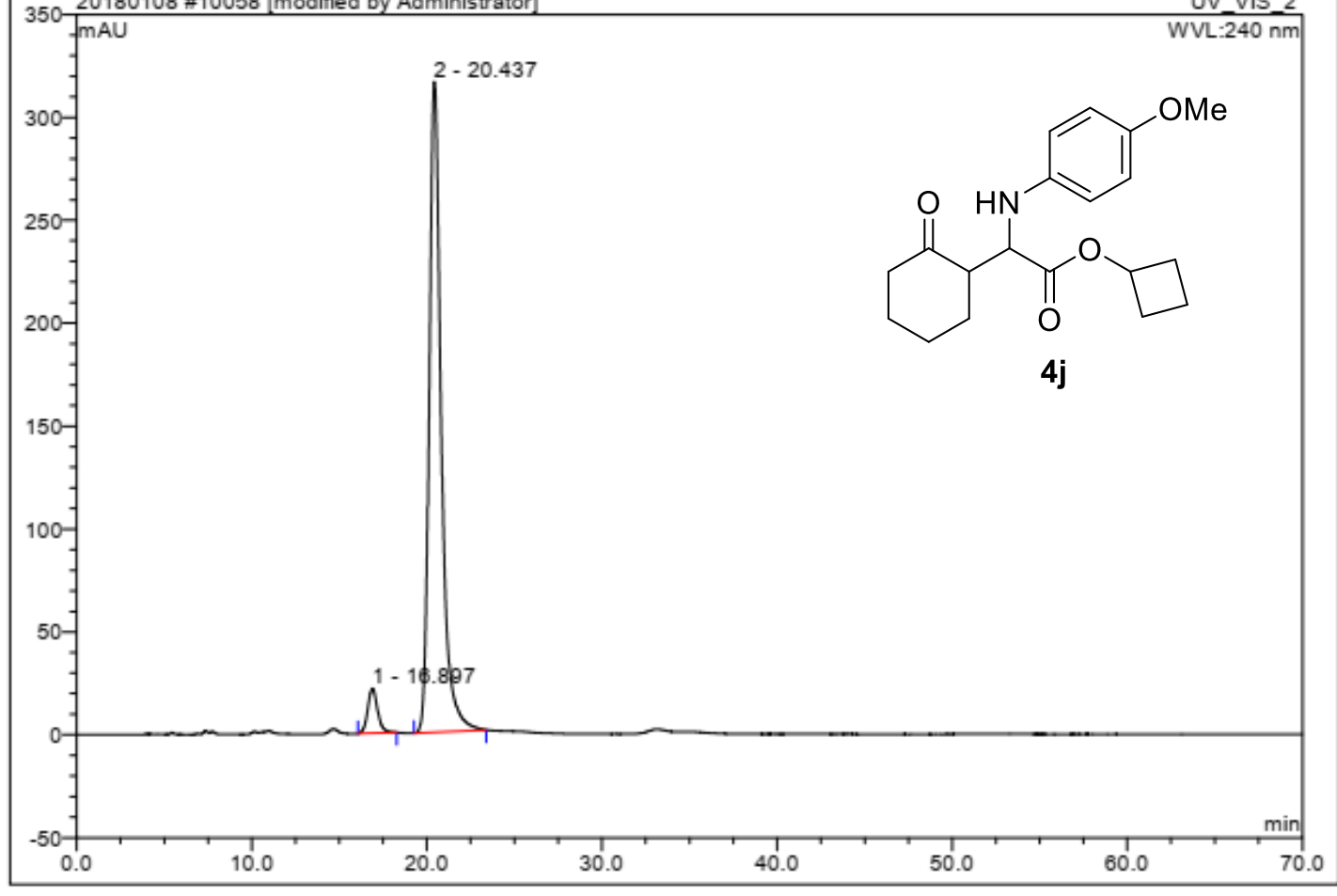

\begin{tabular}{|r|ccrrrrr|}
\hline No. & $\begin{array}{c}\text { Ret.Time } \\
\text { min }\end{array}$ & Peak Name & $\begin{array}{c}\text { Height } \\
\text { mAU }\end{array}$ & $\begin{array}{c}\text { Area } \\
\text { mAU*min }\end{array}$ & $\begin{array}{c}\text { Rel.Area } \\
\%\end{array}$ & Amount & Type \\
\hline 1 & 16.90 & n.a. & 21.805 & 14.300 & 5.40 & n.a. & BMB \\
2 & 20.44 & n.a. & 316.182 & 250.763 & 94.60 & n.a. & BMB \\
\hline Total: & & & 337.988 & 265.063 & 100.00 & 0.000 & \\
\hline
\end{tabular}




\section{WZH1201-5-1+5-2 ASH 912140.7}

\begin{tabular}{llll}
\hline Sample Name: & WZH1201-5-1+5-2 ASH 91 214 0.7 & Injection Volume: & 3.0 \\
Vial Number: & RB5 & Channel: & UV_VIS_2 \\
Sample Type: & unknown & Wavelength: & $\mathbf{2 1 4 . 0}$ \\
Control Program: & test-dad3 & Bandwidth: & 4 \\
Quantif. Method: & $\mathbf{2 0 1 7 0 6 0 8}$ & Dilution Factor: & 1.0000 \\
Recording Time: & $\mathbf{2 0 2 0 - 1 2 - 1 5 ~ 1 8 : 4 3}$ & Sample Weight: & 1.0000 \\
Run Time (min): & $\mathbf{4 0 . 0 0}$ & Sample Amount: & $\mathbf{1 . 0 0 0 0}$ \\
\hline
\end{tabular}

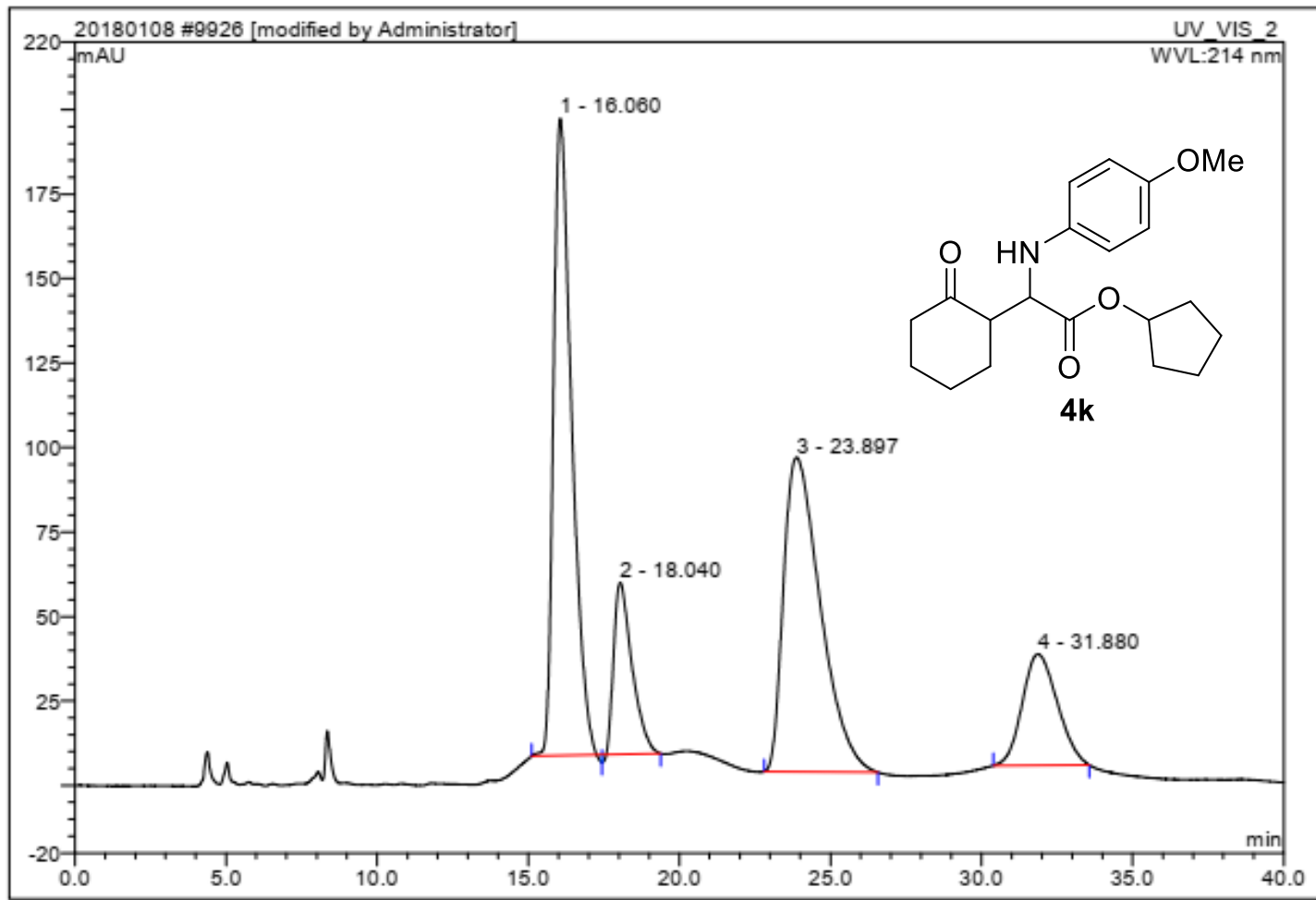

\begin{tabular}{|r|ccrrrrr|}
\hline No. & $\begin{array}{c}\text { Ret.Time } \\
\text { min }\end{array}$ & Peak Name & $\begin{array}{c}\text { Height } \\
\text { mAU }\end{array}$ & $\begin{array}{c}\text { Area } \\
\text { mAU*min }\end{array}$ & $\begin{array}{r}\text { Rel.Area } \\
\%\end{array}$ & Amount & Type \\
\hline 1 & 16.06 & n.a. & 188.670 & 131.916 & 38.35 & n.a. & BM $^{*}$ \\
2 & 18.04 & n.a. & 50.891 & 35.676 & 10.37 & n.a. & MB $^{*}$ \\
3 & 23.90 & n.a. & 93.096 & 131.921 & 38.35 & n.a. & BMB \\
4 & 31.88 & n.a. & 33.026 & 44.494 & 12.93 & n.a. & BMB \\
\hline Total: & & & 365.682 & 344.006 & 100.00 & 0.000 & \\
\hline
\end{tabular}




\begin{tabular}{|lllll|}
\hline \#\#\# WZH-1207-1 ASH 912140.7 & & \\
& & & \\
\hline Sample Name: & WZH-1207-1 ASH 912140.7 & Injection Volume: & 1.0 \\
Vial Number: & RD6 & Channel: & UV_VIS_2 \\
Sample Type: & unknown & Wavelength: & $\mathbf{2 4 0 . 0}$ \\
Control Program: & test-dad2 & Bandwidth: & 4 \\
Quantif. Method: & $\mathbf{2 0 1 7 0 6 0 8}$ & Dilution Factor: & 1.0000 \\
Recording Time: & $\mathbf{2 0 2 0 - 1 2 - 3 0 ~ 9 : 4 0}$ & Sample Weight: & $\mathbf{1 . 0 0 0 0}$ \\
Run Time (min): & $\mathbf{7 0 . 0 0}$ & Sample Amount: & $\mathbf{1 . 0 0 0 0}$ \\
\hline
\end{tabular}

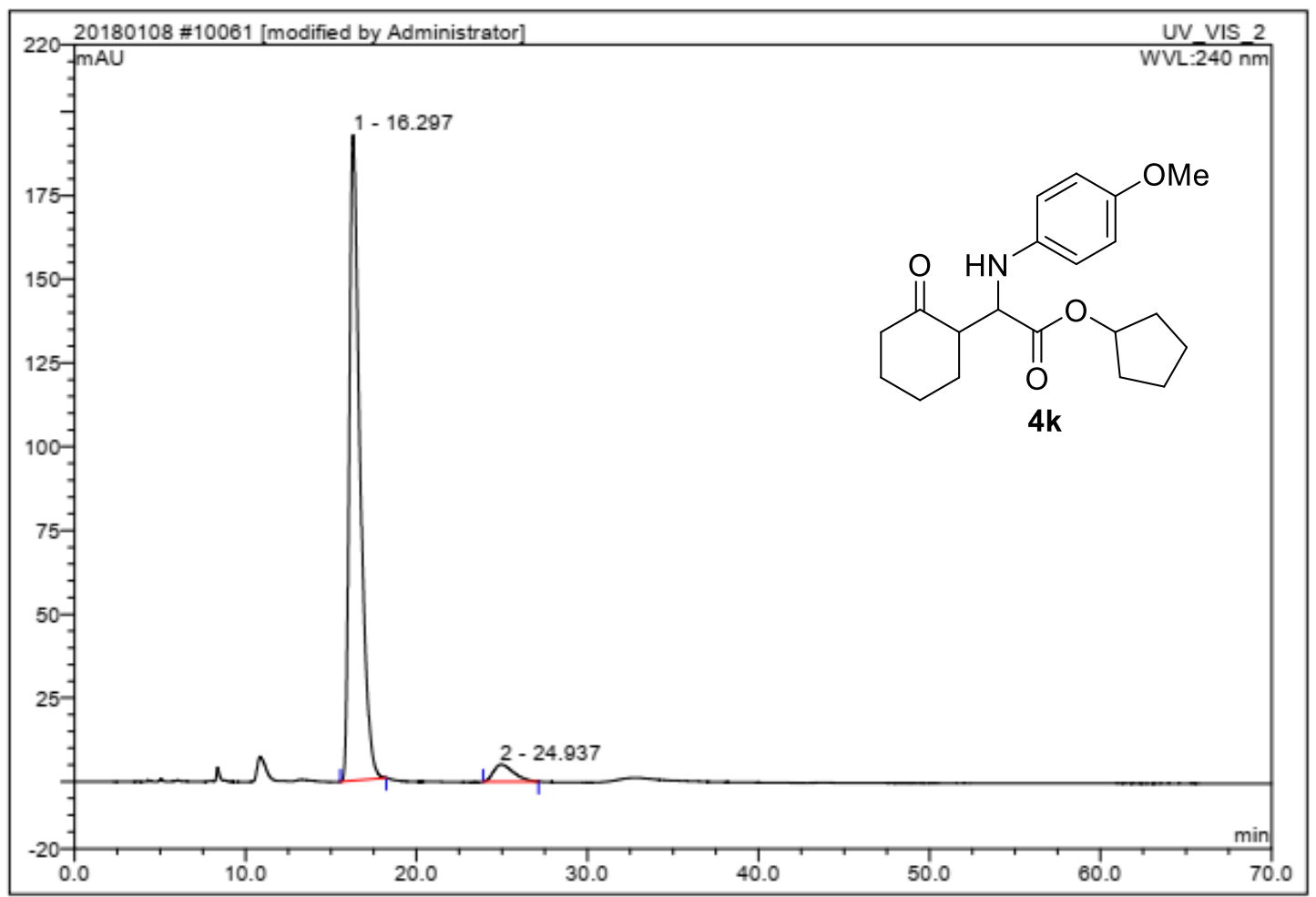

\begin{tabular}{|r|rrrrrrr|}
\hline No. & $\begin{array}{c}\text { Ret.Time } \\
\text { min }\end{array}$ & Peak Name & $\begin{array}{c}\text { Height } \\
\text { mAU }\end{array}$ & $\begin{array}{c}\text { Area } \\
\text { mAU*min }\end{array}$ & $\begin{array}{c}\text { Rel.Area } \\
\%\end{array}$ & Amount & Type \\
\hline 1 & 16.30 & n.a. & 192.930 & 139.532 & 95.21 & n.a. & BMB \\
2 & 24.94 & n.a. & 5.139 & 7.021 & 4.79 & n.a. & BMB $^{\star}$ \\
\hline Total: & & & 198.069 & 146.552 & 100.00 & 0.000 & \\
\hline
\end{tabular}




\section{\#\#\# WZH-1201-6+- PC2 912140.7}

\begin{tabular}{|llll|}
\hline Sample Name: & WZH-1201-6+- PC2 912140.7 & Injection Volume: & 2.0 \\
Vial Number: & RE5 & Channel: & UV_VIS_2 \\
Sample Type: & unknown & Wavelength: & $\mathbf{2 1 4 . 0}$ \\
Control Program: & test-dad4 & Bandwidth: & 4 \\
Quantif. Method: & $\mathbf{2 0 1 7 0 6 0 8}$ & Dilution Factor: & $\mathbf{1 . 0 0 0 0}$ \\
Recording Time: & $\mathbf{2 0 2 0 - 1 2 - 2 2 ~ 1 1 : 0 9}$ & Sample Weight: & $\mathbf{1 . 0 0 0 0}$ \\
Run Time (min): & $\mathbf{7 2 . 4 4}$ & Sample Amount: & $\mathbf{1 . 0 0 0 0}$ \\
\hline
\end{tabular}

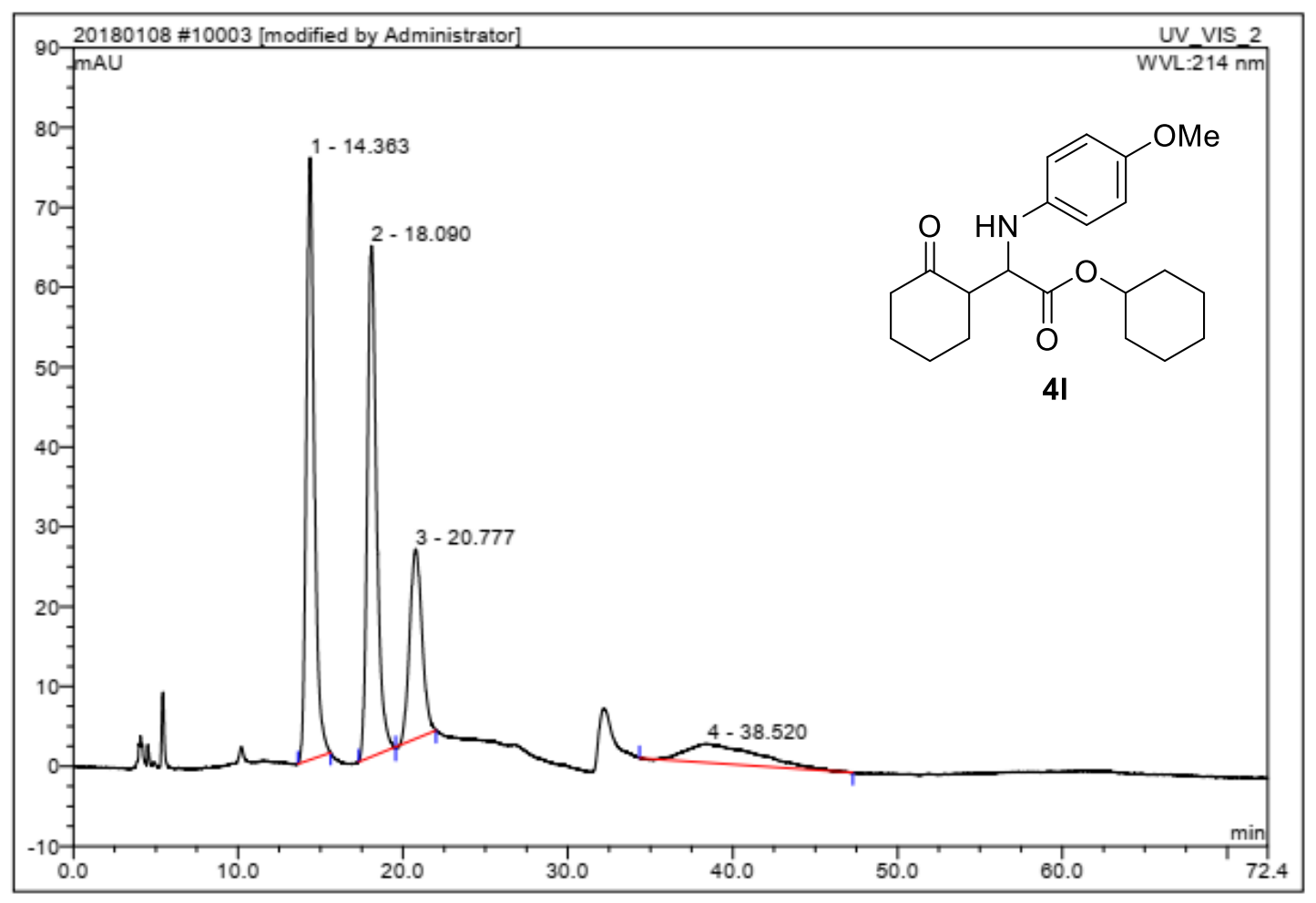

\begin{tabular}{|r|ccrrrrc|}
\hline No. & $\begin{array}{c}\text { Ret.Time } \\
\text { min }\end{array}$ & Peak Name & $\begin{array}{c}\text { Height } \\
\text { mAU }\end{array}$ & $\begin{array}{c}\text { Area } \\
\text { mAU*min }\end{array}$ & $\begin{array}{c}\text { Rel.Area } \\
\%\end{array}$ & Amount & Type \\
\hline 1 & 14.36 & n.a. & 75.367 & 42.559 & 36.24 & n.a. & BMB \\
2 & 18.09 & n.a. & 64.027 & 41.709 & 35.52 & n.a. & BM \\
3 & 20.78 & n.a. & 23.806 & 20.711 & 17.64 & n.a. & MB \\
4 & 38.52 & n.a. & 2.357 & 12.450 & 10.60 & n.a. & BMB $^{\star}$ \\
\hline Total: & & & 165.557 & 117.429 & 100.00 & 0.000 & \\
\hline
\end{tabular}




\begin{tabular}{|lllll|}
\hline \#\#\# WZH-1221-3 PC2 912140.7 & & \\
\hline Sample Name: & WZH-1221-3 PC2 912140.7 & Injection Volume: & 1.0 \\
Vial Number: & RD7 & Channel: & UV_VIS_2 \\
Sample Type: & unknown & Wavelength: & $\mathbf{2 1 4 . 0}$ \\
Control Program: & test-dad2 & Bandwidth: & 4 \\
Quantif. Method: & 20170608 & Dilution Factor: & 1.0000 \\
Recording Time: & $2020-12-319: 21$ & Sample Weight: & 1.0000 \\
Run Time (min): & 75.00 & Sample Amount: & 1.0000 \\
\hline
\end{tabular}

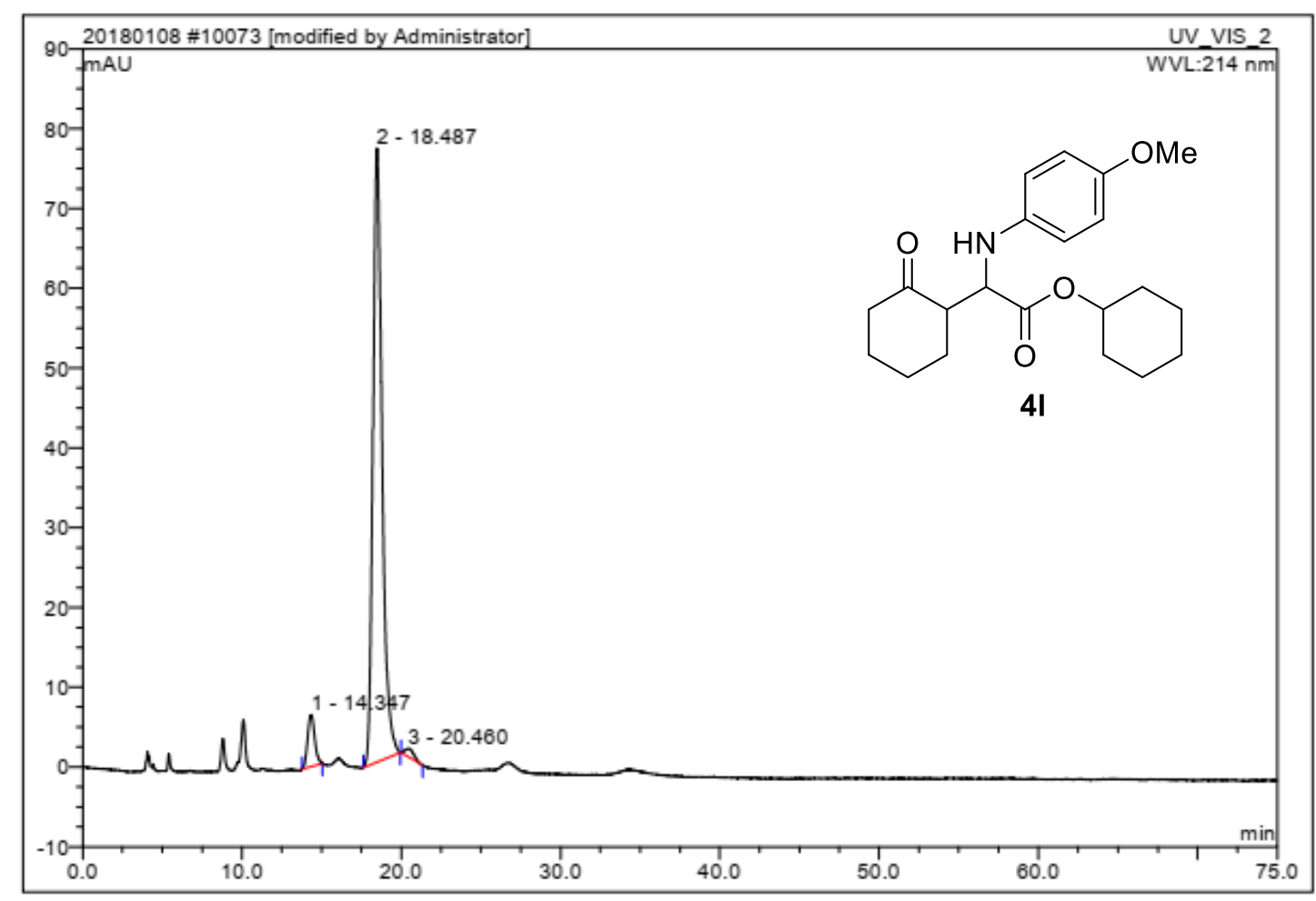

\begin{tabular}{|r|ccrrrrr|}
\hline No. & $\begin{array}{c}\text { Ret.Time } \\
\text { min }\end{array}$ & Peak Name & $\begin{array}{c}\text { Height } \\
\text { mAU }\end{array}$ & $\begin{array}{r}\text { Area } \\
\text { mAU*min }\end{array}$ & $\begin{array}{r}\text { Rel.Area } \\
\%\end{array}$ & Amount & Type \\
\hline 1 & 14.35 & n.a. & 6.485 & 3.343 & 6.06 & n.a. & BMB \\
2 & 18.49 & n.a. & 76.897 & 51.162 & 92.70 & n.a. & BMB \\
3 & 20.46 & n.a. & 1.043 & 0.684 & 1.24 & n.a. & BMB $^{*}$ \\
\hline Total: & & & 84.425 & 55.189 & 100.00 & 0.000 & \\
\hline
\end{tabular}




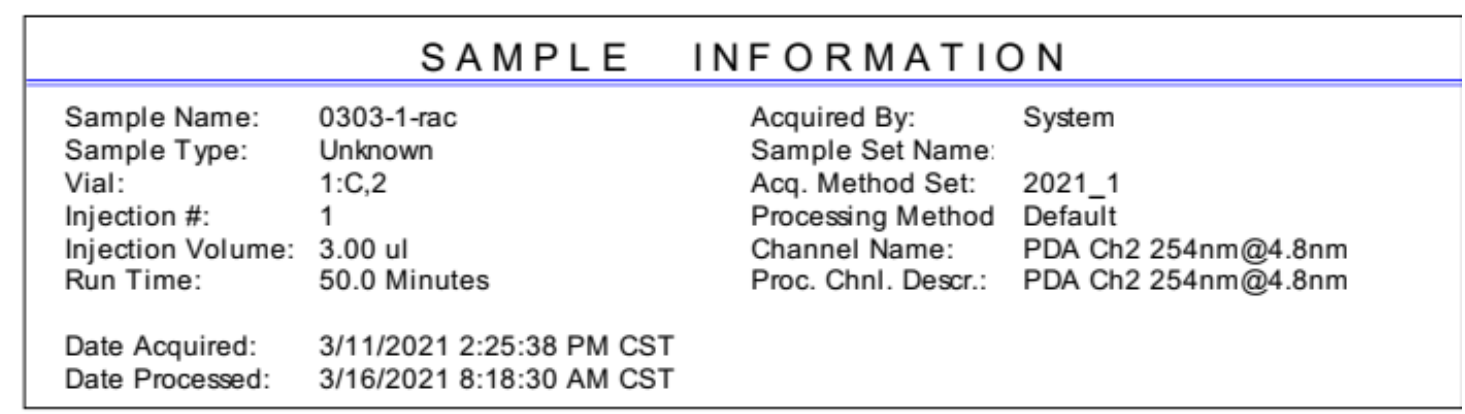

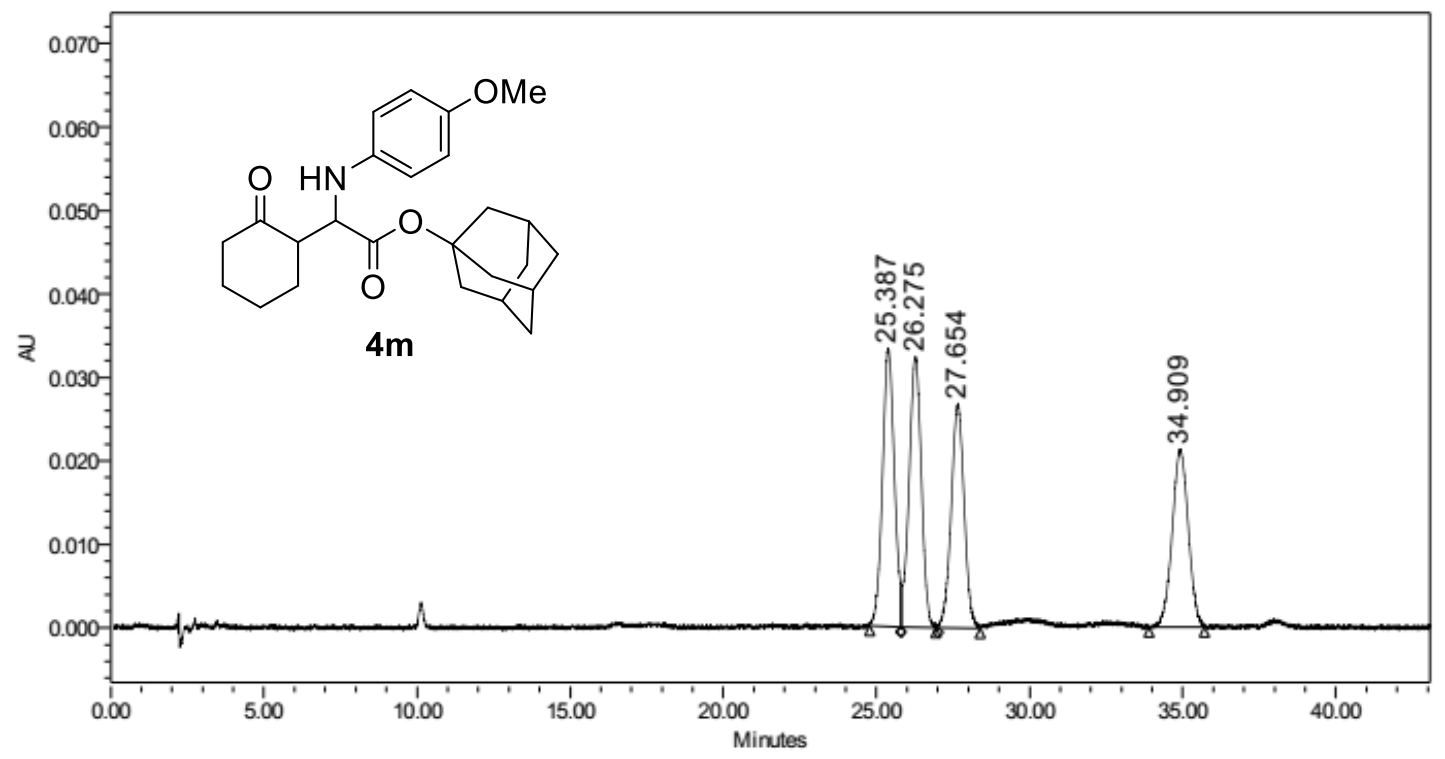

\begin{tabular}{|c|c|c|c|c|c|}
\hline & RT & $\begin{array}{c}\text { Width } \\
(\mathrm{sec})\end{array}$ & Height & Area & $\%$ Area \\
\hline 1 & 25.387 & 61.600 & 33297 & 895909 & 26.50 \\
\hline 2 & 26.275 & 68.350 & 32383 & 907663 & 26.85 \\
\hline 3 & 27.654 & 82.750 & 26892 & 792621 & 23.45 \\
\hline 4 & 34.909 & 109.250 & 21329 & 784057 & 23.20 \\
\hline
\end{tabular}

Reported by User: System

Report Method: Default Individual Report

Report Method I[ 2063

Page: 1 of 1
Project Name: 2021

Date Printed:

$3 / 16 / 2021$

8:24:14 AM PRC 


\begin{tabular}{|c|c|c|c|}
\hline \multicolumn{2}{|r|}{ SAMPLE } & \multicolumn{2}{|c|}{ INFORMATION } \\
\hline Sample Name: & $1118-1$ & Acquired By: & System \\
\hline Sample Type: & Unknown & Sample Set Name: & \\
\hline Vial: & $1: C, 4$ & Acq. Method Set: & 2021_1 \\
\hline Injection \#: & 1 & Processing Method & Defaūt \\
\hline Injection Volume: & $3.00 \mathrm{ul}$ & Channel Name: & PDA Ch2 254nm@4.8nm \\
\hline Run Time: & 50.0 Minutes & Proc. Chnl. Descr.: & PDA Ch2 254nm@4.8nm \\
\hline Date Acquired: & 3/11/2021 1:28:57 PM CST & & \\
\hline Date Processed: & $3 / 16 / 2021$ 8:20:00 AM CST & & \\
\hline
\end{tabular}

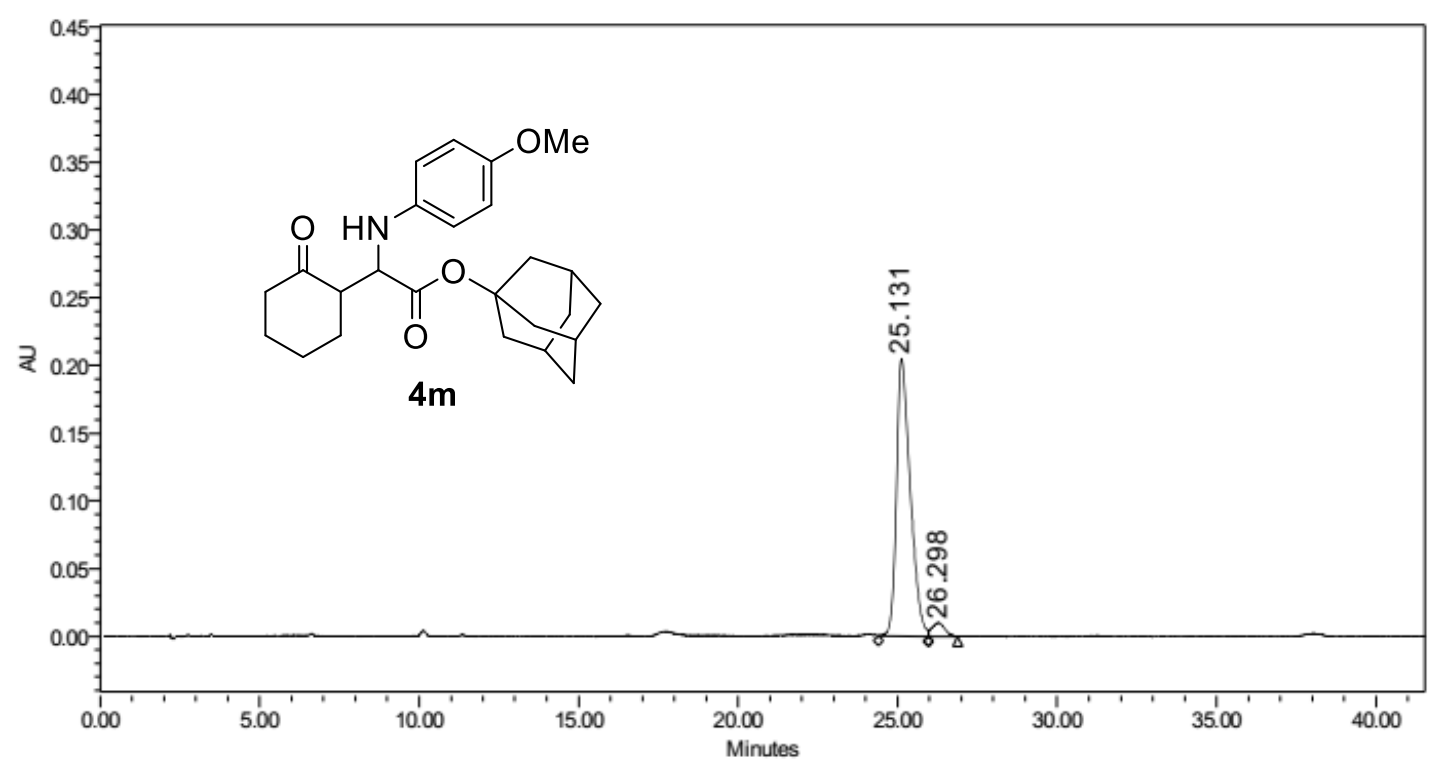

\begin{tabular}{|r|c|r|r|r|r|}
\hline & RT & $\begin{array}{c}\text { Width } \\
(\mathrm{sec})\end{array}$ & Height & Area & $\%$ Area \\
\hline 1 & 25.131 & 93.900 & 204868 & 6162124 & 95.67 \\
\hline 2 & 26.298 & 55.150 & 9886 & 278766 & 4.33 \\
\hline
\end{tabular}




\begin{tabular}{|c|c|c|c|}
\hline \multicolumn{2}{|r|}{ SAMPLE } & \multicolumn{2}{|c|}{ INFORMATION } \\
\hline Sample Name: & $0321-2$ & Acquired By: & System \\
\hline Sample Type: & Unknown & Sample Set Name: & \\
\hline Vial: & $1: F, 5$ & Acq. Method Set: & 2021 \\
\hline Injection \#: & 1 & Processing Method & Default \\
\hline Injection Volume: & $2.00 \mathrm{ul}$ & Channel Name: & PDA Ch2 254nm@4.8nm \\
\hline Run Time: & 40.0 Minutes & Proc. Chnl. Descr.: & PDA Ch2 254nm@4.8nm \\
\hline Date Acquired: & 4/13/2021 2:12:08 PM CST & & \\
\hline Date Processed: & 4/13/2021 3:49:29 PM CST & & \\
\hline
\end{tabular}

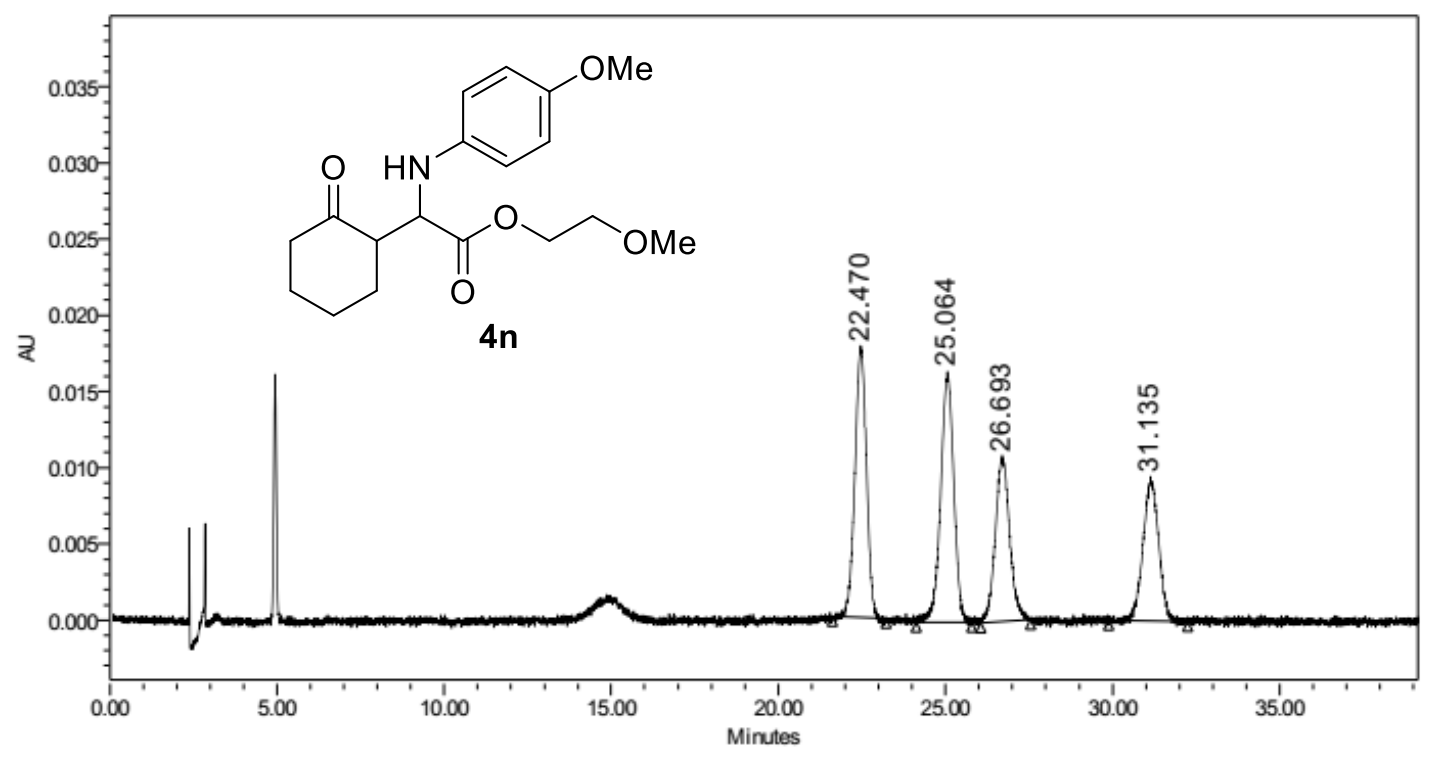

\begin{tabular}{|r|c|r|r|r|r|}
\hline & RT & $\begin{array}{c}\text { Width } \\
(\mathrm{sec})\end{array}$ & Height & Area & $\%$ Area \\
\hline 1 & 22.470 & 96.700 & 17822 & 430725 & 28.43 \\
\hline 2 & 25.064 & 99.300 & 16467 & 450994 & 29.77 \\
\hline 3 & 26.693 & 88.900 & 10896 & 324821 & 21.44 \\
\hline 4 & 31.135 & 141.800 & 9481 & 308592 & 20.37 \\
\hline
\end{tabular}

Reported by User: System

Report Method: Default Individual Report

Report Method I[ 4871

Page: 1 of 1
Project Name: 2021

Date Printed:

4/13/2021

3:54:31 PM PRC 


\begin{tabular}{|c|c|c|c|}
\hline \multicolumn{2}{|r|}{ SAMPLE } & \multicolumn{2}{|c|}{ INFORMATION } \\
\hline Sample Name: & $0320-2$ & Acquired By: & System \\
\hline $\begin{array}{l}\text { Sample Type: } \\
\text { Vial: }\end{array}$ & $\begin{array}{l}\text { Unknown } \\
\text { 1:F.6 }\end{array}$ & $\begin{array}{l}\text { Sample Set Name: } \\
\text { Acq. Method Set: }\end{array}$ & 20211 \\
\hline Injection \#: & 1 & Processing Method & Default \\
\hline Injection Volume: & $1.30 \mathrm{ul}$ & Channel Name: & PDA Ch2 254nm@4.8nm \\
\hline Run Time: & 40.0 Minutes & Proc. Chnl. Descr.: & PDA Ch2 254nm@4.8nm \\
\hline Date Acquired: & 4/13/2021 2:52:09 PM CST & & \\
\hline Date Processed: & 4/13/2021 3:49:54 PM CST & & \\
\hline
\end{tabular}

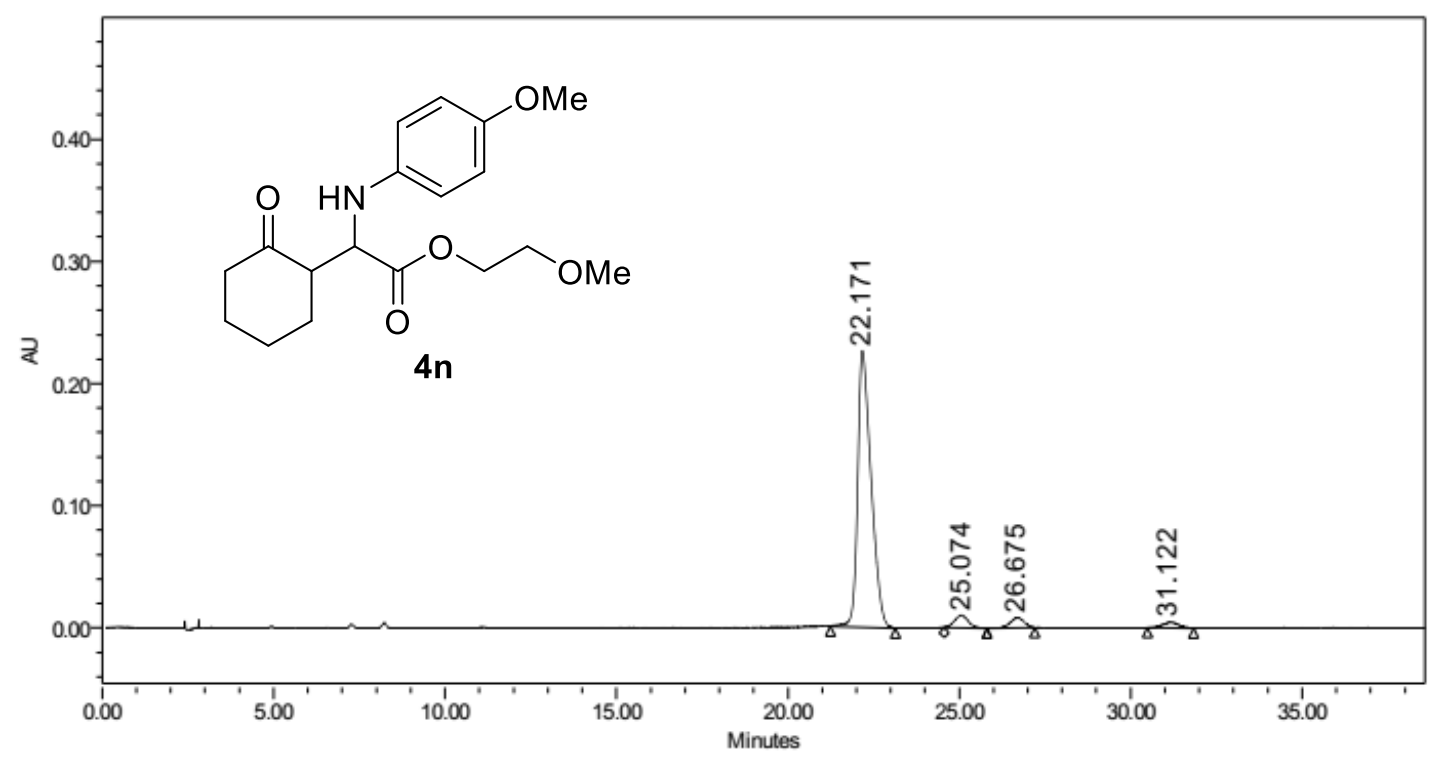

\begin{tabular}{|r|c|r|r|r|r|}
\hline & RT & $\begin{array}{c}\text { Width } \\
(\mathrm{sec})\end{array}$ & Height & Area & $\%$ Area \\
\hline 1 & 22.171 & 113.600 & 226297 & 6177935 & 89.98 \\
\hline 2 & 25.074 & 75.050 & 10499 & 291555 & 4.25 \\
\hline 3 & 26.675 & 83.400 & 8551 & 247740 & 3.61 \\
\hline 4 & 31.122 & 80.600 & 4664 & 148519 & 2.16 \\
\hline
\end{tabular}

Reported by User: System

Report Method: Default Individual Report

Report Method I[ 4871

Page: 1 of 1
Project Name: 2021

Date Printed:

4/13/2021

3:54:48 PM PRC 


\begin{tabular}{|c|c|c|c|}
\hline \multicolumn{2}{|r|}{ SAMPLE } & \multicolumn{2}{|c|}{ INFORMATION } \\
\hline Sample Name: & wzh-0314-1 & Acquired By: & System \\
\hline $\begin{array}{l}\text { Sample Type: } \\
\text { Vial: }\end{array}$ & $\begin{array}{l}\text { Unknown } \\
1: A .1\end{array}$ & $\begin{array}{l}\text { Sample Set Name: } \\
\text { Acq. Method Set: }\end{array}$ & 20211 \\
\hline Injection \#: & 1 & Processing Method & Default \\
\hline Injection Volume: & $2.00 \mathrm{ul}$ & Channel Name: & PDA Ch1 214nm@4.8nm \\
\hline Run Time: & 30.0 Minutes & Proc. Chnl. Descr.: & PDA Ch1 214nm@4.8nm \\
\hline Date Acquired: & 3/19/2021 3:33:43 PM CST & & \\
\hline Date Processed: & 3/25/2021 8:05:11 AM CST & & \\
\hline
\end{tabular}

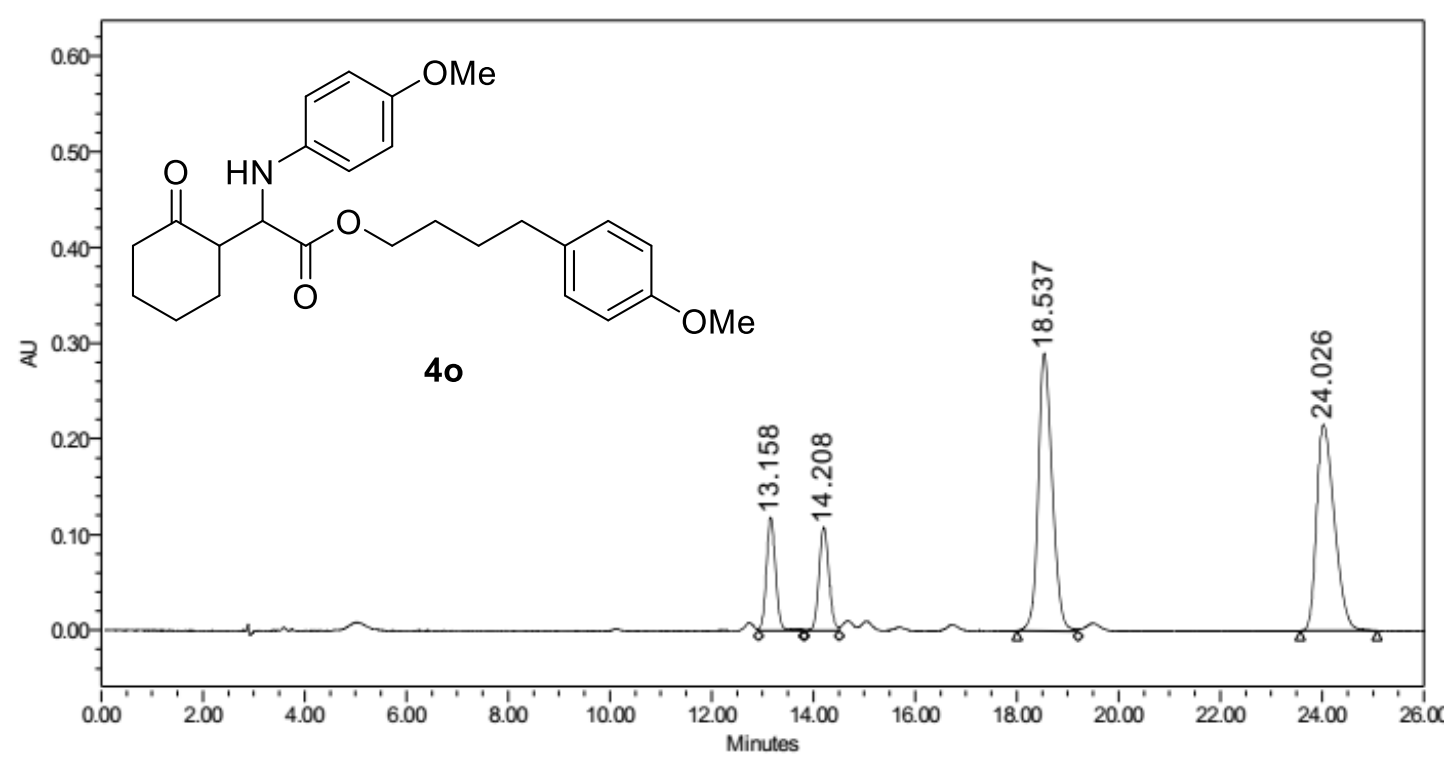

\begin{tabular}{|c|c|c|c|c|c|}
\hline & RT & $\begin{array}{c}\text { Width } \\
(\mathrm{sec})\end{array}$ & Height & Area & $\%$ Area \\
\hline 1 & 13.158 & 53.900 & 118459 & 1490167 & 10.85 \\
\hline 2 & 14.208 & 41.300 & 108483 & 1481421 & 10.78 \\
\hline 3 & 18.537 & 71.950 & 290062 & 5580985 & 40.62 \\
\hline 4 & 24.026 & 90.450 & 215582 & 5185302 & 37.74 \\
\hline
\end{tabular}

Reported by User: System

Report Method: Default Individual Report

Report Method I[ 3141

Page: 1 of 1
Project Name: 2021

Date Printed:

$3 / 25 / 2021$

3:31:10 PM PRC 


\begin{tabular}{|c|c|c|c|}
\hline \multicolumn{2}{|r|}{ SAMPLE } & \multicolumn{2}{|c|}{ INFORMATION } \\
\hline Sample Name: & wzh-0313-1 & Acquired By: & System \\
\hline Sample Type: & Unknown & Sample Set Name: & \\
\hline Vial: & 1:A,2 & Acq. Method Set: & $2021 \_1$ \\
\hline Injection \#: & 1 & Processing Method & Default \\
\hline Injection Volume: & $2.00 \mathrm{ul}$ & Channel Name: & PDA Ch1214nm@4.8nm \\
\hline Run Time: & 30.0 Minutes & Proc. Chnl. Descr.: & PDA Ch1 214nm@4.8nm \\
\hline Date Acquired: & 3/19/2021 4:00:38 PM CST & & \\
\hline Date Processed: & 3/25/2021 8:05:49 AM CST & & \\
\hline
\end{tabular}

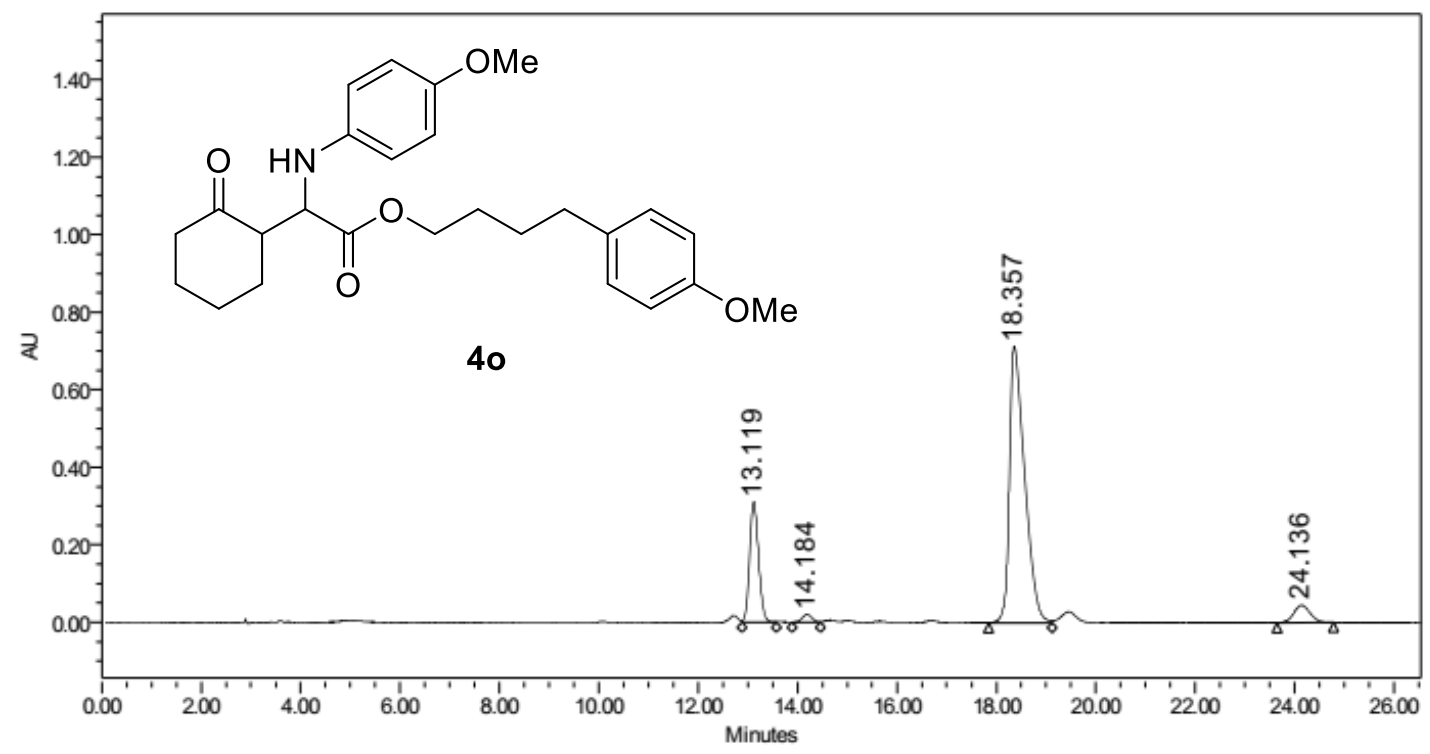

\begin{tabular}{|r|c|r|r|r|r|}
\hline & RT & $\begin{array}{c}\text { Width } \\
(\mathrm{sec})\end{array}$ & Height & Area & $\%$ Area \\
\hline 1 & 13.119 & 41.600 & 310462 & 3835154 & 18.86 \\
\hline 2 & 14.184 & 34.050 & 20419 & 286159 & 1.41 \\
\hline 3 & 18.357 & 76.650 & 714114 & 15182128 & 74.67 \\
\hline 4 & 24.136 & 68.150 & 44786 & 1029821 & 5.06 \\
\hline
\end{tabular}


9654 WZH-1029-4-1+4-2 IA 912140.7

\begin{tabular}{llll|}
\hline Sample Name: & WZH-1029-4-1+4-2 IA 91 214 0.7 & Injection Volume: & 3.0 \\
Vial Number: & RC5 & Channel: & UV_VIS_2 \\
Sample Type: & unknown & Wavelength: & $\mathbf{2 1 4 . 0}$ \\
Control Program: & test-dad3 & Bandwidth: & 4 \\
Quantif. Method: & $\mathbf{2 0 1 7 0 6 0 8}$ & Dilution Factor: & 1.0000 \\
Recording Time: & $\mathbf{2 0 2 0 - 1 1 - 4 ~ 1 0 : 4 5}$ & Sample Weight: & 1.0000 \\
Run Time (min): & $\mathbf{7 9 . 7 1}$ & Sample Amount: & $\mathbf{1 . 0 0 0 0}$ \\
\hline
\end{tabular}

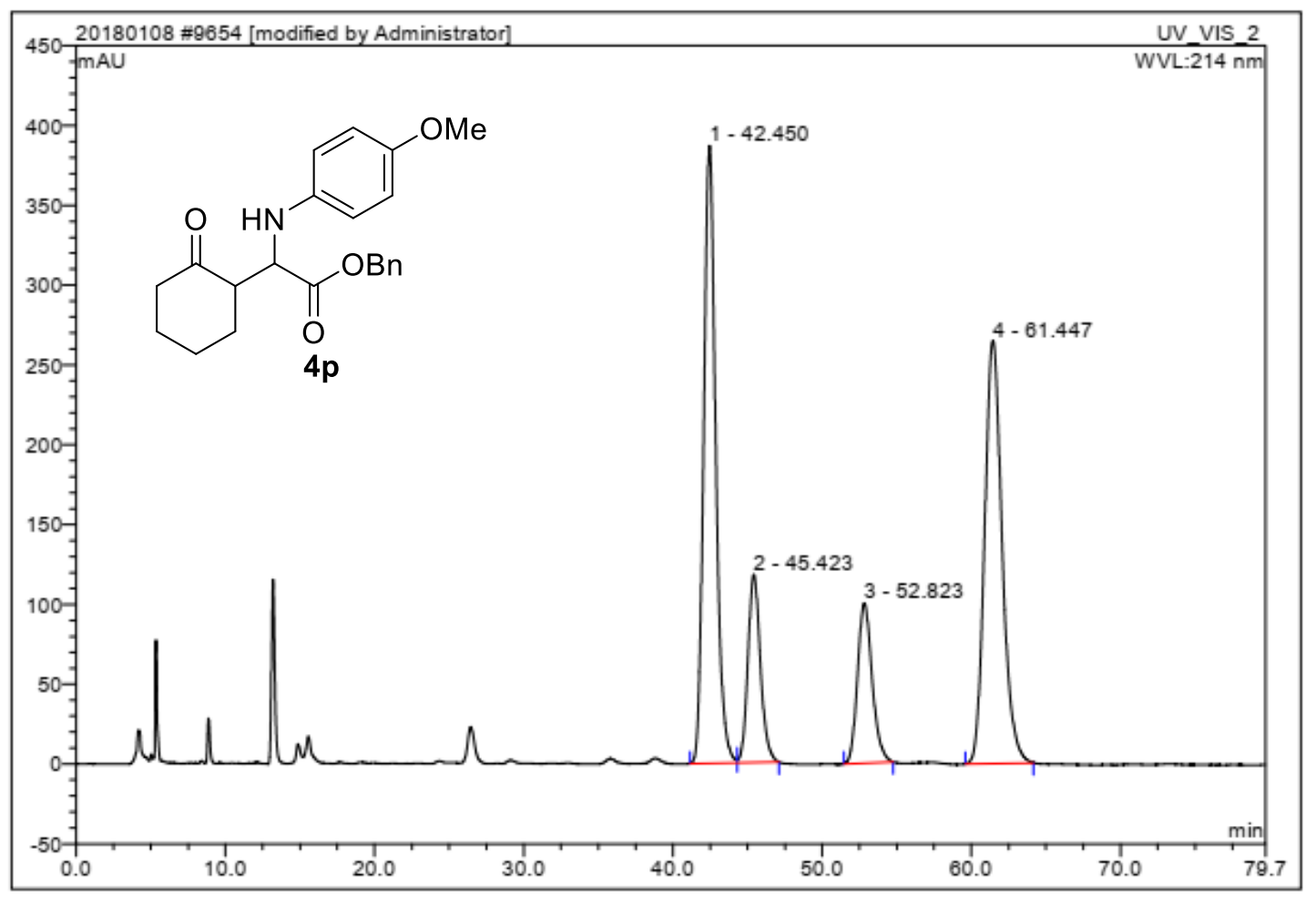

\begin{tabular}{|r|ccccccc|}
\hline No. & $\begin{array}{c}\text { Ret.Time } \\
\text { min }\end{array}$ & Peak Name & $\begin{array}{c}\text { Height } \\
\text { mAU }\end{array}$ & $\begin{array}{c}\text { Area } \\
\text { mAU*min }\end{array}$ & $\begin{array}{c}\text { Rel.Area } \\
\%\end{array}$ & Amount & Type \\
\hline 1 & 42.45 & n.a. & 387.035 & 359.992 & 37.94 & n.a. & BM \\
2 & 45.42 & n.a. & 118.056 & 114.903 & 12.11 & n.a. & MB \\
3 & 52.82 & n.a. & 100.355 & 114.141 & 12.03 & n.a. & BMB \\
4 & 61.45 & n.a. & 264.821 & 359.852 & 37.92 & n.a. & BMB \\
\hline Total: & & & 870.267 & 948.889 & 100.00 & 0.000 & \\
\hline
\end{tabular}




\section{WZH-1114 IA 912140.7}

Sample Name: Vial Number:

Sample Type:

Control Program:

Quantif. Method:

Recording Time:

Run Time (min):

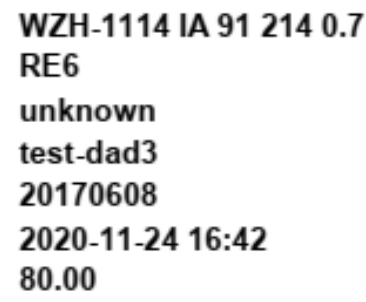

unknown

test-dad3

20170608

2020-11-24 16:42

80.00

$\begin{array}{ll}\text { Injection Volume: } & \mathbf{2 . 0} \\ \text { Channel: } & \text { UV_VIS_2 } \\ \text { Wavelength: } & 214.0 \\ \text { Bandwidth: } & 4 \\ \text { Dilution Factor: } & 1.0000 \\ \text { Sample Weight: } & 1.0000 \\ \text { Sample Amount: } & 1.0000\end{array}$

Injection Volume

Bandwidth:

Dilution Factor:

Sample Amount:

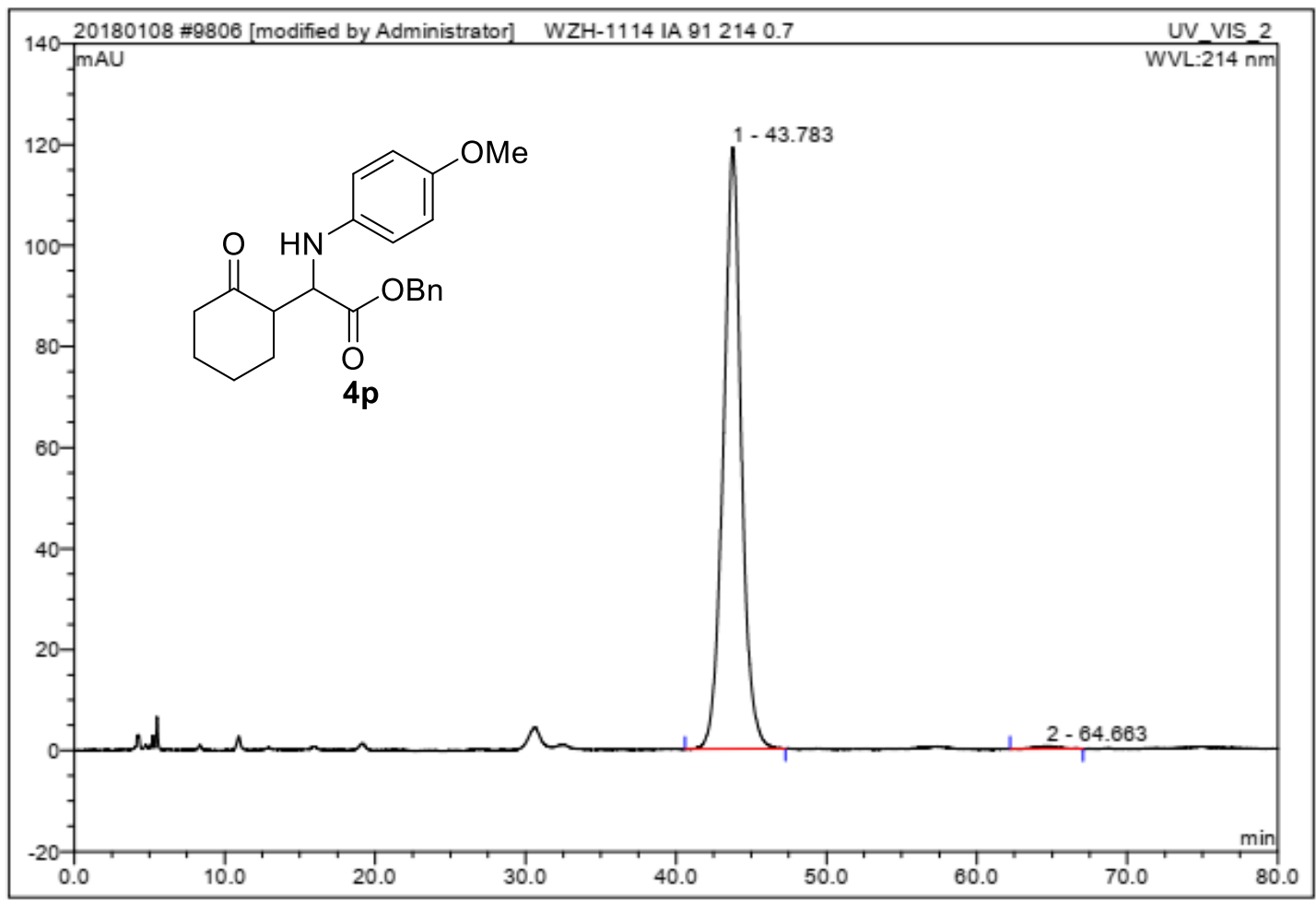

\begin{tabular}{|r|ccrrrrr|}
\hline No. & $\begin{array}{c}\text { Ret.Time } \\
\text { min }\end{array}$ & Peak Name & $\begin{array}{c}\text { Height } \\
\text { mAU }\end{array}$ & $\begin{array}{c}\text { Area } \\
\text { mAU*min }\end{array}$ & $\begin{array}{c}\text { Rel.Area } \\
\%\end{array}$ & Amount & Type \\
\hline 1 & 43.78 & n.a. & 119.106 & 164.469 & 99.48 & n.a. & BMB $^{*}$ \\
2 & 64.66 & n.a. & 0.530 & 0.864 & 0.52 & n.a. & BMB $^{*}$ \\
\hline Total: & & & 119.636 & 165.332 & 100.00 & 0.000 & \\
\hline
\end{tabular}




\begin{tabular}{|c|c|c|c|}
\hline \multicolumn{2}{|r|}{ SAMPLE } & \multicolumn{2}{|c|}{ INFORMATION } \\
\hline Sample Name: & $0317-2$ & Acquired By: & System \\
\hline Sample Type: & Unknown & Sample Set Name: & \\
\hline Vial: & $1: \mathrm{C}, 1$ & Acq. Method Set: & 20211 \\
\hline Injection \#: & 3 & Processing Method & Default \\
\hline Injection Volume: & $2.00 \mathrm{ul}$ & Channel Name: & PDA Ch2 254nm@4.8nm \\
\hline Run Time: & 30.0 Minutes & Proc. Chnl. Descr.: & PDA Ch2 254nm@4.8nm \\
\hline Date Acquired: & $3 / 29 / 2021$ 10:12:31 AM CST & & \\
\hline Date Processed: & $3 / 29 / 2021$ 1:40:44 PM CST & & \\
\hline
\end{tabular}

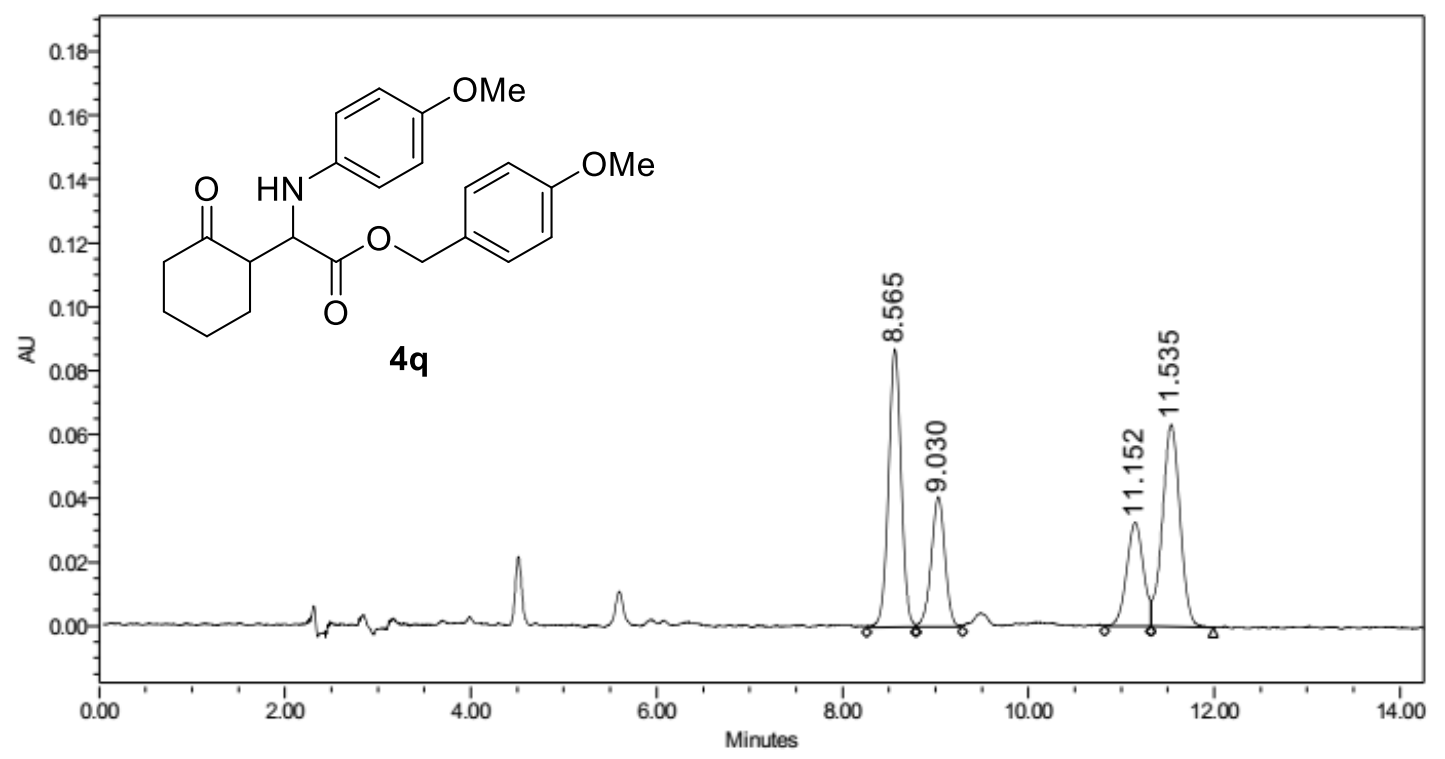

\begin{tabular}{|c|c|c|c|c|c|}
\hline & RT & $\begin{array}{c}\text { Width } \\
(\mathrm{sec})\end{array}$ & Height & Area & $\%$ Area \\
\hline 1 & 8.565 & 31.900 & 87106 & 828887 & 33.26 \\
\hline 2 & 9.030 & 30.150 & 40699 & 418409 & 16.79 \\
\hline 3 & 11.152 & 29.800 & 32575 & 408055 & 16.37 \\
\hline 4 & 11.535 & 40.350 & 63352 & 836711 & 33.58 \\
\hline
\end{tabular}

Reported by User: System

Project Name: 2021

Date Printed:

Report Method: Default Individual Report

$3 / 31 / 2021$

Page: 1 of 1

3:43:16 PM PRC 


\begin{tabular}{|c|c|c|c|}
\hline \multicolumn{2}{|r|}{ S A M P L E } & \multicolumn{2}{|c|}{ I NFORMATION } \\
\hline Sample Name: & $0317-1$ & Acquired By: & System \\
\hline Sample Type: & Unknown & Sample Set Name: & \\
\hline Vial: & $1: C, 2$ & Acq. Method Set: & 2021_1 \\
\hline Injection \#: & 1 & Processing Method & Defaūt \\
\hline Injection Volume: & $2.00 \mathrm{ul}$ & Channel Name: & PDA Ch2 254nm@4.8nm \\
\hline Run Time: & 30.0 Minutes & Proc. Chnl. Descr.: & PDA Ch2 254nm@4.8nm \\
\hline Date Acquired: & 3/29/2021 10:27:45 AM CST & & \\
\hline Date Processed: & 3/29/2021 1:40:29 PM CST & & \\
\hline
\end{tabular}

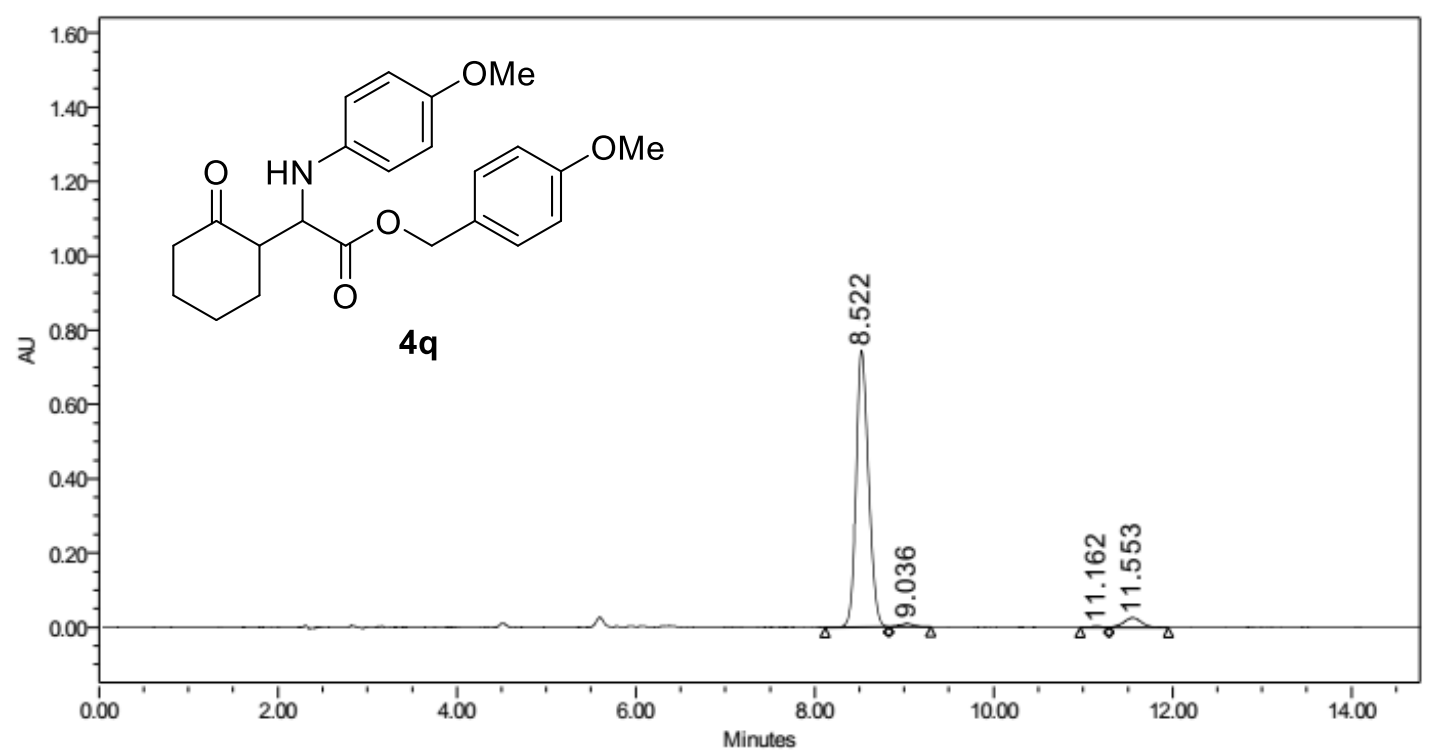

\begin{tabular}{|r|c|r|r|r|r|}
\hline & RT & $\begin{array}{c}\text { Width } \\
(\mathrm{sec})\end{array}$ & Height & Area & $\%$ Area \\
\hline 1 & 8.522 & 42.600 & 744901 & 7222696 & 94.05 \\
\hline 2 & 9.036 & 28.150 & 8538 & 90040 & 1.17 \\
\hline 3 & 11.162 & 19.350 & 2291 & 26641 & 0.35 \\
\hline 4 & 11.553 & 39.650 & 25122 & 340554 & 4.43 \\
\hline
\end{tabular}

Reported by User: System

Report Method: Default Individual Report

Report Method I[ 3821

Page: 1 of 1
Project Name: 2021

Date Printed:

$3 / 31 / 2021$

3:43:01 PM PRC 


\begin{tabular}{|c|c|c|c|}
\hline \multicolumn{2}{|r|}{ SAMPLE } & \multicolumn{2}{|c|}{ INFORMATION } \\
\hline Sample Name: & 0309-2-rac-IJ & Acquired By: & System \\
\hline Sample Type: & Unknown & Sample Set Name: & \\
\hline Vial: & $1: B, 3$ & Acq. Method Set: & $2021 \_1$ \\
\hline Injection \#: & 1 & Processing Method & Default \\
\hline Injection Volume: & $2.00 \mathrm{ul}$ & Channel Name: & PDA Ch1214nm@4.8nm \\
\hline Run Time: & 25.0 Minutes & Proc. Chnl. Descr.: & PDA Ch1 214nm@4.8nm \\
\hline Date Acquired: & 3/15/2021 8:38:05 AM CST & & \\
\hline Date Processed: & 3/16/2021 8:14:46 AM CST & & \\
\hline
\end{tabular}

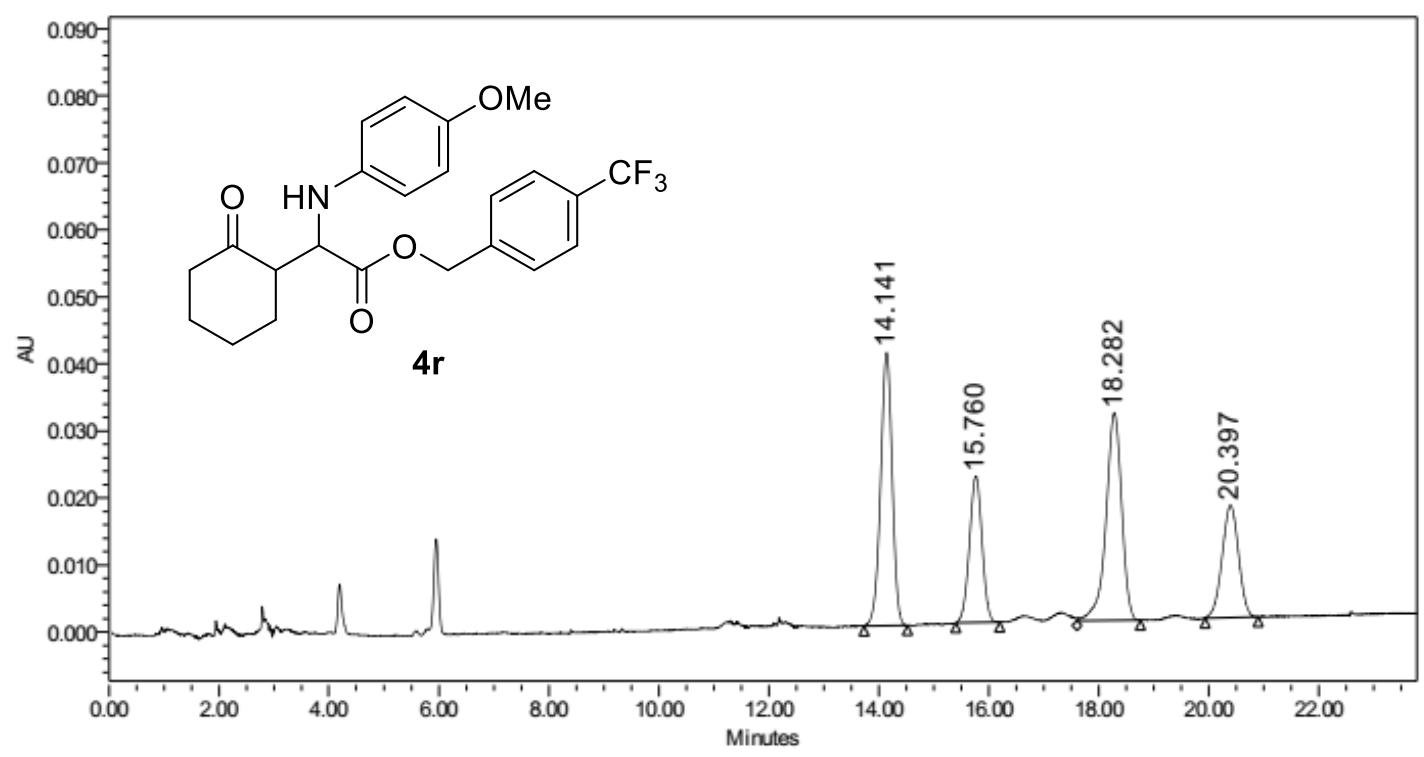

\begin{tabular}{|c|c|c|c|c|c|}
\hline & RT & $\begin{array}{c}\text { Width } \\
(\mathrm{sec})\end{array}$ & Height & Area & $\%$ Area \\
\hline 1 & 14.141 & 47.550 & 40773 & 595129 & 31.20 \\
\hline 2 & 15.760 & 48.050 & 21878 & 348455 & 18.27 \\
\hline 3 & 18.282 & 69.600 & 30967 & 614734 & 32.22 \\
\hline 4 & 20.397 & 57.900 & 16754 & 349363 & 18.31 \\
\hline
\end{tabular}

Reported by User: System

Report Method: Default Individual Report

Report Method I[ 2063

Page: 1 of 1
Project Name: 2021

Date Printed:

$3 / 16 / 2021$

8:23:10 AM PRC 


\begin{tabular}{|c|c|c|c|}
\hline \multicolumn{2}{|r|}{ SAMPLE } & \multicolumn{2}{|c|}{ INFORMATION } \\
\hline Sample Name: & $0309-1$ & Acquired By: & System \\
\hline Sample Type: & Unknown & Sample Set Name: & \\
\hline Vial: & $1: B, 4$ & Acq. Method Set: & 2021 \\
\hline Injection \#: & 1 & Processing Method & Default \\
\hline Injection Volume: & $2.00 \mathrm{ul}$ & Channel Name: & PDA Ch1214nm@4.8nm \\
\hline Run Time: & 25.0 Minutes & Proc. Chnl. Descr.: & PDA Ch1214nm@4.8nm \\
\hline Date Acquired: & $3 / 15 / 2021$ 9:05:33 AM CST & & \\
\hline Date Processed: & 3/16/2021 8:15:12 AM CST & & \\
\hline
\end{tabular}

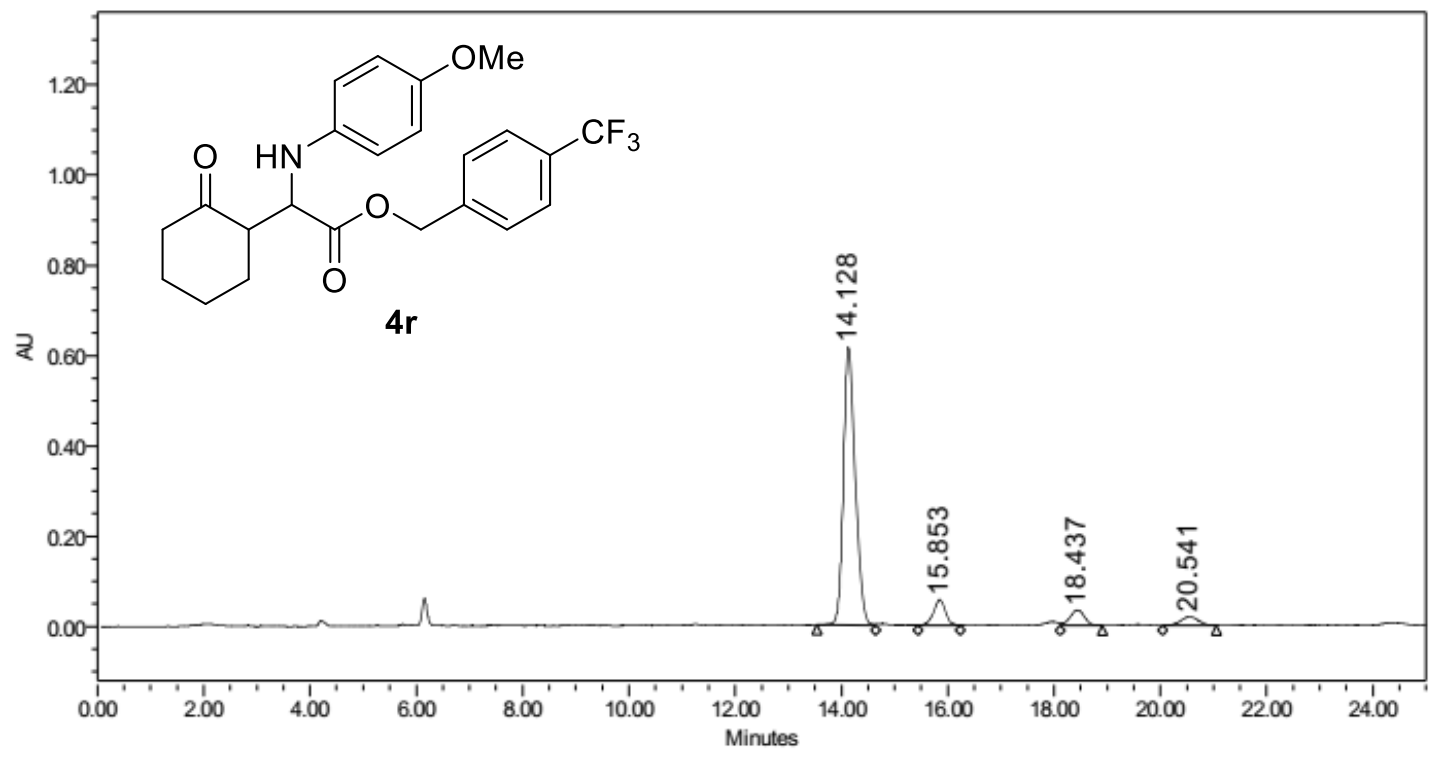

\begin{tabular}{|c|c|c|r|r|r|}
\hline & RT & $\begin{array}{c}\text { Width } \\
(\mathrm{sec})\end{array}$ & Height & Area & $\%$ Area \\
\hline 1 & 14.128 & 66.250 & 614909 & 9371724 & 82.80 \\
\hline 2 & 15.853 & 47.450 & 56260 & 879222 & 7.77 \\
\hline 3 & 18.437 & 47.650 & 33295 & 645841 & 5.71 \\
\hline 4 & 20.541 & 60.850 & 18939 & 421782 & 3.73 \\
\hline
\end{tabular}

Reported by User: System

Project Name: 2021

Date Printed:

$3 / 16 / 2021$

Report Method I[ 2063

8:23:27 AM PRC

Page: 1 of 1 


\begin{tabular}{|c|c|c|c|}
\hline \multicolumn{2}{|r|}{ S A M P L E } & \multicolumn{2}{|c|}{ INFORMATION } \\
\hline Sample Name: & $0317-4$ & Acquired By: & System \\
\hline Sample Type: & Unknown & Sample Set Name: & \\
\hline Vial: & $1: C, 5$ & Acq. Method Set: & 2021_1 \\
\hline Injection \#: & 1 & Processing Method & Default \\
\hline Injection Volume: & $2.00 \mathrm{ul}$ & Channel Name: & PDA Ch1214nm@4.8nm \\
\hline Run Time: & 20.0 Minutes & Proc. Chnl. Descr.: & PDA Ch1 214nm@4.8nm \\
\hline Date Acquired: & 4/1/2021 8:15:02 AM CST & & \\
\hline Date Processed: & 4/1/2021 2:23:15 PM CST & & \\
\hline
\end{tabular}

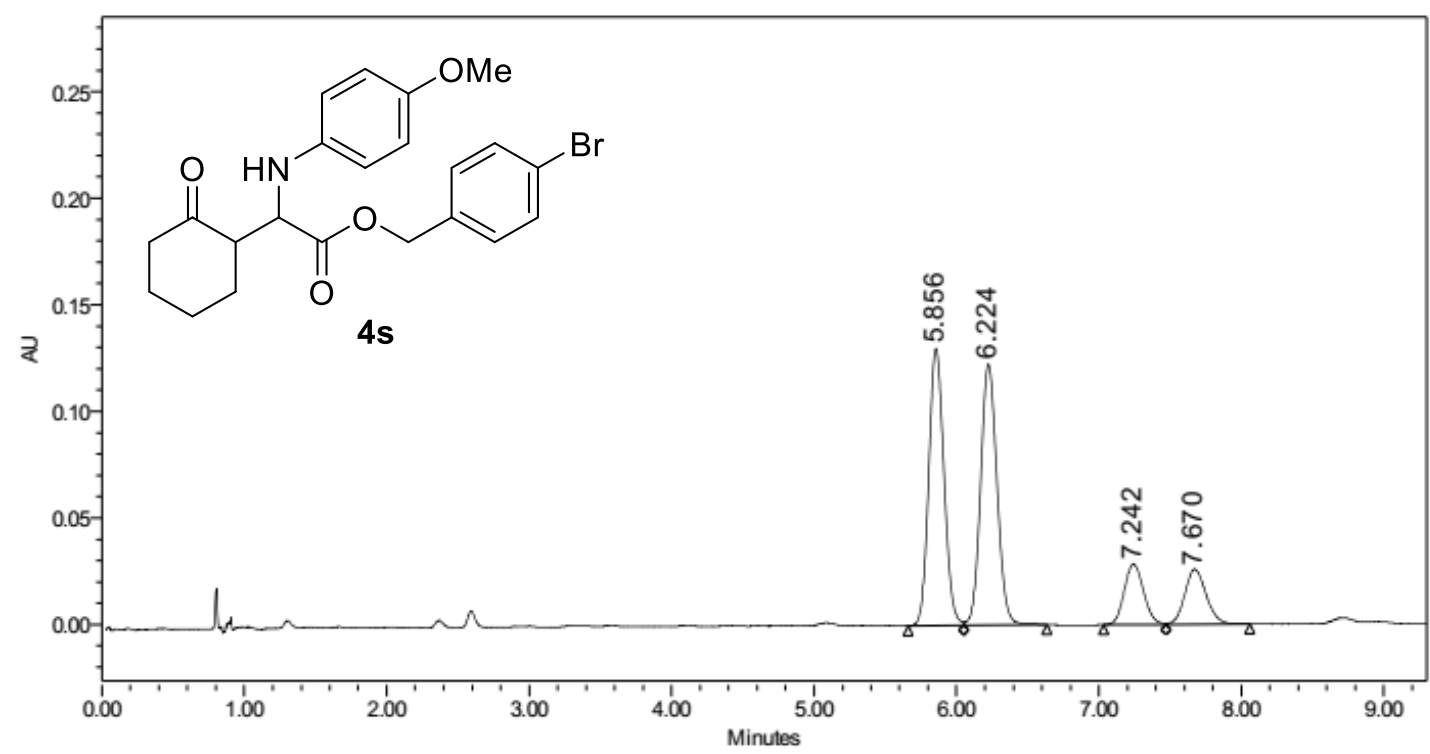

\begin{tabular}{|c|c|c|c|c|c|}
\hline & RT & $\begin{array}{c}\text { Width } \\
(\mathrm{sec})\end{array}$ & Height & Area & $\%$ Area \\
\hline 1 & 5.856 & 23.350 & 129941 & 974717 & 38.90 \\
\hline 2 & 6.224 & 34.950 & 122434 & 989484 & 39.49 \\
\hline 3 & 7.242 & 26.500 & 28527 & 268405 & 10.71 \\
\hline 4 & 7.670 & 35.200 & 25948 & 273033 & 10.90 \\
\hline
\end{tabular}

Reported by User: System

Report Method: Default Individual Report

Report Method I[ 4010

Page: 1 of 1
Project Name: 2021

Date Printed:

4/1/2021

2:30:57 PM PRC 


\begin{tabular}{|c|c|c|c|}
\hline \multicolumn{2}{|r|}{ S A M P L E } & \multicolumn{2}{|c|}{ INFORMATION } \\
\hline Sample Name: & $0317-3$ & Acquired By: & System \\
\hline Sample Type: & Unknown & Sample Set Name: & \\
\hline Vial: & 1:C,6 & Acq. Method Set: & 2021_1 \\
\hline Injection \#: & 1 & Processing Method & Defaūt \\
\hline Injection Volume: & $2.00 \mathrm{ul}$ & Channel Name: & PDA Ch1214nm@4.8nm \\
\hline Run Time: & 20.0 Minutes & Proc. Chnl. Descr.: & PDA Ch1 214nm@4.8nm \\
\hline Date Acquired: & 4/1/2021 8:25:15 AM CST & & \\
\hline Date Processed: & 4/1/2021 2:26:48 PM CST & & \\
\hline
\end{tabular}

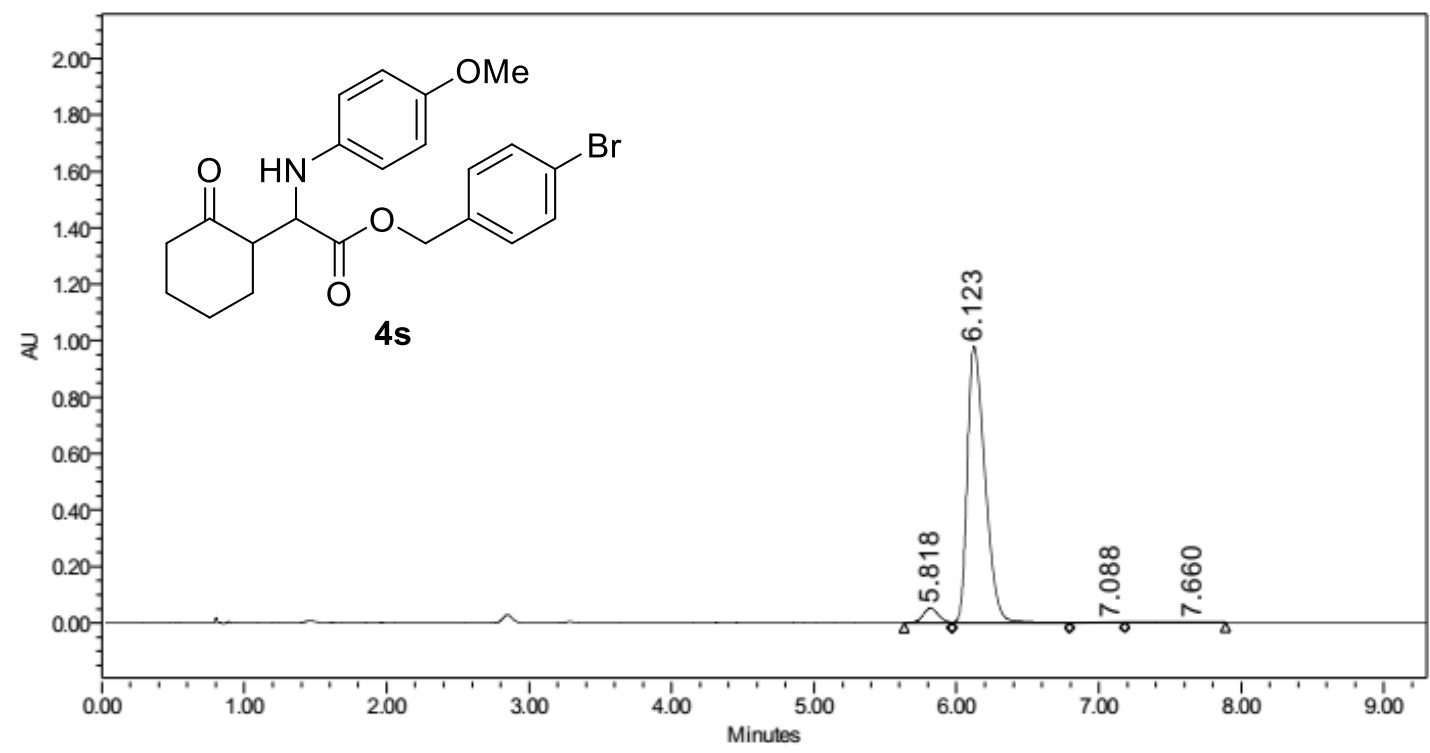

\begin{tabular}{|r|c|c|r|r|r|}
\hline & RT & $\begin{array}{c}\text { Width } \\
(\mathrm{sec})\end{array}$ & Height & Area & $\%$ Area \\
\hline 1 & 5.818 & 20.050 & 51197 & 369660 & 4.19 \\
\hline 2 & 6.123 & 49.450 & 978774 & 8413804 & 95.44 \\
\hline 3 & 7.088 & 23.400 & 509 & 6819 & 0.08 \\
\hline 4 & 7.660 & 42.450 & 1099 & 25826 & 0.29 \\
\hline
\end{tabular}

Reported by User: System

Report Method: Default Individual Report

Report Method I[ 4010

Page: 1 of 1
Project Name: 2021

Date Printed:

$4 / 1 / 2021$

2:30:35 PM PRC 


\begin{tabular}{|c|c|c|c|}
\hline \multicolumn{2}{|r|}{ SAMPLE } & \multicolumn{2}{|c|}{ INFORMATION } \\
\hline Sample Name: & $0322 \mathrm{R}$ & Acquired By: & System \\
\hline Sample Type: & Unknown & Sample Set Name: & \\
\hline Vial: & $1: C, 3$ & Acq. Method Set: & 20211 \\
\hline Injection \#: & 1 & Processing Method & Default \\
\hline Injection Volume: & $2.00 \mathrm{ul}$ & Channel Name: & PDA Ch1214nm@4.8nm \\
\hline Run Time: & 20.0 Minutes & Proc. Chnl. Descr.: & PDA Ch1 214nm@4.8nm \\
\hline Date Acquired: & $3 / 31 / 20214: 26: 32$ PM CST & & \\
\hline Date Processed: & 3/31/2021 4:44:39 PM CST & & \\
\hline
\end{tabular}

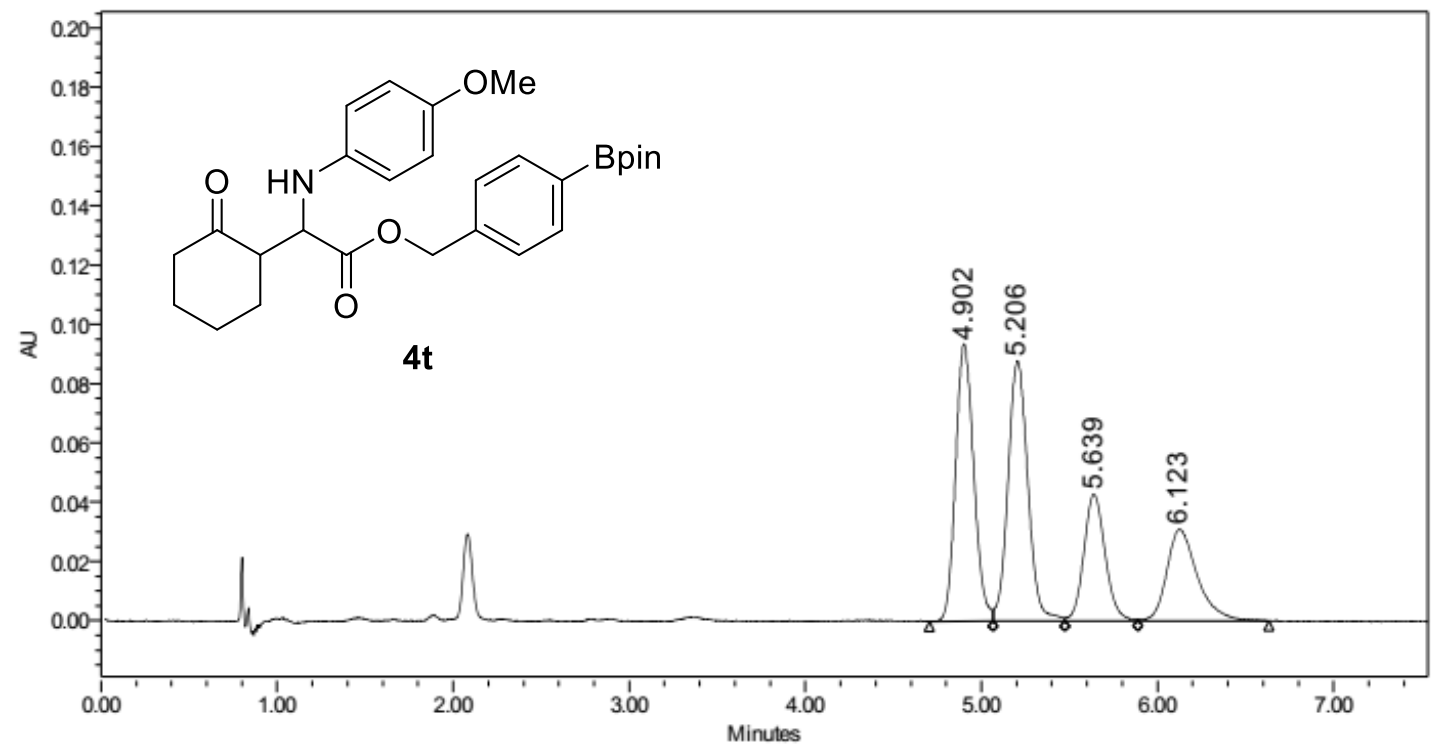

\begin{tabular}{|c|c|c|c|c|c|}
\hline & RT & $\begin{array}{c}\text { Width } \\
(\mathrm{sec})\end{array}$ & Height & Area & $\%$ Area \\
\hline 1 & 4.902 & 21.650 & 93591 & 667651 & 32.00 \\
\hline 2 & 5.206 & 24.500 & 87872 & 682350 & 32.70 \\
\hline 3 & 5.639 & 24.950 & 42696 & 364166 & 17.45 \\
\hline 4 & 6.123 & 44.550 & 30940 & 372378 & 17.85 \\
\hline
\end{tabular}

Reported by User: System

Report Method: Default Individual Report

Report Method I[ 3821

Page: 1 of 1
Project Name: 2021

Date Printed:

$4 / 1 / 2021$

2:25:30 PM PRC 


\begin{tabular}{|c|c|c|c|}
\hline \multicolumn{2}{|r|}{ SAMPLE } & \multicolumn{2}{|c|}{ INFORMATION } \\
\hline Sample Name: & 0318 & Acquired By: & System \\
\hline Sample Type: & Unknown & Sample Set Name: & \\
\hline Vial: & $1: C, 4$ & Acq. Method Set: & 2021 \\
\hline Injection \#: & 1 & Processing Method & Default \\
\hline Injection Volume: & $2.00 \mathrm{ul}$ & Channel Name: & PDA Ch1214nm@4.8nm \\
\hline Run Time: & 20.0 Minutes & Proc. Chnl. Descr.: & PDA Ch1214nm@4.8nm \\
\hline Date Acquired: & 3/31/2021 4:34:58 PM CST & & \\
\hline Date Processed: & $3 / 31 / 2021$ 4:45:02 PM CST & & \\
\hline
\end{tabular}

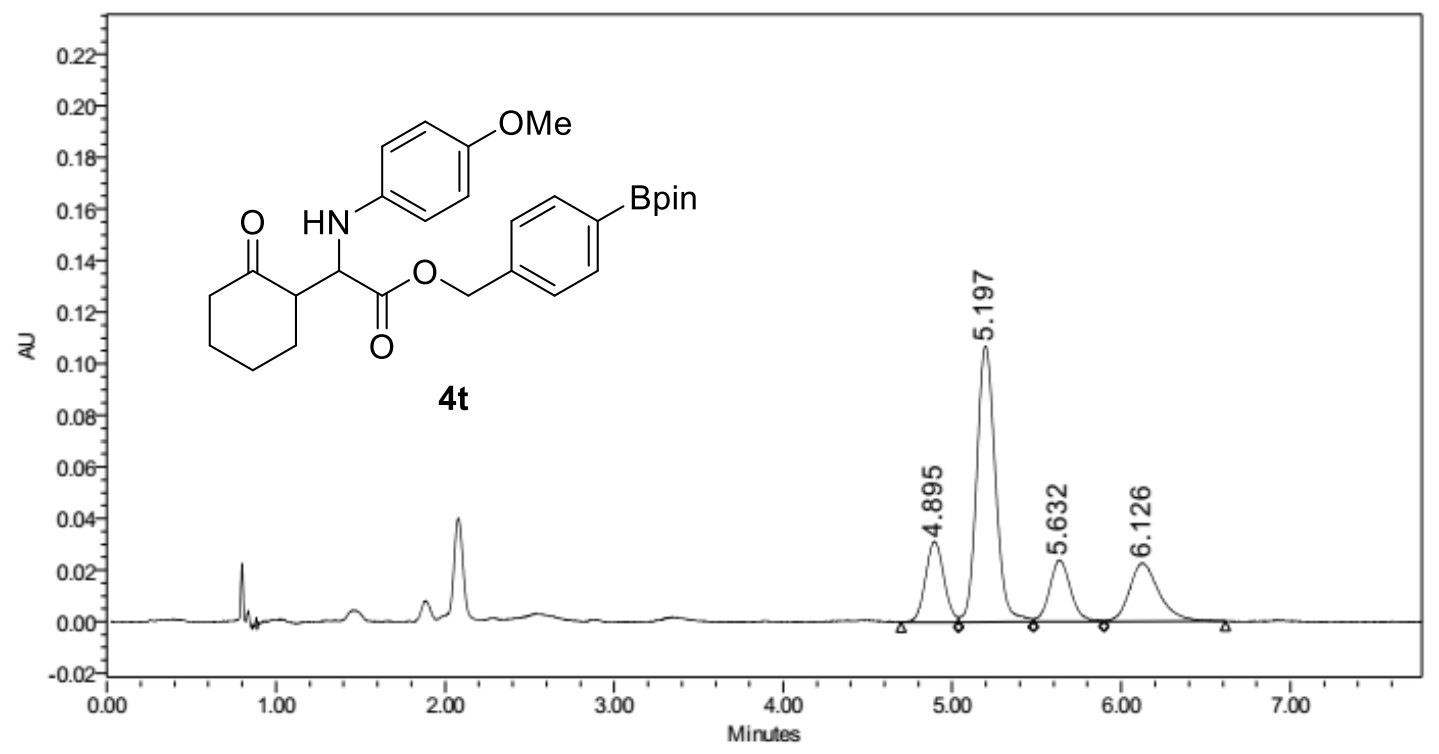

\begin{tabular}{|c|c|r|r|r|r|}
\hline & RT & $\begin{array}{c}\text { Width } \\
(\mathrm{sec})\end{array}$ & Height & Area & $\%$ Area \\
\hline 1 & 4.895 & 20.450 & 31225 & 235000 & 15.26 \\
\hline 2 & 5.197 & 26.450 & 107054 & 823499 & 53.47 \\
\hline 3 & 5.632 & 25.100 & 23895 & 206500 & 13.41 \\
\hline 4 & 6.126 & 43.150 & 22569 & 275050 & 17.86 \\
\hline
\end{tabular}

Reported by User: System

Report Method: Default Individual Report

Report Method I[ 3821

Page: 1 of 1
Project Name: 2021

Date Printed:

$4 / 1 / 2021$

2:25:44 PM PRC 


\begin{tabular}{|c|c|c|c|}
\hline \multicolumn{2}{|r|}{ SAMPLE } & \multicolumn{2}{|c|}{ INFORMATION } \\
\hline Sample Name: & $0321-1$ & Acquired By: & System \\
\hline Sample Type: & Unknown & Sample Set Name: & \\
\hline Vial: & $1: C, 1$ & Acq. Method Set: & 2021_1 \\
\hline Injection \#: & 2 & Processing Method & Defaūt \\
\hline Injection Volume: & $2.00 \mathrm{ul}$ & Channel Name: & PDA Ch1214nm@4.8nm \\
\hline Run Time: & 20.0 Minutes & Proc. Chnl. Descr.: & PDA Ch1 214nm@4.8nm \\
\hline Date Acquired: & 3/31/2021 4:15:30 PM CST & & \\
\hline Date Processed: & 3/31/2021 4:26:24 PM CST & & \\
\hline
\end{tabular}

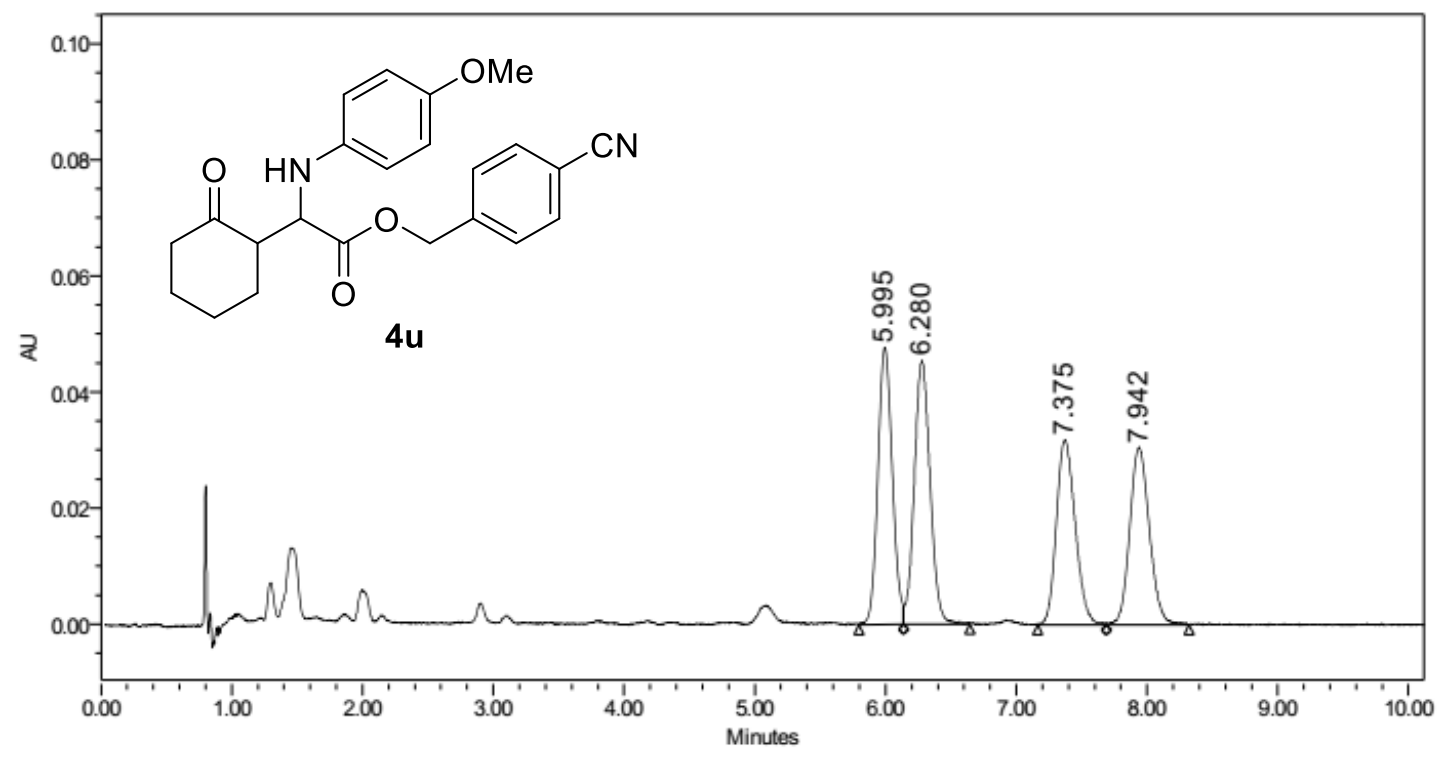

\begin{tabular}{|c|c|c|c|c|c|}
\hline & RT & $\begin{array}{c}\text { Width } \\
(\mathrm{sec})\end{array}$ & Height & Area & $\%$ Area \\
\hline 1 & 5.995 & 20.500 & 47769 & 368643 & 26.57 \\
\hline 2 & 6.280 & 30.400 & 45426 & 379542 & 27.36 \\
\hline 3 & 7.375 & 31.500 & 31889 & 318002 & 22.92 \\
\hline 4 & 7.942 & 38.050 & 30591 & 321059 & 23.14 \\
\hline
\end{tabular}

Reported by User: System

Report Method: Default Individual Report

Report Method I[ 3821

Page: 1 of 1
Project Name: 2021

Date Printed:

$4 / 1 / 2021$

2:25:16 PM PRC 


\begin{tabular}{|c|c|c|c|}
\hline \multicolumn{2}{|r|}{ S A MPLE } & \multicolumn{2}{|c|}{ INFORMATION } \\
\hline Sample Name: & $0320-1$ & Acquired By: & System \\
\hline Sample Type: & Unknown & Sample Set Name: & \\
\hline Vial: & 1:C,2 & Acq. Method Set: & 2021_1 \\
\hline Injection \#: & 1 & Processing Method & Defaūt \\
\hline Injection Volume: & $2.00 \mathrm{ul}$ & Channel Name: & PDA Ch1214nm@4.8nm \\
\hline Run Time: & 20.0 Minutes & Proc. Chnl. Descr.: & PDA Ch1 214nm@4.8nm \\
\hline Date Acquired: & 3/31/2021 3:54:51 PM CST & & \\
\hline Date Processed: & 3/31/2021 4:12:17 PM CST & & \\
\hline
\end{tabular}

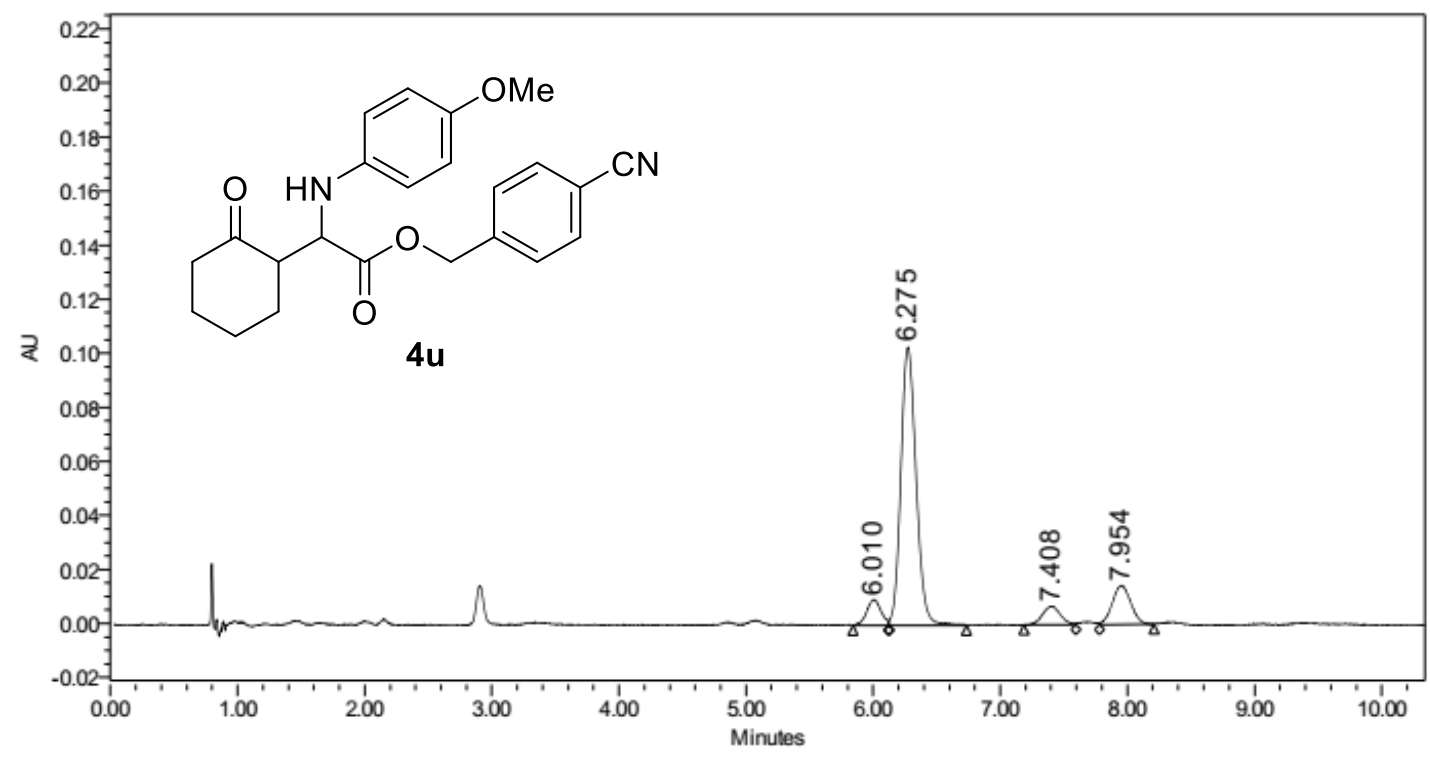

\begin{tabular}{|r|c|r|r|r|r|}
\hline & RT & $\begin{array}{c}\text { Width } \\
(\mathrm{sec})\end{array}$ & Height & Area & $\%$ Area \\
\hline 1 & 6.010 & 16.750 & 9434 & 71723 & 6.31 \\
\hline 2 & 6.275 & 36.750 & 102927 & 854839 & 75.17 \\
\hline 3 & 7.408 & 24.450 & 6841 & 66564 & 5.85 \\
\hline 4 & 7.954 & 25.700 & 14372 & 144125 & 12.67 \\
\hline
\end{tabular}

Reported by User: System

Project Name: 2021

Date Printed:

Report Method: Default Individual Report

4/1/2021

Report Method I[ 3821

2:25:00 PM PRC 


\begin{tabular}{|c|c|c|c|}
\hline \multicolumn{2}{|r|}{ SAMPLE } & \multicolumn{2}{|c|}{ INFORMATION } \\
\hline Sample Name: & 0316-1 & Acquired By: & System \\
\hline Sample Type: & Unknown & Sample Set Name: & \\
\hline Vial: & 1:A,3 & Acq. Method Set: & 2021_1 \\
\hline Injection \#: & 1 & Processing Method & Defaūt \\
\hline Injection Volume: & $2.00 \mathrm{ul}$ & Channel Name: & PDA Ch1214nm@4.8nm \\
\hline Run Time: & 30.0 Minutes & Proc. Chnl. Descr.: & PDA Ch1 214nm@4.8nm \\
\hline Date Acquired: & 3/22/2021 9:50:01 AM CST & & \\
\hline Date Processed: & $3 / 25 / 2021$ 8:04:06 AM CST & & \\
\hline
\end{tabular}

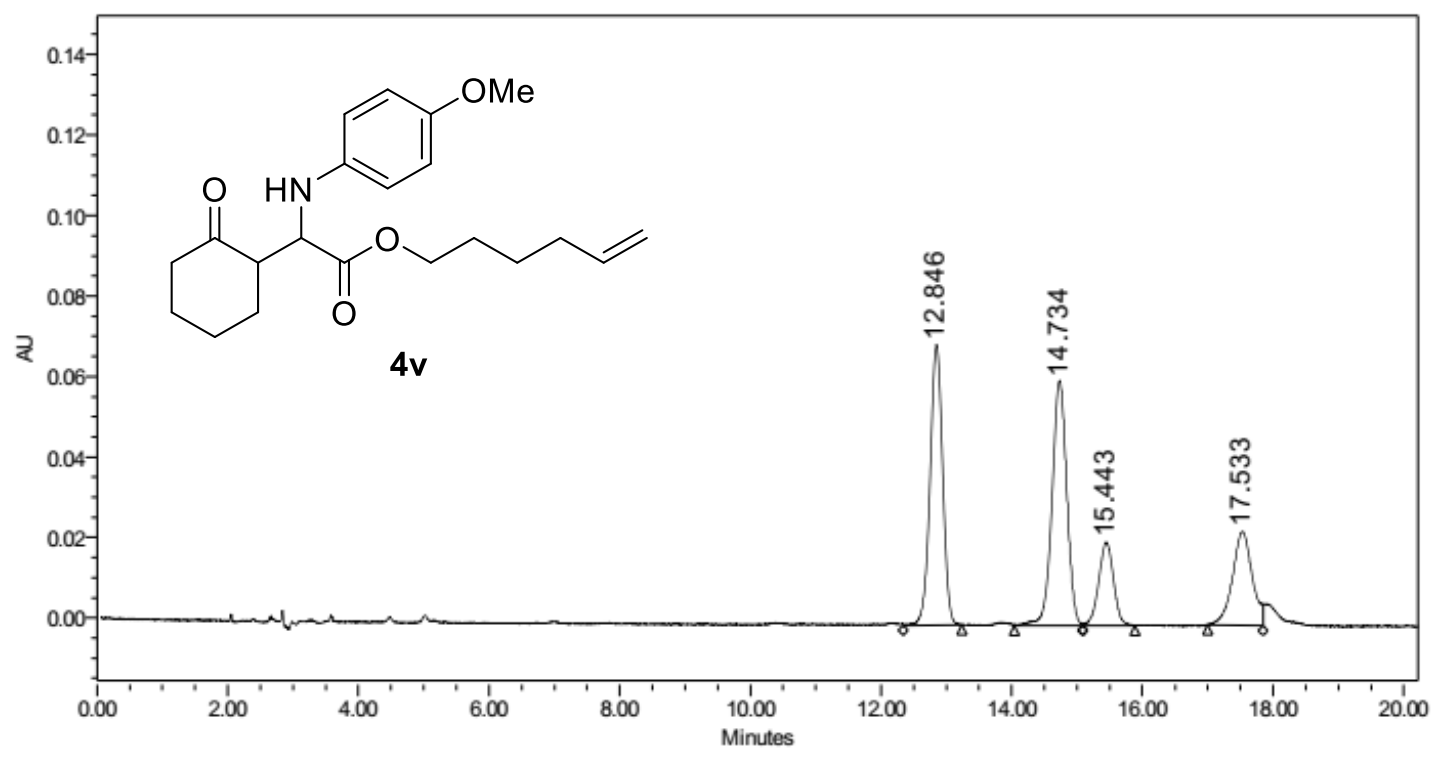

\begin{tabular}{|c|c|c|c|c|c|}
\hline & RT & $\begin{array}{c}\text { Width } \\
(\mathrm{sec})\end{array}$ & Height & Area & $\%$ Area \\
\hline 1 & 12.846 & 53.800 & 69672 & 938457 & 34.68 \\
\hline 2 & 14.734 & 62.900 & 60838 & 950149 & 35.11 \\
\hline 3 & 15.443 & 47.800 & 20680 & 329077 & 12.16 \\
\hline 4 & 17.533 & 50.900 & 23310 & 488304 & 18.05 \\
\hline
\end{tabular}

Reported by User: System

Report Method: Default Individual Report

Report Method I[ 3049

Page: 1 of 1
Project Name: 2021

Date Printed:

3/25/2021

3:30:25 PM PRC 


\begin{tabular}{|c|c|c|c|}
\hline \multicolumn{2}{|r|}{ S A M P L E } & \multicolumn{2}{|c|}{ INFORMATION } \\
\hline $\begin{array}{l}\text { Sample Name: } \\
\text { Sample Type: }\end{array}$ & $\begin{array}{l}0315-1 \\
\text { Unknown }\end{array}$ & $\begin{array}{l}\text { Acquired By: } \\
\text { Sample Set Name }\end{array}$ & System \\
\hline Vial: & $1: A, 4$ & Acq. Method Set: & 20211 \\
\hline Injection \#: & 1 & Processing Method & Defaūt \\
\hline Injection Volume: & $2.00 \mathrm{ul}$ & Channel Name: & PDA Ch1214nm@4.8nm \\
\hline Run Time: & 30.0 Minutes & Proc. Chnl. Descr.: & PDA Ch1214nm@4.8nm \\
\hline Date Acquired: & 3/22/2021 10:11:11 AM CST & & \\
\hline Date Processed: & 3/25/2021 8:04:48 AM CST & & \\
\hline
\end{tabular}

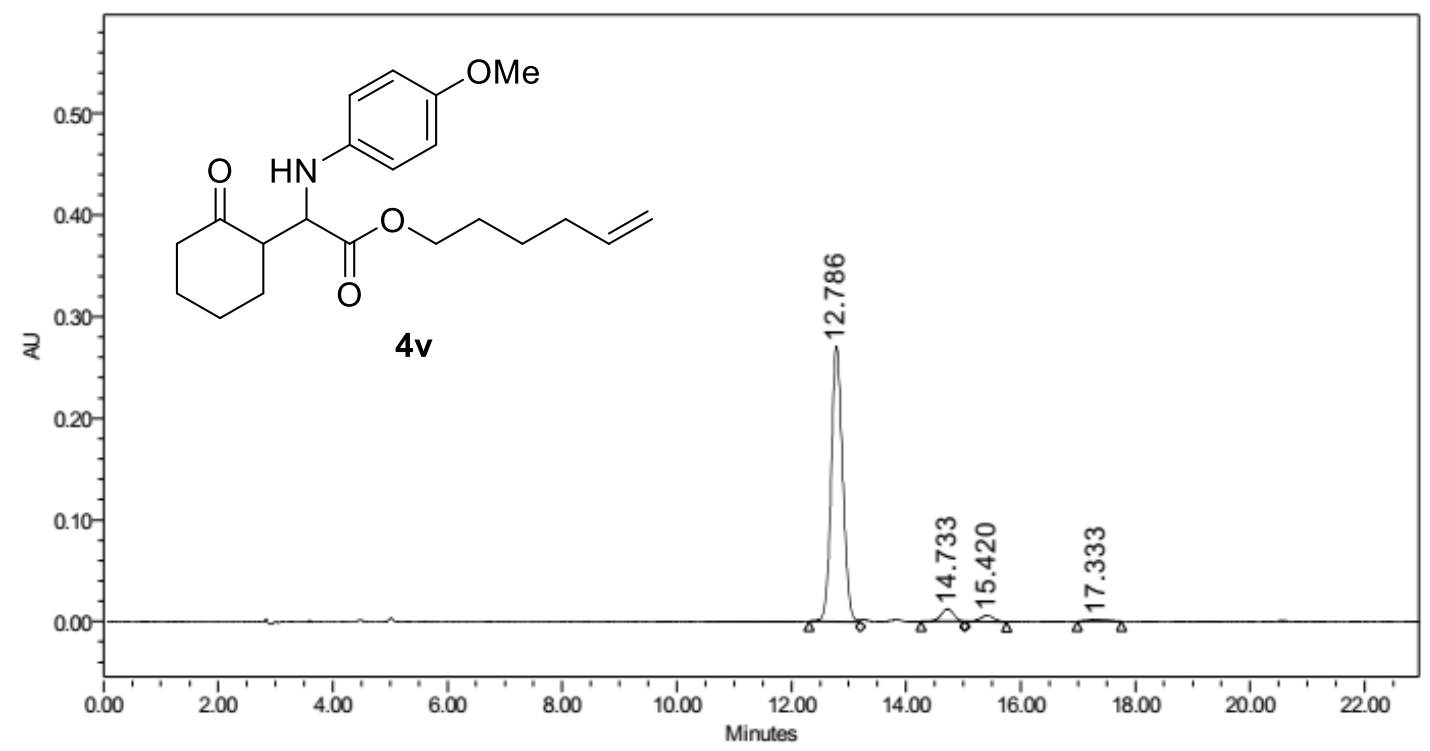

\begin{tabular}{|r|c|r|r|r|r|}
\hline & RT & $\begin{array}{c}\text { Width } \\
(\mathrm{sec})\end{array}$ & Height & Area & $\%$ Area \\
\hline 1 & 12.786 & 53.550 & 271350 & 3685460 & 91.94 \\
\hline 2 & 14.733 & 45.650 & 12395 & 187172 & 4.67 \\
\hline 3 & 15.420 & 43.650 & 6044 & 96276 & 2.40 \\
\hline 4 & 17.333 & 45.800 & 2159 & 39481 & 0.98 \\
\hline
\end{tabular}

Reported by User: System

Report Method: Default Individual Report

Report Method I[ 3049

Page: 1 of 1
Project Name: 2021

Date Printed:

$3 / 25 / 2021$

3:30:38 PM PRC 


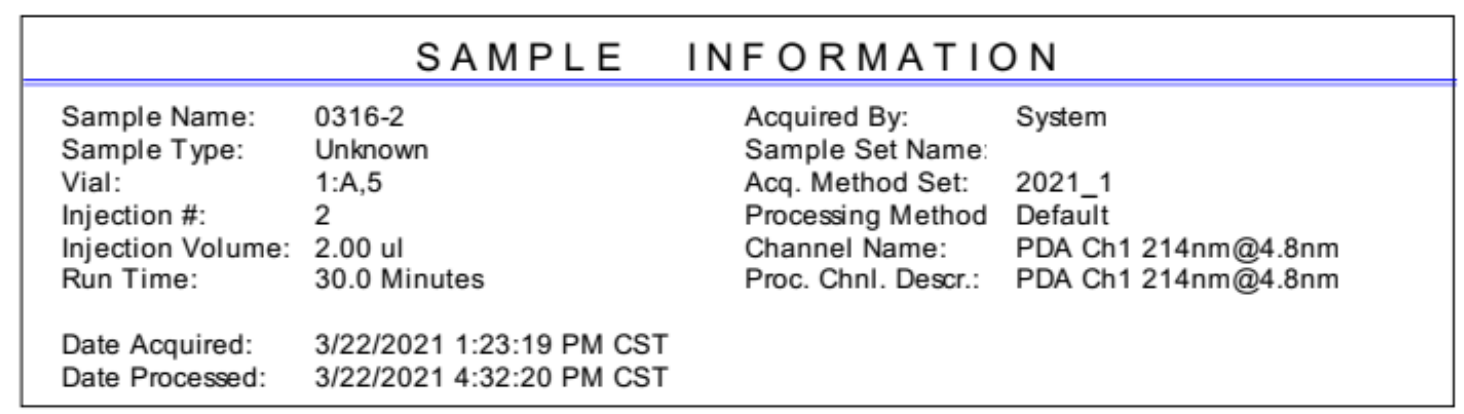

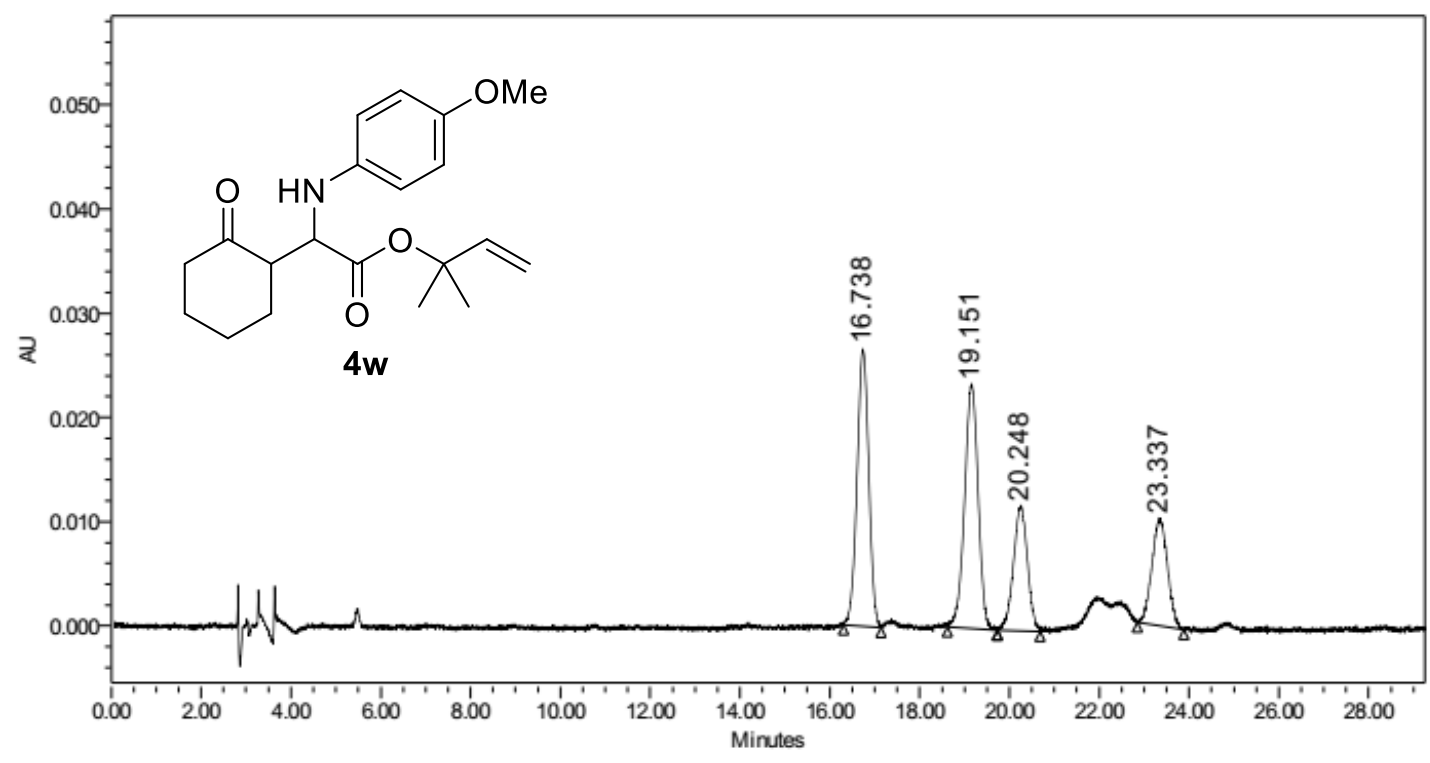

\begin{tabular}{|c|c|c|c|c|c|}
\hline & RT & $\begin{array}{c}\text { Width } \\
(\mathrm{sec})\end{array}$ & Height & Area & $\%$ Area \\
\hline 1 & 16.738 & 50.250 & 26623 & 483013 & 32.42 \\
\hline 2 & 19.151 & 66.700 & 23500 & 494141 & 33.17 \\
\hline 3 & 20.248 & 57.000 & 12070 & 263211 & 17.67 \\
\hline 4 & 23.337 & 61.650 & 10342 & 249339 & 16.74 \\
\hline
\end{tabular}




\begin{tabular}{|c|c|c|c|}
\hline \multicolumn{2}{|r|}{ SAMPLE } & \multicolumn{2}{|c|}{ INFORMATION } \\
\hline Sample Name: & $0315-3$ & Acquired By: & System \\
\hline Sample Type: & Unknown & Sample Set Name: & \\
\hline Vial: & $1: A, 6$ & Acq. Method Set: & 2021_1 \\
\hline Injection \#: & 1 & Processing Method & Default \\
\hline Injection Volume: & $2.00 \mathrm{ul}$ & Channel Name: & PDA Ch1214nm@4.8nm \\
\hline Run Time: & 30.0 Minutes & Proc. Chnl. Descr.: & PDA Ch1 214nm@4.8nm \\
\hline Date Acquired: & 3/22/2021 1:53:36 PM CST & & \\
\hline Date Processed: & 3/22/2021 4:33:05 PM CST & & \\
\hline
\end{tabular}

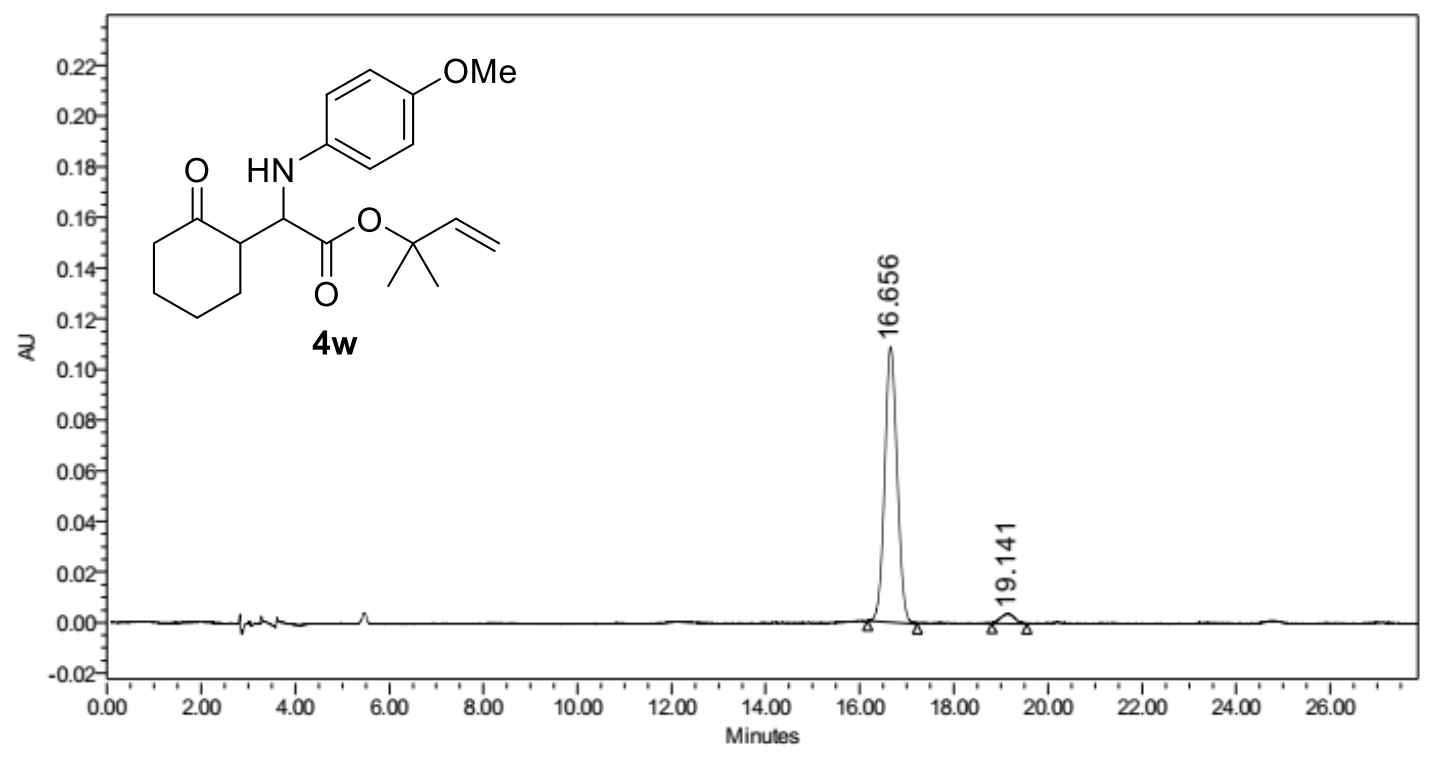

\begin{tabular}{|r|c|r|r|r|r|}
\hline & RT & $\begin{array}{c}\text { Width } \\
(\mathrm{sec})\end{array}$ & Height & Area & $\%$ Area \\
\hline 1 & 16.656 & 63.700 & 108845 & 2007285 & 96.18 \\
\hline 2 & 19.141 & 44.500 & 4023 & 79666 & 3.82 \\
\hline
\end{tabular}


\#\#\# WZH-1122-3+- PA2 732140.7

\begin{tabular}{llll}
\hline Sample Name: & WZH-1122-3+- PA2 73214 0.7 & Injection Volume: & $\mathbf{2 . 0}$ \\
Vial Number: & RE1 & Channel: & UV_VIS_2 \\
Sample Type: & unknown & Wavelength: & $\mathbf{2 4 0 . 0}$ \\
Control Program: & test-dad2 & Bandwidth: & 4 \\
Quantif. Method: & $\mathbf{2 0 1 7 0 6 0 8}$ & Dilution Factor: & $\mathbf{1 . 0 0 0 0}$ \\
Recording Time: & $\mathbf{2 0 2 0 - 1 2 - 2 4 1 5 : 4 5}$ & Sample Weight: & $\mathbf{1 . 0 0 0 0}$ \\
Run Time (min): & $\mathbf{9 3 . 0 6}$ & Sample Amount: & $\mathbf{1 . 0 0 0 0}$ \\
\hline
\end{tabular}

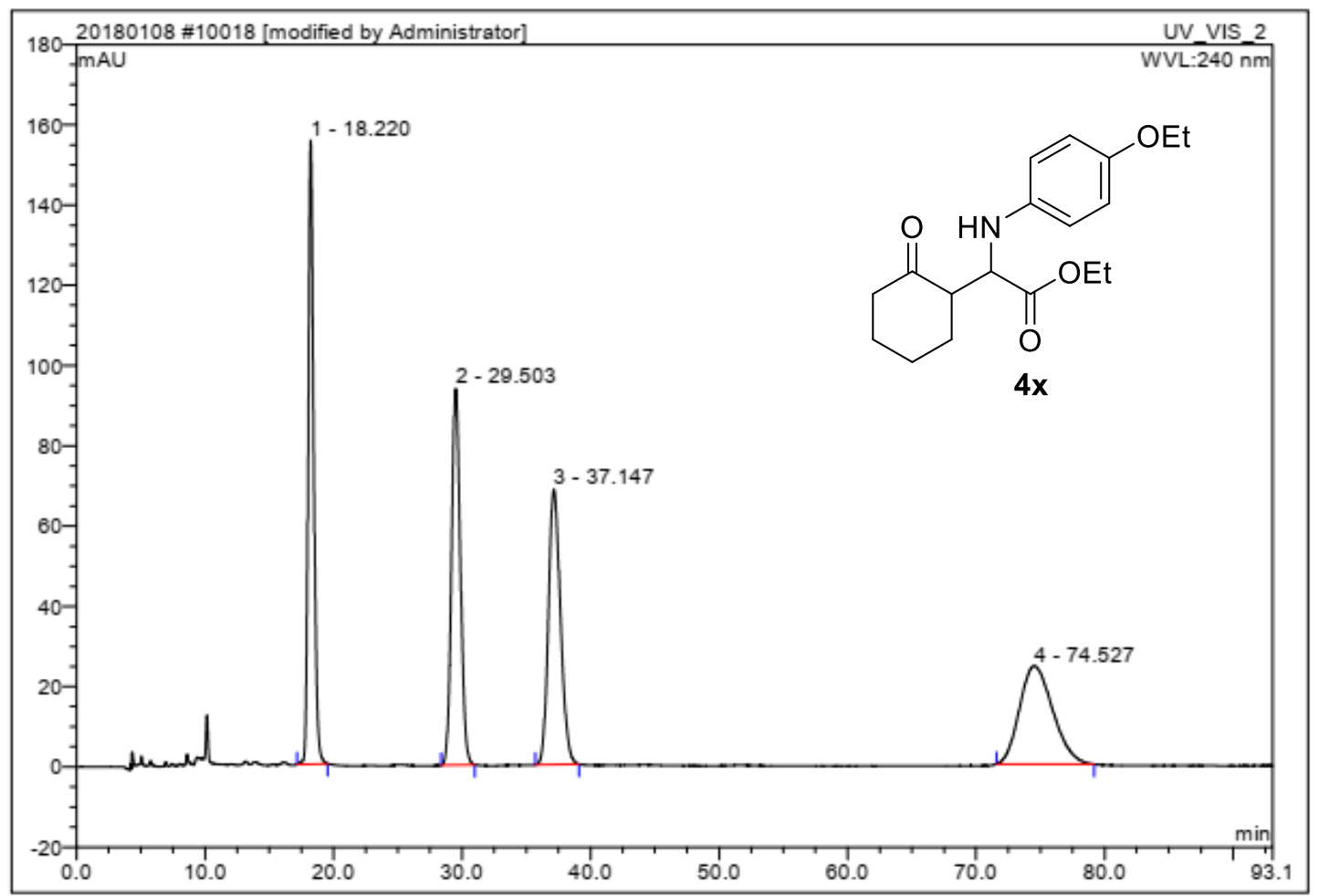

\begin{tabular}{|r|ccrrrrr|}
\hline No. & $\begin{array}{c}\text { Ret.Time } \\
\text { min }\end{array}$ & Peak Name & $\begin{array}{c}\text { Height } \\
\text { mAU }\end{array}$ & $\begin{array}{c}\text { Area } \\
\text { mAU*min }\end{array}$ & $\begin{array}{c}\text { Rel.Area } \\
\%\end{array}$ & Amount & Type \\
\hline 1 & 18.22 & n.a. & 155.423 & 77.027 & 25.64 & n.a. & BMB \\
2 & 29.50 & n.a. & 93.859 & 75.209 & 25.03 & n.a. & BMB \\
3 & 37.15 & n.a. & 68.592 & 74.706 & 24.86 & n.a. & BMB \\
4 & 74.53 & n.a. & 24.544 & 73.522 & 24.47 & n.a. & BMB $^{\star}$ \\
\hline Total: & & & 342.417 & 300.464 & 100.00 & 0.000 & \\
\hline
\end{tabular}




\begin{tabular}{|lllll|}
\hline \#\#\# WZH-1122-1 PA2 732140.7 & & \\
& & & \\
\hline Sample Name: & WZH-1122-1 PA2 732140.7 & Injection Volume: & 1.0 \\
Vial Number: & RD6 & Channel: & UV_VIS_2 \\
Sample Type: & unknown & Wavelength: & $\mathbf{2 4 0 . 0}$ \\
Control Program: & test-dad2 & Bandwidth: & 4 \\
Quantif. Method: & $\mathbf{2 0 1 7 0 6 0 8}$ & Dilution Factor: & 1.0000 \\
Recording Time: & $\mathbf{2 0 2 0 - 1 2 - 2 9} 12: 37$ & Sample Weight: & $\mathbf{1 . 0 0 0 0}$ \\
Run Time (min): & $\mathbf{1 2 1 . 0 5}$ & Sample Amount: & $\mathbf{1 . 0 0 0 0}$ \\
\hline
\end{tabular}

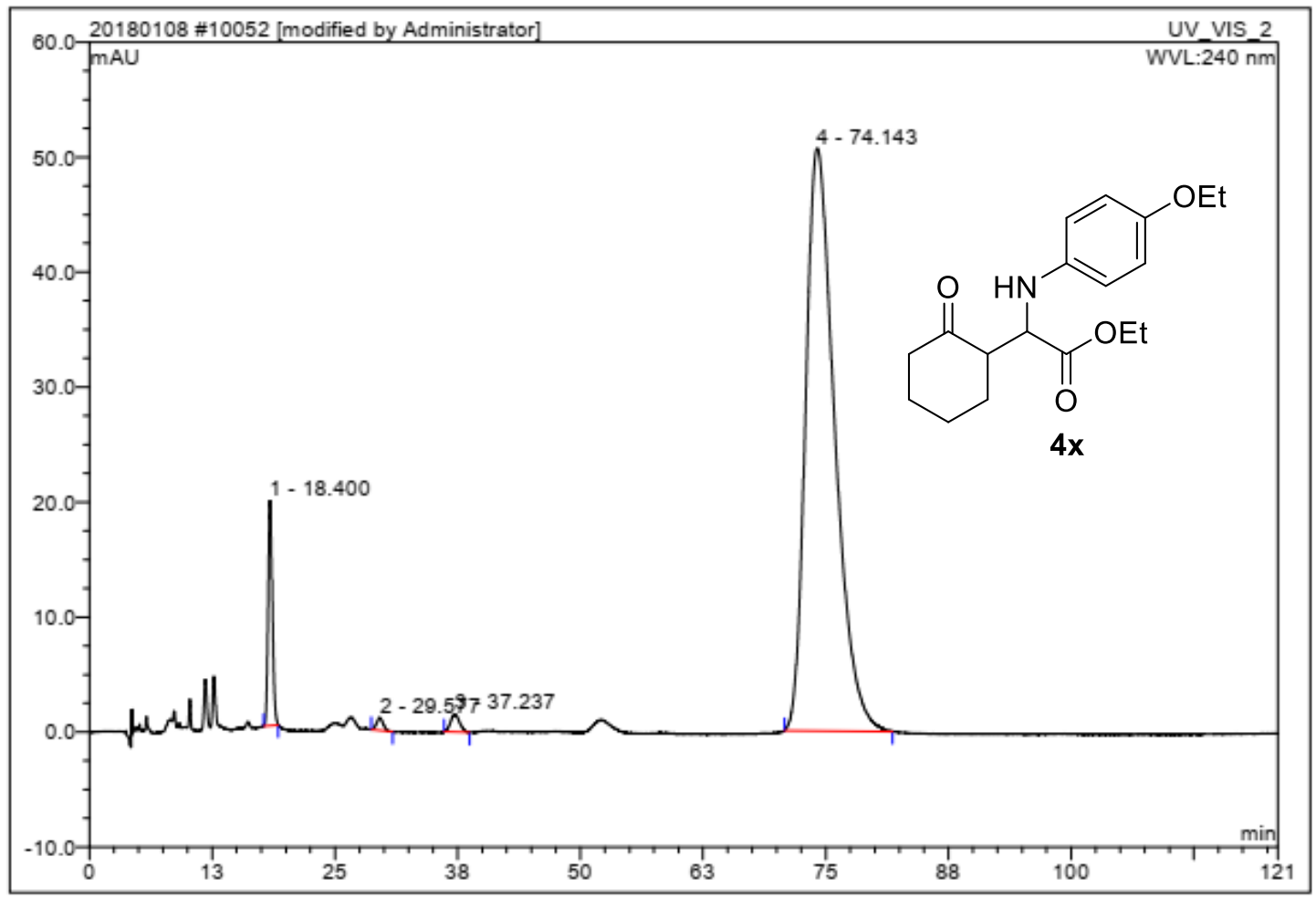

\begin{tabular}{|r|ccrrrrr|}
\hline No. & $\begin{array}{c}\text { Ret.Time } \\
\text { min }\end{array}$ & Peak Name & $\begin{array}{c}\text { Height } \\
\text { mAU }\end{array}$ & $\begin{array}{c}\text { Area } \\
\text { mAU*min }\end{array}$ & $\begin{array}{r}\text { Rel.Area } \\
\%\end{array}$ & Amount & Type \\
\hline 1 & 18.40 & n.a. & 19.563 & 10.033 & 5.38 & n.a. & BMB $^{*}$ \\
2 & 29.58 & n.a. & 1.088 & 0.840 & 0.45 & n.a. & BMB $^{\star}$ \\
3 & 37.24 & n.a. & 1.524 & 1.627 & 0.87 & n.a. & BMB $^{\star}$ \\
4 & 74.14 & n.a. & 50.685 & 173.837 & 93.29 & n.a. & BMB $^{\star}$ \\
\hline Total: & & & 72.860 & 186.337 & 100.00 & 0.000 & \\
\hline
\end{tabular}


9999 WZH-1126-2+- PC2 912140.7

\begin{tabular}{|llll|}
\hline Sample Name: & WZH-1126-2+- PC2 912140.7 & Injection Volume: & $\mathbf{2 . 0}$ \\
Vial Number: & RE3 & Channel: & UV_VIS_2 \\
Sample Type: & unknown & Wavelength: & $\mathbf{2 1 4 . 0}$ \\
Control Program: & test-dad4 & Bandwidth: & 4 \\
Quantif. Method: & $\mathbf{2 0 1 7 0 6 0 8}$ & Dilution Factor: & $\mathbf{1 . 0 0 0 0}$ \\
Recording Time: & $\mathbf{2 0 2 0 - 1 2 - 2 2 ~ 1 3 : 3 5}$ & Sample Weight: & $\mathbf{1 . 0 0 0 0}$ \\
Run Time (min): & $\mathbf{1 1 4 . 7 9}$ & Sample Amount: & $\mathbf{1 . 0 0 0 0}$ \\
\hline
\end{tabular}

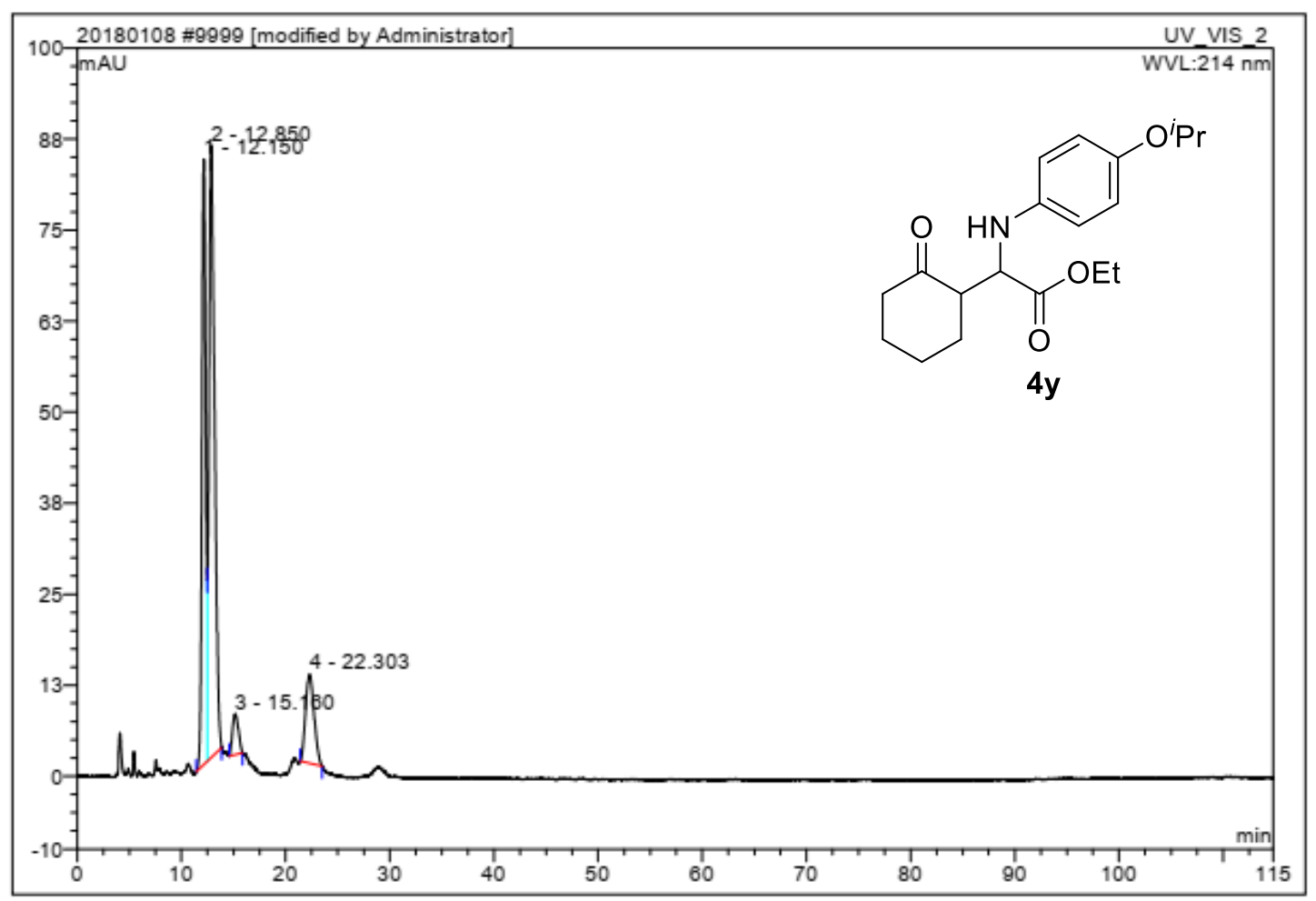

\begin{tabular}{|r|ccrrrrr|}
\hline No. & $\begin{array}{c}\text { Ret.Time } \\
\text { min }\end{array}$ & Peak Name & $\begin{array}{c}\text { Height } \\
\text { mAU }\end{array}$ & $\begin{array}{c}\text { Area } \\
\text { mAU*min }\end{array}$ & $\begin{array}{r}\text { Rel.Area } \\
\%\end{array}$ & Amount & Type \\
\hline 1 & 12.15 & n.a. & 83.165 & 34.087 & 33.57 & n.a. & BM \\
2 & 12.85 & n.a. & 84.151 & 52.128 & 51.33 & n.a. & MB \\
3 & 15.16 & n.a. & 5.581 & 3.697 & 3.64 & n.a. & BMB \\
4 & 22.30 & n.a. & 12.238 & 11.636 & 11.46 & n.a. & BMB \\
\hline Total: & & & 185.134 & 101.547 & 100.00 & 0.000 & \\
\hline
\end{tabular}




\begin{tabular}{|c|c|c|c|}
\hline \multicolumn{4}{|c|}{ \#\#\# WZH-1129-1+- PC2 912140.7} \\
\hline Sample Name: & WZH-1129-1+- PC2 912140.7 & Injection Volume: & \\
\hline Vial Number: & RC6 & Channel: & UV_VIS_2 \\
\hline Sample Type: & unknown & Wavelength: & 240.0 \\
\hline Control Program: & test-dad2 & Bandwidth: & 4 \\
\hline Quantif. Method: & 20170608 & Dilution Factor: & 1.0000 \\
\hline Recording Time: & 2020-12-29 17:59 & Sample Weight: & 1.0000 \\
\hline Run Time (min): & 70.00 & Sample Amount: & 1.0000 \\
\hline
\end{tabular}

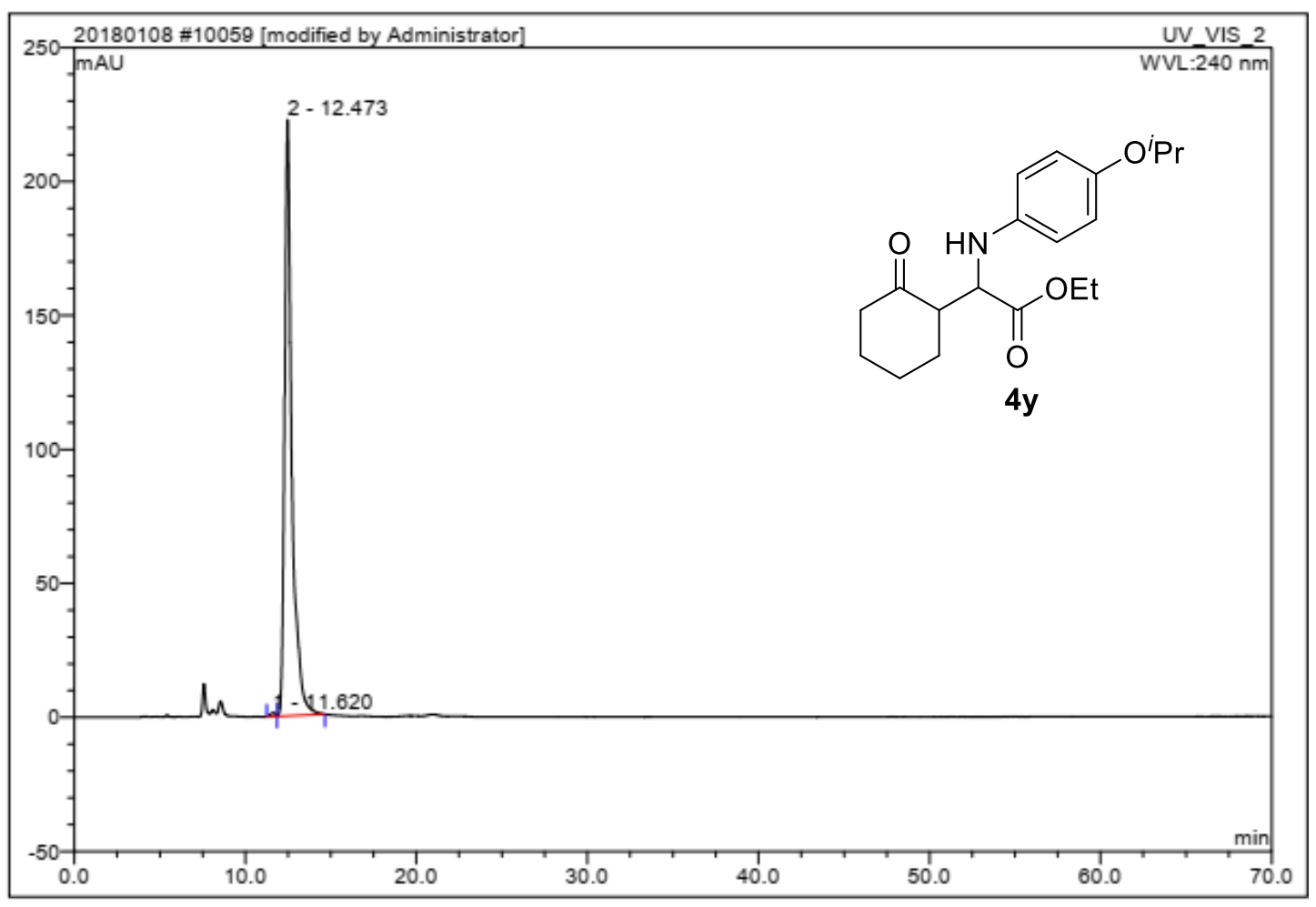

\begin{tabular}{|r|ccrrrrr|}
\hline No. & $\begin{array}{c}\text { Ret.Time } \\
\text { min }\end{array}$ & Peak Name & $\begin{array}{c}\text { Height } \\
\text { mAU }\end{array}$ & $\begin{array}{c}\text { Area } \\
\text { mAU*min }\end{array}$ & $\begin{array}{r}\text { Rel.Area } \\
\%\end{array}$ & Amount & Type \\
\hline 1 & 11.62 & n.a. & 1.439 & 0.466 & 0.42 & n.a. & BM \\
2 & 12.47 & n.a. & 222.669 & 109.782 & 99.58 & n.a. & MB \\
\hline Total: & & & 224.109 & 110.249 & 100.00 & 0.000 & \\
\hline
\end{tabular}




\begin{tabular}{|c|c|c|c|}
\hline \multicolumn{2}{|r|}{ SAMPLE } & \multicolumn{2}{|c|}{ INFORMATION } \\
\hline Sample Name: & 0304-rac & Acquired By: & System \\
\hline Sample Type: & Unknown & Sample Set Name: & \\
\hline Vial: & $1: C, 1$ & Acq. Method Set: & 2021_1 \\
\hline Injection \#: & 2 & Processing Method & Defaūt \\
\hline Injection Volume: & $3.00 \mathrm{ul}$ & Channel Name: & PDA Ch2 254nm@4.8nm \\
\hline Run Time: & 50.0 Minutes & Proc. Chnl. Descr.: & PDA Ch2 254nm@4.8nm \\
\hline Date Acquired: & 3/11/2021 3:41:47 PM CST & & \\
\hline Date Processed: & 3/16/2021 8:16:13 AM CST & & \\
\hline
\end{tabular}

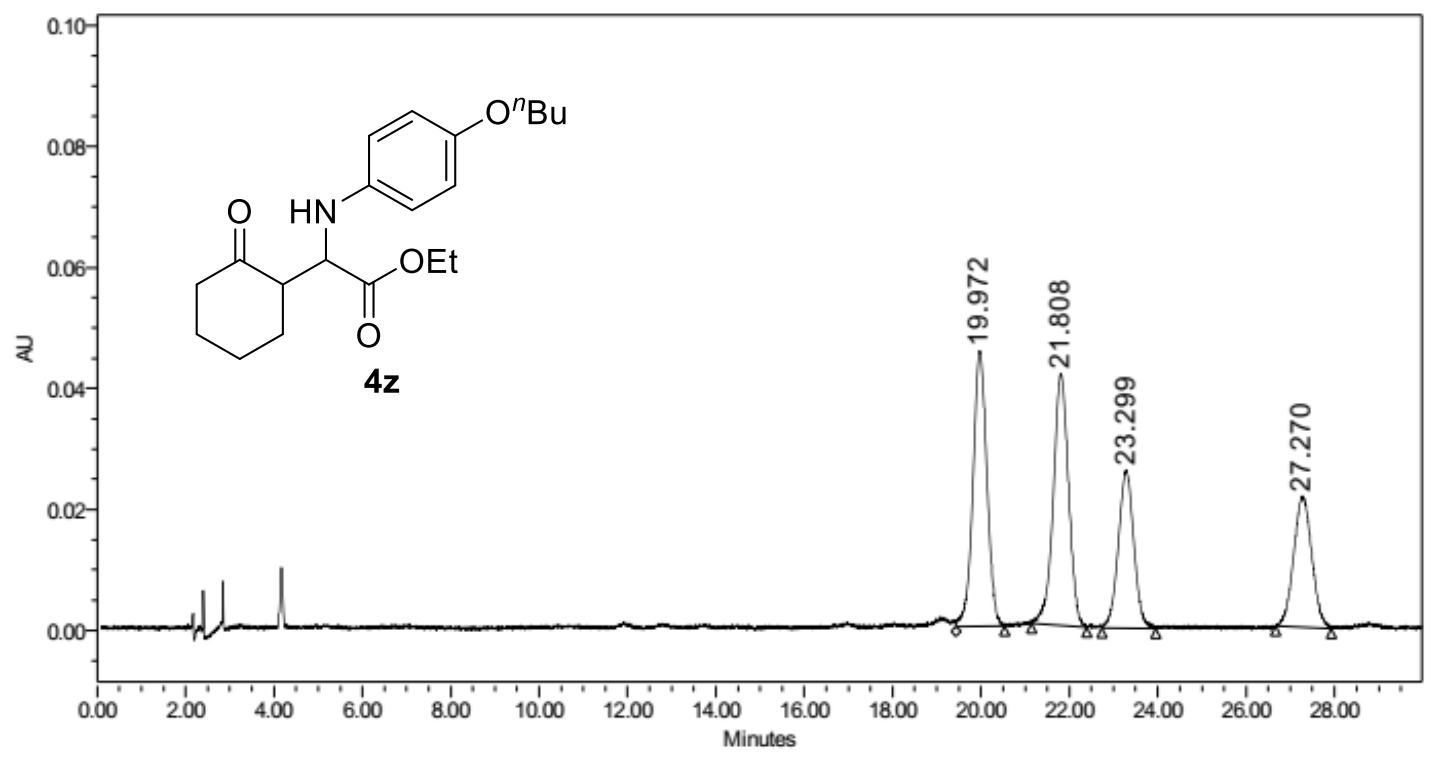

\begin{tabular}{|c|c|c|c|c|c|}
\hline & RT & $\begin{array}{c}\text { Width } \\
(\mathrm{sec})\end{array}$ & Height & Area & $\%$ Area \\
\hline 1 & 19.972 & 65.850 & 45561 & 1001969 & 30.69 \\
\hline 2 & 21.808 & 75.150 & 41740 & 1000188 & 30.64 \\
\hline 3 & 23.299 & 73.350 & 26092 & 643387 & 19.71 \\
\hline 4 & 27.270 & 75.300 & 21676 & 618958 & 18.96 \\
\hline
\end{tabular}

Reported by User: System

Project Name: 2021

Date Printed:

Report Method: Default Individual Report

$3 / 16 / 2021$

Page: 1 of 1

8:23:41 AM PRC 


\begin{tabular}{|c|c|c|c|}
\hline \multicolumn{2}{|r|}{ SAMPLE } & \multicolumn{2}{|c|}{ INFORMATION } \\
\hline Sample Name: & 0104-chiral & Acquired By: & System \\
\hline Sample Type: & Unknown & Sample Set Name: & \\
\hline Vial: & $1: C, 3$ & Acq. Method Set: & 2021_1 \\
\hline Injection \#: & 1 & Processing Method & Default \\
\hline Injection Volume: & $3.00 \mathrm{ul}$ & Channel Name: & PDA Ch2 254nm@4.8nm \\
\hline Run Time: & 50.0 Minutes & Proc. Chnl. Descr.: & PDA Ch2 254nm@4.8nm \\
\hline Date Acquired: & 3/11/2021 4:12:41 PM CST & & \\
\hline Date Processed: & 3/16/2021 8:17:13 AM CST & & \\
\hline
\end{tabular}

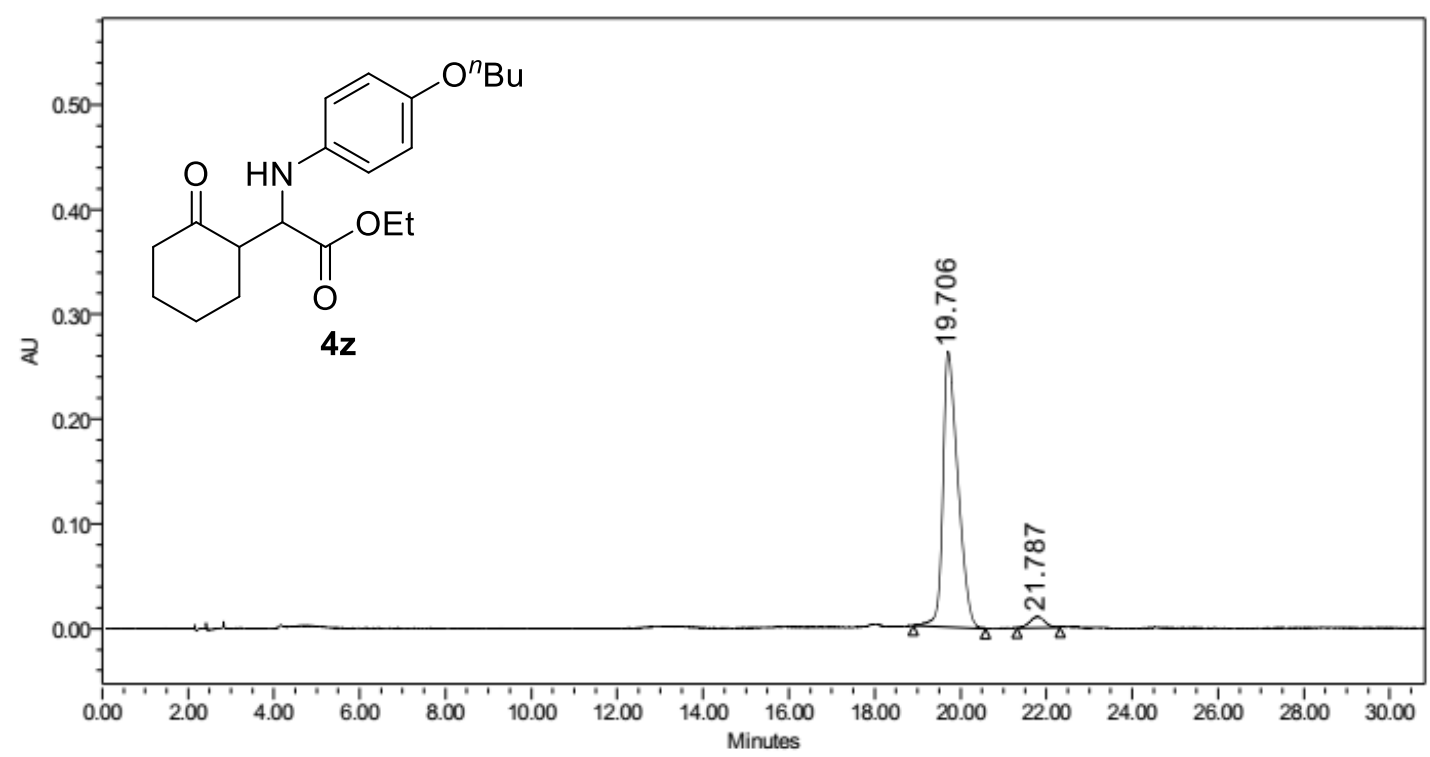

\begin{tabular}{|c|c|r|r|r|r|}
\hline & RT & $\begin{array}{c}\text { Width } \\
(\mathrm{sec})\end{array}$ & Height & Area & $\%$ Area \\
\hline 1 & 19.706 & 100.800 & 263132 & 6409467 & 96.16 \\
\hline 2 & 21.787 & 60.000 & 10731 & 256237 & 3.84 \\
\hline
\end{tabular}

Reported by User: System

Report Method: Default Individual Report

Report Method I[ 2063

Page: 1 of 1
Project Name: 2021

Date Printed:

$3 / 16 / 2021$

8:23:56 AM PRC 


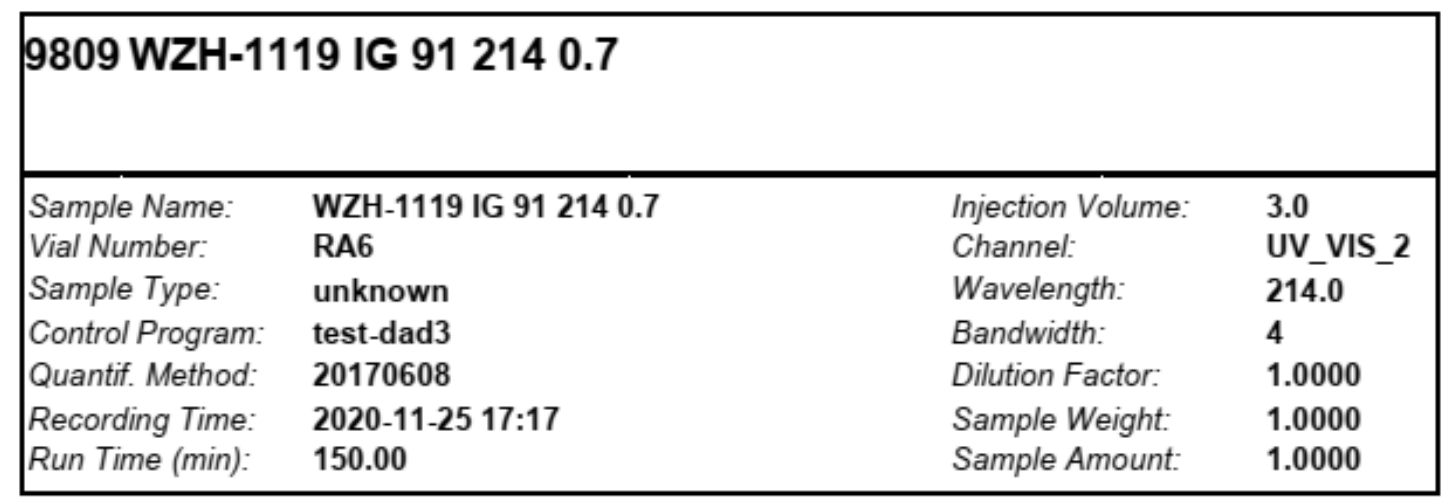

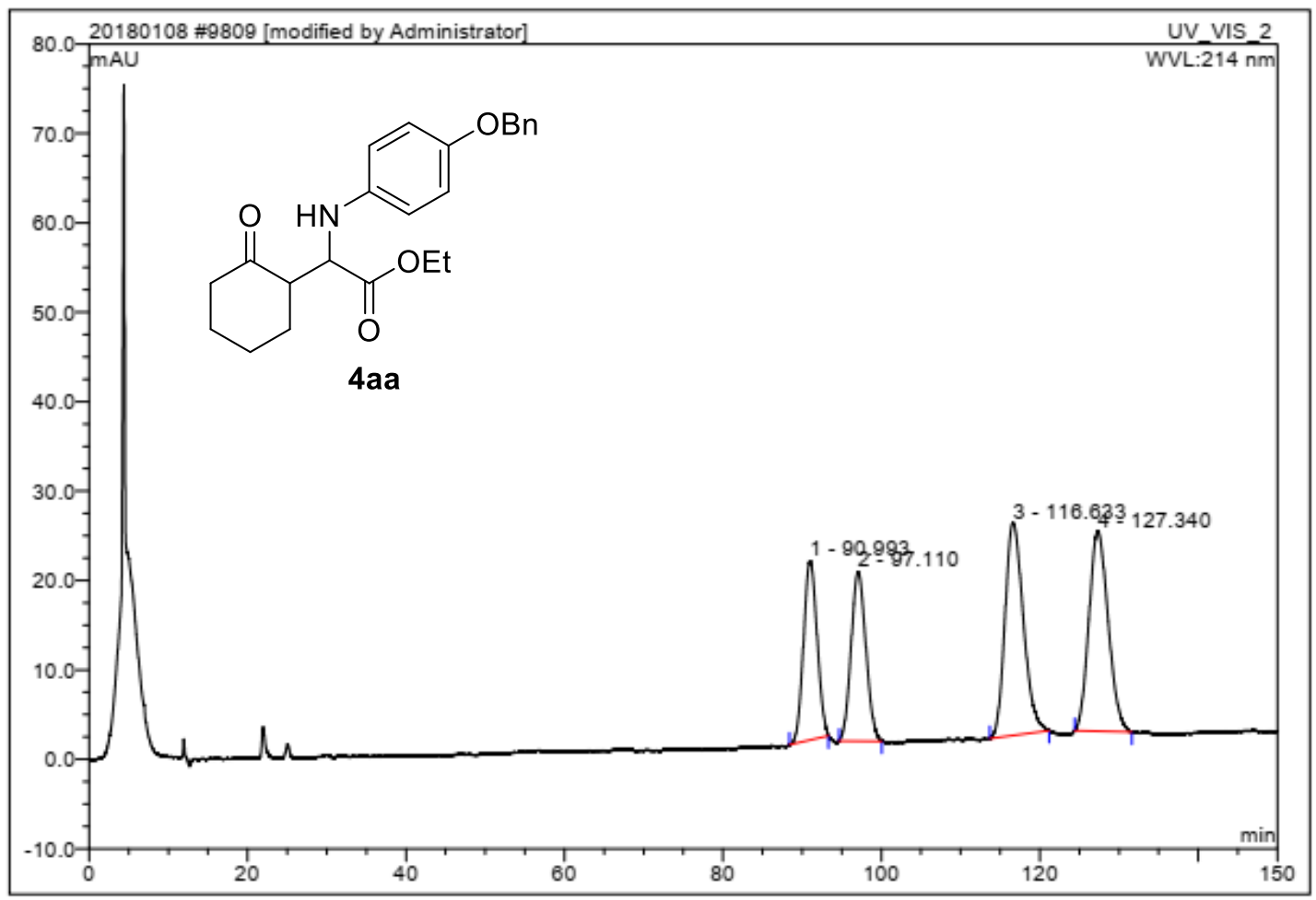

\begin{tabular}{|r|rrrrrrr|}
\hline No. & $\begin{array}{c}\text { Ret.Time } \\
\text { min }\end{array}$ & Peak Name & $\begin{array}{c}\text { Height } \\
\text { mAU }\end{array}$ & $\begin{array}{c}\text { Area } \\
\text { mAU*min }\end{array}$ & $\begin{array}{c}\text { Rel.Area } \\
\%\end{array}$ & Amount & Type \\
\hline 1 & 90.99 & n.a. & 20.089 & 39.986 & 19.19 & n.a. & BMB $^{\star}$ \\
2 & 97.11 & n.a. & 18.924 & 41.152 & 19.75 & n.a. & BMB $^{\star}$ \\
3 & 116.63 & n.a. & 23.778 & 64.119 & 30.78 & n.a. & BMB $^{*}$ \\
4 & 127.34 & n.a. & 22.329 & 63.083 & 30.28 & n.a. & BMB $^{*}$ \\
\hline Total: & & & 85.119 & 208.340 & 100.00 & 0.000 & \\
\hline
\end{tabular}


9810 WZH-1116 IG 912140.7

Sample Name:

Vial Number:

Sample Type:

Control Program:

Quantif. Method:

Recording Time:

Run Time ( $\mathrm{min})$ :

WZH-1116 IG 912140.7
RE5
unknown
test-dad3
20170608
$2020-11-269: 17$
150.00

$\begin{array}{ll}\text { Injection Volume: } & 3.0 \\ \text { Channel: } & \text { UV_VIS_2 } \\ \text { Wavelength: } & 214.0 \\ \text { Bandwidth: } & 4 \\ \text { Dilution Factor: } & 1.0000 \\ \text { Sample Weight: } & 1.0000 \\ \text { Sample Amount: } & 1.0000\end{array}$

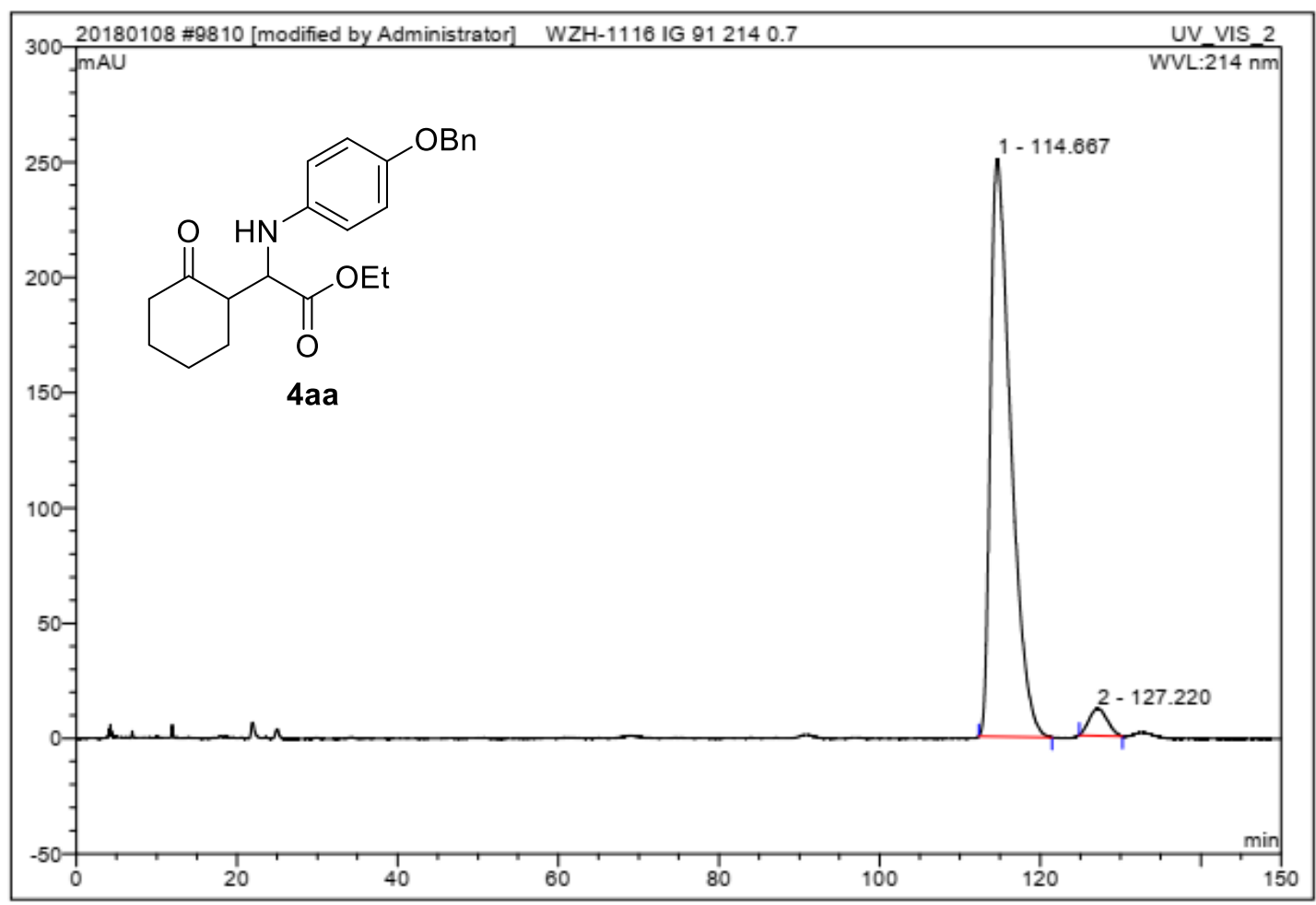

\begin{tabular}{|r|ccrrrrr|}
\hline No. & $\begin{array}{c}\text { Ret.Time } \\
\text { min }\end{array}$ & Peak Name & $\begin{array}{c}\text { Height } \\
\text { mAU }\end{array}$ & $\begin{array}{c}\text { Area } \\
\text { mAU*min }\end{array}$ & $\begin{array}{r}\text { Rel.Area } \\
\%\end{array}$ & Amount & Type \\
\hline 1 & 114.67 & n.a. & 250.545 & 735.813 & 95.97 & n.a. & BMB $^{*}$ \\
2 & 127.22 & n.a. & 11.769 & 30.915 & 4.03 & n.a. & BMB $^{\star}$ \\
\hline Total: & & & 262.314 & 766.728 & 100.00 & 0.000 & \\
\hline
\end{tabular}




\begin{tabular}{|lllll|}
\hline \#\#\# WZH-1224-2+- ADH 912140.7 & & \\
& & & \\
\hline Sample Name: & WZH-1224-2+- ADH 912140.7 & Injection Volume: & 3.0 \\
Vial Number: & RC3 & Channel: & UV_VIS_1 \\
Sample Type: & unknown & Wavelength: & $\mathbf{2 1 4}$ \\
Control Program: & $\mathbf{2 0 1 7 0 1 - 2}$ & Bandwidth: & n.a. \\
Quantif. Method: & 201701 & Dilution Factor: & 1.0000 \\
Recording Time: & $2021 / 1 / 2516: 21$ & Sample Weight: & 1.0000 \\
Run Time (min): & 64.68 & Sample Amount: & 1.0000 \\
\hline
\end{tabular}

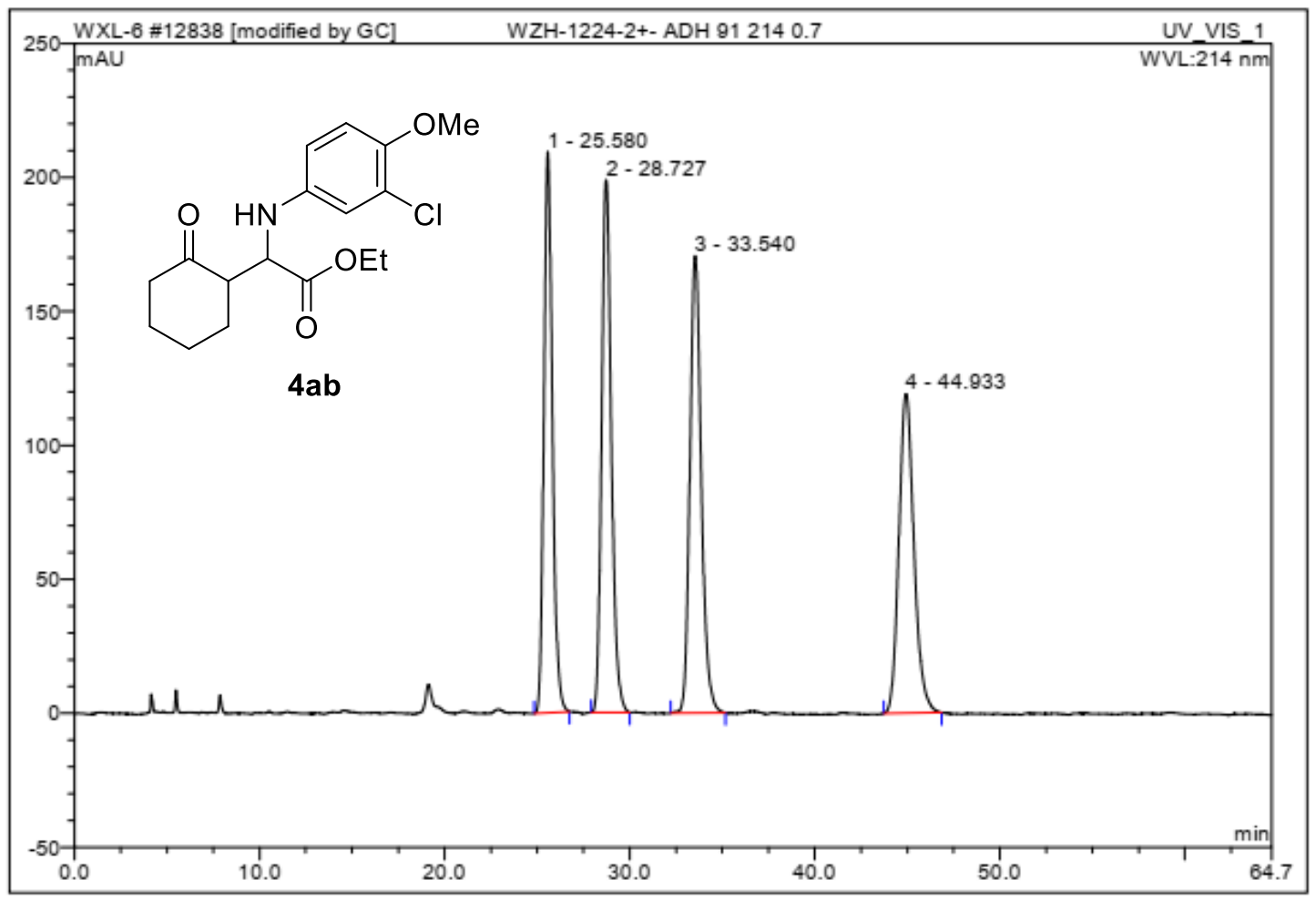

\begin{tabular}{|r|ccccccc|}
\hline No. & $\begin{array}{c}\text { Ret.Time } \\
\text { min }\end{array}$ & Peak Name & $\begin{array}{c}\text { Height } \\
\text { mAU }\end{array}$ & $\begin{array}{c}\text { Area } \\
\text { mAU*min }\end{array}$ & $\begin{array}{c}\text { Rel.Area } \\
\%\end{array}$ & Amount & Type \\
\hline 1 & 25.58 & n.a. & 209.502 & 111.883 & 23.97 & n.a. & BMB \\
2 & 28.73 & n.a. & 198.849 & 120.607 & 25.84 & n.a. & BMB \\
3 & 33.54 & n.a. & 170.637 & 121.640 & 26.06 & n.a. & BMB \\
4 & 44.93 & n.a. & 119.134 & 112.577 & 24.12 & n.a. & BMB \\
\hline Total: & & & 698.121 & 466.707 & 100.00 & 0.000 & \\
\hline
\end{tabular}




\begin{tabular}{|llll|}
\hline \#\#\# WZH-0108-2 ADH 912140.7 & & \\
& & & \\
\hline Sample Name: & WZH-0108-2 ADH 912140.7 & Injection Volume: & 2.0 \\
Vial Number: & RA1 & Channel: & UV_VIS_1 \\
Sample Type: & unknown & Wavelength: & $\mathbf{2 1 4}$ \\
Control Program: & $\mathbf{2 0 1 7 0 1 - 3}$ & Bandwidth: & n.a. \\
Quantif. Method: & 201701 & Dilution Factor: & $\mathbf{1 . 0 0 0 0}$ \\
Recording Time: & $\mathbf{2 0 2 1 / 2 / 1} 18: 52$ & Sample Weight: & $\mathbf{1 . 0 0 0 0}$ \\
Run Time (min): & $\mathbf{5 1 . 2 3}$ & Sample Amount: & $\mathbf{1 . 0 0 0 0}$ \\
\hline
\end{tabular}

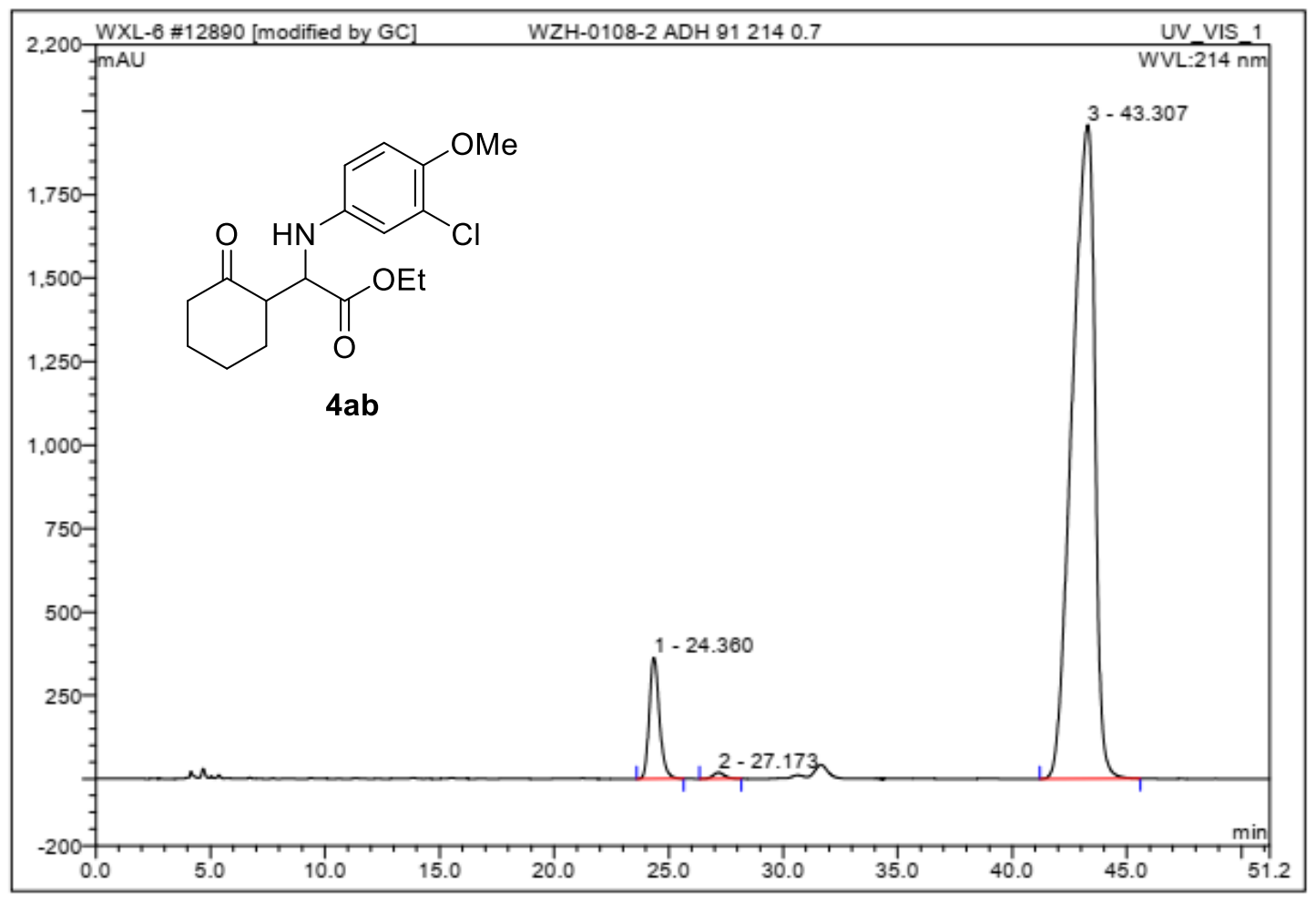

\begin{tabular}{|r|ccrrrrr|}
\hline No. & $\begin{array}{c}\text { Ret.Time } \\
\text { min }\end{array}$ & Peak Name & $\begin{array}{c}\text { Height } \\
\text { mAU }\end{array}$ & $\begin{array}{r}\text { Area } \\
\text { mAU*min }\end{array}$ & $\begin{array}{r}\text { Rel.Area } \\
\%\end{array}$ & Amount & Type \\
\hline 1 & 24.36 & n.a. & 363.777 & 186.451 & 7.62 & n.a. & BMB \\
2 & 27.17 & n.a. & 18.720 & 10.848 & 0.44 & n.a. & BMB \\
3 & 43.31 & n.a. & 1958.762 & 2248.028 & 91.93 & n.a. & BMB \\
\hline Total: & & & 2341.259 & 2445.327 & 100.00 & 0.000 & \\
\hline
\end{tabular}


\#\#\# WZH-1115-1-1+1-2 IC 9552140.7

\begin{tabular}{|llll|}
\hline Sample Name: & WZH-1115-1-1+1-2 IC 9552140.7 & Injection Volume: & $\mathbf{1 . 0}$ \\
Vial Number: & GC1 & Channel: & UV_VIS_1 \\
Sample Type: & unknown & Wavelength: & $\mathbf{2 5 4}$ \\
Control Program: & $\mathbf{2 0 1 7 0 1 - 5}$ & Bandwidth: & n.a. \\
Quantif. Method: & $\mathbf{2 0 1 7 0 1}$ & Dilution Factor: & $\mathbf{1 . 0 0 0 0}$ \\
Recording Time: & $\mathbf{2 0 2 0 / 1 1 / 2 3 ~ 1 1 : 5 9}$ & Sample Weight: & $\mathbf{1 . 0 0 0 0}$ \\
Run Time (min): & $\mathbf{9 0 . 8 8}$ & Sample Amount: & $\mathbf{1 . 0 0 0 0}$ \\
\hline
\end{tabular}

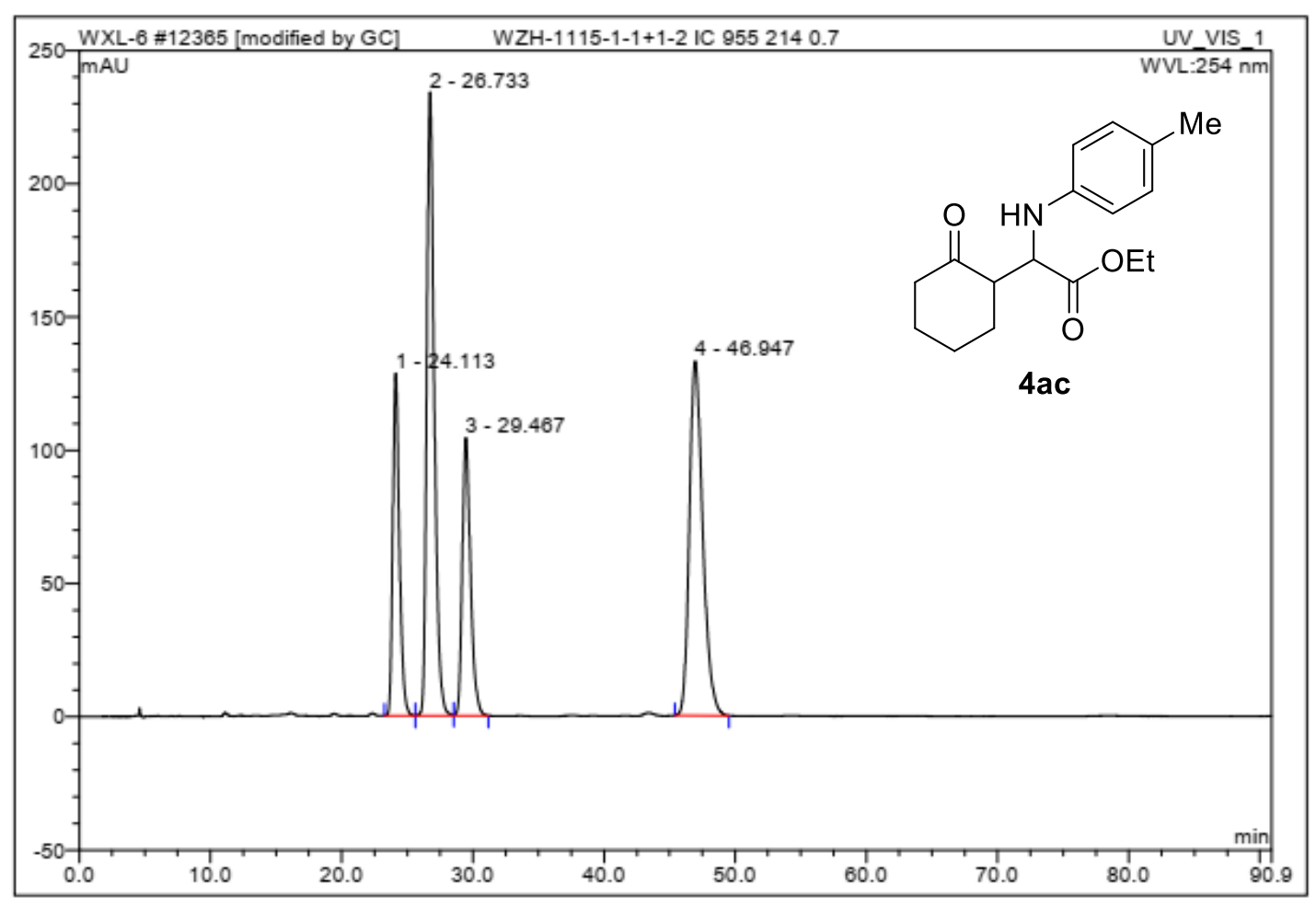

\begin{tabular}{|r|ccrrrrc|}
\hline No. & $\begin{array}{c}\text { Ret.Time } \\
\text { min }\end{array}$ & Peak Name & $\begin{array}{c}\text { Height } \\
\text { mAU }\end{array}$ & $\begin{array}{c}\text { Area } \\
\text { mAU*min }\end{array}$ & $\begin{array}{c}\text { Rel.Area } \\
\%\end{array}$ & Amount & Type \\
\hline 1 & 24.11 & n.a. & 128.423 & 74.881 & 16.12 & n.a. & BM \\
2 & 26.73 & n.a. & 233.971 & 156.609 & 33.70 & n.a. & M \\
3 & 29.47 & n.a. & 104.257 & 75.904 & 16.34 & n.a. & MB \\
4 & 46.95 & n.a. & 132.996 & 157.254 & 33.84 & n.a. & BMB \\
\hline Total: & & & 599.646 & 464.648 & 100.00 & 0.000 & \\
\hline
\end{tabular}




\section{\#\#\# WZH-1113-1 IC 9552540.7}

Sample Name:

Vial Number:

Sample Type:

Control Program:

Quantif. Method:

Recording Time:

Run Time (min):

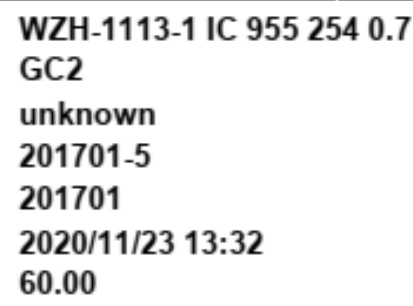

201701

2020/11/23 13:32

60.00

$\begin{array}{ll}\text { Injection Volume: } & 1.0 \\ \text { Channel: } & \text { UV_VIS_1 } \\ \text { Wavelength: } & 254 \\ \text { Bandwidth: } & \text { n.a. } \\ \text { Dilution Factor: } & 1.0000 \\ \text { Sample Weight: } & 1.0000 \\ \text { Sample Amount: } & 1.0000\end{array}$

Injection Volume:

Channel:

Wavelength:

Bandwidth:

Dilution Factor:

Sample Amount:

1.0000

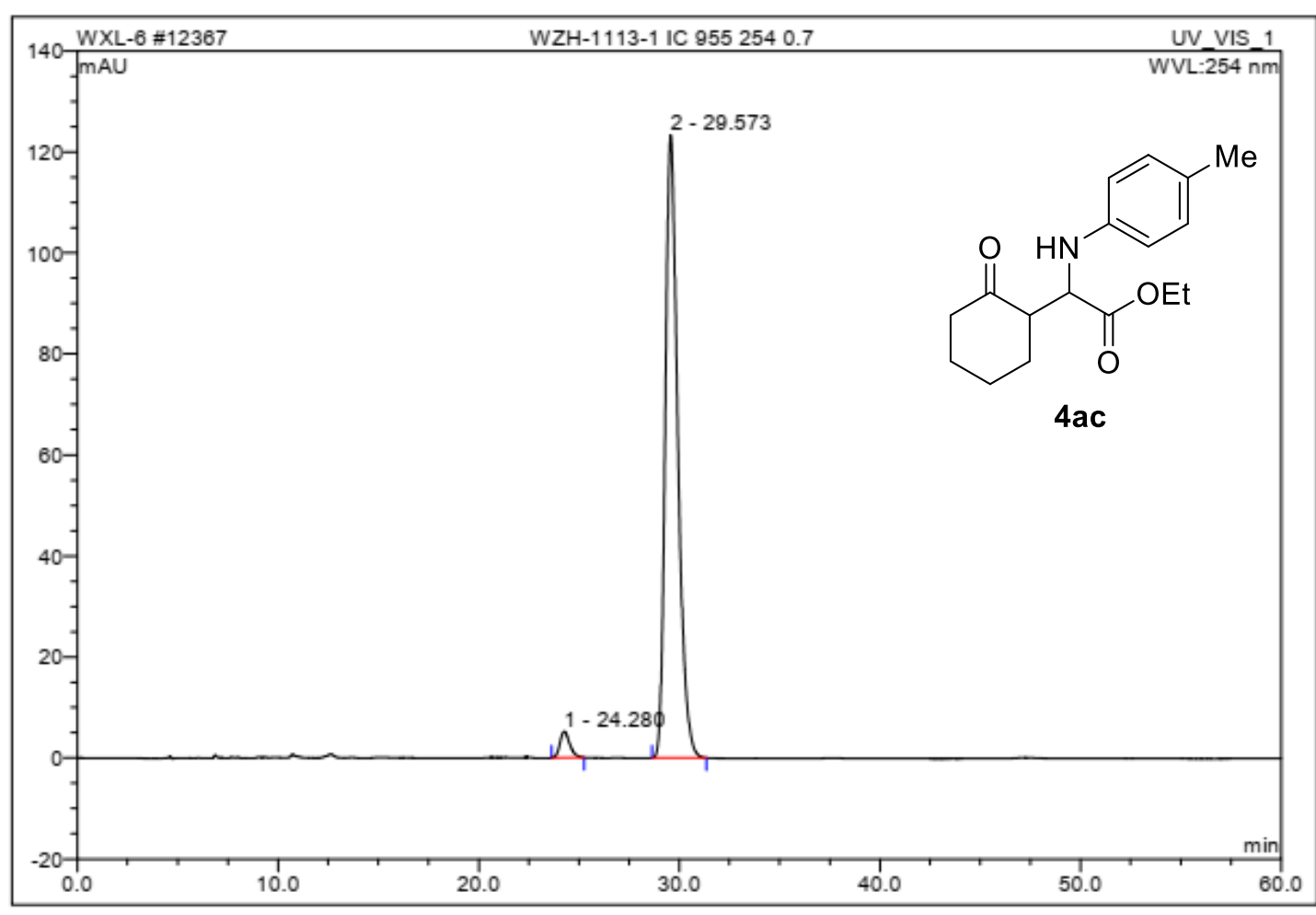

\begin{tabular}{|c|c|c|c|c|c|c|c|}
\hline No. & $\begin{array}{c}\text { Ret.Time } \\
\text { min }\end{array}$ & Peak Name & $\begin{array}{c}\text { Height } \\
\text { mAU }\end{array}$ & $\begin{array}{c}\text { Area } \\
\mathrm{mAU}^{*} \mathrm{~min}\end{array}$ & $\begin{array}{c}\text { Rel.Area } \\
\%\end{array}$ & Amount & Type \\
\hline 1 & 24.28 & n.a. & 5.219 & 2.956 & 3.21 & n.a. & $\mathrm{BMB}$ \\
\hline 2 & 29.57 & n.a. & 123.375 & 89.113 & 96.79 & n.a. & $\mathrm{BMB}$ \\
\hline Total: & & & 128.594 & 92.068 & 100.00 & 0.000 & \\
\hline
\end{tabular}


\#\#\# WZH-1224-1-1+2 IE3 822140.7

\begin{tabular}{|llll|}
\hline Sample Name: & WZH-1224-1-1+2 IE3 822140.7 & Injection Volume: & 3.0 \\
Vial Number: & RC2 & Channel: & UV_VIS_1 \\
Sample Type: & unknown & Wavelength: & $\mathbf{2 1 4}$ \\
Control Program: & $\mathbf{2 0 1 7 0 1 - 5}$ & Bandwidth: & n.a. \\
Quantif. Method: & $\mathbf{2 0 1 7 0 1}$ & Dilution Factor: & $\mathbf{1 . 0 0 0 0}$ \\
Recording Time: & $\mathbf{2 0 2 1 / 1 / 2 0 ~ 2 : 2 2}$ & Sample Weight: & $\mathbf{1 . 0 0 0 0}$ \\
Run Time (min): & $\mathbf{6 5 . 0 1}$ & Sample Amount: & $\mathbf{1 . 0 0 0 0}$ \\
\hline
\end{tabular}

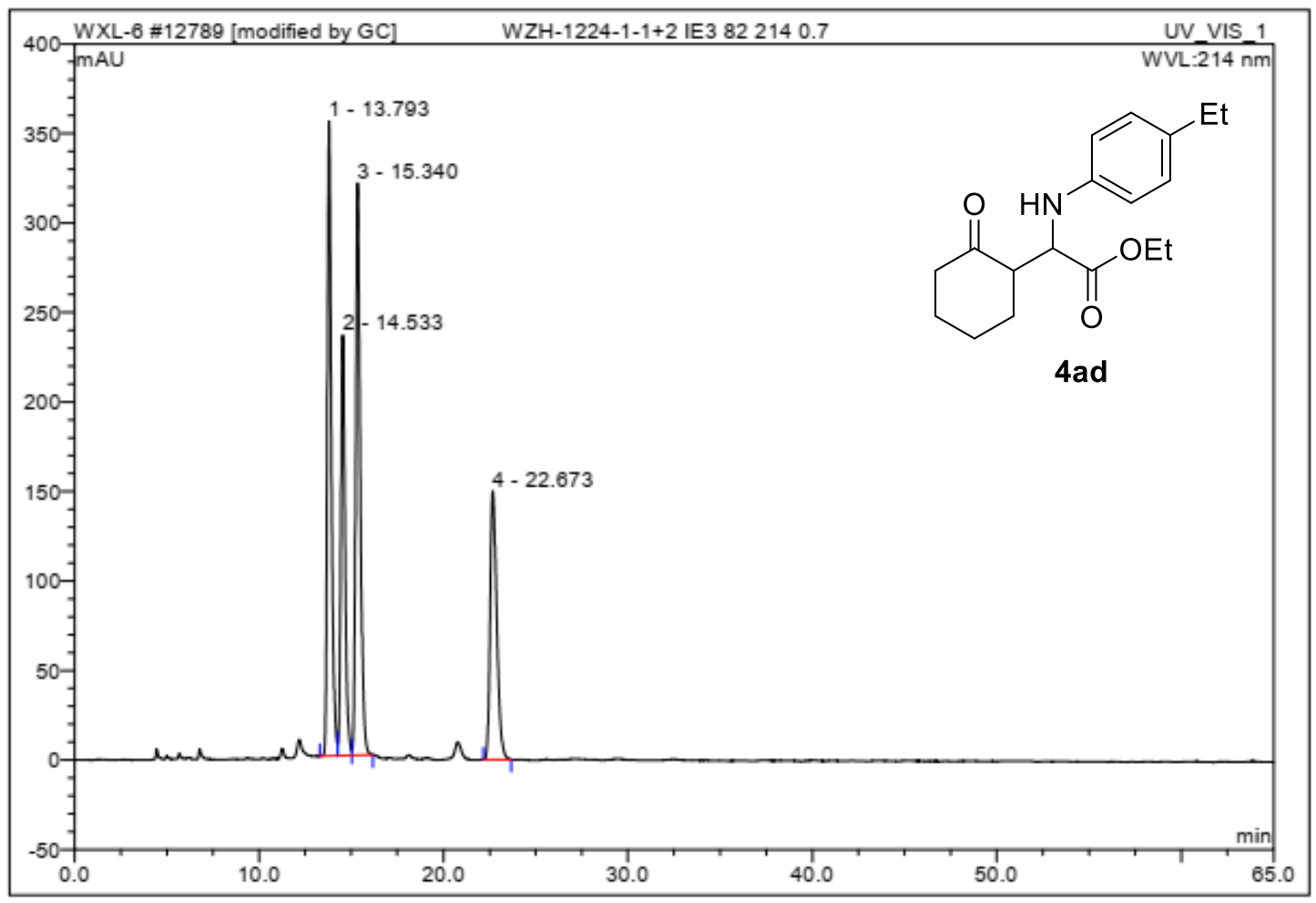

\begin{tabular}{|r|ccccccc|}
\hline No. & $\begin{array}{c}\text { Ret.Time } \\
\text { min }\end{array}$ & Peak Name & $\begin{array}{c}\text { Height } \\
\text { mAU }\end{array}$ & $\begin{array}{c}\text { Area } \\
\text { mAU*min }\end{array}$ & $\begin{array}{c}\text { Rel.Area } \\
\%\end{array}$ & Amount & Type \\
\hline 1 & 13.79 & n.a. & 354.656 & 91.614 & 29.63 & n.a. & BM \\
2 & 14.53 & n.a. & 234.965 & 62.409 & 20.19 & n.a. & M \\
3 & 15.34 & n.a. & 319.815 & 92.454 & 29.90 & n.a. & MB \\
4 & 22.67 & n.a. & 150.163 & 62.687 & 20.28 & n.a. & BMB \\
\hline Total: & & & 1059.599 & 309.163 & 100.00 & 0.000 & \\
\hline
\end{tabular}




\section{\#\#\# WZH-0108-1 IE3 822140.7}

\begin{tabular}{llll|}
\hline Sample Name: & WZH-0108-1 IE3 82 214 0.7 & Injection Volume: & 3.0 \\
Vial Number: & RB5 & Channel: & UV_VIS_1 \\
Sample Type: & unknown & Wavelength: & $\mathbf{2 1 4}$ \\
Control Program: & $\mathbf{2 0 1 7 0 1 - 2}$ & Bandwidth: & n.a. \\
Quantif. Method: & $\mathbf{2 0 1 7 0 1}$ & Dilution Factor: & $\mathbf{1 . 0 0 0 0}$ \\
Recording Time: & $\mathbf{2 0 2 1 / 1 / 2 7 ~ 1 7 : 1 4}$ & Sample Weight: & $\mathbf{1 . 0 0 0 0}$ \\
Run Time (min): & $\mathbf{4 2 . 1 1}$ & Sample Amount: & $\mathbf{1 . 0 0 0 0}$ \\
\hline
\end{tabular}

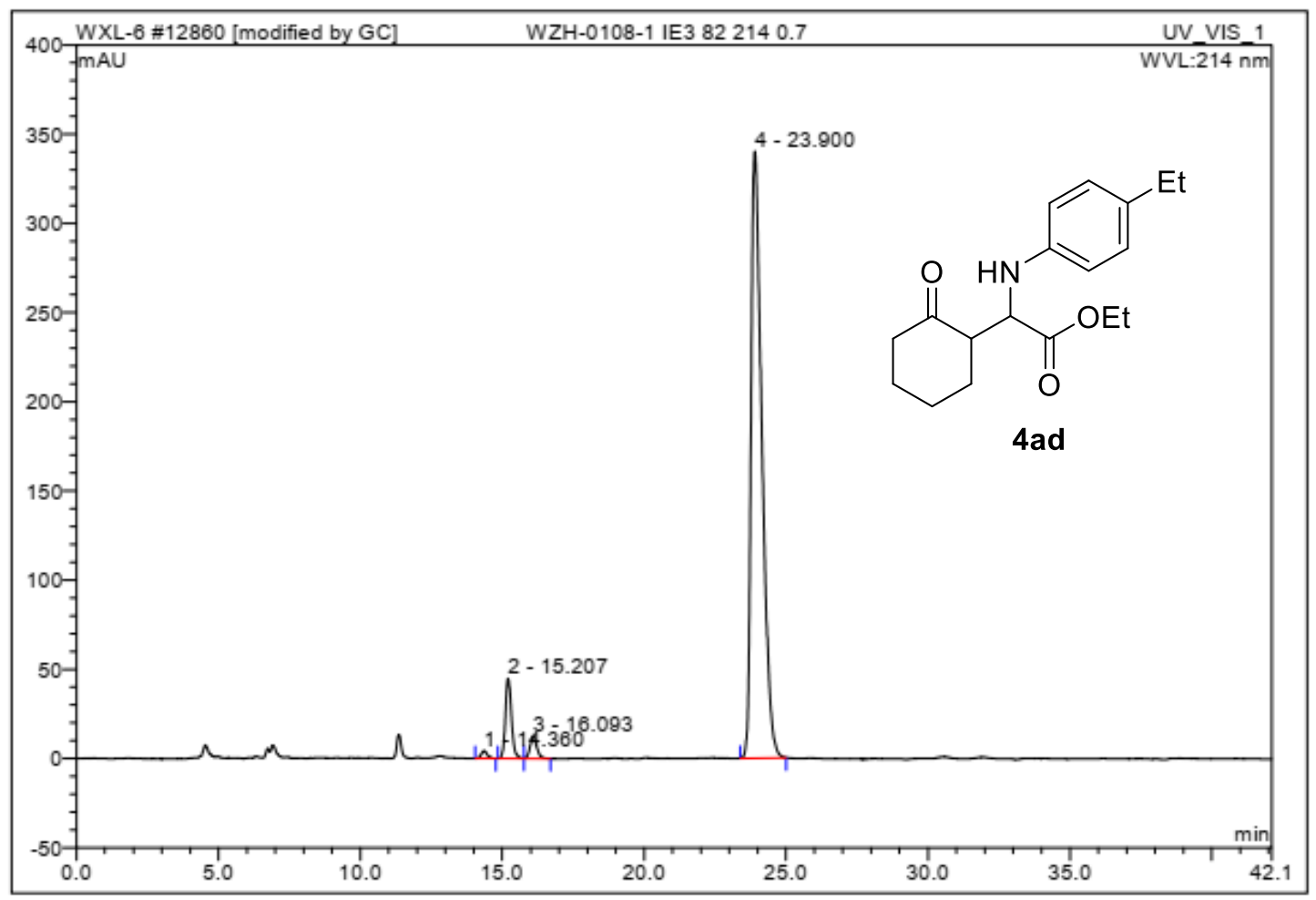

\begin{tabular}{|r|ccrrrrc|}
\hline No. & $\begin{array}{c}\text { Ret.Time } \\
\text { min }\end{array}$ & Peak Name & $\begin{array}{c}\text { Height } \\
\text { mAU }\end{array}$ & $\begin{array}{c}\text { Area } \\
\text { mAU*min }\end{array}$ & $\begin{array}{r}\text { Rel.Area } \\
\%\end{array}$ & Amount & Type \\
\hline 1 & 14.36 & n.a. & 4.336 & 1.074 & 0.65 & n.a. & BMB \\
2 & 15.21 & n.a. & 45.163 & 11.237 & 6.78 & n.a. & BM \\
3 & 16.09 & n.a. & 12.644 & 3.472 & 2.09 & n.a. & MB \\
4 & 23.90 & n.a. & 340.321 & 150.073 & 90.48 & n.a. & BMB \\
\hline Total: & & & 402.463 & 165.856 & 100.00 & 0.000 & \\
\hline
\end{tabular}


\#\#\# WZH-1211-3-1+2 PA2 912140.7

\begin{tabular}{llll}
\hline Sample Name: & WZH-1211-3-1+2 PA2 912140.7 & Injection Volume: & 2.0 \\
Vial Number: & RD6 & Channel: & UV_VIS_2 \\
Sample Type: & unknown & Wavelength: & $\mathbf{2 4 0 . 0}$ \\
Control Program: & test-dad2 & Bandwidth: & 4 \\
Quantif. Method: & $\mathbf{2 0 1 7 0 6 0 8}$ & Dilution Factor: & $\mathbf{1 . 0 0 0 0}$ \\
Recording Time: & $\mathbf{2 0 2 0 - 1 2 - 2 8 ~ 1 2 : 5 8}$ & Sample Weight: & $\mathbf{1 . 0 0 0 0}$ \\
Run Time (min): & $\mathbf{1 3 3 . 6 1}$ & Sample Amount: & $\mathbf{1 . 0 0 0 0}$ \\
\hline
\end{tabular}

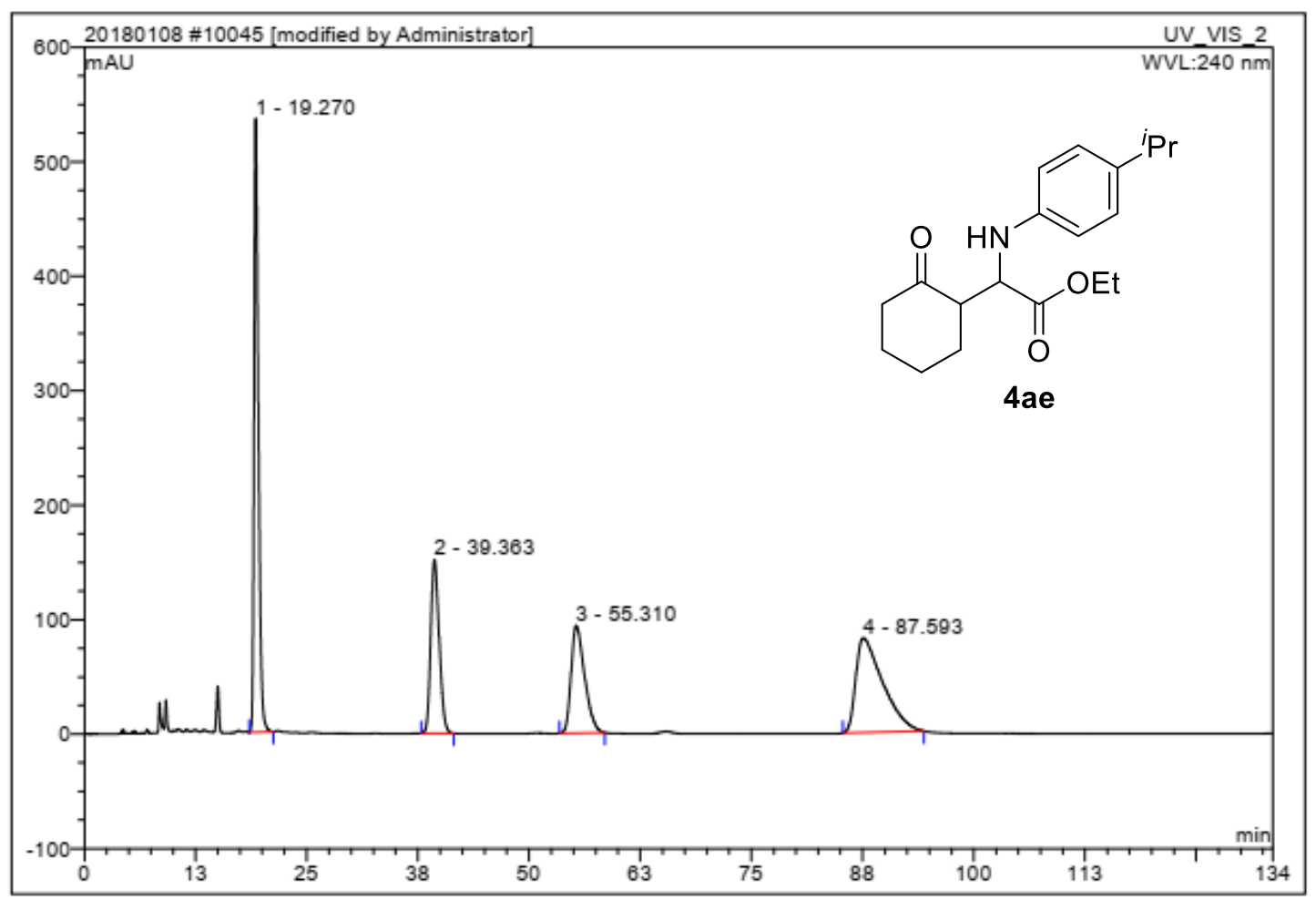

\begin{tabular}{|r|ccrrrrr|}
\hline No. & $\begin{array}{c}\text { Ret.Time } \\
\text { min }\end{array}$ & Peak Name & $\begin{array}{c}\text { Height } \\
\text { mAU }\end{array}$ & $\begin{array}{c}\text { Area } \\
\text { mAU*min }\end{array}$ & $\begin{array}{c}\text { Rel.Area } \\
\%\end{array}$ & Amount & Type \\
\hline 1 & 19.27 & n.a. & 536.219 & 303.688 & 32.69 & n.a. & BMB \\
2 & 39.36 & n.a. & 152.132 & 168.057 & 18.09 & n.a. & BMB \\
3 & 55.31 & n.a. & 94.304 & 165.750 & 17.84 & n.a. & BMB \\
4 & 87.59 & n.a. & 82.857 & 291.611 & 31.39 & n.a. & BMB \\
\hline Total: & & & 865.513 & 929.106 & 100.00 & 0.000 & \\
\hline
\end{tabular}




\begin{tabular}{|lllll|}
\hline \#\#\# WZH-1215-1 PA2 912140.7 & & \\
& & & \\
\hline Sample Name: & WZH-1215-1 PA2 912140.7 & Injection Volume: & 1.0 \\
Vial Number: & RD6 & Channel: & UV_VIS_2 \\
Sample Type: & unknown & Wavelength: & $\mathbf{2 1 4 . 0}$ \\
Control Program: & test-dad2 & Bandwidth: & 4 \\
Quantif. Method: & 20170608 & Dilution Factor: & 1.0000 \\
Recording Time: & $2021-1-610: 25$ & Sample Weight: & 1.0000 \\
Run Time (min): & 126.40 & Sample Amount: & 1.0000 \\
\hline
\end{tabular}

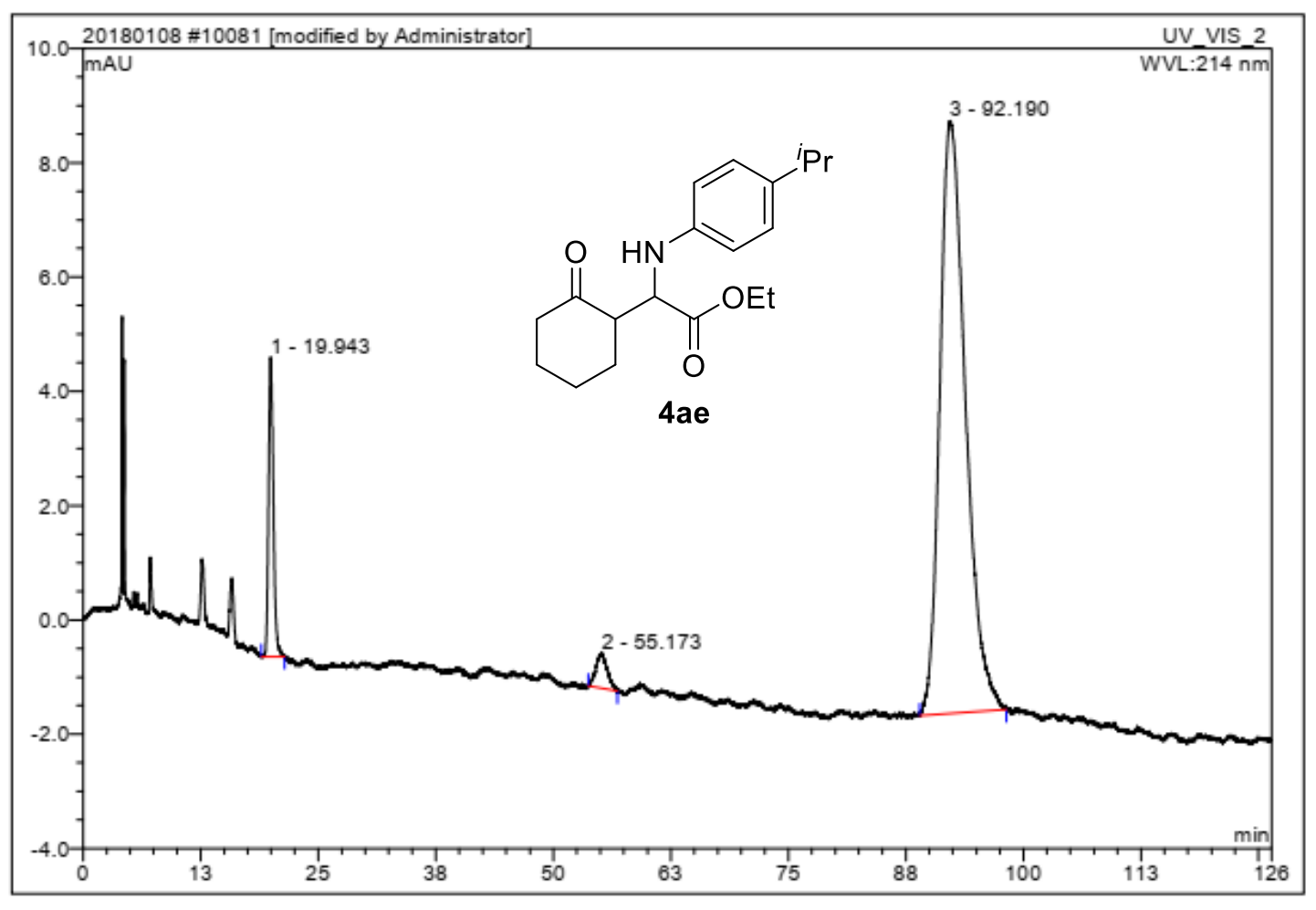

\begin{tabular}{|c|c|c|c|c|c|c|c|}
\hline No. & $\begin{array}{c}\text { Ret.Time } \\
\text { min }\end{array}$ & Peak Name & $\begin{array}{c}\text { Height } \\
\text { mAU }\end{array}$ & $\begin{array}{c}\text { Area } \\
\mathrm{mAU}^{*} \min \end{array}$ & $\begin{array}{c}\text { Rel.Area } \\
\%\end{array}$ & Amount & Type \\
\hline 1 & 19.94 & n.a. & 5.231 & 3.128 & 8.49 & n.a. & $\mathrm{BMB}^{*}$ \\
\hline 2 & 55.17 & n.a. & 0.621 & 0.858 & 2.33 & n.a. & $\mathrm{BMB}^{*}$ \\
\hline 3 & 92.19 & n.a. & 10.378 & 32.864 & 89.18 & n.a. & $\mathrm{BMB}^{*}$ \\
\hline Total: & & & 16.230 & 36.850 & 100.00 & 0.000 & \\
\hline
\end{tabular}


\#\#\# WZH-1211-4-1+2 PA2 912140.7

\begin{tabular}{llll|}
\hline Sample Name: & WZH-1211-4-1+2 PA2 91 214 0.7 & Injection Volume: & $\mathbf{1 . 0}$ \\
Vial Number: & RB6 & Channel: & UV_VIS_2 \\
Sample Type: & unknown & Wavelength: & $\mathbf{2 1 4 . 0}$ \\
Control Program: & test-dad2 & Bandwidth: & 4 \\
Quantif. Method: & $\mathbf{2 0 1 7 0 6 0 8}$ & Dilution Factor: & 1.0000 \\
Recording Time: & $\mathbf{2 0 2 0 - 1 2 - 3 0 1 8 : 2 3}$ & Sample Weight: & 1.0000 \\
Run Time (min): & $\mathbf{1 1 5 . 0 0}$ & Sample Amount: & $\mathbf{1 . 0 0 0 0}$ \\
\hline
\end{tabular}

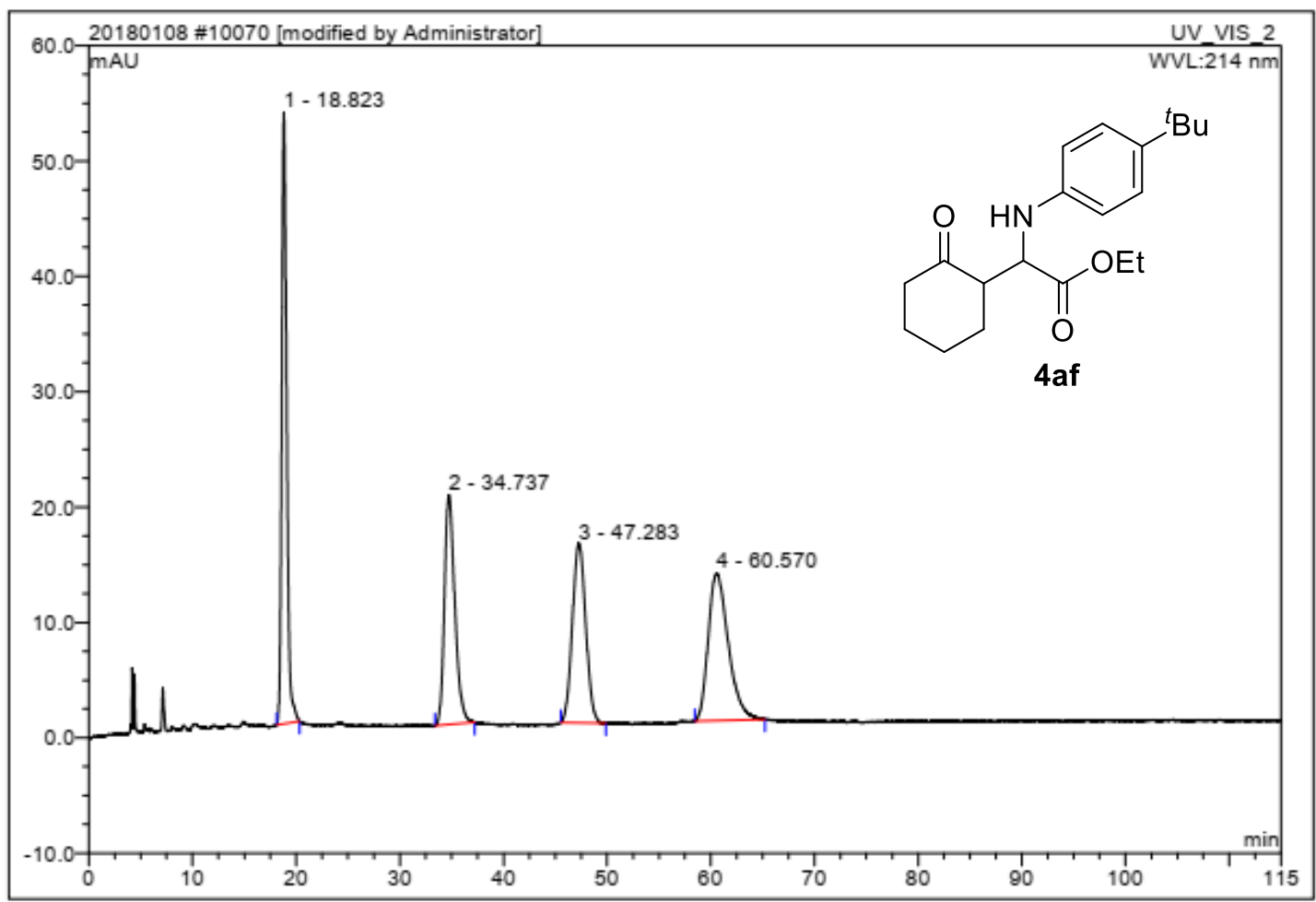

\begin{tabular}{|r|ccrrrrr|}
\hline No. & $\begin{array}{c}\text { Ret.Time } \\
\text { min }\end{array}$ & Peak Name & $\begin{array}{c}\text { Height } \\
\text { mAU }\end{array}$ & $\begin{array}{c}\text { Area } \\
\text { mAU*min }\end{array}$ & $\begin{array}{c}\text { Rel.Area } \\
\%\end{array}$ & Amount & Type \\
\hline 1 & 18.82 & n.a. & 53.008 & 29.551 & 28.24 & n.a. & BMB $^{*}$ \\
2 & 34.74 & n.a. & 19.917 & 23.144 & 22.11 & n.a. & BMB $^{\star}$ \\
3 & 47.28 & n.a. & 15.557 & 23.255 & 22.22 & n.a. & BMB $^{\star}$ \\
4 & 60.57 & n.a. & 12.805 & 28.710 & 27.43 & n.a. & BMB $^{\star}$ \\
\hline Total: & & & 101.287 & 104.661 & 100.00 & 0.000 & \\
\hline
\end{tabular}


\#\#\# WZH-1215-2 PA2 912140.7

\begin{tabular}{llll}
\hline Sample Name: & WZH-1215-2 PA2 912140.7 & Injection Volume: & 1.0 \\
Vial Number: & RC6 & Channel: & UV_VIS_2 \\
Sample Type: & unknown & Wavelength: & $\mathbf{2 1 4 . 0}$ \\
Control Program: & test-dad2 & Bandwidth: & 4 \\
Quantif. Method: & $\mathbf{2 0 1 7 0 6 0 8}$ & Dilution Factor: & 1.0000 \\
Recording Time: & $\mathbf{2 0 2 0 - 1 2 - 3 0 2 0 : 1 9}$ & Sample Weight: & 1.0000 \\
Run Time (min): & $\mathbf{1 1 5 . 0 0}$ & Sample Amount: & $\mathbf{1 . 0 0 0 0}$ \\
\hline
\end{tabular}

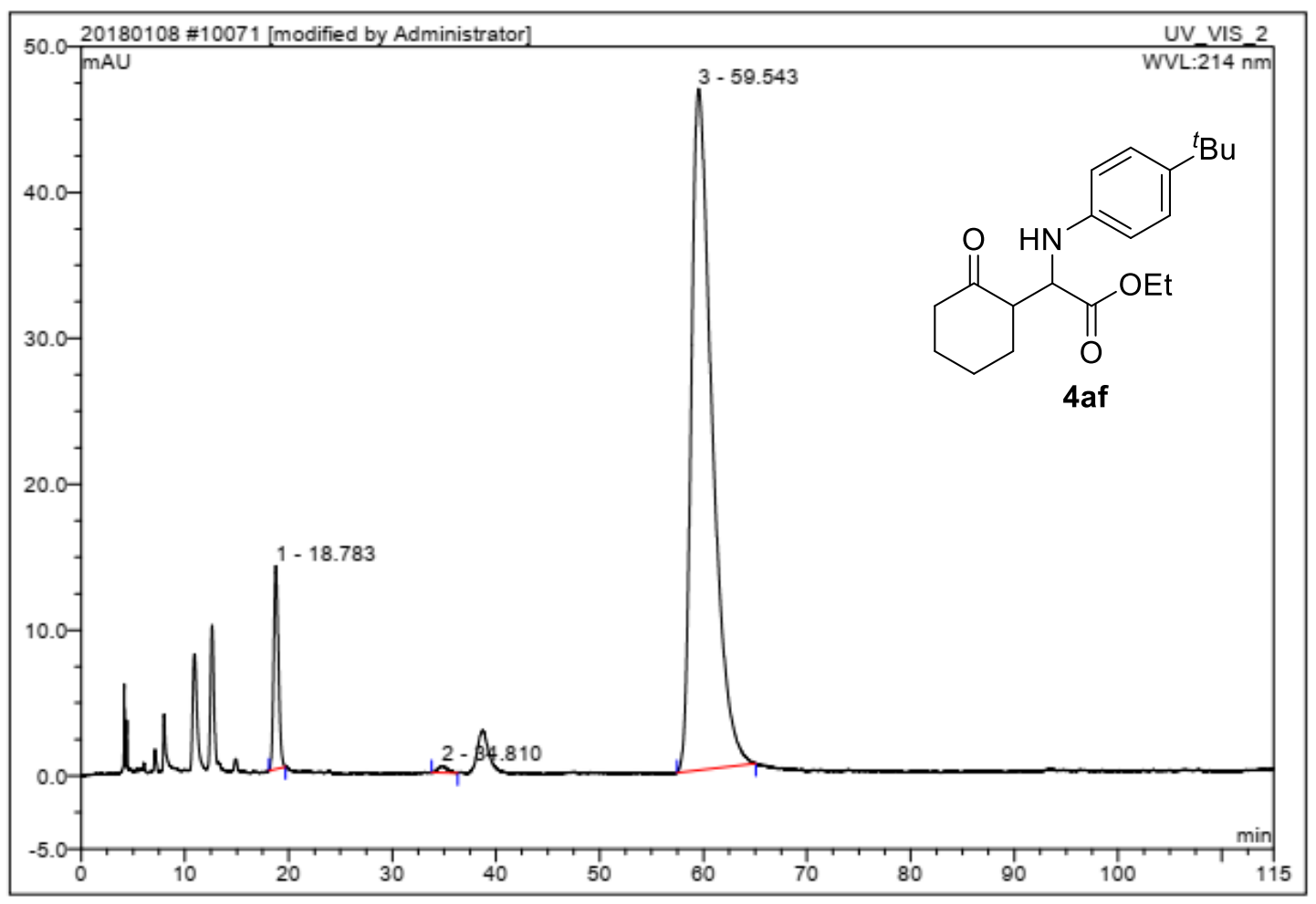

\begin{tabular}{|r|ccrrrrr|}
\hline No. & $\begin{array}{c}\text { Ret.Time } \\
\text { min }\end{array}$ & Peak Name & $\begin{array}{c}\text { Height } \\
\text { mAU }\end{array}$ & $\begin{array}{c}\text { Area } \\
\text { mAU*min }\end{array}$ & $\begin{array}{r}\text { Rel.Area } \\
\%\end{array}$ & Amount & Type \\
\hline 1 & 18.78 & n.a. & 13.944 & 7.484 & 6.42 & n.a. & BMB \\
2 & 34.81 & n.a. & 0.467 & 0.486 & 0.42 & n.a. & BMB $^{\star}$ \\
3 & 59.54 & n.a. & 46.730 & 108.672 & 93.17 & n.a. & BMB $^{*}$ \\
\hline Total: & & & 61.142 & 116.642 & 100.00 & 0.000 & \\
\hline
\end{tabular}




\section{WZH-1126-4 ASH 822140.7}

\begin{tabular}{|llll|}
\hline Sample Name: & WZH-1126-4 ASH 82 214 0.7 & Injection Volume: & $\mathbf{1 . 0}$ \\
Vial Number: & RB7 & Channel: & UV_VIS_2 \\
Sample Type: & unknown & Wavelength: & $\mathbf{2 1 4 . 0}$ \\
Control Program: & test-dad3 & Bandwidth: & 4 \\
Quantif. Method: & $\mathbf{2 0 1 7 0 6 0 8}$ & Dilution Factor: & 1.0000 \\
Recording Time: & $\mathbf{2 0 2 0 - 1 2 - 9 1 4 : 4 2}$ & Sample Weight: & 1.0000 \\
Run Time (min): & 37.98 & Sample Amount: & $\mathbf{1 . 0 0 0 0}$ \\
\hline
\end{tabular}

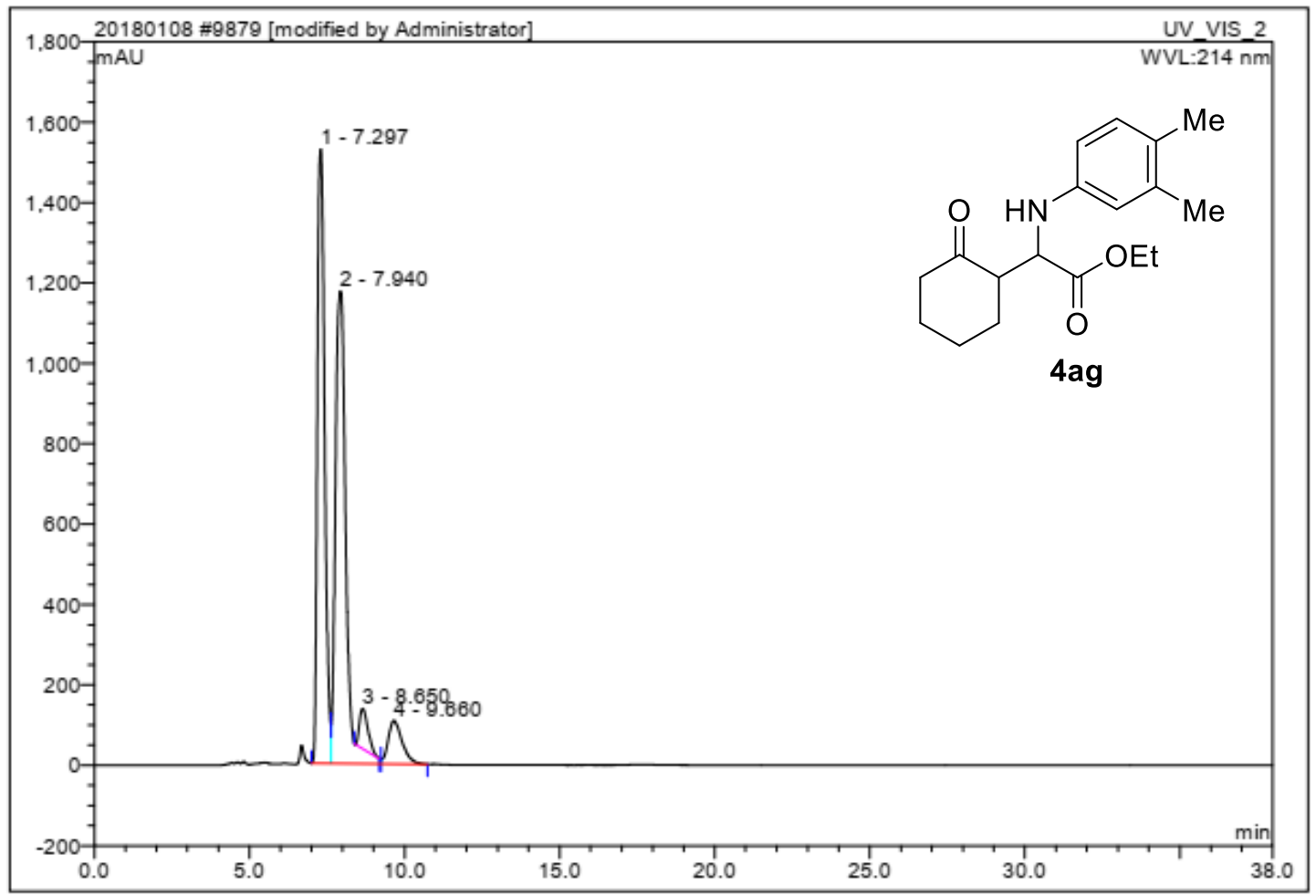

\begin{tabular}{|r|ccrrrrr|}
\hline No. & $\begin{array}{c}\text { Ret.Time } \\
\text { min }\end{array}$ & Peak Name & $\begin{array}{c}\text { Height } \\
\text { mAU }\end{array}$ & $\begin{array}{c}\text { Area } \\
\text { mAU*min }\end{array}$ & $\begin{array}{r}\text { Rel.Area } \\
\%\end{array}$ & Amount & Type \\
\hline 1 & 7.30 & n.a. & 1527.794 & 420.554 & 43.41 & n.a. & BM $^{*}$ \\
2 & 7.94 & n.a. & 1175.340 & 459.399 & 47.42 & n.a. & M $^{*}$ \\
3 & 8.65 & n.a. & 99.979 & 32.453 & 3.35 & n.a. & Rd $^{*}$ \\
4 & 9.66 & n.a. & 107.669 & 56.283 & 5.81 & n.a. & MB $^{*}$ \\
\hline Total: & & & 2910.781 & 968.690 & 100.00 & 0.000 & \\
\hline
\end{tabular}




\begin{tabular}{|lllll|}
\hline \#\#\# WZH-1129-33 ASH 822140.7 & & \\
& & & \\
\hline Sample Name: & WZH-1129-33 ASH 822140.7 & Injection Volume: & 2.0 \\
Vial Number: & RE6 & Channel: & UV_VIS_2 \\
Sample Type: & unknown & Wavelength: & $\mathbf{2 4 0 . 0}$ \\
Control Program: & test-dad2 & Bandwidth: & 4 \\
Quantif. Method: & 20170608 & Dilution Factor: & $\mathbf{1 . 0 0 0 0}$ \\
Recording Time: & $2020-12-24$ 9:30 & Sample Weight: & $\mathbf{1 . 0 0 0 0}$ \\
Run Time (min): & 22.73 & Sample Amount: & $\mathbf{1 . 0 0 0 0}$ \\
\hline
\end{tabular}

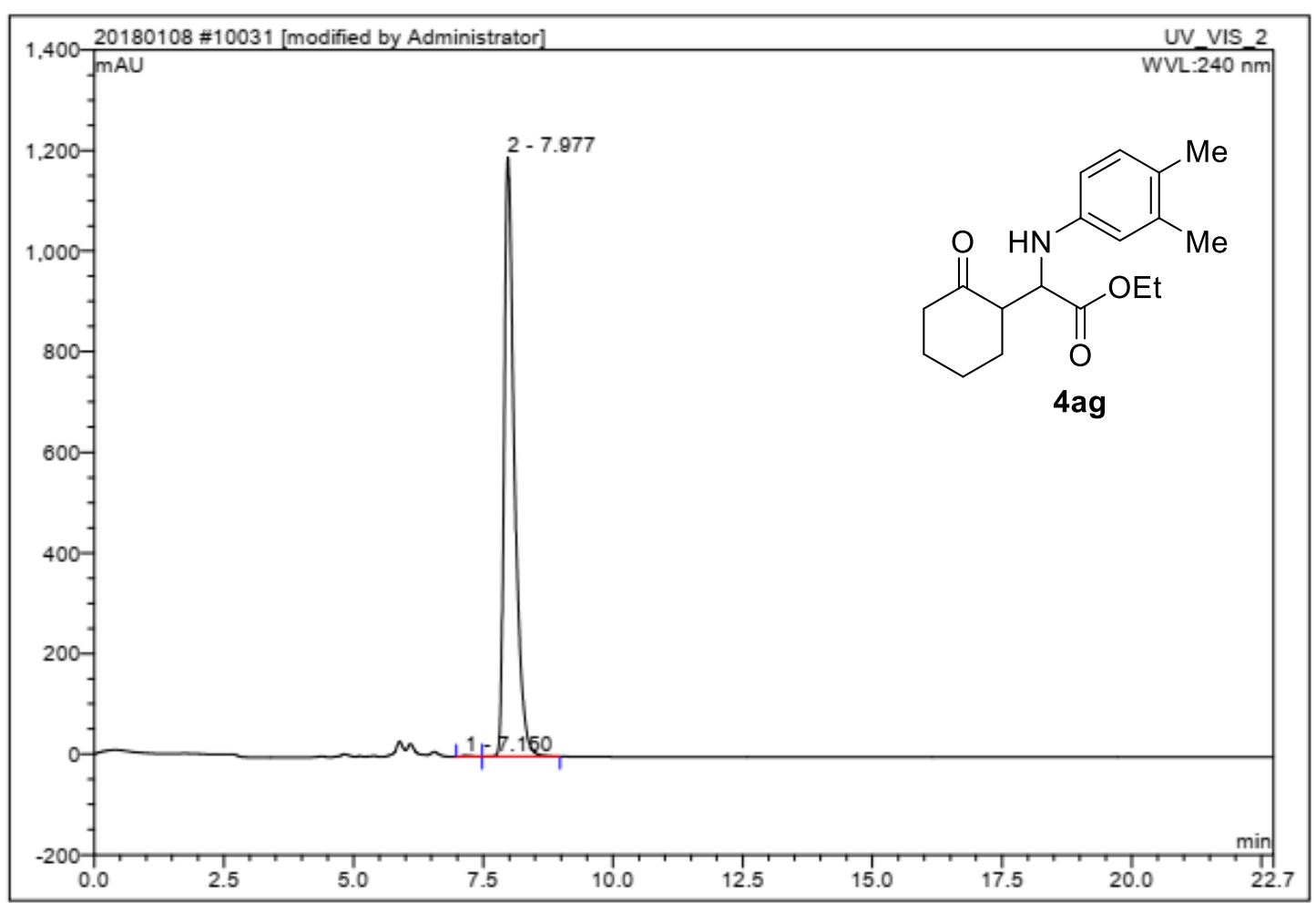

\begin{tabular}{|r|ccrrrrr|}
\hline No. & $\begin{array}{c}\text { Ret.Time } \\
\text { min }\end{array}$ & Peak Name & $\begin{array}{c}\text { Height } \\
\text { mAU }\end{array}$ & $\begin{array}{c}\text { Area } \\
\text { mAU*min }\end{array}$ & $\begin{array}{r}\text { Rel.Area } \\
\%\end{array}$ & Amount & Type \\
\hline 1 & 7.15 & n.a. & 2.414 & 0.512 & 0.19 & n.a. & BM $^{*}$ \\
2 & 7.98 & n.a. & 1190.688 & 274.840 & 99.81 & n.a. & MB $^{*}$ \\
\hline Total: & & & 1193.103 & 275.353 & 100.00 & 0.000 & \\
\hline
\end{tabular}


\#\#\# WZH-1126-3+- IE3 822400.5

\begin{tabular}{llll}
\hline Sample Name: & WZH-1126-3+- IE3 82240 0.5 & Injection Volume: & $\mathbf{2 . 0}$ \\
Vial Number: & GD3 & Channel: & UV_VIS_1 \\
Sample Type: & unknown & Wavelength: & $\mathbf{2 4 0}$ \\
Control Program: & $\mathbf{2 0 1 7 0 1 - 3}$ & Bandwidth: & n.a. \\
Quantif. Method: & $\mathbf{2 0 1 7 0 1}$ & Dilution Factor: & $\mathbf{1 . 0 0 0 0}$ \\
Recording Time: & $\mathbf{2 0 2 0 / 1 2 / 9} 14: 55$ & Sample Weight: & $\mathbf{1 . 0 0 0 0}$ \\
Run Time (min): & $\mathbf{5 4 . 8 7}$ & Sample Amount: & $\mathbf{1 . 0 0 0 0}$ \\
\hline
\end{tabular}

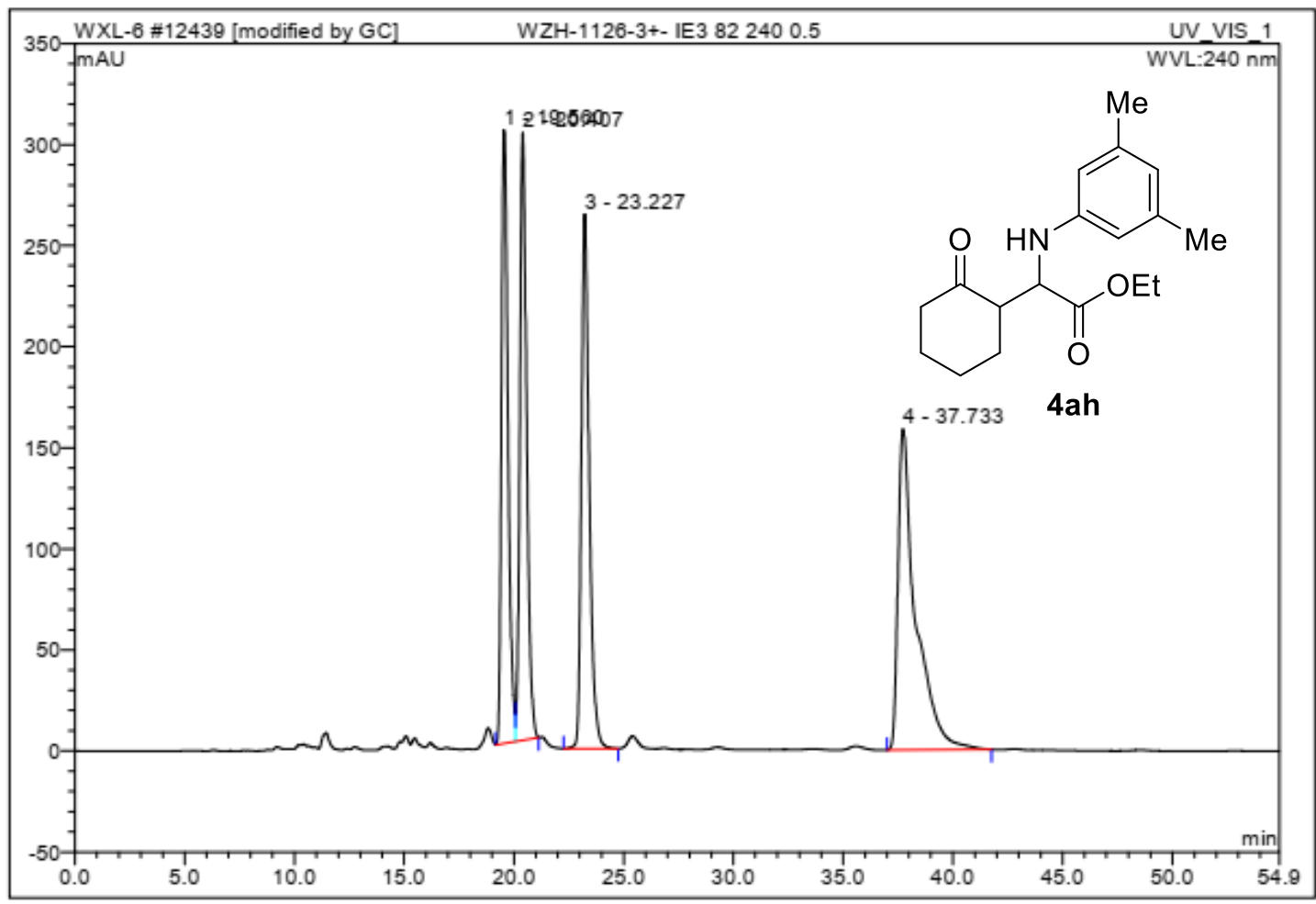

\begin{tabular}{|r|ccccccc|}
\hline No. & $\begin{array}{c}\text { Ret.Time } \\
\text { min }\end{array}$ & Peak Name & $\begin{array}{c}\text { Height } \\
\text { mAU }\end{array}$ & $\begin{array}{c}\text { Area } \\
\text { mAU*min }\end{array}$ & $\begin{array}{c}\text { Rel.Area } \\
\%\end{array}$ & Amount & Type \\
\hline 1 & 19.56 & n.a. & 303.462 & 104.347 & 22.15 & n.a. & BM $^{*}$ \\
2 & 20.41 & n.a. & 300.817 & 108.208 & 22.97 & n.a. & MB $^{*}$ \\
3 & 23.23 & n.a. & 264.497 & 110.359 & 23.42 & n.a. & BMB $^{*}$ \\
4 & 37.73 & n.a. & 158.897 & 148.236 & 31.46 & n.a. & BMB \\
\hline Total: & & & 1027.673 & 471.149 & 100.00 & 0.000 & \\
\hline
\end{tabular}




\begin{tabular}{|c|c|c|c|}
\hline \multicolumn{4}{|c|}{ \#\#\# WZH-1129-22 IE3 822400.5} \\
\hline Sample Name: & WZH-1129-22 IE3 822400.5 & Injection Volume: & 2.0 \\
\hline Vial Number: & BE5 & Channel: & UV_VIS_1 \\
\hline Sample Type: & unknown & Wavelength: & $240^{-}$ \\
\hline Control Program: & 201701-3 & Bandwidth: & n.a. \\
\hline Quantif. Method: & 201701 & Dilution Factor: & 1.0000 \\
\hline Recording Time: & 2020/12/22 11:23 & Sample Weight: & 1.0000 \\
\hline Run Time (min): & 60.01 & Sample Amount: & 1.0000 \\
\hline
\end{tabular}

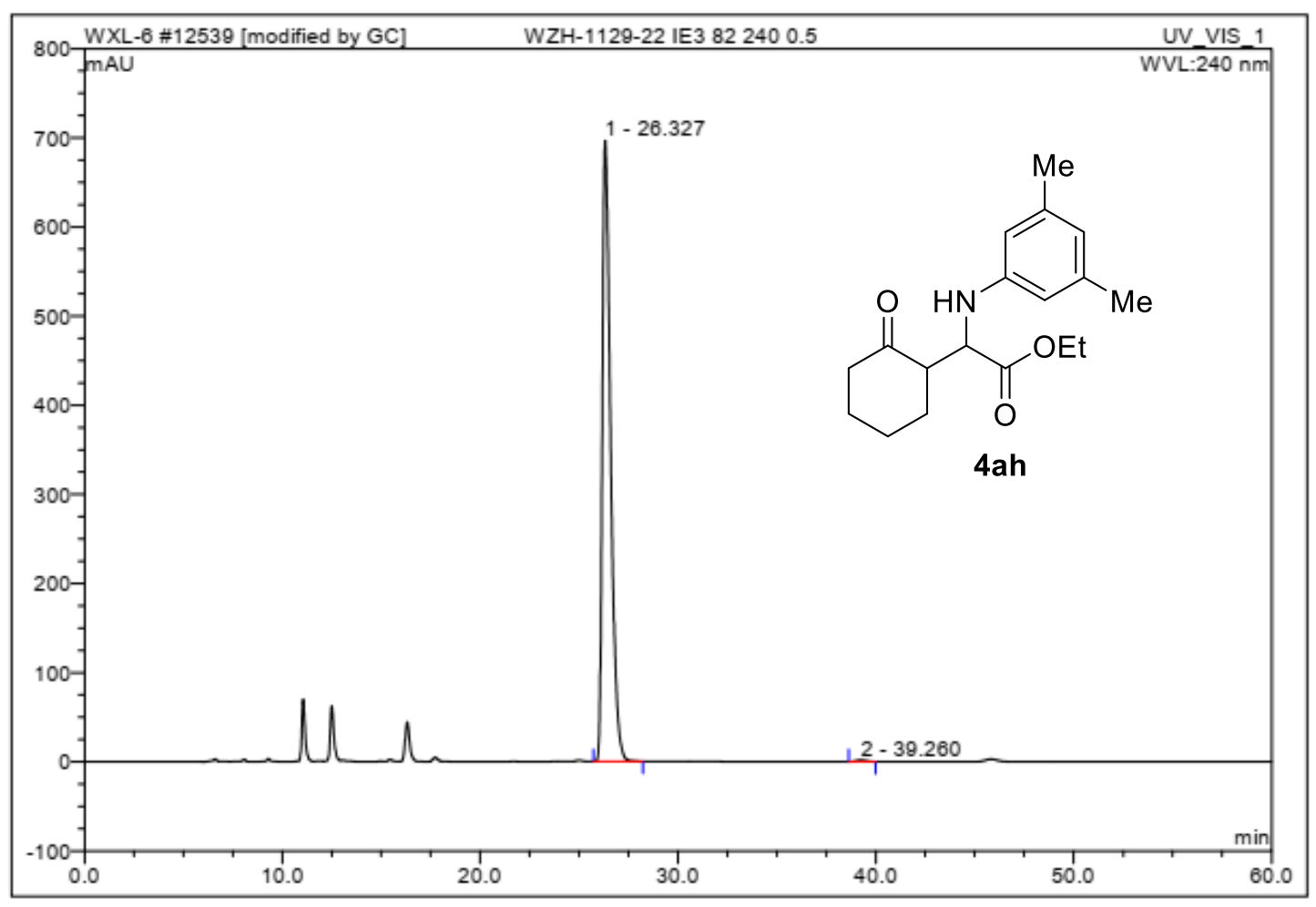

\begin{tabular}{|c|c|c|c|c|c|c|c|}
\hline No. & $\begin{array}{c}\text { Ret.Time } \\
\text { min }\end{array}$ & Peak Name & $\begin{array}{c}\text { Height } \\
\text { mAU }\end{array}$ & $\begin{array}{c}\text { Area } \\
\mathrm{mAU}^{*} \min \end{array}$ & $\begin{array}{c}\text { Rel.Area } \\
\%\end{array}$ & Amount & Type \\
\hline 1 & 26.33 & n.a. & 696.718 & 338.323 & 99.69 & n.a. & BMB \\
\hline 2 & 39.26 & n.a. & 1.778 & 1.068 & 0.31 & n.a. & BMB \\
\hline Total: & & & 698.497 & 339.391 & 100.00 & 0.000 & \\
\hline
\end{tabular}




\section{\#\#\# WZH-1115-1-1+1-2 IC 9552140.7}

\begin{tabular}{llll}
\hline Sample Name: & WZH-1115-1-1+1-2 IC 9552140.7 & Injection Volume: & $\mathbf{1 . 0}$ \\
Vial Number: & GC1 & Channel: & UV_VIS_1 \\
Sample Type: & unknown & Wavelength: & $\mathbf{2 5 4}$ \\
Control Program: & $\mathbf{2 0 1 7 0 1 - 5}$ & Bandwidth: & n.a. \\
Quantif. Method: & $\mathbf{2 0 1 7 0 1}$ & Dilution Factor: & $\mathbf{1 . 0 0 0 0}$ \\
Recording Time: & $\mathbf{2 0 2 0 / 1 1 / 2 3 ~ 1 1 : 5 9}$ & Sample Weight: & $\mathbf{1 . 0 0 0 0}$ \\
Run Time (min): & $\mathbf{9 0 . 8 8}$ & Sample Amount: & $\mathbf{1 . 0 0 0 0}$ \\
\hline
\end{tabular}

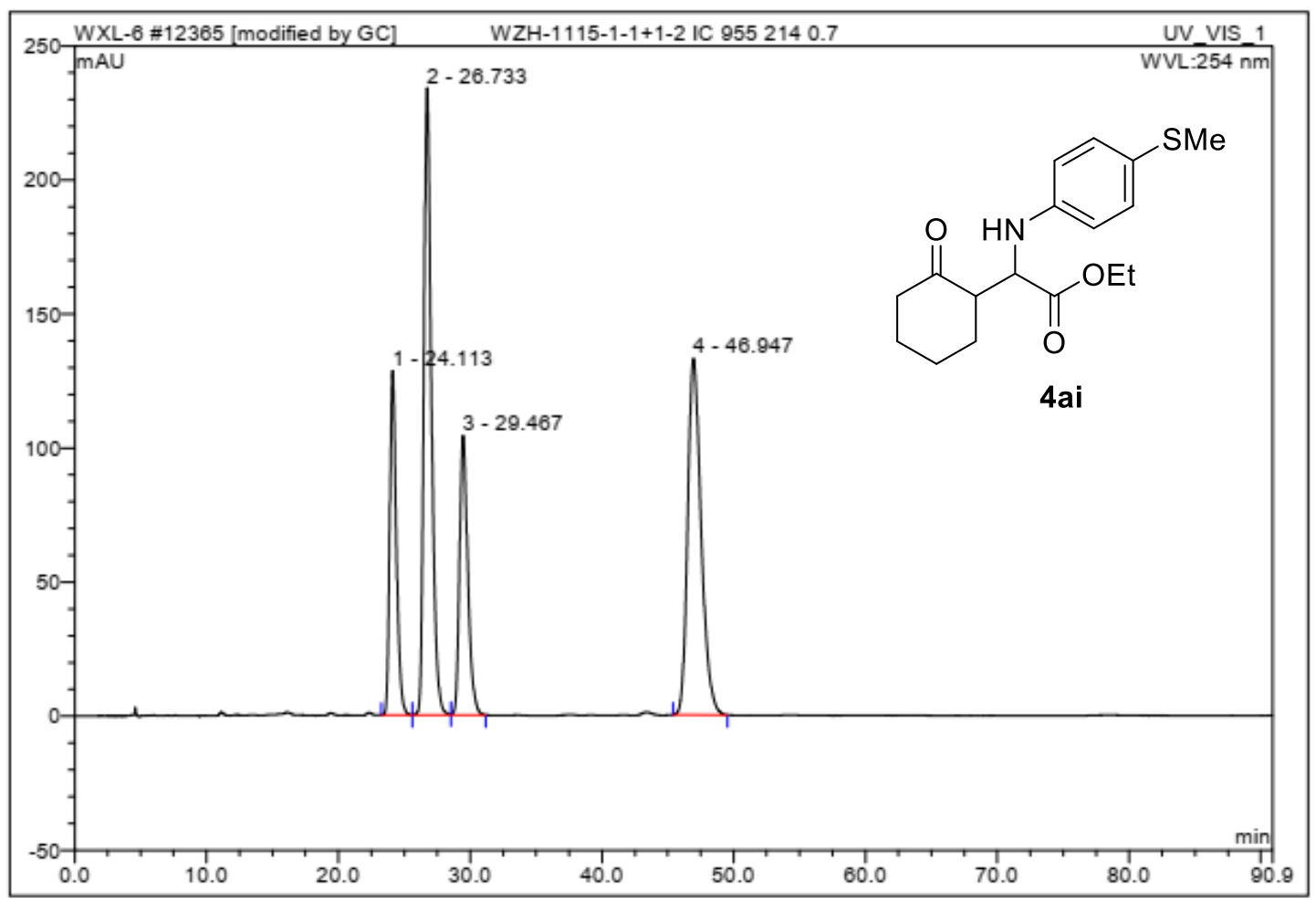

\begin{tabular}{|r|cccrccc|}
\hline No. & $\begin{array}{c}\text { Ret.Time } \\
\text { min }\end{array}$ & Peak Name & $\begin{array}{c}\text { Height } \\
\text { mAU }\end{array}$ & $\begin{array}{c}\text { Area } \\
\text { mAU*min }\end{array}$ & $\begin{array}{c}\text { Rel.Area } \\
\%\end{array}$ & Amount & Type \\
\hline 1 & 24.11 & n.a. & 128.423 & 74.881 & 16.12 & n.a. & BM \\
2 & 26.73 & n.a. & 233.971 & 156.609 & 33.70 & n.a. & M \\
3 & 29.47 & n.a. & 104.257 & 75.904 & 16.34 & n.a. & MB \\
4 & 46.95 & n.a. & 132.996 & 157.254 & 33.84 & n.a. & BMB \\
\hline Total: & & & 599.646 & 464.648 & 100.00 & 0.000 & \\
\hline
\end{tabular}




\begin{tabular}{|llll|}
\hline \multicolumn{1}{|l|}{ \#\#\# WZH-0108-3 IE3 822140.7} & & \\
\hline Sample Name: & WZH-0108-3 IE3 822140.7 & Injection Volume: & 3.0 \\
Vial Number: & RA5 & Channel: & UV_VIS_1 \\
Sample Type: & unknown & Wavelength: & $\mathbf{2 1 4}$ \\
Control Program: & $201701-2$ & Bandwidth: & n.a. \\
Quantif. Method: & 201701 & Dilution Factor: & 1.0000 \\
Recording Time: & $2021 / 1 / 27$ 18:01 & Sample Weight: & 1.0000 \\
Run Time (min): & 56.01 & Sample Amount: & 1.0000 \\
\hline
\end{tabular}

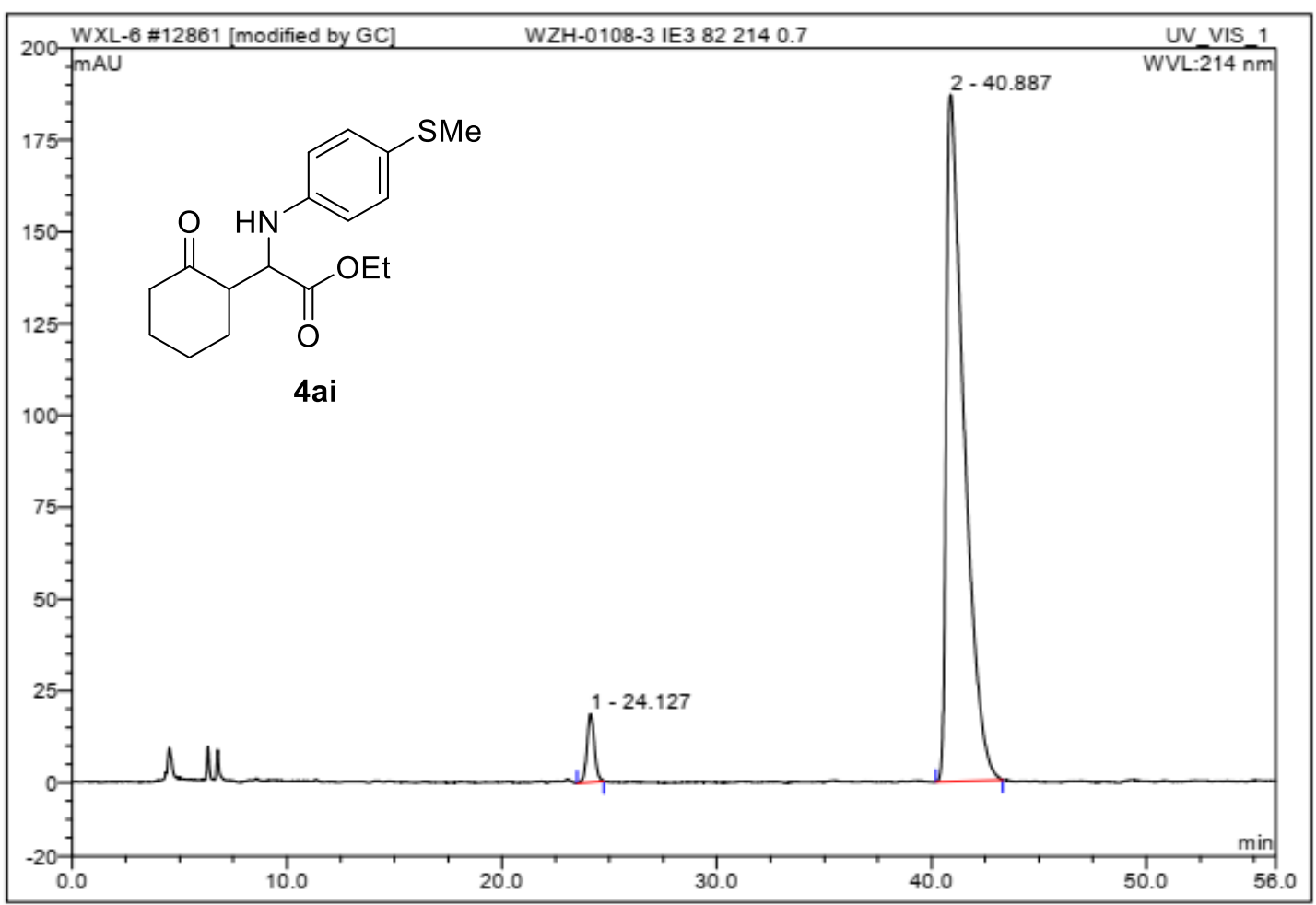

\begin{tabular}{|r|ccrrrrr|}
\hline No. & $\begin{array}{c}\text { Ret.Time } \\
\text { min }\end{array}$ & Peak Name & $\begin{array}{c}\text { Height } \\
\text { mAU }\end{array}$ & $\begin{array}{c}\text { Area } \\
\text { mAU } \text { min }^{*}\end{array}$ & $\begin{array}{c}\text { Rel.Area } \\
\%\end{array}$ & Amount & Type \\
\hline 1 & 24.13 & n.a. & 18.545 & 7.385 & 3.94 & n.a. & BMB \\
2 & 40.89 & n.a. & 187.101 & 179.921 & 96.06 & n.a. & BMB \\
\hline Total: & & & 205.646 & 187.305 & 100.00 & 0.000 & \\
\hline
\end{tabular}




\begin{tabular}{|llll|}
\hline \multicolumn{2}{c|}{ S A M P L E } & IN F O R M A T I O N \\
\hline \hline Sample Name: & $0512-2$ & Acquired By: & System \\
Sample Type: & Unknown & Sample Set Name & \\
Vial: & 2 & Acq. Method Set: & 2695PDA \\
Injection \#: & 1 & Processing Method & Default \\
Injection Volume: & $2.00 \mathrm{ul}$ & Channel Name: & 2998 Ch1 230nm@1.2nm \\
Run Time: & 130.0 Minutes & Proc. Chnl. Descr.: 2998 Ch1 230nm@1.2nm \\
& & & \\
Date Acquired: & $2021 / 5 / 25$ 10:51:16 CST & & \\
Date Processed: & $2021 / 5 / 2515: 50: 37$ CST & & \\
\hline
\end{tabular}

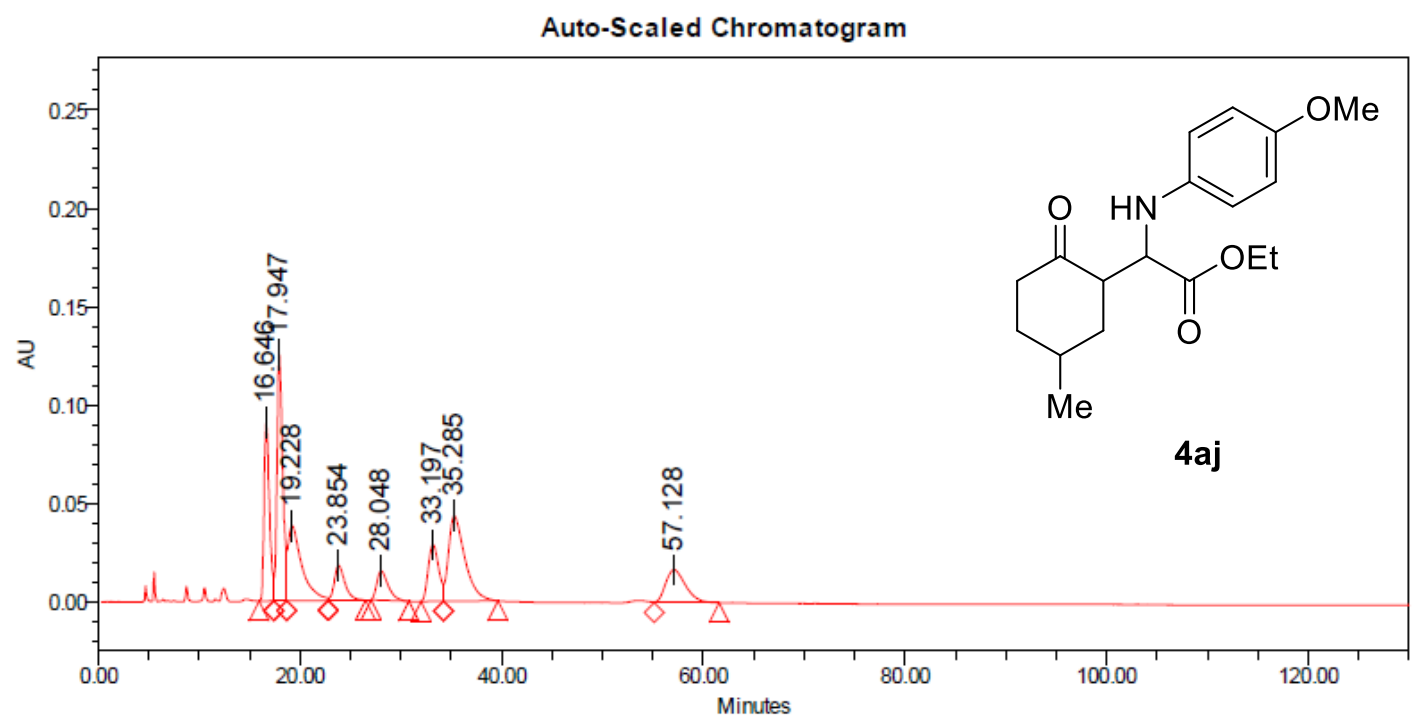

Peak Results
\begin{tabular}{|r|l|r|r|r|r|r|}
\hline & SampleName & RT & $\begin{array}{r}\text { Width } \\
\text { (sec) }\end{array}$ & Height & Area & $\%$ Area \\
\hline 1 & $0512-2$ & 16.646 & 89.200 & 90549 & 3333231 & 14.08 \\
\hline 2 & $0512-2$ & 17.947 & 76.400 & 125176 & 4967979 & 20.99 \\
\hline 3 & $0512-2$ & 19.228 & 246.600 & 38016 & 3629245 & 15.33 \\
\hline 4 & $0512-2$ & 23.854 & 222.300 & 17667 & 1289989 & 5.45 \\
\hline 5 & $0512-2$ & 28.048 & 223.400 & 14836 & 1167488 & 4.93 \\
\hline 6 & $0512-2$ & 33.197 & 133.400 & 28729 & 2080561 & 8.79 \\
\hline 7 & $0512-2$ & 35.285 & 323.200 & 43348 & 4899760 & 20.70 \\
\hline 8 & $0512-2$ & 57.128 & 387.200 & 16732 & 2302002 & 9.73 \\
\hline
\end{tabular}

Reported by User. System

Report Method: Default Individual Report: Report Method ID: 33974

Page: 1 of 1
Project Name: installation Date Printed: $2021 / 5 / 25$

15:54:11 PRC 


\begin{tabular}{|llll|}
\hline & \multicolumn{2}{c|}{ S A M P L E } & IN F O R M A T I O N \\
\hline \hline Sample Name: & $0512-1$ & Acquired By: & System \\
Sample Type: & Unknown & Sample Set Name & \\
Vial: & 3 & Acq. Method Set: & 2695PDA \\
Injection \#: & 2 & Processing Method & Default \\
Injection Volume: & $2.00 \mathrm{ul}$ & Channel Name: & 2998 Ch1 230nm@1.2nm \\
Run Time: & 130.0 Minutes & Proc. Chnl. Descr.: 2998 Ch1 230nm@1.2nm \\
& & \\
Date Acquired: & $2021 / 5 / 25$ 9:09:22 CST & & \\
Date Processed: & $2021 / 5 / 25$ 15:53:29 CST & & \\
\hline
\end{tabular}

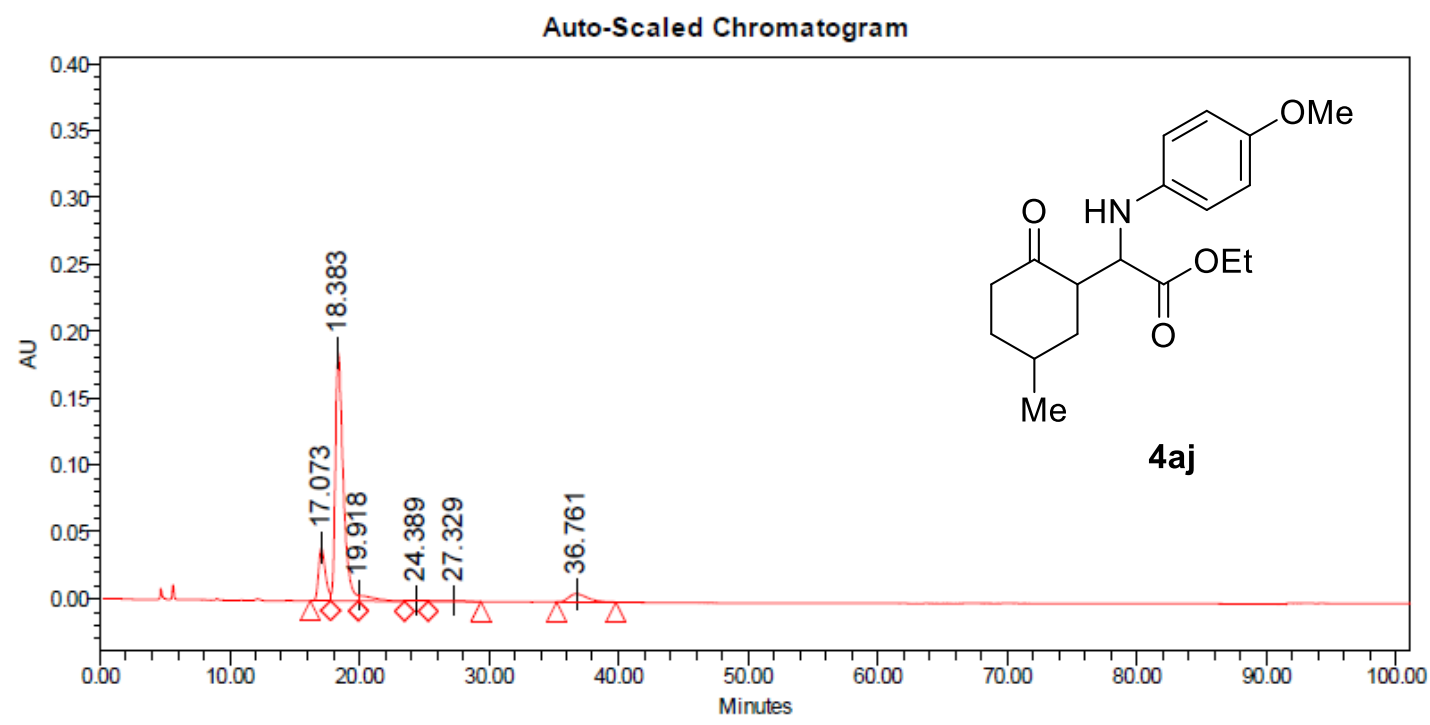

\begin{tabular}{|l|l|r|r|r|r|r|}
\hline \multicolumn{7}{|c|}{ Peak Results } \\
\hline & SampleName & RT & $\begin{array}{c}\text { Width } \\
(\mathrm{sec})\end{array}$ & Height & Area & $\%$ Area \\
\hline 1 & $0512-1$ & 17.073 & 93.800 & 39518 & 1457950 & 13.95 \\
\hline 2 & $0512-1$ & 18.383 & 127.500 & 185555 & 7875916 & 75.38 \\
\hline 3 & $0512-1$ & 19.918 & 214.500 & 4318 & 364291 & 3.49 \\
\hline 4 & $0512-1$ & 24.389 & 110.000 & 466 & 34887 & 0.33 \\
\hline 5 & $0512-1$ & 27.329 & 243.400 & 642 & 92855 & 0.89 \\
\hline 6 & $0512-1$ & 36.761 & 273.900 & 6262 & 622814 & 5.96 \\
\hline
\end{tabular}

Reported by User. System

Report Method: Default Individual Report:

Report Method ID: 33974

Page: 1 of 1
Project Name: installation

Date Printed:

2021/5/25

15:54:26 PRC 


\begin{tabular}{|llll|}
\hline & \multicolumn{2}{c|}{ S A M P L E } & IN F O R M A T I O N \\
\hline \hline Sample Name: & $0514-2$ & Acquired By: & System \\
Sample Type: & Unknown & Sample Set Name & \\
Vial: & 73 & Acq. Method Set: & 2695PDA2 \\
Injection \#: & 1 & Processing Method & Default \\
Injection Volume: $1.50 \mathrm{ul}$ & Channel Name: & 2998 Ch3 254nm@1.2nm \\
Run Time: & 50.0 Minutes & Proc. Chnl. Descr.: 2998 Ch3 254nm@1.2nm \\
& & & \\
Date Acquired: & $2021 / 6 / 18$ 8:31:23 CST & & \\
Date Processed: & $2021 / 6 / 18$ 14:35:52 CST & & \\
\hline
\end{tabular}

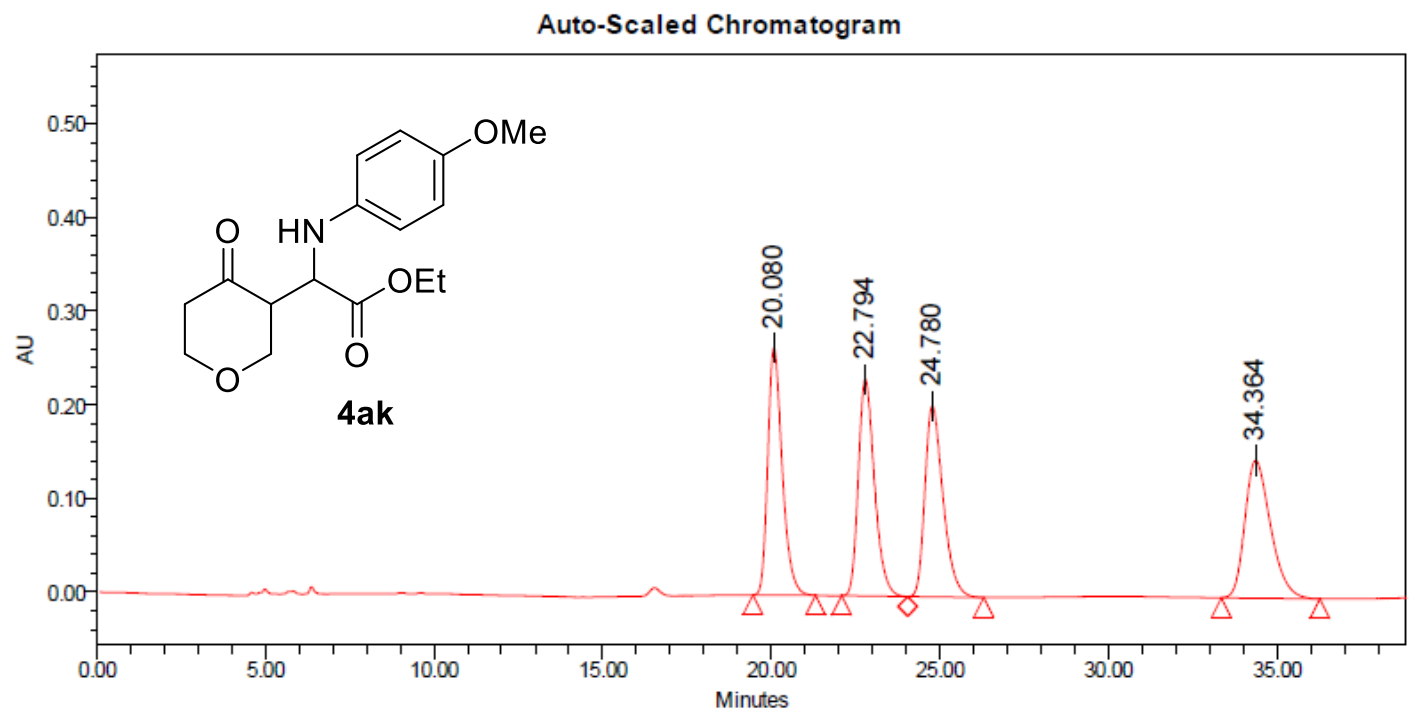

Peak Results

\begin{tabular}{|c|l|c|c|c|c|c|}
\hline & SampleName & RT & $\begin{array}{c}\text { Width } \\
(\mathrm{sec})\end{array}$ & Height & Area & \% Area \\
\hline 1 & $0514-2$ & 20.080 & 111.700 & 263817 & 7877333 & 25.38 \\
\hline 2 & $0514-2$ & 22.794 & 117.500 & 231488 & 7708192 & 24.84 \\
\hline 3 & $0514-2$ & 24.780 & 135.300 & 203970 & 7644229 & 24.63 \\
\hline 4 & $0514-2$ & 34.364 & 174.900 & 147024 & 7807244 & 25.15 \\
\hline
\end{tabular}

Reported by User. System

Report Method: Default Individual Report:

Report Method ID: 33974

Page: 1 of 1
Project Name: installation Date Printed:

2021/6/18

14:36:17 PRC 


\section{SAMPLE INFORMATION}

\begin{tabular}{|c|c|c|c|}
\hline Sample Name: & $0514-1$ & Acquired By: & System \\
\hline Sample Type: & Unknown & Sample Set Name & \\
\hline Vial: & 74 & Acq. Method Set: & 2695PDA2 \\
\hline Injection \#: & 1 & Processing Method & Default \\
\hline Injection Volume: & $3.00 \mathrm{ul}$ & Channel Name: & 2998 Ch3254nm@1.2nm \\
\hline Run Time: & 50.0 Minutes & Proc. Chnl. Descr.: & 2998 Ch3 254nm@1.2nm \\
\hline Date Acquired: & 2021/6/18 9:11:07 CST & & \\
\hline Date Processed: & 2021/6/18 14:35:39 CST & & \\
\hline
\end{tabular}

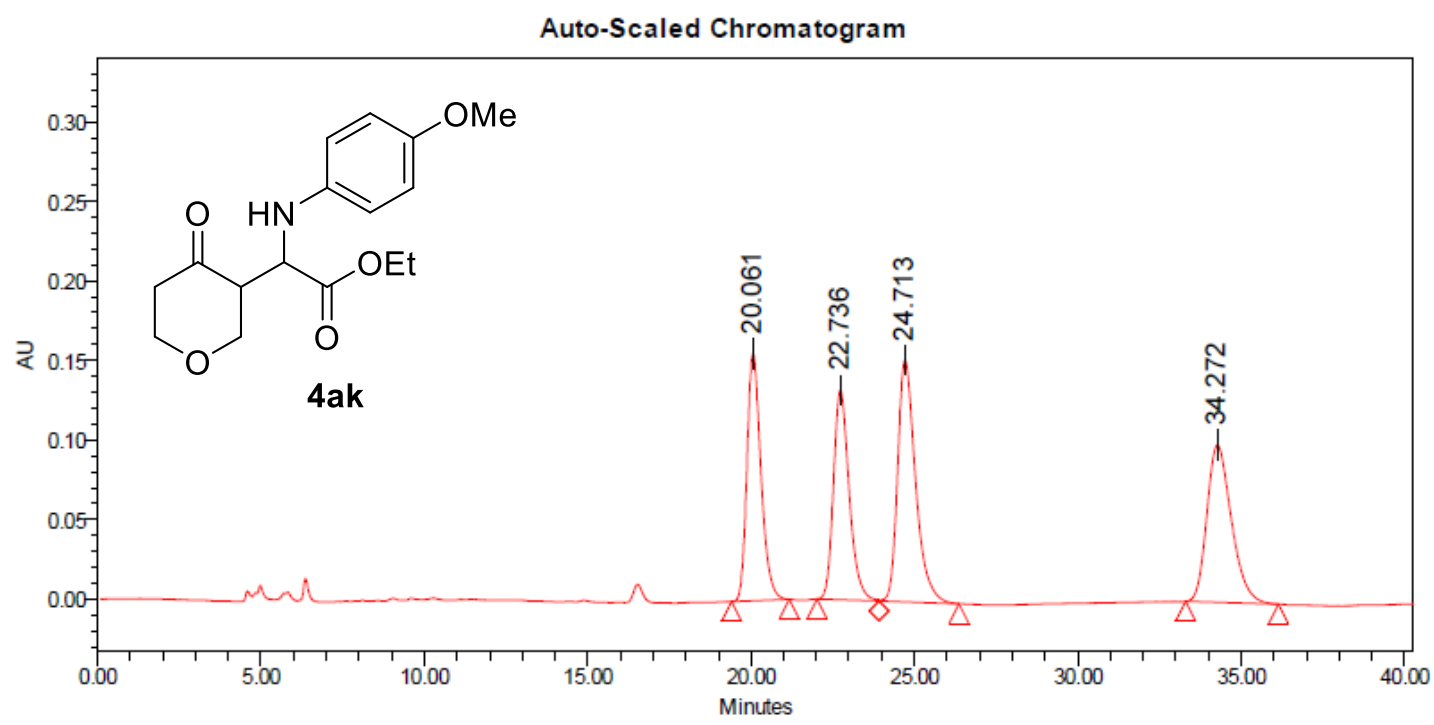

\section{Peak Results}

\begin{tabular}{|r|l|c|r|r|r|r|}
\hline & SampleName & RT & $\begin{array}{c}\text { Width } \\
(\mathrm{sec})\end{array}$ & Height & Area & \% Area \\
\hline 1 & $0514-1$ & 20.061 & 105.400 & 155315 & 4607633 & 23.05 \\
\hline 2 & $0514-1$ & 22.736 & 113.800 & 131731 & 4427893 & 22.15 \\
\hline 3 & $0514-1$ & 24.713 & 147.200 & 151986 & 5796662 & 28.99 \\
\hline 4 & $0514-1$ & 34.272 & 170.000 & 98803 & 5161451 & 25.82 \\
\hline
\end{tabular}

Reported by User. System

Report Method: Default Individual Report Report Method ID: 33974

Page: 1 of 1
Project Name: installation Date Printed:

2021/6/18

14:36:04 PRC 


\begin{tabular}{|c|c|c|c|}
\hline \multicolumn{2}{|r|}{ SAMPLE } & \multicolumn{2}{|c|}{ INFORMATION } \\
\hline Sample Name: & 0628-1-RAC & Acquired By: & System \\
\hline Sample Type: & Unknown & Sample Set Name & \\
\hline Vial: & 15 & Acq. Method Set: & 2695PDA2 \\
\hline Injection \#: & 1 & Processing Method & Default \\
\hline Injection Volume: & $1.00 \mathrm{ul}$ & Channel Name: & 2998 Ch1230nm@1.2nm \\
\hline Run Time: & 50.0 Minutes & Proc. Chnl. Descr.: & 2998 Ch1230nm@1.2nm \\
\hline Date Acquired: & $2021 / 7 / 28: 39: 50$ CST & & \\
\hline Date Processed: & 2021/7/2 13:07:26 CST & & \\
\hline
\end{tabular}

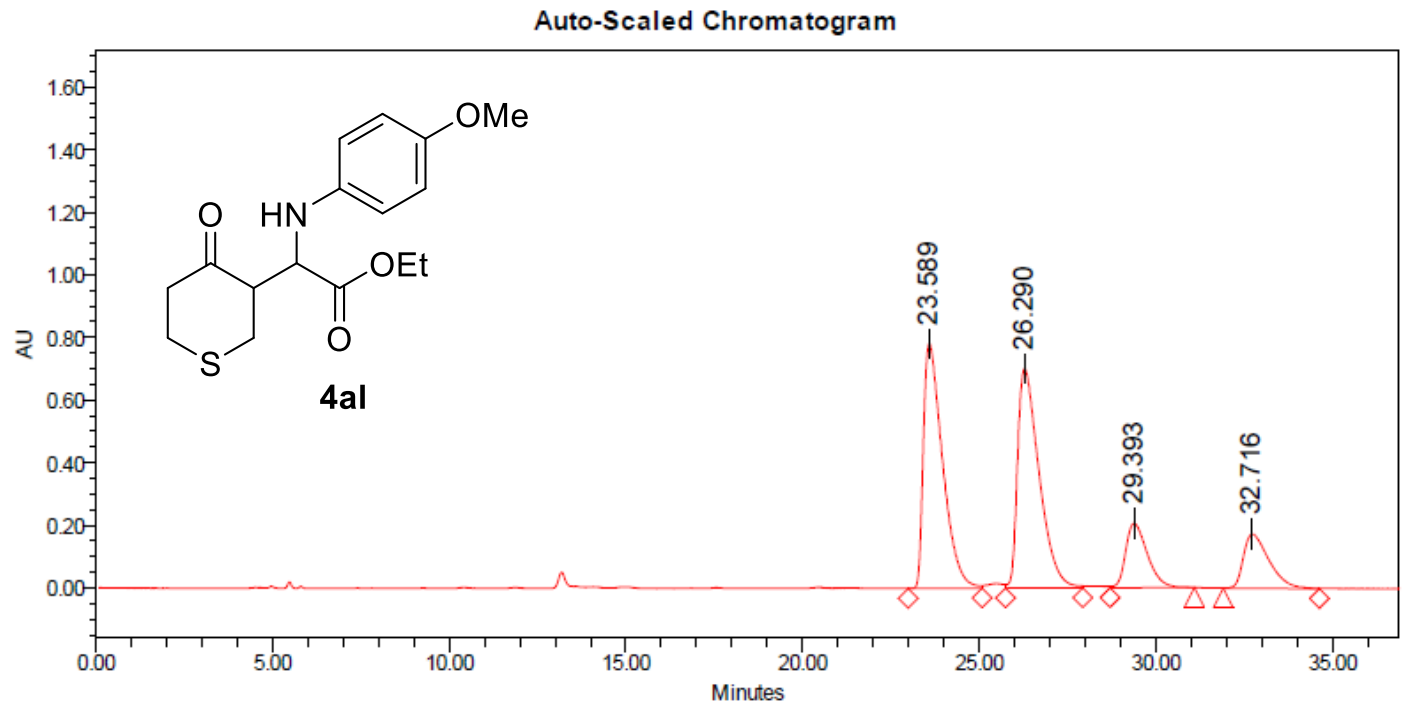

\section{Peak Results}

\begin{tabular}{|c|c|c|c|c|c|r|}
\hline & SampleName & RT & $\begin{array}{c}\text { Width } \\
(\mathrm{sec})\end{array}$ & Height & Area & $\%$ Area \\
\hline 1 & $0628-1-R A C$ & 23.589 & 125.800 & 782199 & 29526880 & 38.45 \\
\hline 2 & $0628-1-R A C$ & 26.290 & 130.700 & 701128 & 29762769 & 38.76 \\
\hline 3 & $0628-1-R A C$ & 29.393 & 143.300 & 206273 & 8801560 & 11.46 \\
\hline 4 & $0628-1-R A C$ & 32.716 & 163.300 & 172653 & 8700194 & 11.33 \\
\hline
\end{tabular}

Reported by User. System

Report Method: Default Individual Report:

Report Method ID: 33974

Page: 1 of 1
Project Name: installation Date Printed:

2021/7/2

14:10:05 PRC 


\section{SAMPLE INFORMATION}

\begin{tabular}{|c|c|c|c|}
\hline $\begin{array}{l}\text { Sample Name: } \\
\text { Sample Type: }\end{array}$ & $\begin{array}{l}\text { 0618-1 } \\
\text { Unknown }\end{array}$ & $\begin{array}{l}\text { Acquired By: } \\
\text { Sample Set Name }\end{array}$ & System \\
\hline $\begin{array}{l}\text { Sample Iype: } \\
\text { Vial: }\end{array}$ & 14 & Acmplet Name & \\
\hline $\begin{array}{l}\text { Vial: } \\
\text { Injection \#: }\end{array}$ & 1 & Acq. Method Set: & 2695PDA2 \\
\hline Injection \#: & & Processing Method & Default \\
\hline Injection Volume: & $1.00 \mathrm{ul}$ & Channel Name: & 2998 Ch1 230nm@1.2nm \\
\hline Run Time: & 50.0 Minutes & Proc. Chnl. Descr.: & 2998 Ch1230nm@1.2nm \\
\hline Date Acq & 2021/7/2 9:18:0 & & \\
\hline Date Processed: & 2021/7/2 13:08:00 CST & & \\
\hline
\end{tabular}

Auto-Scaled Chromatogram

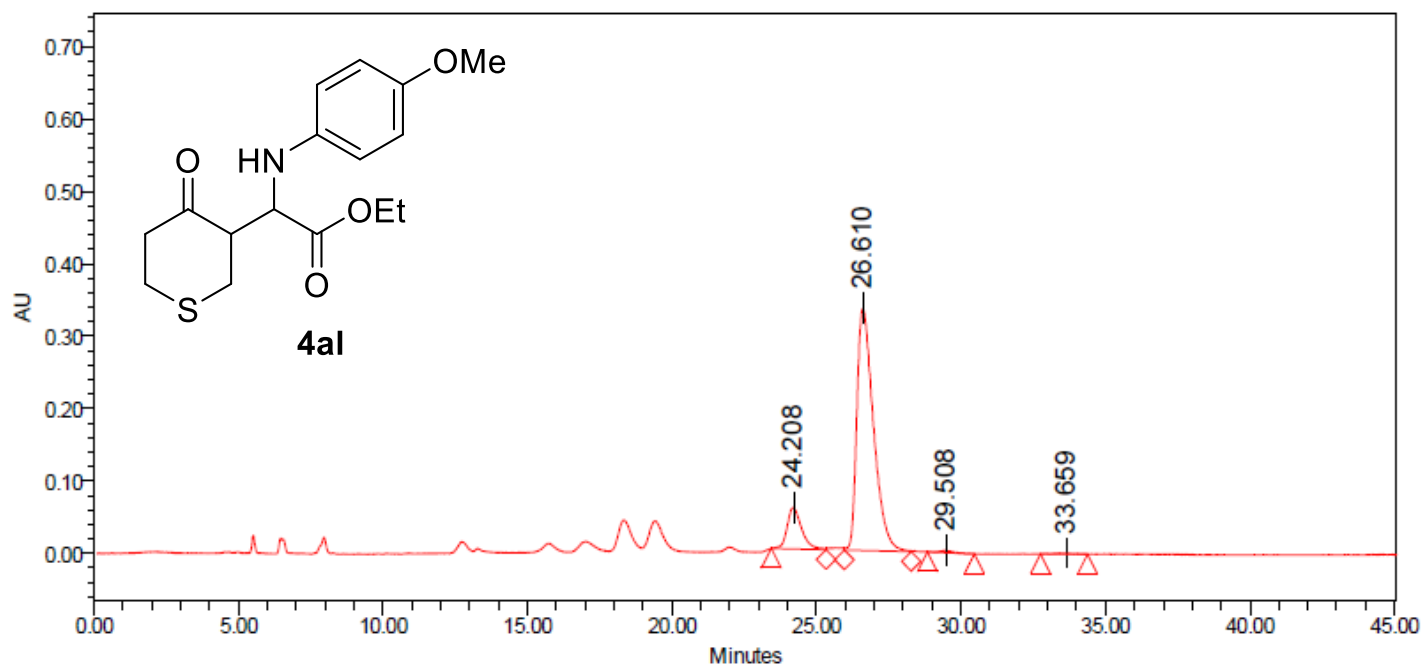

Peak Results

\begin{tabular}{|r|l|c|r|r|r|r|}
\hline & SampleName & RT & $\begin{array}{c}\text { Width } \\
(\mathrm{sec})\end{array}$ & Height & Area & \% Area \\
\hline 1 & $0618-1$ & 24.208 & 113.900 & 57114 & 1975692 & 12.82 \\
\hline 2 & $0618-1$ & 26.610 & 139.100 & 334761 & 13294429 & 86.26 \\
\hline 3 & $0618-1$ & 29.508 & 98.500 & 3302 & 93933 & 0.61 \\
\hline 4 & $0618-1$ & 33.659 & 97.300 & 1342 & 47925 & 0.31 \\
\hline
\end{tabular}

Reported by User. System

Project Name: installation

Report Method: Default Individual Report:

Date Printed:

Report Method ID: 33974

$2021 / 7 / 2$

Page: 1 of 1

14:09:26 PRC 


\begin{tabular}{|llll|}
\hline \multicolumn{2}{c|}{ S A M P L E } & I N F O R M A T I O N \\
\hline \hline Sample Name: & 0628-2-RAC & Acquired By: & System \\
Sample Type: & Unknown & Sample Set Name & \\
Vial: & 11 & Acq. Method Set: & 2695PDA2 \\
Injection \#: & 1 & Processing Method & Default \\
Injection Volume: & $3.00 \mathrm{ul}$ & Channel Name: & 2998 Ch1 230nm@1.2nm \\
Run Time: & 50.0 Minutes & Proc. Chnl. Descr.: 2998 Ch1 230nm@1.2nm \\
& & \\
Date Acquired: & $2021 / 7 / 212: 49: 24$ CST & & \\
Date Processed: & $2021 / 7 / 213: 38: 02$ CST & & \\
\hline
\end{tabular}

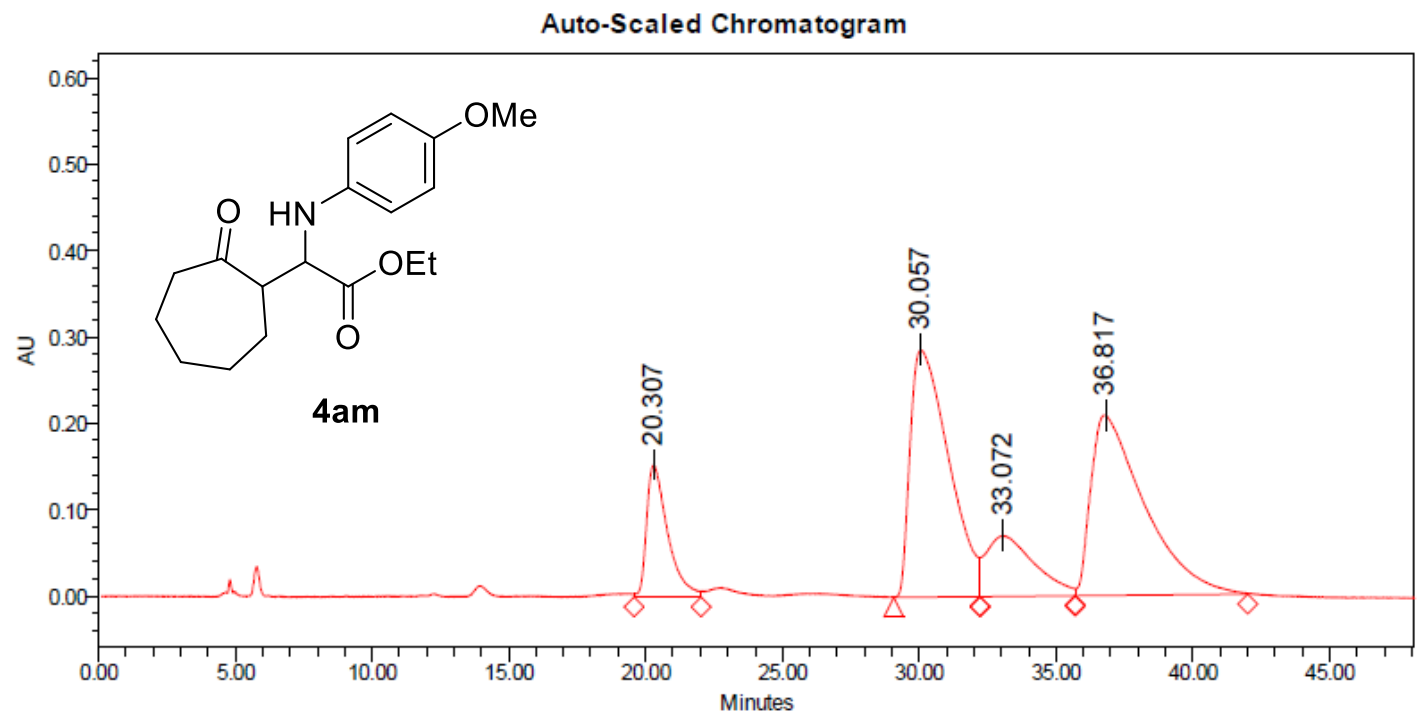

\section{Peak Results}

\begin{tabular}{|r|c|c|c|r|r|r|}
\hline & SampleName & RT & $\begin{array}{c}\text { Width } \\
(\mathrm{sec})\end{array}$ & Height & Area & $\%$ Area \\
\hline 1 & $0628-2-$ RAC & 20.307 & 147.000 & 152694 & 8160859 & 11.12 \\
\hline 2 & $0628-2-\mathrm{RAC}$ & 30.057 & 189.400 & 286397 & 28137313 & 38.33 \\
\hline 3 & $0628-2-\mathrm{RAC}$ & 33.072 & 209.200 & 70675 & 8633803 & 11.76 \\
\hline 4 & $0628-2-\mathrm{RAC}$ & 36.817 & 377.300 & 208846 & 28478320 & 38.79 \\
\hline
\end{tabular}

Reported by User. System

Report Method: Default Individual Report Report Method ID: 33974

Page: 1 of 1
Project Name: installation Date Printed:

2021/7/2

13:38:22 PRC 


\begin{tabular}{|llll|}
\hline & \multicolumn{2}{c|}{ S A M P L E } & I N F O R M A T I O N \\
\hline \hline Sample Name: & $0615-1$ & Acquired By: & System \\
Sample Type: & Unknown & Sample Set Name & \\
Vial: & 12 & Acq. Method Set: & 2695PDA2 \\
Injection \#: & 1 & Processing Method & Default \\
Injection Volume: & $3.00 \mathrm{ul}$ & Channel Name: & 2998 Ch1 230nm@1.2nm \\
Run Time: & 50.0 Minutes & Proc. Chnl. Descr.: & 2998 Ch1 230nm@1.2nm \\
Date Acquired: & $2021 / 7 / 211: 58: 11 \mathrm{CST}$ & & \\
Date Processed: & $2021 / 7 / 213: 34: 50 \mathrm{CST}$ & & \\
\hline
\end{tabular}

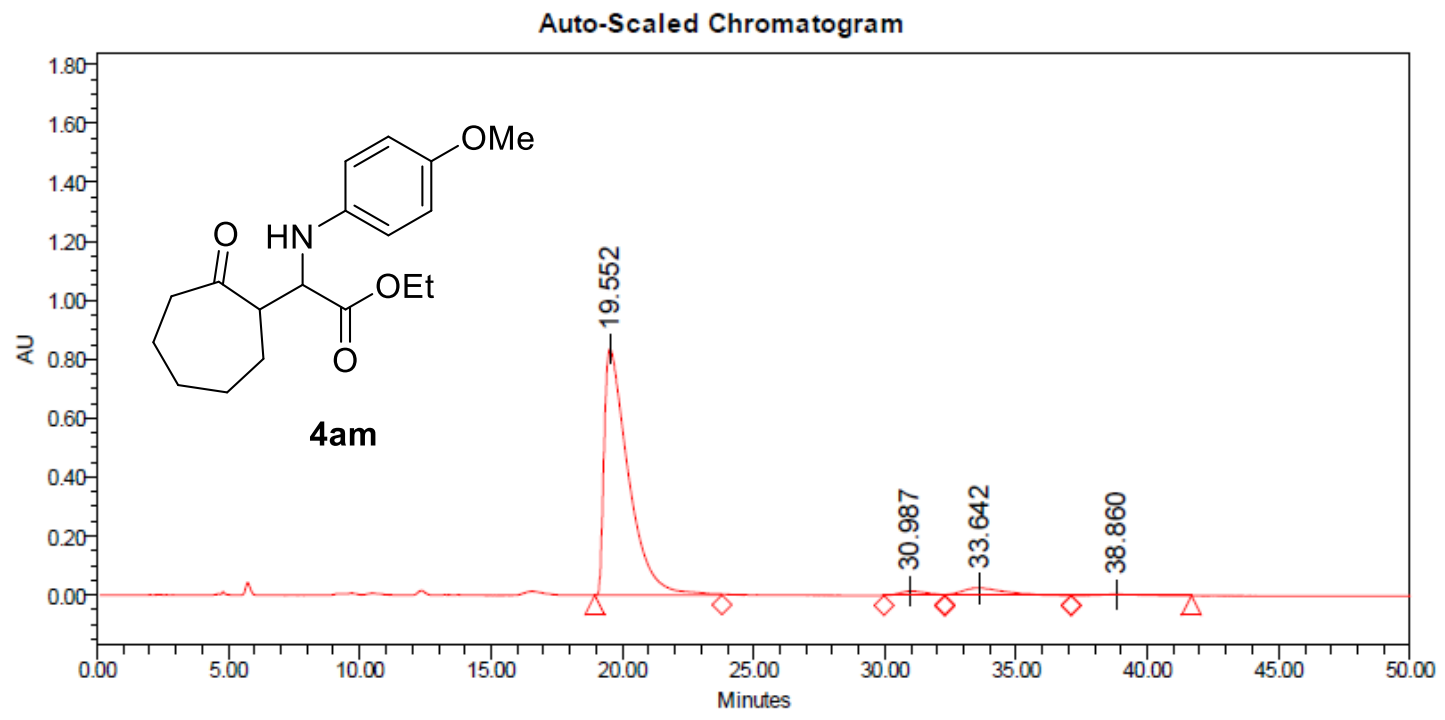

\section{Peak Results}

\begin{tabular}{|r|l|r|r|r|r|r|}
\hline & SampleName & RT & $\begin{array}{c}\text { Width } \\
(\mathrm{sec})\end{array}$ & Height & Area & $\%$ Area \\
\hline 1 & $0615-1$ & 19.552 & 290.800 & 835634 & 53673509 & 91.80 \\
\hline 2 & $0615-1$ & 30.987 & 138.600 & 15725 & 1146501 & 1.96 \\
\hline 3 & $0615-1$ & 33.642 & 289.300 & 26106 & 3121395 & 5.34 \\
\hline 4 & $0615-1$ & 38.860 & 275.200 & 4826 & 526542 & 0.90 \\
\hline
\end{tabular}

Reported by User. System

Report Method: Default Individual Report:

Report Method ID: 33974

Page: 1 of 1
Project Name: installation Date Printed:

2021/7/2

13:38:37 PRC 


\begin{tabular}{|c|c|c|c|}
\hline \multicolumn{2}{|r|}{ SAMPLE } & \multicolumn{2}{|c|}{ INFORMATION } \\
\hline Sample Name: & 0701-1-AD-3--9-1 & Acquired By: & System \\
\hline Sample Type: & Unknown & Sample Set Name & \\
\hline Vial: & 53 & Acq. Method Set: & 2695PDA \\
\hline Injection \#: & 1 & Processing Method & Default \\
\hline Injection Volume: & $1.50 \mathrm{ul}$ & Channel Name: & 2998 Ch3 254nm@1.2nm \\
\hline Run Time: & 50.0 Minutes & Proc. Chnl. Descr.: & 2998 Ch3 254nm@1.2nm \\
\hline Date Acquired: & 2021/7/21 12:43:47 CST & & \\
\hline Date Processed: & $2021 / 7 / 21$ 14:09:41 CST & & \\
\hline
\end{tabular}

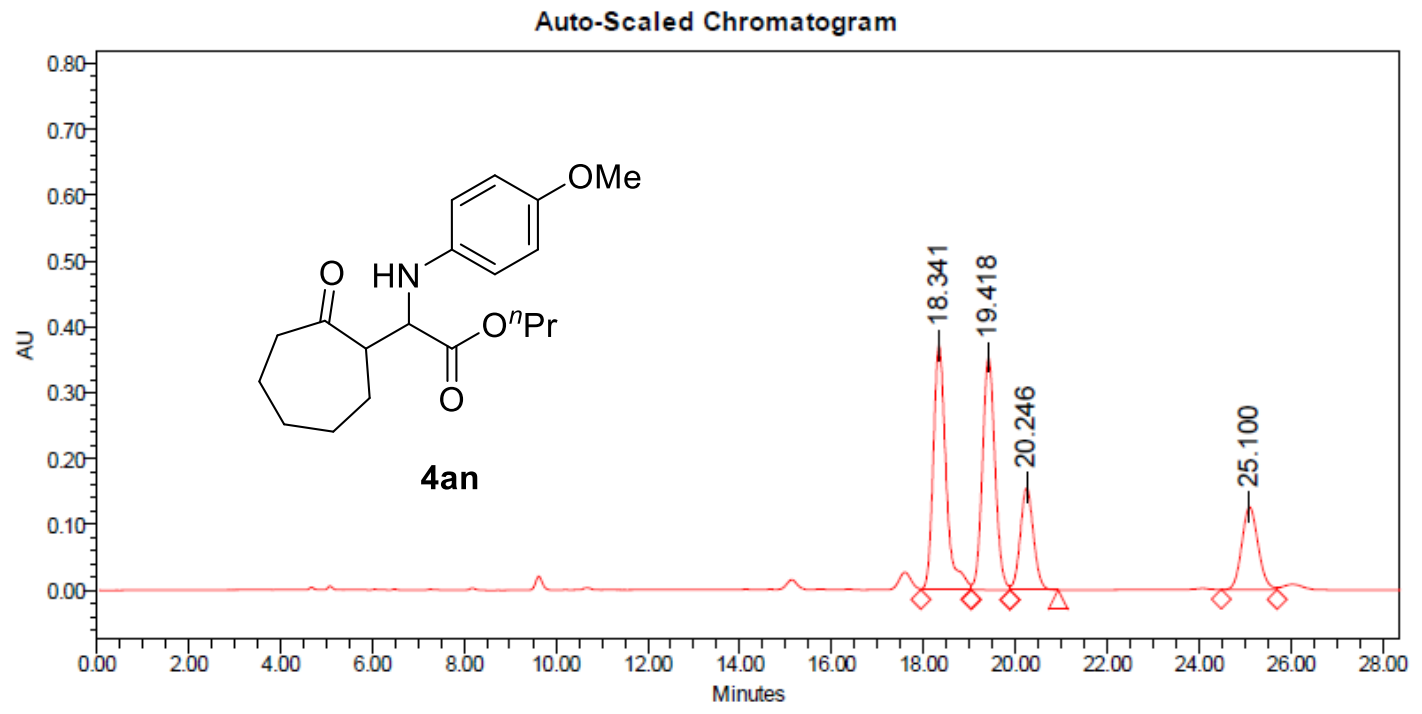

Peak Results
\begin{tabular}{|c|c|c|c|c|c|c|}
\hline & SampleName & RT & $\begin{array}{c}\text { Width } \\
(\mathrm{sec})\end{array}$ & Height & Area & $\%$ Area \\
\hline 1 & $0701-1-$ AD-3--9-1 & 18.341 & 65.800 & 371014 & 7182432 & 35.69 \\
\hline 2 & $0701-1-$ AD-3--9-1 & 19.418 & 50.700 & 352431 & 6743562 & 33.51 \\
\hline 3 & $0701-1-A D-3-9-1$ & 20.246 & 62.500 & 154966 & 3095200 & 15.38 \\
\hline 4 & $0701-1-A D-3-9-1$ & 25.100 & 73.000 & 125316 & 3102715 & 15.42 \\
\hline
\end{tabular}

Reported by User. System

Project Name: installation

Report Method: Default Individual Report:

Date Printed

Report Method ID: 38136

$2021 / 7 / 21$

Page: 1 of 1

14:18:40 PRC 


\begin{tabular}{|llll|}
\hline & \multicolumn{1}{c|}{ S A M P L E } & I N F O R M A T I O N \\
\hline \hline Sample Name: & $0705-1$ & Acquired By: & System \\
Sample Type: & Unknown & Sample Set Name & \\
Vial: & 55 & Acq. Method Set: & 2695PDA \\
Injection \#: & 1 & Processing Method & Default \\
Injection Volume: & $1.50 \mathrm{ul}$ & Channel Name: & 2998 Ch3 254nm@1.2nm \\
Run Time: & 50.0 Minutes & Proc. Chnl. Descr.: 2998 Ch3 254nm@1.2nm \\
& & \\
Date Acquired: & $2021 / 7 / 21$ 13:13:04 CST & & \\
Date Processed: & $2021 / 7 / 2114: 10: 08$ CST & & \\
\hline
\end{tabular}

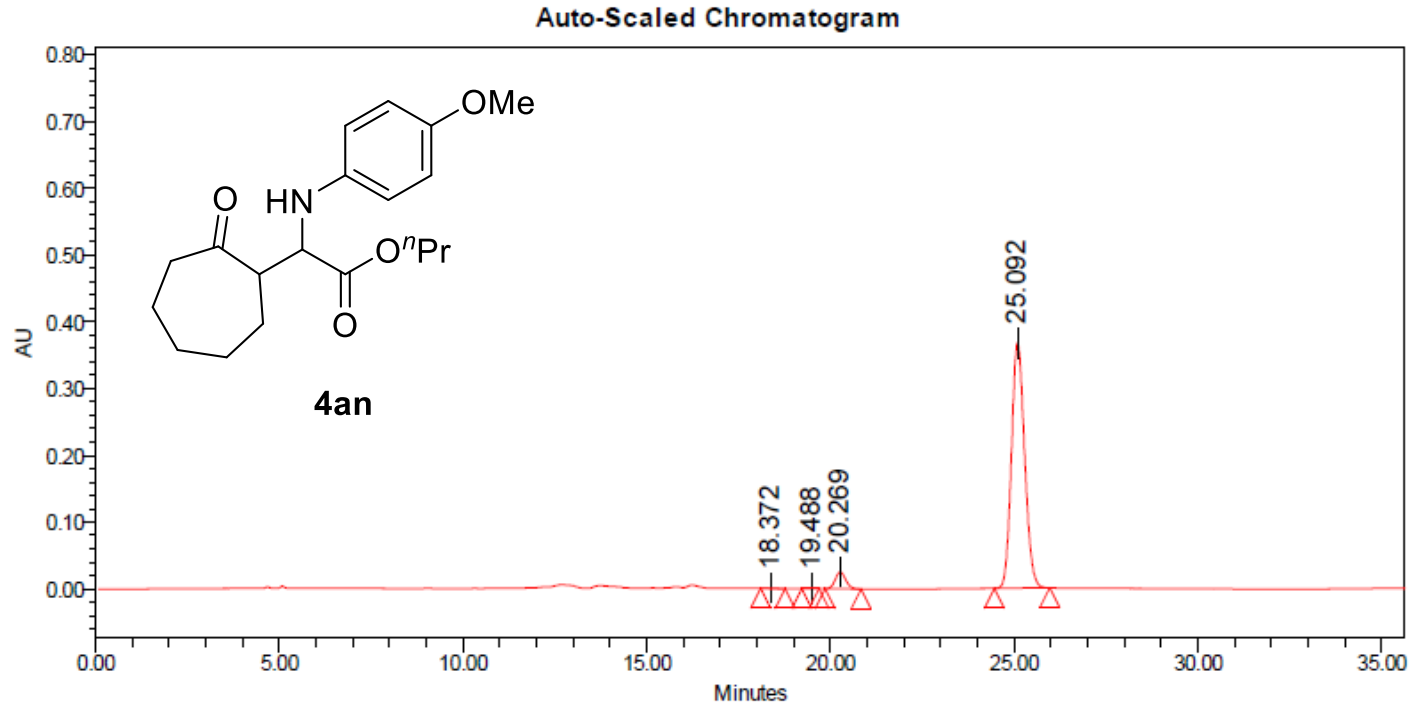

Peak Results

\begin{tabular}{|r|l|c|c|r|r|r|}
\hline & SampleName & RT & $\begin{array}{l}\text { Width } \\
(\mathrm{sec})\end{array}$ & Height & Area & $\%$ Area \\
\hline 1 & $0705-1$ & 18.372 & 40.000 & 464 & 7381 & 0.08 \\
\hline 2 & $0705-1$ & 19.488 & 28.200 & 200 & 2464 & 0.03 \\
\hline 3 & $0705-1$ & 20.269 & 58.500 & 25222 & 495779 & 5.14 \\
\hline 4 & $0705-1$ & 25.092 & 90.400 & 367278 & 9135876 & 94.76 \\
\hline
\end{tabular}

Reported by User. System

Report Method: Default Individual Report:

Report Method ID: 38136

Page: 1 of 1
Project Name: installation Date Printed:

2021/7/21

14:18:57 PRC 


\section{SAMPLE INFORMATION}

\begin{tabular}{|c|c|c|c|}
\hline Sample Name: & 0701-2-RAC & Acquired By: & System \\
\hline Sample Type: & Unknown & Sample Set Name & \\
\hline Vial: & 54 & Acq. Method Set: & 2695PDA \\
\hline Injection \#: & 2 & Processing Method & Default \\
\hline Injection Volume: & $1.00 \mathrm{ul}$ & Channel Name: & 2998 Ch3 254nm@1.2nm \\
\hline Run Time: & 50.0 Minutes & Proc. Chnl. Descr.: & 2998 Ch3 254nm@1.2nm \\
\hline
\end{tabular}

Date Acquired: $\quad$ 2021/7/21 14:28:56 CST

Date Processed: $\quad$ 2021/7/21 15:54:53 CST

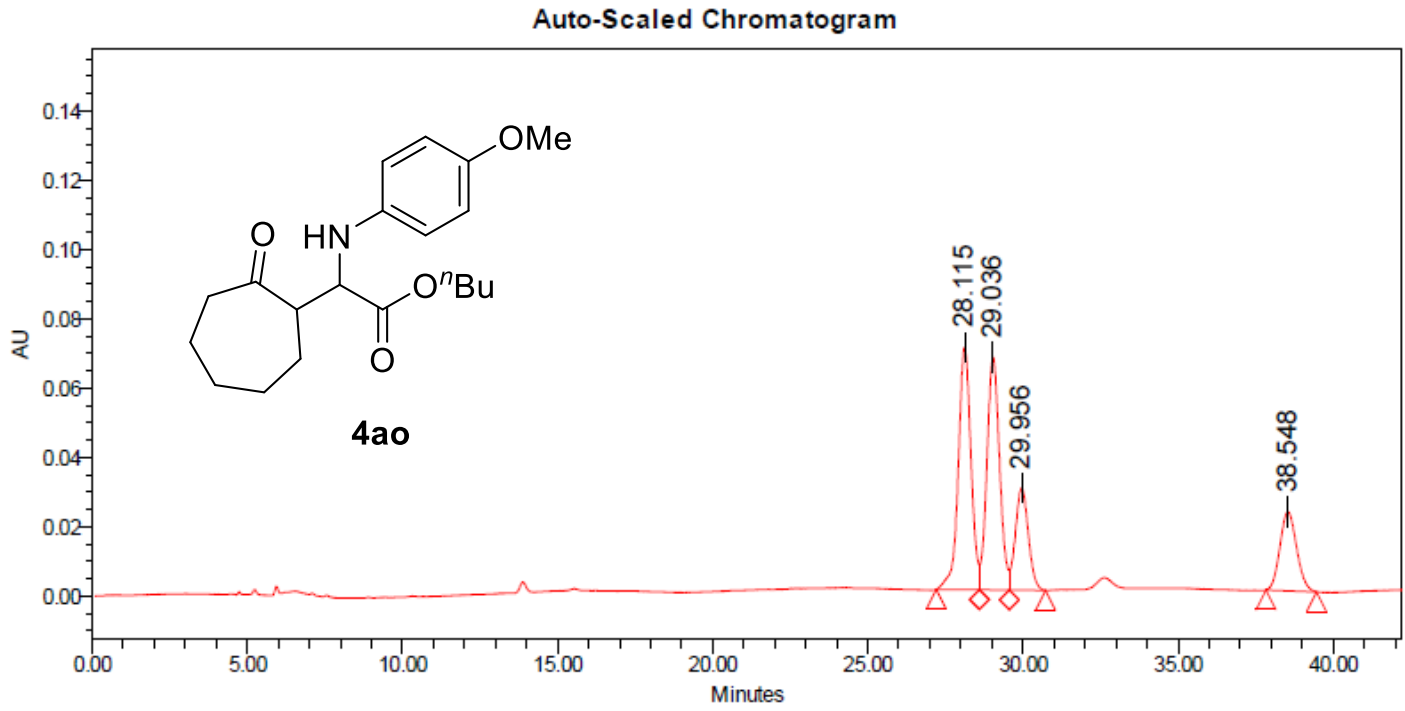

Peak Results

\begin{tabular}{|c|c|c|c|c|c|c|}
\hline & SampleName & RT & $\begin{array}{c}\text { Width } \\
(\mathrm{sec})\end{array}$ & Height & Area & \% Area \\
\hline 1 & $0701-2-\mathrm{RAC}$ & 28.115 & 83.600 & 69934 & 1983325 & 35.45 \\
\hline 2 & $0701-2-\mathrm{RAC}$ & 29.036 & 57.500 & 67064 & 1898793 & 33.94 \\
\hline 3 & $0701-2-\mathrm{RAC}$ & 29.956 & 70.200 & 29482 & 859790 & 15.37 \\
\hline 4 & $0701-2-\mathrm{RAC}$ & 38.548 & 98.100 & 22799 & 853294 & 15.25 \\
\hline
\end{tabular}

Reported by User. System

Report Method: Default Individual Report:

Report Method ID: 38136

Page: 1 of 1
Project Name: installation Date Printed:

$2021 / 7 / 21$

15:56:40 PRC 


\begin{tabular}{|llll|}
\hline & \multicolumn{1}{c|}{ S A M P L E } & I N F O R M A T I O N \\
\hline \hline Sample Name: & $0705-2$ & Acquired By: & System \\
Sample Type: & Unknown & Sample Set Name & \\
Vial: & 56 & Acq. Method Set: & 2695PDA \\
Injection \#: & 1 & Processing Method & Default \\
Injection Volume: $1.00 \mathrm{ul}$ & Channel Name: & 2998 Ch3 254nm@1.2nm \\
Run Time: & 50.0 Minutes & Proc. Chnl. Descr.: 2998 Ch3 254nm@1.2nm \\
& & \\
Date Acquired: & $2021 / 7 / 21$ 15:12:17 CST & & \\
Date Processed: & $2021 / 7 / 2115: 56: 23$ CST & & \\
\hline
\end{tabular}

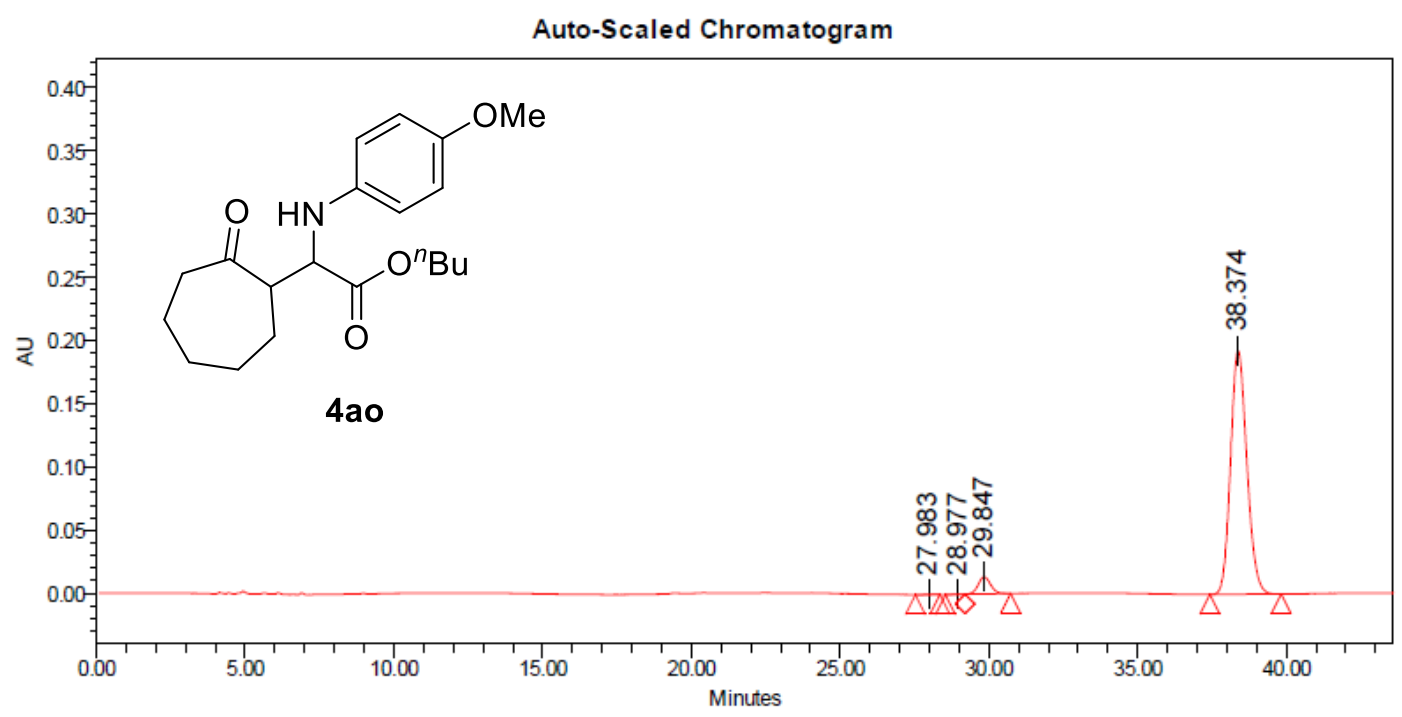

Peak Results
\begin{tabular}{|r|l|c|r|r|r|r|}
\hline & SampleName & RT & $\begin{array}{c}\text { Width } \\
(\mathrm{sec})\end{array}$ & Height & Area & $\%$ Area \\
\hline 1 & $0705-2$ & 27.983 & 49.300 & 413 & 8050 & 0.10 \\
\hline 2 & $0705-2$ & 28.977 & 39.300 & 326 & 7561 & 0.10 \\
\hline 3 & $0705-2$ & 29.847 & 92.400 & 13495 & 395766 & 5.10 \\
\hline 4 & $0705-2$ & 38.374 & 144.200 & 192784 & 7350790 & 94.70 \\
\hline
\end{tabular}

Reported by User. System

Report Method: Default Individual Report:

Report Method ID: 38136

Page: 1 of 1
Project Name: installation Date Printed

2021/7/21

15:56:54 PRC 